Eva Maria Lohner

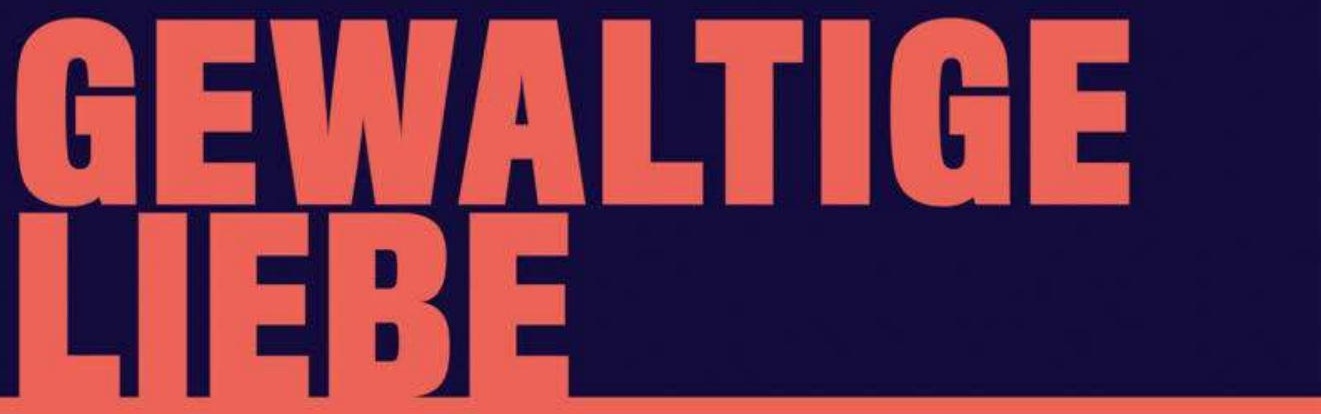

Praktiken und Handlungsorientierungen junger Frauen in gewaltgeprägten Paarbeziehungen

[transcript] Pädagogik 
Eva Maria Lohner

Gewaltige Liebe

Pädagogik 
Eva Maria Lohner (Dipl.-Päd.) ist wissenschaftliche Mitarbeiterin am Institut für Erziehungswissenschaft der Universität Tübingen. Ihre Arbeitsschwerpunkte sind Sozialpädagogische Beratung, Qualitative Forschung, Biografieforschung sowie das Theorie-Praxis-Verhältnis. 
Eva Maria LoHner

\section{Gewaltige Liebe}

Praktiken und Handlungsorientierungen junger Frauen

in gewaltgeprägten Paarbeziehungen

[transcript] 
Diese Arbeit wurde unter dem Titel »Gewaltige Liebe - Eine empirische Studie zur Rekonstruktion von Praktiken und Handlungsorientierungen jugendlicher und junger Frauen in gewaltgeprägten Paarbeziehungen « von der Universität Tübingen 2018 als Dissertation angenommen.

\section{Bibliografische Information der Deutschen Nationalbibliothek}

Die Deutsche Nationalbibliothek verzeichnet diese Publikation in der Deutschen Nationalbibliografie; detaillierte bibliografische Daten sind im Internet über http://dnb.d-nb.de abrufbar.

\section{(c) $(1) \Theta$}

Dieses Werk ist lizenziert unter der Creative Commons Attribution-NonCommercial-NoDerivs 4.o Lizenz (BY-NC-ND). Diese Lizenz erlaubt die private Nutzung, gestattet aber keine Bearbeitung und keine kommerzielle Nutzung. Weitere Informationen finden Sie unter https://creativecommons.org/licenses/by-nc-nd/4.o/deed.de Um Genehmigungen für Adaptionen, Übersetzungen, Derivate oder Wiederverwendung zu kommerziellen Zwecken einzuholen, wenden Sie sich bitte an rights@ transcript-verlag.de

Die Bedingungen der Creative-Commons-Lizenz gelten nur für Originalmaterial. Die Wiederverwendung von Material aus anderen Quellen (gekennzeichnet mit Quellenangabe) wie z.B. Schaubilder, Abbildungen, Fotos und Textauszüge erfordert ggf. weitere Nutzungsgenehmigungen durch den jeweiligen Rechteinhaber.

\section{(C) 2019 transcript Verlag, Bielefeld}

Umschlaggestaltung: Maria Arndt, Bielefeld

Lektorat: Eva Maria Lohner

Korrektorat: Anja Lochner

Druck: Majuskel Medienproduktion GmbH, Wetzlar

Print-ISBN 978-3-8376-4800-3

PDF-ISBN 978-3-8394-4800-7

https://doi.org/10.14361/9783839448007

Gedruckt auf alterungsbeständigem Papier mit chlorfrei gebleichtem Zellstoff. Besuchen Sie uns im Internet: https://www.transcript-verlag.de

Unsere aktuelle Vorschau finden Sie unter www.transcript-verlag.de/vorschau-download 


\section{Inhalt}

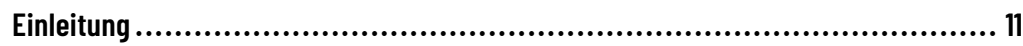

2 Paarbeziehung im gesellschaftlichen Wandel ............................................17

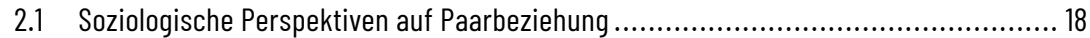

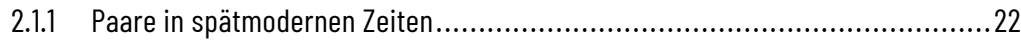

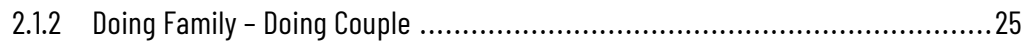

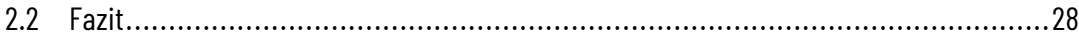

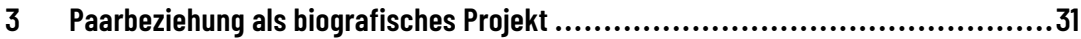

3.1 Biografie als wissenschaftliches Konzept........................................................ 32

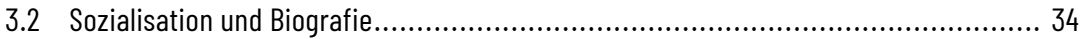

3.3 Subjekttheoretische Perspektiven in der Biografieforschung .................................37

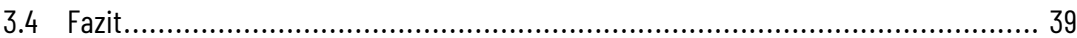

4 Gewalt in Paarbeziehungen Jugendlicher und junger Erwachsener ....................43

4.1 Liebesbeziehungen im Jugend- und jungen Erwachsenenalter ..................................45

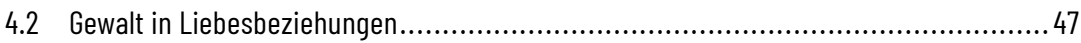

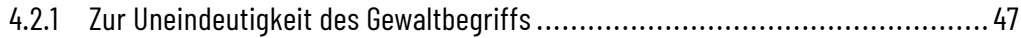

4.2.2 Definition und Formen von Gewalt in jungen Paarbeziehungen ........................50

4.2.3 Zur Entstehung von Gewalt in Paarbeziehungen .......................................52

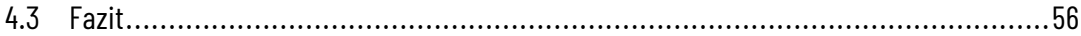

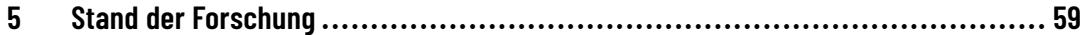

5.1 Forschungsergebnisse zu Beziehungskonzepten und Lebensthemen junger Frauen .....60

5.2 Forschungsstand zu Gewalt in den ersten eigenen Paarbeziehungen..........................62

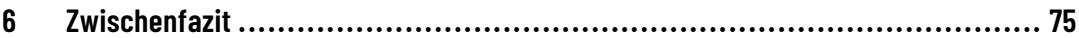

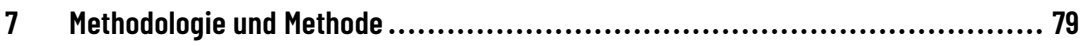

7.1 Qualitatives Forschungsdesign und dokumentarische Methode................................79 
7.1.1 Immanenter und dokumentarischer Sinngehalt - Konjunktive und kommunikative Erfahrung ................................................................. 81

7.1.2 Orientierungsmuster: Orientierungsschemata und Orientierungsrahmen .84

7.2 Methodische und methodologische Überlegungen zur empirischen Erhebung von

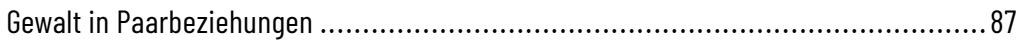

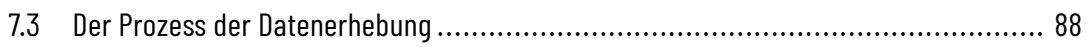

7.3.1 Das autobiografisch-narrative Interview........................................... 88

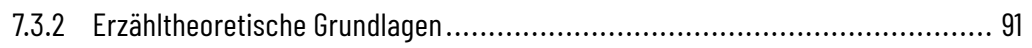

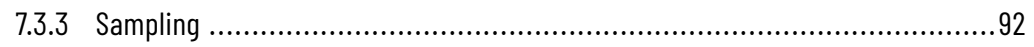

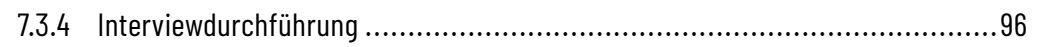

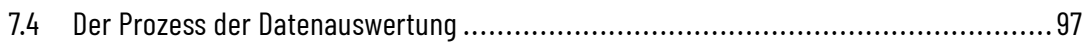

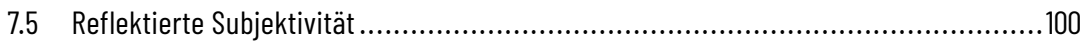

7.6 Grenzen und Chancen der narrativen Gesprächsführung bei Menschen mit Traumatisierungen und Umgang mit Traumatisierungen im narrativen Interview ................................................... 102

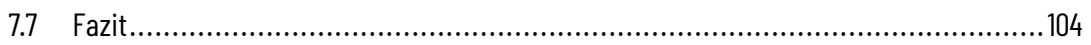

8 Ergebnisse der empirischen Untersuchung................................... 107

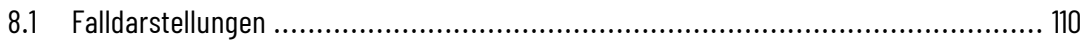

8.1.1 Anna - »immer wieder die gleichen Männer immer wieder [...] das gleiche Schema« (169f.) ........................................................ 110

8.1.1.1 Fallporträt ...................................................... 110

8.1.1.2 Analyse der Erzählstruktur ......................................... 112

8.1.1.3 Herkunftsfamilie und Bedingungen des Aufwachsens ......... 113

8.1.1.4 Kernthemen des Interviews...................................... 116

8.1.1.5 Argumentative Stellungnahmen ................................... 137

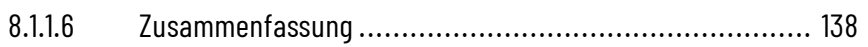

8.1.2 Amy - »es hat ja alles immer so $n$ Kreis so $n$ Lebenskreis « (293f.) ................. 140

8.1.2.1 Fallporträt ....................................................... 140

8.1.2.2 Analyse der Erzählstruktur ........................................... 142

8.1.2.3 Herkunftsfamilie und Bedingungen des Aufwachsens ........ 143

8.1.2.4 Kernthemen des Interviews....................................... 147

8.1.2.5 Argumentative Stellungnahmen .....................................158

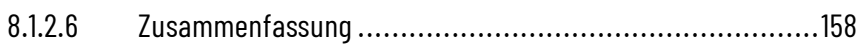

8.1.3 Zofia - »irgendwann ging mir das: [...] soweit dass ich dann wirklich auch für mich entschieden hab [...] und jetzt wirklich geh« (114-116) ................... 160

8.1.3.1 Fallporträt ......................................................... 160

8.1.3.2 Analyse der Erzählstruktur ........................................ 162

8.1.3.3 Herkunftsfamilie und Bedingungen des Aufwachsens ........ 163

8.1.3.4 Kernthemen des Interviews....................................... 166 
8.1.3.5 Argumentative Stellungnahmen ................................. 177

8.1.3.6 Zusammenfassung ................................................... 180

8.1.4 Daria - »wenn er handgreiflich wird geht's schon mal gar nich « (587f.)......... 183

8.1.4.1 Fallporträt ................................................ 183

8.1.4.2 Analyse der Erzählstruktur ......................................... 184

8.1.4.3 Herkunftsfamilie und Bedingungen des Aufwachsens .........186

8.1.4.4 Kernthemen des Interviews........................................ 191

8.1.4.5 Argumentative Stellungnahme ...................................201

8.1.4.6 Zusammenfassung ................................................ 202

8.1.5 Alina - »ich hab immer gedacht ja okay, er liebt mich « (61) ...................... 203

8.1.5.1 Fallporträt ................................................... 203

8.1.5.2 Analyse der Erzählstruktur...................................... 204

8.1.5.3 Herkunftsfamilie und Bedingungen des Aufwachsens ........ 205

8.1.5.4 Kernthemen des Interviews..................................... 207

8.1.5.5 Argumentative Stellungnahme ................................ 216

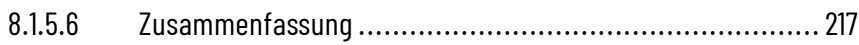

8.1.6 Jasmin - » wenn's dann nich gepasst hat hab ich Schläge gekriegt und des war eigentlich jeden Tag so « (312f.) ................................................... 220

8.1.6.1 Fallporträt ................................................... 220

8.1.6.2 Analyse der Erzählstruktur ........................................... 221

8.1.6.3 Herkunftsfamilie und Bedingungen des Aufwachsens ....... 222

8.1.6.4 Kernthemen des Interviews....................................... 225

8.1.6.5 Argumentative Stellungnahme ................................. 235

8.1.6.6 Zusammenfassung .................................................. 236

8.2 Typengenese: Praktiken und Handlungsorientierungen im Umgang mit Gewalt.......... 238

8.2.1 Der Typus »Pertinenz « - »die Liebe, die ich immer gesucht habe« .............. 240

8.2.2 Der Typus »Autonomie« - »sich allein als wertvoll erfahren ohne die Bestätigung des Partners «

8.2.3 Der Typus "Abhängigkeit « - »und wenns dann nicht gepasst hat hab ich Schläge gekriegt «.............................................................. 245

9 Diskurstheoretische Betrachtungen ......................................... 249

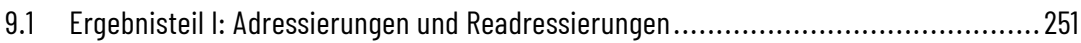

9.1.1 Exkurs zur Subjekttheorie Judith Butlers ...............................................251

9.1 .2 Adressierung ...................................................................... 253

9.2 Ergebnisteil II:

Gewalt zwischen Wandel und Persistenz: Widerstandsstrategien und Ansatzpunkte für ein reformuliertes Gewaltverständnis

9.2.1 Beziehungsgestaltung als biografisches Projekt: die Konzepte des biografischen Eigensinns und der Biografizität. 260

9.2.2 Wandel und Persistenz von Gewalt.... 
9.3 Anschlusspunkte für sozialpädagogische Unterstützung und Ausblick

10 Literatur ................................................................. 273

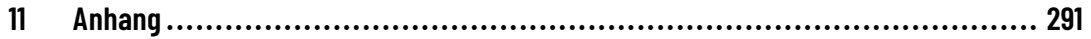

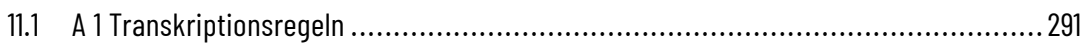

11.2 A 2 Anschreiben im Rahmen der Datenerhebung........................................... 293

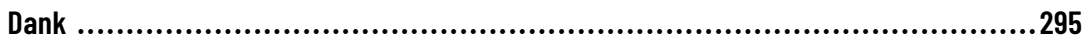


»Mein Gott ja okay da war's halt nur mal an die Wand gedrückt und mal ne Faust ... aber da denk ich auch so okay wenn's jetzt zweimal vor kam jetzt auch noch 'n drittes Mal vorkommt ... ja am Anfang war's echt okay aber dann am Ende hatt' ich einfach kein' Bock mehr"

(Daria, 18 Jahre) 



\section{Einleitung}

Zur Aktualität des Themas und zur Genese der Fragestellung Im Rahmen meiner Tätigkeit als Vorstandsmitglied eines kleinen freien Jugendhilfeträgers begleitete ich unter wissenschaftlicher Perspektive von 2008 bis 2010 ein EU-Projekt zur »Prävention von Gewalt in intimen Teenagerbeziehungen " (Köberlein u.a. 2010). In diesem Rahmen setzte ich mich mit Fragen zur Situation Jugendlicher in Deutschland und Europa auseinander, die von Gewalt innerhalb ihrer Beziehung betroffen sind. Bei der Literaturrecherche zeigte sich, dass sowohl im deutschsprachigen als auch im europäischen Raum kaum thematisch einschlägige Literatur und Forschungsergebnisse vorzufinden waren. Einzig die Studien der Forschungsgruppe um Barbara Krahé (Krahé u.a. 1999, Krahé/Scheinberger-Olwig 2002) behandelten unter dem Fokus der sexuellen Aggression das Thema. Inzwischen liegen einige weitere deutsche (Blättner u.a. 2016; Maschke/Stecher 2017) und europäische (Barter 2009; Ministère de la Communauté française 2009; Averdijk u.a. 2011) Studien zur Häufigkeit von Gewalt in jugendlichen Paarbeziehungen vor. Dagegen befasst sich die US-amerikanische Forschungsliteratur seit über zwanzig Jahren unter dem Begriff »Dating Violence« oder »Teen Dating Violence« mit der Thematik (z.B. Kann u.a. 2014; Liz Claiborne Inc. 2008; Davies 2008; Glass u.a. 2008), und es liegen eine Vielzahl an praktischen Ratgebern und allgemeiner Literatur zum Thema vor (z.B. Pirog-Good/Stets 1998; Levy 1990; Levy 2006; Levy/Occhiuzzo Giggans 1995). Allerdings ist die Übertragbarkeit der Ergebnisse aus US-amerikanischen Studien aufgrund kultureller Unterschiede problematisch.

Im Unterschied zum gut beforschten Thema »Häusliche Gewalt«, das seit der Frauenbewegung in den 1970er-Jahren zunehmend sowohl in der öffentlichen Wahrnehmung und Diskussion als auch in Wissenschaft und Forschung eine breite Berücksichtigung fand, ist die Tatsache, dass auch Jugendliche bereits Gewalt bei Dates oder in den ersten eigenen Paarbeziehungen erleben können, bislang kaum in den Blick genommen worden.

Die vorliegenden Untersuchungen zur Frage nach Gewalterfahrungen in den ersten eigenen Paarbeziehungen sind fast ausschließlich quantitativ ausgerichtet. Im Unterschied dazu liegt dieser Studie ein qualitatives Forschungsdesign zugrunde. Im Gegensatz zu Fragen nach Prävalenz und Formen der erlebten Gewalt 
werden hier besonders die »reflexiven, gendergeprägten und situierten Aspekte menschlicher Erfahrung « (Denzin 2013, S. 144) fokussiert. Ziel ist es, die Subjektperspektive in den Blick zu nehmen und weibliche Jugendliche und junge Frauen nach ihren Erfahrungen zu befragen. Und weil sich Erfahrungen nicht direkt und unmittelbar erforschen lassen, nimmt die Studie lebensgeschichtliche Erzählungen junger Frauen in den Blick, da sich in ihnen Alltagserfahrungen in Form von narrativen Repräsentationen zeigen (vgl. ebd., S. 146).

Die zentrale Fragestellung der vorliegenden Arbeit lautet daher:

Welche Praktiken und Handlungsorientierungen zeigen junge Frauen im Umgang mit Gewalt während der ersten eigenen Paarbeziehung(en)?

Damit verbunden sind folgende Fragen:

Wie erleben junge Frauen Gewalt in den ersten eigenen Paarbeziehungen?

Wie wird Paarbeziehung konstruiert? Welche Vorstellungen formulieren die jungen Frauen über das partnerschaftliche Zusammenleben und wie gestalten sie angesichts dieser gewaltvollen Erfahrungen ihre Beziehungen?

Welche Rolle spielen Cewalterfahrungen in der Herkunftsfamilie? Wie prägen diese Gewalterfahrungen die Beziehungsvorstellungen?

Welche Ressourcen stehen den jungen Frauen zur Verfügung?

Ziel der Arbeit ist es, diese Fragen aus der Perspektive der Subjekte zu beantworten und so zu einem vollständigeren Bild des Erlebens von Gewalt in den ersten eigenen Paarbeziehungen, dem Umgang damit und den daraus resultierenden $\mathrm{Be}$ ziehungskonzepten zu gelangen.

In die Erhebung wurden Frauen einbezogen, die über ihre Erfahrungen in hetero- und homosexuellen Beziehungen berichten. Die Entscheidung dafür war auch forschungspragmatisch begründet, da eine Ausdehnung der Befragung auf junge Männer den Rahmen der Forschungsarbeit gesprengt hätte.

Die Studie verfolgt zwei theoretische Zugänge: Zum einen richtet sie den Blick auf (veränderte) gesellschaftliche Bedingungen und analysiert, wie sich diese heute auf Beziehungen auswirken. Dabei wird die Auseinandersetzung mit den gesellschaftlichen Veränderungen im Zuge des sozialen Wandels insbesondere unter individualisierungstheoretischer Perspektive betrachtet. Das bedeutet, dass Widersprüche in den Planungs- und Orientierungsentwürfen und somit in den Beziehungskonzepten auch als Symptom gesellschaftlicher Individualisierung angesehen werden müssen.

Interaktionshandeln und Bewältigungshandeln sind aber auch immer gesamtbiografisch ausgebildet und auf den in der Sozialisation etablierten Handlungsmustern aufgebaut. Somit reproduzieren sich biografische Bewältigungsmechanismen oder Muster biografischer Arbeit in Paarbeziehungen. Deshalb rekurriert die vorliegende Arbeit zum anderen auf biografietheoretische Perspektiven. Die 
Frage, inwiefern familiäre Einflüsse und Sozialisationsbedingungen spätere Erfahrungen in den eigenen Paarbeziehungen beeinflussen, erfordert es, sich mit dem Zusammenhang von Biografie und Sozialisation zu beschäftigen.

Mit den Fragen nach Handlungsmöglichkeiten von Frauen in Gewaltbeziehungen sowie nach der möglichen Reproduktion und Transformation von Verhaltensweisen und sozialen Verhältnissen werden des Weiteren Fragen zu Subjektivität aufgeworfen. In Anlehnung an aktuelle Diskussionen um eine Neuthematisierung des subjekttheoretischen Verständnisses innerhalb der Biografieforschung wird der biografietheoretische Zugang um eine subjekttheoretische Perspektive ergänzt.

Für das vorliegende Thema der Gewalt in den ersten eigenen Paarbeziehungen gilt es außerdem - im Unterschied und in Abgrenzung zu häuslicher Gewalt - zu berücksichtigen, dass die Aufnahme von Paarbeziehungen in die vulnerable Phase der Jugend und Adoleszenz fällt, die besondere Herausforderungen an Jugendliche und junge Erwachsene stellt.

Im Fokus des empirischen Vorgehens stehen sechs biografisch-narrative Interviews mit jungen Frauen im Alter zwischen 18 und 27 Jahren. Diese lebensgeschichtlichen Erzählungen wurden in der vorliegenden Studie mit der dokumentarischen Methode analysiert. Eine Besonderheit liegt dabei darin, dass die Arbeit in einem ersten Schritt eine individuelle, familienspezifische und damit biografisch ausgerichtete Sicht sowohl auf die Genese von Beziehungsorientierungen als auch auf die Handlungspraxis der Beziehungsgestaltung einnimmt. Im zweiten Schritt fokussiert sie kollektive Alltagsorientierungen der beforschten Akteurinnen als Ausdruck gesellschaftlicher Prägung. Jenseits des subjektiv gemeinten Sinns rücken hier sozial geprägte Muster und Sinnstrukturen in den Blick, die das Wissen um das Zusammenwirken von gesellschaftlichen Strukturen und individuellen Handlungen erweitern. So konstituieren sich etwa geschlechtstypische Erfahrungsräume »über die Kombination der Handlungs- bzw. Interaktionspraxis geschlechtsspezifischer Sozialisation und das Erleben geschlechtstypischer (Fremd-)Zuschreibungen und Interpretationen « (Przyborski/Slunecko 2010, S. 63). Die Rekonstruktion dieses Erfahrungswissens, das sich in der Alltagspraxis niederschlägt, steht im Mittelpunkt der Arbeit und zielt auf die Generierung einer Typologie, die die Variationsbreite der Orientierungen der befragten Akteurinnen aufzeigt.

Dabei wird im Laufe der rekonstruktiven Analyse der Blick insbesondere auch auf widerständiges und eigensinniges Verhalten der jungen Frauen gerichtet, da davon ausgegangen wird, dass »alltäglichen Widerstandspotentialen« (Dausien 1994, S. 141) »transformatorisches Potential« (Thon 2016, S. 187) innewohnen kann. Mit diesem Fokus wird eine erweiterte Perspektive auf Gewalt eingenommen, die Frauen nicht nur bzw. nicht ausschließlich als Opfer von Gewalt versteht. 
Biografische Konstruktionen werden dabei als veränderbar, nie abgeschlossen und nicht linear verstanden. Das bedeutet auch, dass am Ende der Adoleszenz kein fertiges Endprodukt eines Lebens- oder Beziehungsentwurfs vorliegt, an dem sich junge Frauen orientieren (vgl. Keddi 2003, S. 118). Stattdessen ist davon auszugehen, dass junge Frauen in unterschiedlichen Situationen, Zusammenhängen und Lebensphasen verschiedene, möglicherweise sich widersprechende Projekte verfolgen (vgl. ebd., S. 119). Damit wird deutlich, dass in der vorliegenden Arbeit immer nur ein biografischer Ausschnitt wiedergegeben werden kann, der immer abhängig von den retrospektiven und prospektiven Elementen der Biografin ist und der nicht für die gesamte Biografie stehen kann.

Aufbau der Arbeit Die vorliegende Studie gliedert sich in drei Teile: den theoretischen Zugang mit den notwendigen Begriffsbestimmungen, dem Überblick zum aktuellen Stand der Forschung sowie einem Zwischenfazit (Kapitel 2 bis 6), die Darstellung des Forschungszugangs und der methodischen Herangehensweise sowie die Darstellung der empirischen Ergebnisse (Kapitel 7 und 8) und das Fazit der Arbeit, das die Ergebnisse verdichtet und an theoretische Analysen rückbindet sowie Anschlusspunkte für Soziale Arbeit diskutiert (Kapitel 9).

Das zweite Kapitel nimmt eine gesellschaftstheoretische Perspektive auf Paarbeziehungen ein, um gesellschaftliche Bedingungen, insbesondere veränderte Anforderungen an Paarbeziehungen in modernen Gesellschaften, für den Lebensweg einzelner Akteurinnen in den Blick zu nehmen.

Mit der Frage, wie mit diesen Anforderungen individuell umgegangen wird, beschäftigt sich das dritte Kapitel. Mit einem biografietheoretischen Zugang werden sozialisationstheoretische Fragen unter subjekttheoretischer Perspektive gestellt. So wird die Sozialisationsgeschichte als Hintergrundfolie verstanden, auf der die Genese individueller Orientierungsrahmen analysiert werden kann.

In Kapitel vier werden zunächst verschiedene Begrifflichkeiten erörtert und geklärt, die für die vorliegende Arbeit Relevanz besitzen. Dies sind: die Lebensphasen Jugend und junges Erwachsenenalter sowie die Herausforderungen in Liebesbeziehungen während dieser Zeit; die Problematik des Gewaltbegriffs sowie Definitionen und Formen zu Gewalt in jugendlichen Paarbeziehungen und zuletzt die Frage nach dem Zusammenhang von Gewalt und Geschlecht.

Kapitel fünf gibt einen Überblick zum bescheidenen Stand der Forschung auf internationaler und europäischer Ebene, für Deutschland zu den Themenkomplexen der Prävalenz von Gewalt in jungen Paarbeziehungen sowie zu Risikofaktoren, Unterstützung und Prävention. An diese Übersicht schließt sich die Begründung der vorliegenden Fragestellung im Kontext eines qualitativen Forschungsdesigns an. Kapitel sechs schließt die theoretische Rahmung des Forschungsthemas mit einem Zwischenfazit ab. 
Im siebten Kapitel werden der Forschungszugang, die methodologischen Grundüberlegungen sowie die methodische Herangehensweise dargelegt. Im Fokus stehen dabei biografisch-narrative Interviews mit jungen Frauen, die mithilfe der dokumentarischen Methode ausgewertet wurden. Im Zuge des Forschungsvorhabens hat sich gezeigt, dass die Erläuterung und Begründung des qualitativen Zugangs und der Erhebungs- und Auswertungsmethode zwar aus handwerklich-theoretischen Gründen geboten bleibt. In theoretischer wie forschungsmethodischer Hinsicht stellen jedoch Überlegungen $\mathrm{zu}$ Grenzen und Chancen der narrativen Gesprächsführung bei Menschen mit Traumatisierungen sowie der Umgang mit Traumatisierungen im narrativen Interview einen entscheidenden Schwerpunkt dieses Kapitels dar. Die Notwendigkeit der Beschäftigung mit diesen Fragen hatte sich im Laufe des Forschungsprozesses immer mehr herauskristallisiert und führte $\mathrm{zu}$ der Erkenntnis, dass entsprechendes Wissen sowohl in theoretischer als auch methodologischer Hinsicht unabdingbar ist, um einerseits - auch aus forschungsethischer Sicht - in Forschungen zu Gewalt nicht (erneut) gesellschaftliche und soziale Tabuisierungsprozesse mit aufrechtzuerhalten und andererseits adäquat mit dem empirischen Material umgehen zu können.

Das achte Kapitel beinhaltet die Ergebnisse der empirischen Untersuchung und bildet das Kernstück der Arbeit. Hier wird zunächst anhand sechs ausführlicher Falldarstellungen die Rekonstruktion unterschiedlicher Praktiken und Handlungsorientierungen junger Frauen im Umgang mit Gewalt innerhalb ihrer ersten Paarbeziehungen aufgezeigt. Im Anschluss daran werden die aus diesen rekonstruierten Denk- und Handlungsmustern generierten Typen konturiert.

Im neunten Kapitel werden die Ergebnisse der verschiedenen Auswertungsschritte zusammengefasst. Dabei zeigt sich am Ende des Forschungsprozesses, inspiriert durch neuere Auseinandersetzungen mit diskurs- und subjekttheoretischen Perspektiven, dass es sich lohnt, die bisherigen Ergebnisse durch eine "subjekttheoretische Brille« (Rose 2012, S. 120), im Sinne einer methodologischen Reflexion, zu betrachten. Deshalb werden in Kapitel 9.1 zunächst diskurstheoretische Betrachtungen im narrativ-biografischen Interview angestellt. Dabei wird der Frage nachgegangen, wie über die Partizipation an Diskursen immer schon vorfindbare Kategorien der (Selbst-)Beschreibung aufgegriffen und gegebenenfalls transformiert werden (vgl. ebd.). Anschließend wird dann in Kapitel 9.2 mit einer performativ-dekonstruktiven Perspektive analytisch aufgezeigt, wo dominante Diskurse unterlaufen werden und wo sich (damit) Phänomene von Widerstand zeigen. Dies ermöglicht es, am Ende der Arbeit ein erweitertes Verständnis von Gewalt gegen Frauen vorzuschlagen.

Im letzten Kapitel 9.3 werden im Anschluss daran Anknüpfungspunkte für sozialpädagogische Hilfe und Unterstützung diskutiert. 



\section{Paarbeziehung im gesellschaftlichen Wandel}

Die Forschungsperspektive der vorliegenden Arbeit fragt auf der Basis narrativer biografischer Interviews nach den Vorstellungen von Paarbeziehungen bzw. den Beziehungskonzepten von Frauen, die Gewalt im Rahmen der ersten eigenen Paarbeziehungen erlebt haben, sowie nach dem Umgang mit der erfahrenen Gewalt. Dieser Frage ist inhärent, in den Blick zu nehmen, wie gesellschaftliche und subjektive Erfahrungen individuell verarbeitet werden, um »anhand dieser Beschäftigung Aussagen über - lebensgeschichtlich vollzogene oder sich im Erzählen vollziehende - Bildungsprozesse Einzelner treffen zu können «(Rose 2012, S. 111). Insofern halte ich für die vorliegende Arbeit eine gesellschaftstheoretische Rahmung notwendig, um die daraus resultierenden Bedingungen für den biografischen Lebensweg der einzelnen Akteurinnen in den Blick zu nehmen und in der Analyse mit zu rekonstruieren.

Moderne Gesellschaften, und so auch die Bundesrepublik Deutschland, unterliegen seit einigen Jahrzehnten immensen kulturellen, ökonomischen und sozialstrukturellen Veränderungen, die auch Einfluss auf die Beziehungs- und Familienentwicklung nehmen. Die sogenannte "Emotionalisierungsthese« hat sich als eine allgemeine These des Wandels von Paarbeziehungen etabliert. Sie behauptet, dass die Ansprüche an die Qualität von Paarbeziehung gestiegen und diese zum Mittelpunkt von Beziehung geworden sind (vgl. Nave-Herz 2004, S. 170).

Vor allem innerhalb der familiensoziologischen Forschung, aber auch darüber hinaus, hat sich die These der Individualisierung zu einer Schlüsselkategorie soziologischer Gegenwartsdeutung entwickelt. Das folgende Kapitel 2 setzt sich daher mit dem sozialen Wandel, den damit verbundenen gesellschaftlichen Veränderungen sowie den sich daraus ergebenden Veränderungen für Beziehung (und Familie) ${ }^{1}$, auch unter individualisierungstheoretischer Perspektive, auseinander. Das Problem der Unschärfe und Mehrdeutigkeit, das dem Individualisierungsbegriff

1 Ehe wurde lange Zeit aus der Perspektive der Familie und damit innerhalb der Familienforschung diskutiert und thematisiert. Karl Lenz hat mit dem ersten Erscheinen seiner Veröffentlichung "Soziologie der Zweierbeziehung «im Jahre 1998 wesentlich dazu beigetragen, dass sich die Paarforschung als eigenständiges Forschungsgebiet innerhalb und außerhalb der Familienforschung etablieren konnte. Da jedoch der Begriff Paar (bzw. Ehe) lange Zeit innerhalb der Familienfor- 
vorgeworfen wird und das in Bezug auf die vernachlässigte subjektive Seite von Individualisierung - also die Art des individuellen Umgangs mit der Optionsvielfalt (vgl. Hainz 2012) - für die vorliegende Arbeit relevant ist, greift Kapitel 3 auf, indem subjekttheoretische Perspektiven, wie sie insbesondere die Geschlechterforschung derzeit diskutiert, thematisiert werden.

\subsection{Soziologische Perspektiven auf Paarbeziehung}

Liebe und Paarbeziehung weisen innerhalb der Soziologie eine lange Tradition auf, wenn auch eher randständig. So führt Niklas Luhmann in seinem Werk »Liebe als Passion « (1982) den Liebesbegriff wieder systematisch in die soziologische Theorie ein und betont die Bedeutung von Liebe als kulturellem Muster: "In diesem Sinne ist das Medium Liebe selbst kein Gefühl, sondern ein Kommunikationscode, nach dessen Regeln man Gefühle ausdrücken, bilden, simulieren, anderen unterstellen, leugnen und sich mit all dem auf die Konsequenzen einstellen kann, die es hat, wenn entsprechende Kommunikation realisiert wird (Luhmann 1982, S. 23). Anthony Giddens analysiert in seinem 1992 erschienenen Buch »The Transformation of Intimacy« ein neues Beziehungsmodell der »reinen Beziehung«, die nach dem Ideal einer gleichberechtigten Beziehung, insbesondere unabhängig von Geschlechterrollen, fungiert. Hervorzuheben sind auch die Arbeiten von Karl Lenz zu einer »Soziologie der Zweierbeziehung« (2009), in denen er das Individuum in den Mittelpunkt stellt und somit einen Individuums-zentrierten Ansatz vertritt. Häufig Bezug genommen wird in der wissenschaftlichen Literatur auch auf die Arbeiten der israelischen Soziologin Eva Illouz, die sich zum einen mit den Veränderungen von Gefühlen im Zuge des Kapitalismus und zum anderen mit dem Leiden an der Liebe beschäftigt (Illouz 2011, 2015).

Burkart (2018) skizziert in seinem Werk »Soziologie der Paarbeziehung« eine »Theorie des Paares«, mit der er zu erklären versucht, weshalb sich der Großteil der Individuen in der biografischen Lebensphase Paarbeziehung wiederfindet, und weshalb dieser eine so hohe Bedeutung zukommt. Der theoretische Bezugsrahmen umfasst dabei sowohl die Makro- als auch die Mikroperspektive, wodurch Paarbildung durch die Verknüpfung verschiedener Ebenen analysiert werden kann (vgl. Burkart 2018, S. 25). In Anlehnung an Giddens geht er dabei davon aus, dass Makroprozesse auf Mikroprozesse einwirken und umgekehrt. Das folgende Schaubild verdeutlicht die vier Ebenen, die sich innerhalb dieser Theorie gegenseitig beeinflussen:

schung diskutiert wurde und teils auch noch wird, beziehe ich mich in meinen Ausführungen an manchen Stellen entsprechend auf Paar und Familie. 
Tabelle 1: Theoretischer Bezugsrahmen zur Analyse von Paarbeziehungen, nach Burkart 2018, S. 25

\begin{tabular}{|c|c|c|c|}
\hline Kultur & Struktur & Interaktion/Praxis & $\begin{array}{l}\text { Sozialisation/ } \\
\text { Persönlichkeit }\end{array}$ \\
\hline $\begin{array}{l}\text { Wertmuster, Wis- } \\
\text { sen, Symbolisierun- } \\
\text { gen zu Liebe, Paar, } \\
\text { Intimität, Sexualität } \\
\text { usw. }\end{array}$ & $\begin{array}{l}\text { Paarbildungs- } \\
\text { strukturen, } \\
\text { (Orte, Märkte, } \\
\text { Regeln) } \\
\text { Institution des } \\
\text { Paares (Strukturen } \\
\text { und Normen) }\end{array}$ & $\begin{array}{l}\text { Paardynamik, Aus- } \\
\text { handlungsprozesse } \\
\text { Leibliche Interaktion } \\
\text { (Intimität) }\end{array}$ & $\begin{array}{l}\text { Habitus } \\
\text { Strategische, } \\
\text { emotionale } \\
\text { und leibliche } \\
\text { Dispositionen }\end{array}$ \\
\hline $\begin{array}{l}\text { Legitimierung durch } \\
\text { »Naturalisierung" }\end{array}$ & $\begin{array}{l}\text { Normative Regeln } \\
\text { der Gestaltung des } \\
\text { Alltags als Paar }\end{array}$ & $\begin{array}{l}\text { Liebe als Praxis } \\
\text { (Gabentausch) }\end{array}$ & $\begin{array}{l}\text { Körperliche } \\
\text { Basis (Sexuali- } \\
\text { tät) }\end{array}$ \\
\hline
\end{tabular}

Kulturelle Vorstellungen zu Liebe und Paar stellen auf der makrotheoretischen Ebene Normalitäten dar, die in der Regel nicht hinterfragt werden und als gewollt und natürlich wahrgenommen werden. Auf der strukturellen Ebene entsprechen ihnen die normativen Regeln der Paarbildung. Das Paar stellt dabei eine gesellschaftliche Institution dar, das seine Bedürfnisse und Interaktionen in bestimmte Strukturen überführt (z.B. Bedürfnisse nach sexueller Erfüllung, Wohlbehagen in Gemeinschaft, aber auch Selbstverwirklichung). Auch normative Regeln, nach denen der Alltag gestaltet wird, gehören zu dieser Institutionalisierung der Paarbeziehung. Diese gehört demnach zu den starken normativen Forderungen an Einzelne durch die Gesellschaft. Ermöglicht wird Paarbildung zudem erst durch das kulturelle Wertmuster der Liebe sowie der sozialen Institution des Paares. (Vgl. ebd., S. 26)

Bei der Betrachtung der kulturellen Ebene hinsichtlich des Legitimationsaspektes wird ein »Prozess der Naturalisierung des Sozialen« (ebd.) sichtbar. Gemeint ist damit die Erklärung sozialer Normierungen als etwas scheinbar Natürliches. So erscheint die Neigung zur Paarbildung als elementar menschliches Grundbedürfnis und damit als natürliche Eigenschaft. Auf der Ebene Interaktion/Praxis werden in der Literatur v.a. die Themen der Partnerwahl sowie des Heiratsmarktes thematisiert. Wenig berücksichtigt wird dabei bislang die Interaktionsebene zwischen den einzelnen Individuen. Burkart zufolge wären gerade solche Studien wichtig, da die Bedeutung von Aushandlungsprozessen in Paarbeziehungen zunimmt (vgl. dazu auch Kap. 3). Deshalb plädiert er hier auch für die Verwendung des Begriffs »Praxis«, da dieser die leiblich-körperliche Dimension in Interaktionen stärker betont und damit ein anderes Verständnis von Interaktion und sozialem Austausch zugrunde legt. Und letztlich finden sozialisatorische Entwicklungsprozesse statt, die zum einen »nicht nur eine mehr oder weniger stabile Geschlechts- 
identität, sondern auch eine Neigung zur Paarbildung« (Burkart 2018, S. 27) hervorbringen. Liebes- und Bindungsfähigkeit sind demnach in der Sozialisation angelegt und stellen affektive Voraussetzungen dar, um kulturelle Wertmuster auszubilden und anzueignen. (Vgl. ebd., S. 25ff.)

Historisch lassen sich drei Phasen ausmachen, in denen Individualität und $\mathrm{Au}$ tonomie innerhalb von Paarbeziehungen jeweils unterschiedlich ausgeprägt sind. Im Folgenden werden die ersten beiden Phasen nur knapp skizziert; der Schwerpunkt der Darstellung liegt auf der dritten und damit gegenwärtigen Phase.

In der Vormoderne, bzw. in der traditionalen Gesellschaft, waren die gesellschaftlichen Sphären von Frauen und Männern klar getrennt und Privatheit war wenig entwickelt:

»Das Paar hatte keine große Bedeutung, jedenfalls nicht im Sinne einer Sphäre der Privatheit oder gar einer Verschmelzung der liebenden Partner. Die Autonomie der Partner war daher größer - nicht zu verwechseln mit individueller >Freiheit‘, denn sie waren eingebunden in ständische Strukturen und Strukturen der Geschlechtersphären. Die Ehefrau war stärker als heute in die weibliche Sphäre der anderen Ehefrauen ... integriert: der Ehemann stärker in die männliche Sphäre ... Die Sphärentrennung zwischen den Geschlechter-Klassen war also relativ stark ausgeprägt - allerdings war sie weit weniger naturalisiert als heute, sie war eher gedacht als ständische Differenzierung. «(Burkart 2018, S. 31f., Herv. im Original)

Kennzeichnend für das Leitbild der Moderne, bzw. der bürgerlichen Gesellschaft, war die Annahme einer naturalisierten Geschlechterdifferenz ${ }^{2}$, die aufgrund einer »entsprechend geschlechterkomplementären Ordnung der beziehungsinternen Verhältnisse ... vereinfacht: den Tausch männlicher Versorgungsleistungen gegen weibliche Fürsorge vorsah" (Hirseland/Leuze 2010, S. 192). Es bildete sich eine Intimsphäre heraus, in die sich das Paar zurückziehen konnte. Die normative Leitidee gründet sich hier auf der Vorstellung einer »lebenslangen, die Partner ... verpflichtenden Lebensgemeinschaft, die gegenüber den individuellen Interessen und/oder Begehren der so vergemeinschafteten Individuen einen an sich schützenswerten Wert darstellt« (ebd.). Somit stellte ein Zwangszusammenhalt, der

2 Karin Hausen zeigt in ihrem Beitrag »Die Polarisierung der Ceschlechtscharaktere - Eine Spiegelung der Dissoziation von Erwerbs- und Familienleben« (1976) eindrücklich auf, wie mit dem - heute in Vergessenheit geratenen - Begriff der »Ceschlechtscharaktere«im 19. Jahrhundert »die mit den physiologischen korrespondierend gedachten psychologischen Geschlechtsmerkmale«bezeichnet wurden (Hausen 1976, S. 363) und wie mithilfe der Herausbildung der sog. »Ceschlechtercharaktere « ein neues Orientierungsmuster gesetzt wurde, um die bestehenden Verhältnisse zu stabilisieren. Das, was als »Ceschlechtercharaktere« gedeutet wurde, bezeichnete "sowohl die in der bürgerlichen Familie konstitutive prinzipielle Verschiedenartigkeit der geschlechtsspezifisch geteilten Arbeit als auch die Dissoziation und Kontrastierung von Erwerbsund Familienleben, von Öffentlichkeit und Privatheit« (ebd., S. 390). 
durch geteilte Ökonomie, polare Geschlechtercharaktere und eine hierarchische Struktur bestimmt war, die Basis der Geschlechterverhältnisse dar, nur noch abgepuffert durch das Ideal der romantischen Liebe (vgl. Jurczyk 2001, S. 14). Während sich die erste Phase der Individualisierung auf den Beginn des Industrialisierungsprozesses bis Mitte des 20. Jahrhunderts bezieht, zeichnet sich seit den 1960er-Jahren ein zweiter gesellschaftlicher Individualisierungsschub ab. Dieser wirkt sich vor allem auf den weiblichen Lebenslauf aus. Demnach setzt sich auch in der weiblichen Normalbiografie immer mehr die Logik individueller Lebensentwürfe durch (vgl. Peuckert 2012, S. 661). Seit den späten 1960er-Jahren führen gesellschaftliche Entwicklungen, die sich unter anderem mit den Stichworten Bildungsexpansion, Wirtschaftswachstum und kompensatorische Sozialpolitik beschreiben lassen, zu einer Ausdifferenzierung horizontaler Ungleichheiten, die sich in einer Pluralisierung von Lebenslagen (Hradil 1997) widerspiegeln. Diese Entwicklungen haben massiven Einfluss auf das Geschlechterverhältnis bzw. die Beziehung der Geschlechter untereinander genommen.

Inzwischen ist der Strukturwandel vollzogen und die daraus resultierenden Herausforderungen bezüglich des Beziehungswandels und der Pluralisierung von Lebensformen werden intensiv diskutiert (Nave-Herz 2002, 2007; BMFSFJ 2006, 2012; Peuckert 2012). Pierre Bourdieu, Anthony Giddens, Ulrich Beck und Elisabeth Beck-Gernsheim sowie Manuel Castells haben mit ihren soziologischen Theorien die Familienforschung nachhaltig beeinflusst. Im Folgenden wird vor allem Bezug genommen auf die Theorie der »Reflexiven Moderne« von Ulrich Beck und Elisabeth Beck-Gernsheim, die auch in Erweiterung der Theorie Bourdieus herangezogen werden kann. Zum einen, weil die Individualisierungstheorie den größten Erkenntnisgewinn, neben den Theorien des Wertewandels und der Theorie der gesellschaftlichen Differenzierung, verspricht (vgl. Peuckert 2012, S. 659). Zum anderen, weil mit ihr auch die biografischen Modi und Verläufe, die durch die Individualisierung entstehen, in den Blick genommen werden können und diese Perspektive für das vorliegende Forschungsinteresse von Bedeutung ist. Nach dieser Theorie ist ein wesentlicher Aspekt des sozialen Wandels die Pluralisierung von Lebensformen. Peuckert fasst diesen wie folgt zusammen:

»Die demographischen und familialen Veränderungen seit Mitte der 1960er Jahre werden analog als Ergebnis eines langfristig stattfindenden Modernisierungsund Individualisierungsprozesses gedeutet, der sich beschleunigt und eine neue Qualität gewonnen hat. In der vormodernen Gesellschaft (der vorindustriellen Zeit) wurde das Leben der Menschen durch eine Vielzahl traditionaler Bindungen bestimmt. Ein zentrales Moment des Übergangs in die Moderne ist - als Folge der Ausbreitung rechtlich freier Lohnarbeit, der Durchsetzung der bürgerlichen Grundrechte, der Zunahme des Wohlstandes, der Ausweitung der Bildungschancen und vielem anderen mehr - die Freisetzung der Individuen aus traditional 
gewachsenen Bindungen, Glaubenssystemen und Sozialbeziehungen.«(Peuckert 2012, S 659)

Individualisierung meint dabei

»zum einen die Auflösung vorgegebener sozialer Lebensformen - zum Beispiel das Brüchigwerden von lebensweltlichen Kategorien wie Klasse und Stand, Geschlechtsrollen, Familie, Nachbarschaft usw.; oder auch ... der Zusammenbruch staatlich verordneter Normalbiographien, Orientierungsrahmen und Leitbilder.« (Beck/Beck-Gernsheim 1994, S. 11)

Individualisierung bedeutet zwar einerseits einen Zugewinn an Handlungsmöglichkeiten und Handlungsspielräumen, da alte Abhängigkeiten und Zwänge an Bedeutung verlieren (Freisetzungsdimension). Andererseits bedeutet Individualisierung aber auch einen zunehmenden Geltungsverlust der Sicherheit und sozialer Normen (Entzauberungsdimension). Indem sich das traditionelle Familien- und Berufsmodell auflöst, können sich Individuen immer weniger an einer Normalbiografie orientieren. Sie sehen sich mit einer Vielzahl konkurrierender Orientierungsmuster in der biografischen Abfolge konfrontiert, die in einen sinnhaften Lebensentwurf münden sollen. Gleichzeitig begegnen ihnen neue Widersprüche, Unfreiheiten und Zwänge (Kontrolldimension). (Vgl. Peuckert 2012, S. 661f.)

Individualisierungsprozesse beruhen nicht auf individuellen Entscheidungen, sondern erfordern neue institutionelle Anforderungen, Kontrollen und Zwänge von Einzelnen. Dabei ist die Regelungsdichte moderner Gesellschaften hoch und die institutionellen Vorgaben der modernen westlichen Gesellschaften stellen Leistungsangebote und Handlungsanreize dar, um die man sich jedoch aktiv bemühen muss (vgl. Beck/Beck-Gernsheim 1994, S. 12).

\subsubsection{Paare in spätmodernen Zeiten}

Mit der zweiten, reflexiven Moderne ging ein Wandel der Geschlechterverhältnisse einher, der zu einer Entkoppelung von Ehe und Liebe sowie von Ehe und legitimer Elternschaft führte. Nicht nur das Liebesverhältnis muss neu ausgehandelt werden, auch die alte Institution Familie reguliert und legitimiert nicht mehr das Paar und ihre Liebe (vgl. Herma 2009, S. 52). Individualisierung bedeutet damit auch:

»Die Menschen werden freigesetzt aus den verinnerlichten Ceschlechterrollen, wie sie im Bauplan der Industriegesellschaft für die Lebensführung nach dem Modell der Kleinfamilie vorgesehen sind, und sie sehen sich (dieses setzt jenes voraus und verschärft es) zugleich gezwungen, bei Strafe materieller Benachteiligung eine eigene Existenz über Arbeitsmarkt, Ausbildung, Mobilität aufzubauen und diese notfalls gegen Familien-, Partnerschafts- und Nachbarschaftsbindungen durchzusetzen und durchzuhalten.«(Beck/Beck-Cernsheim 2015, S. 13) 
Die Verzahnung zweier Arbeitsmarktbiografien, anstelle der bisherigen Verzahnung einer Arbeitsmarktbiografie mit einer Hausarbeitsbiografie, erfordert ein »Dauerkunststück, ein[en] Drahtseildoppelakt, der so pauschal keiner Generation zuvor zugemutet wurde« (vgl. ebd.). Genau in diesem Punkt liegt der Widerspruch zwischen den Anforderungen des Arbeitsmarktes und den Anforderungen der Partnerschaft. Zudem haben sich epochale Veränderungen in den Bereichen Sexualität, Recht und Bildung vollzogen, denen eine Konstanz im Verhalten und in der Lage von Frauen und Männern, vor allem auf dem Arbeitsmarkt und in der sozialen Sicherung, gegenübersteht:

»Die jungen Frauen haben - in der Angleichung der Bildung und in der Bewußtwerdung ihrer Lage - Erwartungen auf mehr Cleichheit und Partnerschaft in Beruf und Familie aufgebaut, die auf gegenläufige Entwicklungen auf dem Arbeitsmarkt und im Verhalten der Männer treffen. Die Männer umgekehrt haben eine Rhetorik der Cleichheit eingeübt, ohne ihren Worten Taten folgen zu lassen.« (Beck/Beck-Gernsheim 2015, S. 24)

Die im modernen Individualisierungsprozess eingebettete Problematik, dass Einzelne die Vervielfältigung biografischer Optionen selbst bewältigen müssen, führt seit der zweiten Hälfte des 20. Jahrhunderts zu einer Zunahme an Beziehungskonflikten (vgl. Herma 2007, S. 39):

»In Paarbeziehungen, die sich am Leitbild der romantischen Liebe orientieren, sei diese Problematik ... zugespitzt, da die Bewältigung der Beziehung verlagert wird auf die Einzelnen mit verminderter Rückendeckung äußerer Regeln. Diese jede Beziehung prägende `Janusköpfigkeit‘ von Individualisierungschancen und Individualisierungsdruck hat nun eine zentrale, für die Ceschlechter diachrone Komponente: Denn mit der allmählichen Entwertung der alten Stützkomponenten im traditionellen Lebens- und Liebesarrangement fließt nun in die Handlungsökonomie des heterosexuellen Paares auch ein neues Individualisierungsbegehren von Frauen mit ein.«(Herma 2007, S. 40)

Die beschriebenen Veränderungen im Geschlechterverhältnis führen zu einem widersprüchlichen Nebeneinander »unterschiedlicher Ausprägungen straditionaler und >moderner Elemente in Geschlechterverhältnissen « (Jurczyk 2001, S. 15). Indem Frauen ihr Recht auf Eigenständigkeit und Selbstbestimmung ernst nehmen und umsetzen, kommt es zu komplexen Ausprägungen weiblicher Individualisierung (vgl. ebd.). Die Pluralisierung von Lebensformen führt dazu, dass verschiedene Lebensformen zum einen nebeneinander existieren (z.B. nichteheliche Lebensgemeinschaften mit und ohne Kinder, gleichgeschlechtliche Partnerschaften, Alleinerziehende, Lebensformen des »living apart together « in Form von Wochenendbeziehungen oder getrennten Haushalten), und dass sich diese verschiedenen Le- 
bensformen zum anderen innerhalb eines Lebenslaufs häufiger ändern (vgl. ebd., S. 17).

Paradoxerweise steigt die Bedeutung von Liebe parallel zur Vervielfältigung der Lebensformen. Liebe ist in spätmodernen Zeiten wichtiger, aber auch schwieriger denn je geworden. Die Ansprüche an sie sind gewachsen; sie gilt als Gradmesser für soziale Anerkennung und Erfolg. »Liebe wird nötig wie nie zuvor und unmöglich gleichermaßen.«(Beck/Beck-Gernsheim 2015; S. 9, Herv. im Original). Indem Liebe eine solche Aufwertung erfährt, wird sie auch zur Überforderung, da sie zur wichtigsten Basis von Paarbeziehung wird. Spätmoderne Paarbeziehungen erfordern eine höhere und persönlichere Verbindlichkeit als frühere Ehe-Beziehungen und sind dabei gleichzeitig mit dem Problem konfrontiert, dass ihre Dauerhaftigkeit nicht mehr institutionell gesichert ist und deshalb ebenfalls auf die Ebene der persönlichen Beziehung verlagert wird (vgl. Burkart 2018, S. 29). Gemeinsamkeit wird also zur Verhandlungssache, zum Beispiel bei der Frage, wer wann wie und warum in Beruf, Haushalt und Freizeit arbeitet. Beide Individuen müssen einen Teil ihrer Individualität und Autonomie aufgeben und sich gemeinsam neu definieren.

Es müssen heute also neue Arrangements von Beziehung, Familie und Beruf sowie neue Umgangsformen entwickelt werden. Partnerschaftliche und familiäre Lebensformen sind zunehmend Ergebnis gemeinsamer Aushandlungen, bis hin zu bewussten Entscheidungen für die jeweilige Lebensweise. Diese Entscheidungen und Aushandlungen finden aber oft innerhalb bestimmter (institutioneller) Strukturen statt, werden von diesen beeinflusst und kommen somit nicht nur und nicht immer bewusst zustande. Oft haben die Beteiligten daher das Gefühl, all das hätte sich einfach so ergeben (vgl. Maihofer 2014, S. 317). Diese Prozesse und Aushandlungen verlaufen dabei nicht zwingend einvernehmlich, und auch bestehende Arrangements sind nicht fix, sondern ständig in Bewegung, müssen neu bestätigt oder gar neu erfunden werden (vgl. ebd.).

Der Druck, neue Lebensformen auszuprobieren, geht dabei mehrheitlich von Frauen aus, da diese heute ihre Perspektive in einer dauerhaften, qualifizierten Berufstätigkeit sehen und traditionellen Beziehungsformen skeptisch gegenüberstehen (vgl. Peuckert 2012, S. 663). Dabei ergeben sich wesentliche Koordinationsprobleme bei Frauen mit Doppelorientierung an familialem und partnerschaftlichem Leben einerseits sowie an beruflicher Ausrichtung andererseits, was kennzeichnend für den gegenwärtigen weiblichen Lebenszusammenhang in den westlichen Gesellschaften ist (vgl. ebd., S. 665). Das Dilemma für Frauen heute »besteht darin, dass sie zwei Lebensbereiche (Familie und Beruf) in Einklang bringen wollen, dass es hierfür aber kein biografisches Modell, keinen >Normallebenslauf^gibt. Sie müssen individuelle Lösungen suchen, neue weibliche Lebenslaufmodelle konstruieren.«(Ebd.) 


\subsubsection{Doing Family - Doing Couple}

Es mag zunächst verwunderlich erscheinen, weshalb ich in diesem Kapitel das Konzept des »Doing-Family« thematisiere. Doing Family als aktiver Gestaltungsprozess von Familien und Familienbeziehungen im gesellschaftlichen Kontext rückt das Alltagshandeln von und die Aushandlungsprozesse in Familien in den Vordergrund (Jurczyk u.a. 2009). Familiale Praktiken folgen individual- und familienbiografischen Prozessen und verändern sich mit dem Alter der Kinder und Eltern und deren Erfahrungen (vgl. ebd., S. 6). So müssen familiale Praktiken an neue Situationen angepasst oder ganz neue Praktiken entwickelt werden.

»>Doing Family < beginnt aber nicht erst, wenn eigene Kinder vorhanden sind, sondern viel früher: Bereits in der eigenen Herkunftsfamilie und durch biografische Erfahrungen entstehen (Wunsch-)Bilder vom späteren Leben. Sie beeinflussen, bewusst und unbewusst, auch die Partnerwahl und das Beziehungsverhalten." (Jurczyk u.a. 2009, S. 6)

Und hierin liegt auch die Begründung, weshalb ich mich auf dieses Konzept beziehe: Der Blick auf biografische Erfahrungen während der (familialen) Sozialisation ist für das vorliegende Forschungsinteresse notwendig, um die subjektiven Denkund Handlungsmuster der befragten jungen Frauen in ihrem Entstehungskontext betrachten und analysieren zu können. Zudem stellen Paare ein familiales Subsystem dar, für das verschiedene Dimensionen des »Doing Family« ebenfalls gelten (z.B. die Herstellung von Gemeinsamkeit, Formen der Arbeitsteilung, Routinen und Rituale).

Der Begriff »Doing Family«, dessen Anleihe beim wissenschaftlichen Konzept des Doing Gender (West/Zimmermann 1987) unübersehbar ist, betont den Akteursstatus der Familie und ihrer Mitglieder und bringt damit zum Ausdruck, dass

»Familie heute als Ressource für Cesellschaft und Individuen nicht mehr selbstverständlich gegeben ist. (...) Familie konstituiert sich damit als zwar gesellschaftlich geformter, aber selbst hervorgebrachter und gestalteter Tätigkeits- und Erfahrungszusammenhang im Privatbereich moderner Gesellschaften«(Jurczyku.a. 2009, S. 1).

Das Konzept schließt wesentlich an den Ansatz der alltäglichen Lebensführung an, nach dem Lebensführung als aktive Leistung begriffen wird - auch wenn dies den Akteur_innen selbst nicht immer bewusst ist (vgl. ebd.). Das Herstellen eines sinnstiftenden Familienlebens erfolgt in Abhängigkeit von den spezifischen Bedingungen der reflexiven Moderne, die mit einem Verlust an Selbstverständlichkeit, Pluralisierung, Optionssteigerung und Ambivalenz verbunden sind (vgl. Lange 2009, S. 439). 
Dem Konzept liegt ein praxeologischer Blick zugrunde, der seine Perspektive auf die Praktiken der Herstellung und Gestaltung persönlicher Beziehungen zwischen den Geschlechtern (und auch zwischen den Generationen) richtet. Es geht also nicht darum, Werte und Einstellungen oder bestimmte Formen des Zusammenlebens zu betrachten, sondern die aktiven Herstellungsleistungen der Einzelnen und zwar im gesellschaftlichen Kontext. Folgende drei wissenschaftliche Referenzpunkte lassen sich nach Jurczyk u.a. (2014) für den Begriff Doing Family festhalten:

1. Die bereits erwähnte Anleihe beim Konzept des Doing Gender hebt hervor, dass es kein natürliches Familienhandeln gibt. Stattdessen entscheiden soziokulturelle Kontexte und Vorstellungen mit darüber, wie Familie gelebt wird.

2. Die im angloamerikanischen Raum stattfindende Beschäftigung mit den Aspekten Raum, Zeit, Körper und Emotion, die in der bisherigen Familienforschung lange Zeit unberücksichtigt blieben, hat verschiedene Autor_innen da$\mathrm{zu}$ angeregt, neue Wege bei der Theoretisierung des Konzepts »Doing Family« $\mathrm{zu}$ beschreiten.

3. Im deutschsprachigen Raum hat das Konzept der Alltäglichen Lebensführung wichtige Impulse für eine alltagsorientierte Familienforschung geliefert, indem es Praktiken als sinnhafte Alltagsgestaltung begreift und Lebensführung insgesamt als aktive Leistung ansieht. (Vgl. Jurczyk u.a. 2014, S. 11)

Das Konzept enthält außerdem eine zeitdiagnostische These, die besagt, »dass unter den Bedingungen der späten Moderne Familie(nleben) weniger denn je einfach 'geschieht

Das Konzept des Doing Family versucht also, in seiner praxeologischen Ausrichtung erstens die Komplexität des Alltags zu erfassen, zweitens die Beziehungen und Interaktionen zwischen den einzelnen Familienmitgliedern zu erfassen und Familie nicht nur aus der Perspektive Einzelner und bezogen auf Einzelne zu sehen, und drittens den Einfluss sich wandelnder sozialökologischer Umwelten, wie beispielsweise des Bildungssystems, auf Familie sowie ihre Wechselwirkungen $\mathrm{zu}$ berücksichtigen (vgl. ebd., S. 12f.).

Lenz (2014) wiederum führt in Anlehnung an den von Jurczyk und Lange (Jurczyk u.a. 2009) eingeführten Begriff des Doing Family sowie im Anschluss an die Arbeiten von Morgan (1996) den Begriff des »Doing Couple« ein. Demnach konstituiert sich der Alltag von Paaren, so wie auch der Alltag von Familien, als selbst hergestellter und gestalteter Erfahrungsraum:

»Die Muster alltäglicher Lebensführungen sind Sedimentierungen des fortlaufenden Prozesses des doing couple. Die Gewohnheiten als wiederkehrende Ablaufmuster in der Paarbeziehung werden dabei nicht ein für alle Mal hergestellt, son- 
dern haben eine Geltung bis auf weiteres. Sie bleiben fragil und können [bzw. müssen, Anm. d. Verf.] bei Bedarf verändert werden. Doing couple umfasst als Teil des Doing Family jene Herstellungsleistungen, die ausschließlich auf die Paargemeinschaft bezogen sind. Dabei wird davon ausgegangen, dass das Paar in eine Familie eingebettet sein kann bzw. auch unabhängig von einer Familie bestehen kann.« (Lenz 2014, S. 115)

Lenz formuliert in seinem Beitrag zu »Zeit(en) in der alltäglichen Lebensführung von Paaren« (2014) das Konzept des Doing Couple unter der - bislang vernachlässigten - Perspektive von Paaren als Subsystem von Familie; zudem nimmt er dabei Bezug darauf, dass auch Paarbeziehung spezifische zeitliche Voraussetzungen erfordert. So sieht er ein Mindestmaß an gemeinsamer Zeit als Grundbedingung für Paar-Stabilität an, und Paare haben den Anspruch, Zeit zusammen zu verbringen und diese miteinander zu gestalten. Nicht zuletzt erfolgt der Schritt zur Familiengründung auf der Basis verfügbarer Zeit. (Vgl. Lenz 2014, S. 113)

In Paarbeziehungen können sich Einzelne gegenseitig in ihrer einzigartigen Individualität bzw. als ganze Person erfahren. Lenz führt dazu aus, dass "gerade in einer Gesellschaft, in der Kommunikation auf der Basis zugeschriebener sozialer Identität (z.B. Polizistin/Polizist, Verkäufer/Verkäuferin) dominant ist, ... die besondere Qualität von Paarbeziehungen umso wichtiger« wird (ebd., S. 114). Für die ganzheitliche Wahrnehmung des/der anderen benötigt es Nähe, Geborgenheit und Vertrauen. Und diese können wiederum nur in gemeinsam verbrachter Zeit aufgebaut und aufrechterhalten werden (vgl. ebd.). Ergänzend lässt sich hier hinzufügen, dass gerade auch vor dem Hintergrund eines gestiegenen Bedürfnisses nach Liebe (vgl. Kap. 2.1.1) sowie deren fundamentaler Bedeutung für die Basis von Paarbeziehung gemeinsame Zeit elementar für die Gestaltung der Beziehung ist.

Auch hier zeigen sich Veränderungen in den Anforderungen an Paare, die sich aus der individualisierten Gegenwartsgesellschaft der späten Moderne ergeben: Kulturelle Vorgaben, die in der Vergangenheit die Aufgaben der Geschlechter vordefinierten, haben an Gültigkeit verloren. Deshalb werden Paare vor die Notwendigkeit gestellt, von Anfang an in einen gemeinsamen Aushandlungsprozess zu treten (vgl. Lenz 2014, S. 115). Diese Verständigungs- und Aushandlungsprozesse finden dabei zum einen auf sprachlicher Ebene, zum anderen »über die Tricks des Geredes und die non-verbale Kommunikation« statt (Kaufmann 1994, S. 227, zit. in Lenz 2014). Dabei wird in der alltäglichen Lebensführung, im Doing Couple, auf kulturell vorgegebene Handlungs- und Deutungsmuster zurückgegriffen. Der sich auf dieser kulturellen Ebene vollzogene Wandel zeigt sich in einem veränderten Leitbild von Beziehung. Danach sind die prinzipielle Gleichwertigkeit der Geschlechter sowie die Zuerkennung der gleichen Rechte und Pflichten fest in diesem Leitbild verankert (vgl. Lenz 2014, S. 121): 
»Die Partnerschaft als Leitbild impliziert die feste Erwartung, dass viel miteinander unternommen wird, dass Kommunikationsoffenheit besteht und dies impliziert, dass mehr individuelle Lebenszeit zur gemeinsamen Paarzeit wird. Der mit diesem Leitbild einhergehende Wegfall geschlechtsstereotyper Vorgaben legt die kulturellen Grundlagen dafür, dass die alltägliche Lebensführung des Paares vor allem das Resultat des doing couple ist. Nicht mehr die bloße Übernahme von starren Rollenvorgaben legt die häusliche Arbeitsteilung fest, sondern gefordert sind - zumindest in bestimmten sozialen Milieus - die Paare selbst, die sich darüber verständigen müssen.«(Lenz 2014, S. 121)

Diese Aushandlungsprozesse stellen eine zunehmende Anforderung an Paare dar, die bewältigt werden muss. Gelingt dies, tragen die Aushandlungsprozesse zur Sicherung der Kontinuität als Paar bei.

\subsection{Fazit}

Folgende zusammenfassende Gedanken erachte ich für das vorliegende Forschungsinteresse als zentral:

- Individualisierung ist nicht gleichzusetzen mit freier Entscheidung der Individuen oder einem Zuwachs an Freiheiten, sondern Individualisierungsprozesse sind durch Widersprüche und Ambivalenzen gekennzeichnet, die Einzelne vor Entscheidungsdilemmata und biografische Unsicherheiten stellen. In Bezug auf Paarbeziehung bedeutet dies, dass Gemeinsamkeit innerhalb der Beziehung verhandelt werden muss und partnerschaftliche Lebensformen das Ergebnis gemeinsamer Aushandlungen sind. Hier eignet sich das Konzept des Doing Family, mit dem die Forschungsperspektive auf die Praktiken der Herstellung und Gestaltung intimer Beziehungen zwischen Beziehungspartner_innen gerichtet wird.

- Zur Erforschung subjektiver Beziehungskonzepte und Umgangsweisen mit Gewalterfahrungen in den ersten eigenen Paarbeziehungen fehlt bislang ein subjektorientierter Zugang, der auch die Erfahrungsverarbeitung von Frauen als gesellschaftlichen Akteurinnen in den Blick nimmt. Deshalb nimmt die vorliegende Forschungsarbeit in den Blick, in welchem Verhältnis der soziale Wandel - mit den entsprechenden Veränderungen und Anforderungen an Paarbeziehung (Kap. 2) - zu den jeweiligen biografischen Erfahrungsaufschichtungen und Handlungsorientierungen (Kap. 3) der befragten jungen Frauen der heutigen Generation steht.

- Gesellschaftstheoretische Ansätze stellen wichtige Erklärungsangebote für weiterhin bestehende (Geschlechter-)Ungleichheiten dar. Die Bedeutung von 
Geschlechternormen für die (Re-)Produktion struktureller Ungleichheit sowie subjektive Orientierungs- und Handlungsmuster im Umgang mit Gewalt sind bislang wenig erforscht. Der Blick auf die Bedeutung normativer Anforderungen für die Handlungsorientierungen von jungen Frauen sowie der Blick darauf, wie sie sich mit diesen auseinandersetzen, versprechen eine Perspektivenerweiterung.

Biografisches Handeln von jungen Frauen geschieht immer in Auseinandersetzung mit gesellschaftlichen Anforderungen, wenn auch im Rahmen konkreter Kontexte. Dabei bildet die Sozialisationsgeschichte die Hintergrundfolie, auf der Denk- und Handlungsmuster entstehen. Um eine einseitige Betrachtung zu vermeiden, gilt es, die Wechselbeziehungen von (sozialisatorischen) Kontexten bzw. gesellschaftlichen Rahmenbedingungen und biografischem Handeln in den Blick zu nehmen. Dies erfolgt im anschließenden Kapitel. 



\section{Paarbeziehung als biografisches Projekt}

In der vorliegenden Arbeit wird die Perspektive auf das biografische Handeln junger Frauen in ihren Paarbeziehungen gerichtet. Dabei verweist Handeln immer auch darauf, »dass junge Frauen in Strukturen und Geschlechterverhältnisse eingebunden sind, die sie als Akteurinnen täglich und lebensgeschichtlich reproduzieren, neutralisieren, aussetzen, modifizieren oder transformieren. Biografisch bedeutet, dass Handeln sich auf biografische Erfahrungsräume, Erwartungshorizonte, die immer auch konstruiert und diskursiv sind, bezieht und verflochten ist mit biografischen Konstruktionen. (...) Erfahrungen werden dabei erst durch die Deutungen der jungen Frauen zugänglich.«(Keddi 2003, S. 119) Die Rekonstruktion lebensgeschichtlicher Texte zielt auf die Herausarbeitung des Dokumentsinns, der sich auf habitualisierte Stile bezieht und sich vom »intendierten Ausdruckssinn " (Mannheim 1964a, S. 104) unterscheidet. Dieser subjektiv gemeinte Sinn wird nicht wörtlich oder explizit, sondern "gestalterisch, metaphorisch oder sstilistisch zum Ausdruck gebracht « (Bohnsack 2010, S. 66). Er unterliegt dem biografischen Handeln latent und kann somit nicht direkt abgefragt werden. Das biografische Handeln junger Frauen ist außerdem in gesamtbiografische Zusammenhänge eingebettet und damit nicht nur Ausdruck von subjektiven Erfahrungen. Damit wird die Frage aufgeworfen, wie sich die Subjekte in Auseinandersetzung mit gesellschaftlichen Verhältnissen konstituieren. Mit Bezug auf die Fragestellung der vorliegenden Arbeit, die nach der biografischen Entwicklung von Beziehungskonzepten fragt, bedeutet dies, den Blick auch auf sozialisatorische Erfahrungen zu richten. Der Studie liegt die Annahme zugrunde, dass es eine individuierte Struktur von Haltungen und Dispositionen gibt. Damit rücken individuelle biografische Haltungen in den Vordergrund. Ziel der Studie ist es, die individuelle Genese von Orientierungsrahmen bzw. die Entstehung individueller Beziehungsvorstellungen $\mathrm{zu}$ betrachten und $\mathrm{zu}$ analysieren.

Das vorliegende Kapitel befasst sich zunächst mit den grundlagentheoretischen Annahmen zu Biografie als wissenschaftlichem Konzept. Im Anschluss werden kurz die Gründe für den Relevanzverlust skizziert, den sozialisationstheoretische Fragestellungen seit längerer Zeit innerhalb der Geschlechterforschung erfahren haben, um dann anhand einiger ausgewählter subjekttheoretischer Kon- 
zepte eine Möglichkeit aufzuzeigen, wie (notwendige) sozialisationstheoretische Fragen aufgebrochen, korrigiert und neu gestellt werden können.

\subsection{Biografie als wissenschaftliches Konzept}

Vorab soll kurz festgehalten werden, welche grundlagentheoretischen Voraussetzungen ich der vorliegende Studie zugrunde lege: Ausgehend von der Annahme, dass es eine grundsätzliche Verschränkung des Sozialen mit dem Subjektiven gibt, bedarf es theoretischer Ansätze, die das Wechselverhältnis zwischen gesellschaftlichen Strukturen und subjektivem Handeln in den Blick nehmen und die

»nicht nur zeigen können, wie sich z.B. gesellschaftliche Strukturen oder gesellschaftlich dominante Diskurse in individuellen Praktiken spiegeln, sondern auch umgekehrt, wie sich individuelle, kollektive, zumeist informelle Praktiken strukturell und diskursiv niederschlagen können«(Stauber 2014, S. 26).

Auch wenn Biografie in die verschiedensten Fachdisziplinen Einzug gehalten hat und der Fokus auf Biografie je nach Fachinteresse variiert, besteht Konsens darüber, Biografie als »soziales Konstrukt « (Fischer/Kohli 1987) zu begreifen, in dem Individuum und Gesellschaft interagieren. ${ }^{1}$ Mit dem Anliegen, den einzelnen Menschen in seinen sinnhaft-interpretativ vermittelten Bezügen zur alltäglichen Lebenswelt und in seinem biografischen Gewordensein zu verstehen, gewinnen die »Dimensionen der Prozessualität und Historizität« (Marotzki 1995, S. 58) an Bedeutung. Dabei wird Biografie als Schnittstelle zwischen gesellschaftlich Vorgefundenem und individuell konstruierten Prozessen gesehen. Biografische Forschung eröffnet damit einen »Zugang zur sozialen Wirklichkeit, bei dem die Individualität des Akteurs berücksichtigt bleibt und diese Individualität sozial verursacht und strukturiert gedacht wird« (Lamnek 1995, S. 329).

Zwei Aspekte sind insbesondere hervorzuheben: Einerseits kann Biografie, mit Bourdieu gedacht, als Konstrukt im Sinne eines »Modus Operatum« (Bourdieu 1987, zit. in Dausien 2006, S. 34) verstanden werden. Demnach ist Biografie ein Produkt gesellschaftlichen und individuellen Handelns. Darunter fallen »alle Formen und Formate der Präsentation und Bearbeitung individueller Identität und gesellschaftlicher Zugehörigkeit, und zwar in der verzeitlichten Form biografischer Ablaufmuster« (Dausien 2006, S. 34). Andererseits meint die Figur von Biografie als

1 Die inzwischen übliche Differenzierung zwischen Biografie und Lebenslauf sieht die Unterscheidung darin, dass unter dem Begriff Lebenslauf eher die objektiven, sozial-strukturell validen Fakten des Lebens und unter Biografie die mit Sinn versehenen Erfahrungen verstanden werden (vgl. Marotzki u.a. 2005, S. 115). 
Konstrukt aber auch den Prozess des Konstruierens im Sinne eines "Modus Operandi« (Bourdieu 1987, zit. in ebd., S. 35). Danach sind Lebensgeschichten

»immer wieder neu hervorgebrachte Konstruktionsleistungen sozialer Subjekte, die ihre Erfahrungen reflexiv verarbeiten und in Interaktion mit anderen kommunizieren. Subjekte handeln und machen Erfahrungen in je konkreten sozialen Welten. Sie greifen dabei je spezifische Aspekte ihrer Handlungsumwelten und konkrete Situationen auf und >bauen s sie aktiv in ihre Erfahrungsstruktur >ein<. Der Vorgang der biographischen 〉Erfahrungsaufschichtung < ist also keine passive Ablagerung erlebter Situationen >im Individuum<, sondern ein aktiver Prozess biographischer Arbeit, in dem Vergangenes und Zukünftiges, Erfahrung und Erwartung ineinandergreifen.«(Dausien 2006, S. 35, Herv. im Original)

Bei dieser Arbeit handelt es sich nicht um eine bewusste oder bewusst gesteuerte Arbeit der Subjekte, sondern um eine soziale Praxis, die in der Interaktion mit anderen vollzogen wird und im impliziten Wissen reflexiv zugänglich sein kann, aber nicht muss (vgl. ebd.). Innerhalb des Prozesses biografischer Arbeit findet einerseits die Aneignung von Selbst und Welt und andererseits die Konstruktion biografischen Sinns, als performativer Akt der Selbstpräsentation und -konstruktion, statt (vgl. ebd., S. 36). So gesehen sind Biografien »konkrete Konfigurationen, die in konkreten >Handlungsumwelten konstruiert werden« (ebd., Herv. im Original). Biografie kann also sowohl unter einer Produktperspektive (erzählte Geschichten) als auch unter einer Prozessperspektive (Erzählen und andere Praktiken des Doing Biography) untersucht werden (vgl. Dausien 2008, S. 354f.). Biografien sind unter der Produktperspektive dann auch Dokumente eines Subjektivierungsprozesses, die unter der Prozessperspektive einer Analyse zugänglich gemacht werden können. Damit ermöglicht Biografie als Konstruktion den Anschluss an Subjektivierung.

Sozialwissenschaftliche Fragestellungen sind an die Erfahrungen von Menschen gebunden, so auch die Frage nach biografisch entwickelten Vorstellungen zu Beziehungen vor dem Hintergrund des Erlebens von Gewalt, die dieser Arbeit zugrunde liegt. Diese menschlichen Erfahrungen haben eine biografische Bedeutung, die im Rahmen der Analyse im Gesamtzusammenhang der Lebensgeschichte interpretiert wird (vgl. Rosenthal 2008, S. 164).

Rosenthal führt dazu folgende grundlagentheoretische Vorannahmen an, die die Rekonstruierbarkeit sowohl von biografischen Verläufen als auch von gegenwärtigen biografischen Konstruktionen der Subjekte begründen:

- Um soziale oder psychische Phänomene verstehen und erklären zu können, bedarf es der Rekonstruktion ihrer Genese. 
- Um das Handeln von Individuen verstehen und erklären zu können, bedarf es des Blicks sowohl auf die Perspektive der Handelnden als auch auf die Handlungsabläufe selbst.

- Um die Aussagen in biografischen Erzählungen verstehen und erklären zu können, bedarf es deren Interpretation im Kontext des Gesamtzusammenhangs des gegenwärtigen Lebens. (Vgl. ebd., S. 165)

Für die vorliegende Arbeit bedeutet dies, ein vergangenes Phänomen, wie das Erleben von Gewalt in den ersten eigenen Paarbeziehungen, im Kontext der gesamten Biografie zu beleuchten. Die Frage ist, welche Erfahrungen dem benannten Phänomen vorausgingen, welcher Umgang damit und welche weiteren Erfahrungen folgten. Darüber hinaus interessiert die Frage nach dem Einfluss dieser Erfahrungen auf die Beziehungskonzepte der jungen Frauen. Die Frage nach der Subjektbildung ist dabei in der vorliegenden Arbeit außerdem unter dem Gesichtspunkt »Geschlecht « zentral. Die leitende Frage lautet demnach, »wie im biographischen Prozess Konstruktionen von ,Selbst und ,Welt entstehen und wie im gleichen Zug Geschlechterverhältnisse im biographischen Format aufgegriffen, reproduziert, variiert und transformiert werden « (Dausien 2006, S. 37, Herv. im Original). Darin wird eine neue theoretische Perspektive auf Subjektbildung deutlich.

Abschließend kann festgehalten werden, dass ein biografietheoretischer $\mathrm{Zu}$ gang zum Ziel hat, nicht in einer dualistischen Verknüpfung von Individuum und Gesellschaft zu verharren, sondern versucht, Biografie sowie die Konstruktion von Geschlecht als Produkt und Prozess zu denken. (Vgl. ebd., S. 30).

\subsection{Sozialisation und Biografie}

Das dieser Arbeit zugrunde liegende Forschungsinteresse fragt nach dem »Werden und Gewordensein geschlechtlich positionierter und identifizierter Subjekte« (Dausien 2006, S. 17) und bezieht sich damit auf sozialisationstheoretische Fragestellungen, indem das Interesse auch biografisch entwickelten Orientierungen und Erfahrungen während der Sozialisation gilt.

Nachdem allerdings seit den 1990er-Jahren das Konzept der geschlechtsspezifischen Sozialisation vor dem Hintergrund dekonstruktivistischer Theorien, insbesondere der Arbeiten Judith Butlers, massive Kritik erfuhr, kam es zu einer Abkehr von Sozialisationsgedanken. Forscher_innen, die weiterhin sozialisationstheoretische Fragen stellten, mussten sich mit terminologischen Verunsicherungen auseinandersetzen (vgl. Bilden/Dausien 2006, S. 8).

Das Sozialisationskonzept versucht einerseits die Vergesellschaftung der Individuen und andererseits die Individualisierung des Sozialen als dialektischen Prozess zusammenzudenken und zwar »in der Perspektive individueller Biographien« 
(Dausien/Walgenbach 2015, S. 19). Der auf Durkheim zurückgehende Begriff der "Sozialisation« stellte die Antwort auf die Frage dar, wie und unter welchen Bedingungen in modernen Gesellschaften, in denen traditionale Vorstellungen und äußere Zwänge an Wirkung verlieren, noch Integration und Kohärenz hergestellt werden können. Damit entfaltet sich eine neue Perspektive auf Vergesellschaftung, die nach der Verinnerlichung von gesellschaftlichen Regeln und Strukturen fragt (vgl. ebd.). Elias und Bourdieu haben diesen Gedanken der Inkorporation äußerer gesellschaftlicher Strukturen in die innere Struktur der handelnden Subjekte übernommen und versuchten damit, die Dichotomie zwischen Individuum versus Gesellschaft zu überwinden (vgl. ebd.).

In den 1970er-Jahren etablierte sich dann die Forschung zur Geschlechtersozialisation im wissenschaftlichen Feld und ermöglichte als Konzept, Geschlecht nicht mehr als Naturtatsache zu verstehen, sondern als etwas sozial Hervorgebrachtes. Der Aufschwung des Sozialisationsparadigmas während der 1970er- und 1980erJahre war mit einer breiten gesellschaftlich-kulturellen Thematisierung von Geschlecht verbunden und erfüllte als Erklärungsmuster für soziale Ungleichheit wie auch für pädagogische Probleme die Funktion eines gesellschaftlichen Deutungsmusters (vgl. ebd., S. 23f.).

Zwei wissenschaftliche Strömungen trugen dann in den 1980er-Jahren dazu bei, dass es zu einer breiten Diskussion und schließlich zur Abkehr vom Sozialisationsparadigma kam: das Konzept des Doing Gender sowie die Debatten um Dekonstruktion. Das Konzept des Doing Gender ermöglicht(e) »nicht nur, substanzialisierende Vorstellungen von Geschlecht, Geschlechtsidentität oder >weibliche/männliche`Eigenschaften zu kritisieren, sondern bietet eine theoretisch und methodologisch überzeugende Alternative an: Geschlecht wird als eine fortlaufende soziale Praxis verstanden, als etwas, was Akteur_innen in konkreten sozialen Situationen tun« (ebd., S. 25, Herv. im Original). Hinzu kam die dekonstruktivistische Debatte im Anschluss an Derrida, Butler und Foucault, die die Idee des autonomen Subjekts und die Identitätskonzepte in der Geschlechterforschung kritisierten:

»Die dekonstruktivistische Debatte setzt sich zwar nicht direkt mit dem Sozialisationsparadigma auseinander, bewirkt aber eine Perspektivenverschiebung in Richtung der gesellschaftlichen Diskurse und kulturellen Ordnungen, die geschlechtliche Subjekte machtvoll hervorbringen. Vorrangiges Ziel ist die Dekonstruktion kultureller Denksysteme verbunden mit einem zunächst eher theoretischen und politischen, weniger empirisch-forschenden Interesse an irritierenden und subversiven Praktiken.«(Dausien/Walgenbach 2015, S. 26, Herv. im Original)

Die Folge ist, dass geschlechtsspezifische Sozialisation in den Verdacht gerät, die kritisierte Idee eines relativ stabilen weiblichen bzw. männlichen Subjekts weiterzuverfolgen. Die genannten Entwicklungen ziehen zwei problematische Folgen 
nach sich: Zum einen kommt es zu dem Vorwurf, innerhalb des Forschungsfeldes zu substanzialisieren und zu dichotomisieren. Zum anderen geraten nach Walgenbach und Dausien aber auch die den sozialisationstheoretischen Erklärungssätzen zugrunde liegenden Fragen aus dem Blick, nämlich »die Fragen nach dem Werden und Gewordensein konkreter gesellschaftlicher Individuen« (ebd., S. 27).

Die Autorinnen sehen zudem noch eine dritte Entwicklung, die zum Bedeutungsverlust der Sozialisationsforschung führte: die schnelle Entfaltung des Individualisierungstheorems als dominantes Deutungsmuster. Die These der Auflösung oder Relativierung bisheriger Klassen- und Geschlechterlagen bedeutete in Bezug auf die Geschlechterpositionen zwar Verunsicherung, aber auch Befreiung aus der traditionellen geschlechtsspezifischen Sozialisation (vgl. ebd., S. 28). Geschlechterverhältnisse haben damit vermeintlich an Bedeutung verloren, denn »das individualisierte Subjekt scheint nicht mehr auf bestimmte weibliche oder männliche Rollenmuster festgelegt, sondern hat Spielräume, die eigene Biographie zu gestalten und dabei auch sein individuelles Konzept von >Frausein entwerfen und zu erproben « (ebd., S. 29). Es bleibt also festzuhalten, dass das Individualisierungstheorem das Sozialisationsparadigma abgelöst hat. Allerdings erfolgt seine Rezeption verkürzt, indem sich Forschung auf die Individualisierungsprozesse von Subjekten bezieht, aber gesellschaftsbezogene Fragen unzureichend berücksichtigt (vgl. ebd., S. 31). Damit einhergeht, dass »spezifische blinde Flecken in Bezug auf die Bedeutung gesellschaftlicher Transformationen von Geschlechterverhältnissen entstanden sind« (Dausien u.a. 2015, S. 10). Die Frage nach der Vergesellschaftung von Individuen wird nicht gestellt, denn

»die Individuen, die mit der Beck'schen Diagnose in den Blick rücken, werden als gesellschaftliche Akteure gedacht, die sich weitgehend aus den gesellschaftlichen Strukturen gelöst haben und in einer bis dato unbekannten, freilich sriskanten Freiheit ihre Biographie gestalten können, aber auch die >Haftung ‘ für ihre Lebensführung übernehmen müssen. Wie dieser widersprüchliche Prozess theoretisch konzeptionalisierbar wird, welche biographischen Leistungen die Subjekte erbringen und welche sinneren< Erfahrungsstrukturen sie dabei aufbauen, bleibt allerdings weitgehend undiskutiert im Hintergrund der soziologischen Analyse." (Dausien 2006, S. 21, Herv. im Original)

Diese These der Individualisierung wird insbesondere aus subjektivierungstheoretischer Sicht kritisiert. Im kommenden Kapitel wird aufgezeigt, wie sich Forscher_innen in der jüngeren erziehungswissenschaftlichen Debatte mit diesen Fragen nach dem Werden und Gewordensein geschlechtlich positionierter Subjekte unter subjekttheoretischer Perspektive auseinandersetzen. 


\subsection{Subjekttheoretische Perspektiven in der Biografieforschung}

Inzwischen wurde - angestoßen durch gendertheoretische Infragestellungen des problematischen Subjektverständnisses - eine Neuthematisierung des subjekttheoretischen Verständnisses sowie dessen begrifflich-theoretische Klärung innerhalb der Biografieforschung notwendig.

Verschiedene Autor_innen (Rose 2012, Thon 2012, Hanses 2013, Hartmann 2015, Rendtorff 2015) nehmen aktuelle Transformationen gesellschaftlicher Geschlechterverhältnisse zum Ausgangspunkt, bisherige Annahmen von Geschlecht und Sozialisation neu zu denken und weiterzuentwickeln. Im Folgenden beziehe ich mich auf einige ausgewählte Arbeiten, die für mich Anknüpfungspunkte an die vorliegende Forschungsarbeit bieten.

So zeigt Jutta Hartmann (2015) in ihren Überlegungen, wie die Arbeiten Butlers Anschlussmöglichkeiten für grundlegende sozialisationstheoretische Fragestellungen bieten. Dabei geht sie davon aus, dass

»Butlers Konzentration auf die Cleichzeitigkeit von normativer Beschränkung und verändernder Handlungsfähigkeit des Subjekts, mit ihrer Frage, in welcher Art und Weise die Konstruktion heterosexueller Zweigeschlechtlichkeit als ein Ineinander von normativen Zwängen und von Transformationen derselben begriffen werden kann ... Anschlussmöglichkeiten für grundlegende sozialisationstheoretische Fragestellungen auf diskurs- und praxistheoretischer Ebene« (Hartmann 2015, S. 55)

bietet. Sie zeigt zentrale Berührungspunkte zwischen poststrukturalistischen Perspektiven und dem sozialisationstheoretischen Paradigma auf und macht anhand von Studien, die im Anschluss an Butlers Theorien durchgeführt wurden, deutlich, wie anhand diskurs- und praxistheoretischer Überlegungen der Diskussion um Sozialisation und Geschlecht konstruktiv begegnet werden kann (vgl. Hartmann 2015, S. 72). Danach kommt es zu einer Hervorhebung der gesellschaftlich-kulturellen Dimension der Subjektbildung, und »das in seiner Psyche wie in seinem Körper entessentialisierte Subjekt [erscheint; Anm. d. Verf.] mit Blick auf seine Handlungsfähigkeit gleichwohl nicht überdeterminiert« (ebd.). Das Konzept der Performativität ermöglicht, die Dominanz von Strukturen, aber auch Aspekte wie Gewalt und Verletzlichkeit als bedeutsame Sozialisationsfaktoren zu diskutieren und Fragen auf das Wie und Warum von Differenzen zu richten.

»Das in den Praxen liegende Aufrufen wie Untergraben normativer Vorlagen, die Gleichzeitigkeit im Hervorbringen und Verwerfen spezifischer Subjektpositionen, die wechselseitige Wirkkraft verschiedener Differenzkategorien wie die Berücksichtigung damit zusammenhängender Diskriminierungserfahrungen markieren Facetten einer poststrukturalistischen reformulierten Sozialisationstheorie, die 
geeignet dafür erscheint, sich der Komplexität des Geschlechtlichwerdens und -seins anzunähern. Sichtbar werden Spielräume und Ceschlechterordnungen, die mit den bisherigen Theorieperspektiven nicht in den Blick gekommen sind.« (Hartmann 2015, S. 73)

Entgegen den bisherigen sozialisationstheoretischen Ansätzen stehen sich innerhalb der poststrukturalistischen Theorien Subjekt und Diskurs nicht als zwei getrennte Dimensionen gegenüber, sondern das Subjekt wird als stets diskursiv verstanden, und Diskurs wird subjektiv hervorgebracht (vgl. ebd., S. 73). Damit wird das Verhältnis von Autonomie und Heteronomie mit dem Konzept der Performativität anders als in den Sozialisationstheorien theoretisiert. Indem »Subjektivität als entscheidender Ansatzpunkt von Macht und Subjektivierung als zentraler Ort gesellschaftlich-kultureller Aushandlung« (ebd.) angesehen wird, ist das Individuum mittels dieser Struktur als Subjekt konstituiert und nicht in die heteronormative Zweigeschlechtlichkeit integriert (vgl. ebd.). Die Handlungsfähigkeit von Subjekten kann sich danach auch nur im Kontext gesellschaftlich-kultureller Diskurse und Praxen entwickeln. Ansatzpunkte zur Emanzipation bieten die Überlegungen im Anschluss an Foucault (1977, zit. in ebd.) zum "gesellschaftlichen Individuum, das sich seiner Vielfältigkeit gewahr wird und sich zu seinen Weisen der Unterwerfung reflektierend verhält« (ebd.).

Auch Rose (2012) diskutiert diskurstheoretische Anregungen und Herausforderungen biografischer Forschung, die sich aus der Konfrontation mit Implikationen aus der Butlerschen Diskurstheorie ergeben:

»Mit Butler könnte man also sagen, dass das Subjekt in Abhängigkeit von und in Unterwerfung unter normative Diskurse konstituiert wird, die regulieren, was das Subjekt überhaupt sein kann (und damit auch implizit, was es nicht sein kann und darf). Subjektivierung bezeichnet in Butlers Perspektive vor allem die Gleichzeitigkeit der Ermöglichung und der Restriktion des Subjekts im Rahmen von Diskursen, denen das Subjekt in seiner Existenz immer verpflichtet und auf sie verwiesen bleibt. Insofern kann Subjektivierung als normierende und formierende Bildung eines Subjekts in Abhängigkeit von einem Diskurs verstanden werden, der immer schon festlegt, unter welchen Umständen etwas überhaupt zum `Subjekt werden kann, ohne damit subjektive Handlungsfähigkeit gänzlich zu unterminieren.«(Rose 2012, S. 115, Herv. im Original)

Das Subjekt ist also einerseits diskursiven Normen unterworfen, wird aber dennoch von Butler als handlungsfähiges Subjekt entworfen. Handlungsfähigkeit versteht sich dabei als »begrenzte Ermächtigung eines Subjekts im Diskurs« (ebd., S. 116). Durch wiederholendes, performatives Bestätigen werden nach Butler Normen und Ordnungen erst wirksam. Und genau an dieser Stelle, der performativen Herstellung von Normen, sieht Butler die Möglichkeit für Widerstand. Es geht But- 
ler darum, »das normierende und normierte Sprechen gleichsam von innen her zu öffnen und in der Kluft der konstitutiven Nicht-Übereinstimmung, die sich in der Wiederholung ergeben kann, die Möglichkeit für Widerstand anzusiedeln.« (ebd., S. 117). Es sind damit Praktiken gemeint, die durch das nicht ordnungsgemäße Wiederholen diskursive Bedeutungen und Normen verschieben können.

Übertragen auf biografische Forschung bedeutet das, dass nicht erzählte Geschichten über das Werden des Subjekts als Ausgangspunkt genommen werden. Stattdessen werden die diskursiven Regulierungen des Sprechens, also die diesem Sprechen eingelagerten Normen, in die Analyse miteinbezogen, denn »das Erzählen dieser Geschichte lässt das Subjekt erst werden, was es bereits zu sein meint“ (ebd., S. 112, Herv. im Original). Demnach gibt es auch kein Subjekt hinter dem Text, sondern allein der lebensgeschichtliche Text gibt Auskunft über das zur Frage stehende Subjekt und dessen Konstitution. Zudem verweist Rose auf die »Weltverpflichtetheit« (ebd., S. 118) biografischer Texte, die sie als Orte des Aufrufens und Weiterleitens von Diskursen versteht. Aus diesem Grunde folgert sie auch die Notwendigkeit, die dem Sprechen eingelagerten Normen ebenfalls in die Analyse mit einzubeziehen und sich kritisch-reflexiv und dekonstruktiv mit biografischen Texten auseinanderzusetzen. Ziel ist dabei, mithilfe von biografischem Material die »individuellen Formen der Verarbeitung gesellschaftlicher und subjektiver Erfahrungen« (Ehrenspeck 2010, S. 164, zit. in Rose 2012, S. 111) zu analysieren, um Erkenntnisse über biografisch vollzogene oder sich im Erzählen vollziehende Denkund Handlungsmuster zu gewinnen. Das neunte Kapitel dieser Arbeit verfolgt in diesem Zusammenhang empirisch zwei Fragestellungen: Zum einen wird untersucht, wie über die Partizipation an Diskursen Kategorien der (Selbst-)Beschreibung aufgegriffen werden. Zum anderen wird die Perspektive darauf gerichtet, an welchen Stellen dominante Diskurse unterlaufen werden und sich damit Phänomene von Widerständigkeit zeigen.

\subsection{Fazit}

Die vorliegende Arbeit rekurriert aus zwei Gründen auf biografietheoretische Perspektiven:

1. Eine biografietheoretische Perspektive impliziert meines Erachtens immer auch die Annahme individueller Lebensgeschichten, denen eine individuierte Struktur von Haltungen und Dispositionen zugrunde liegt. Biografische Erfahrungsprozesse lassen sich demnach getrennt von kollektiven Prägungen analysieren - wenngleich diese aufeinander bezogen bleiben. Eine biografische Perspektive lohnt sich in der vorliegenden Studie, weil so die adoleszenten und biografischen Verarbeitungsprozesse der befragten jungen Frauen analysiert 
werden können. Zudem ermöglicht der Rückgriff auf biografietheoretische Annahmen, insbesondere in Bezug auf erzähltheoretische Grundlagen (Schütze 1983), Wandlungsprozesse und damit Transformationen der Orientierungsrahmen in den Biografien in den Blick zu nehmen (vgl. dazu auch die Arbeiten von Helsper u.a. 2013; Kramer 2009; Kramer 2013). Konkret bedeutet das, die Perspektive auf die Genese individueller Beziehungsvorstellungen, die Inkorporation von Praktiken und Orientierungen - nicht nur, aber insbesondere auch im Erfahrungsraum Familie -, sowie Transformationen dieser inkorporierten Haltungen und Praktiken zu richten.

2. Biografie wird sowohl als Prozess als auch als Produkt gedacht und verstanden und versucht somit als Konzept, die Schnittstelle zwischen Individuum und Gesellschaft zu erfassen. Mit den thematisierten Fragen nach Handlungsmöglichkeiten von Frauen in Gewaltbeziehungen sowie nach der möglichen Reproduktion und Transformation von Verhaltensweisen und sozialen Verhältnissen werden auch Fragen zu Subjektivität aufgeworfen, weshalb der biografietheoretische Zugang um eine subjekttheoretische Perspektive ergänzt wird. Diesen Fragen liegen diskurstheoretische Überlegungen zugrunde, die davon ausgehen, dass wir den zeitgeschichtlichen Diskursen sowie den strukturellen Bedingungen des Aufwachsens unterliegen bzw. uns mit diesen auseinandersetzen: »Denn eine Biografie wird nicht nur durch die individuelle Erfahrungsgeschichte und durch situative und kommunikative Bedingungen strukturiert, sondern eben auch durch Diskurse, die quer zu den übrigen Konstitutionsprozessen liegen« (Spies 2009, [Absatz 70]). Theorien der Subjektivation sind

"zwingend auf praktikentheoretische bzw. praxeologische Theorien ver- und angewiesen - und das nicht nur, weil praxeologisch betont wird, dass das, was Menschen sind, nicht substantiell zu begreifen ist, sondern auch und in dem entsteht, was sie aus sich selbst - unter naturalen, sozialen und kulturellen Bedingungen machen; sondern vielmehr, weil Subjektivationsprozesse einerseits auf konkrete, materiale, soziale und kulturelle sowie explizit praktische Gestaltungen und deren Dynamik und Prozessualität andererseits verweisen«(Ricken u.a. 2017, S. 205).

Aus den diskurstheoretischen Bezügen leitet sich somit ein praxeologisches Grundverständnis ab, das davon ausgeht, »dass Subjekte aus der Verwirklichung von Körpern in sozialen Praxen entstehen « (Alkemeyer u.a. 2015, S. 25). In diesem Verständnis rückt »das wie vor das was und vor das warum« (Stauber 2014, S. 28, Herv. im Original). Demnach richtet die vorliegende Arbeit ihre Perspektive bei der Frage nach der Herstellung und Gestaltung von Paarbeziehung und dem Umgang mit Gewalterfahrungen darauf, wie die beteiligten Akteurinnen an ihrer eigenen Konstitution mitwirken, wo und wie dominante Normen wiederholt werden. Sie richtet ihren Blick aber auch darauf, wie solche Normen und Diskurse 
unterlaufen und unterbrochen werden können, wie sich individuelle Praktiken also auch diskursiv niederschlagen können, und zeigt damit nicht nur Momente der Unterwerfung, sondern auch der Ermächtigung auf. 



\section{Gewalt in Paarbeziehungen Jugendlicher und junger Erwachsener}

Bevor im kommenden Kapitel die Bedeutung von Liebesbeziehungen im Jugendund jungen Erwachsenenalter sowie Fragen nach Gewalterfahrungen in diesen Beziehungen thematisiert werden, soll vorab eine begriffliche Klärung dessen stattfinden, was in der vorliegenden Forschungsarbeit unter »uggend « und »jungem Erwachsenenalter« sowie unter Liebes- bzw. Paarbeziehung verstanden wird.

Jugendliche und junge Erwachsene Seit mit Norbert Elias (1985) und Ulrich Beck (1986) von der Enttraditionalisierung sozialer Umgangsformen und einer Individualisierung der Lebensgestaltung gesprochen werden kann, durch die die Lebensgestaltung der einzelnen Individuen zunehmend selbstbestimmt und unabhängiger von vorgegebenen Traditionen stattfindet, kommt es zu einer Individualisierung und Pluralisierung von einzelnen Lebensphasen, die sich in ihrem Verhältnis zueinander neu arrangieren (vgl. Hurrelmann/Quenzel 2016, S. 17f.). Demzufolge verliert die für die Persönlichkeitsbildung prägende einzelne Lebensphase, angesichts der Vielzahl von Übergängen zwischen den Lebensphasen, an Bedeutung. Dies ermöglicht eine Zunahme an Chancen der Neugestaltung und -definition des eigenen Lebensentwurfs. Durch diese Optionen sowie die gewachsenen Gestaltungsspielräume »kommt es zu immer offeneren, gewissermaßen fließenden Übergängen zwischen ihnen. Kinder- und Jugendphase, Jugend- und Erwachsenenphase überlagern sich« (ebd., S. 18).

Während Individuen einerseits durch die Freisetzung aus Bindungen immer mehr Entscheidungsmöglichkeiten und Lebensoptionen erhalten, verlieren sie andererseits aber auch zunehmend gesellschaftlich garantierte Verlässlichkeiten, wie bspw. im Hinblick auf einen berechenbaren Lebenslauf (vgl. Hitzler/Niederbacher 2010, S. 12).

>Jugend stellt sich

»mithin nicht mehr als soziostrukturell ıgeregelte`oder zumindest angeleitete Lebensphase dar, die mit dem Ende der Kindheit beginnt, bestimmte Ereignis- und Erlebnisabfolgen impliziert und mit dem Eintritt in das Berufsleben endet. ... Die 
Konturen von `Jugend ‘ als einer Lebensphase verschwimmen.«(Hitzler/Niederbacher 2010, S. 13)

Nach diesem Verständnis wird Jugend als ein Phänomen aufgefasst, das zum einen durch eigenständige Inhalte und Lebensvollzugsformen konturiert ist, zum anderen aber wegen seiner enormen Heterogenität nur schwer zu fassen ist. Das Phänomen >Jugend entstrukturiert sich in fast jeglicher Hinsicht. (Vgl. Hitzler/Niederbacher 2010, S. 9)

Da sich der Lebenslauf »nicht strukturlos denken lässt« (Stauber/Walther 2013, S. 272), sind mit der Entstrukturierung bzw. besser Entstandardisierung des Lebenslaufs Unsicherheiten und Ungewissheiten angesprochen, die die individuellen Handlungsbedingungen beeinflussen:

»Unsicherheit, weil Lebenssituationen entstehen, die wohlfahrtsstaatlich nicht abgesichert sind; Ungewissheit, weil diese Übergangssituationen nicht zuverlässig in einen stabilen und erwartbaren Erwachsenenstatus münden und sich >Normalität< relativiert. «(Stauber/Walther 2013, S. 272)

Demnach wird in der vorliegenden Arbeit das Konzept sjunge Erwachsene in einer »heuristischen Funktion in Bezug auf das Entstehen von Lebenslagen zwischen den herkömmlichen institutionalisierten und abgesicherten Lebensphasen « (Stauber/Walther 2013, S. 270) gesehen. Der entscheidende Bezugspunkt ist die Frage nach den Erfahrungen in den ersten eigenen Paarbeziehungen von Jugendlichen und jungen Erwachsenen. Dabei lässt sich der Zeitraum, in dem diese Erfahrungen gemacht werden, nicht kategorisch in Lebensphasen einteilen.

Mit der vorliegenden Forschungsperspektive werden Jugendliche und junge Erwachsene in engem Bezug auf die Anforderungen und entsprechenden Veränderungen gesehen, die sich durch Prozesse der Entstandardisierung ergeben. Von Interesse sind dabei die besonderen Anforderungen, die sich im Kontext der Aufnahme erster eigener Beziehungen ergeben, sowie der daraus resultierende Umgang mit schwierigen Erfahrungen resp. Gewalterfahrungen.

Paarbeziehungen Eine Paarbeziehung stellt eine Dyade dar und umschreibt somit das Verhältnis von zwei Personen. Karl Lenz (2009) schlägt den Begriff der Zweierbeziehung vor, da er als einheitlicher Begriff für die bestehende Beziehungsvielfalt genutzt werden kann. Im Gegensatz dazu hält er den Begriff der Partnerschaft für ungeeignet, da er seiner Meinung nach bereits mit einem anderen Bedeutungsinhalt besetzt ist, indem unter Partnerschaft »ein kulturelles Ideal für die interne Gestaltung einer Beziehung verstanden« (Lenz 2009, S. 47) wird. Lenz definiert Zweierbeziehung wie folgt:

»Unter einer Zweierbeziehung soll ein Strukturtypus persönlicher Beziehung zwischen Personen unterschiedlichen oder gleichen Ceschlechts verstanden werden, 
der sich durch einen hohen Grad an Verbindlichkeit (Exklusivität) auszeichnet, ein gesteigertes Maß an Zuwendung aufweist und die Praxis sexueller Interaktion oder zumindest deren Möglichkeit - einschließt.«(Lenz 2009, S. 48)

Die Verwendung des Begriffs der Zweierbeziehung grenzt diesen Typus von anderen Begrifflichkeiten wie »Liebesbeziehung«, »Intimbeziehung«, »sexuelle Beziehung« oder »romantische Beziehung « ab, die weniger konkret und konzeptuell ausgearbeitet sind. Dennoch werden in der vorliegenden Arbeit die Begriffe »Paarbeziehung« und »Liebesbeziehung« dem Begriff der »Zweierbeziehung« vorgezogen, da diese sowohl die dyadische Struktur als auch die Aspekte, die mit der Entwicklung von Beziehungsvorstellungen und der Entstehung von Beziehungen zusammenhängen, zum Ausdruck bringen.

\subsection{Liebesbeziehungen im Jugend- und jungen Erwachsenenalter}

Paarbeziehungen im Wandel der Zeit Die (romantische) Liebesbeziehung, wie sie heute sowohl im Alltagswissen als auch in der Familiensoziologie gedacht und verstanden wird, ist eine Entwicklung des Bürgertums im 19. Jahrhundert und dient(e) als Grundlage für die Ehe. Zuvor sicherte die Eheschließung Herrschaftsansprüche, wobei die emotional geprägte Partnerwahl weitgehend unbedeutend war (vgl. Gildemeister/Robert 2008, S. 142).

Liebesbeziehungen sind heute nicht mehr an die Institution Ehe gebunden, und das bürgerliche Modell der »Liebesehe« wird zunehmend durch das Modell der »Partnerschaft« abgelöst. Paarbildung stellt heute stark normative Anforderungen der Gesellschaft an Einzelne. Damit einher geht auch, dass ein Leben mit Partner_in von den meisten als wünschenswerte Perspektive angesehen wird, wobei die Fähigkeiten dazu nicht selbstverständlich gegeben sind, sondern in Kindheit und Jugend erworben werden müssen (vgl. ebd., S. 143).

Die Paarform gilt als Norm und wird in den westlichen Kulturkreisen innerhalb der sogenannten »heterosexuellen Matrix« (Butler 1991) als »exklusive Zweierberziehung« (Gildemeister/Robert 2008, S. 141) institutionalisiert. Diese Institutionalisierung findet dabei als Aneignung, Erlernen und Einüben von Geschlecht ab dem Moment der Geburt statt und bedarf institutioneller Arrangements, die die Zweigeschlechtlichkeit auf sozialstruktureller Ebene reproduzieren und den Beteiligten die Möglichkeit bieten, ihre Geschlechtszugehörigkeit interaktiv zu validieren (vgl. ebd.). Neben Familie, Kindergarten, Schule und Arbeitsmarkt stellt auch das heterosexuelle Paar eines dieser institutionellen Arrangements dar.

Während in der Entwicklungspsychologie die Bedeutung der Aufnahme erster Liebesbeziehungen als wichtige Entwicklungsaufgabe Berücksichtigung findet (Grob/Jaschinski 2003; Oerter/Dreher 2008), sind frühe Paarbeziehungen im Ju- 
gendalter erst in der jüngsten Vergangenheit in den Blick sozialwissenschaftlicher Forschung geraten. Dabei stellt der Aufbau der ersten eigenen Paarbeziehungen eigene Anforderungen dar, die es im Kontext der zahlreichen psychischen, sozialen und biologischen Veränderungen, die das Jugendalter mit sich bringt, zu berücksichtigen gilt. Zu diesen Entwicklungsaufgaben gehören neben der Auseinandersetzung mit den körperlichen Veränderungen der Aufbau von Beziehungen zu Gleichaltrigen, die Aufnahme von Liebes- und sexuellen Beziehungen, der Umbau der Beziehungen zu den Eltern sowie die Vorbereitung auf den späteren Beruf (vgl. Pinquart 2008, S. 4).

Herausforderungen in Liebesbeziehungen Jugendlicher und junger Erwachsener Wendt und Walper (2015) konstatieren, dass sich das Thema Partnerschaften und Sexualität im Jugend- und Erwachsenenalter in den letzten Jahren zunehmend als wichtiges Forschungsfeld etabliert hat (vgl. ebd., S. 227). Demnach widmen sich die Entwicklungspsychologie, die Bindungsforschung, die Sexualforschung, die Gesundheits- und Präventionsforschung sowie die Sozialforschung der Untersuchung von Paarbeziehungen im Jugend- und jungen Erwachsenenalter.

Innerhalb der Entwicklungspsychologie werden Stufen- und Phasenmodelle der Partnerschaftsentwicklung diskutiert, die das Konzept der Entwicklungsaufgaben um die Dimension der Beziehungsgestaltung erweitern (Brown 1999; Furmann/Simon 1999). Dabei wird von einer Entwicklungsfolge ausgegangen, nach der es zu einer Intensivierung der Beziehungen hinsichtlich Emotionalität, Intimität, Sexualität und gegenseitiger Verpflichtung bei gleichzeitigem Umbau der sozialen Beziehungen innerhalb der Peergroup und der Eltern-Kind-Beziehungen kommt (vgl. Wendt 2009, S. 16).

Was allerdings innerhalb der Stufen- und Phasenmodelle nicht sichtbar wird, sind die Erfahrungen, die »in der Realität oftmals mit emotionaler Achterbahnfahrt und teilweise auch großen Herausforderungen verbunden « (Wendt/Walper 2015, S. 228) sind. Ebenso sind die weiter oben konstatierten fließenden Übergänge zwischen einzelnen Erfahrungen in solchen Modellen nicht abzubilden. In der neueren Literatur finden sich deshalb darüber hinaus zwei weitere Perspektiven auf die Frage nach den Herausforderungen in romantischen und sexuellen Beziehungen Jugendlicher und junger Erwachsener: zum einen die Frage nach dem gelingenden und misslingenden Umgang mit (vermeintlich) normativen Anforderungen in intimen Beziehungen und zum anderen die Frage nach dem Umgang mit Abweichungen von diesen vermeintlich normativen Anforderungen (vgl. ebd.). Die Fragestellungen beziehen sich dabei auf konkrete Forschungsfragen, wie bspw. die Frage nach sexuellen Schwierigkeiten in heterosexuellen studentischen Beziehungen (Matthiessen/Dekker 2015) oder die Frage nach dem Einfluss von Bindung und emotionaler Verletzungssensitivität auf das emotionale Erleben von Trennung bei jungen Erwachsenen (Zimmermann/Celik 2015), sodass sie immer nur ausschnitts- 
weise Antworten auf die Frage nach den Herausforderungen von Liebesbeziehungen im Jugendalter liefern können.

Allgemein kann festgehalten werden, dass sich Paarbeziehungen im Jugendalter zunehmend intensivieren und Jugendliche Beziehungskompetenzen erwerben, die ihnen einerseits die Entwicklung eines positiven Selbstbilds von sich als Beziehungspartner_in ermöglichen. Andererseits bilden die erworbenen Fähigkeiten die Grundlage, um im späteren Erwachsenenalter stabile und zufriedenstellende Partnerschaften zu leben und zu gestalten. Die Aufnahme von Paarbeziehungen im Jugendalter geht außerdem mit einem Umbau der sozialen Beziehungen einher, der sich vor allem auf die Neugestaltung der Beziehung zu den Eltern sowie zu den Gleichaltrigen bezieht. So bilden erotische und sexuelle Erfahrungen in Mädchengruppen ein verbindendes Element und wichtige Themen rund um Partnerschaft und Sexualität werden innerhalb der Gruppe ausgetauscht, während in Jungengruppen Paarbeziehungen und Beziehungen zwischen Gleichaltrigen getrennt gelebt werden (vgl. Stich 2005, S. 175).

\subsection{Gewalt in Liebesbeziehungen}

Seit der zweiten Frauenbewegung in den 1970er-Jahren ist das Thema Gewalt gegen Frauen öffentlich und zum Gegenstand politischer Planungen und Interventionen geworden. Der Frauenhausbewegung folgte auch die Forschung über Gewalt im Geschlechterverhältnis.

Gewalt in Liebesbeziehungen Jugendlicher ist dagegen, wie bereits erwähnt, ein Phänomen, das erst jüngst - zumindest im europäischen Raum - in den Blick sozialwissenschaftlicher Forschung gerückt ist (vgl. Kap. 5).

Bei der begrifflichen Klärung des Gewaltbegriffs im folgenden Kapitel sowie der Diskussion der Diskurse um das Phänomen Gewalt beziehe ich mich daher zunächst auf die sozialwissenschaftliche Literatur zum Thema häusliche bzw. familiale Gewalt. Dabei soll als erstes die Problematik des Gewaltbegriffs an sich beleuchtet werden, um dann den Begriff der Partnerschaftsgewalt resp. der häuslichen Gewalt näher zu bestimmen. In einem zweiten Schritt gehe ich dann auf die bisher bestehenden Definitionen zu Gewalt in jugendlichen Paarbeziehungen bzw. zu Dating Violence ein.

\subsubsection{Zur Uneindeutigkeit des Gewaltbegriffs}

Die Verwendung des Gewaltbegriffs in gesellschaftlichen Diskursen birgt verschiedene Gefahren für die wissenschaftliche Analyse, die Heitmeyer und Hagan (2002) als »Thematisierungsfalle« (vgl. ebd., S. 21) bezeichnen. Demnach bestehen unterschiedliche Gefahren wie bspw. die Personalisierung, Pathologisierung, Skandali- 
sierung und Moralisierung sowie Verharmlosung von Gewalt oder auch das Rekurrieren auf vereinfachte Erklärungsschemata zur Erklärung von Gewalt. Heitmeyer und Hagan plädieren aufgrund der Ambivalenz von Gewalt sowie der Unklarheiten über ihre Entwicklungstendenzen zu einem vorsichtigen Umgang mit dem Phänomen Gewalt. Zudem ist »Gewalt « ein problematischer Begriff, da er unscharf ist und verschiedene komplexe Phänomene umfasst. So hält Scherr (2004) fest:

»Die einzige Cemeinsamkeit der vielfältigen Formen der Gewalt liegt wohl darin, dass sie zur Verletzung der physischen und psychischen Integrität der Personen führt.«(Scherr 2004, S. 204)

Imbusch (2002) konstatiert die überlappenden Konnotationen des Gewaltbegriffs mit weiteren inhaltlich ähnlichen Begriffen, wie bspw. Zwang, Aggression, Konflikt und Macht, die die präzise Verwendung des Gewaltbegriffs erschweren (vgl. ebd., S. 27). Die Unschärfe des Gewaltbegriffs hängt mit seiner Abgrenzung zusammen, da sowohl weite als auch enge Definitionen bestehen. Sogenannte enge Definitionen, die sich hauptsächlich auf körperliche Formen von Gewalt beschränken, bergen das Risiko der Untererfassung, da sie subtilere Formen nicht berücksichtigen. Weite Definitionen nehmen neben physischen auch psychische, verbale und weitere Handlungsweisen in den Blick und werden in den Sozialwissenschaften am häufigsten verwendet. Allerdings liegt das Risiko hier in der Übererfassung oder dem Verlust der Ausdifferenzierung nach Form und Schweregrad der Gewalt.

So definiert Galtung Gewalt in einer breit angelegten Definition als

»Diskrepanz zwischen möglicher persönlicher Entfaltung und Verwirklichung und der tatsächlichen, soweit letztere durch die Beeinflussung Dritter verursacht wird « (Reinhold 1992, S. 211).

Zudem erweitert Galtung (1975) die Definition von Gewalt um die strukturelle Dimension und weitet den Gewaltbegriff nicht nur auf das Phänomen soziale Ungleichheit aus, sondern setzt sie mit sozialer Ungerechtigkeit gleich (vgl. Lamnek u.a. 2012, S. 7). Diese Definition greift Phänomene wie Zwang, Herrschaft und Macht, die im Zusammenhang mit Gewalt stehen, auf und erfuhr vor allem im Umfeld sozialer Bewegungen eine breite Rezeption (vgl. Melzer/Schubarth 2015, S. 25).

Einwände löste diese Definition dahingehend aus, dass ihr eine Überladung und Entgrenzung des Gewaltbegriffs vorgeworfen wurde, die dazu führt, dass gewaltfreie Räume kaum noch existent sind (vgl. Lamnek u.a., S. 8). Außerdem wurde die Frage aufgeworfen, inwieweit ein makrosoziologischer Theorieentwurf auch auf der individuellen Ebene greift und ob Umgangsformen in gesellschaftlichen Subsystemen, wie die der Familie, auf diese Weise analysiert und erklärt werden können (vgl. Melzer/Schubarth 2015, S. 25). Eine weitere Unschärfe sehen Lamnek u.a. (2012) in der Wahrnehmung neuer Gewaltthemen, zu der die Gewalt in Part- 
nerschaften zählt, da diese Form der Gewalt erst seit drei Jahrzehnten als solche gesehen und diskutiert wird. Solche neuen Gewaltformen

»sind Gegenstand und Produkt von öffentlich-politischen Diskurszyklen und damit abhängig von Medien, Interessengruppen und jeweils aktuellen politischen Strömungen. Das bedeutet aber auch: Die Phänomene werden öffentlich und nur in dem Umfang diskutiert, der den Interessen der jeweiligen sozialmoralischen Meinungsführer dient«(Lamnek u.a. 2012, S. 8).

Wie die knappe Übersicht zum Gewaltbegriff deutlich macht, kann Gewalt einerseits in personale (physische und psychische Gewalt) und andererseits in institutionelle Gewalt (strukturelle und kulturelle Gewalt) differenziert werden. Kulturelle Gewalt, die Galtung (1990) in Erweiterung seines Konzepts der strukturellen Gewalt definierte, meint dabei diejenigen kulturellen Aspekte, die zur Rechtfertigung oder zur Legitimierung der direkten, illegitimen institutionellen oder strukturellen Gewalt benutzt werden. Das Ziel kultureller Gewalt ist es, andere Formen der Gewalt als rechtmäßig erscheinen zu lassen und sie so zu Formen gesellschaftlich akzeptierter Gewalt zu machen (vgl. Imbusch 2002, S. 40).

An dieser Stelle wird bereits die Schwierigkeit einer einheitlichen Definition von Gewalt deutlich. Außerdem ist es meines Erachtens notwendig, innerhalb der Gewaltforschung auch die subjektive Perspektive der Beteiligten zu berücksichtigen, wie es vor allem in feministischen Diskursen praktiziert wird. Demnach definiert Hagemann-White (1992) Gewalt als »Verletzung der körperlichen oder seelischen Integrität eines Menschen durch einen anderen« (ebd., S. 22) und räumt den betroffenen Frauen das Recht ein, selbst darüber zu bestimmen, was sie in ihrer Integrität verletzt. Mit dieser Auffassung ist die Annahme verbunden, dass ein objektiver Gewaltbegriff nicht existiert (vgl. ebd., S. 24).

Im Hinblick auf häusliche Gewalt bzw. Gewalt in der Partnerschaft bietet es sich für das Verständnis der vorliegenden Arbeit an, sowohl Bezug zu sozialwissenschaftlichen als auch feministischen Definitionen zu nehmen und Gewalt als

"sowohl physische als auch psychische Ausübung bzw. Androhung von Zwang und entsprechenden Verletzungen bzw. Schäden zu thematisieren, nicht nur weil jene nicht selten gemeinsam auftreten, sondern auch weil psychischer Zwang bisweilen als gravierender empfunden wird und psychische Verletzungen langfristigere Auswirkungen auf die Befindlichkeit und das Sozialverhalten der Betroffenen haben können als physische.«(Lamnek u.a. 2012, S. 9)

Neben dem Diskurs um die Definition von Gewalt stellen Rollenzuschreibungen bzw. Rollenfixierungen einen weiteren zentralen Diskurs dar, da diese im Rahmen der Diskussion um Macht ebenfalls eine wichtige Rolle spielen. Dabei geht es immer auch um Fragen der Produktion, Konstruktion und Stigmatisierung von Geschlecht über Gewalt (vgl. Lamnek u.a. 2012, S. 19). 
Die binäre Verwendung der Begrifflichkeiten des Erleidens (Opfer) und des Ausübens (Täter/Täterin) führt in der Forschung einerseits dazu, dass durch die Verwendung des Täter-Begriffs Gewalt personalisiert wird: Der Täter-Begriff »behauptet eine moralische Zurechenbarkeit der Tat gegenüber einer exkulpierenden Auffassung der Gewalt als akteurslosem Geschehnis« (Helfferich u.a. 2016, S. 3).

Andererseits neigen der Opferbegriff ebenso wie der Täterbegriff zu einer Totalisierung, durch die die Heterogenität der Betroffenen, unter dem Merkmal betroffen zu sein, unsichtbar wird. Zudem unterstellt die Verwendung der Täter-OpferTerminologie, dass es eine deutliche Abgrenzung der Rollen der Beteiligten gebe und dass es keine weiteren Rollen wie bspw. Kompliz_innen oder Unterstützer_innen gebe (vgl. Helfferich u.a. 2016, S. 3f.). Die Autorinnen plädieren daher für das Aufbrechen der binären Codierung der Begrifflichkeiten sowie für eine verstärkt prozessuale Sichtweise auf Gewalt.

In Anlehnung an diese Auffassung wird in der vorliegenden Arbeit auf die Verwendung der Begriffe »Opfer« und »Täter« verzichtet. Ausgangspunkt der Fragestellung ist zwar das Erleben von Gewalt innerhalb der ersten eigenen Paarbeziehungen; dennoch wird von Anfang an die komplexe Verwobenheit von Opfer- und Täterpositionen mitgedacht, die sich bspw. in einer interpersonalen Verstrickung der beiden Rollen innerhalb einer Person zeigen können.

Damit Mechanismen der Gewalt weiterführend entschlüsselt werden, bedarf es einerseits der Hinterfragung kategorialer Grundlagen der Zuschreibungen männlich-weiblich und Opfer-Täter, zum Beispiel über die Erfassung des Zusammenhangs von neuen Weiblichkeitskonzepten und der Gewaltbereitschaft junger Frauen in Beziehungen. Andererseits müssen die Wirkung geschlechtsspezifischer Sozialisation sowie Konzepte von Männlichkeit und Weiblichkeit, die Einfluss auf das Erleben von Gewalt nehmen, verstärkt in den Blick genommen werden (vgl. ebd., S. 5). Dem trägt die vorliegende Studie Rechnung, indem sie sich in Kapitel 2 und 3 mit den Veränderungen solcher Konzepte innerhalb der gegenwärtigen Gesellschaft beschäftigt.

\subsubsection{Definition und Formen von Gewalt in jungen Paarbeziehungen}

Während sich innerhalb der Forschungsliteratur zu häuslicher Gewalt unterschiedliche Definitionen finden, lassen sich für Gewalterfahrungen in jungen Paarbeziehungen bzw. in den ersten eigenen Liebesbeziehungen im deutschsprachigen oder auch europäischen Raum bislang kaum Definitionen ausmachen. Das hängt damit zusammen, dass bislang kaum empirische Studien zu diesem Thema vorliegen. In internationalen Forschungsberichten wird der Begriff »Teen Dating Violence« verwendet, wenn auch ohne einheitliche Definition: 
»Researchers of dating violence have utilized a number of definitions. (...) One of the difficulties with these definitions is that the terms >dating ‘ and >courtship ‘ are not adequately defined and seem to apply to a broad range of persons and social activities.«(Pirog-Cood/Stets 1989, S. 5)

Die Autor_innen sehen die Kontaktaufnahme des Datings als dyadische Interaktion, die perspektivisch eine emotionale und/oder sexuelle Beziehung einschließt. Demnach wird Dating Violence wie folgt definiert:

»Our definition of dating violence (1) excludes married individuals and divorced couples who are not attempting to reconcile their relationships; (2) incorporates a range of relationships from the first dates to cohabitation and engagement; and (3) can apply to homosexual as well as to heterosexual relationships.« (PirogCood/Stets 1989, S. 5)

In der »WHO Multi-country study on women's health and domestic violence against women« wird Teen Dating Violence wie folgt definiert:

»Dating violence refers to physical or sexual violence occurring in the context of a sdating relationship is a term that frequently refers in Europe and the United States to neither a marriage nor a long-term cohabiting relationship that occurs during adolescence and young adulthood. These range from casual first encounters to longer-term sexual partnerships. In Asia and other parts of the world where marriage often takes place at a much younger age, the phenomenon of dating violence is rare and intimidate partner violence begins at an earlier age." (WHO/LSHTM 2000, S. 14)

Allerdings ist die Übertragung des Begriffs »Dating Violence«, wie er von den meisten US-amerikanischen Forscher_innen sowie in einer gewissen Breite auch in der internationalen Forschungsliteratur verwendet wird, in den europäischen Kontext aus folgenden Gründen problematisch: Erstens verwenden Jugendliche und junge Erwachsene den Begriff selbst nicht. Und zweitens: »dating seems to imply a degree of formality which does not necessarily reflect the diverse range of young people's intimate encounters and relationships« (Barter 2009, S. 7).

Wie auch bei häuslicher Gewalt werden unter Gewalthandlungen in den Paarbeziehungen Jugendlicher und junger Erwachsener physische, psychische und sexuelle Gewalt bzw. deren Kombinationen verstanden.

In der europäischen und deutschsprachigen Literatur finden sich indessen diverse Umschreibungen des Themengebiets, wie etwa »Negative Partnerschaftserfahrungen im Jugendalter«, "grenzwertige, grenzüberschreitende oder gewalttätige Verhaltensweisen in den ersten Verabredungen oder Liebesbeziehungen unter Jugendlichen« oder »Gewalt in intimen Teenagerbeziehungen«. Innerhalb der so- 
zialpsychologischen Forschung wird einheitlich von »sexueller Aggression unter Jugendlichen« (Krahé 2009, Krahé/Scheinberger-Olwig 2002) gesprochen.

Für die Bestimmung des Gegenstands "Gewalt in jungen Paarbeziehungen«, den ich mit der vorliegenden Studie näher beleuchten möchte, halte ich folgende Aspekte für zentral:

- Erste intime und/oder sexuelle Erfahrungen bzw. die Aufnahme erster Liebesbzw. Paarbeziehungen erfolgen in der Regel im Jugendalter (vgl. BZgA 2006), können aber auch erst zu einem späteren Zeitpunkt, im jungen Erwachsenenalter, stattfinden.

- Im Unterschied zu späteren Partnerschaften geht es bei der Aufnahme erster Liebesbeziehungen darum, erste Erfahrungen mit dem bzw. der Freund_in zu sammeln. Es geht bspw. darum zu lernen, seinen Gefühlen Ausdruck zu verleihen, die Gefühle und Wünsche des Gegenübers zu erkennen sowie erste sexuelle Erfahrungen zu machen.

- Dabei sind Jugendliche mit unterschiedlichen sozialen Erwartungen seitens der Eltern und der Peers konfrontiert, die mit einem Umbau der sozialen Beziehungen einhergehen. Es gilt auszuloten, wie viel Zeit mit dem Freund oder der Freundin verbracht werden kann und wie viel Zeit in die Beziehungen mit anderen Gleichaltrigen investiert wird.

Diese Erfahrungen sind keinesfalls einheitlich positiv geprägt, sondern es kann innerhalb dieser Erfahrungen zu negativen, grenzwertigen, grenzüberschreitenden oder gewalttätigen Erlebnissen bzw. Übergriffen kommen.

Im Mittelpunkt der vorliegenden Arbeit steht das Erleben der jungen Frauen, die retrospektiv von ihren ersten eigenen negativen und gewaltvollen Beziehungserfahrungen berichten. Dabei habe ich bei meinem methodischen Vorgehen bewusst auf Definitionen von Gewalt verzichtet, um, wie weiter oben ausgeführt, die subjektive Deutung der Frauen dessen, was sie selbst als Verletzung ihrer eigenen Integrität erleben, zu erfahren (vgl. ausführlicher dazu auch Kap. 7.2 »Methodische und methodologische Überlegungen zur empirischen Erhebung von Gewalt in Paarbeziehungen«).

\subsubsection{Zur Entstehung von Gewalt in Paarbeziehungen}

Mit der Frage nach der Entstehung und der Ursache von (familialer bzw. partnerschaftlicher) Gewalt beschäftigt sich eine Vielzahl von Theorien (vgl. dazu Albrecht 2002; Eisner 2002; Gelles 2002; Hagemann-White 2002; Tedeschi 2002; Lamnek u.a. 2012). Insgesamt wurden drei allgemeine theoretische Analyseebenen zur Erklärung von Gewalt entwickelt: die intra-individuelle Ebene, die sozialpsychologische Ebene und die soziologische oder soziokulturelle Ebene (vgl. Gelles 2002). 
Das psychiatrische Modell (intra-individuelle Ebene) nimmt vor allem die Persönlichkeitsmerkmale der gewaltausübenden Personen in den Blick und sieht diese als wichtigste Determinante für die Gewaltausübung und Misshandlung nahestehender Personen an. Im Gegensatz dazu geht das sozialpsychologische Modell (Mikroebene) davon aus, dass Gewalt am besten durch die Untersuchung äußerer Umweltfaktoren verstanden werden kann, die auf die alltäglichen Interaktionen zwischen Menschen mit enger Bindung einwirken. Sozialstrukturelle Modelle (Makroebene) analysieren Gewalt im Zusammenhang mit sozial strukturierten Variablen wie Ungleichheit, Patriarchat, kulturellen Normen und Familienbeziehungen. (Vgl. Gelles 2002, S. 1067)

Eine Verknüpfung von makro- und mikrosozialen Dimensionen findet sich in soziologischen Erklärungen für Gewalt: Das sogenannte Makro-Mikro-MakroModell schließt dabei als metatheoretische Klammer eine Vielzahl von theoretischen Konzepten und empirischen Ergebnissen ein:

»Im Rahmen des Makro-Mikro-Makro-Modells der soziologischen Erklärung werden makrosoziale Phänomene (z.B. die Belastungen von Gesellschaften mit familialer Gewalt) auf mikrosoziale Strukturen und Prozesse (u.a. gewaltförmige Interaktionen) und diese wiederum auf Makrophänomene (beispielsweise geschlechts- oder milieutypische Sozialisationsmuster) zurückgeführt. (Lamnek u.a. 2012, S. 87)

Im Folgenden wird nun auf diejenigen Erklärungsansätze eingegangen, die auch zu einem besseren Verständnis von Gewalt in den ersten eigenen Paarbeziehungen beitragen.

Ressourcen- und Machttheorien gehen dabei davon aus, dass jedes soziale System, und damit auch die Familie, in gewissem Maße auf Zwang oder der Androhung von Zwang basiert (vgl. Gelles 2002, S. 1068). Dabei können eigene Interessen gegenüber den Interessen anderer, auch ohne Gewaltanwendung oder Gewaltandrohung, besser durchgesetzt werden, je mehr persönliche, soziale, materielle oder immaterielle Ressourcen dem Einzelnen zur Verfügung stehen. Das Drohen mit Gewalt oder der Einsatz von Gewalt kann als Äquivalent für das Fehlen von Ressourcen angesehen werden und ist umso wahrscheinlicher, je weniger Ressourcen dem Einzelnen zur Erreichung seiner Ziele zur Verfügung stehen (vgl. Lamnek u.a. 2012, S. 89). Auch Böhnisch (2001) sieht die Ursachen von Gewalt in dem Versuch von Individuen, Anforderungen und Spannungszustände zu bewältigen. Er erweitert die Ressourcentheorie um den Bezug auf gesellschaftliche Dimensionen und sieht ebenfalls kulturelle Normen, soziale Lernprozesse und die strukturelle Überforderung der Familie als Ursachen für häusliche Gewalt (vgl. Lamnek u.a. 2012, S. 107). Feministische Ansätze weisen darauf hin, dass vor allem Frauen, die wirtschaftlich und sozial von ihrem Partner abhängig sind, auch eher gefährdet sind, Gewalt durch diesen zu erleben (vgl. ebd., S. 104). 
Lerntheoretische Erklärungen sehen die Entstehung von Gewalt in der kindlichen Sozialisation verankert. Familie wird hier als der erste und zentrale Ort angesehen, an dem der Umgang mit Ärger, Frustration, Krisen und negativen Emotionen erlernt wird. Demnach haben Kinder, die Gewalt erlebt oder zwischen ihren Eltern beobachtet haben, ein höheres Risiko, als Erwachsene zu Gewaltausübung zu neigen. Aussagen wie »Du warst böse, also hast du Prügel verdient « führen da$\mathrm{zu}$, dass Kinder Rechtfertigungen für gewalttätiges Verhalten erlernen (vgl. Gelles 2002, S. 1068). Dabei richtet sich Gewalt als erlernte Reaktion auf eine Frustration oder auf Ärger in der Regel nicht auf den Verursacher dieses Ärgers, solange dieser als überlegen erlebt wird, sondern sie wendet sich auf ein Ersatzobjekt, das einem weniger gefährlich und unterlegen erscheint (vgl. Lamnek u.a. 2012, S. 94). Studien, die die intergenerationale Weitergabe von Gewalt innerhalb von Familien belegen, stützen diese lerntheoretischen Annahmen zur Erklärung familialer Gewalt (vgl. dazu unteren Abschnitt zur intergenerationalen Transmission von Gewalt).

Die Stress- oder Stressbewältigungstheorie befasst sich mit der Frage, weshalb es in manchen Situationen zu Gewalt kommt und in anderen nicht. Zwei Hauptfaktoren werden als Ursachen für die Entstehung von Gewalt angesehen: zum einen strukturbedingter Stress in Verbindung mit mangelnden Bewältigungsmöglichkeiten und zum anderen kulturelle Normen, die der Ausübung von Gewalt zugrunde liegen (vgl. Gelles 2002, S. 1068).

Feministische und gendertheoretische Erklärungsmodelle gehen in ihrer Konzeptualisierung von Gewalt zwischen Intimpartner_innen von einem asymmetrischen Verhältnis aus. Das bedeutet, dass es um eine Machtfrage innerhalb hierarchischer Beziehungen geht und dass diese Form der Gewalt innerhalb des Alltags bis zu einem gewissen Maße allgemein üblich ist und toleriert wird (vgl. Dobash/Dobash 2002, S. 931f.). Im Mittelpunkt der Theorie stehen ökonomische und soziale Prozesse, die die patriarchalische soziale Ordnung und Familienstruktur direkt oder indirekt fördern (vgl. Gelles 2002, S. 1070).

Die Frage nach Gewalt im Kontext des Geschlechterverhältnisses wurde in den 1970er-Jahren im Kontext der Frauenbewegung gestellt und stellte dabei - auch innerhalb der Sozialwissenschaften - einen Verstoß gegen die soziale Ordnung dar (vgl. Hagemann-White 2002, S. 124). Auf diese Anfänge der Forschung zur Problematik der Gewalt in intimen Partnerschaften wird im folgenden Abschnitt eingegangen.

Gewalt im Geschlechterverhältnis Zunächst wurde Gewalt in Familien von der Familiensoziologie thematisiert und ressourcentheoretisch erklärt, wobei »aber eine heute kaum noch vorstellbare Selbstverständlichkeit des männlichen Machtanspruchs über Frau und Kinder« (Hagemann-White 2002, S. 124) unterstellt wurde. Dieser impliziten, nicht hinterfragbaren Ordnung im Geschlechterverhältnis sprach die feministische Bewegung mit ihrem Slogan »Das Private ist politisch!« jede Legi- 
timität ab. Die Illegitimität der Ausübung von Gewalt im Geschlechterverhältnis und die Anwendung von Gewalt als Bestandteil von Machtverhältnissen galt es zu entlarven (vgl. ebd.). Für die Gender-Perspektiven auf Gewalt war somit eine feministische Analyse prägend, die die Verortung von Gewalt im Geschlechterverhältnis ansiedelt. Die Gender-Perspektive auf Gewalt findet sich zudem aufgrund übergreifender internationaler wie auch lokal gewachsener Dimensionen in unterschiedlichen Diskursen (vgl. ebd., S. 125). Mit der Entdeckung der empirischen Häufigkeit, mit der Gewalt von Männern gegen Frauen ausgeübt wurde, der Erkenntnis, dass diese vor allem im Rahmen eines Beziehungskontextes stattfindet, sowie der Tatsache, dass die Taten für den Täter meist sanktionsfrei und folgenlos blieben, bildete sich die These von Gewalt gegen Frauen als Kennzeichen des Patriarchats. Die Analyse nimmt jedoch nicht nur Mädchen und Frauen als Betroffene in den Blick, sondern auch Männer, die Opfer geschlechtsbezogener Gewalt werden, wenn sie der hegemonialen Männlichkeit nicht entsprechen:

»Allgemein wird interpersonelle Gewalt von feministischer Seite danach befragt, welchen Stellenwert sie bei der Fortschreibung einer normativ gestützten Ungleichheit der Geschlechter hat, während umgekehrt die jeweiligen Geschlechterverhältnisse danach beurteilt werden, inwiefern Cewalt mit Männlichkeit assoziiert und zur Normalität gehört.« (Hagemann-White 2002, S. 127)

Der Begriff »Gewalt im Geschlechterverhältnis« wurde erstmals in einer Bestandsaufnahme des Hilfesystems zu Gewalt gegen Mädchen und Frauen (HagemannWhite 1992) verwendet. Der nunmehr neue Begriff sollte einerseits die Erkenntnisse, dass sexueller Missbrauch in engem Zusammenhang mit anderen Formen der Gewalt gegen Frauen steht, sowie andererseits die Feststellung, dass auch Jungen und Männer Opfer werden und Frauen Täterinnen sein können, zum Ausdruck bringen. Gewalt im Geschlechterverhältnis ist demnach

»jede Verletzung der körperlichen oder seelischen Integrität einer Person, welche mit der Ceschlechtlichkeit des Opfers oder Täters zusammenhängt und unter Ausnutzung eines Machtverhältnisses durch die strukturell stärkere Person zugefügt wird.«(Hagemann-White 1992, S. 23)

Hagemann-White führt weiter aus:

»Dazu gehören sowohl die Befriedigung sexueller Wünsche auf Kosten eines Opfers oder gegen dessen Willen, wie auch alle Verletzungen, die aufgrund einer vorhandenen geschlechtlichen Beziehung (oder zwecks Durchsetzung einer solchen) zugefügt werden.«(Hagemann-White 1992, S. 22)

Sie deutet auf die Notwendigkeit hin, aus heutiger Sicht auch diejenigen absichtlichen Verletzungen zu benennen, die der Durchsetzung der heteronormativen Matrix, also der heteronormativen Ordnung der Zweigeschlechtlichkeit, dienen. 
Die Bezugnahme auf ein strukturelles Machtgefälle in der Definition deutet auf die gesellschaftliche und politische Verantwortung für Intervention hin. Der Verweis »strukturell stärker« in der o.g. Definition bezieht sich in der Regel »aufgrund der geschlechtsspezifisch ungleichen Verteilung von Besitz, Einkommen, Arbeit, politischer Macht und symbolischem Kapital« (Stövesand 2010, S. 83) auf Männer. Sie lässt aber eine geschlechtsspezifische Zuschreibung offen. Demnach ist innerhalb der Begrifflichkeit »Gewalt im Geschlechterverhältnis« beides enthalten: die Verwobenheit von Frauen und Männern in die Thematik (vgl. ebd.). Zu betonen ist außerdem, dass es unbestritten Gewalt gibt, die sich vor allem gegen Männer richtet, wie bspw. Straßenkriminalität. Zudem können strukturelle Machtgefälle durch Abhängigkeiten entstehen, z.B. am Arbeitsplatz oder durch Verhältnisse sozialer und rechtlicher Ungleichheit (vgl. Hagemann-White 2016, S. 19).

Abschließend lässt sich festhalten, dass die Entwicklung von Forschung zu geschlechtsbezogener Gewalt im deutschsprachigen Raum meist der Theoriediskussion folgte, die sich auf feministische Analysen von Gewalt im Geschlechterverhältnis bezog. Rein machttheoretische Erklärungen sind dabei jedoch bald in den Hintergrund getreten und sozialisationstheoretische Fragestellungen dazu gekommen (vgl. ebd., S. 134). Insgesamt rücken jedoch komplexere theoretische Fragestellungen in den Vordergrund, die sich auf jeweils unterschiedliche Theorietraditionen beziehen und dementsprechend unterschiedliche Fragestellungen entwickeln. ${ }^{1}$

\subsection{Fazit}

Abschließend lässt sich festhalten, dass nicht nur die inhaltlichen Aspekte des Gewaltbegriffs, sondern auch die Frage nach den Ursachen von Gewalt bis heute Gegenstand anhaltender Diskussionen sind. Heitmeyer und Hagan (2002) konstatieren, dass bereits mit der Festlegung dessen, was als Gewalt gelten soll, die Probleme der Gewaltforschung beginnen (vgl. Heitmeyer und Hagan 2002, S. 16). Zudem ist durch die vielfältige Aufweichung von moralischen, sexuellen, erzieherischen und rechtlichen Normen und Werten eine klare Festlegung, was als Grenzüberschreitung gilt, kaum noch möglich. Aufgrund einer höheren Sensibilität (z.B. hinsichtlich Vergewaltigung in der Ehe) ergeben sich neue Grenzmarkierungen von Gewalt. So ist "gerade weil der Problembereich Gewalt in besonderem Maße uneindeutig ist, ... eine erhöhte Sensibilität und Reflexivität geboten« (ebd., S. 16).

In diesem Sinne wird hinsichtlich einer Begriffsdefinition dessen, was in der vorliegenden Arbeit unter Gewalt verstanden wird, an dieser Stelle nochmals explizit der Bezug auf sozialwissenschaftliche und feministische Definitionen von

1 Eine Übersicht zu diesen unterschiedlichen Theorietraditionen und den aus diesen entwickelten Fragestellungen findet sich bei Hagemann-White 2002, S. $134 \mathrm{ff}$. 
Gewalt hervorgehoben. Demnach wird Gewalt im Hinblick auf physische, psychische, verbale und weitere, vorab nicht näher bestimmbare, Dimensionen in den Blick genommen. Es wird eine offene (Forschungs-)Haltung eingenommen, die es den befragten Frauen ermöglichen soll, selbst darüber zu bestimmen, was sie als Gewalt definieren und was sie in ihrer Integrität verletzt. Albrecht (2002) plädiert mit Blick auf die weitere und zukünftige Forschung zu Gewalt für eine differenzierte Perspektive, in der insbesondere psychische und soziale Faktoren nicht isoliert betrachtet werden und Akteur_innen nicht als Objekte, sondern als Gestalter_innen von Bedingungen gedacht werden (vgl. Albrecht 2002, S. 801). Im Anschluss daran verfolgt die vorliegende Arbeit einen gänzlich offenen Zugang zum untersuchten Phänomen der Gewalt, indem eben nicht vorab festgelegt wird, was als Gewalt verstanden wird und was nicht; sondern indem den interviewten Frauen die Möglichkeit gegeben wird, selbst darzulegen, was sie als Gewalt definieren.

Hinsichtlich der Konzeption von Forschungsprojekten und Gewalt - und diese schließt auch die Fragen nach den Erklärungsversuchen von Gewalt mit ein muss unbedingt kritisch untersucht werden, dass individuelle und soziokulturelle Variablen kombiniert werden.

An diese Forderung knüpft das vorliegende Forschungsvorhaben an, indem es die Wechselbeziehung von individuell-biografischem Handeln und gesellschaftlichen Rahmenbedingungen in den Blick nimmt. 



\section{Stand der Forschung}

Aus der Frauen- und Geschlechterforschung liegt eine Vielzahl an Studien zum weiblichen Lebenszusammenhang und zu weiblichen Lebensentwürfen vor, die sich auf Konzepte des »doppelten Lebensentwurfs« oder der »doppelten Vergesellschaftung « und damit auf Individualisierungsansätze beziehen (Beck-Gernsheim 1983; Becker-Schmidt u.a. 1983; Gilligan 1984; Dausien 1996; Geissler-Oechsle 1996; Flaake 1998; Schwiter 2011). Einigkeit besteht inzwischen darin, dass von Geschlecht als sozialer Konstruktion, also dem »Doing Gender « als interaktive Konstruktion, ausgegangen wird, statt nach Differenzen zwischen den Geschlechtern zu suchen. Mit dem Übergreifen des Individualisierungsprozesses auf weibliche Lebensläufe haben traditionelle Geschlechtervorstellungen an Wirkungskraft eingebüßt und damit auch die primäre Familienorientierung junger Frauen aufgelöst. Das steigende Interesse an Frauen als Arbeitskräften sowie die staatliche Bildungspolitik führten dazu, dass die Qualifizierung von Frauen und infolge davon ihre Selbständigkeit vorangetrieben wurden (vgl. Peuckert 2012, S. 405f.). Aktuelle Modernisierungsprozesse verändern somit wesentlich die Lebenslagen von Frauen und beeinflussen ihre Lebensthemen sowie ihre Erwartungen an Beziehungen. Aber Frauen sind auch Akteurinnen innerhalb dieses sozialen Wandels, und als solche werden sie in der vorliegenden Arbeit verstanden.

Anhand einiger ausgewählter Studien soll im Folgenden zunächst ein Überblick über die empirischen Forschungsergebnisse zu Beziehungskonzepten und Lebensthemen junger Frauen gegeben werden. Dies ist notwendig, da die vorliegende Arbeit an diese Ergebnisse anknüpft, indem sie ebenfalls die Beziehungsvorstellungen von jungen Frauen sowie die Entwicklung dieser Beziehungskonzepte in den Blick nimmt, diese aber zusätzlich vor dem Hintergrund von Gewalterfahrungen analysiert.

In Kapitel 5.2 schließt sich eine Übersicht über den Stand der Forschung zu den Themenbereichen Prävalenz von Gewalt in jungen Paarbeziehungen sowie zu Risikofaktoren, Unterstützung und Prävention an. 


\subsection{Forschungsergebnisse zu Beziehungskonzepten und Lebensthemen junger Frauen}

In einem Forschungsprojekt gingen Micus-Loos u.a. (2016) der Frage nach, mit welchen normativen Anforderungen junge Frauen im Rahmen ihrer Berufsorientierungen, aber auch ihrer Lebensplanung insgesamt konfrontiert sind, inwieweit normative Orientierungsmuster bestätigt, aber auch, wo sie infrage gestellt werden. Die Ergebnisse zeigen, mit welch vielfältigen normativen und dabei teils widersprüchlichen Anforderungen junge Frauen konfrontiert sind. Junge Frauen wollen und versuchen Familie und Beruf zu vereinbaren und ein Sowohl-als-auch zu ermöglichen. Dabei werden die bestehenden Orientierungen und Anforderungen als in einem spannungs- und widerspruchsreichen Verhältnis zueinanderstehend verstanden und diskutiert:

»Während nämlich eine zu starke Orientierung an einer eigenen Berufstätigkeit die Gefahr birgt, mit normativen Anforderungen an >gute<Mutterschaft in Konflikt zu geraten ..., geht eine zu starke Fokussierung auf Mutterschaft mit dem Risiko einher, keine finanzielle und persönliche Unabhängigkeit zu erreichen, die den befragten Frauen sehr wichtig ist.«(Micus-Loos u.a. 2016, S. 202)

In allen Gruppendiskussionen der Studie wurde die Realisierung von Vereinbarkeit als individuelle Herausforderung und selbst $\mathrm{zu}$ lösendes Entscheidungsproblem interpretiert (vgl. ebd.). Der Typus der »doppelten Lebensplanung « findet sich auch in der viel zitierten Studie von Geissler/Oechsle (1996) neben einer unerwarteten Vielfalt von Lebensläufen, die sich im Zuge der Modernisierung des weiblichen Lebenslaufs ergeben.

Hirseland und Leuze (2010) gingen der empirischen Frage nach, inwieweit sich der Übergang vom »Familienernährermodell« zum »Selbsternährermodell« im Beziehungskonzept von Frauen und Männern niederschlägt. Beziehungskonzept meint dabei

»ein Set subjektiver Deutungsmuster, die mehr oder weniger bewusste Vorstellungen darüber enthalten, wie eine gute Beziehung aussehen sollte, welchen Eigenwert Beziehungen annehmen und welche Formen wechselseitiger Verpflichtung oder - die Individualisierungsoption steigernder - Entpflichtungen zwischen den Partnern erwünscht sind.«(Hirseland/Leuze 2010, S. 194)

Die Autor_innen unterscheiden idealtypisch zwei Formen von Beziehungskonzepten, kollektivistische und individualistische Formen, die auf unterschiedliche Formen beziehungspraktischer Alltags- und Lebensgestaltung bei Paaren verweisen. Während der Typ des kollektivistischen Beziehungskonzepts sich an der traditionellen Ehe orientiert und die Beziehung, als das zu schützende Gut, den Referenzrahmen für das individuelle Handeln bildet, steht beim Typ des individualistischen 
Beziehungskonzepts die Wahrung oder Maximierung der persönlichen Freiheit an vorderster Stelle. Dem Forschungsvorhaben lag die These zugrunde, dass gemäß der Individualisierungsthese die Vorstellungen zu Paarbeziehungen durchgängig zum Typus des individualistischen Beziehungskonzeptes hintendieren müssten. Die Ergebnisse zeigten, dass sich »Elemente des traditionalen Beziehungskonzepts durchaus mit individualistischen Formen der Ausgestaltung von Beziehungen vereinbar« (ebd., S. 205) zeigen. Zudem ergaben sich Hinweise, die für eine Erosion des traditionalen Leitbildes mit festgefügten Beziehungsordnungen sprechen. Stattdessen wird eine Vervielfältigung von Beziehungskonzepten deutlich, die nicht eindeutig einem Typus zuzuordnen sind. Insgesamt zeigt sich jedoch, dass die Verbreitung individualistischer Orientierungen mit steigendem Bildungsstatus und mit abnehmendem Einkommensstatus zunimmt.

Die Studie von Helfferich (2004) zu Lebensläufen und Familienplanung von Frauen kommt zu dem Ergebnis, dass feste Partnerschaften und Treue nach wie vor eine hohe Relevanz für die befragten 20 - bis 44-jährigen Frauen besitzen. Hinsichtlich der Familiengründung ist die Auffassung verbreitet, dass man nicht mehr verheiratet sein muss, wenn man ein Kind bekommt. Es wird aber dennoch oft geheiratet, wenn die Frau schwanger ist. Bezüglich der Vorstellungen zu Familie und Beruf halten es nur sehr wenige für ideal, wenn Frauen mit kleinen Kindern den Beruf aufgeben. Hinsichtlich des Engagements des Partners gehen die Meinungen deutlich auseinander. So möchte nur ein Drittel der Frauen mit niedriger Bildung aus den westlichen Bundesländern, dass ihr Partner reduziert arbeitet. Frauen mit der höchsten Bildung plädieren am stärksten für eine Erwerbstätigkeit von Müttern und familiäres Engagement des Partners (72 \%). Frauen aus den östlichen Bundesländern gaben an, auch mit kleinen Kindern arbeiten zu wollen, ohne dass der Partner seine Arbeitszeit reduziert (27\%). (Vgl. Helfferich 2004, S. 7)

Keddi (2003) stellt in ihrer Untersuchung zu Lebensthemen und biografischem Handeln junger Frauen dem Konzept des doppelten Lebensentwurfs eine Perspektive gegenüber, die für eine Offenheit bezüglich der subjektiven Handlungs- und Konstruktionsprozesse von jungen Frauen plädiert und diese nicht vorschnell auf geschlechtstypische Konstruktionen festlegt. Das zentrale Ergebnis ihrer Arbeit ist, dass diverse Lebensthemen für das biografische Handeln der befragten Frauen bedeutsam sind; dass diese Frauen sich aber an einem Lebensthema orientieren und dieses handlungsleitend und strukturierend auf die Biografie wirkt.

Die Ergebnisse der empirischen Studien machen die oben genannten Aspekte, die sich aus den Modernisierungsprozessen ergeben und auf die Lebenslagen und Lebensverläufe von jungen Frauen einwirken, deutlich. Besonders hervorzuheben sind dabei zwei Ergebnisse: zum einen das, ebenfalls bereits erwähnte, gleichzeitige Bestehen von traditionalen und modernen Lebensentwürfen und zum anderen der individualisierte Umgang mit den normativen Anforderungen, insbesondere was die Vereinbarkeit von Familie und Beruf anbelangt. Deutlich wird, dass As- 
pekte des traditionalen Beziehungskonzepts, Werte wie beispielsweise Treue oder Ehe, eine hohe Wertschätzung erfahren; so wird, wenn auch spät, häufig (wieder) geheiratet, wenn gemeinsame Kinder da sind. Allerdings hat sich die Ausgestaltung von Beziehungen verändert. Weil eine Vielzahl an Optionen besteht, müssen Paare intern aushandeln, wie sie gemeinsam leben wollen.

\subsection{Forschungsstand zu Gewalt in den ersten eigenen Paarbeziehungen}

Im Gegensatz zu US-amerikanischer Forschungsliteratur, die sich seit über zwanzig Jahren unter den Begriffen »Dating Violence« oder »Teen Dating Violence« mit dem Thema Gewalt in jugendlichen Paarbeziehungen befasst, ist dieses Themenfeld in Europa erst in den vergangenen Jahren in den Blickpunkt der Forschung geraten. Im Folgenden soll zunächst ein Einblick in die empirischen Befunde der USamerikanischen Studien gegeben werden. Aufgrund der begrenzt möglichen Übertragbarkeit der Erkenntnisse auf den europäischen Kontext (s.u.) werden im Folgenden schwerpunktmäßig die Ergebnisse aktueller Studien vor allem aus Großbritannien, der Schweiz und Deutschland beleuchtet.

US-amerikanische Studien bieten bislang den genausten Einblick in die Gewalterfahrungen Jugendlicher während der ersten eigenen Beziehungserfahrungen. In der repräsentativen Youth Risk Behavior Surveillance System (2014) wurden 13.583 Schüler_innen hinsichtlich ihrer Erfahrungen mit körperlicher und sexueller Gewalt befragt (Kann u.a. 2014). Unter den 73,9\% der Befragten, die in den vergangenen zwölf Monaten vor der Befragung eine Verabredung hatten oder in einer Beziehung waren, erlebten 10,3\% körperliche Gewalt; 10,4 \% erlebten sexualisierte Gewalt, wobei bei beiden Gewaltformen der Anteil der betroffenen weiblichen Jugendlichen fast doppelt so hoch war (von körperlicher Gewalt waren $13 \%$ der Mädchen und 7,4 \% der Jungen, von sexualisierter Gewalt 14,4 \% der Mädchen und 6,2 \% der Jungen betroffen) (vgl. ebd., S. 1of.).

In der Tween and Teen Dating Violence and Abuse Study wurden 1043 Tweens zwischen 11 und 14 Jahren, 532 Eltern von Tweens und 626 Teens zwischen 15 und 18 Jahren erfasst. Die Ergebnisse zeigen, dass jugendliche Paarbeziehungen früher beginnen als bisher angenommen. Demnach gab fast die Hälfte der 11- bis 14-Jährigen an, eine Beziehung zu haben. Die Studie belegt außerdem einen $\mathrm{Zu}$ sammenhang zwischen der frühen Aufnahme sexueller Aktivitäten und dem Erleben von Gewalt. So gaben 69 \% der Befragten, die im Alter von 14 Jahren oder jünger ihre ersten sexuellen Kontakte hatten, an, in irgendeiner Form Gewalt erlebt $\mathrm{zu}$ haben. Bei älteren Jugendlichen, die erst zu einem späteren Zeitpunkt sexuelle Erfahrungen machten, war der Anteil an Gewalterfahrungen geringer. (Vgl. Liz Claiborne Inc. 2008) 
Die Studie des amerikanischen National Council on Crime and Delinquency, die den Schwerpunkt auf körperliche Gewalt in jugendlichen Paarbeziehungen legte, bestätigt die Ergebnisse verschiedener anderer Studien, wonach eine von drei Jugendlichen Opfer interpersonaler Gewalt wird. Außerdem zeigt sie auf, dass weibliche Jugendliche, die Gewalt in Teenagerbeziehungen erleben, auch deutlich häufiger anderen Formen von Gewalt ausgesetzt sind und sie außerdem zu selbstgefährdendem und risikoreichem Verhalten neigen. So zeigen sie häufiger gesundheitsschädigendes Verhalten wie Drogen- oder Alkoholkonsum und haben häufiger Suizidgedanken als junge männliche Erwachsene oder Frauen ohne Gewalterfahrungen (vgl. Davis 2008). Bezüglich der Häufigkeit und des Ausmaßes verschiedener Gewaltformen gibt der Review von Glass u.a. (2003) einen Überblick über 15 Studien bis zum Jahr 2001 und fasst deren Ergebnisse zusammen:

»The prevalence of physical and sexual violence in dating relationships during the middle and high school years has been the focus of several recent investigations. ... the prevalence of lifetime, past year, and current physical dating violence has varied significantly across studies, with ranges reported from $9 \%$ to $46 \%$ of adolescent males and females involved as victims or perpetrators. These wide variations in prevalence of dating violence victimization and perpetration can be attribute to several issues. The lack of uniform definitions for the identification of dating violence, variations in the instruments used to assess dating violence, and the absence of longitudinal data, as well as different populations, ages considered to comprise adolescence, and time periods for which the results are reported ..., make comparisons across studies difficult. Although comparisons are difficult, it is important to synthesize the existing knowledge of dating violence among adolescents to improve the effectiveness and relevance of prevention strategies. «(Class u.a. 2003, S. 228f.)

Insgesamt ist die Übertragung der Ergebnisse auf europäische Länder jedoch aufgrund der kulturell spezifischen Unterschiede (siehe dazu die Ausführungen in Kap. 4.2.2) problematisch. Erste Studien in Großbritannien (Barter 2009), Belgien (Ministère de la Communauté française 2009), der Schweiz (Averdijk u.a. 2011) und Deutschland (Krahé 2003a; Krahé 2008a; Krahé 2008b; Blättner 2015) machen jedoch die Relevanz des Themas auch in Europa deutlich.

Brzank u.a. (2013) verglichen die Ergebnisse des o.g. Reviews sowie nachfolgender Studien innerhalb und außerhalb Europas und kamen zu dem Schluss, dass die Ergebnisse für alle Gewaltformen zwischen den EU- und Nicht-EU-Ländern um zehn bis zwanzig Prozentpunkte abweichen. Auch sind die Ergebnisse unter dem Aspekt der geschlechtsspezifischen Betroffenheit widersprüchlich. Dennoch gibt es Hinweise darauf, dass das Erleiden von Gewalt mit dem Geschlecht verbunden unterschiedlich ist: 
»Für beide Geschlechter ist ein hohes Ausmaß verschiedener Gewaltformen zu beobachten und beide Ceschlechter üben Cewalt aus. In den Studien zeigen sich aber auch Hinweise darauf, dass Mädchen häufiger sexualisierte Gewalt erleiden; d.h. im Umkehrschluss, dass Jungen häufiger sexualisierte Gewalt anwenden und dass Mädchen häufiger von schwerer körperlicher Cewalt als Jungen berichten; d.h. dass Jungen eher schwerere Gewaltformen ausüben.« (Vgl. Brzank u.a. 2013, S. 475)

Aufgrund der begrenzt möglichen Übertragung der US-amerikanischen empirischen Befunde werden im Folgenden die erwähnten Studien aus dem europäischen Kontext näher dargestellt.

In der britischen Studie von Barter u.a. (2009) wurden 1353 Schüler_innen im Alter zwischen 13 und 17 Jahren zu Erfahrungen mit physischer, psychischer und sexueller Gewalt in den ersten eigenen Paarbeziehungen per Fragebogen sowie 62 Mädchen und 29 Jungen mittels Tiefeninterviews befragt. Hinsichtlich allgemeiner Beziehungserfahrungen gaben $88 \%$ der Jugendlichen an, Beziehungserfahrungen zu haben; die meisten Jugendlichen hatten heterosexuelle Beziehungen, $4 \%$ hatten Partner_innen des gleichen Geschlechts. Außerdem hatten Jugendliche mit Gewalterfahrungen in der Familie früher und häufiger Beziehungen und Mädchen mit Gewalterfahrungen in der Familie hatten mit größerer Wahrscheinlichkeit einen älteren Partner. Was die Häufigkeit von Gewalterfahrungen in Teenagerbeziehungen betrifft, berichteten $25 \%$ der Mädchen und $18 \%$ der Jungen über körperliche Gewalterfahrungen in irgendeiner Form; $11 \%$ der Mädchen und $4 \%$ der Jungen berichteten über schwere körperliche Gewalt. 75 \% der Mädchen und 50 \% der Jungen berichteten über Formen psychischer Gewalt, wobei »lächerlich gemacht werden« und »kontrolliert werden« als häufigste Formen psychischer Gewalt benannt wurden. 33 \% der Mädchen und $16 \%$ der Jungen berichteten über sexuelle Gewalt durch ihre_n Partner_in. Dabei handelte es sich meist um einmalige Vorkommnisse, für eine Minderheit jedoch war die sexuelle Gewalt Teil ihrer Beziehungsnormalität.

Durchgehend berichten die Mädchen von schlimmeren und langfristigeren Auswirkungen auf ihr Wohlbefinden. Ebenso berichtete die größere Anzahl der Mädchen davon, dass Gewalt wiederholt auftritt und mit der Zeit auch zunimmt. Jungen tendieren dazu, Gewalt - sowohl selbst erlebte als auch eigene Gewalthandlungen - zu bagatellisieren oder als gegenseitige Gewalt darzustellen.

Als weitere wichtige Ergebnisse der Untersuchung lassen sich folgende Punkte festhalten:

- Besonders bei sexueller Gewalt zeigen Mädchen Schuldgefühle aufgrund der Wahrnehmung, selbst eingewilligt zu haben. Dies belastete die Mädchen langfristig gesehen häufig mehr als die sexuelle Gewalt selbst. 
- Kontrolle wurde als häufigste Form von psychischer Gewalt genannt, verbunden mit der Isolation von Freund_innen. Außerdem findet Kontrolle häufig auch mithilfe der Neuen Medien statt.

- Die Gefahr der Eskalation der Gewalt erhöht sich nach einer Trennung.

- Betroffene Jugendliche erzählen in erster Linie Gleichaltrigen von ihren Erfahrungen und suchen bei ihnen Unterstützung.

In der landesspezifischen repräsentativen Optimus-Studie aus der Schweiz wurden im Jahr 2010 fast 7.000 Schüler_innen im Alter von ca. 15 Jahren sowie öffentliche und private Kinderschutzorganisationen zu sexueller Viktimisierung von Kindern und Jugendlichen befragt. Die Befragung ergab, dass $22 \%$ der Mädchen und $8 \%$ der Jungen mindestens einmal körperliche Viktimisierung (z.B. gegen den eigenen Willen geküsst oder berührt zu werden) und $40 \%$ der Mädchen und $20 \%$ der Jungen nicht-körperliche Viktimisierung (z.B. in Form von Exhibitionismus, Belästigung oder Viktimisierung über elektronische Medien) erlebt haben. Etwa zwei Drittel der Betroffenen gaben an, mehr als einmal gewaltvolle Erfahrungen gemacht zu haben und etwa ein Drittel berichtete von über fünf oder mehr Erfahrungen.

Die Studie konnte außerdem zeigen, dass sexuelle Übergriffe unter Jugendlichen durch Gleichaltrige wesentlich häufiger sind (39\%) als sexuelle Viktimisierung durch Familienangehörige. 42 \% der Betroffenen gaben an, mindestens einen Übergriff innerhalb der Beziehung oder bei einer Verabredung erlebt zu haben (vgl. Averdijk u.a. 2011).

In einer weiteren Schweizer Studie (Ribeaud 2015) wurden 1999, 2007 und 2014 etwa 2.500 Schüler_innen im Alter von ca. 15 Jahren weitestgehend identisch befragt, um Erkenntnisse zur Entwicklung von Jugendgewalt im Dunkelfeld sowie ihren Kontexten und Risikofaktoren zu gewinnen. Die Studie legt damit erstmals Daten aus der Schweiz vor, die über die Untersuchung des Phänomens sexuelle Gewalt hinausgehen. Zudem nahm die Studie auch Fragen zur Überwachung und Kontrolle des Freundes bzw. der Freundin unter dem Begriff »Monitoring« mit auf. In der Befragung gaben mehr Schüler (25,8 \% der Neuntklässler, 37,3 \% der Elftklässler) als Schülerinnen (18,5\% der Neuntklässlerinnen, 29,0\% der Elftklässlerinnen) an, körperliche Gewalt innerhalb der Paarbeziehung erlebt zu haben. Umgekehrt sind jedoch Mädchen häufiger (19,2\% der Neuntklässlerinnen und 16,1\% der Elftklässlerinnen) als Jungen (7,2 \% bzw. 7,4 \%) von sexueller Gewalt betroffen. Die Ergebnisse zur selbstberichteten Delinquenz (Täterperspektive) stimmen mit denen zur Viktimisierung (Opferperspektive) überein. Die insgesamt niedrigeren Täterraten interpretiert der Autor als Ausdruck sozial erwünschten Antwortverhaltens, indem erlebte Gewalt eher angegeben wird als ausgeübte Gewalt. Er sieht daher auch die Opferraten als verlässlichere Indikatoren für die Verbreitung von Gewalt in jugendlichen Paarbeziehungen. (Vgl. Ribeaud 2015, S. 96) Von allen untersuch- 
ten Formen von Gewalt ist in dieser Studie das Monitoring, also die Überwachung und Kontrolle des Partners bzw. der Partnerin, die häufigste Form. So gaben 65,7\% der Elftklässler und 76,9\% der Elftklässlerinnen an, ein solches Kontrollverhalten erlebt zu haben (vgl. ebd., S. 97).

Für Deutschland liegen bislang wenig Daten zur Gewalt in den ersten eigenen Paarbeziehungen vor. In der Repräsentativbefragung von Müller/Schröttle (2004) zur Lebenssituation, Sicherheit und Gesundheit von Frauen in Deutschland berichten rund $25 \%$ der Befragten im Alter von 16 bis 85 Jahren, Formen körperlicher oder sexueller Gewalt, oder beides, durch aktuelle Beziehungspartner_innen erlebt zu haben. In der Untersuchung wurden von $99 \%$ der Frauen männliche Beziehungspartner und nur $1 \%$ weibliche Beziehungspartnerinnen als Gewalt ausübende Partner benannt (vgl. ebd., S. 9f.). 12 \% der 16- bis 24-jährigen Frauen und Mädchen gaben an, sexuelle Gewalt seit dem Alter von 16 Jahren erlebt zu haben, und $70 \%$ dieser Befragten gaben an, Formen sexueller Belästigung erlebt zu haben (vgl. Schröttle 2009, S. 101). 39 \% der Betroffenen nannten als Täter den Partner und $36 \%$ benannten Freunde oder Bekannte (vgl. ebd.).

Für Deutschland lagen lange Zeit nur Studien zu sexuell aggressivem Verhalten $^{1}$ oder sexualisierter Gewalt vor (Krahé/Scheinberger-Olwig 2002; Krahé 2003a, Krahé 2003b; Krahé 2009; BZgA 2006; BZgA 2010; BZgA 2015; Maschke/Stecher 2017). Die jüngste Studie der BZgA aus dem Jahr 2015 bestätigt, dass sexuelle Gewalterfahrung für Mädchen und junge Frauen zwischen 14 und 25 Jahren kein Einzelfall ist. Eine von fünf Frauen berichtet - unter den sexuell aktiven ist es jede vierte Frau - im Laufe ihres Lebens mindestens einmal sexuelle Gewalt erlebt zu haben. Im Gegensatz dazu ist ein kleiner Teil der männlichen Jugendlichen betroffen. Allerdings sind homosexuell oder bisexuell orientierte Jungen und junge Männer fast ebenso häufig betroffen (21\%) wie junge Frauen. Insgesamt ist die Gruppe der nicht (eindeutig) heterosexuell orientierten Jugendlichen und jungen Erwachsenen sexueller Gewalt überdurchschnittlich stark ausgesetzt. Auch homooder bisexuelle Mädchen und junge Frauen berichteten häufiger (32\%), sexuelle Gewalt zu erleben als der Schnitt der Mädchen/Frauen (20\%). (Vgl. BZgA 2015, S. 196) Bezüglich der Täterkreise konzentrieren sich die Angaben der betroffenen Mädchen/Frauen auf den (Ex-)Freund (7\%) und eine neue Bekanntschaft (7 \%) (vgl. ebd., S. 198).

In einer Befragung von 456 sexuell erfahrenen weiblichen Jugendlichen mit einem Durchschnittsalter von 18,4 Jahren ergab sich eine Prävalenzrate von 31,3\% für sexuelle Viktimisierungserfahrungen durch einen Beziehungspartner. In 1,8\%

1 Krahé und Scheinberger-Olwig definieren sexuelle Aggression als die Durchsetzung sexueller Interessen gegen den Willen der Partnerin bzw. des Partners. Aggressive Strategien reichen dabei von verbalem Druck über körperliche Bedrohung bis hin zur Ausübung körperlicher Gewalt (vgl. ebd. 2002). 
der Fälle kam es zu erzwungenem Geschlechtsverkehr unter Androhung oder Ausübung von körperlicher Gewalt (vgl. Krahé/Scheinberger-Olwig 2002). Außerdem berichtete mehr als jede_r zehnte Jugendliche, durch verbalen Druck zu sexuellen Handlungen gezwungen worden zu sein, wobei in 3,3\% der Fälle der Partner die Wehrlosigkeit des Opfers, z.B. aufgrund des Einflusses von Alkohol, ausnutzte (vgl. ebd., S. 128). Eine weitere Befragung von 524 männlichen Jugendlichen, mit einem Durchschnittsalter von 18,7 Jahren, zum Täterhandeln ergab eine Prävalenzrate von $0,2 \%$ für vollzogenen und $0,4 \%$ für versuchten Geschlechtsverkehr gegen den Willen der Partnerin. Über ein Drittel der Befragten gab an, ihre Partnerin durch verbale Aggression zu sexuellen Handlungen gezwungen zu haben (vgl. ebd., S. 141). Die Autorinnen folgern aus diesen Befunden, dass die befragten Jugendlichen bereits über ein Repertoire an aggressiven Strategien verfügen, um ihre sexuellen Interessen in einer Beziehung umzusetzen. In einer weiteren Studie von Krahé und Scheinberger-Olwig wurde die sexuelle Aggression von Frauen gegenüber Männern untersucht. Dabei wurden 248 Frauen mit einem Altersmittelwert von 18,1 Jahren befragt. Dabei ergab sich über alle Strategien der Druckausübung hinweg eine Prävalenz von 9,3\% für sexuell übergriffiges Verhalten durch Frauen. Dazu kommt ein Anteil von 2,4 \% der Frauen, die versuchten, einen Mann gegen seinen Willen, aber ohne vollzogene sexuelle Aggression, zu sexuellem Kontakt zu bringen (vgl. Krahé/Scheinberger-Olwig 2002, S. 191ff.). Die Studie macht außerdem deutlich, dass sexuelle Aggression von Frauen gegenüber Männern ausgeübt wird, zu denen zumindest eine Bekanntschaft oder vormals eine Partnerschaft bestand oder besteht. Über sexuelle Aggression gegenüber unbekannten Männern wird selten berichtet. Diese Ergebnisse bestätigen somit das Muster, das sich auch bezüglich der sexuellen Aggression von Männern gegenüber Frauen empirisch bestätigt hat; sexuelle Übergriffe gegenüber Unbekannten stellen die Ausnahme dar (vgl. Krahé 2004, S. 30).

In einer weiteren deutschen Studie, der sogenannten »Speak!-Studie«, wurden 2016 in Hessen alle Schüler_innen in den Jahrgangsstufen 9 und 10 an allen allgemeinbildenden Schulen mittels standardisierter Fragebögen zu sexualisierten Gewalterfahrungen unter Gleichaltrigen befragt. Über die Hälfte der Befragten (52 \%) hat mindestens eine Form sexualisierter Gewalt (nicht-körperliche oder körperliche sexualisierte Gewalt) erlebt. $48 \%$ der Jugendlichen haben mindestens eine Erfahrung mit nicht-körperlicher sexualisierter Gewalt (sexuelle Kommentare, Beleidigungen; Witze; Opfer exhibitionistischer Handlungen; sexuelle Anmache oder Belästigung im Internet). Drei Viertel aller Befragten berichten, bereits mehrfach nicht-körperliche sexuelle Gewalt erlebt zu haben. Fast jede_r vierte Jugendliche (23\%) hat bislang mindestens eine Form körperlicher sexueller Gewalt (z.B. Berührungen oder Körperkontakt gegen den eigenen Willen) erlebt; wobei Mädchen deutlich stärker von sexualisierter körperlicher Gewalt betroffen sind. Außerdem haben 81 \% irgendeine Erfahrung mit sexualisierter Gewalt gemacht (direkt betroffen; be- 
obachtet; davon gehört; ausgeübt). Zudem nimmt der Anteil der Jugendlichen, die irgendeine Erfahrung mit sexualisierter Gewalt gemacht haben, zwischen 14 und 17 Jahren signifikant zu. (Vgl. Maschke/Stecher 2017)

Für die vorliegende Forschungsarbeit sind außerdem die Befunde zu sexualisierter Gewalt in Paarbeziehungen interessant: $28 \%$ der Betroffenen von sexualisierter Gewalt im nicht-körperlichen Bereich gaben ihren Freund als die Gewalt ausübende Person an; 9 \% gaben die Freundin an. Differenziert nach Geschlecht wird deutlich, dass die höchstplatzierten Personen bei Mädchen wie Jungen männlichen Geschlechts sind. $18 \%$ der betroffenen Mädchen nennen den Freund und Bekannten und 11 \% den Ex-Partner als Täter. Bei den betroffenen Jungen nennen $13 \%$ die Mitschülerin oder Freundin als Täterin. Bei den Formen körperlich-sexualisierter Gewalt geben ein Drittel der betroffenen Mädchen den Freund und $15 \%$ den Ex-Freund als Täter an. Bei den Jungen nennen knapp ein Drittel der Betroffenen die Freundin und $12 \%$ die Ex-Freundin. (Vgl. ebd.) Insgesamt wird anhand dieser Zahlen deutlich, dass die Gruppe der Gleichaltrigen die größte Gruppe derjenigen darstellt, die sexualisierte Übergriffe ausüben.

2012 und 2013 wurde die erste repräsentative Studie innerhalb Deutschlands unter hessischen Schüler_innen durchgeführt. Diese Studie stellt bislang auch die einzige deutsche Studie zur Häufigkeit von Gewalt in jugendlichen Paarbeziehungen dar. Die Schüler_innen zwischen 14 und 18 Jahren wurden hier nicht nur zu sexualisierter Gewalt (vgl. Speak!-Studie), sondern allgemein zu Gewalterfahrungen in den eigenen Paarbeziehungen oder bei Dates befragt. Ziel der Studie war es zum einen, Daten zum Ausmaß von körperlicher, emotionaler und sexualisierter Gewalt, den Risikofaktoren und Auswirkungen zu erhalten; und zum anderen auch Empfehlungen für die Präventionsarbeit zu entwickeln (vgl. Blättner u.a. 2015, S. 174).

Von den 354 Schüler_innen, die angaben, Erfahrungen mit Dates oder Beziehungen zu haben, gaben 65,7 \% der Mädchen und 60,1 \% der Jungen an, mindestens einmal eine Form von Grenzüberschreitungen oder Gewalt erlebt zu haben. 61,3\% der Mädchen und 56,6\% der Jungen berichteten von emotional schwierigen Situationen, insbesondere von Kontrollversuchen. Von sexualisierter Gewalt waren Mädchen signifikant häufiger (26,0\%) als Jungen (12,7\%) betroffen. Körperliche Gewalt erlebten Mädchen (10,5 \%) und Jungen (10,5 \%) in etwa gleichem Maße. Die Studie kommt außerdem zu dem Ergebnis, dass die große Mehrheit der Betroffenen Gewalt in heterosexuellen Beziehungen erlebte. Nur in wenigen Fällen wurde

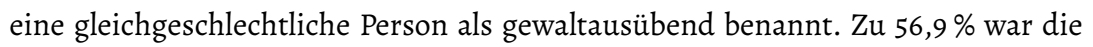
gewaltausübende Person männlich. Außerdem waren die grenzüberschreitenden oder gewaltausübenden Partner_innen bei den Mädchen überwiegend älter und bei den Jungen überwiegend gleichalt oder jünger. (Vgl. ebd., S. 175f.) 
Die Forschungslage zu Gewalt in gleichgeschlechtlichen Beziehungen sieht dagegen noch magerer aus. ${ }^{2}$ In der Studie von Krahé und Scheinberger-Olwig wurde auch nach sexueller Aggression in gleichgeschlechtlichen Partnerschaften zwischen Männern gefragt. Dabei gaben 12,6\% der befragten 310 Männer an, durch verbale oder körperliche Gewalt oder das Ausnutzen einer wehrlosen Situation ihren Beziehungspartner zu sexuellen Handlungen gebracht zu haben. Weiter gaben 11 \% der befragten Männer an, sexuelle Gewalt durch ihren Partner erlebt zu haben. (Vgl. Krahé/Scheinberger-Olwig 2002, S. 173)

Zusammenfassend lässt sich zum Stand der Prävalenz von Gewalt in den ersten eigenen Paarbeziehungen festhalten, dass die bestehenden Studien aufgrund uneinheitlicher Forschungsdesigns nur schwer vergleichbar sind. Krahé kommt zu folgendem Schluss:

»Daher sind die Zahlen am besten so zu deuten, dass ein erheblicher Anteil von Jugendlichen bereits zu Beginn ihrer Partnerschaftserfahrungen aggressive Formen der Auseinandersetzung einsetzt und erlebt, die nicht nur die aktuelle Partnerschaft belasten, sondern auch Weichen für künftige aggressionsbelastete Beziehungen stellen.« (Krahé 2008a, S. 132)

Zudem liegt der Schwerpunkt der vorliegenden Studien auf heterosexuellen Paarbeziehungen. Die wenigen Ergebnisse zu Gewalt in gleichgeschlechtlichen Beziehungen lassen allerdings auf ein ähnliches Ausmaß von Gewalt schließen.

Eine aktuelle Studie von Blättner/Schultes (2018) kritisiert den bisherigen Fokus vorhandener Studien auf die Art der Gewalthandlung, weil »dieser weniger gut geeignet zu sein [scheint, Anm. d. Verf.], das Phänomen zu verstehen und die geschlechterdifferente Verteilung zwischen Verletzungsoffenheit und Verletzungsmächtigkeit ... zu verwischen« (Blättner u.a. 2018, S. 325f.). Infolge der genannten Kritik führten die Forscherinnen acht Fokusgruppen und vier Einzelinterviews mit 50 Jugendlichen durch. Diese wurden aufgefordert, von schwierigen Situationen innerhalb ihrer Paarbeziehungen zu berichten. Im Anschluss wurden 46 grenzüberschreitende oder gewaltgeprägte Situationen aus dem Datenmaterial entnommen, kontrastierend verglichen und daraus vier Typen hinsichtlich der Art des Gewaltgeschehens generiert (vgl. ebd., S. 326). Die Studie macht deutlich, dass die Erfassung von Gewalt nicht nur anhand von Skalen erfasst werden sollte, sondern dass auch die spezifischen Kontexte, in denen das Phänomen Gewalt auftritt, sowie die darin enthaltende Dynamik ebenfalls in den Blick genommen werden

2 Die Studie von Constance Ohms zur häuslichen Cewalt in Intimbeziehungen von Frauen leistet einen wichtigen Beitrag, indem sie zur Enttabuisierung von Cewalt in gleichgeschlechtlichen Beziehungen beiträgt. Sie nimmt jedoch die Beziehungen erwachsener Frauen in den Blick, weshalb ich hier nicht weiter auf konkrete Befunde der Studie eingehe (Ohms 2008). 
sollten. Damit leistet sie einen ersten Beitrag an qualitativen Untersuchungen zur Erforschung des Phänomens.

Risikofaktoren Im Gegensatz zu der uneinheitlichen Forschungslage zur Prävalenz von Gewalt in jugendlichen Paarbeziehungen lassen sich hinsichtlich der risikoerhöhenden Bedingungen, Gewalt auszuüben bzw. Gewalt zu erleben, einige klare Befunde nennen. Generell zeigen die vorliegenden Studien, dass jede weibliche Jugendliche unabhängig von Sozialstatus, Ethnie oder Kultur von Gewalt in den ersten eigenen Paarbeziehungen betroffen sein kann (vgl. Temple/Freeman 2001). Einen breiten Überblick über den Forschungsstand zu Risikofaktoren geben Brzank u.a. (2013). Folgende Risikofaktoren wurden von ihnen auf der Grundlage der vorliegenden Studien identifiziert: Missbrauchserfahrungen in der Kindheit oder das Erleben häuslicher Gewalt; Akzeptanz von Gewalt im Kontext der Peergroup; jüngeres Alter der Mädchen, höheres Alter des Partners bzw. höhere Altersdifferenz für die Viktimisierung von Mädchen; riskanter Lebensstil durch häufige Partnerwechsel und risikoreiches Sexualverhalten oder Alkohol- und Drogenkonsum (vgl. ebd., S. 476). Die Beziehung zu einem älteren Partner, besonders zu einem »sehr viel älteren Partner« (mindestens zwei Jahre Altersunterschied), sowie Gewalterfahrungen in der Herkunftsfamilie bzw. die Zeugenschaft häuslicher Gewalt wurden auch in der weiter oben dargestellten Studie von Barter u.a. (2009) als wesentliche Risikofaktoren identifiziert.

Die genannten Faktoren sind jedoch nicht zwangsläufig als Ursache zu verstehen, sondern können gleichzeitig mit Gewalt in jungen Paarbeziehungen bestehen. So kann ein risikoreicher Lebensstil auch als Bewältigungsstrategie von Gewalterfahrungen verstanden werden. (Vgl. ebd.) Die Studie von Blättner u.a. (2015) verweist auf eine Tendenz, nach der sich das Risiko von Gewalt in jugendlichen Paarbeziehungen bei Jugendlichen erhöht, die unter dem Druck stehen, eine Beziehung haben zu sollen (vgl. ebd., S. 176). Auch das aggressive Verhalten in der Peergroup scheint Einfluss zu nehmen, denn Grenzüberschreitungen oder Gewalt waren unter den Befragten mit einem aggressiven Freundeskreis höher (vgl. ebd.). Allerdings erwies sich Gewalt in der Herkunftsfamilie auch in dieser Studie als deutlicher Einflussfaktor. Dieses Ergebnis deckt sich damit mit anderen internationalen Befunden. Außerdem bestätigt die Studie internationale Forschungsergebnisse hinsichtlich der Chronifizierung von Partnergewalt: Wer bereits in frühen Paarbeziehungen Gewalt erlebt, hat ein wesentlich höheres Risiko, auch Partnergewalt im Erwachsenenalter zu erleben oder auszuüben (vgl. Blättner u.a. 2016).

Bezüglich der gesundheitlichen Folgen von Gewalt in Paarbeziehungen belegen internationale Studien ähnliche Folgen für weibliche Jugendliche. So berichten Jugendliche von körperlichen Verletzungen und psychischen Störungen wie Depressionen, Angststörungen oder posttraumatischen Belastungsstörungen. Betroffene greifen außerdem in erhöhtem Maße zu Alkohol oder anderen Drogen. Auch be- 
richten Jugendliche, die Gewalt erlebten, von einem geringeren Selbstwertgefühl, einem negativen Selbstbild und einer pessimistischen Grundstimmung (vgl. den ausführlichen Überblick zum Forschungsstand hierzu bei Brzank u.a. 2013, S. 477).

Unterstützung und Prävention International und national gibt es verschiedenste Präventionsprogramme (Avery-Leaf u.a. 1997; Wolfe u.a. 1997; Avery-Leaf/Cascardi 2002; Schewe 2002; Kohlhofer u.a. 2008; Weisz/Black 2009; Köberlein u.a. 2010), wobei vor allem schulische und digitale Prävention die verbreiteten Formen darstellen. Der Review von La Rue u.a. (2014) zeigt auf, dass schulische Präventionsprogramme zur Veränderung von Wissen und Einstellungen beitragen. Allerdings lassen sich keine Effekte auf das Ausüben oder Erleben von Gewalt festmachen.

Blättner u.a. (2014) untersuchten digitale Präventionsangebote und analysierten deren inhaltliche und mediale Gestaltung sowie das Vorliegen von Evaluationen. Die Studie kommt zu dem Schluss, dass der hohe Anteil der genutzten OnlinePlattformen darauf verweist, dass Jugendliche dort gut erreichbar sind (vgl. ebd., S. 525). Allerdings liegen kaum Erkenntnisse darüber vor, wer die Angebote nutzt und wie wirksam sie sind. Da das Internet mit einer Komm-Struktur verbunden ist und damit weniger eine Form primärer Prävention darstellt, liegt der Schwerpunkt digitaler Prävention mehr auf dem Aufzeigen von Handlungsstrategien, um zukünftige schwierige Situationen besser bewältigen oder eine Chronifizierung von Gewalt innerhalb der Beziehung vermeiden zu können (vgl. ebd., S. 528).

Bezüglich des Hilfesuchverhaltens wenden sich Jugendliche primär an Freund_innen und an zweiter Stelle an die Eltern. Als weitere Möglichkeiten der Unterstützung wurden Geschwister und das Internet genannt (Ashley/Foshee 2005; Barter u.a. 2009; Blättner u.a. 2015). Wissen über das Hilfesuchverhalten von Betroffenen ist relevant, um entsprechende Strukturen in der Unterstützung etablieren zu können.

Intergenerationale Transmission Bezüglich der intergenerationalen Transmission früherer Beziehungserfahrungen auf die Gestaltung der eigenen Paarbeziehung finden sich in der psychologischen Literatur und Forschung verschiedene Inhalte und Erklärungen (s. dazu den Überblick bei Walper u.a. 2010, S. 292). Intergenerationale Transmissionsprozesse lassen sich dabei in horizontale und vertikale Transmissionen einteilen. Horizontale Transmission meint die direkten Einflüsse der elterlichen Paarbeziehung auf die Paarbeziehung der folgenden Generation und wird z.B. über die Beobachtung des Elternmodells erlernt. Vertikale Transmission sind Einflüsse der Eltern-Kind-Beziehung, wie bspw. Sozialisationsprozesse innerhalb der Eltern-Kind-Beziehung oder die Qualität der Eltern-Kind-Beziehung (vgl. 
ebd.). Wie die deutsche pairfam-Studie ${ }^{3}$ zeigen konnte, beeinflusst sowohl die Beziehung zu den Eltern als auch deren Erziehungsverhalten die Beziehungsqualität in jungen Paarbeziehungen (vgl. ebd., S. 309). Dabei prägt sowohl die aktuelle als auch die erinnerte Beziehung zu den Eltern verschiedene Bereiche der Beziehungsqualität in den ersten eigenen Paarbeziehungen. Die Studie konnte außerdem zeigen, dass die Transmissionseffekte auch geschlechtsspezifisch ausfallen, indem die Daten überwiegend auf gegengeschlechtliche Einflüsse im Übertragungsprozess verweisen (vgl. ebd.). So zeigt sich bspw. ein deutliches Muster überwiegend gegengeschlechtlicher Transmissionseffekte bezüglich emotionaler Unsicherheit und der Übertragung von Intimität. Dagegen zeigt sich bei der Transmission von Autonomie für Jungen ein stärkerer Einfluss des Vaters (vgl. ebd., S. 309f.).

Verschiedene Studien belegen zudem die intergenerationale Transmission von Gewalt (vgl. dazu Kalmuss 1984; Albrecht 2002; Grossmann/Grossmann 2004; Müller/Schröttle 2004). So stellt bspw. das Miterleben häuslicher Gewalt ein Risiko dar, in den eigenen Paarbeziehungen zu Gewalt zu greifen (vgl. Lamnek u.a. 2012). Laut Swinford u.a. (2000) hat nach neuesten Befunden das Erleben harter körperlicher Gewalt in der Herkunftsfamilie Folgen für gewalttätiges Verhalten in intimen Paarbeziehungen,

»und zwar direkt und indirekt über die Verursachung von generellen Verhaltensproblemen und Cewaltdelikten in der Jugend und im jungen Erwachsenenalter, die das Risiko gewalttätiger Auseinandersetzungen in der Partnerschaft erhöhen. (...) Der Zyklus von Cewalt und Sozialisation ist also doppelt gesichert: Gewalterfahrung erhöht die Wahrscheinlichkeit von Delinquenz, und Delinquenz erhöht die Wahrscheinlichkeit von Cewalt in Beziehungen, in denen typischerweise Sozialisation erfolgt.«(Albrecht 2002, S. 780)

Aber auch wenn verschiedene Studien das Erleben von Gewalt in der Herkunftsfamilie als Risiko erkannt haben, ist darauf hinzuweisen, dass dies nicht der einzige auslösende Faktor ist, später selbst zu gewalttätigem Verhalten zu neigen oder Gewalt zu erleben. Außerdem besteht hinsichtlich der Erforschung der tatsächlichen Mechanismen der intergenerationalen Übertragung von Gewalt noch erheblicher Forschungsbedarf (vgl. Gelles 2002, S. 1063).

Fazit Insgesamt lässt sich aus den internationalen Erkenntnissen sowie den bislang ersten Daten aus Deutschland zur Prävalenz von Gewalt in jugendlichen Paarbeziehungen folgern, dass Gewalt in den ersten eigenen Paarbeziehungen kein Einzelphänomen darstellt. Die empirischen Befunde aus Großbritannien und der

3 Die pairfam-Studie (»Panel Analysis of Intimate Relationships and Family Dynamics«) ist eine multidisziplinäre Längsschnittstudie zur Erforschung partnerschaftlicher und familialer Lebensformen in Deutschland. Sie startete im Jahr 2008. 
Schweiz zur Prävalenz von Gewalt in jugendlichen Paarbeziehungen sowie erste Ergebnisse aus Deutschland machen den Forschungsbedarf deutlich. Des Weiteren zeigt die dargestellte Übersicht zum Forschungsstand, dass es sich, zumindest im europäischen Kontext, fast ausschließlich um quantitativ ausgerichtete Forschungsdesigns handelt ${ }^{4}$

Die vorliegende Forschungsarbeit verfolgt im Unterschied zu den meisten bisherigen Studien einen qualitativen Zugang. Sie nimmt biografische Faktoren und damit auch die Sozialisationsgeschichte der befragten Frauen in den Blick. Von dieser Perspektive ausgehend, wird in der vorliegenden Studie nach Praktiken und Handlungsorientierungen gefragt, die junge Frauen im Umgang mit Gewalt während der ersten eigenen Paarbeziehung(en) zeigen. Damit verbunden sind Fragen, wie junge Frauen Gewalt innerhalb der ersten Paarbeziehungen erleben, welche Rolle Gewalterfahrungen in der Herkunftsfamilie spielen und welche Vorstellungen junge Frauen zu Paarbeziehungen entwickeln. Biografische Erfahrungen werden dabei in der Auswertung berücksichtigt und analysiert. Die Studie legt somit einen Schwerpunkt auf die subjektiven Erfahrungen und Lebenswelten der betroffenen Frauen.

4 Ausnahmen stellen die Studie von Barter u.a. 2009 dar, die neben einer Fragebogen-Erhebung auch Interviews in die Untersuchung mit einbezog, sowie die aktuelle Studie von Blättner/Schultes (2018). 



\section{Zwischenfazit}

Erste Liebesbeziehungen Jugendlicher sind ebenso wie Gewalt als spezifischer Aspekt von Paarbeziehungen Jugendlicher und junger Erwachsener erst in jüngster Vergangenheit in den Blick sozialwissenschaftlicher Forschung geraten. Die vorhandenen empirischen Studien zur Prävalenz von Gewalt in den ersten eigenen Paarbeziehungen machen den Forschungsbedarf deutlich. Die vorliegende Forschungsarbeit widmet sich damit nicht nur einem bislang -zumindest in Europa - kaum in den Blick genommenen Phänomen. Sie verfolgt zudem, im Unterschied zu den meisten bisherigen Studien, einen qualitativen Zugang und legt damit den Schwerpunkt auf subjektive Erfahrungen und Lebenswelten. Den zentralen Fokus bilden dabei Praktiken und Handlungsorientierungen junger Frauen im Umgang mit Gewalt während der ersten eigenen Paarbeziehungen. Ziel der Arbeit ist es, zu einem vollständigeren Bild des Erlebens von Gewalt in den ersten eigenen Paarbeziehungen, des Umgangs mit dieser Gewalt sowie der daraus resultierenden Beziehungskonzepte beizutragen.

Wie die vorausgehenden Kapitel aufzeigen, nähert sich die vorliegende Arbeit ihrer zentralen Forschungsfrage nach den Praktiken und Handlungsorientierungen junger Frauen im Umgang mit Gewalt während der ersten eigenen Paarbeziehungen im Wesentlichen auf der Grundlage zweier theoretischer Perspektiven: einer gesellschaftstheoretischen und einer biografischen Perspektive. Die Verbindung dieser beiden Zugänge hat zum Ziel, die Wechselbeziehung von gesellschaftlichen Rahmenbedingungen und biografischem Handeln in den Blick zu nehmen.

Die folgende Grafik verdeutlicht die Annäherung an das Forschungsthema sowie die genannten theoretischen Zugänge: 
Abbildung 1: Theoretische Zugänge

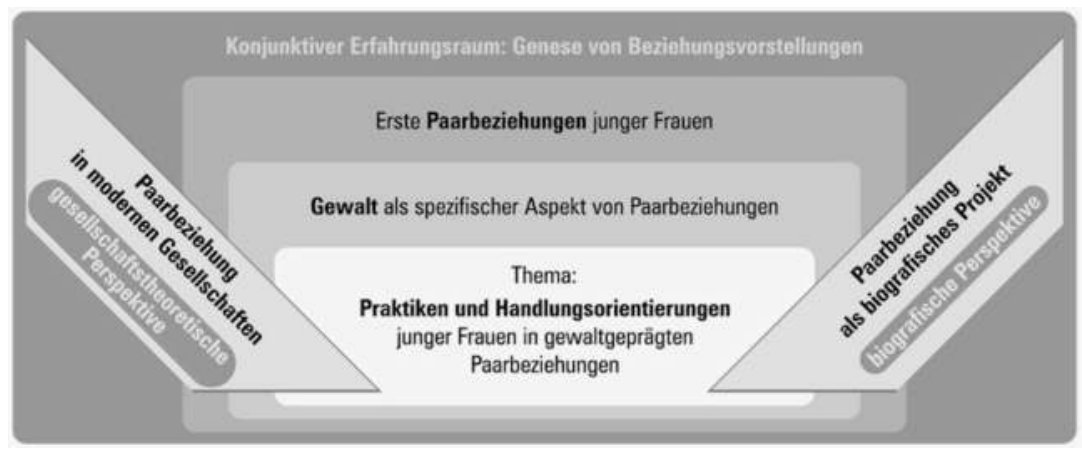

Der gesellschaftstheoretische Zugang thematisiert insbesondere unter individualisierungstheoretischer Perspektive die veränderten Anforderungen an Paarbeziehungen in modernen Gesellschaften. Gravierende gesellschaftliche Veränderungen im Bildungsbereich sowie in den Bereichen Sexualität und Recht führen zu Veränderungen im Geschlechterverhältnis und zu einem widersprüchlichen $\mathrm{Ne}$ beneinander traditionaler und moderner Elemente. Besonders deutlich wird dies im Widerspruch zwischen den Anforderungen des Arbeitsmarktes und den Anforderungen der Partnerschaft.

Die Frage, wie dieses »Dauerkunststück« (Beck/Beck-Gernsheim 2015, S. 13) individuell be- und verarbeitet wird, wie sich Paarbeziehung als individuelles Projekt gestaltet, wird unter biografischer Perspektive beleuchtet.

Der Fokus ist dabei zum einen auf individuelle Haltungen und Handlungen der befragten jungen Frauen gerichtet, indem die individuelle Genese von Orientierungsrahmen bzw. die Entstehung individueller Beziehungsvorstellungen sowie die Inkorporation von Praktiken, insbesondere im Erfahrungsraum Familie, betrachtet und analysiert werden. Im Rahmen von Einzelfallanalysen werden biografische Erfahrungsprozesse zunächst getrennt von kollektiven Prägungen analysiert - wenngleich sie auf diese bezogen bleiben. Auf diese Weise können adoleszente und biografische Verarbeitungsprozesse der befragten jungen Frauen analysiert werden. Zudem ermöglicht der biografische Zugang neben der Perspektive auf die Genese individueller Beziehungsvorstellungen auch, den Blick auf Transformationen dieser inkorporierten Haltungen zu richten.

Zum anderen analysiert die vorliegende Arbeit biografisches Handeln junger Frauen im gesamtbiografischen Zusammenhang, womit sich die Frage verbindet, wie sich Subjekte in Auseinandersetzung mit gesellschaftlichen Verhältnissen konstituieren. Diese subjekttheoretische Ergänzung des biografietheoretischen $\mathrm{Zu}$ gangs ermöglicht es, Fragen nach Handlungsmöglichkeiten von Frauen in Gewaltbeziehungen $\mathrm{zu}$ thematisieren. In diesem Zusammenhang werden für die vorlie- 
gende Arbeit auch sozialisationstheoretische Fragen relevant, indem das Interesse biografisch entwickelten Orientierungen während der Sozialisation gilt.

Insgesamt wird deutlich, dass biografisches Handeln dabei immer in Auseinandersetzung mit gesellschaftlichen Rahmenbedingungen geschieht. Diese Wechselbeziehung nimmt die vorliegende Arbeit in den Blick. 



\section{Methodologie und Methode}

\subsection{Qualitatives Forschungsdesign und dokumentarische Methode}

Eine Studie, die nach dem Erleben von Gewalt, dem Umgang mit dieser erlebten Gewalt und den daraus resultierenden biografischen Vorstellungen von Paarbeziehungen fragt, ist mit verschiedenen methodischen Ansprüchen konfrontiert.

Wie lassen sich Aussagen über die biografische Entwicklung von Beziehungsvorstellungen machen, ohne diese im Vorfeld durch implizite Annahmen über den Forschungsgegenstand zu beeinflussen? Inwiefern lassen sich aufgrund der Aussagen der Beforschten über ihre Beziehungsvorstellungen Rückschlüsse auf ihre Erfahrungen ziehen? Wie kann mit gesellschaftlichen Tabuisierungen des Themas Gewalt in Beziehungen angemessen umgegangen werden?

Rekonstruktive Forschung hat den Anspruch, durch das Prinzip der Offenheit

»denjenigen, die Cegenstand der Forschung sind, die Strukturierung der Kommunikation im Rahmen des für die Untersuchung relevanten Themas so weit wie möglich zu überlassen, damit diese ihr Relevanzsystem und ihr kommunikatives Regelsystem entfalten können und auf diesem Wege die Unterschiede zum Relevanzsystem der Forschenden überhaupt erst erkennbar werden.«(Bohnsack 2010, S. 21)

Nach Christa Hoffmann-Riem sind das Prinzip der Offenheit sowie das Prinzip der Kommunikation die zentralen methodischen Prinzipien interpretativer Verfahren. Sie ermöglichen zum einen, dass sich die Strukturierung des Forschungsgegenstandes durch die beforschten Subjekte herausbildet und die theoretische Strukturierung durch die Forschenden zurückgestellt wird. Zum anderen gewinnen Forschende nur Zugang zu Daten, wenn sie eine Forschungsbeziehung zu den Beforschten eingehen. (Vgl. Hoffmann-Riem 1980, S. 343ff.)

Im Hinblick auf den gesamten Forschungsprozess gibt es eine besondere Beziehung zwischen sozialwissenschaftlichen Konstruktionen und ihrem Gegenstand: Sozialwissenschaftliche Konstruktionen bzw. wissenschaftliche Begriffsbildungen stellen sogenannte sekundäre Konstruktionen dar. Sie schließen immer an die im alltäglichen Handeln bereits vollzogenen Konstruktionen und Typenbildungen, den 
Common Sense, an. Jede (alltägliche) Handlung setzt Hintergrundwissen, einen Entwurf oder eine Orientierung voraus. Sozialwissenschaftliche Konstruktionen richten den Blick auf die impliziten Konstruktionen und Orientierungen, in die die Handlungspraxis eingebettet ist (vgl. Przyborski/Wohlrab-Sahr 2010, S. 26). Bohnsack formuliert dies folgendermaßen:

»Die Besonderheit sozialwissenschaftlichen Denkens besteht also darin, dass sich nicht nur dieses Denken selbst aus Interpretationen, Typenbildungen, Konstruktionen zusammensetzt, sondern dass bereits der Gegenstand dieses Denkens, eben das soziale Handeln, das Alltagshandeln auf unterschiedlichen Ebenen durch sinnhafte Konstruktionen, durch Typenbildungen und Methoden vorstrukturiert ist. «(Bohnsack 2010a, S. 23, Herv. im Original)

Nicht nur das Nachdenken oder Reflektieren über Handeln, sondern auch das Handeln selbst ist »typengeleitet, wissensgeleitet und entwurfsorientiert« (ebd.).

Zudem bringen die Handelnden selbst Interpretationen hervor, die es im Forschungsprozess zu rekonstruieren gilt. Dabei werden die Interpretationen der Handelnden zunächst als Konstruktionen ersten Grades rekonstruiert. In einem weiteren Schritt werden daraus Theorien und Typen gebildet. Diese stellen dann Konstruktionen zweiten Grades dar. (Vgl. Przyborski/Wohlrab-Sahr 2010, S. 26)

Bei interpretativen oder rekonstruktiven Verfahren ist bereits $\mathrm{zu}$ Beginn des Interpretationsprozesses, der Rekonstruktion der Konstruktion ersten Grades, eine »kontrollierte Methode des Fremdverstehens «" (ebd., S. 30) erforderlich. Methodische Kontrolle bezieht sich dabei auf die Kontrolle der Unterschiede in den Darstellungsformen der Untersuchten und der Forschenden, indem der Differenz zwischen Relevanzsystemen und Interpretationsrahmen systematisch Rechnung getragen wird (vgl. ebd., S. 31). Während der Datenerhebung wird methodisch kontrolliertes Fremdverstehen durch die Zurückhaltung der Forschenden ermöglicht. Indem die Fragestellung im Interview möglichst offen formuliert wird, werden die Erforschten zu Erzählungen ermutigt. Sie bekommen die Gelegenheit, das Thema in ihrer eigenen Sprache mit ihren eigenen Schwerpunkten und damit ihr eigenes Relevanzsystem zu entfalten. Auf diese Weise werden Sprache und Interpretationsrahmen von Erforschten und Forschenden methodisch kontrolliert (vgl. Bohnsack 2010, S. 20). Im Prozess der Auswertung gilt es, von den Kontextuierungen der Erforschten auszugehen und nicht von Vorab-Annahmen der Forschenden, indem Äußerungen im Kontext seiner oder ihrer Erzählung interpretiert werden (vgl. Przyborski/Wohlrab-Sahr 2010, S. 31).

Die Rekonstruktion der hier beschriebenen sinnhaften Konstruktionen, die dem sozialen Handeln zugrunde liegen und dieses vorstrukturieren, ist das Ziel

1 Eine kontrollierte Methode des Fremdverstehens wurde von Schütze u.a. 1973 entwickelt. Er rekurrierte dabei auf Alfred Schütz und dessen Konzept des Fremdverstehens. 
der vorliegenden Arbeit. Es geht also um die Rekonstruktion des Herstellungsprozesses bzw. des Orientierungsrahmens, in dem Paarbeziehung gestaltet wird. In einem zweiten Schritt wird erforscht, wie mit Gewalterfahrungen innerhalb dieser Paarbeziehungen umgegangen wird. Die dokumentarische Methode (vgl. Kap. 7.1), die für die Auswertung der erhobenen Daten ausgewählt wurde, ermöglicht dabei, die Perspektive auf die Verschränkung von individualbiografischer Erfahrung und kollektiver Einbettung zu richten (vgl. Nohl 2016, S. 335). Indem es um die »Schnittflächen von Interaktion, Biografie und Sozialstruktur» (Denzin 2013, S. 149) geht, soll zu einem besseren Verständnis des subjektiven Erlebens von Gewalt im Kontext gesellschaftlicher (Macht-)Strukturen beigetragen werden.

\subsubsection{Immanenter und dokumentarischer Sinngehalt - Konjunktive und kommunikative Erfahrung}

Die dokumentarische Methode knüpft in ihrer heutigen Ausrichtung und Verwendung an drei methodologisch-theoretische Zugänge an: zum ersten an die Wissenssoziologie Karl Mannheims, zum zweiten an die Ethnomethodologie (Harold Garfinkel) und zum dritten an die praxeologische Kultursoziologie Pierre Bourdieus mit seiner Konzeption des Habitus (vgl. Bohnsack 2012, S. 120).

Begründet und erkenntnistheoretisch geprägt wurde der Begriff »dokumentarische Methode« 1922 von Karl Mannheim als zentraler Begriff seiner Wissenssoziologie (vgl. Bohnsack 2010, S. 57). Den drei genannten Traditionen folgend steht die Analyse der Praxis des Handelns bzw. das diese Praxis orientierende, implizite inkorporierte oder »atheoretische" (Mannheim 1980, S. 73) Wissen im Mittelpunkt. Die dokumentarische Methode geht davon aus, dass für die empirische Analyse nicht nur wörtlich Mitgeteiltes relevant ist, sondern vor allem der Sinngehalt, der diesen Äußerungen zugrunde liegt und den es als sogenanntes implizites Wissen $\mathrm{zu}$ rekonstruieren gilt (vgl. Nohl 2005, Kap. 4). Dabei differenziert die dokumentarische Methode nicht zwischen subjektiv und objektiv, sondern sie unterscheidet zwischen der im Erleben verankerten Wirklichkeit, dem handlungspraktischen Wissen und dem kommunikativ-generalisierten Wissen, das normalerweise in explizierter Form zugänglich ist (vgl. Przyborski/Wohlrab-Sahr 2010, S. 275).

Durch die Unterscheidung zwischen einem reflexiven oder theoretischen Wissen der Akteur_innen einerseits und dem handlungsleitenden und inkorporierten Wissen andererseits will die dokumentarische Methode die Aporie von Subjektivismus und Objektivismus überwinden.

Dabei wird das Wissen der Handelnden als empirische Ausgangsbasis berücksichtigt, ohne an deren subjektive Intentionen gebunden zu bleiben. So wird ein Zugang zur Handlungspraxis sowie zu den Strukturen ermöglicht, die dieser Praxis zugrunde liegen und welche den Akteur_innen nicht zugänglich sind (vgl. Bohnsack/Nentwig-Gesemann/Nohl 2007, S. 12). Der Wechsel in der Analyseeinstellung 
erfolgt darin, dass nicht mehr danach gefragt wird, was die gesellschaftliche Realität aus der Perspektive der Handelnden ist, sondern wie diese Praxis hergestellt wird (vgl. ebd.). Die Frage danach, wie Alltagswissen unterschiedlich ausgeformt wird, verweist immer auch auf die verschiedenen sozialen Bedingungen der Akteur_innen. In der Rekonstruktion des Modus Operandi der Herstellung, der für eine Persönlichkeit oder ein Kollektiv typisch ist, dokumentiert sich der individuelle oder kollektive Habitus (vgl. Bohnsack 2010, S. 60). Bohnsack u.a. (1995) unterschieden mit Bezug auf Bourdieu zwischen "persönlichem Habitus«, wie er vor allem in narrativ-biografischen Interview zu finden ist, sowie dem »kollektiven milieuspezifischen Habitus«, der sich besonders in Gruppendiskussionen rekonstruieren lässt. Das Verhältnis zwischen Kollektivität und Individualität wurde aber in den letzten Jahren in verschiedenen Forschungsarbeiten (z.B. Helsper u.a. 2013; Kramer u.a. 2009; Kramer u.a. 2013) im Zuge der Weiterentwicklung der dokumentarischen Methode neu beleuchtet. ${ }^{2}$

Im Zuge dieser Weiterentwicklung wird die dokumentarische Interpretation von narrativ-biografischen Interviews zunehmend dafür genutzt, den bisher der dokumentarischen Methode eigenen Fokus auf kollektive Erfahrungsräume »um die Berücksichtigung der in der Lebensgeschichte von Menschen gelagerten individuellen Erfahrungsaufschichtungen zu erweitern« (Nohl 2016, S. 331). So schließen Helsper und Kollegen in ihren Forschungsarbeiten an den von Bohnsack angedeuteten, aber nicht weiter ausgeführten »individuellen « bzw. "persönlichen « (Bohnsack 2010a) Habitus an und formulieren, in Bezugnahme auf Pierre Bourdieu, Ansatzpunkte für (immer auch individuelle) Bildungsprozesse und Transformationen innerhalb der dokumentarischen Methode. Zudem formulieren sie Überlegungen einer sozialisationstheoretischen Bestimmung der Genese des Habitus (vgl. Helsper u.a. 2013). Indem sie die »Genese des Habitus als individuelle Strukturbildung ( (ebd., S. 134) fassen und damit aufzeigen, dass der Habitus bzw. Orientierungsrahmen, von seiner Genese her gedacht, individuell sein muss, verdeutlichen sie, dass der Habitus "immer beides zugleich - individuell und kollektiv« (ebd., S. 135) ist.

Für die Interpretation des Datenmaterials bildet die Unterscheidung zwischen immanentem und dokumentarischem Sinngehalt den Mittelpunkt der methodologischen Grundbegriffe, auf den sich dann auch die Auswertungsschritte beziehen. Während sich der immanente Sinngehalt auf seine Richtigkeit überprüfen lässt, nimmt der dokumentarische Sinngehalt »den soziokulturellen Entstehungszusammenhang bzw. das, was sich davon manifestiert hat, in den Blick« (Przyborski/Wohlrab-Sahr 2010, S. 278).

Bohnsack schreibt dazu:

2 Einen Überblick über den aktuellen Stand der Weiterentwicklung der dokumentarischen Methode bietet Nohl 2016. 
»Die dokumentarische Methode ist ... darauf gerichtet, einen Zugang zum handlungspraktischen, zum impliziten und konjunktiven Erfahrungswissen zu erschließen. Das konjunktive (Orientierungs-)Wissen als ein in die Handlungspraxis eingelassenes und diese Praxis orientierendes und somit vorreflexives Erfahrungswissen ist dem Interpreten nur zugänglich, wenn er sich den je individuellen oder kollektiven Erfahrungsraum erschließt. Das heißt eine Äußerung oder Handlung wird mir nur verständlich, wenn ich den dazugehörigen Erfahrungsraum kenne.«(Bohnsack 2007, S. 15)

Menschen durchlaufen während ihrer Entwicklung verschiedene Erfahrungsräume, von denen manche wieder verlassen werden; andere Erfahrungsräume hingegen strukturieren ein Leben lang alltägliche Handlungsvollzüge (vgl. Przyborski/Slunecko 2010, S. 633). So konstituieren sich etwa geschlechtstypische Erfahrungsräume »über die Kombination der Handlungs- bzw. Interaktionspraxis geschlechtsspezifischer Sozialisation und das Erleben geschlechtstypischer (Fremd-)Zuschreibungen und Interpretationen« (ebd.). Die dokumentarische Methode zielt auf dieses Erfahrungswissen, das sich in der Alltagspraxis abbildet. Die grundlegende Perspektive ist eine praxeologische, bei der habituelles Handeln, konjunktive Erfahrung und Orientierungsmuster im Mittelpunkt stehen. Przyborski und Slunecko (2010) konstatieren, dass sich die Weiterentwicklung der dokumentarischen Methode sich nicht zuletzt der abstrakten Idee der konjunktiven Erfahrung verdankt, die nicht in der konkreten Gruppe verankert ist, sondern »eine von der konkreten Gruppe gelöste Kollektivität [erfasst], indem sie all diejenigen miteinander verbindet, die an die Handlungspraxen und damit an Wissensund Bedeutungsstrukturen teilhaben, die in einem bestimmten Erfahrungsraum gegeben sind« (ebd., S. 632).

Die dokumentarische Methode ist also darauf gerichtet, einen Zugang zu konjunktiven Erfahrungsräumen bzw. zum konjunktiven Wissen zu erschließen. Mit dieser "grundlagentheoretischen Kategorie des konjunktiven Erfahrungsraums wird das menschliche Miteinander, das sich in der gelebten Praxis fraglos und selbstverständlich vollzieht, gefasst « (Przyborski/Wohlrab-Sahr 2010, S. 279). Im Gegensatz dazu ist die Erschließung des kommunikativen Wissens unproblematisch, da es abgefragt werden kann und den Akteur_innen unmittelbar zugänglich ist. Dieses kommunikative Wissen entspricht dem, was Schütze (1987, S. 145ff.) in der Rekonstruktion narrativer Interviews als Textsorten der Argumentation und Bewertung herausgearbeitet hat. In detaillierten Beschreibungen und noch deutlicher in Erzählungen wird dagegen vor allem das implizite bzw. atheoretische Wissen der Erzähler_innen aktualisiert. Allerdings lässt sich dieses Wissen nicht in jedem Fall als »konjunktiv« beschreiben, denn Erzählungen sind nicht nur durch kollektives, sondern auch durch individuelles Wissen geprägt (vgl. Nohl 2016, S. 332). 
Die dokumentarische Methode wird diesem Verhältnis von Individualität und Kollektivität dadurch gerecht, indem sie bei biografischen Interviews zunächst den Fall als solchen interpretiert und in einem zweiten Schritt anhand fallübergreifender Regelmäßigkeiten eine Typenbildung generiert. Diese »typisierbaren Regelmäßigkeiten lassen sich, da sie ja auch bei anderen Akteuren zu finden sind, dann nicht mehr als rein individuell bezeichnen; vielmehr verweisen sie auf die Einbettung dieser Akteure in kollektive bzw. konjunktive Erfahrungsräume« (ebd.).

\subsubsection{Orientierungsmuster: Orientierungsschemata und Orientierungsrahmen}

Die dokumentarische Methode geht davon aus, dass handelnde Subjekte gesellschaftlichen Strukturen nicht einfach nur ausgesetzt sind, sondern auf Grundlage ihres Alltagswissens und in Relation zu gesellschaftlichen Bedingungen ihre soziale Wirklichkeit durch ihr praktisches Handeln konstruieren. Dabei werden in Bezugnahme auf die Handlungstheorie von Alfred Schütz zwei Wissensformen handlungsleitenden Erfahrungswissens unterschieden: Orientierungsschemata und Orientierungsrahmen (vgl. Bohnsack 2012).

Orientierungsschemata repräsentieren kommunikatives Wissen über institutionalisierte Verläufe, mit denen sich Individuen auseinandersetzen und in denen sie agieren. Sie werden von den jeweils einzelnen Akteur_innen als soziale Anforderungen erlebt, die eine normative Wirkung haben. Solche institutionalisierten normativen Vorgaben der Gesellschaft zeigen sich bspw. in der Schule, die die Anerkennung gewisser Regeln voraussetzt, oder in den allgemeinen Regeln des Umgangs miteinander.

Explizites bzw. kommunikativ-generalisiertes Wissen und damit Handlungsentwürfe finden sich als Orientierungsschemata in theoretisierenden Wissensbeständen wieder, die den Erzähler_innen direkt zugänglich sind. In argumentativen Passagen, in denen Handeln im Zuge der Konstruktion von »Um-zu-Motiven ${ }^{3}$ erläutert wird und in denen Orientierungsschemata deutlich werden, zeigen sich bspw. Erwartungen, die die Erzählerinnen an ihre Beziehungspartner_in haben.

Dagegen verweist der Begriff des Orientierungsrahmens auf den je eigentümlichen, konjunktiven Erlebnis- und Erfahrungszusammenhang. Er setzt sich »aus in der eigenen Sozialisation gemachten konkreten Erfahrung zusammen, die in der Summe zu einem eigenen Fundus an Alltagswissen und einer bestimmten Art des Handelns führt« (Kleemann u.a. 2009, S. 157). Implizite Wissensbestände und

3 Alfred Schütz (1971 und 1974) hat im Anschluss an Max Weber den subjektiv gemeinten Sinn als Grundbaustein der Handlungstheorie verwendet und ihn als Entwurf verstanden, an dem sich das Handeln orientiert. Er hat diesen Entwurf auch als »Um-zu-Motiv« bezeichnet (vgl. Bohnsack 2012, S. 121). 
mentale Bilder, die als Orientierungsrahmen bezeichnet werden, ergeben als Produkt den sogenannten Modus Operandi (vgl. Bohnsack 2012, S. 125). Die Rekonstruktion dieser mentalen Bilder erfolgt in der empirischen Analyse von Erzählungen und Beschreibungen der Handlungspraktiken durch die Erzähler_innen. Allerdings kann der Modus Operandi auch das Ergebnis inkorporierter Praktiken sein. Hier sind die Orientierungsrahmen dann durch die direkte Beobachtung der Performanz von Interaktion und Gesprächen zugänglich:

»Das atheoretische Wissen und der darin fundierte Orientierungsrahmen umfassen also sowohl das inkorporierte Wissen, welches in Form materialer (Ab-)Bilder empirisch-methodisch in valider Weise zugänglich ist, wie auch das implizite oder metaphorische Wissen im Medium des Textes, für welche mentale Bilder, also Metaphern, von zentraler Bedeutung sind.«(Bohnsack 2012, S. 126)

Häufig wird der Begriff des Orientierungsrahmens synonym mit dem des Habitus verwendet. Bohnsack (2012) schreibt jedoch dem Orientierungsrahmen eine weitergreifende Bedeutung zu, da er sowohl Bezüge zu grundlegenden Kategorien der Mannheimschen Wissenssoziologie als auch zu Kategorien der Ethnomethodologie und in Teilen zur Chicagoer Schule herstellt. Die in diesen genannten Traditionen entwickelten Kategorien, die sich auch unter dem Begriff interpretatives Paradigma zusammenfassen lassen, werden mit dem Oberbegriff Orientierungsschemata bezeichnet (vgl. ebd., S. 126).

Im Vergleich zum Habitusbegriff erweitert der Begriff des Orientierungsrahmens diesen um den Aspekt, »dass und wie der Habitus sich in der Auseinandersetzung mit den Orientierungsschemata, also u.a. den normativen Anforderungen und denjenigen der Fremd- und Selbstidentifizierung, immer wieder reproduziert und konturiert «(Bohnsack 2012, S. 126). Der Begriff des Orientierungsrahmens bezeichnet im engeren Sinne, wie auch der des Habitus, die Struktur der Handlungspraxis und ist damit der Gegenbegriff $\mathrm{zu}$ dem des Orientierungsschemas. Im weiteren Sinne ist der Orientierungsrahmen jedoch auch als übergeordneter Begriff zu demjenigen des Orientierungsschemas zu verstehen, denn die Orientierungsschemata erhalten »ihre eigentliche Bedeutung erst durch die Rahmung, d.h. die Integration der >Brechung in und durch die fundamentale existentielle Dimension der Handlungspraxis, ... wie sie sich im modus operandi des Habitus oder eben Orientierungsrahmens vollzieht« (Bohnsack 2013, S. 181, Herv. im Original). 


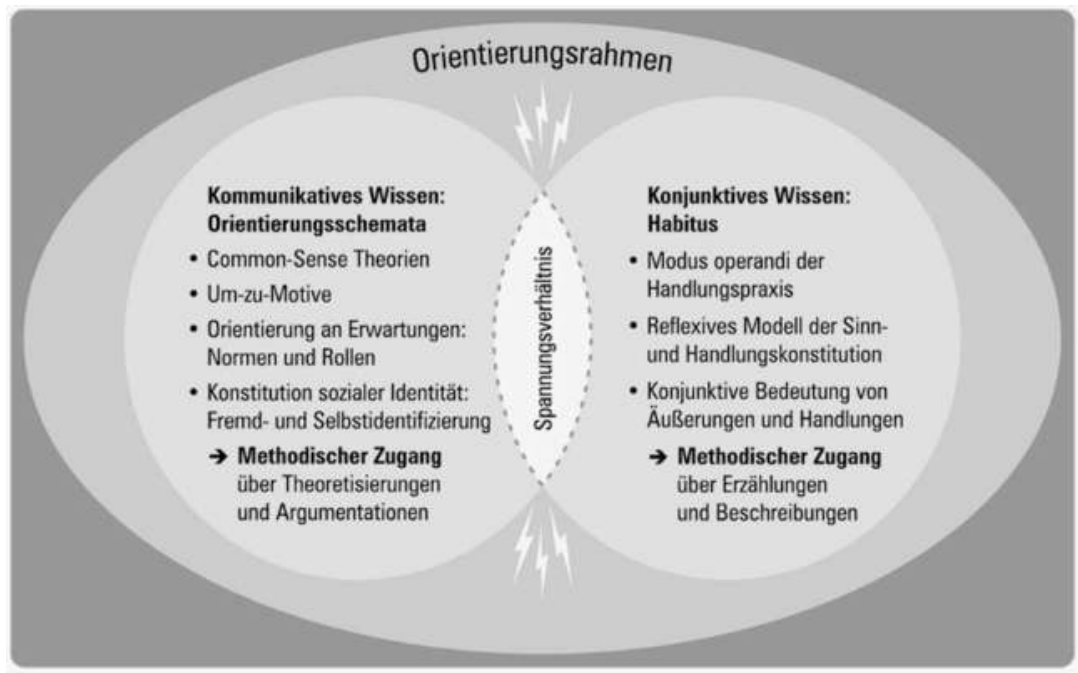

Aus der Übertragung der beschriebenen grundlagentheoretischen Annahmen ergeben sich für die vorliegende Studie zwei Ziele:

1. Der Fokussierung auf die Genese von Beziehungsvorstellungen, die aus der Fragestellung resultiert, liegt die Annahme individueller Lebensgeschichten zugrunde. Das bedeutet, dass von einer individuierten Struktur von Haltungen und Dispositionen ausgegangen wird, wonach die individuell-biografischen Haltungen in den Vordergrund rücken. Die Frage nach der individuellen Genese von Orientierungsrahmen setzt also eine Fokussierung auf den fallbezogenen individuellen Orientierungsrahmen voraus und erfordert zugleich eine biografische Perspektive. Deshalb wurde in der vorliegenden Arbeit von der üblicherweise verfolgten methodischen Umsetzung der dokumentarischen Methode, zügig in die komparative Analyse der Fälle einzusteigen, abgewichen. Stattdessen verfolgt die Arbeit, der aus der Fragestellung und theoretischen Verortung resultierenden Gewichtung der individuell-biografischen Haltung, auf methodischer Ebene einen konsequenten Fallbezug ${ }^{4}$.

2. Zum zweiten fokussiert die Arbeit auf kollektive Orientierungsrahmen und Erfahrungshintergründe - im Sinne des bisher der dokumentarischen Methode eigenen Fokus - und richtet den Blick darauf, wie sich diese insbesondere im

4 Werner Helsper u.a. (2013) nutzten die dokumentarische Interpretation narrativer Interviews dazu, individuelle Orientierungsrahmen herauszuarbeiten, und erweiterten damit den Anwendungsbereich der dokumentarischen Methode erheblich. 
Rahmen des konjunktiven Erfahrungsraums der Familie herausbilden. Die hier fallübergreifend rekonstruierten Regelmäßigkeiten lassen sich dann in eine Typenbildung überführen. Diese typisierbaren Regelmäßigkeiten im Umgang mit Gewalt innerhalb der ersten eigenen Paarbeziehungen, die sich auch bei anderen Akteurinnen finden lassen, sind nicht mehr als rein individuell zu bezeichnen, sondern verweisen auf die Einbettung in konjunktive Erfahrungsräume.

\subsection{Methodische und methodologische Überlegungen zur empirischen Erhebung von Gewalt in Paarbeziehungen}

Cornelia Helfferich u.a. (2016) argumentieren, dass es keine spezielle Methodologie der Gewaltforschung gibt. Vielmehr sei es erforderlich, unterschiedliche sozialwissenschaftliche Forschungszugänge für je unterschiedliche Fragestellungen zu nutzen (vgl. ebd., S. 5). Unabhängig von der gewählten Methode ergeben sich jedoch für alle Forschenden gemeinsam besondere Anforderungen an eine ethische und reflektierende Haltung:

»Aus dieser Verantwortung heraus müssen sich alle Forschungstraditionen daraufhin kritisch hinterfragen lassen, wie sie es mit dem gesellschaftlichen Umgang mit Gewalt, in den sie eingebunden sind, und mit den wissenschaftlichen Konstruktionen von Gewalt halten, an denen sie mitbeteiligt sind. Forschung kann Gewalt bagatellisieren oder spezifische Aspekte skandalisieren, sie kann differenzieren oder pauschalisieren, sie kann Erkenntniskategorien fortschreiben oder kritisieren und weiterentwickeln und in der Regel fokussiert sie bestimmte Aspekte und blendet andere aus. Da der gesellschaftliche Umgang mit Cewalt einem historischen Wandel unterliegt und die Erkenntnismöglichkeiten stets zeithistorisch gebunden sind, muss die Forschung ihre Ergebnisse stets an den gesellschaftlichen Entwicklungen abgleichen und das `Cui bo?<, also den Nutzen und die Folgen der Forschung, auf den Prüfstand stellen.«(Helfferich u.a. 2016, S. 6f.)

Die vorliegende Forschungsarbeit versucht, den genannten Anforderungen gerecht $\mathrm{zu}$ werden, indem sie über ihren qualitativ-rekonstruktiven Zugang die Rekonstruktion des subjektiven Herstellungsprozesses bzw. Orientierungsrahmens, in dem Paarbeziehung gestaltet wird, in den Mittelpunkt stellt. Diese subjektiven Orientierungen liegen den wissenschaftlichen Konstruktionen zugrunde, die im Verlauf des Analyseprozesses entwickelt wurden. Zudem kommt der (Selbst-)Reflexion während des gesamten Forschungsprozesses ein hoher Stellenwert zu (vgl. dazu Kap. 7.5).

In der Forschung zu Gewalt im Geschlechterverhältnis bzw. zu Gewalt in sozialen nahen Beziehungen, wie sie der vorliegenden Arbeit auch zugrunde liegt, gilt es eine Reihe von ethischen Aspekten zu berücksichtigen. So habe ich mich 
zum einen bei der methodischen Gestaltung meines Forschungsdesigns für eine offene Erzählaufforderung entschieden, weil diese es ermöglicht, Gewalterfahrungen während des Interviews zu überspringen. Damit wurde versucht, eine zu starke psychische Belastung zu verhindern. Zum anderen hatte ich mich im Vorfeld über verschiedene Beratungs- und Unterstützungsangebote informiert, um ggf. an diese vermitteln zu können. Auch die Reflexion der Machtdimensionen im Forschungsprozess stellt einen Aspekt dar, den es einzubeziehen und zu reflektieren gilt (vgl. auch dazu Kap. 7.5).

\section{Der Prozess der Datenerhebung}

\subsubsection{Das autobiografisch-narrative Interview}

In der vorliegenden Untersuchung wird ein rekonstruktiver Zugang verfolgt. Die Entscheidung dafür beruht auf grundlagentheoretischen Vorannahmen, die im Folgenden kurz erläutert werden:

1. Erstens müssen die Genese, die Aufrechterhaltung und die Veränderung von sozialen oder psychischen Phänomenen rekonstruiert werden, um diese Phänomene verstehen und erklären zu können. Auch Schütze (1983) schlug unabhängig vom thematischen Schwerpunkt der Untersuchung vor, zur Erzählung der gesamten Biografie aufzufordern. Denn dies ermöglicht, einzelne Lebensbereiche und -phasen im Gesamtzusammenhang des Lebens und in ihrer Entstehung betrachten zu können.

2. Zweitens müssen die Sichtweise der Handelnden und ihre Handlungsabläufe sichtbar gemacht werden, um das Handeln selbst verstehen und erklären $\mathrm{zu}$ können.

3. Und drittens müssen Aussagen einer Interviewten in den Gesamtzusammenhang ihres gegenwärtigen Lebens eingebettet werden, um sie verstehen zu können (vgl. Rosenthal 2008, S. 165).

Diesen Vorannahmen folgend, bedeutet dies für die vorliegende Untersuchung: Um ein (gegenwärtiges oder vergangenes) soziales Phänomen wie das Erleben von Gewalt in der eigenen Paarbeziehung während des Jugend- bzw. jungen Erwachsenenalters verstehen und erklären zu können, braucht es den Einblick in die gesamte Biografie. So lässt sich ermitteln, welche Erfahrungen dem Gewalterleben vorausgingen und welche ihm folgten. Auf diese Weise lassen sich die Erfahrungen im Prozess des Werdens rekonstruieren. Autobiografische Stegreiferzählungen sind dabei prädestiniert "für die Darstellung jener Dimension, in der der persönliche Habitus sich dokumentiert« (Bohnsack 2010a, S. 66). In biografisch-narrativen In- 
terviews konzipiert sich also der dokumentarische Sinn in Form des Modus Operandi als Prozessstruktur des Habitus (vgl. Helsper u.a. 2013, S. 122). Damit wird deutlich, dass der Gegenstand der dokumentarischen Interpretation nicht allein der kollektive, sondern auch der »individuelle, der persönliche Habitus« (Bohnsack 2010a, S. 65) ist.

Ein weiterer Grund für die Wahl des autobiografisch-narrativen Interviews ergibt sich aus der Tatsache, dass die Thematik »Gewalt in jugendlichen und adoleszenten Paarbeziehungen « bislang wenig erforscht ist. Da es sich hier also um noch recht unbekannte Phänomene handelt, für die bisher explizit noch keine theoretischen Konzepte vorliegen, wird eine qualitative Untersuchung angelegt (vgl. Rosenthal 2008, S. 18). Um einen möglichst umfassenden methodisch-wissenschaftlichen Zugang zu den individuellen Orientierungsrahmen zu erhalten, werden in Anlehnung an Schütze (vgl. Schütze 1983) biografisch-narrative Interviews durchgeführt. Ziel dabei ist es, »Sinnkonstruktionen und Handlungen aus der Perspektive der handelnden und erleidenden Individuen $\mathrm{zu}$ erfassen und einer Analyse zugänglich zu machen« (Jakob 1997, S. 335). Im Sinne der rekonstruktiven Forschungslogik ermöglicht diese Form des offenen Interviews den Erforschten die Strukturierung des Gesprächs, sodass Vorannahmen der Forschenden dieses so wenig wie möglich beeinflussen. Der Interviewpartnerin wird eine autobiografische Darstellung, ausgehend von den eigenen Relevanzsetzungen, ermöglicht. Eine solche Gesprächsführung, die der Offenlegung alltagsweltlicher Konstruktionen und subjektiver Deutungssysteme sowie dem Nachvollzug von Handlungsabläufen dient, vermeidet vorzeitige Themensetzungen und Definitionen seitens der Forscherin (vgl. Loch/Rosenthal 2002, S. 221). Gerade bei Fragen nach dem Erleben von Gewalt ist eine grundlegende Offenheit gegenüber den eigenen Relevanzsetzungen und Definitionen der Erzählerinnen erforderlich:

»Die Wahl des narrativen Interviews als Erhebungsinstrument begründet sich vor allem in seiner prinzipiellen Offenheit, welche den Cesprächspartnerinnen ein Einlassen auf ihre Erinnerungen jenseits der gesellschaftlichen und individuell konstituierten Kommunikationszwänge ermöglicht. Hierdurch können Erzählprozesse in Gang gesetzt werden, deren inhaltliche Darstellung näher am Erleben und damit oftmals auch weiter entfernt von verinnerlichten gesellschaftlichen Cesprächserwartungen sind. Das heißt, mit dem narrativen Interview werden Daten erhoben, die den Forschenden eine Unterscheidung zwischen den Erzähl- und Erlebensperspektiven ermöglichen.«(Loch 2002, S. 234)

Bei der Frage nach dem Erleben von Gewalt ist davon auszugehen, dass die befragten Frauen traumatisierende Erlebnisse erfahren haben. Dies kann dazu führen, dass an Stellen im Interview, an denen (unbearbeitete) Traumatisierungen angesprochen werden, Ausführungen verwirrend oder bruchstückhaft sind. Die mittels des narrativen Interviews erhobenen Daten ermöglichen den Forschenden dann 
die notwendige Unterscheidung zwischen den Erzähl- und Erlebensperspektiven (vgl. Loch 2002, S. 234).

Dieser Zugang zu unterschiedlichen Ebenen der Erfahrungsbildung im Alltag bildet das Zentrum der von Fritz Schütze entwickelten Methodologie des narrativen Interviews. Anknüpfend an die Phänomenologische Soziologie von Alfred Schütz sowie an den Symbolischen Interaktionismus (Herbert Blumer) sucht Fritz Schütze den Zugang zu der für die Alltagswirklichkeit und Alltagshandeln konstitutiven Erfahrungen auf dem Wege der Erzählungen (vgl. Bohnsack 2010, S. 91). Einerseits entwickelte Schütze eine sprachsoziologisch fundierte Theorie des Erzählens, andererseits beinhaltet die Methodologie des narrativen Interviews aber auch eine Theorie über das, was in der Erzählung inhaltlich mitgeteilt wird (vgl. ebd., S. 92):

- Mit dem narrativen Interview lässt sich die soziale Wirklichkeit aus der Perspektive der handelnden und erleidenden Subjekte erfassen. Das Forschungsinteresse ist immer darauf gerichtet, wie die Subjekte die soziale Wirklichkeit erfahren und an ihrer Herstellung beteiligt sind.

- Das narrative Interview ermöglicht durch das freie Erzählenlassen von Geschichten Zugang zu subjektiven Bedeutungsstrukturen, die sich einem systematischen Abfragen versperren würden.

- Weiter lassen sich anhand von lebensgeschichtlichen Darstellungen Veränderungsprozesse im Zusammenhang mit biografischen Erfahrungen herausarbeiten (Jakob 1997, S. 445). In der vorliegenden Arbeit interessiert dabei auch, inwiefern familiäre Einflüsse und Sozialisationsbedingungen spätere Erfahrungen in den eigenen Paarbeziehungen beeinflussen.

- Mithilfe des narrativen Interviews lassen sich sowohl Veränderungen in den Gefühlszuständen, Identitätsentwürfen usw. als auch äußere soziale und gesellschaftliche Veränderungen rekonstruieren (vgl. ebd., S. 447). Die vorliegende Arbeit nimmt hierbei in den Blick, wie sich die Gestaltung von Paarbeziehungen im gesellschaftlichen Wandel vollzieht: »Mit der Analyse individueller Biografien werden damit auch soziale Rahmen und kollektiv-historische $\mathrm{Ab}$ läufe in ihren Auswirkungen auf die Lebensführung und die Lebensgeschichte sichtbar.« (Ebd.)

- Die Rekapitulation des eigenen Handelns in der Erzählung geht mit einer Rekonstruktion der Orientierungsstrukturen des Handelns einher (Schütze 1977, S. 1). Hier verknüpft sich der Anspruch der dokumentarischen Methode mit dem Anliegen des biografisch-narrativen Interviews, indem sich beide dafür interessieren, wie die Erfahrungen, Theoretisierungen und Orientierungen der Interviewten in deren Handlungsgeschichte eingebettet werden. 
Der Zugang über biografisch-narrative Interviews bietet eine Annäherung an die Perspektive der von Gewalt betroffenen jungen Frauen und deren individuelle Orientierungsrahmen und ermöglicht eine individuelle, familienspezifische, biografisch ausgerichtete Sicht auf die Handlungspraxis der Beziehungsgestaltung junger Frauen.

\subsubsection{Erzähltheoretische Grundlagen}

Eine wesentliche methodologische Grundannahme des narrativen Interviews ist, dass die Erzählerin ihre Lebensgeschichte so reproduziert, wie sie sie auch erfahren hat und wie sie für ihre Identität konstitutiv und somit auch handlungsleitend ist. Damit wird von einer Homologie von Erzähl- und Erfahrungskonstitution ausgegangen. Diese Homologie von Erzählung und Erfahrung findet sich in der (biografischen) Stegreiferzählung wieder: »Das Stegreiferzählen ist ein schöpferischer Akt, es gestaltet den Strom der gemachten Erfahrungen weit über die anfänglichen Erwartungen und Vorabbilder des Erzählers hinaus. «(Schütze 1987, S. 184) Indem die Erzählerin mittels einer offenen, erzählgenerierenden Aufforderung zu einer autobiografischen Stegreiferzählung eingeladen wird, wird eine Dynamik in Gang gesetzt, die nicht mehr gesteuert werden kann. Die Erzählerin/der Erzähler muss sich nun »auf die zusammenhängende Reproduktion bereits abgearbeiteter (und in ihrer Selbsterfahrung und Selbstkonstitution verankerter) sowie theoretisch-reflexiv wenig überformter Erfahrungen einlassen« (Bohnsack 2010, S. 93).

Die Stegreiferzählung folgt in ihrem formalen Aufbau den sogenannten »Zugzwängen des Erzählens«, wie sie Kallmeyer und Schütze (1977) ausgearbeitet haben und die sich auch auf Erkenntnisse der Konversationsanalyse beziehen:

- Kondensierungszwang: Die Darstellung muss verdichtet, kondensiert erfolgen, damit sie überschaubar bleibt und innerhalb der verfügbaren Zeit (eine Interviewsituation ist i.d.R. begrenzt, schon allein aufgrund der begrenzten Konzentrationsfähigkeit von Erzählerin und Zuhörerin) erfolgen kann.

- Detaillierungszwang: Bestimmte erwähnte lebensgeschichtliche Ereignisse müssen zum Zweck der Plausibilisierung in Zusammenhang mit anderen Ereignissen gebracht werden. Die Darstellung erfolgt dabei so detailliert wie notwendig.

- Gestaltschließungszwang: Inhaltliche Darstellungen in Erzählungen müssen gegenüber anderen Darstellungen in sich, in ihrer Gestalt, geschlossen sein (vgl. ebd. S. 162).

Die durch diese Zugzwänge innerhalb der Stegreiferzählung entwickelte Eigendynamik ist von der Selbstdarstellung der Erzählenden relativ unabhängig und trägt zur Freisetzung der gemachten Erfahrungen bei. Diese finden sich dann in den 
narrativen Passagen, die nicht theoretisch-reflexiv überformt sind (vgl. Bohnsack 2010, S. 94).

Die Haupterzählung wird i.d.R. mit einer Erzählkoda beendet, an die sich zunächst die »immanenten Nachfragen« (Schütze 1983) anschließen, bei dem die Interviewer_innen Unklarheiten ansprechen oder Fragen zu den angesprochenen Themen stellen. Den Abschluss bilden die »exmanenten Nachfragen« (ebd.) oder die Bilanzierungsphase (Friebertshäuser 1997, S. 387), in der auch Fragen gestellt werden können, die auf Erklärungen oder eine Bilanz der Geschichte abzielen.

\subsubsection{Sampling}

Eine der Voraussetzungen für die Verallgemeinerbarkeit einer empirischen Studie ist die Repräsentativität der erhobenen Daten. Bei qualitativ-rekonstruktiven Untersuchungen geht es, im Unterschied zu quantitativen Studien, nicht um die Gewährleistung einer statistischen Repräsentativität, sondern darum, die im untersuchten Feld vorhandene Heterogenität zu berücksichtigen und zu dokumentieren (vgl. Kelle/Kluge 2010, S. 109).

In der vorliegenden Untersuchung erfolgte die erste Auswahl der Stichprobe anhand vorab definierter äußerer Merkmale (vgl. Fuchs-Heinritz 1998, S. 10):

- Bei den befragten Frauen, im Alter zwischen 18 und 35 Jahren, ${ }^{5}$ sollte die Gewaltbeziehung beendet sein bzw. das Gewalterleben in der Vergangenheit liegen. Dies ermöglicht eine Distanz im Erzählen, gleichzeitig liegen die Erfahrungen aber nicht zu weit zurück, sodass retrospektive Verzerrungen eher vermieden werden.

- Das Sample sollte ein Alter von 18 Jahren nicht unter- und ein Alter von 35 Jahren nicht überschreiten. Damit soll die inhaltliche Abgrenzung des Forschungsgegenstandes zu häuslicher Gewalt geschaffen werden. Die Forschungsperspektive der vorliegenden Arbeit richtet sich darauf, die Erfahrung von Gewalt in den ersten eigenen Beziehungen in den Kontext der Entwicklung von Paarbeziehungen einzuordnen. In diesem Lebensabschnitt sind andere Themen wie bspw. die Ablösung von der Herkunftsfamilie, Auseinandersetzung mit Peers, Aufnahme von Liebesbeziehungen, Zukunftsvorstellungen und eigene Beziehungsvorstellungen relevant.

5 Die Altersspanne von 18 bis 35 Jahren ergab sich erst im Prozess der Datenerhebung. Bei der Suche nach Interviewpartnerinnen verzichtete ich bewusst auf Alterseingrenzungen. Der Fokus war immer auf die ersten eigenen Beziehungserfahrungen gerichtet. Als Obergrenze wurde erst im Laufe der Erhebung das Alter von 35 Jahren festgelegt, um einerseits die Abgrenzung zu häuslicher Cewalt zu gewährleisten. Andererseits sollten die Biografien innerhalb der aktuellen »Cenerationslagerung (Mannheim 1964) verortet sein, um Bezüge zu den und Einordnungen in die aktuellen gesellschaftlichen Rahmenbedingungen und Entwicklungen herstellen zu können. 
- Die Entscheidung, nur Frauen nach ihrem Erleben von Gewalt zu befragen, beruht auf zwei Aspekten: Zum einen müssen Fragen nach Gewalt immer im Kontext von Gewalt im Geschlechterverhältnis betrachtet werden (vgl. ausführlicher dazu Kap. 4.2). Dabei ist Gewalt gegen Frauen in ein strukturelles Machtverhältnis eingebunden, das spezifische verbreitete Gewaltformen ermöglicht und fördert (vgl. Hagemann-White 2016, S. 19). Auf genau diesen Kontext richtet die vorliegende Forschungsarbeit ihr Augenmerk. Zum anderen unterliegt auch ein Dissertationsvorhaben einem gewissen begrenzten Rahmen, weshalb hier letztlich auch aus forschungspragmatischer Perspektive der Fokus auf Frauen gerichtet ist. Eine Erweiterung des Samples um Männer wäre ebenfalls wichtig und spannend, kann aber in dieser Arbeit nicht mit abgedeckt werden.

- Dem Einbezug von hetero- und homosexuellen Beziehungserfahrungen liegt die Absicht zugrunde, ein Sample zu bilden, das nicht der heterosexuellen Matrix (Butler 1991) entspricht.

Nach Festlegung dieser äußeren Merkmale wurde die Stichprobe nach der von Glaser und Strauss vorgeschlagenen Strategie des "theoretical sampling" (Strauss/Corbin 1996) zusammengestellt. Theoretisch relevante Konzepte und Phänomene, die aufgrund der Auswertung der ersten Fälle entwickelt werden, leiten dann die Auswahl weiterer Fälle an. Beim theoretical Sampling werden Fälle miteinander verglichen, die »hinsichtlich theoretisch bedeutsamer Merkmale entweder relevante Unterschiede oder große Ähnlichkeit aufweisen« (Kelle/Kluge 2010, S. 48, Herv. im Original), sie werden also minimal oder maximal kontrastiert. Allerdings ist es, wie Strübing einwendet, in der Praxis empirischer Forschung mitunter kaum möglich, die Generierung von Daten über einen langen Zeitraum zu strecken und nach den im Theoriebildungsprozess gewonnenen Erkenntnissen neue Daten zu erheben (vgl. Strübing 2014, S. 30). Diese Einschränkung verhindert jedoch nicht die Anwendung des theoretical Sampling. Stattdessen ist im Zweifelsfall, wenn die Auswahlkriterien nicht immer eindeutig bestimmbar sind oder der Feldzugang zeitlich begrenzt ist, »eine begründungspflichtige Abweichung von der prinzipiellen Vorgehensweise« (ebd., S. 19) offenzulegen. Die theoretische Sättigung ist dann erreicht, wenn keine neuen Eigenschaften der Kategorie resp. keine neuen Phänomene im Material gefunden werden.

Wenn, wie in der vorliegenden Arbeit, Personen befragt werden, die für eine sozialwissenschaftliche Untersuchung schwer zu erreichen sind, ist zwangsläufig ein eingeschränkter Feldzugang gegeben und die Fallauswahl beruht nicht unbedingt auf bewussten Entscheidungen (vgl. Schittenhelm 2012, S. 407). Daher muss die Qualität eines Samplings immer auch im Kontext von der Beschaffenheit des Untersuchungsfeldes und den vorhandenen Zugangsvoraussetzungen gesehen werden. 
Nach der Methode des »theoretical sampling« werden nach theoretischen Gesichtspunkten, die sich im Laufe des Analyseprozesses ergeben, nach und nach Interviewpartner_innen gesucht (vgl. Przyborski/Wohlrab-Sahr 2010, S. 177). Die Entscheidung für die ersten Fälle wird zunächst auf der Basis einer vorläufigen Problemdefinition oder Gegenstandsbeschreibung getroffen. Dafür wählte ich verschiedene Kanäle, die mir zur Verfügung standen: Ich entwickelte ein Informationsschreiben, das ich an mir bekannte Kolleginnen aus Praxis und Hochschulen weiterleitete, die teils explizit zum Thema Gewalt, teils aber auch in ganz anderen sozialen Arbeitsfeldern arbeiteten. Diese wiederum leiteten mein Anliegen an die ihnen bekannten Netzwerke, Gruppen etc. weiter. Mit dem Vorgehen, über verschiedene Zugangswege Interviewpartnerinnen zu gewinnen, war das Ziel verbunden, nicht nur Frauen zu erreichen, die ihre Erfahrungen bereits im Kontext von Therapie, Beratung o. Ä. thematisiert hatten. Die Erhebungsphase erstreckte sich über einen Zeitraum von einem Jahr und fand zwischen August 2011 und September 2012 statt. Da der Rücklauf begrenzt war, führte ich mit allen Frauen, die sich bei mir meldeten, ein Interview. Lediglich Frauen in deutlich höherem Alter als 30 Jahre nahm ich nicht ins Sample mit auf.

Von fünf Frauen bekam ich den Kontakt über eine Fachkraft in der Sozialen Arbeit, woraufhin ich die Frauen telefonisch kontaktierte. Drei weitere Frauen, die sich per Mail oder telefonisch bei mir meldeten, schloss ich wegen ihres zu hohen Alters aus. Zwei vereinbarte Interviews kamen nicht zustande, da die Frauen zum Interviewtermin nicht erschienen. Hier kann nur vermutet werden, dass die Frauen das Interview kurzfristig als zu belastend erlebten.

Innerhalb dieser Fallgruppe zeigte sich im Laufe des Auswertungsprozesses, dass sich die Fälle hinsichtlich verschiedener Aspekte sowohl minimal als auch maximal kontrastieren ließen (Dauer der Gewaltbeziehung, Form und Intensität der Gewalt, Alter, Beendigung der Gewalt, eigentheoretische Erklärungen), sodass ich lediglich ein Interview zu Gewalterfahrungen in homosexuellen Beziehungen im Juli 2016 nacherhoben habe, um eine diesbezüglich größere Varianz zu erreichen.

Da auf der Basis der erhobenen Daten auch zu einem späteren Zeitpunkt entschieden werden kann, welche der vorliegenden Fälle einer intensiven Analyse unterzogen werden, wird auch von einem mehrstufigen Samplingverfahren gesprochen (Schittenhelm 2012). So habe ich nach Beginn der Interpretation der ersten Fälle und dem damit verbundenen zunehmenden systematischen Fallvergleich (Bohnsack 2010) auf Basis der dokumentarischen Methode einen Eckfall (Anna) ausgewählt und ausgewählte Fälle einer intensiveren Analyse unterzogen.

Darstellung des Samples In den insgesamt acht durchgeführten Interviews hatten drei Frauen einen Migrationshintergrund, sieben Frauen erlebten Gewalt in heterosexuellen Beziehungen, eine Frau in homosexueller Beziehung. Alle Frauen erlebten psychische und physische Gewalt innerhalb der ersten oder in einer der ersten 
Paarbeziehungen im Alter zwischen 15 bis Anfang 20. Der Zeitraum, in dem Gewalt erlebt wurde, variierte zwischen einem Jahr und mehreren Jahren. Die meisten Frauen hatten bereits Gewalt auf der Elternebene und/oder der Eltern-Kind-Ebene in der Herkunftsfamilie erlebt.

Tabelle 2: Sample der Untersuchung

\begin{tabular}{|c|c|c|c|c|c|}
\hline Name & Alter & $\begin{array}{l}\text { Migrations- } \\
\text { hintergrund }\end{array}$ & Tätigkeit & $\begin{array}{l}\text { Formen } \\
\text { der } \\
\text { Gewalt }\end{array}$ & $\begin{array}{l}\text { Gewalt in der } \\
\text { Herkunftsfamilie }\end{array}$ \\
\hline Anna & 26 & - & Studentin & $\begin{array}{l}\text { physisch } \\
\text { psychisch }\end{array}$ & Eltern-Kind-Ebene \\
\hline Zofia & 31 & Tschechoslowakei & Studentin & $\begin{array}{l}\text { physisch } \\
\text { psychisch }\end{array}$ & Eltern-Kind-Ebene \\
\hline Amy & 27 & - & Auszubildende & $\begin{array}{l}\text { physisch } \\
\text { psychisch } \\
\text { sexualisiert }\end{array}$ & Eltern-Kind-Ebene \\
\hline Alina & 18 & - & Auszubildende & $\begin{array}{l}\text { physisch } \\
\text { psychisch } \\
\text { sexualisiert }\end{array}$ & $\begin{array}{l}\text { häusliche Gewalt } \\
\text { Eltern-Kind-Ebene }\end{array}$ \\
\hline Emira & 27 & Kosovo & erwerbstätig & $\begin{array}{l}\text { physisch } \\
\text { psychisch } \\
\text { sexualisiert }\end{array}$ & 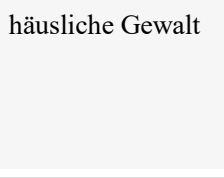 \\
\hline Daria & 18 & Ukraine & Auszubildende & $\begin{array}{l}\text { physisch } \\
\text { psychisch }\end{array}$ & - \\
\hline Jasmin & 23 & - & erwerbstätig & $\begin{array}{l}\text { physisch } \\
\text { psychisch }\end{array}$ & $\begin{array}{l}\text { häusliche Gewalt } \\
\text { Eltern-Kind-Ebene }\end{array}$ \\
\hline Angela & 35 & - & erwerbstätig & $\begin{array}{l}\text { physisch } \\
\text { psychisch }\end{array}$ & - \\
\hline
\end{tabular}




\subsubsection{Interviewdurchführung}

Bei den Vorgesprächen erläuterte ich nochmals mein Forschungsvorhaben und betonte die Freiwilligkeit der Teilnahme. Die Entscheidung, an welchem Ort die Frauen die Gespräche durchführen wollen, überließ ich ihnen und machte nur Vorschläge, wenn sie äußerten, es sei ihnen egal. Angesichts des belastenden Themas wollte ich den Interviewpartnerinnen die Möglichkeit geben, einen für sie geschützten Ort wählen zu können. Alle Frauen entschieden sich für einen neutralen Ort, sodass die Interviews entweder an einem öffentlichen Ort, in einem Park oder Café stattfanden. Zwei Interviews fanden in einem Beratungsraum statt, den ich organisierte. Ein weiteres Gespräch führte ich in einer Tagesklinik, in der die Interviewpartnerin zum Zeitpunkt des Interviews betreut wurde.

Die Interviews erfolgten in einem zeitlich offenen Rahmen, was bedeutete, dass ich sowohl für die Interviews selbst als auch für die Small-Talk-Phase davor und danach einige Stunden Zeit eingeplant hatte. Vor Ort erläuterte ich nochmals mein Forschungsanliegen, sicherte erneut die Anonymisierung zu und wies auf die Möglichkeit hin, jederzeit Pausen einlegen oder das Interview abbrechen zu können.

Im Sinne der rekonstruktiven Forschungslogik ist in der Interviewdurchführung darauf $\mathrm{zu}$ achten, dass Vorannahmen der Forschenden nicht dazu führen, das Gespräch durch ihre Fragen zu beeinflussen. Die Strukturierung des Interviews erfolgt entlang der Relevanzsetzungen und Orientierungen der Befragten. Zudem orientierte ich mich an den von Rosenthal u.a. (2006) erarbeiteten Anleitungen zu einer konsequent biografisch-narrativen Gesprächsführung.

Wenn die Frauen keine Nachfragen mehr hatten, formulierte ich meine Erzählaufforderung, die in der Regel wie folgt lautete:

»Ich möchte dich bitten, mir deine Lebensgeschichte zu erzählen, alle Erlebnisse, die dir einfallen. Du kannst dir so viel Zeit nehmen, wie du möchtest. Ich werde dich erst mal nicht unterbrechen und mir nur einige Notizen machen, auf die ich später zurückkomme.«

Auch wenn ich mit diesem Erzählimpuls selbstläufige Erzählungen ermöglichen wollte, gelang dies nicht in jedem Interview gleichermaßen gut. Teilweise war es für die Frauen schwierig, von bestimmten Erfahrungen zu berichten. Sei es, weil diese unangenehm oder auch dissoziiert waren. So weisen manche Interviews immer wieder dialogische Passagen auf oder Abschnitte, in denen die Erzählerinnen über sich selbst und ihre Lebensgeschichte reflektieren und theoretisieren. Mir fiel es insbesondere in den ersten Interviews schwer, Nachfragen zu konkreten Gewalterlebnissen oder anderen schwierigen Erfahrungen zu stellen, da ich Sorge hatte, dass diese Fragen zu einer Retraumatisierung führen könnten (vgl. dazu Kap. 7.6, in dem beschrieben wird, wie ich mich mit dem Thema Traumatisierungen auseinandergesetzt habe). Dennoch bilden die Interviews eine ausreichende Grundlage, 
um etwas über den Stellenwert von Beziehungen in der Adoleszenz und dem jungen Erwachsenenalter und damit verbundenen Gewalterfahrungen innerhalb der Biografie zu erfahren. Grenzen zu respektieren und Auslassungen in Erzählungen zu akzeptieren, sehe ich dabei auch als ethisches Prinzip innerhalb von Forschung an.

\subsection{Der Prozess der Datenauswertung}

Im Folgenden werden die aufeinander aufbauenden Arbeitsschritte der dokumentarischen Interpretation vorgestellt und deren Umsetzung in der vorliegenden Forschungsarbeit dargelegt. Dabei beziehe ich mich vor allem auf Arnd-Michael Nohl (2009), der in kritischer Auseinandersetzung mit der dokumentarischen Methodik, aber auch auf deren Grundlage, ein alternatives Auswertungsverfahren für (biografisch-)narrative Interviews entwickelt hat.

Die dokumentarische Interpretation von Interviews beruht demnach auf einer strikten Trennung zwischen der »formulierenden « Interpretation und der »reflektierenden « Interpretation. Diese Trennung zielt auf die Rekonstruktion des Orientierungsrahmens, in dem die Themen bearbeitet werden. Weiter wird von Beginn an eine konsequente komparative Analyse verfolgt, die am Ende auf die Generierung von Typen zielt. (Vgl. Nohl 2009, S. 45)

Die formulierende Interpretation zielt auf den »immanenten « Sinngehalt, indem der jeweilige Abschnitt sequenziell nach Ober- und Unterthemen gegliedert und $\mathrm{zu}$ jedem Unterthema eine thematische Zusammenfassung erstellt wird. Durch die Reformulierung des thematischen Gehalts gewinnen die Interpret_innen eine Übersicht über den Text, wobei die begrifflich-theoretische Explikation bereits eine erste Interpretation dessen darstellt, was im Text implizit ist (vgl. Bohnsack 2010, S. 134). Zwar schlägt die Methodenliteratur ${ }^{6}$ vor, zunächst durch das Abhören der Audioaufnahmen einen »thematischen Verlauf « (ebd., S. 135) zu erstellen, aus dem dann jene Passagen ausgewählt werden, die der formulierenden und reflektierenden Interpretation unterzogen werden; dennoch bin ich bereits zu Beginn des Forschungsprozesses dazu übergegangen, alle Interviews zu transkribieren. Meine Erfahrung war, dass sich im Verlauf des Interpretationsprozesses immer wieder neue Themen - zum Teil auch erst im Nachfrageteil - ergeben haben, die die Transkription der entsprechenden Passagen erforderten. Um den laufenden Interpretationsprozess dadurch nicht immer wieder unterbrechen zu müssen, habe ich

6 Mit Ausnahme von Nohl (2009), der für biografische Interviews eine vollständige Transkription zumindest der Stegreiferzählung für notwendig erachtet. 
mich für eine komplette Transkription ${ }^{7}$ der Interviews entschieden. ${ }^{8}$ Anhand der nun vorliegenden Transkripte erstellte ich thematische Verläufe, die mir ermöglichten, jene Themen zu identifizieren, die in unterschiedlichen Fällen behandelt werden und sich daher für die komparative Analyse eignen.

Der nächste Schritt, die reflektierende Interpretation, zielt auf die Rekonstruktion und Explikation des Rahmens, in dem ein Thema abgehandelt wird, und fragt somit nach dem Wie: Wie wird ein Thema bearbeitet? In welchem (Orientierungs-)Rahmen wird ein Thema bearbeitet? (vgl. Nohl 2009, S. 47).

Dabei trägt dieser Auswertungsschritt der Tatsache Rechnung, dass die Frage nach der Art und Weise, mit der ein Thema bearbeitet wird, auf eine entsprechende Semantik verweist. Daher wird zunächst eine Textsortentrennung, wie sie von Fritz Schütze für die Narrationsstrukturanalyse entwickelt worden ist, durchgeführt (vgl. ebd.). In Erzählungen und Beschreibungen werden die erlebten Handlungspraktiken der Akteur_innen deutlich, wodurch die Explikation des »atheoretischen« und »konjunktiven« Wissens möglich wird (vgl. Bohnsack/Pfaff 2010, S. 32). In Argumentationen verbergen sich dagegen Eigentheorien der Erzählenden, die Zugang zum »kommunikativen« Wissen ermöglichen.

Der nächste Auswertungsschritt der semantischen Interpretation zielt auf den dokumentarischen Sinngehalt und damit auf die Handlungsorientierungen und $\mathrm{Ha}$ bitusformen der Akteurinnen. Im »Dokumentsinn« zeigt sich, »was eine Äußerung implizit über die Orientierungen, Relevanzen, Normalitätsannahmen, Weltsichten - kurz: über das Alltagswissen von Sprechenden« (Kleemann u.a. 2009, S. 160) aussagt. Orientierungen meinen dabei Sinnmuster, die unterschiedliche Handlungen hervorbringen (Przyborski/Wohlrab-Sahr 2010, S. 289).

Die Interpret_innen gehen dabei »nicht davon aus, dass sie mehr wissen als die Akteure und Akteur_innen, sondern davon, dass letztere selbst nicht wissen, was sie da eigentlich alles wissen, somit also über ein implizites Wissen verfügen, welches ihnen reflexiv nicht so ohne weiteres zugänglich ist « (Bohnsack u.a. 2007, S. 11). Forschende haben damit die Aufgabe, Wissen, das von den Erforschten selbst nicht expliziert wird, (abduktiv) zur Explikation zu bringen. Damit wird ein Zugang zu einer Handlungspraxis und den ihr zugrunde liegenden Strukturen gewonnen, die sich der Perspektive der Akteure selbst entziehen (vgl. ebd., S. 12).

Für die vorliegende Untersuchung bedeutet dies, dass in einem ersten Schritt, konsequent fallbezogen der jeweilige individuelle Orientierungsrahmen rekon-

7 Die verwendeten Transkriptionsregeln befinden sich im Anhang.

8 Bei allen Transkriptionen wurde in Anlehnung an Rosenthal u.a. (2006) alles, was auf der Aufnahme zu hören war, ohne Berücksichtigung der Regeln der Schriftsprache wortwörtlich niedergeschrieben. Da die Verwendung von Satzzeichen bereits eine Interpretation bedeutet, wurde im Sinne der grammatikalischen Regeln auf diese verzichtet. Kommata wurden entsprechend der hörbaren Interpunktion gesetzt; auf Einteilung in Sätze durch Punkte, Ausrufe- und Fragezeichen habe ich verzichtet (vgl. ebd., S. 21f.) 
struiert wird. Hierbei ist die Perspektive auf die biografische Genese dieses individuellen Orientierungsrahmens gerichtet. Neben der Rekonstruktion des Orientierungsrahmens, in dem Paarbeziehung gestaltet wird, ist der Fokus auch auf die Auseinandersetzung mit normativen Erwartungen, also den Orientierungsschemata, gerichtet. Nur so kann aufgezeigt werden, wie sich der Habitus in Auseinandersetzungen mit den Orientierungsschemata immer wieder reproduziert und konturiert. In einem zweiten Schritt werden die aus den Fällen herausgearbeiteten Handlungsorientierungen komparativ analysiert und zu einer Typik des Umgangs mit Gewalterfahrungen verdichtet.

Für die dokumentarische Methode ist der fortlaufende Fallvergleich zentral. Interviews werden daraufhin verglichen, wie die Interviewten mit einem Thema umgehen, also in welchem Orientierungsrahmen sie ein Thema bearbeiten. Dabei strukturiert das sogenannte Tertium Comparationis, das gemeinsame Dritte, den Vergleich (vgl. Nohl 2009, S. 56).

Es werden sowohl mehrere Passagen innerhalb eines Falles als auch thematisch gleiche Passagen verschiedener Fälle sowie bereits identifizierte Orientierungen miteinander verglichen. Allerdings ist bei biografischen Interviews die Identifizierung gemeinsamer Themen vergleichsweise aufwändig und muss im thematischen Verlauf sowie in der formulierenden Feininterpretation erarbeitet werden (vgl. ebd.).

Aus diesem Grund habe ich bei allen sechs Interviews, die ausführlich interpretiert wurden, die jeweils komplette Stegreiferzählung der semantischen Interpretation unterzogen. Zudem habe ich weitere Passagen aus dem immanenten und exmanenten Nachfrageteil nach folgenden Kriterien ausgewählt und ebenfalls semantisch interpretiert:

1. Passagen, in denen sich Themen zeigen, die für meine Forschungsfrage inhaltlich relevant sind,

2. Passagen, die durch eine hohe narrative Dichte geprägt sind und bei denen die Interviewpartnerinnen Themen besonders ausführlich, detailliert oder engagiert berichtet haben. Diese Passagen werden auch als »Fokussierungsmethaphern« (Bohnsack 2010, S. 137) bezeichnet.

Dabei habe ich zunächst innerhalb der Einzelfälle verschiedene Äußerungen zu einem Thema fallimmanent kontrastiert. Nach und nach, im Prozess des zirkulären Auswertens, kristallisierten sich zunehmend die Themen heraus, die als Tertium Comparationis eine fallvergleichende Kontrastierung ermöglichten (z.B. erste Dates, erste Beziehungserfahrungen/der erste Freund, Gewalterfahrungen, Peers). Damit wird auch deutlich, dass die komparative Analyse in der dokumentarischen Methode bereits bei der Analyse der Sequenzen beginnt (vgl. Nohl 2007, S. 266). 
Nachdem homologe Sinnstrukturen bzw. Orientierungsrahmen innerhalb einer Passage identifiziert und weitere Orientierungsrahmen in anderen Passagen desselben Interviews gefunden wurden, gilt es, diese auch in anderen Fällen herauszuarbeiten (vgl. Przyborski/Wohlrab-Sahr 2010, S. 296). Indem die kontrastierenden Orientierungsrahmen der Vergleichsfälle eine eigenständige Bedeutung erhalten, werden sie zunehmend vom Einzelfall abstrahiert und zu Typen ausformuliert (vgl. Nohl 2009, S. 57). Durch diese komparative Analyse der Sequenzanalyse lassen sich aus den rekonstruierten Orientierungsrahmen sinngenetische Typen bilden. Wird eine komplexere Analyse verfolgt, und das Tertium Comparationis variiert, ist eine mehrdimensionale soziogenetische Typenbildung möglich, indem Bezüge zwischen spezifischen Orientierungen und deren Erlebnishintergrund, in dem die Genese der Orientierung zu suchen ist (vgl. Bohnsack 2010, S. 141), herausgearbeitet werden.

Ziel der vorliegenden Arbeit ist es, auf Grundlage der biografisch-narrativen Interviews und mithilfe der formulierenden und reflektierenden Interpretation Orientierungsrahmen zu identifizieren, die dann von den Einzelfällen abstrahiert und zu einer Typik des Umgangs mit Gewalterfahrungen auf der Ebene der sinngenetischen Typenbildung verdichtet werden.

\subsection{Reflektierte Subjektivität}

In diesem Kapitel reflektiere ich meine Rolle der Forscherin als Subjekt und als Teil der Welt, die ich erforsche, soweit als möglich. Dabei stelle ich auch dar, wie ich bereits während des Forschungsprozesses anhand verschiedener Maßnahmen und Strategien meine Subjektivität methodisch reflektierte.

Zunächst stellt eine Forschung zum Thema Gewalt besondere Anforderungen dar. Gewalt ist ein emotionales und moralisch aufgeladenes Thema, das eine reflektierte Haltung erfordert, da eine scheinbar neutrale Position dazu nicht möglich ist - mehr noch: Diese würde »das Gewaltgeschehen vom Blickwinkel des dominanten Teiles sehen « (Hagemann-White 2016, S. 13). Insofern ist eine sorgfältige Klärung der Begriffe (Kap. 4), ein hohes Maß an Reflexivität bezüglich der eigenen Vorannahmen und Gefühle sowie Achtsamkeit in der Forschungsethik (vgl. ebd.) unabdingbar.

Bezüglich meiner eigenen Vorannahmen und in Auseinandersetzung mit meiner eigenen Haltung zum Phänomen der Gewalt wurde mir bei der Erstellung des Forschungsdesigns bewusst, dass sich meine Sichtweisen über Gewalt, wie ich diese definiere, wo ich selbst Grenzen sehe, von denen anderer und damit auch von meinen Interviewpartnerinnen selbstverständlich unterscheiden (können). Deshalb habe ich mich erstens bei der Auswahl meiner Erhebungsmethode für ein biografisch-narratives Interview entschieden, das den Erzählerinnen ermöglicht, 
ihre subjektiven Sichtweisen zu entfalten, und von mir als Interviewerin ein hohes Maß an Zurückhaltung fordert. Zweitens habe ich im Zuge der Kontaktaufnahme vermieden, Gewalt zu definieren und mein Forschungsinteresse sehr offen formuliert, indem ich bewusst von meinem Interesse an »allem, was für dich in Beziehungen schwierig ist « und von "Gewalt in Beziehungen« sprach.

Hinsichtlich forschungsethischer Fragen war für mich neben der ausdrücklichen und wiederholten Betonung der Freiwilligkeit die Beschäftigung mit dem Umgang (möglicher) Traumatisierungen essenziell. So beschäftigte ich mich im Vorfeld der Datenerhebung intensiv mit Chancen und Grenzen der narrativen Gesprächsführung bei Menschen mit traumatischen Erlebnissen in der Kindheit (vgl. ausführlicher dazu das nachfolgende Kapitel).

Die Erforschung von Gewalt erfordert außerdem, sorgfältig die Machtdimensionen im Forschungsprozess zu reflektieren (vgl. ebd., S. 23). Denn jedem Gewalthandeln liegt ein Machtgefälle zugrunde, welches gleichzeitig dazu beiträgt, die Gewalt zu verfestigen (vgl. ebd.). Konkret bedeutete dies, mir meine dominante Position als Forscherin bewusst zu machen, die die Fragestellung, das Forschungsdesign und die Interpretation der Daten bestimmt. Weiter bedeutete dies auch, die Zielgruppe der Forschung nicht ausschließlich nach einer Dimension der Machtungleichheit zusammenzusetzen, weil dadurch andere Dimensionen meist Positionen der relativen Privilegierung - unreflektiert die Zusammensetzung der Fallauswahl bestimmen (vgl. ebd., S. 24). Es geht also darum zu bedenken, »wo sich Hierarchien der Machtungleichheit in der Gesellschaft auf eine Weise kreuzen, die es besonders schwer macht, Gewalt überhaupt als solche zu erkennen« (ebd.). In der vorliegenden Untersuchung versuchte ich demzufolge, aus der Perspektive der Intersektionalität denkend, Interviewpartnerinnen sowohl aus verschiedenen Milieus als auch, der heteronormativen Matrix entgegengesetzt, aus gleichgeschlechtlichen Beziehungen in den Blick zu nehmen. Dass sich der $\mathrm{Zu}$ gang insgesamt als nicht einfach erwies, wird in Kap. 7.3.3 näher beleuchtet. Kein empirisches Projekt ist in der Lage,

»systematisch alle Machthierarchien der Gesellschaft zu berücksichtigen, und das ist auch nicht erforderlich. Geht es doch darum, an den Stellen, wo diese Linien sich schneiden, die spezifische Verwundbarkeit und die spezifischen Ressourcen in den Blick zu bekommen, ... Augen und Ohren für die Erfahrungen zu öffnen, die aus der relativ mächtigen Position der Forschenden allzu leicht unsichtbar bleiben.« (Hagemann-White 2016, S. 24)

Während des Auswertungsprozesses verfolgte ich zwei methodische Strategien der Selbstreflexivität: Zum einen wählte ich einige Passagen aus, anhand derer ich die Interaktionsprozesse zwischen Interviewerin und Erzählerin analysierte - und die an einigen Stellen auch in die Darstellung der Ergebnisse einbezogen wurden (z.B. Kap. 8.1.1.2; Kap. 8.1.4.2). Trotz des Wissens darum, wie prägend die Interaktio- 
nen in der Interviewsituation auf das Erzählgeschehen sind, werden in den meisten Publikationen Äußerungen oder Gesprächsanteile der Interviewenden fast nie mitinterpretiert (vgl. Jensen/Welzer 2003, [Absatz 5]).

Zum anderen führte ich während des gesamten Forschungsprozesses ein Forschungstagebuch (Flick 2007, S. 377), in dem neben der Dokumentation des laufenden Prozesses insbesondere Erfahrungen und Probleme im Kontakt mit dem Feld oder Interviewpartnerinnen festgehalten und reflektiert wurden. So füllte ich bspw. nach jedem Interview einen Interviewprotokollbogen (Helfferich 2005) aus; darin hielt ich fest, welche Angaben zur Erzählperson im Interview möglicherweise nicht zur Sprache gekommen waren, und notierte formale Angaben zum Interview wie Datum, Ort, Kontaktaufnahme sowie Stichworte zur Interviewatmosphäre und zur personalen Beziehung zwischen Interviewerin und Interviewpartnerin (vgl. ebd., S. 172).

Ausgehend von der Annahme, dass Interpretationen von wissenschaftlichen Subjekten abhängen, stellt sich die »Frage nach den Möglichkeiten und Grenzen einer Reflexion der Subjekt- und Standortgebundenheit von Deutung im Rahmen des qualitativen Forschungsprozesses, die über die Arbeit einsamer Textwissenschaftler(innen) hinausweist (Mruck/Mey 1998, S. 287). Dieser Annahme Rechnung tragend, war ich in zwei Forschungsgruppen eingebunden, mit denen ich mich regelmäßig, über meinen gesamten Forschungsprozesse hinweg, getroffen habe. Durch die Organisation qualitativer Deutungsarbeit in Gruppen konnten zum einen die anderen Interpret_innen entlang meiner Fragen und Instruktionen das vorliegende qualitative Material deuten und in der Gruppe besprechen. Zum anderen konnten meine Interpretationen diskutiert werden und so mögliche Verzerrungen erkannt und vermieden werden. Nicht zuletzt boten diese Arbeitszusammenhänge neben der Diskussion weiterer relevanter Fragen theoretisch-inhaltlicher oder auch praktischer Art wertvolle Unterstützung - vor allem auch bezüglich supervisorischer Aufgaben im Sinne einer Entlastung und Unterstützung, besonders im Hinblick auf die emotionale Thematik der Arbeit.

\subsection{Grenzen und Chancen der narrativen Gesprächsführung bei Menschen mit Traumatisierungen und Umgang mit Traumatisierungen im narrativen Interview}

Die Vorbereitung der Datenerhebung mittels biografisch-narrativer Interviews, die sich mit dem Erleben von sowie dem Umgang mit Gewalt beschäftigen, erfordert auch die Beschäftigung damit, wie mit (möglichen) Retraumatisierungen während des Interviews umgegangen werden kann. Ein weiterer wichtiger Schritt besteht dann darin, Spuren von Traumatisierungen bei der Analyse narrativer Interviews $\mathrm{zu}$ erkennen und adäquat $\mathrm{zu}$ interpretieren. 
Die narrative Gesprächsführung bietet verschiedene Chancen im Umgang mit Menschen, die Traumatisierungen erfahren haben. Zunächst kann davon ausgegangen werden, dass die Bereitschaft zu einem Interview generell vom Bedürfnis getragen wird, schwierige oder tabuisierte Bereiche der Lebensgeschichte zu thematisieren (vgl. Loch 2002, S. 235). Zudem sorgt das eigene Abwehrsystem dafür, dass traumatische Erfahrungen nur soweit erzählt werden, wie es für die Erzählerin noch erträglich ist (vgl. ebd.). Hier möchte ich aufgrund meiner eigenen Erfahrungen anfügen, dass für viele meiner Interviewpartnerinnen die Bereitschaft $\mathrm{zu}$ einem Interview sich auch darin begründete, einen Beitrag zu einem bislang kaum erforschten Thema zu leisten. Dieser Wunsch war verknüpft mit der Idee, dass durch das Forschungsvorhaben das Thema Gewalt in den ersten eigenen Beziehungen allgemein bekannter würde und Frauen besser geholfen werden könnte bzw. Hilfsangebote für betroffene Frauen besser entwickelt werden könnten.

Zunächst zum ersten Punkt der Datenerhebung und Durchführung mittels narrativer Interviews. Neben der in Kap.7.3.1 ausgeführten Begründungen hinsichtlich der prinzipiellen Offenheit für die Wahl des autobiografisch-narrativen Interviews kommt im Hinblick auf die Gewaltthematik in der vorliegenden Arbeit ein weiteres Begründungsmoment hinzu: Die durch das narrative Interview ermöglichte Unterscheidung zwischen Erzähl- und Erlebensperspektive in den erhobenen Daten ist vor allem dort relevant, »wo Interviewausführungen eher verwirrend, (scheinbar) widersprüchlich und/oder bruchstückhaft sind« (ebd., S. 234). Solche Ausführungen liefern Hinweise auf (unbearbeitete) Traumatisierungen und verbergen möglicherweise die erlebten Erfahrungen oder Handlungen, die gesellschaftlich und sozial mit Schweigegeboten belegt sind (vgl. ebd.). Aus diesem Grund habe ich zu Beginn des Auswertungsprozesses bei der Auswahl der zu interpretierenden Passagen auch diejenigen in den Blick genommen, die sich gerade auch durch Brüche und erzählerische Lücken kennzeichnen.

Interviewpartnerinnen haben meist einen ambivalenten Umgang mit dem Sprechen über akute traumatische Erfahrungen oder erlittene Traumatisierungen in der Kindheit. Einerseits gibt es das Bedürfnis, über die Situation zu sprechen, andererseits besteht auch ein hoher Erzählwiderstand aufgrund der erfahrenen oder angedrohten Situationen, der gemachten Stigmatisierungserfahrungen sowie antizipierter Überforderung anderer Gesprächspartner (Loch 2008, Absatz 3). Wie sich beispielsweise im Interview mit Anna deutlich zeigt, versichern sich manche über sogenanntes »thematisches Nachfragen« (Loch 2002, S. 236), wie die Haltung oder Meinung der Interviewerin sich verhält (vgl. Loch 2007, S. 207). So fragte Anna mich, ob sie jetzt erzählen soll, wie es zu der Gewalterfahrung kam (Anna, Transkript, Z. 690f.). Daraufhin signalisierte ich ihr deutlich, offen für ihre Erzählungen und Erfahrungen zu sein und diese ggf. auch auszuhalten. Diese neuen Erkenntnisse an Handlungsmöglichkeiten, wie intensives In-Kontakt-Bleiben 
mit der Interviewpartnerin, die Möglichkeit, einen Ausstieg aus dem Thema oder Pausen anzubieten, waren für mich förderlich und umsetzbar.

Insgesamt kann ich rückblickend festhalten, dass die Beschäftigung mit narrativen Gesprächstechniken für die Vorbereitung der Interviews außerordentlich hilfreich war. Zum einen war ich sensibilisiert, Spuren von dissoziierten ${ }^{9}$ trauma- $^{-}$ tischen Erfahrungen in Erzählungen zu erkennen, zum anderen wurde mir bewusst, dass eine grundsätzliche Vermeidung schwieriger Lebenssituationen mit dazu beitragen kann, gesellschaftliche und soziale Tabuisierungsprozesse mit aufrechtzuerhalten. Mit dem Wissen, dass dissoziative Fähigkeiten das Erzählen von schwierigen Lebenssituationen ermöglichen (vgl. Loch 2008, Absatz 19), traute ich mich zunehmend, entsprechende Nachfragen zu stellen. Vor allem dann, wenn in der Stegreiferzählung seitens der Interviewpartnerin Situationen oder Ereignisse nur angedeutet und nicht näher ausgeführt wurden, sprach ich diese während des immanenten Nachfrageteils an. Dennoch erfordert jedes Nachfragen ein Abwägen darüber, ob die Erzählaufforderung eher die heilsame Integration unterstützen oder eher retraumatisierend wirken kann.

Hinsichtlich des zweiten Schrittes, Spuren von Traumatisierungen während der Interpretation $\mathrm{zu}$ erkennen und adäquat zu interpretieren, lenkte ich mein Augenmerk gerade auf die Passagen, in denen sich Brüche, erzählerische Lücken, Lachen, Weinen oder die Inkonsistenz einer Erzählung zeigten. Auch Ausführungen, die verwirrend und widersprüchlich schienen (v.a. im Interview mit Amy), interpretierte ich besonders sorgfältig, da dies Hinweise auf Spaltungsprozesse sind, die sich so auf der Textebene manifestieren. Diese Interpretationen finden Eingang in die Analyse der Einzelfälle.

\subsection{Fazit}

Die dieser Arbeit zugrunde liegenden theoretischen Perspektiven bilden sich in den Entscheidungen hinsichtlich der Methodik ab. Dabei stellt der methodische Zuschnitt der vorliegenden Studie eine Besonderheit dar, indem sie die dokumentarische Methode dazu nutzt, neben dem Fokus auf kollektive Erfahrungsräume dennoch die individuelle Erfahrungsaufschichtung der befragten jungen Frauen $\mathrm{zu}$ berücksichtigen. Demnach werden die rekonstruierten Orientierungsrahmen immer sowohl individuell als auch kollektiv betrachtet.

Dies wird im folgenden Schaubild verdeutlicht:

9 Betroffene versuchen alle Gefühle, Vorstellungen und Handlungen, die mit der traumatischen Situation assoziiert sind bzw. die sie erlebt haben, zu vermeiden. Dadurch kommt es zu Dissoziationen, die diese Gefühle und Vorstellungen abspalten (vgl. Haubl 2003, S. 70). 
Abbildung 3: Theoretische und methodische Zugänge

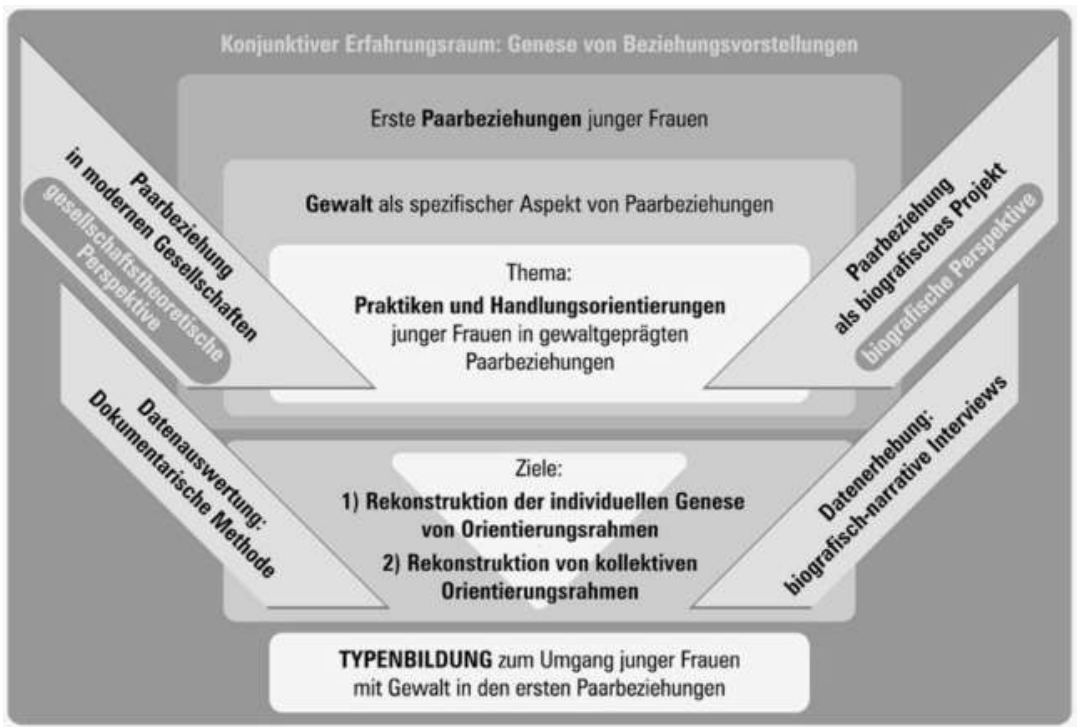

Die Fortführung der theoretischen Zugänge auf methodischer Ebene zeigt sich sowohl hinsichtlich der Datenerhebung (1) als auch der Datenauswertung (2):

1. Die Wahl des autobiografisch-narrativen Interviews als Instrument zur Datenerhebung ermöglicht die Annäherung an die Perspektive der von Gewalt betroffenen jungen Frauen und deren individuelle Orientierungsrahmen. Diese Perspektive erlaubt eine individuelle, familienspezifische und damit biografisch ausgerichtete Sicht sowohl auf die Genese von Beziehungskonzepten als auch auf die Handlungspraxis der Beziehungsgestaltung junger Frauen.

2. Mithilfe der dokumentarischen Methode wird zunächst die individuelle Erfahrungsaufschichtung in den Biografien der befragten jungen Frauen berücksichtigt. In einem zweiten Schritt wird die Perspektive dann auf kollektive Erfahrungsräume, insbesondere auf denjenigen der Familie, gerichtet. Die rekonstruierten Regelmäßigkeiten werden letztlich in eine Typenbildung hinsichtlich der Praktiken und Handlungsorientierungen im Umgang mit Gewalt überführt.

Die Hauptfragestellung der vorliegenden Arbeit lautet demnach:

Welche Praktiken und Handlungsorientierungen zeigen junge Frauen im Umgang mit Cewalt während der ersten eigenen Paarbeziehung(en)?

Damit verbunden sind folgende Fragen: 
Wie erleben junge Frauen Gewalt in den ersten eigenen Paarbeziehungen?

Wie wird Paarbeziehung konstruiert? Welche Vorstellungen formulieren die jungen Frauen über das partnerschaftliche Zusammenleben und wie gestalten sie angesichts dieser gewaltvollen Erfahrungen ihre Beziehungen?

Welche Rolle spielen Gewalterfahrungen in der Herkunftsfamilie? Wie prägen diese Gewalterfahrungen die Beziehungsvorstellungen?

Welche Ressourcen stehen den jungen Frauen zur Verfügung?

Ziel dieser Arbeit ist es, die Perspektive auf die Verschränkung von individualbiografischer Erfahrung und kollektiver Einbettung zu richten. Dies geschieht zum einen durch das Einnehmen einer biografischen Perspektive, die auf den individuellen, fallbezogenen Orientierungsrahmen fokussiert. Auf methodischer Ebene zeigt sich dies, indem ein konsequenter Fallbezug verfolgt wird. Auf dieser Ebene kann die Genese des Habitus bzw. des Orientierungsrahmens auch unter sozialisationstheoretischer Perspektive gefasst und aufgezeigt werden. Zum anderen werden kollektive Erfahrungsräume, insbesondere der konjunktive Erfahrungsraum der Familie, in den Blick genommen. Die hier rekonstruierten Regelmäßigkeiten hinsichtlich des Umgangs mit Gewalt sind nicht mehr als rein individuell zu begreifen, sondern verweisen auf konjunktive Erfahrungsräume. Diese herausgearbeiteten Handlungsorientierungen werden schließlich zu einer Typik des Umgangs mit Gewalterfahrungen verdichtet.

Die Arbeit trägt insgesamt dazu bei, die o.g. Fragen aus der Perspektive der Subjekte zu beantworten und so zu einem vollständigeren Bild des Erlebens von Gewalt in den ersten eigenen Paarbeziehungen, dem Umgang damit und den daraus resultierenden Beziehungskonzepten zu gelangen. 


\section{Ergebnisse der empirischen Untersuchung Falldarstellung und Typengenese - Praktiken und Handlungsorientierungen junger Frauen in gewaltgeprägten Paarbeziehungen}

Bei der Darstellung der Rekonstruktion der Ergebnisse gilt es, die Gesamtgestalt des Einzelfalls in seinen Besonderheiten mit der fallübergreifenden Analyse zu vereinbaren, die zur Entwicklung einer Typik führt. Um diesem Anspruch gerecht zu werden, habe ich mich für ein mehrstufiges Vorgehen bei der Darstellung der Ergebnisse entschieden, das zunächst die Darstellung von sechs ausgewählten Einzelfällen und daran anschließend die Generierung von Typen vorsieht. Das achte Kapitel. »Ergebnisse der empirischen Untersuchung« ist wie folgt aufgebaut:

A) Falldarstellung Zunächst wurde aus dem gesamten Material heraus der Lebenslauf rekonstruiert und in einem Fallporträt festgehalten. Es wurde bewusst auf Interpretationen verzichtet und eine chronologische Abfolge anhand relevanter Fakten und markanter biografischer Ereignisse erstellt, die den entsprechenden Interviewpassagen entnommen wurden. Innerhalb der Fallporträts wurde außerdem der Fokus auf die Übergänge in Paarbeziehung und die dort gemachten Erfahrungen gerichtet.

Im Kapitel Analyse der Erzählstruktur werden die Gesamtgestalt des Interviews, die Art und Weise der Erzählungen sowie die jeweiligen Besonderheiten jedes einzelnen Interviews hinsichtlich der formalen Struktur analysiert. Dabei geht es darum, autobiografisches Erzählen als Herstellung und Darstellung von narrativer Identität im Interview zu rekonstruieren. ${ }^{1}$ Die Perspektive auf die Gesamtstruktur des Interviews nimmt in den Blick, wie die Erzählerin erzählt, wie

1 Ich beziehe mich hier auf ein Verständnis des narrativen Interviews als eine situierte und durch persönliche Bedürfnisse motivierte Konstruktionsleistung, wie es Lucius-Hoene und Deppermann (2002) in ihrem Werk »Rekonstruktion narrativer Identität« entfalten. Demnach wird das narrative Interview als eine »sich vollziehende Identitätskonstruktion verstanden, in der sich die autobiografische Darstellung von Identität mit der performativen und interaktiven Herstellung von Identität verbindet«(Lucius-Hoene/Deppermann 2002, S. 10). 
sie ihre Erzählungen aufbaut, welche Themen platziert werden, wie sie sich selbst präsentiert. Die Formalstruktur von Texten gibt Aufschluss über die performative Kontextuierung der Orientierungsrahmen. Die Validität des Orientierungsrahmens bzw. des Typus ist auch davon abhängig, inwieweit Homologien zwischen propositionalen und performativen Ebenen aufgezeigt werden können (vgl. Bohnsack 2010, S. 57). Auf diese Analyse wird im theoretisierenden Teil der Arbeit unter Kapitel 9 wieder Bezug genommen. Für die Einzelfalldarstellungen wurden diesbezüglich einzelne Aspekte ausgewählt, die mir relevant erschienen; diese erheben damit nicht den Anspruch auf Vollständigkeit. Im anschließenden Kapitel zu Herkunftsfamilie und Bedingungen des Aufwachsens stand die Perspektive auf sozialisatorische Bedingungen des Heranwachsens im Vordergrund. Hierzu wurden thematisch entsprechende Passagen und deren Analyse für die Darstellung ausgewählt.

Den Schwerpunkt der Falldarstellungen stellt das Kapitel Kernthemen des Interviews dar. Dabei wurden in Anlehnung an die Forschungsfrage zunächst Passagen zu den Themen »Erste Dates/der erste Freund « sowie »Erfahrungen und Umgang mit Gewalt « als Ausgangspunkt für die Generierung des Orientierungsrahmens ausgewählt. Anschließend wurden aber auch, die Gesamtgestalt der jeweiligen Einzelfälle berücksichtigend, die weiteren Kernthemen der jeweiligen Einzelfälle identifiziert. Auf Grundlage dieser ausgewählten thematischen Passagen wurde schrittweise der jeweilige Orientierungsrahmen entfaltet. Dabei habe ich bewusst aus Gründen der besseren Lesbarkeit an einzelnen Stellen auf die Dokumentation entsprechend weiterer Belegstellen verzichtet. Die Darstellung der Kernthemen erfolgte entlang der Chronologie der biografischen Erzählung und unter Hinzunahme bzw. Einbezug der thematisch passenden Passagen aus dem Nachfrageteil.

Zusammenfassend werden einzelne Aspekte bezüglich des zu rekonstruierenden Orientierungsrahmens oder auch den rekonstruierten Orientierungsrahmen ${ }^{2}$ hervorgehoben. Dabei werden sowohl Elemente des Orientierungsrahmens im engeren Sinne, also im Hinblick auf konjunktives Erfahrungswissen, als auch Elemente von Orientierungsschemata, in denen sich kommunikatives Wissen repräsentiert, aufgezeigt. Außerdem werden vereinzelt Hinweise auf intergenerationale Transmissionsprozesse aufgezeigt.

Obwohl bereits bei der Interpretation der Kernthemen eigentheoretische Äußerungen nicht vollständig außen vorgelassen wurden, aber entsprechend der methodischen Anforderungen vor allem Erzählungen und Beschreibungen in den Fokus der Analyse rückten, wurden für das anschließende Kapitel Argumentationen

2 Der Begriffdes Orientierungsrahmens wird hier im weiteren Sinne, als übergeordneter Begriff zu demjenigen der Orientierungsschemata verstanden (vgl. dazu Abbildung 1 und Erläuterungen in Kap. 7.1.2). 
zentrale argumentative Stellungnahmen der Erzählerinnen ausgewählt und analysiert. Meines Erachtens ist es essenziell, auch theoretische Textsorten in die Auswertung miteinzubeziehen, da diese Aufschluss darüber geben, wie jemand seine Handlungsweisen rechtfertigt oder bewertet und zwar nicht nur zum heutigen Zeitpunkt, sondern auch zu einem damaligen, zurückliegenden Zeitpunkt in der Lebensgeschichte (vgl. Schütze 1987, S. 128). Nach Nohl (2009) kann der Modus Operandi des Theoretisierens auch Aufschluss über den Orientierungsgehalt geben, innerhalb dessen die Erzählerin ihre Themen bearbeitet (vgl. ebd., S. 50). Nicht zuletzt sind Aussagen hinsichtlich der Orientierung zu Normen und Werten aus heutiger Sicht auch deshalb zentral, um Aussagen darüber machen zu können, wie die Erzählerinnen in der Gegenwart gegenüber Gewalt in Beziehungen eingestellt sind. Auch hier wurden einzelne relevante eigentheoretische Äußerungen für die Analyse ausgewählt.

In den Zusammenfassungen wurden nun hinsichtlich vergleichbarer Themen (Erleben von Gewalt in Paarbeziehungen; Umgang mit erlebter Gewalt, Bewältigungsstrategien und Ressourcen; Beziehungsmuster und Beziehungsgestaltung sowie Gewalt(-Beziehungen) und Sozialisation) die wesentlichen Ergebnisse gebündelt. Traten in einzelnen Interviews Themen auf, die bezüglich der Forschungsfrage zentral waren, sich jedoch nicht in den anderen Interviews wiederholten, habe ich dieses unter der zusätzlichen Überschrift »Weitere Themen« resümiert. Diese Zusammenfassungen bildeten die inhaltlich-thematische Grundlage für das folgende Kapitel Typenbildung.

B) Typengenese Auch die Darstellung der sinngenetischen Typenbildung erfolgt in der genannten expliziten und impliziten Form. In Kap. 8.2 werden die Ergebnisse der komparativen Analyse und der Typenbildung zusammenfassend dargestellt.

In den folgenden Falldarstellungen sind die privaten Daten, Jahreszahlen und Orte codiert. Dabei habe ich, um die Anonymisierung zu gewährleisten, bestimmte Daten oder Ereignisse, die auf konkrete Personen verweisen oder deren Anonymität gefährden würden, verändert oder weggelassen, bspw. habe ich tatsächliche Berufe durch vergleichbare ersetzt. ${ }^{3}$

3 Um die Anonymität der Interviewpartnerinnen zu gewährleisten, wurden für die Veröffentlichung der Arbeit zudem Dialekte geglättet und einzelne Passagen, die Rückschlüsse auf Personen ermöglicht hätten, gelöscht. 


\subsection{Falldarstellungen ${ }^{4}$}

\subsubsection{Anna - »immer wieder die gleichen Männer immer wieder [...] das gleiche Schema« (169f.)}

\subsubsection{Fallporträt}

Anna wächst bei ihrer alleinerziehenden Mutter ohne Geschwister in einer deutschen Großstadt auf. Ihren Vater lernt sie erst als erwachsene Frau kennen. Anna beschreibt ihr Umfeld, in dem sie aufwächst, als sehr chaotisch. Ihre Mutter, die selbst eine schwierige Jugend erlebte, hat starke psychische Probleme. Das Verhältnis zwischen Anna und ihrer Mutter ist ambivalent, Anna erlebt keine Verlässlichkeit in der Beziehung zu ihrer Mutter. Sie leidet einerseits unter der Vernachlässigung durch die Mutter, die sie vor allem dann erlebt, wenn diese Drogen konsumiert. Andererseits ist ihre Mutter auch um Anna bemüht und besucht mit ihr bspw. kulturelle Veranstaltungen oder liest ihr viel vor. Anna hat bereits als Kind immer wieder depressive Phasen, weshalb sie auch häufig in der Schule fehlt.

Annas Mutter nimmt sie häufig am Wochenende auf Rockkonzerte mit. Mit 13 lernt Anna dort einen deutlich älteren Mann kennen, mit dem sie eine Beziehung eingeht. Sie treffen sich über ein Jahr lang, wobei es bei den Treffen vor allem um Reden und Knutschen geht. Anna erfährt irgendwann, dass er verheiratet ist, trifft sich aber weiterhin mit ihm. Als er von Anna mehr möchte, lässt sie dies nicht zu. Eines Tages bedrängt er Anna, weil er mit ihr schlafen möchte. Anna willigt nicht ein, womit die Beziehung beendet ist.

Als Anna 15 Jahre alt ist, spitzen sich die Konflikte mit ihrer Mutter zu. Anna bringt immer wieder Männer mit nach Hause, die bei ihr übernachten. Die Mutter wendet sich zu diesem Zeitpunkt an das Jugendamt und bittet um Hilfe. Anna kommt daraufhin in eine Wohngruppe. Sie hat immer wieder depressive Phasen und es kommt es zu einem Suizidversuch.

Ihren ersten Freund hat Anna mit 15 Jahren. Die Beziehung dauert etwa ein Jahr. Da ihr Freund Asylbewerber ist, verbringt sie ihre Wochenenden bei ihm im Asylbewerberheim. Dass ihr Freund mit Drogen dealt, stellt für Anna einen gewissen vertrauten Rahmen dar, den sie von zu Hause bereits kennt. Als ihr Freund nach einem Jahr Beziehung der Meinung ist, sie sollten heiraten, damit sein Aufenthaltsstatus gesichert wird, und Anna nicht einwilligt, beendet er die Beziehung.

4 In den Einzelfallanalysen verwende ich bei Zitaten der Interviewpartnerinnen im Fließtext lediglich die Zeilenzahlen in Klammern und verzichte auf das »Z.«. Da sich die angegebenen Zeilenzahlen immer auf den aktuellen Fall beziehen, verzichte ich auch auf das wiederholte Ausschreiben des jeweiligen Namens der jeweiligen Erzählerin. Selbstverständlich sind in allen anderen Kapiteln die Zitate auch mit Namen gekennzeichnet. 
Anna hat in dieser Zeit viele Dates und One-Night-Stands. Sie fühlt sich häufig allein und verzweifelt.

Mit 16 Jahren lernt sie einen Mann kennen, der sie recht hartnäckig umwirbt und von dem sie nach kurzer Zeit schwanger wird. Anna wechselt von der Wohngruppe in eine Mutter-Kind-Einrichtung und absolviert, einen Monat vor der Geburt, ihren Realschulabschluss. Kurz nach der Geburt ihrer Tochter wird ihr Freund, der auch Asylbewerber ist, nach Afghanistan abgeschoben. Sie wartet, bis sie 18 Jahre alt ist, und reist gemeinsam mit ihrer Tochter nach Afghanistan, um ihn zu heiraten. Anna versucht, sich dort eine Existenz aufzubauen, und arbeitet in einer sozialen Einrichtung. Zwischen ihrem Mann und ihr kommt es immer wieder zu Konflikten, ihr Mann zeigt sich extrem eifersüchtig. Anna schreibt die Differenzen vor allem den kulturellen Unterschieden zu. Nach einigen Monaten entscheidet sie sich aufgrund der prekären Lebenssituation in Afghanistan und der Beziehungskonflikte, mit ihrer Tochter nach Deutschland zurückzukehren.

Einige Monate später beginnt sie eine Affäre mit einem Mann, von dem sie erneut schwanger wird. Da der Vater ihres zweiten Kindes ihr seine Hilfe zusichert, entschließt sie sich, dieses zu bekommen. Als Anna erfährt, dass ihr neuer Freund mit harten Drogen dealt, trennt sie sich sofort von ihm. Allein mit zwei kleinen Kindern geht es Anna psychisch zunehmend schlechter. Mit Unterstützung der sie betreuenden Familienhelferin beginnt sie eine Psychotherapie. Wenige Zeit nach Therapiebeginn versucht sie ein weiteres Mal, sich das Leben zu nehmen. Nach einem kurzen Aufenthalt in der Psychiatrie besucht sie über einen längeren Zeitraum die psychiatrische Tagesklinik, in der sie sich deutlich stabilisiert.

Nach zwei Jahren darf ihr Mann von Afghanistan nach Deutschland einreisen. Sie unternehmen einen kurzen Versuch zusammenzuleben, der allerdings scheitert. Da Anna den Großteil der Abschiebekosten bezahlt hat, schuldet ihr Mann ihr einen großen Geldbetrag. Aufgrund der finanziell prekären Situation eskaliert eine Auseinandersetzung, in der Anna ihren Mann körperlich angreift, woraufhin die Polizei einschreitet und gegenüber ihrem Mann einen Platzverweis ausspricht. Anna reicht daraufhin die Scheidung ein.

Sie beginnt ein sechsmonatiges Praktikum in einem Kinderhort und absolviert anschließend die Ausbildung zur Floristin. In dieser Zeit geht Anna verschiedene Affären oder kurze Beziehungen ein. Sie beendet ihre Ausbildung mit einem sehr guten Abschluss, macht anschließend die Fachhochschulreife und beginnt ein Lehramtsstudium.

Zum Zeitpunkt des Interviews lebt sie mit ihren Töchtern zusammen und hat einen neuen Partner. 


\subsubsection{Analyse der Erzählstruktur}

Anna zeigt von Beginn an eine hohe Bereitschaft für das Interview, was sich insbesondere im flüssigen Erzählstil zeigt. Die biografische Stegreiferzählung entwickelt sie überwiegend entlang einer chronikartigen Darstellung, in der autobiografische Abschnitte und Ereignisse in der chronologischen Reihenfolge ohne die Benennung oder Hervorhebung besonderer Höhepunkte erzählt werden. Der erzählerische Auflösegrad findet vor allem in der Raffung resp. Verdichtung statt, indem größere Zeitabschnitte überwiegend summarisch zusammengefasst und in der $\mathrm{Ab}$ folge einzelner Ereignisse oder Etappen dargestellt werden (Geburt, Bedingungen des Aufwachsens, Pubertät, Wohngruppe, Schwangerschaft usw.). Einzelne Epochen oder Ereignisse werden dabei unter einer Sinn- oder Zeitgestalt zusammengefasst (»das war für mich so n Schlüssel-(.)dings« (150); »s:: muss jetzt muss was passieren (3) an meiner inneren Geschichte (153f.)) und so anhand einer bestimmten sinnstiftenden Deutungsfigur charakterisiert (vgl. Lucius-Hoene/Deppermann 2002, S. 118). Sie beendet die Darstellung mit der metanarrativen Schlussformulierung »also das war jetzt so ne Kurzzusammenfassung meiner @Biografie@«(189f.).

Während des immanenten Nachfrageteils wurde über eine konsequent narrative Gesprächsführung zur Erzählung von Lebensphasen oder einzelnen Situationen zu bereits Erwähntem aufgefordert. Anna greift dies auf, und es kommt zu vielen einzelnen szenisch-episodischen Darstellungen, in der die Herstellung einer Szene mit dramatisierender Form aus der Perspektive des damaligen Handelns resp. Geschehens re-inszeniert wird (vgl. Lucius-Hoene/Deppermann 2002, S. 146).

Im Voraus einer solchen Erzählung im szenisch-episodischen Duktus zum Gewalterleben innerhalb einer Paarbeziehung zeigt sich ein verbreitetes Phänomen in Interviews, indem über thematisches Nachfragen (vgl. Loch 2002, S. 236) der Erzählerin die Haltung der Interviewerin zum Thema Gewalt abgefragt wird:

A: und $\operatorname{des}^{\circ}$ war auch der (1) der Mann (1) der einzige und auch der ((stockend)) erste $^{*}$ der mich geschlagen hat//mmh//ja (3) $)$ soll ich da mehr davon erzählen oder lieber nich

I: Wenn du möchtest
A: Ähm
I: nur wenn du möchtest

A: Ja also (2) d e r (1) also einmal hat er mich geschlagen (688-699)

Deutlich wird in dieser Passage, dass die Interviewerin einerseits die Freiwilligkeit und Entscheidungsmacht der Erzählerin überlässt und anderseits ihre Bereitschaft zuzuhören signalisiert.

Ein weiteres Merkmal, welches das Interview mit Anna kennzeichnet, ist die Verwendung eines Vokabulars, das vermutlich aus der Psychotherapieerfahrung 
übernommen wurde und das in einigen Erzählsequenzen mal mehr, mal weniger stark in Erscheinung tritt. ${ }^{5}$ Folgendes Zitat stellt ein solches Beispiel dar:

des musst ich mir halt schon seit frühster Kindheit anhören, also ich war quasi die Ther- die Therapeutin meiner Mutter ((holt Luft)) u:nd des hat mich sehr stark belastet (1) des hat mich wesentlich reifer auch gemacht als ich eigentlich war damals, also ich musste mir als Kind Sachen anhören ((holt Luft)) die normalerweise äh Psychotherapeuten oder@Psychologen@ sich anhören, (233-238)

Die verwendeten psychologischen Theorien werden als Interpretationsmuster genutzt, um eigene Erfahrungen durch Rückgriff auf ein wissenschaftlich begründetes Erklärungssystem zu deuten und zu bewerten ${ }^{6}$ (vgl. Lucius-Hoene/Deppermann 2002, S. 131).

\subsubsection{Herkunftsfamilie und Bedingungen des Aufwachsens Eingangsnarration}

A: Gut//mmh//(1) also dann fang ich mal an=ich wurd ahm bevor ich (1) vo:n mir erzähl erstmal (2) meine familiären Hintergründe (1) ${ }^{\circ}$ so $n$ bisschen ${ }^{\circ}$ (1) erklären, und zwar a::hm (2) bin ich ä::h (2) [...] mit meiner [...] (1) Mutter (.) äh aufgewachsen [...] ((holt Luft)) (1) meine Mutter hatte eine sehr schwierige Kindheit, (1) wurde (1) mhh (2) ähm:: also ist quasi s- mit vierzehn auf die Straße gekommen und wurde zwangsprostituiert und so weiter und (.) war dann auch schwer drogenabhängig, (2) $(12-19)^{7}$

Für Annas Darstellung ihrer Lebensgeschichte ist es zentral, zuerst einmal auf ihre familiären Hintergründe einzugehen, bevor sie über sich selbst weitererzählen kann. Nach Annas Auffassung lässt sich somit ihre Geschichte nur vor dem Hintergrund der folgenden Schilderungen verstehen. Damit schreibt sie gleichzeitig diesen Ereignissen eine große Bedeutung für ihre eigene Lebensgeschichte zu.

Anna erzählt, dass sie bei ihrer Mutter aufgewachsen ist, und betont damit das Merkmal alleinerziehend. Der Vater bleibt in ihrer Erzählung implizit.

In einer Rückblende vollzieht Anna nun einen zeitlichen Sprung zurück in die Vorgeschichte dessen, was sie gerade begonnen hat zu erzählen: Sie verweist auf ein höchst prekäres Aufwachsen ihrer Mutter, die im Alter von 14 Jahren zur Prostitution gezwungen wurde und »schwer drogenabhängig« (18f.) war. Durch die Ver-

5 In der Einzelfallanalyse werden diese Informationen entsprechend kenntlich gemacht.

6 Die Studie von Kühnlein und Mutz (1996) belegt diesen Befund, indem sie aufzeigt, wie sich Erfahrungen aus Psychotherapie als Expert_innenwissen im Alltagshandeln niederschlagen.

7 In den folgenden Analysen werden Zitate im Fließtext, die sich auf das angegebene Blockzitat beziehen, lediglich in Anführungszeichen gesetzt, nicht aber nochmals mit Zeilennummern versehen. Bei Zitaten darüber hinaus werden diese selbstverständlich mit den entsprechenden Zeilennummern angegeben. 
wendung der Vorgangspassivform »wurde zwangsprostituiert « (17f.) betont Anna das Ausgeliefertsein der Mutter. Zum anderen liefert sie damit vorweg eine Erklärung für ihre familiären Hintergründe, die dazu dient, das (zukünftige) Verhalten der Mutter zu erläutern.

\section{Geburt}

und dann kam ich $@(). @{ }^{\circ}$ ich wurde quasi ahm in ziemliches Chaos reingeboren, ((holt Luft)) u::nd (1) meine Mutter war=genau meine Mutter war alleinerziehend, (1) mein Vater war schon längst wieder in Land 1 [...], ((holt Luft)) ich bin dann mit meiner Mutter aufgewachsen die se:hr (.) starke psychische Probleme immer wieder hatte und ah ihre Drogenvergangenheit auch nicht wirklich abgeschlossen hatte, (5) (21-27)

Die Umgebung, in die sie geboren wird, beschreibt Anna als "ziemliches Chaos«, die Mutter alleinerziehend, der Vater »längst « wieder in sein Herkunftsland zurückgekehrt. Sie bezeichnet ihr Aufwachsen weiter als »sehr ambivalent« (30). Diese Ambivalenz bezieht sich auf ein Heranwachsen zwischen »behütet« $(29,31)$ und »chaotisch« (31) und »unbehütet« (31). Es dokumentiert sich hier, dass Anna, obwohl sie in einem chaotischen Umfeld aufwächst, trotzdem Gefühle von Sicherheit und Geborgenheit erlebt.

Die Art und Weise, mit der Anna über ihre familialen Wurzeln, hier insbesondere die Vergangenheit ihrer Mutter und ihr eigenes Aufwachsen mit dieser berichtet, lässt einen therapeutischen Zugang Annas zur Biografie ihrer Mutter erkennen. In ihrer Deutung der Mutter-Tochter-Beziehung zeigt sich ein psychotherapeutisches Erklärungsmuster. Verstärkt wird diese Lesart durch die Kontrastierung mit anderen Sequenzen, in denen das Mutter-Tochter-Verhältnis thematisiert wird: Einerseits beschreibt Anna, in die Rolle der »Therapeutin« (234) gedrängt worden zu sein, indem sie als Zuhörerin für die Probleme ihrer Mutter instrumentalisiert wird. Andererseits übt ihre Mutter einen »starken Beschützerinstinkt « (240) aus, um Anna vor der »bösen Welt zu schützen« (242). Es wird deutlich, dass Anna in der Beziehung zu ihrer Mutter sehr ambivalente Erfahrungen macht, wobei sich durchgängig Elemente von Verständnis zeigen, die Anna ihrer Mutter entgegenbringt.

\section{Konflikte mit der Mutter}

im Jugendalter fings dann an dass ich ähm angefangen hab zu rebellieren, (2) [...] $\mathrm{u}$ :nd mit f-also genau, dann bin ich (.) dann wurds: (.) ähm sehr sehr (2) also als ich dann vierzehn fünfzehn wurde is=es dann völlig eskaliert mit meiner Mutter (1) also da gabs nur noch Streit und Auseinandersetzungen und meine Mutter war auch total überfordert (.) mit mir und ich war mit meiner Mutter überfordert, (37-50) 
Als Anna etwa 14, 15 Jahre alt ist, verschärft sich die Situation mit ihrer Mutter. Die Formulierung »da gabs nur noch Streit und Auseinandersetzungen« verweist auf eine dauerhaft angespannte Situation, in der eine konstruktive Auseinandersetzung um die bestehenden Meinungsverschiedenheiten, die nicht weiter ausgeführt werden, nicht mehr möglich erscheint. Die aus heutiger Sicht angebrachte Erklärung »meine Mutter war auch total überfordert (.) mit mir« deutet erneut auf ein verständnisorientiertes empathisches Einfinden Annas gegenüber ihrer Mutter hin (s.o.).

\section{Suizidversuch}

aber ich war immer depressiv ((holt Luft)) hab auch schon mit vierzehn das erste Mal versucht ähm (.) mir das Leben zu nehmen (.) (61-62)

Fast schon beiläufig, in einer weiteren Hintergrundkonstruktion erwähnt Anna einen Suizidversuch, den sie im Alter von 14 Jahren begeht. Sie kommt daraufhin in die Kinder- und Jugendpsychiatrie. Zeitlich liegen die Ereignisse kurz vor dem Zeitpunkt, als Anna in die Wohngruppe kommt.

\section{Aufnahme in eine Wohngruppe}

dann (2) ähm GENAU wir hatten schon immer mit dem Jugendamt Kontakt (.) meine Mutter hat auch Hilfe vom Jugendamt (2) äh:: hat um Hilfe gebeten (.) da war ich zwölf oder so und mit:: $\mathrm{fü} \mathrm{n} f \mathrm{z}$ e $\mathrm{h}$ n gings dann ga:r nicht mehr, da wollte meine Mutter dann nicht mehr dass ich bei ihr lebe und ich wollte auch nicht mehr bei meiner Mutter leben (51-55)

Nachdem sich Annas Mutter angesichts der aktuellen Konflikte hilfesuchend an das Jugendamt wendet - der Kontakt zwischen dem Jugendamt und Anna sowie ihrer Mutter besteht bereits seit einigen Jahren - , zieht Anna in eine Jugendwohngruppe.

Während der Kontakt zwischen Mutter und Tochter zu dieser Zeit zunächst als »absolut (.) offen« (57f.) beschrieben wird, verändert sich die Beziehung mit der Zeit: »irgendwie zu der Zeit hat sie es nicht geschafft n:: engeren Kontakt zu mir zu halten« (58f.). Das heißt, trotz vereinfachter Bedingungen ist es der Mutter nicht möglich, die Beziehung zu ihrer Tochter in dem Maße aufrecht zu erhalten, wie es sich diese wünscht. Und trotz dieser verringerten Anforderungen verteidigt Anna das Verhalten ihrer Mutter, was durch die Formulierung des »Nicht-Schaffens« zum Ausdruck kommt und angesichts der Tatsache des gemeinsamen Aufenthalts in derselben Stadt an Bedeutung gewinnt. Gleichzeitig wird dadurch ihr Wunsch nach einem weiteren intensiven Kontakt mit der Mutter zum Ausdruck gebracht. Insgesamt kommt hier wieder eine verständnisorientierte Haltung gegenüber der Mutter zum Ausdruck. 


\section{Mutter-Kind-Einrichtung}

hab ich aber=dann inzwischen schon in Stadt 1 gewohnt ((atmet tief)) in ner Mutter-Kind-Einrichtung (1) weil ich ja quasi kein richtiges Zuhause hatte (2)) (91-93)

Zum Zeitpunkt der Geburt ihrer Tochter lebt Anna in einer Mutter-KindEinrichtung. Sie begründet ihren Aufenthalt dort damit, kein »richtiges Zuhause« mehr gehabt zu haben, da sie, nachdem sie schwanger war, nicht mehr in der Wohngruppe bleiben konnte.

Anna lebt etwa eineinhalb Jahre in der Mutter-Kind-Einrichtung. Sie beschreibt, dass es ihr in dieser Zeit psychisch nicht gut ging und schließt das Segment mit der bewertenden theoretisch-reflektierenden Stellungnahme ab, die Situation insgesamt wirklich gut gemeistert zu haben. Auch diese Aussage lässt eine reflexive Auseinandersetzung mit der eigenen Lebensgeschichte vermuten.

Zusammenfassend lässt sich festhalten, dass Anna immer wieder im Laufe ihrer Kindheit und Jugend die Erfahrung macht, ihr Zuhause verlassen zu müssen, und damit von wichtigen Bezugspersonen, Zurückweisung erfährt. Sie erklärt sich dieses Verhalten mit einer Überforderung der Erziehungspersonen, worin sich eine verständnisvolle Perspektive diesen gegenüber dokumentiert.

Hier ist vermutlich die Suche nach Zugehörigkeit angelegt, die sich generell durch die weitere Biografie zieht und aus der auch die Suche nach Zugehörigkeit resultiert, die Anna im weiteren Verlauf ihrer Biografie in der Beziehung mit Männern sucht. Eine weitere Parallele besteht in der Unzuverlässigkeit der Beziehungen, die sich ebenfalls in der Beziehung mit Männern zeigt.

\subsubsection{Kernthemen des Interviews}

Erste Dates

\section{Erster intimer Kontakt}

ich hab sehr frü:h ne Beziehung zu einem wesentlich älteren Mann (.) angefangen=damals war ich dreizehn und er war (.) Mitte zwanzig (2) es war keine wirkliche Beziehung es war eher so:: $\mathrm{n}$ Treffen (1) wo er immer wieder versucht hat mich rumzukriegen und ich's dann aber nicht gemacht hab (39-43)

Als Anna 13 Jahre alt ist, geht sie eine Beziehung zu einem deutlich älteren Mann ein. Die Aussage, es habe sich um eine »Beziehung« gehandelt, relativiert Anna kurz darauf: Es wären eher »Treffen« gewesen, bei denen dieser Mann versuchte, sie »rumzukriegen « - zu was genau expliziert sie an dieser Stelle nicht - , sie jedoch ihre Grenzen deutlich machen konnte. Anna begründet die Kontaktaufnahme zu diesem Mann damit, »männliche Nähe« (44), konkreter »nen Vater in ihm« (45), gesucht zu haben. Es scheint ihr wichtig, vor allem aber erklärungsbedürftig zu sein, ihr Bedürfnis nach männlicher Nähe zu einem älteren Mann zu begründen. Mögli- 
cherweise scheint auch hier wieder eine psychologisierende Erklärungsperspektive durch, die aus therapeutischen Erfahrungen resultiert.

Die hier ausgeführte Begründung zielt auf eine normativ geprägte Vorstellung von Paarbeziehung, nach der ein hoher Altersunterschied unüblich und daher begründungsbedürftig ist. Damit dokumentiert sich hier auf der Ebene kommunikativen Wissens eine Orientierung an einem normativen Beziehungsmodell.

\section{Kennenlernen}

$\mathrm{Ja}=\mathrm{ja}$ ähm also kennengelernt also des=is=so meine Mutter hat mich früher immer zu Konzerten mitgenommen [...] (1) des war halt so des Milieu in dem ich aufgewachsen bin (2) und da war ich schon als Kind sehr oft fast jedes Wochenende dabei (2) u::nd mit dreizehn waren wir dann auch wieder gemeinsam bei einem Konzert meine Mutter und ich (2) und da hab ich diesen Mann kennengelernt (2) mit dem hab ich da irgendwie ein bisschen getanzt ich fand den halt ganz toll war halt groß und ja halt war halt ich hab halt so für den geschwärmt so wie halt so Dreizehn- Vierzehnjährige dann sind, (593-601)

Die erste Begegnung mit diesem Mann findet auf einem Konzert statt, zu dem Anna von ihrer Mutter - wie »fast jedes Wochenende« - mitgenommen wird. Dass Anna hier den Verweis auf die Häufigkeit der Konzertbesuche einschiebt, lässt deutlich werden, dass für sie der Aufenthalt auf diesen Konzerten erklärungsbedürftig erscheint. Daraus kann geschlossen werden, dass Anna bewusst ist, dass Konzerte eher unübliche Orte für Kinder sind. Ihre Aussage »des war halt so des Milieu in dem ich aufgewachsen bin« impliziert eine Erklärung, der durch die Wortwahl »Milieu« eine analysierende Bedeutung zukommt, wodurch eine Distanz gegenüber dem erlebten Umfeld ausgedrückt wird.

Anna tanzt auf diesem Konzert mit einem Mann und begründet ihr Verhalten mit der Argumentation, sie habe ihn »toll« gefunden, »war halt groß«, »hab halt so für den geschwärmt so wie halt so Dreizehn- Vierzehnjährige dann sind«. Er gibt ihr seine Telefonnummer, woraufhin sie ihn anruft.

In ihrem Verhalten zeigt sich ein Bedürfnis nach (männlicher) Nähe und Fürsorge, dem sie über die Annäherung an einen älteren Mann versucht nachzukommen. Es proponiert sich hier außerdem Annas aktives Zugehen auf (ältere) Männer.

\section{Erstes Date}

dann haben wir uns irgendwo getroffen [...] (.) da haben=wir uns=dann in irgendeiner Bude getroffen (.) wo lauter ausländische Männer gewohnt haben (.) ich weiß nicht was des war ((holt Luft 2)) und hab ich ihn dann immer wieder getroffen und geredet und hat viel geknutscht und er wollt halt jedes Mal mehr und ich habs dann halt so: (.) nich passieren lassen und er war halt ganz wild nach mir und wollt 
halt unbedingt und ich wollt halt nich und so gings dann halt jedes Treffen immer so, (1) (615-621)

Die Treffen finden in sogenannten »Bude[n] « statt, in denen überwiegend oder ausschließlich Männer mit Asylbewerberstatus leben. Durch die ungenaue Ortsbezeichnung wird den Treffpunkten eine gewisse Anonymität zugeschrieben. Annas Ausführungen zu den Orten sowie ihre Versuche diese zu beschreiben, zeigen, dass diese erklärungsbedürftig erscheinen. Die »Bude« als ausschließlicher Ort des Treffens spiegelt möglicherweise den Charakter der Beziehung wider. Der Mann entscheidet über den Ort und vielleicht, wie das folgende Zitat zeigt, auch darüber, ob und welche Aktivitäten unternommen werden.

\section{Verlauf der Beziehung}

wir waren nur einmal zusammen Bowlen mit Freunden sonst waren wir nie draußen, ich mein er konnte mich ja nicht mit in die Disco nehmen oder so ich war ja erst dreizehn ((holt Luft)) genau, (1) (621-624)

Anna trifft den Mann immer wieder. Bei diesen Treffen wird geredet, aber auch »viel geknutscht« (618), wobei er mehr möchte als sie. Anna beschreibt, dass sie das jedoch »nicht passieren« (619) lässt, auch wenn sich sein Verlangen nach körperlicher Intimität von Mal zu Mal steigert. Anna führt hier nicht weiter aus, wie ihr Umgang mit seinem offensichtlichen Drängen aussieht. Indem sie allerdings zu sexuellen Aktivitäten nicht bereit ist und diese auch abwehrt, zeigt sie ihre Grenze auf und setzt diese auch durch.

Gleichzeitig deutet ihre Formulierung »nur einmal« mit Freunden etwas unternommen zu haben, auf ein Bedauern hin, dass solche gemeinsamen Unternehmungen nicht häufiger stattfanden. Möglicherweise standen sich die unterschiedlichen Bedürfnisse diametral entgegen und Anna wünschte sich mehr Aktivitäten mit Gleichaltrigen bzw. Freunden in der »äußeren Sphäre«, während ihr Freund Treffen in den sog. »Buden« also in einer »inneren Sphäre« favorisierte.

Als Anna ihren Freund eines Tages mit einer anderen Frau sieht, vermutet sie, dass er verheiratet ist, was sich dann auch bestätigt. Sie trifft sich dennoch weiterhin mit ihm. In einer Hintergrundkonstruktion schildert sie ihr Bedürfnis, ihn immer dann, wenn sie »Sehnsucht hatte nach dieser Aufmerksamkeit« (629), anzurufen. Hier bestätigt sich Annas Bedürfnis nach Nähe, in Form von Aufmerksamkeit und Beachtung, das sie zur Initiierung dieser Treffen veranlasst. Dabei findet die erfahrene Zuwendung in erster Linie auf intimer bzw. sexueller Ebene statt.

\section{Ende der Beziehung}

ja und irgendwann wars dann soweit dass er dann (1) aufdringlich wurde also da wurde er dann (.) wollte eindeutig mit mir schlafen (3) u:nd (.) ich hab gesagt (.) 
ob er überhaupt Kondome dabei hat dann hat er gesagt »Nö«dann hab ich gesagt »]a also erstens will ich gar nicht mit dir schlafen ohne Kondom sowieso nicht« ich mein ich war damals vierzehn dann muss man schon sagen dass ich des gepackt hab des zu sagen bin ich heut noch stolz (1) aber auf jeden Fall dann war=er sauer (2) und dann hat=er mich heimgefahren (1) @und das wars letzte Mal@ dasswir uns gesehen haben (630-637)

Anna schildert hier etwas detaillierter eine Situation, die gleichzeitig auch das letzte Treffen mit ihrem Freund markiert. Ihre Äußerung »irgendwann wars dann soweit« deutet darauf hin, dass sie mit dem nun Folgenden bereits gerechnet hatte oder die Erfahrung im Nachhinein aus ihrer Position der Erzählerin im Heute als absehbar deutet und darlegt. Ihr Freund wird aufdringlich, indem er ihr signalisiert, er wolle Sex mit ihr. Ihre Frage, ob er Kondome dabeihabe, verneint er, woraufhin sie, erzählerisch in der direkten Rede wiedergegeben, antwortet, sie wolle erstens nicht mit ihm schlafen und ohne Kondome ohnehin nicht. In Form einer Hintergrundkonstruktion im Modus der Argumentation stellt sie erneut aus ihrer Position der Erzählerin im Heute fest, stolz auf ihr Verhalten zu sein, insbesondere in Anbetracht ihres Alters.

Hinsichtlich des unterliegenden Orientierungsrahmens werden verschiedene Aspekte deutlich: Zum einen drückt sich in Annas Kontaktaufnahme zu einem älteren Mann ihre Orientierung nach Nähe und Aufmerksamkeit aus. Sie ist diejenige, die Kontakt zu ihm aufnimmt, wenn sie das Bedürfnis danach hat. Darin dokumentiert sich seitens Anna ein aktives Handeln bezüglich der Beziehungsgestaltung.

Weiter wird deutlich, dass es für Anna innerhalb einer Beziehung wichtig ist, sich mit ihrem Freund auch innerhalb des Freundeskreises zu zeigen. Im Gegensatz dazu ist ihr Freund vor allen auf sexueller Eben an Anna interessiert. Trotz dieser divergierenden Beziehungsvorstellungen, und auch der Tatsache, dass er verheiratet ist, hält Anna am Zusammensein mit ihm fest.

Als es dann allerdings zu einer sexuellen Grenzverletzung durch ihren Freund kommt, beendet sie den Kontakt zu ihm.

Der erste Freund Mit 15 Jahren lernt Anna einen Mann kennen, den sie als ihren »erste[n] Freund « (68) bezeichnet und mit dem sie etwa ein Jahr lang eine Beziehung führt. Diese beschreibt Anna als »ziemlich chaotisch« (69) und »übel chaotisch» (669) und begründet dies damit, dass ihr Freund »Asylbewerber« (69) ist und mit »Drogen gedealt« (70) hat. Sowohl ihre Wochenenden als auch die Schulferien verbringt sie im Asylbewerberheim.

Auffällig sind in dieser Sequenz die Charakterisierung und Rahmung der Beziehung als »chaotisch«. Hier fällt die Parallele zur Beschreibung der Umgebungsbedingungen auf, in denen Anna aufwuchs und die sie ebenfalls als »teils total 
chaotisch « (31) bezeichnet. Zudem zeigt sich eine weitere Parallele hinsichtlich ihres aktuellen Freunds, der dealt sowie Annas sozialisatorischem Umfeld, das u.a. durch den Drogenkonsum der Mutter geprägt war.

Annas Freund hat einen ungesicherten Aufenthaltsstatus als Asylbewerber, was für beide bedeutet, mit der Ungewissheit über die weitere Zukunft leben zu müssen.

In einer argumentativen Darstellung verweist sie selbst auf die aufgezeigten Analogien: »es war schon des was ich auch gewohnt war ja was ich kannte« (675f.). Ein weiteres Argument in ihrer Eigentheorie bezieht sich auf den Reiz, den die Besonderheit der Situation auf sie ausübt: nämlich die Einzige in der Klasse zu sein, die bereits einen Freund hat, und immer wieder weg zu sein, sich mit ihm zu treffen, ohne dass jemand den Ort kennt. Für Anna war dieses Verhalten mit Rebellion verknüpft.

Anna bilanziert abschließend, dass ihr Freund »auch ziemlich (.) grob« (71) war und beendet die Sequenz mit dem ergebnissichernden Schlusskommentar »also des würd ich nicht als Liebesbeziehung bezeichnen« (72).

Insgesamt wird in diesem Segment deutlich, dass Anna sich einen Mann aussucht, der sich in einem Umfeld bewegt, das eine gewisse Ähnlichkeit zu ihrem bisherigen Umfeld aufweist und ihr somit vertraut ist: chaotische Strukturen, in denen Drogen eine relevante Rolle spielen. Die abschließende Reflexion, in der ihre Eigentheorie über das geschilderte Erleben zum Ausdruck kommt, weist auf die bereits erfolgte therapeutische Aufarbeitung ihrer Vergangenheit hin.

\section{Kennenlernen}

Also den hab ich auch bei einer bei einem Fest oder auf einem Konzert kennengelernt (2) u::nd (3) der hat mir auch seine Nummer gegeben ich hab ihn dann angerufen und dann hamn wir uns ((holt Luft 2)) also des=is ziemlich schräg aber ich erzähl's jetzt @einfach@, und zwar hamn wir uns verabredet in ner anderen Stadt (3) er hat da bei irgendner Familie gelebt (1) und dann bin ich da hingegangen hat er mich vom Bahnhof abgeholt und dann=kamen wir da zu dieser Familie (1) dann saß ich da wurde mir was zu trinken angeboten auf einmal hat er gesagt »Komm wir gehn rüber« (1) also des war unser erstes Date da war ich (.) fünfzehn »Komm wir gehn rüber« dann geh ich da rüber ne hat er im Kinderzimmer mitten auf $n$ Boden ne Matratze hingelegt und=hat gesagt »So jetzt legst dich hin «//mmh//(2) hab ich gesagt »Nee ich leg mich jetzt nich hin«ja (1) und war dann einerseits total angeekelt und andrerseits hab ich mich auch geschmeichelt gefühlt dass der unbedingt mit mir jetzt schlafen will//mmh//ja (2) also so $n$ Mischmasch//mmh//(1) und mit dem war ich dann aber tatsächlich noch $\mathrm{n}$ gutes Jahr zusammen (.) mit diesem Mann,//mmh mmh//(1) genau des war mein erster Freund, (653-668) 
Anna beginnt ihre Erzählung über das Kennenlernen mit der Benennung des Ortes, an dem dieses stattfand. Wie in der Beziehung zuvor lernt sie »den« - er bleibt, wie auch alle anderen Männer, namenlos - ebenfalls an einem öffentlichen Ort kennen. Sie erinnert sich allerdings nicht mehr genau, ob die erste Begegnung »bei nem Fest oder auf nem Konzert« stattfand. Dass sich Anna nicht mehr genau an den Ort des Kennenlernens erinnern kann, deutet auf eine eher oberflächliche und unverbindliche Kontaktaufnahme hin. Indem der Mann Anna seine Telefonnummer gibt, weist er Anna eine aktive Rolle zu: Die Entscheidung liegt bei ihr, ob der Kontakt fortgeführt wird. Anna nimmt Kontakt zu ihm auf - hier zeigt sich eine Parallele zur Kontaktaufnahme in der Beziehung zuvor - und es kommt zu einem Treffen, wobei Anna, bevor sie Näheres hierzu erzählt, in einem Abstract, der inhaltlich zusammenfassend das Kommende ankündigt, anfügt: »also des=is ziemlich schräg aber ich erzähls jetzt @einfach@«. Der Vorgriff darauf, dass das nachfolgend Erzählte »schräg« ist, macht deutlich, dass das Erlebte für Anna in der nachträglichen Erinnerung nicht mehr nachvollziehbar ist.

Hinsichtlich der Orientierung, die Ort, Zeit, Personen und die Situation charakterisiert, schildert sie, dass sie zu der Verabredung in eine andere Stadt fuhr, da er dort bei einer Familie lebte. Die Einbettung der Situation vor Ort in einen familiären Rahmen - Anna bekommt etwas zu trinken angeboten - mutet ebenfalls "schräg« an hinsichtlich eines ersten Dates. Über die weiteren Umstände, weshalb er bei einer Familie untergebracht ist, äußert sich Anna nicht. Seine Aufforderung "Komm wir gehn rüber « scheint für Anna plötzlich zu erfolgen (»auf einmal«). Auch ihre Wiederholung seiner Aufforderung sowie ihr Einschub, es habe sich um ihr erstes "Date« gehandelt und sie sei damals erst "fünfzehn« gewesen, verdeutlichen ihr Unverständnis bzw. ihre Empörung über seinen Vorschlag und den weiteren Verlauf.

Es folgt nun die Darstellung der Handlungskomplikation: Sie kommt seiner Aufforderung nach, mit ihm ins »Kinderzimmer « zu gehen. Die Wahl des Wortes »Kinderzimmer« erscheint bei der Beschreibung dieser Situation skurril. Auch wird nicht klar, weshalb er in einem »Kinderzimmer« wohnt. Er weist Anna an, sich auf eine Matratze zu legen, was sie ablehnt. In einer abschließenden Evaluation der Episode schildert sie ihre ambivalenten Gefühle: Sie fühlt sich »einerseits total angeekelt und andererseits hab ich mich auch geschmeichelt gefühlt dass er unbedingt mit mir jetzt schlafen will«. Ihre ambivalenten Empfindungen fasst sie nochmals in dem Ausdruck »so n Mischmasch" zusammen. Der weitere Verlauf wird von Anna nicht erzählt. Es bleibt somit unklar, wie sich die Situation fortsetzt.

Der Verweis in der Koda der Erzählung, mit diesem Mann »tatsächlich« noch ein Jahr eine Beziehung geführt zu haben, zeigt, dass dies für Anna aus heutiger Perspektive nicht mehr nachvollziehbar ist. 
Der hier unterliegende Orientierungsrahmen verweist auf eine Handlungspraxis, die auf Anerkennung und Zuwendung ausgerichtet ist. Dies zeigt sich in Annas Interpretation, das Verlangen ihres Freundes nach Sex mit ihr als Kompliment aufzufassen.

\section{Ende der Beziehung}

und der hat dann irgendwann mal nach nem Jahr [...] hat=er gesagt »]a (.) also jetzt sind wir schon ein Jahr zusammen (2) jetzt wirds dann schon langsam mal Zeit dass du mich heiratest «ja=also wegen seinem Aufenthaltstitel (1) ${ }^{\circ}$ und dann hab ich gesagt »Nee ich bin sechzehn ich will nicht heiraten« [...] und dann hat er gesagt »Okay wenn du mir nich helfen kannst dann mach ich jetzt Schluss« @(.)@//mmh//ja des=war meine erste so war dann meine erste Beziehung $(680-688)$

Anna schildert erneut eine konkrete Situation, die sich auf das Ende der Beziehung bezieht. Nach einem Jahr Beziehung fordert Annas Freund sie in Bezugnahme auf die Dauer der Beziehung (»jetzt sind wir schon ein Jahr zusammen«) auf, ihn zu heiraten. Für Anna steht fest, dass der Hintergrund für diese Forderung der Aufenthaltsstatus ihres Freundes ist, den dieser durch die Heirat sichern würde. Sie begründet ihre ablehnende Antwort mit ihrem Alter. Daraufhin beendet ihr Freund die Beziehung mit der Begründung, sie helfe ihm nicht. Anna schließt die Schilderung der Situation mit der metanarrativen Schlussformulierung "ja des=war meine erste so war dann meine erste Beziehung«.

Insgesamt werden in der gesamten Sequenz erneut Parallelen hinsichtlich Annas sozialisatorischem Umfeld und ihrer ersten Paarbeziehung bezüglich eins chaotischen Umfeldes sowie des Vorhandenseins von Drogen deutlich.

Erfahrung physischer Gewalt

\section{Metaebene: Rückversicherung}

A: und $\operatorname{des}^{\circ}$ war auch der (1) der Mann (1) der einzige und auch der ((stockend)) erste* $^{*}$ der mich geschlagen hat//mmh//ja (3) () soll ich da mehr davon erzählen oder lieber nich

I: Wenn du möchtest

\section{A: Ähm}

I: nur wenn du möchtest (688-697)

Nach der leise gesprochenen Aussage, dies sei der einzige und erste Mann gewesen, der sie geschlagen hat, fragt Anna die Interviewerin, ob sie »mehr davon erzählen« solle oder lieber nicht. Anna vergewissert sich hier, ob die Interviewerin die Bereitschaft mitbringt, die angedeutete Erfahrung zu hören. Damit wird der ambivalente Umgang mit schwierigen bzw. krisenhaften Erfahrungen deutlich, bei 
denen es sich möglicherweise auch um traumatische Erlebnisse handelt. Einerseits gibt es ein Bedürfnis, über die Erfahrung zu sprechen, andererseits ist das Schildern solcher Erfahrungen auch mit einem Erzählwiderstand belegt.

Die Antwort der Interviewerin »wenn du möchtest« drückt einerseits ihre Offenheit im Hinblick auf die mögliche Erzählung schwieriger Erfahrungen aus. Gleichzeitig gibt sie die Verantwortung und damit die Entscheidung, ob Anna von diesen Erfahrungen erzählen möchte, an diese zurück. Anna setzt bereits vor Ende der Aussage der Interviewerin mit »ähm« ein, woraufhin die Interviewerin nochmals die Freiwilligkeit sowie ihre Bereitschaft zuzuhören, betont.

\section{Schlag ins Gesicht}

Ja also (2) d e r (1) also einmal hat er mich geschlagen da ham=wir ne ga- ne kleine Diskussion gehabt ich weiß gar nicht mehr um was es ging ((holt Luft)) einfach nur ne Meinungsverschiedenheit (2) und dann hat er mir halt voll eine gepfeffert ins Gesicht (1) damals war schon die Zeit da hab ich nicht immer bei meiner Mutter gewohnt da hab ich bei Freunden [...] teilweise gewohnt [...] der hat mich dort quasi besucht $/ / \mathrm{mmh} / /(1)$ und dann hat er mich voll ins Cesicht geschlagen und ähm (.) was für mich (2) und die Freunde bei denen ich damals gewohnt habe das waren Landsleute von ihm//mmh//(1) also der Mann war $n$ Landsmann (.) und da waren zwei Landsmänner noch quasi in der gleichen Wohnung die war- haben des zwar nicht gesehen aber die waren dabei und ich war dann natürlich total schockiert

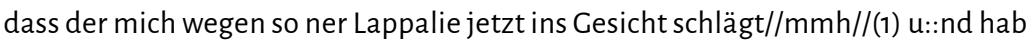
des dann den andern auch völlig empört erzählt dass der mich grad geschlagen hat ohne Grund (1) und so wie ich mich erinnere kam da aber keine Reaktion von denen (1)//mmh//ja (.) die haben den jetzt nich irgendwie rausgeschmissen und gesagt »Sag mal schlag doch die nich «oder so ja (1) haben ihn jetzt=zwar auch nich verteidigt aber (2) haben einfach nichts gemacht//mmh//und des fand ich damals schon irgendwie schlimm, (2) (699-717)

Anna beginnt ihre Erzählung mit einem Abstract, in dem sie darauf verweist, dass ihr Freund sie »einmal " geschlagen habe. Dabei wird, vor dem Hintergrund der folgenden Sequenz, deutlich, dass das Adverb in der Bedeutung von szum einen, erstens - und nicht im Sinne von ein seinziges Mak - verwendet wird. Das heißt, Anna kündigt an, zunächst eine Situation zu schildern, der möglicherweise noch eine weitere folgen wird. Zunächst verweist Anna auf den Hintergrund der Situation: Sie hätten eine »kleine Diskussion« gehabt. Der Verweis, es sei eine »kleine« Debatte gewesen, macht deutlich, dass es ihrer Meinung nach eher um eine Lappalie ging. Diese Auffassung wird nochmals durch die Äußerung »einfach nur ne Meinungsverschiedenheit« verstärkt. Die Handlungskomplikation besteht darin, dass Anna eine Ohrfeige bekommt (»und dann hat er mir halt voll eine gepfeffert ins Gesicht«). Es folgt die Orientierung in Form einer Hintergrundkonstruktion im 
Modus der Beschreibung: Anna verweist hier darauf, dass sie zum damaligen Zeitpunkt nicht mehr dauerhaft zu Hause lebt, sondern zum Teil bei Freunden. Als Anna von ihrem Freund geschlagen wird, befinden sich zwei »Landsmänner« von ihm ebenfalls in der Wohnung, auch wenn sie den Vorfall nicht direkt sehen können. Die Aussage »aber die waren dabei« deutet darauf hin, dass sich Anna möglicherweise Unterstützung von diesen erwartet hätte, und sei es weniger im Sinne des Eingreifens als vielmehr in ihrer Reaktion der Empörung über den Schlag. Anna selbst ist in der Situation "natürlich total schockiert dass der mich wegen so ner Lappalie jetzt ins Gesicht schlägt«.

Sie berichtet den anderen Anwesenden davon, die jedoch weiterhin nicht eingreifen: Weder wird Annas Freund »rausgeschmissen« noch wird ihm deutlich gemacht, dass er seine Freundin nicht schlagen soll. Sie »haben einfach nichts gemacht«. Annas detaillierte Erzählung über das Ausbleiben einer Reaktion der Anderen macht deutlich, wie zentral diese Erfahrung für sie war. Sie selbst beschreibt, dass sie dieses Verhalten als »irgendwie schlimm« erlebt habe. Das veranschaulicht sowohl ihre Erwartungen in Bezug auf Hilfe und Unterstützung als auch gegenüber dem Verhalten ihres Freundes.

Es dokumentiert sich hier einerseits Annas Haltung gegenüber körperlichen Übergriffen: Sie ist schockiert und empört. Gleichzeitig erwartet sie Hilfe von $\mathrm{Au}$ ßenstehenden. Damit wird andererseits auch hinsichtlich ihrer Vorstellungen von Beziehungen deutlich, dass körperliche Gewalt ein No-Go darstellt. Dass sie eine Reaktion ihrerseits gegenüber ihrem Freund nicht thematisiert, lässt vermuten, dass es eine solche auch nicht gegeben hat. In der Situation selbst verhält sie sich angepasst.

Auf die Schilderung dieser Situation folgt unmittelbar eine weitere, die sich auf die erste, und damit auf eine Fortsetzung der Erfahrung von Gewalt, bezieht.

\section{Drohung und Verfolgung}

und die zweite Situation war (1) da waren wir bei Freunden von ihm (.) da gabs dann so ne Art Hausparty da war einer im Ausland und hat dann Klamotten und Mützen und sowas mitgebracht aus Land 1 oder so und hat die dann da halt für $n$ paar Mark damals noch verkauft ((holt Luft)) und ich hatte halt (.) des=war halt so dass dieser erste Freund von mir finanziell in Anführungsstrichen profitiert hat ich hab im Monat zwanzig Mark Taschengeld gekriegt und mein ganzes Taschengeld alles was ich hatte hab ich ihm immer gegeben,//mmh//und er hat mir dann quasi noch zwanzig Mark geschuldet und für mich mit meinen fünfzehn Jahren war des halt viel//mmh//(2) und dann hat er halt dort was gekauft bei diesem Freund dann=hat er sich ne Mütze gekauft (2) und dann war ich halt sauer und hab zu ihm gesagt »Du also ich find des jetzt schon echt unverschämt du schuldest mir hier Celd ist mein Taschengeld (1) ich geb dir immer mein ganzes Taschengeld du sagst 
immer du hast mei-kein Celd und jetzt kaufst du dir hier ne Mütze«//mmh//(1) und hab ich halt auch geschimpft hab vielleicht gesagt »Arschloch «oder so ich weiß es nich also ich hab auf jeden Fall n=Schimpfwort zu ihm gesagt (4) und dann war er noch (.) während wir bei in der Anwesenheit von diesen anderen Leuten waren war er ganz ruhig//mmh//(2) und dann sind wir rausgekommen (1) äh sind wir ähm aus der Tür rausgegangen und=auf einmal ist er voll ausgerastet, dann hat er hat er irgendwie gesagt »Okay jetzt bring ich dich um « so nach dem Motto (1) ja er hat gesagt »]etzt bring ich dich um « [...] dann hab ichs hab ich gedacht des::० @kann doch jetzt nich sein@ ja wegen so was ja (.) ich=hab dann halt zuerst gedacht der meint des nich ernst oder so (1) und dann wars aber tatsächlich so dass wir (.) des=war so am Stadtrand das wir quasi (1) ich musste rennen weil da kam kein Bus (1) der is mir dann wi:rklich (.) ich bin gerannt ja ich bin gerannt und der ist mir hinterhergerannt und da war grad Sperrmüll und=dann hat er irgendwie so ne riesen Holzstange genommen und hat gebrüllt »lch bring dich um ich bring dich um«, weil ich hab ihn glaub ich vor seinen Freunden bloßgestellt weil ich ihn halt vor seinen Freunden beleidigt hab//mmh//(1) und=des war für den so schlimm dann hab ich gesagt »lch ruf gleich die Polizei« dann hat er ges- hat er mir hinterhergerufen »Ist mir egal wen du rufst ich bring dich um«also@wirklich@er mich hat mich gejagt ja//mmh//und da hab ich wirklich auch Angst gehabt (1) ich hab schon Angst gehabt dass der mir jetzt was tut weil ich wusste der meint des ernst, (1) $(717-752)$

Anna kündigt erneut in einem Abstract die Erzählung einer weiteren Situation an. In der Orientierung schildert sie den Hintergrund der Situation: Sie ist gemeinsam mit ihrem Freund bei einer Hausparty, auf der aus dem Ausland importierte Kleidung für wenig Geld verkauft wird. In einer Hintergrundkonstruktion wirft Anna aus ihrer gegenwärtigen Perspektive die Erklärung ein, ihr Freund habe damals finanziell von ihr profitiert, indem sie ihm regelmäßig ihr monatliches Taschengeld gegeben habe. In der darauffolgenden dargestellten Handlungskomplikation erzählt Anna nahezu isochron, d.h. die Lebenszeit wird in Form einer szenischen Darstellung wiedergegeben. Durch die wörtliche Rede wird der erzählerische Höhepunkt unterstrichen. Der Konflikt liegt darin begründet, dass Annas Freund sich von ihrem Taschengeld eine Mütze kaufe. Sie streitet mit ihm und äußert ihm gegenüber auch ein Schimpfwort, an das sie sich allerdings nicht mehr genau erinnern kann. Die von Annas Freund äußerlich demonstrierte Ruhe in Anwesenheit der anderen ändert sich schlagartig, als sie das Haus verlassen.

Für Anna völlig unerwartet »ist er voll ausgerastet« und droht ihr, sie umzubringen. Die direkte Wiederholung der Drohung in Annas Erzählung, die bestätigenden Charakter hat (»ja er hat gesagt«), verstärkt deren Vehemenz. Anna schildert, wie sie zunächst nach verschiedenen Erklärungen für die geäußerte Drohung sucht. Lachend erzählt sie, gedacht zu haben »des:: @kann doch jetzt nicht sein@ 
ja wegen so was«. Damit wird deutlich, wie unangemessen sie die Reaktion ihres Freundes auf die zuvor erfolgte Auseinandersetzung empfindet. Diese Auffassung setzt sich auch in der von ihr folgenden Erklärung fort, sie habe gedacht, »der meint des nich ernst oder so (1)«.

In der darauffolgenden Sequenz bricht Anna mit der bis dahin recht chronologisch erfolgten Darstellung der Geschehnisse. Dies lässt darauf schließen, dass ihre Schilderungen nun ab dem Zeitpunkt einsetzen, als sie begreift, dass ihr Freund seine Drohung ernst meint. Entscheidend ist zunächst für sie die Erwähnung des Stadtrandes; »ich musste rennen weil da kam kein Bus«. Die Tatsache, dass sich die gesamte Situation am Stadtrand abspielt und sie genötigt ist $\mathrm{zu}$ rennen, macht Annas Notlage deutlich. Offensichtlich gibt es keine andere Möglichkeit, sich zu schützen als diese. Anna Freund folgt ihr, ergreift eine Holzstange und brüllt wiederholt »ich bring dich um ich bring dich um«.

An dieser Stelle erfolgt erneut ein Erklärungsversuch Annas für die Eskalation der Situation. Sie vermutet, sie habe ihren Freund vor dessen Freunden sowohl blamiert (indem sie ihre Meinung äußert) als auch beleidigt (sie äußert ein Schimpfwort). Sie deutet ihr Verhalten als unerträgliches Erlebnis für ihren Freund.

Als Anna sagt, sie rufe die Polizei, wiederholt ihr Freund ein weiteres Mal seine Drohung (»ist mir egal wen du rufst ich bring dich um«). An dieser Stelle steigt Anna aus der chronologischen Erzählung aus und fasst die Situation resümierend zusammen (Evaluation): Ihr Freund habe sie gejagt, sie habe auch »wirklich Angst gehabt«, »ich hab schon Angst gehabt«, da sie seine Drohung als ernst erlebte. Die Botschaft in dieser Evaluation bringt deutlich zum Ausdruck, dass Anna die hier dargestellte Situation als lebensbedrohlich erlebte.

Im Resultat, das den Ausgang der Geschichte erzählt, berichtet Anna, sie habe ihren Freund, trotz dieser Erfahrung, »nicht gehen lassen« (757) wollen. Sie schließt mit der Koda »'so war des ${ }^{\circ} «(758)$ ab.

In einer erneuten Evaluation fasst Anna ihr Verhalten zusammen. Dabei erscheint es ihr aus heutiger Perspektive nicht mehr plausibel, weshalb sie, trotz der dargestellten Eskalation mit ihm zusammenbleiben möchte.

In einer abschließenden Koda resümiert Anna, dass dies »die einzige äh so Gewalterfahrung« (762) war, die sie erlebte. Interessant ist hier der Zusatz »die von nem andern ausging (762f.). Damit eröffnet sie den Gegensatz zu Gewalt, die sie selbst auf Andere ausübt bzw. möglicherweise auch gegen sich selbst richtet.

Hier dokumentiert sich Annas Definition von dem, was sie unter Gewalt versteht: Sie bezeichnet die hier erzählte Situation - die sich mit den Merkmalen Morddrohung, Einschüchterung, Androhung körperlicher Gewalt charakterisieren lässt - explizit als »Gewalterfahrung« (762). Gleichzeitig nimmt sie eine Einschränkung vor, indem sie sagt, es sei »eigentlich« (761) die einzige Gewalterfahrung gewesen. Die Pause, die sie vor Verwendung des Begriffs »Gewalterfahrung« (762) mit den Füllwörtern »äh so« (762) füllt, deutet darauf hin, dass ihr die Begriffswahl 
nicht leichtfällt. Möglicherweise nimmt sie in der Verwendung dieser Begrifflichkeit Bezug zum Forschungsvorhaben der Interviewerin, die diesen Begriff im Vorfeld des Interviews verwendete. Anna schließt evaluierend mit den Worten ab, die Erfahrung habe sie »sehr geprägt« (763).

Insgesamt zeigt sich hier bezüglich des Orientierungsrahmens erneut Annas Ambivalenz innerhalb der Beziehung: trotz der erlebten Bedrohung und Verfolgung entscheidet sie sich, die Beziehung fortzusetzen.

Die dritte Beziehung mit dem zukünftigen Ehemann

\section{Kennenlernen des Ehemanns}

Ähm ihn hab ich auch@(.)@ in der Disco kennengelernt in Stadt 1//mmh//(5) u:nd ich fand den eigentlich gar nicht anziehend oder so der hat mir eigentlich gar nich gefallen ich fand den eher abstoßend und (2) genau, wir ham dann halt gemeinsam getanzt und er hat halt versucht mich so $n$ bisschen anzumachen aber ich fand den (2) pfff (1) der hat mich gar nicht interessiert und=dann hat er mir quasi seine Nummer gegeben//mmh//[...] ich hab den aber nie angerufen und dann=ham wir uns irgendwie wieder getroffen und dann hat er mich wieder angesprochen und is halt irgendwie hartnäckig geblieben ((holt Luft)) und dann hab ich ihm meine Nummer gegeben und dann hat er wirklich auch um mich gekämpft in Anführungsstrichen hat mich sehr oft angerufen (1) (854-870)

Anna ratifiziert ihre vorausgegangene Äußerung hinsichtlich einer Ähnlichkeit beider Beziehungen, indem sie betont, diesen Mann auch in einer Disco kennengelernt $\mathrm{zu}$ haben (ihren ersten Freund hatte Anna ebenfalls auf einem Konzert kennengelernt). Ihr kurzes Auflachen direkt nach der Feststellung dieser Gemeinsamkeit signalisiert möglicherweise ein Schmunzeln ihrerseits aus heutiger Sicht über diese Parallele. In der direkt anschließenden Beschreibung äußert Anna, diesen Mann als »nicht anziehend« und sogar »eher abstoßend« empfunden zu haben.

Anna greift ihre Vordergrunderzählung wieder auf und berichtet, dass sie gemeinsam getanzt haben und er versucht hat, sie »anzumachen«. Wieder betont sie ihr Desinteresse gegenüber diesem Mann. Vor diesem Hintergrund ist es umso erklärungsbedürftiger, weshalb sie sich dann doch auf ihn einlässt. Er gibt ihr seine Telefonnummer (eine weitere Parallele zur Situation des Kennenlernens ihres ersten Freundes).

Anna setzt ihre Erzählung mit dem Verweis fort, ihn dennoch nie angerufen $\mathrm{zu}$ haben. Sie trifft ihn jedoch - wohl zufällig - wieder und gibt ihm, nachdem er sie anspricht und »hartnäckig geblieben« ist, ihre Telefonnummer. Anna erlebt ihn als einen Mann, der um sie wirbt und sich um sie bemüht. In einem beschreibenden Einschub verweist Anna darauf, dass diese Ereignisse in eine Zeit fallen, in der sie viele Männerbekanntschaften und One-Night-Stands hat. Der Hinweis, dies wäre aus »Verzweiflung« (874) geschehen, lässt auf ein Bedürfnis nach Anerkennung und 
Wertschätzung schließen. Der Hinweis in einer weiteren Hintergrundkonstruktion, zu dieser Zeit kein Zuhause gehabt zu haben, verdeutlicht ihre Situation des Alleinseins und der fehlenden Zugehörigkeit.

Weniger seine Attraktivität oder Annas Interesse an ihm als vielmehr die Tatsache, dass er immer wieder anruft, scheinen Anna letztlich dazu zu bewegen, sich auf ein Treffen mit ihm einzulassen:

und irgendwann=hab ich mich halt doch mit ihm verabredet und dann gings halt ziemlich schnell, also ich war da früher auch immer ganz (1) hopplahopp//mmh//ja man trifft sich//mmh//u:nd landet halt gleich im Bett und so (877-880)

Anna bestätigt erneut, dass dieser Mann ihr nicht gefällt, sie sich aber aufgrund seiner Hartnäckigkeit, die sie als sein Interesse an ihr wertet, immer wieder mit ihm trifft. Sie beschreibt seine Wohnsituation in einer sogenannten »Bude« (883), die sie als »komisch « (884) bezeichnet, ebenso wie die »komischen Menschen« (884), die dort wohnen und mit Drogen handeln. Die Bezeichnung »komisch « verweist auf Annas Abgrenzung gegenüber dem beschriebenen Umfeld. Mit ihrer folgenden Äußerung, dass ihr der dort stattfindende Handel mit Drogen damals nicht bewusst war, erklärt sie aus heutiger Sicht ihre Besuche in diesem Umfeld.

Die folgenden bewertenden und theoretisch-reflektierenden Äußerungen er habe »nen ganz netten Eindruck gemacht « (888f.), »wir waren schon verliebt!« (889) und »der war jetzt auch nicht so:: viel älter « (889f.) sind aus Annas heutiger, an der Gegenwart orientierten Sicht zu verstehen und lassen auf ihre Erklärung schließen, weshalb sie die Beziehung zu diesem Mann fortgesetzt hat.

\section{Schwangerschaft}

irgendwann wurd ich dann halt schwanger (2) eigentlich wars so nich geplant (oder so) ich weiß natürlich wie man Kinder macht aber ((holt Luft)) s=hat halt gut in die chaotische Zeit reingepasst und ich hab mir auch ne Familie gewünscht//mmh//(2) und: (.) hab gedacht vielleicht wenn ich $n$ Kind hab und so $n$ tollen Mann wie ihn dann wird mein Leben (.) gut verlaufen, (1) (897-901)

Anna fährt in ihrer Erzählung mit der Aussage fort, irgendwann schwanger geworden zu sein, die sie aber sofort mit einem argumentativen Einschub wieder unterbricht, indem sie die Gründe für die Schwangerschaft erläutert: Zunächst führt sie auf, dass die Schwangerschaft nicht geplant war. Das folgende Argument $» s=$ hat halt gut in die chaotische Zeit reingepasst « begründet die spontane Schwangerschaft und macht diese damit für Anna erklärbar.

Annas Mutter reagiert auf die Nachricht mit einem »richtigen Nervenzusammenbruch« (906). Anna scheint diese Reaktion erwartet zu haben, denn in der folgenden Hintergrundkonstruktion erläutert sie, die Schwangerschaft so lange für sich behalten zu haben, bis eine Abtreibung nicht mehr möglich war. Damit wird 
deutlich, dass sie von Anfang an nicht mit Unterstützung ihrer Mutter und/oder der Wohngruppe rechnet. Vielmehr hat sie Sorge, möglicherweise zur Abtreibung des Kindes überredet zu werden. Für sie selbst ist jedoch klar, dass sie das Kind behalten möchte und daher die entsprechende Zeit abwartet, bis sie ihrem Umfeld davon erzählt.

Bereits während der Schwangerschaft gibt es »sehr viele Konflikte [...] mit dem Kindsvater « (938). Dass Anna hier vom Kindsvater spricht, deutet ihr - möglicherweise auch schon zur damaligen Zeit - distanziertes Verhältnis an. Sie führt drei Gründe als Erklärung für die Auseinandersetzungen auf: Zum einen leben sie permanent in der Unsicherheit, ob ihr Freund in Deutschland bleiben darf, zum zweiten verhält er sich als sehr unzuverlässig und zum dritten ist er »in irgendwelche illegalen Dinge verwickelt« (941), über die Anna aber nicht näher Bescheid weiß. Sie bilanziert diese Zeit so, dass sie von ihrem Freund nicht unterstützt wurde. Sie relativiert ihre Aussage damit, dass es "Zwar schon regelmäßige Besuche gegeben habe« (943f.), äußert aber bestimmt, dass die Situation keinesfalls so war, wie sie es sich gewünscht hätte.

In dieser Sequenz wird Annas biografische Konstruktion deutlich, die auf einen Orientierungsrahmen hinweist, innerhalb dessen Familie als einziger Möglichkeitsraum fungiert, der ein gutes resp. besseres Leben ermöglichen soll. Dem Orientierungsrahmen zugrunde liegt die Suche nach Zugehörigkeit, die über die Konstituierung der Familie zu erreichen versucht wird. Es dokumentiert sich hier außerdem, dass Annas Vorstellung einer positiven bzw. gelungenen Beziehungsgestaltung Zuverlässigkeit und Unterstützung umfasst.

Es kommt zu einem einschneidenden Erlebnis, just in einer »ziemlich guten Phase« (946) der Beziehung. Anna erhält nachts einen Anruf, ihr Freund werde abgeschoben. Zunächst kommt er für drei Monate in Abschiebehaft und Anna bemüht sich auch mit Unterstützung eines Anwalts um Aufhebung der Abschiebung. Zudem besucht sie ihn regelmäßig. Trotz aller Bemühungen wird ihr Freund abgeschoben.

\section{Ausreise}

ich hab dann alles für die Reise geplant//mmh//(2) ä:hm (1) und=dachte mir als erstes ich war schon irgendwie () die Sehnsucht nach diesem Mann war einfach $\mathrm{da} / / \mathrm{mmh} / /(1) \mathrm{u}$ :nd ich wollte auch mh (1) ich wusst nich wenn ich jetzt nich wenn ich ihn nich heirate wusst ich nich ob meine Tochter ihn irgendwann überhaupt wieder sieht (960-965)

Anna bereitet die Reise $\mathrm{zu}$ ihrem Freund vor. Sie begründet die Reise einerseits mit der »Sehnsucht nach diesem Mann«. Zum anderen geht sie davon aus, dass nur über eine Heirat ihre Tochter weiterhin Kontakt zu ihrem Vater haben könne. Möglicherweise wird ihre Entscheidung, nach Afghanistan zu reisen, um dort die 
Familie zusammenzuführen, auch durch ihr Gefühl bestärkt, in Deutschland kein Zuhause mehr zu haben (»weil ich ja quasi kein richtiges Zuhause hatte« (93)). Vor dem Hintergrund, dass sie selbst ohne Vater aufwuchs, kann ihr Motiv auch dadurch bestärkt worden sein, dass sie sich für ihre Tochter einen präsenten Vater wünscht.

\section{Beziehungsprobleme}

hatten wir auch sehr starke äh (.) Probleme, ((holt Luft)) aber=des hatte halt auch ähm kulturelle Hintergründe gehabt, also des hab ich dann schon bemerkt also (.) er ist halt muslimisch und (2) er=hat da halt schon so n paar (1) Ansichten (1) wo ich ich glaub nich jeder moslim- muslimische Men-Mann is so oder so auf keinen Fall aber er hat halt schon so $n$ paar Ansichten (1) da stützt er sich dann schon sehr auf seine Religion aber halt nur da wo's ihm dann passt ja also, ((holt Luft)) und war auch do:rt extrem eifersüchtig (970-978)

Nach Annas Ankunft in Afghanistan heiraten die beiden. Anna sucht Arbeit in einer sozialen Einrichtung. Doch in ihrer Paarbeziehung treten erneut »sehr starke äh (.) Probleme« auf, die Anna insbesondere den kulturellen Unterschieden zwischen ihr und ihrem Mann zuschreibt. Als Moslem habe er »schon so n paar Ansichten«, wobei Anna nicht näher ausführt, worin diese bestehen. Allerding erwähnt sie seine starke Eifersucht, die ebenfalls zu Konflikten führt. Zudem sind die Wohnverhältnisse prekär: Sie leben äußerst beengt, die finanziellen Mittel sind knapp. Ihr Mann findet keine Arbeit, und für Anna ist es schwierig, ohne Ausbildung Geld zu verdienen. Sie entschließt sich, nach Deutschland zurückzukehren.

In Bezug auf den unterliegenden Orientierungsrahmen wird Annas Wunsch nach der Zusammenführung ihrer Familie im Hinblick auf zwei Aspekte deutlich: Erstens formuliert sie eine Sehnsucht ihrerseits nach ihrem Freund. Zweitens ist es für sie wichtig, dass ihre Tochter nicht ohne ihren Vater aufwächst. Für sie steht fest, ihrem Freund nach dessen Abschiebung nachzureisen, um ihn in seinem Herkunftsland zu heiraten und damit seine Rückkehr nach Deutschland zu ermöglichen.

In Annas Handlungspraxis dokumentiert sich die aktive und zielstrebige Umsetzung ihrer Pläne, die ganz von dem Wunsch nach einem Leben mit Familie getragen wird. Damit wird auch Annas Orientierung deutlich, ihr Leben aktiv zu gestalten und selbst in die Hand zu nehmen.

\section{Neuer Beziehungsversuch in Deutschland}

dann durfte er nach zwei Jahren einreisen ich hatte ja in der Zwischenzeit dann nochmal $\mathrm{n}$ Kind von nem andern Mann//mmh//(.) mit dem ich mich da: (.) zusammengetan hatte (.) leider $u$ :nd: wir haben=aber trotzdem gesagt okay wir versu- 
chen es trotzdem//mmh//((holt Luft 2)) aber als er=dann hierherkam ${ }^{\circ}$ wars dann ganz $\mathrm{klar} / / \mathrm{mh} / /$ dass $^{\circ} / /$ genau//er kam dann hierher und dann (2) also=s war völlig (.) also ich war schon nach zwei Wochen beim (Anwalt)@was@ich tun muss um=mich scheiden zu lassen, (1046-1052)

Annas Mann kann erst zwei Jahre später wieder nach Deutschland einreisen. Anna, die inzwischen ein weiteres Kind mit einem anderen Mann bekommen hat, wagt mit ihrem Mann dennoch einen neuen Beziehungsversuch. Allerdings misslingt dieser nach wenigen Wochen und Anna sucht einen Anwalt auf, um die Scheidung einzureichen.

In der anschließenden Argumentation erläutert Anna die Gründe für das Scheitern der Beziehung: Einerseits sei sie älter und vor allem auch durch ihr Kind reifer geworden. Andererseits vermutet sie, ihr Mann sei weniger an seiner Familie als an einem Leben in Deutschland interessiert gewesen.

Ihrer Erzählung zum weiteren Verlauf der Trennung nimmt Anna eine Erläuterung vorweg, in der sie erzählerisch die Dramatik der bevorstehenden zu berichtenden Ereignisse steigert: Zunächst deutet Anna in einem Abstract an, dass ihr Mann ins Ausland gereist sei, bevor sie in der Orientierung der Erzählung mit einem beschreibenden Einschub die Beziehungssituation charakterisiert: Ihr Mann verbringt die Wochenenden mit seinen Freunden; sowohl er als auch sie leben ein eigenes Leben. Es folgt die Handlungskomplikation: Ihr Mann kündigt an, ins Ausland zu reisen, da ihm dort jemand noch Geld schulde. Er verreist für zwei Wochen und nimmt das gesamte Arbeitslosengeld mit, das beiden zur Verfügung steht. Anna versucht vergeblich, ihn zu erreichen; er selbst meldet sich nur sporadisch bei ihr, was erneut seine Unzuverlässigkeit zeigt. Annas Aussage »ich wusst nich lebt er überhaupt=noch « (1077) verdeutlicht, wie sporadisch der Kontakt zwischen beiden ist. In der Handlungskomplikation schildert Anna dann die eskalierende Situation, die sich ereignet, als ihr Mann nach zwei Wochen zurückkommt: Zunächst hat sie das Bedürfnis, mit ihm zu reden, um die Hintergründe für seine Reise zu erfahren. Vor allem aber fordert sie das mitgenommene Arbeitslosengeld zurück. In diesem Zusammenhang erwähnt sie auch, dass sie aufgrund des fehlenden Geldes sich und ihre Tochter nicht mehr ernähren könne. Diese Aussage veranschaulicht die existenziell bedrohliche Lage, in der sie sich in diesem Moment befindet und die ihr folgendes Verhalten bedingt. In dieser Situation fordert sie dann auch die Schulden zurück, die sie aufgenommen hatte, um die Abschiebekosten ihres Mannes zu bezahlen.

Die Formulierung »ich fands halt einfach ne Frechheit dass ich hab halt einfach viel Geld (1) bezahlt für alles« (1086f.) bringt - zum ersten Mal - Annas Wut zum Ausdruck, die sie angesichts des Verhaltens ihres Mannes verspürt, der sich nicht auf ein Gespräch mit ihr einlässt und sich schlafen legt. Annas Bedürfnis ist dagegen diametral: Sie möchte mit ihm sprechen, erwartet eine Erklärung. Als sie aber 
ignoriert wird und sich dadurch vermutlich zurückgesetzt, gekränkt und verletzt fühlt, eskaliert die Situation:

\section{Eigene Gewaltaktivität}

und dann bin ich halt echt ausgerastet, (1)//mmh//also des war dann auch da bin ich auf meinen Mann losgegangen auf mein damaligen (1) also ich hab ihn jetzt nich irgendwie ins Gesicht geboxt aber ich hab halt ich bin echt ausgerastet ja, (.) also ich hab ihn schon angegriffen (.) (1090-1093)

In der darauffolgenden Argumentation erklärt sie sich ihr Verhalten mit dem Gefühl der Hilflosigkeit, das sich keine Alternative erkennen lässt. Als ihr Mann sagt, er rufe die Polizei, erwidert Anna, sie rufe selbst die Polizei. Als diese erscheint, nimmt sie Annas Mann mit und spricht einen Platzverweis aus (Resultat).

Anna drückt in einer bewertenden und theoretisch-reflektierenden Stellungnahme ihre Erleichterung über die Mitnahme ihres Mannes aus (»zum Glück« (1098f.) »froh« (1101)), denn es ist für sie nicht selbstverständlich, dass er die Wohnung verlassen muss und nicht sie, da sie diejenige war, die ihn angegriffen hatte. Auch über den Platzverweis ist sie erleichtert, da er in ihren Augen einen Schutz sowohl für ihren Mann als auch für sie darstellt.

Anna schließt diese erzählerische Episode mit der Koda »und seither sind wir nicht mehr zusammen« (1105) ab.

In Annas Bereitschaft, nochmals einen Beziehungsversuch zu wagen, dokumentiert sich ihr Wunsch nach erneuter Herstellung einer (vollständigen) Familie. Auch zeigt sich in ihrem Versuch, zunächst das Gespräch mit ihrem Mann zu suchen, eine egalitäre Beziehungsvorstellung, in der versucht wird, Konflikte verbal $\mathrm{zu}$ lösen.

Es zeigt sich jedoch im weiteren Verlauf ein Wendepunkt, an dem Anna nicht mehr bereit ist, sich in allem ihrem Mann anzupassen. In der für sie existenziell bedrohlichen Situation, die sich insbesondere aus der Verantwortung für ihre beiden Kinder ergibt, überkommt sie eine große Wut, aus der heraus sie sich in Form eines körperlichen Angriffs gegen die Vernachlässigung und Unverantwortlichkeit, die sie bei ihrem Mann spürt, wehrt. In dieser Handlungspraxis dokumentiert sich eine starke Orientierung an einem autonomen und selbstbestimmten Leben.

Bildungsaspiration

\section{Schulabschluss}

da wurde ich dann schwanger (.) mit sechzehn Jahren, da war ich auf der Realschule (2) hab (.) meinen Realschulabschluss abgeschlossen hochschwanger (.) u::nd (3) hab (1) einen Monat nach meinem Schulabschluss mein erstes Kind bekommen (88-91) 
Als Anna zum ersten Mal schwanger wird, besucht sie die Realschule. Da Anna von der Wohngruppe in eine Mutter-Kind-Einrichtung umzieht, hat sie einen weiteren Schulweg zu bewältigen als vorher. Im schulischen Umfeld macht sie sowohl mit Mitschüler_innen als auch mit Lehrer_innen negative Erfahrungen hinsichtlich ihrer Schwangerschaft. Es wird über sie geredet, sie wird »fertiggemacht « (922) und es wird ihr »wenig Verständnis« (923f.) entgegengebracht. Trotz dieser erschwerten Bedingungen absolviert sie hochschwanger ihren Abschluss und bringt einen Monat später ihre Tochter zur Welt. Dies zeugt von einem hohen Maß an Willen, Energie und Ehrgeiz und veranschaulicht die Bedeutsamkeit, die Anna dem Schulabschluss beimisst.

Insgesamt offenbart sich in Annas Handlungsorientierung ihre Orientierung nach Autonomie, indem sie hochschwanger die Schule abschließt und so ihr Leben aktiv in die Hand nimmt und gestaltet.

\section{Praktika}

aber ich habe wirklich mein Bestes versucht und war dann ziemlich motiviert $\mathrm{ja}, / / \mathrm{mmh} / /(1)$ als sie zwei Monate alt war hab ich dann im Kinderhort gegenüber $n$ Praktikum gemacht und//mmh//(1) war da schon: (1) also hab ich dann auch an meine Zukunft gedacht ja (2) was ich da jetzt so mach mit meinem Realschulabschluss als junge Mutter (933-937)

Anna beschreibt die Zeit nach der Geburt ihrer Tochter als sehr anstrengend, was sie auch darauf zurückführt, dass es ihr selbst nicht gut ging. Dennoch gibt sie ihr »Bestes « und fühlt sich »motiviert«. Sie beginnt kurze Zeit später ein Praktikum in einem Kinderhort und macht sich generell Gedanken über ihre Zukunft.

Durch ihre Reise nach Afghanistan wird Annas Bildungsaspiration zunächst unterbrochen. Allerdings sucht sie sich auch dort unmittelbar eine Arbeit, ebenfalls in einer sozialen Einrichtung.

Zurück in Deutschland kommt es zunächst zu einer erneuten Schwangerschaft und der Geburt ihres zweiten Kindes. Nachdem Annas zweite Tochter geboren wurde, wird die Frage nach ihrer Zukunft für Anna wieder drängender. Sie entschließt sich, eine Ausbildung zur Floristin zu machen.

\section{Ausbildung}

ich bin halt jemand wenn ich was anfange//mmh//(holt Luft)) ich wollts fertig machen ja das wa- das is mir immer wichtig ich fang nix an//mmh//(1) ich will keine halben Sachen machen, und dann ((holt Luft)) hab ich da echt fertiggemacht aber//mmh//@(.)@hinterherwarich echt@froh@//ja//(1589-1593) 
Obwohl die Zeit nicht einfach ist und es während ihrer Praxiszeit verschiedene Probleme innerhalb des Arbeitsteams gibt, die Anna die Arbeit dort erschweren, bewältigt sie die Ausbildung und schließt sie ab.

Hier zeigt sich in allen Bereichen der formalen Bildung Annas Orientierung nach Autonomie und Unabhängigkeit, die sie aktiv gestaltet. Hat sie eine Entscheidung getroffen, verfolgt sie diese mit hohem Engagement.

In einer Theorie zum Selbst evaluiert sie die Bereiche "Arbeiten oder Lernen« in ihrem Leben als sehr positiv:

\section{Theorie zum Selbst}

also ich habe schon immer//mmh//des war immer so des äh was mich dann was mir auch gut getan hat in meiner ganzen Biografie=alles $/ / \mathrm{mmh} / /$ was mit Arbeiten oder Lernen zu tun hat//mmh//war immer gut//mmh//(1) (980-983)

Professionelle Hilfe und Unterstützung

Anna erfährt im Laufe ihrer Biografie verschiedene Formen professioneller Hilfe, die sie unterschiedlich erlebt.

\section{Jugendamt}

ähm GENAU wir hatten schon immer mit dem Jugendamt Kontakt (.) meine Mutter hatt auch Hilfe vom Jugendamt (2) äh:: hat um Hilfe gebeten (.) da war ich zwölf oder so (51-53)

Bereits im Kindes- und Jugendalter erhält ihre Mutter Unterstützung vom Jugendamt bzw. wendet sich bei Konflikten an dieses. So kommt es auch, dass Anna mit 15 Jahren in eine Wohngruppe wechselt:

\section{Wohngruppe}

und mit:: $f \ddot{u} \mathrm{n} f \mathrm{z}$ e h $\mathrm{n}$ gings dann ga:r nicht mehr, da wollte meine Mutter dann nicht mehr dass ich bei ihr lebe und ich wollte auch nicht mehr bei meiner Mutter leben, ((holt Luft)) und da bin ich dann in ne Wohngruppe gekommen (1) (53-56)

Die Zeit, in der Annas Töchter sehr klein sind und während der sie keinerlei Unterstützung von den Vätern erhält, beschreibt sie als eine Zeit, in der sie spürt, dass sie Hilfe braucht. Sie beantragt eine Familienhilfe, da sie sich mit der Situation »überfordert« fühlt.

\section{Familienhilfe}

das=heißt ich saß dann alleine in ner Wohnung mit zwei Kleinkindern, (2) u::nd (.) zu dieser Zeit gings mir psychisch sehr schlecht (.) also da hab ich 
auch gemerkt=dass ich psychisch (.) Unterstützung brauch (1) (das heißt) ich hatte in der ganzen Zeit nie wirklich professionelle Therapie oder so was gemacht $/ / \mathrm{mmh} / /\left(\right.$ holt Luft 2) ${ }^{\circ} \mathrm{u}: \mathrm{nd}^{\circ}$ genau, hab dann aber auch ne Familienhilfe beantragt weil ich sehr überfordert war ((holt Luft)) (123-128)

An dieser Stelle dokumentiert sich ein weiteres Mal Annas selbstinitiatives Handeln, um ihr Leben zu organisieren und in den Griff zu bekommen. Das selbstinitiative Handeln wird vor allem vor dem Hintergrund einer akuten Notsituation entwickelt.

\section{Kinder- und Jugendpsychotherapie}

und die hat dann gesagt also ich müsste auch was für meine Psyche tun (.) dass es so nicht weitergeht, (1) und dann hab ich ne ähm Kinder- und Jugendlichentherapie angefangen (128-131)

Durch den Anstoß der Familienhelferin beginnt Anna eine Kinder- und Jugendtherapie. Sie begründet die Art der Therapie damit, damals noch unter 21 Jahre alt gewesen zu sein, und erklärt in einem Vorgriff auf die Zukunft, die Therapie insgesamt fünf Jahre in Anspruch genommen zu haben. Diese wurde aufgrund von Annas Bedarf immer wieder verlängert.

Angesichts der Dauer von fünf Jahren Therapie kann davon ausgegangen werden, dass die Beziehung zum Therapeuten oder zur Therapeutin, möglicherweise auch zur Familienhelferin, von Anna als sehr tragfähige Beziehungen erlebt wurden.

Theorie zum Selbst Anna resümiert abschließend, sie habe »keine normale Jugend« (1222) gehabt, da sie bereits mit 16 Jahren schwanger wurde. Nach ihrer Rückkehr aus dem Ausland beginnt sie, exzessiv zu feiern, und zeigt ähnliche »Tendenzen« (1233) in ihrem Verhalten wie ihre Mutter.

Anna selbst fühlt sich »in dieser Rolle« (1239) zunächst wohl, da es das ihr Bekannte widerspiegelt. Möglicherweise erfuhr sie durch die Therapie wichtige Unterstützungsimpulse, die ihr halfen, die genannten Parallelen zu erkennen und einen Weg aus diesen heraus zu finden.

Hier zeigt sich im Hinblick auf den unterliegenden Orientierungsrahmen einerseits, dass Anna sich in ihren Beziehungen an ähnlichen Milieus orientiert wie denjenigen, die sie aus ihrer Kindheit kennt (Drogen, chaotisches Umfeld, Unzuverlässigkeit in Beziehungen); andererseits dokumentiert sich Annas Anstrengung, sich von diesem Milieu zu befreien, was ihr u.a. mithilfe ihrer Bildungsaspiration und professioneller Unterstützung auch gelingt. 


\section{Psychiatrie und Tagesklinik}

genau diese Trennung dann=ging mir dann auch ziemlich nah $/ / \mathrm{mmh} / /(4)$ ah (.) weil das quasi so (.) damals dacht=ich das wär die Liebe meines Lebens und so weiter, ((holt Luft 2)) u:nd ${ }^{\circ}$ wie war $\mathrm{n}$ des noch was ist dann passiert wo war ich jetzt alleine zwei Kinder (2) genau Suizid ${ }^{\circ}$ (.) dann war ich erstmal in in der Psychiatrie ein paar Tage und dann drei:: Monate oder sogar noch länger in der Tages$\mathrm{klinik} / / \mathrm{mmh} / /(1)$ in der psychiatrischen Tagesklinik ((holt Luft)) (142-148)

Anna begeht einen zweiten Suizidversuch kurz nach Beginn der Therapie. In einer Rückblende in Form einer Hintergrundkonstruktion schildert Anna ihre damalige Situation: Ihr Mann, mit dem sie trotz der großen Entfernung versucht zusammenzubleiben, kann nach zwei Jahren wieder nach Deutschland einreisen. Anna stellt jedoch bereits nach einer Woche fest, dass die »Erwartungen die ich an ne Ehe hab und die er an ne Ehe hat komplett anders sind « (141f.), woraufhin es zur Trennung kommt. Sie ringt um Worte und endet schließlich mit einem evaluativen Schlusskommentar: »damals dacht=ich das wär die Liebe meines Lebens und so weiter«.

Hinsichtlich Annas normativen Beziehungsvorstellungen dokumentiert sich hier auf der Ebene des Orientierungsschemas eine romantische Vorstellung von Liebe bzw. der Wunsch nach der großen Liebe, die im besten Fall ein Leben lang hält (»Liebe meines Lebens«).

Anna wird nach dem Suizidversuch zunächst in der Psychiatrie aufgenommen und wechselt anschließend für einige Wochen in die psychiatrische Tagesklinik. Sie bewertet den Aufenthalt in der Tagesklinik als sehr positiv, da sie insbesondere dort feststellt, dass sich in ihrem Leben etwas verändern sollte. Sie fasst ihre Erfahrung diesbezüglich als Schlüsselerlebnis zusammen, durch das ihr klar wird: »ich kann mehr« (151). Eine weitere prägende Erfahrung, die damit einhergeht, ist die Erkenntnis, nicht so werden bzw. enden zu wollen wie manche ihrer Mitpatient_innen.

In diesem Segment dokumentiert sich erneut Annas Initiative, ihr Leben in die Hand zu nehmen. Ausschlaggebend ist hierfür ihre Erkenntnis, mehr zu können. Insbesondere das Voraugenführen der Mitpatient_innen stellt einen Negativhorizont für sie dar, durch den sie erkennt, wie sie nicht werden möchte. Hier bestätigt sich erneut Annas Fähigkeit, in einer Notsituation Kräfte zu mobilisieren, um sich aus dieser zu befreien.

Zusammenfassend lässt sich folgendes festhalten: Obwohl die Zeit während der Ausbildung nicht einfach ist, schließt Anna diese ab. Wesentlich hierbei ist die Unterstützung der Therapeutin. Darin dokumentiert sich die Erfahrung von professionellen Beziehungen als tragfähigen Beziehungen, in denen Anna insbesondere Kontinuität und Verlässlichkeit erlebt. Diese Erfahrungen stehen im Gegensatz zu denen, die Anna innerhalb von Paarbeziehungen macht bzw. mit Männern erlebt. 
Diese neuen Erfahrungen von Kontinuität und Verlässlichkeit, Wertschätzung und Anerkennung im Hinblick auf Beziehungsgestaltung, ermöglichen Anna perspektivisch einen anderen Zugang zu Beziehungen und deren Gestaltung. Sie erkennt das immer wiederkehrende Muster, das sich in ihren Kontakten zu Männern zeigt, was ihr ermöglicht, es auf der Grundlage ihrer Reflexion zu durchbrechen. In den argumentativen Passagen des Interviews wird dieser Prozess deutlich, weshalb im folgenden Kapitel das Augenmerk auf diese eigentheoretischen Äußerungen gerichtet werden soll.

\subsubsection{Argumentative Stellungnahmen}

In der vorausgehenden Falldarstellung wurden bereits eigentheoretische Äußerungen der Erzählerin in der Analyse berücksichtigt. Darüber hinaus wird im Folgenden anhand zweier ausgewählter argumentativer Stellungnahmen der Erzählerin die theoretische und evaluative Haltung der Erzählerin zu den berichteten Ereignissen verdeutlicht. Es wird dabei die gegenwärtige bewertende und theoretische Haltung der Erzählerin fokussiert, um die »Ablösefähigkeit gegenüber Handlungsund Erleidensverwobenheiten « (Schütze 1987, S. 138) und damit die Prozesshaftigkeit der Biografie deutlich zu machen.

\section{Wiederkehrendes Muster}

ich bin immer wieder in dieses Ding zurückgegangen [...] also immer wieder ((holt Luft)) die gleichen Männer immer wieder das gleiche Schema einfach (167-170)

In dieser theoretisch-reflektierenden Stellungnahme bringt Anna in Explizitform ihre reflektierende Stellungnahme gegenüber den narrativen Darstellungsteilen aus heutiger Perspektive zum Ausdruck. Die Wiederholung ihrer Reflexion, die sich direkt anschließt, verstärkt deren Ausdruck:

ja also s: (.) hat sich wiederholt nur mit@anderen Männern@ja, (.) aber zum Clück wurde ich nicht mehr@schwanger@(170f.)

Die analytische Ablösefähigkeit, die sich in dieser Reflexion zeigt, fußt auf einer Distanzierung gegenüber der erzählten Geschichte. Darin zeigt sich, wie sich aufgrund der Auseinandersetzung mit der eigenen Biografie Reflexionsprozesse in Gang setzen, die zukunftsorientierte Änderungen der eigenen Handlungspraxis ermöglichen.

Anders gesagt: In diesen argumentativen Äußerungen kommt die Biografizität (vgl. Kap. 9) der Erzählerin zum Ausdruck.

Mit folgender Evaluation, die den Kernpunkt der Erzählung - gleichsam die »Moral von der Geschichte - auf den Punkt bringt, beendet Anna die biografische Stegreiferzählung: 
und kann aber sagen dass es mir gut geht (1)//mmh//ja (.) und dass ich die (.) Zeit

(1) gut überstanden hab (188f.)

Hier bringt Anna abschließend deutlich zum Ausdruck, dass es ihr heute insgesamt gut geht. Die Wahl des Begriffs »überstanden« illustriert nochmals, dass die Vergangenheit alles andere als leicht zu bewältigen war. Dass sie diese jedoch "gut « überstanden hat, macht ihre subjektive Sicht und Bilanzierung einer erfolgreichen Bewältigung des bisherigen Lebens deutlich.

\subsubsection{Zusammenfassung}

Erleben von Gewalt in Paarbeziehungen Anna erlebt innerhalb ihrer ersten Beziehungserfahrungen verschiedene Dimensionen von Gewalt, die von groben Verhaltensweisen, über Bedrängt-Werden bis hin zu konkreter physischer und psychischer Gewalt, die episodischen Charakter annimmt, reichen.

In ihrer letzten Beziehung kommt es zu einem Gewaltausbruch ihrerseits, indem sie ihren Partner körperlich angreift.

Umgang mit erlebter Gewalt, Bewältigungsstrategien und Ressourcen Während Anna Grobheiten und sexuelles Bedrängt-Werden vergleichsweise lange innerhalb ihrer Beziehungen toleriert, reagiert sie auf eine Ohrfeige bestürzt. Aber weder diese Situation des körperlichen Übergriffs noch die darauffolgende Verfolgung mit Drohungen führen dazu, dass sie die Ereignisse innerhalb der Beziehung thematisiert oder sich trennt. Sie hält trotz der erfahrenen Gewalt an der Beziehung mit ihrem Freund fest.

Hinsichtlich ihrer Ressourcen zeigt sich bei Anna ein enormes Potenzial an Bildungsaspiration, die von dem Wunsch nach einem autonomen Leben getragen wird.

Die rekonstruierbare Funktion von Bildung zur Befreiung aus einengenden Rahmenbedingungen sowie zur Ermöglichung eines selbstbestimmten Lebens geht einher mit der Fokussierung auf den Entschluss, die Schule kurz vor der Geburt des ersten Kindes abzuschließen, auf den Abschluss der Berufsausbildung trotz schwieriger Bedingungen sowie auf die Aufnahme eines Studiums als alleinerziehende Mutter. Insgesamt wird deutlich, dass sich verschiedene Bildungsaktivitäten durch die gesamte Biografie ziehen.

Eine weitere Ressource, die Anna nutzt, ist die Annahme professioneller Hilfe in verschiedenen Lebensphasen. Im Gegensatz zu der erfahrenen professionellen Hilfe bereits im Kindes- und Jugendalter (Jugendamt, Wohngruppe), die sie überwiegend als von außen vermittelt erlebte, nimmt sie als junge Frau aktiv Familienhilfe und anschließend auch therapeutische Hilfe in Anspruch. Die selbstinitiative Organisation von Unterstützung zeigt das Bestreben nach Autonomie und führt letztlich mit dazu, dass sich Annas Leben insgesamt stabilisiert. 
Beziehungsmuster und Beziehungsgestaltung In der Analyse des Interviews mit Anna lässt sich eine Orientierung an einem normativen Beziehungs- und Familienmodell rekonstruieren. Hinsichtlich Annas Bild von Familie wird in ihrer Erzählung ein Orientierungsschema deutlich, das sich an einer behüteten Kindheit und intakten Familie orientiert.

Dieser Orientierung liegt eine biografische Konstruktion zugrunde, innerhalb der Familie als Möglichkeitsraum fungiert, der zu einem besseren Leben verhelfen soll. Das Bedürfnis nach Zugehörigkeit, das sich als grundlegende Orientierungsfigur in der Analyse herausschält, wird über die Konstituierung von Familie zu erfüllen versucht. Diese Herstellung von Zugehörigkeit über Familie stellt den zentralen Orientierungsrahmen dar, mit dem das oben genannte Orientierungsschema bearbeitet wird.

Annas Beziehungsmuster weist Vorstellungen hinsichtlich bestimmter Werte auf: So sind für sie Zuverlässigkeit und gegenseitige Unterstützung innerhalb einer gelungenen Beziehungsgestaltung unabdingbar. Hier zeigt sich allerdings eine Diskrepanz zur gelebten und erfahrenen Realität, in der genau diese Bedürfnisse nicht befriedigt werden.

Die Beziehungen zu Männern konstelliert Anna so, dass sie unabhängig bleibt. Ihre Grunderfahrung ist, dass Beziehungen nicht verlässlich sind. In der Analyse des Interviews wird ein immer wiederkehrendes Muster deutlich: In den Beziehungen zu ihren Partnern spiegeln sich die Umstände und Verhältnisse wider, die Anna aus ihrer Kindheit und Jugend kennt und die von Unzuverlässigkeit und Unbeständigkeit geprägt sind. Besonders deutlich wird das habitualisierte Beziehungsverständnis auf der kommunikativen Ebene innerhalb der Paarbeziehung: hier zeigt sich, dass es keine verbale Aushandlung, keine Verständigung über Wünsche und Bedürfnisse gibt.

Gleichzeitig besteht ein existenzielles Angewiesensein auf diese Beziehungen, in denen Zugehörigkeit gesucht wird. Besonders deutlich wird dies in den Beziehungen, an denen Anna trotz erfahrener Abwertung und Nötigung festhält.

(Gewalt-)Beziehungen und Sozialisation Annas sozialisatorisches Umfeld ist durch chaotische Verhältnisse und das Aufwachsen in einer Ein-Eltern-Familie geprägt. Die Erfahrung, nicht erwünscht zu sein, zieht sich durch die Biografie (kein Kontakt zum Vater, Aufnahme in eine Wohngruppe nach Eskalation der Konflikte mit der Mutter, Wechsel in Mutter-Kind-Wohnheim). Das Bedürfnis nach Zugehörigkeit und die Suche nach dieser im weiteren Verlauf der Biografie gründet auf diesen lebensgeschichtlichen Erfahrungen.

Ihre Orientierung innerhalb von Paarbeziehungen findet zu einem frühen Zeitpunkt eine familiale Ausrichtung, was durch die frühe Schwangerschaft verstärkt wird resp. wodurch das bis dahin bestehende Familienkonzept sich konkretisiert. 
Hinsichtlich der Mobilisation von Kräften in existenziell bedrohlichen Situationen zeigt Anna ein enormes Potenzial; in der zugrunde liegenden Handlungspraxis wird eine klare Zukunftsorientierung deutlich.

\subsubsection{Amy - »es hat ja alles immer so $\mathrm{n}$ Kreis so $\mathrm{n}$ Lebenskreis « (293f.)}

\subsubsection{Fallporträt}

Amy wird als älteste Tochter von insgesamt vier Kindern in einer deutschen Großstadt geboren. Nach der Geburt ihrer Schwester wandern ihre Eltern mit beiden Kindern nach Kanada aus. Amy ist zu diesem Zeitpunkt zweieinhalb Jahre alt. Dort werden nochmals zwei Geschwister geboren. Amy wird bereits im Kindesalter Verantwortung für ihre Geschwister und deren Versorgung übertragen, außerdem ist sie für einen Großteil der Haushaltsführung zuständig.

Als Amy acht Jahre alt ist, kehrt die Mutter mit allen vier Kindern zurück nach Deutschland, nachdem sie erfahren hat, dass ihr Mann sie betrügt. Für alle Kinder ist der Start in Deutschland schwierig, zumal sie nur Englisch sprechen. Auch die Wohnsituation ist zunächst prekär. Als Amys Mutter Arbeit findet, zieht die Familie in eine »schönere Wohnung schönere Gegend « (21) um. Allerdings leiden die Kinder unter der Alkoholsucht der Mutter und der daraus resultierenden Vernachlässigung. Amys Mutter verbringt viel Zeit in Kneipen, wohin sie Amy bereits als Mädchen mitnimmt. Amy erlebt diese Unternehmungen einerseits als »cool« (496). Andererseits macht sie viele einschneidende und traumatisierende Erfahrungen. So erlebt sie ihre Mutter häufig in stark betrunkenem Zustand, in dem diese auch davon spricht, sie wolle sich umbringen (391).

In dieser Zeit beginnt Amy, nach älteren Männern Ausschau zu halten. Mit zwölf Jahren verliebt sie sich in Taavi. Es bleibt offen, wie alt er ist. In jedem Fall ist er volljährig, da er den Führerschein besitzt. Bei ihm erlebt Amy ein Stück heile Welt, indem er ihr »die gerade Linie« (1078), »den Weg, der's richtig geht« (1078f.) zeigt. Zwischen beiden entwickelt sich mit der Zeit eine intensive Beziehung, die, als Amy 16 Jahre alt wird, auch die Aufnahme sexueller Aktivitäten miteinschließt. Mit 17 Jahren beendet Amy die Beziehung zu Taavi, da sie keine gemeinsame $\mathrm{Zu}$ kunft sieht.

Mit 14 Jahren besucht sie zum letzten Mal ihren Vater in Kanada. Das Verhältnis zwischen Amy und ihrem Vater ist jedoch so angespannt, dass kein weiterer Besuch folgt.

Amy hat wenig gleichaltrige Freund_innen; sie ist meistens mit älteren unterwegs. Über ihre Schulzeit berichtet sie wenig, allerdings erzählt sie, dass sie bis zur achten Klasse häufig Schläge von ihrer Mutter erhält (»ich hab mich ja immer nur schlagen lassen« (781)).

Über eine Einrichtung der Jugendberufshilfe beginnt sie eine Ausbildung zur Schreinerin. In dieser Zeit hat sie viele wechselnde Bekanntschaften mit Männern. 
Als sie sich von ihrem Chef gemobbt fühlt, bricht Amy die Ausbildung ab. Zu dieser Zeit pflegt sie eine lose Beziehung zu Sascha, den sie kennt, seit sie zwölf Jahre alt ist, und der deutlich älter ist als sie. Die Beziehung hat einen unverbindlichen Charakter (»ich war nicht richtig fest mit ihm zusammen« (601)), dauert aber immerhin zweieinhalb Jahre. Sascha ist vor allem an einer sexuellen Beziehung interessiert (»du nimmst alles was nicht bei drei auf'm Baum is « (594); »= ${ }^{\circ}$ wollt früher halt auch (.) ${ }^{\circ}$ klar junges Hüpferl und so« (592)). In der Beziehung kommt es oft zu Streitigkeiten und Sascha rastet häufig aus (742). Amy trennt sich von Sascha, als dieser ins Gefängnis kommt.

Kurze Zeit später wird sie von Daniel schwanger. Amy ringt lange mit der Entscheidung, ob sie das Kind bekommen soll, und entscheidet sich letztlich dafür. Als Amy 20 Jahre alt ist, wird ihre Tochter geboren. Sie lebt zunächst zwei Jahre allein mit ihr, bis sich Daniel, der Vater ihrer Tochter, bei ihr meldet. Erst jetzt beginnen beide, sich näher kennenzulernen. Allerdings kommt es bereits nach wenigen Wochen zu Konflikten, weil Daniel von ihr erwartet, dass Amy nicht arbeiten geht, sondern zu Hause bleibt. Sie trennt sich daraufhin von ihm, da sie keine gemeinsame Zukunft mit ihm sieht.

Die anschließende Zeit ist geprägt von Konflikten, die sich vor allem auch auf die Erziehung und den gemeinsamen Umgang mit der Tochter beziehen. Es eskaliert immer wieder zwischen beiden, wobei es neben Streitereien auch zu körperlichen Auseinandersetzungen kommt.

Als Amy 24 Jahre alt ist, wendet sie sich ans Arbeitsamt, weil sie wieder arbeiten möchte. Zunächst beginnt sie mit einem Ein-Euro-Job. Dann nimmt sie, erneut mit Unterstützung durch den Jugendberufshilfeträger, wieder ihre Ausbildung zur Schreinerin auf. Zudem erhält sie über die Jugendberufshilfe Unterstützung in ihrer Situation als alleinerziehende Mutter und Auszubildende (»Mutterkurs« (928)).

Es folgt eine Beziehung zu einem Mann, über die Amy jedoch nichts Näheres berichtet. Sie trennt sich nach vier Jahren von ihm. Obwohl sie zu dieser Zeit nichts mehr von Männern wissen will, lernt sie Robert kennen. Sie weiß, dass er eine pädophile Neigung hat und kinderpornografische Bilder konsumiert. Dennoch zieht sie mit ihm zusammen. Sie hat die Hoffnung, dass er damit aufhört und zerstört im Laufe der Beziehung drei Laptops, als sie kinderpornografische Bilder bei ihm entdeckt. Um die Belastungen zu bewältigen, fängt Amy in dieser Zeit an, Cannabis zu konsumieren.

Eines Tages entdeckt sie auf dem Laptop ihres Freundes Fotos ihrer Tochter. Sie wirft ihren Freund daraufhin umgehend aus der gemeinsamen Wohnung und zieht einige Monate später in eine andere Wohnung um. Amy plagen seither massive Schuldgefühle sowie die Frage, ob ihre Tochter sexuell missbraucht wurde. Da sie zunehmend gewisse Parallelen zwischen dem Leben ihre Mutter und ihrem eigenen erkennt, formuliert sie ihre Sorge darüber, möglicherweise so $\mathrm{zu}$ werden 
wie ihre Mutter. Mit Unterstützung einer Freundin sucht sich Amy therapeutische Hilfe.

Zum Zeitpunkt des Interviews finden keine regelmäßigen Kontakte zwischen Amys Tochter und deren Vater statt. Sie versucht, sich auf ihr eigenes Leben und ihre Tochter zu konzentrieren und formuliert den Wunsch, bis auf Weiteres keine neuen Affären oder Beziehungen einzugehen.

\subsubsection{Analyse der Erzählstruktur}

Amy beginnt ihre biografische Stegreiferzählung zusammenfassend-retrospektiv. Durch die durchgängig eingestreuten knappen Argumentationen wird ihre heutige Wissens- und Bewertungsperspektive deutlich. Auffallend ist die Raffung größerer Zeitabschnitte, die summarisch zusammengefasst und lediglich durch die Erwähnung der Abfolge einzelner Etappen (Geburt, Reise nach Kanada, Rückkehr nach Deutschland) dargestellt werden. Werden die suprasegmentalen Gliederungszusammenhänge betrachtet, wird jedoch deutlich, dass Amy in ihrer Erzählung eine grundsätzliche Erlebnishaltung zum Geschehensablauf zeigt, die sich durch das gesamte Interview durchzieht: die Erfahrung, belogen und betrogen worden zu sein, sowie die damit verbundene Erfahrung, dass das Leben von Anfang an schwierig ist.

Eine weitere Auffälligkeit im Interview mit Amy besteht darin, dass sie nach der verdichteten Darstellung größerer Zeitabschnitte relativ schnell in der Gegenwart ankommt und über ihre aktuelle Lebenssituation erzählt. Auf der erzählerischen Ebene zeigt sich dies, indem Amy im Anschluss an ihre narrativen Ausführungen das Erzählte sofort bewertet oder erklärt. Damit wird ihre aktuelle Verstrickung in Schwierigkeiten und Herausforderungen, die sich zum Zeitpunkt des Interviews an sie stellen, deutlich. Zudem sind ihre Darstellungen von Brüchen, erzählerischen Leerstellen und einer immer wieder auftauchenden Inkonsistenz der Erzählung geprägt. Dies deutet auf erlernte Abwehrmechanismen der Dissoziation hin, die als Folge traumatischer Erfahrungen in der Kindheit anzusehen sind und sich auf Textebene manifestieren. Wie sich im Fall Amy Spuren von Traumatisierungen im Text zeigen, wird im Laufe der Einzelfallanalyse näher beleuchtet.

Zudem habe ich aufgrund der Auffälligkeit, dass die Passagen, in denen Amy über ihren erlebten sexuellen Missbrauch in Kanada sowie über den Missbrauchsverdacht bei ihrer Tochter spricht, von vielen Argumentationen durchzogen sind, zwei Hypothesen zum Zusammenhang von theoretischen Aktivitäten in der Stegreiferzählung und Traumatisierungen entwickelt:

1. Argumentation als Schutz vor Trigger: Die Erzählerin kann sich an die Erlebnisse erinnern, aber sie kann nicht darüber sprechen. Sie spürt, dass sie sich schützen muss, um den Schmerz nicht zu groß werden zu lassen und um nicht 
von Gefühlen überflutet und überwältigt (Dissoziation) zu werden. Mittels der Verwendung von Argumentationen stellt sie eine Distanz zu den erlebten Erfahrungen und den damit zusammenhängenden Emotionen her. Argumentationen werden immer auf Ebene der Gegenwart erzählt. Die Verortung in der Gegenwart ist notwendig, um sich vor der Gefahr der Überflutung und damit der Möglichkeit der Retraumatisierung zu schützen. Mithilfe von Argumentationen begibt sich die Erzählerin auf eine Metaebene der Reflexion.

2. Amnesie: Die zweite Hypothese geht davon aus, dass die Erinnerungen so abgespalten sind, dass die Erzählerin keinen Zugang mehr dazu hat. In diesem Fall handelt es sich um eine Amnesie, da der Anteil der Erinnerungen abgespalten ist. Die Erzählerin fragt sich, wie es gewesen sein könnte. Da Erfahrungen nicht erzählt werden können, verfällt die Erzählerin in den Modus der Argumentation, das »ein wichtiges Mittel der analytischen Verarbeitung der Handlungs- und Erleidensprozesse des Erzählers als Geschichtenträger und des Gewinnens von analytischen Einsichten aus den Ereignisverstrickungen anderer Geschichten- und Ereignisträger« (Schütze 1987, S. 143) ist.

Auch wenn nicht abschließend festgestellt werden konnte, wann welche Hypothese im vorliegenden Fall zutrifft, erschien es mir wichtig, beide bei der Interpretation mitzudenken und sie entsprechend bei der Analyse zu berücksichtigen. So konnten bspw. Leerstellen in Erzählungen besser identifiziert und in die Interpretation mit eingebunden werden.

\subsubsection{Herkunftsfamilie und Bedingungen des Aufwachsens}

Amy präsentiert nach dem offenen Erzählstimulus in der Eingangserzählung eine kurze narrative Passage, die mit Aussagen zu ihrer Geburt und ihrer Familie beginnt:

\section{Eingangsnarration}

A: Gu:t (1) also ich bin hier geboren in Stadt 1, (.) ahm dann is meine Schwester auch hier geboren (1) ((holt Luft)) dann sind wi:r zu viert meine Mama Papa und (.) wir zwei Kinder nach Kanada (.)//mmh//gezogen u:n ah, (1) ja dann sind die [...] noch auf die Welt gekommen ((holt tief Luft 2)) war relativ schön dort ahm (.) dann ah (1) hat meine Mutter rausbekommen dass mein Papa meine Mama betrogen hat dann sind wir alle vier Kinder mit meiner Mama wieder nach Deutschland (12-18)

Amy bestätigt durch »Gu:t« die Aufforderung der Interviewerin, ihre Lebensgeschichte zu erzählen. Damit signalisiert sie ihre Bereitschaft, sich auf das Interview einzulassen. Außerdem proponiert sich eine hohe Bereitschaft, die eigene Lebensgeschichte zu erzählen. Durch die Vermittlung des Interviews über die Kon- 
taktperson Anke ${ }^{8}$ könnte außerdem die Bereitschaft verstärkt worden sein, eine vertrauensvolle Beziehung, hier mit der Interviewerin, einzugehen.

Amy beginnt ihre Erzählung mit Aussagen zu ihrer Geburt und ihrer Familie. Damit zeigt sie auch den ersten Orientierungsgehalt: Durch die regionale Verortung ihrer Geburt »hier« und der Geburt ihrer Schwester »auch hier« konstituiert sie Zugehörigkeit zu denen, die am gleichen Ort geboren sind; die Familie wird dadurch geteilt, dass ein Teil »hier geboren« und ein anderer Teil in Kanada geboren ist. Im Gegensatz dazu wird Zugehörigkeit, die auf einer emotionalen Basis beruht, nicht thematisiert.

Deutlich wird an dieser Stelle die Strukturierung der Biografie mittels Orten (Stadt 1, Kanada, Deutschland). Hierin dokumentiert sich einerseits, dass Amys familiale Orientierung regional verortet ist; gleichzeitig proponiert sich das Thema »Zugehörigkeit« bereits in der Eingangsnarration.

Amy bezieht sich in der Darstellung ihrer Biografie auf größere Zeiträume, indem sie wesentliche Lebensabschnitte wie frühe Kindheit und Kindheit erst gar nicht näher ausführt. Anhand der biografischen Stationen »Geburt in Stadt 1«, »Kanada«, »Deutschland «, »schönere Gegend« werden die Ereignisse gerafft und nur in ihren zentralen Veränderungsaspekten dargestellt. Die Ereignisabfolgen und Erfahrungen sind nicht re-inszeniert aus der Perspektive des damaligen Erlebens, sondern rückblickend mit zusammenfassenden und aus dem heutigen Wissen heraus evaluierenden Begriffen (»war relativ schön dort«, "war schwer am Anfang», »alles soweit okay«). Es finden sich in dieser Erzählpassage keine Höhepunkte, auch Anzeichen von Emotionalität sind nicht erkennbar. Dies spricht für einen erhöhten Grad an Distanzierung gegenüber dem Erlebten. Neben der Benennung von Orten und einzelnen, jedoch nicht namentlich genannten Familienangehörigen fällt auf, dass weitere biografisch relevante Stationen oder Personen (wie Kindergarten, Schule, Freundinnen etc.) nicht auftauchen.

Amy ist es nicht möglich, ihre Lebensgeschichte chronologisch zu erzählen, die Erzählung bleibt fragmentarisch, was erste Hinweise auf mögliche Traumatisierungen liefert. Es kann davon ausgegangen werden, dass die genannten Ortschaften für Amy so etwas wie Heimat resp. Orte der Sicherheit repräsentieren und sich auf die Erzählung konsolidierend auswirken: So, wie sie die Erzählung stabilisieren, stabilisieren sie die Biografie.

8 Anke arbeitet in einer Einrichtung der Jugendberufshilfe, über die Amy ihre Ausbildung zur Schreinerin absolviert. Außerdem bietet die Einrichtung sogenannte »Mütterkurse« an, in denen junge Mütter sozialpädagogische Begleitung und Unterstützung erfahren. Das Interview mit Amy kam über die Sozialpädagogin des Mutterkurses, Anke, zustande. 


\section{Aufwachsen in Kanada}

also s- (1) mein Vater kam heim ich hab alles gemacht, (1) der hat sich auf die Couch gelegt, ich hab mit ((holt Luft)) fünf Jahren meine Ceschwister wickeln müssen, (1) bettfertig machen müssen Essen fertig machen müssen ((zieht Nase hoch)) u:nd ja:, da ich dann immer so: groß (.) gemacht worden bin, (.) war ich halt jetzt immer noch die Große (342-346)

Hier kommt es bereits zu einem frühen Zeitpunkt in Amys Biografie zu einer Parentifizierung, einer Rollenumkehr zwischen Erwachsenen und Kindern, indem sie früh in eine Erwachsenenrolle gegenüber ihren Geschwistern gedrängt wird. Amy wird Verantwortung für ihre Geschwister und deren Versorgung übertragen, indem sie diese bspw. wickeln oder für sie das Essen zubereiten muss. Außerdem ist sie für einen Großteil der Haushaltsführung verantwortlich.

\section{Alkoholsucht der Mutter}

meine Mutter is irgendwie abgesackt (1) die hat Alkoholprobleme (.) dadurch sind wir Kinder halt vernachlässigt worden (.) ahm merk ich jetzt halt auch erst im (.) Alter, und wenn man älter wird, (.)) (22-24)

$\mathrm{Zu}$ Beginn dieser Sequenz folgt zunächst die Präambel (Gliederungsmarkierer) "meine Mutter is irgendwie abgesackt«, mit der die Bedeutung der kommenden Zeit angekündigt wird. Damit wird auch ein Bruch zu dem bisher Erzählten (»alles soweit okay« (21f.)) deutlich. Die Erzählung wird hier weiter durch das Verhalten der Mutter strukturiert, die abdriftet. Der Ausdruck »irgendwie« macht deutlich, dass kein Zusammenhang hergestellt wird, weshalb oder wie dies geschieht. Das weist darauf hin, dass Amy keinen Bezug zu diesem Geschehen hat. Aufgrund der »Alkoholprobleme« der Mutter sind »wir Kinder« nach Amys eigentheoretischer Erklärung aus heutiger Sicht vernachlässigt worden. Erst in der heutigen Gegenwart ist es Amy möglich zu verstehen, was in ihrer Kindheit passiert ist. Insgesamt zeigt sich hier, dass Amy Kind-Sein, auch heute noch, an der Mutter festmacht.

Im Folgenden verlässt Amy die allgemeine Ebene des Erzählens und fokussiert einen Ausschnitt der Lebensphase Kindheit, nämlich die Zeit, in der sie von ihrer Mutter in Kneipen mitgenommen wurde:

\section{Aufenthalt in Kneipen}

dann ahm ja: war ich halt öfters dabei bei meiner Mama in den Kneipen, (1) hab relativ a:hm viel Ausschau gehalten (von) älteren Männern, also so einfach vielleicht Papaersatz oder so:, (24-27)

Erst dieses Segment lässt erahnen, worauf sich das Abdriften der Mutter bezieht: Diese verbringt viel Zeit in Kneipen, wohin sie auch ihre älteste Tochter mitnimmt. 
Die Tatsache, dass ein Kind in Kneipen mitgenommen wird, ist in jeder Hinsicht begründungsbedürftig. Amy sieht hier jedoch keinen Erklärungsbedarf; genauso bleibt die Frage nach dem Verbleib der Geschwister offen.

Insgesamt zeigt sich hier, dass Amy keine Vorstellung davon hat, was ihre Kindheit ausmachte; möglicherweise auch generell nicht, was Kindheit bedeutet. Weiter wird deutlich, dass sich Generationengrenzen vermischen: Die Differenzierung zwischen Kind und Erwachsenem löst sich auf.

In den Kneipen sieht sich Amy insbesondere nach älteren Männern um. Dieses Verhalten erklärt sie mit der eigentheoretischen Begründung, dass sie vermutlich nach einem »Papaersatz« suchte.

Hier dokumentiert sich die Kontaktaufnahme zu älteren Männern: Bei der Betrachtung der sequenzanalytischen Abfolge fällt auf, dass Amy mit der generellen Beschreibung beginnt, »relativ a:hm viel Ausschau « nach älteren Männern gehalten zu haben. Dies wird konkreter anhand des Sich-Verliebens im folgenden Segment ausgeführt. Der Begriff >Ausschau halten impliziert das Bedürfnis nach Kontakt mit jemandem; das Wort »viel« deutet auf die Stärke des Wunsches hin. Die Aussage wird ergänzt durch die Erklärung »also so einfach vielleicht Papaersatz oder so:«. Mit dieser eigentheoretischen Begründung drückt Amy den Wunsch nach einer vollständigen Familie - also inklusive Vater - aus.

Gleichzeitig impliziert die Aussage "Ausschau gehalten« den Wunsch oder die Suche nach einem Beziehungspartner. Dies macht deutlich, dass sich in Amys Vorstellungen die Rolle des Beziehungspartners und die des Vater(-ersatzes) vermischen. Hinzu kommt, dass sie sich, indem sie nach Männern Ausschau hält, als sexuell aktiv präsentiert.

Weiter dokumentiert sich in dieser Aussage das Bild eines aktiv handelnden Mädchens, indem sie Ausschau hält. Allerdings bleibt offen, in welcher Weise sich Amy präsentiert oder ob sie vielmehr von außen angesprochen wird.

Hinsichtlich des hier unterliegenden Orientierungsrahmens lässt sich dieser dahingehend konkretisieren, dass es für Amy schwierig ist, sich als abgegrenzte Person zu beschreiben und sich auch so zu erleben. Sie passt sich ihrem Umfeld an, wird mehr als Erwachsene denn als Kind wahrgenommen und angesprochen. Familie stellt ein zentrales Thema dar und bleibt einerseits durch den Fokus auf die Mutter und andererseits in der Suche nach einem Vaterersatz auch weiter zentral. Bezüglich ihrer Beziehungsvorstellungen dokumentiert sich hier, dass sie über keine Vorstellungen darüber verfügt, was eine Erwachsenen-Beziehung ausmacht.

Exkurs zu Amys Großeltern mütterlicherseits Amys Mutter wächst mit acht Geschwistern auf und erlebt bereits früh Gewalt zwischen ihren Eltern. Ihr Vater (Amys Großvater) ist seiner Frau gegenüber körperlich gewalttätig, sodass Amys Mutter diese immer wieder zu einer Trennung auffordert. Der Vater nimmt Amys Mutter häufig in Kneipen und Wirtschaften mit. Als er stirbt, kommen die Kinder in 
ein Kinderheim. Das Verhältnis zwischen Amys Mutter und deren Mutter bleibt schwierig. Amy beschreibt die Mutter-Tochter-Beziehung vor allem als eine Beziehung, die von wenig Akzeptanz und Zutrauen der Mutter in ihre Tochter geprägt war. Hier werden bereits erste Hinweise auf die intergenerationale Übertragung häuslicher Gewalt sichtbar.

\subsubsection{Kernthemen des Interviews}

Das erste Verliebtsein

\section{Verlieben}

dann hab ich mich mit zwölf in irgendeinen (.) Taavi, damals hieß der, verliebt abis ich achtzehn neunzehn war, also das war so (1) teilweise auch Papaersatz dann wurd's immer mehr immer mehr ((holt Luft)) (.) dann a:hm (1) ja mit neunzehn also achtzehn (.) neunzehn a:hm (2) nee mit achtzehn war's=siebzehn achtzehn hab ich's beendet, (.) weil ich sag des geht nich mehr so (27-32)

Mit zwölf Jahren verliebt sich Amy in einen Mann namens Taavi. Auch wenn sie an dieser Stelle nicht explizit ausführt, dass sie mit ihm eine Beziehung führt, so ist doch von einer solchen auszugehen, denn sie »beendet« dieses nicht näher bezeichnete Zusammensein, als sie 17 oder 18 Jahre alt ist. Sie begründet diese Entscheidung mit der Erklärung »des geht nich mehr so«. Es bleibt jedoch offen, was damit gemeint ist. Offen bleibt auch, wie sie Beziehung für sich definiert.

In diesem Segment fällt die Wiederholung der eigentheoretischen Erklärung auf, in der Beziehung oder dem Kontakt zu (älteren) Männern einen Vaterersatz zu suchen.

\section{Verlauf der Beziehung}

und irgendwann sitzen wir da und der kommt rei::n, ein Lockenkopf ein großer Mann [...] und er kommt reingelaufen und sagt zu mir und alles klar, und ich kuck ihn an und sag und dich heirat ich irgendwann, (.) [...] ja:: und dann (.) irgendwann wurd's von mir halt aus me::hr, ich wollt ich hab mich dann richtig verli:ebt, und dann hat er immer gesagt nee, jetzt wart ab (.) wenn du sechzehn bist und ((atmet ein)) des ge::ht nich ${ }^{\circ}$ und ja:, (.) ein Tag nach meinem Sechzehnten kam er dann, ${ }^{\circ}$ DANN hab ich gewusst@wieso@ich jetzt sechzehn werden muss, also >s war an richtig harter Tiefschlag, ich hab gemerkt nee, (.) des kann's jetzt nich sein oder, (.) und des war's dann ja, (1) wir hatten danach auch ne schöne Zeit war alles toll uns so, aber (2) ja,(1) (530-545)

Mit 16 Jahren hat Amy ihr erstes Mal, was eine negative Erfahrung für sie bedeutete (»richtig harter Tiefschlag«; »das kann's jetzt nich sein«). Sie blendet diese negative Erfahrung jedoch weitgehend aus und beschreibt den weiteren Verlauf der Beziehung als eine »schöne Zeit«. 
Vor dem Hintergrund des sexuellen Missbrauchs, den Amy in ihrer Kindheit erlebte, setzt sich hier möglicherweise eine Retraumatisierung fort. Zudem zeigt sich auch im weiteren Verlauf der Biografie die Fortsetzung negativer Erfahrungen mit Sexualität.

In Bezug auf Amys Handlungspraxis dokumentiert sich hier eine Akzeptanz der für sie negativ konnotierten Sexualität. Auch wenn Sexualität zunächst als »harter Tiefschlag« erlebt wird, überwiegen noch eine Zeitlang die positiven Erfahrungen in der Beziehung bezüglich der Nähe und Geborgenheit und damit der Zugehörigkeit, die sie bei Taavi erlebt. Amy deutet ein angepasstes Verhalten an die - sexuellen - Bedürfnisse ihres Freundes an, um die positiven Erfahrungen von Zuneigung und Zugehörigkeit aufrechtzuerhalten. Sie selbst kann jedoch keine positiven sexuellen Erfahrungen machen und blendet die negativen Erfahrungen aus. Die Hypothese, dass sie nach der Devise lebt, dass Sex nun mal zu einer Beziehung dazugehört und sie diesen deshalb in Kauf nehmen muss, kann im weiteren Verlauf der fallimmanenten Kontrastierung bestätigt werden.

Ein bis zwei Jahre später beendet Amy die Beziehung:

\section{Trennung}

aber mit siebzehn (.) [...] na hab ich gesagt des geht nich, ich kann nich mehr, (.) ich mach mich kaputt (1) ich mach jetzt meine Ausbildung und(.) des bringt's au nich, (573-579)

In dieser Sequenz dokumentiert sich in Amys Handlungspraxis die Fokussierung auf ihre eigene Zukunft, die dazu führt, die Beziehung, die für sie nicht zukunftsträchtig ist, $\mathrm{zu}$ beenden. Interessant dabei ist, dass Amy ihre Entscheidung mit dem Hinweis auf den Beginn einer Ausbildung verknüpft. Dies lässt vermuten, dass die Option auf Ausbildung, die eine Zukunftsperspektive beinhaltet, Amys Entscheidung begründet.

In Bezug auf den hier unterliegenden Orientierungsrahmen wird deutlich, dass Amy eine Beziehung eingeht, in der sie sich den sexuellen Bedürfnissen des Partners anpasst, um ihr eigenes Bedürfnis nach Nähe und Geborgenheit und damit nach Zugehörigkeit zu erfahren. Gleichzeitig zeigt sie sich zukunftsorientiert, wünscht sie sich auch eine gemeinsame Zukunft. Als sie erkennt, dass diese in der Beziehung mit Taavi nicht zu realisieren ist, beendet sie die Beziehung.

Unverbindliche Dates

Während der Zeit ihrer Ausbildung als Schreinerin schildert Amy, dass sie beruflich viel unterwegs ist. Sequenzanalytisch betrachtet knüpft sie an das Thema der Gestaltung der Beziehungen mit Männern an, für die nun keine Zeit mehr bleibt. 
hab meine Ausbildung angefangen als Schreinerin ((schluckt)) und a.hm ja: wa:r (.) eigentlich nur beruflich unterwegs (.) die Zeit hat mir ja auch gefehlt, (1) a:hm ja hatte da mal wieder [...] einen Mann da mal wieder einen anderen (32-35)

Auffällig ist die namenlose Benennung der Männer, die Amy »hatte«. Die Beiläufigkeit, mit der diese hier erwähnt werden, deutet darauf hin, dass Amy diese Kontakte als selbstverständlich erlebt und sie ihnen keine größere Bedeutung beimisst. Gleichzeitig impliziert die begriffliche Verwendung »hatte da mal wieder [...] einen Mann« auch etwas Gebrauchendes.

Damit zeigt sich ein aktives Verhalten Amy hinsichtlich ihrer Beziehungsgestaltung zu Männern, die durch eine hohe Unverbindlichkeit und Abwechslung geprägt ist. Die vielen unverbindlichen Kontakte mit Männern deuten auf eine ständige Suche seitens Amy hin.

Durch Amys Erfahrung, dass ihre Partner vor allem Sex mit ihr haben wollen, erfährt sie eine Begrenzung auf ihre Körperlichkeit. Sie ist aber auf der Suche nach Zugehörigkeit, nach Nähe und Zuwendung.

In der folgenden Theorie zum eigenen Selbst wird deutlich, welche Orientierungen Amys Beziehungsmotiven zugrunde liegen:

ja hab ich irgendwie versucht, also ich weiß es jetzt mit meinen Erfahrungen=irgendwie Lie:be gesucht Ceborgenheit die Nu- Nä:he (.) Zuneigung die ich klar nich in den Männern gefunden hab, (40-42)

Damit lässt sich der bisher skizzierte Orientierungsrahmen im Hinblick auf die Suche nach Anerkennung und Zuwendung konkretisieren, die sie zunächst durch Anpassung besonders an die sexuellen Bedürfnisse der Männer und Partner zu erreichen versucht.

Allerdings zeigt sich Amy in ihrer Handlungspraxis auch aktiv: Sie reagiert auf die Nicht-Erfüllung ihres Bedürfnisses nach Nähe und Zugehörigkeit, indem sie häufig ihre Partner wechselt, in der Hoffnung, diese bei einem anderen Mann oder in einer neuen Beziehung zu finden.

Wunsch nach Familie

Amy bricht ihre Ausbildung ab, als sie sich von ihrem Chef gemobbt fühlt, und wird kurze Zeit später schwanger:

\section{Schwangerschaft}

daraufhin bin ich (.) also kurze Zeit später schwanger geworden ((holt Luft) (.) hab dann die Kleine ahm ja: bekommen, auch mit viel (1) hin und wieder is es richtig is es nich richtig, (.) dann hab ich sie bekommen (46-49) 
Für Amy stellt sich die Frage, ob sie das Kind behalten will bzw. soll. Sie entscheidet sich letztlich für das Kind. Auffällig ist hier wieder die Beiläufigkeit, mit der ein solch einschneidendes biografisches Ereignis geschildert wird. Lediglich ihr Ringen um die Entscheidung, schließlich für das Kind, wird deutlich. In dieser Passage fällt erneut die Nicht-Benennung des Vaters auf. In dieser Anonymität zeigt sich abermals das Nicht-Greifbarwerden des Mannes.

In Amys Entscheidung, das Kind zu behalten, wird ihr Wunsch nach Normalität, die sie mit der Idee, eine Familie zu haben verbindet, deutlich. In der folgenden Passage bestätigt sich diese familiale Orientierung:

war dann (1) mit einem zusammen (1) de::r (1) ja:: (2) also so: eigentlich immer nur abends kam, (1) da nie so die richtige Familienleben oder so gezeigt hat, (.) ich hab's halt nie irgendwie wahrgenommen, und die Zuneigung und die Liebe hab ich halt immer gemei::nt ahja schö: toll tralala ((holt Luft)) dann hab ich mich auch von ihm getrennt und hab dann rausbekommen dass er mich fast die vier Jahre lang wo wir miteinander (.) zusammen=waren, auch betrogen hat und belogen und (1) ja: (49-56)

Der Wunsch nach einer Familie, der in Zusammenhang steht mit dem Bedürfnis, Liebe und Geborgenheit zu erfahren, dokumentiert sich auch in Amys Handlungspraxis bezüglich der Aufnahme einer neuen Paarbeziehung. Die Konkretisierung erfolgt anhand eines positiven Horizontes mit den Stichworten des Ziels eines »richtigen Familienlebens« sowie der Suche nach »Liebe« und »Zuneigung«. Demgegenüber steht der negative Horizont eines Partners, der immer nur abends $\mathrm{zu}$ Hause ist und sich somit nicht am gemeinsamen Familienalltag beteiligt, wie es Amys Wunsch wäre.

Nach der Trennung bleibt bei Amy das Gefühlt zurück, »betrogen [...] und belogen« (55) worden zu sein. Sie beschließt, keine Beziehung mehr einzugehen, da sie keinen Mann mehr braucht. Dieses Muster wiederholt sich in Amys Beziehungsgeschichte immer wieder: Sie geht eine Beziehung ein, macht negative Erfahrungen, fühlt sich enttäuscht und getäuscht und zieht den Schluss, sich auf keinen Mann mehr einzulassen. Dann lernt sie jedoch wieder einen kennen, den sie als anders erlebt als die bisherigen, wo sie neue Hoffnungen entwickelt, und versucht es erneut.

\section{Neue Beziehung - neue Hoffnung}

dann ka:m mein letzter ${ }^{\circ}$ Freund $^{\circ}$ (1) ((holt Luft)) und bei dem war es, (1) am Anfang wow ich hab gedacht es gibt doch Männer die normal sind (1) (56-58)

Die Aufnahme dieser Beziehung ist erneut von dem Wunsch nach einem familiären Zusammenleben geprägt. Dies dokumentiert sich insbesondere in Amys Hand- 
lungspraxis, die trotz des Wissens um den Konsum kinderpornografischer Bilder ihres Freundes mit ihm zusammenzieht.

Aus heutiger Perspektive ist diese Entscheidung für Amy nicht mehr nachvollziehbar. Rückblickend erklärt sie, davon ausgegangen zu sein, dass er keine kinderpornografischen Bilder mehr konsumieren würde. Möglicherweise verstellte ihre Hoffnung auf ein Leben als Familie ihren Blick auf Roberts pädophile Neigung.

Physische Gewalt

geschlagen von nem Mann wurde ich einmal, aber jetzt nicht geschlagen geschlagen, ich hab ne Ohrfeige bekommen (2) wa:r, ich sag jetzt nicht dass es ge:rechtfertigt war, (2) nur ich in dem Moment hab des halt gesagt weil ich gesagt hab du lügst mir immer ins Cesicht warum lügst $d u$ mir bei diesem Thema nicht ins Cesicht, (106-110)

Mit dieser Sequenz beginnt Amy eine thematisch neue Erzählung, in der sie darauf verweist, von einem Mann einmal geschlagen worden zu sein. Ihre Aussage »nicht geschlagen geschlagen, ich hab ne Ohrfeige bekommen« bringt eine Differenzierung hinsichtlich der Art von Schlägen zum Ausdruck. Eine Ohrfeige sieht Amy zwar nicht als gerechtfertigt, dennoch wertet sie eine solche als weniger schlimm als andere körperliche Schläge.

ja:, irgendwie beim Streit wieder mal war des Thema und dann hab ich zu ihm gesagt mensch ich glaub dir des und dann (.) ((holt Luft)) so schnell (.) hab ich gar nich reagieren können als ich des gesagt hab war sie schon ge-hangen, (116-119)

Amy weiß von Robert, dass er als Kind vergewaltigt wurde, und beschreibt seine Kindheit insgesamt als »schlimme Kindheit« (113). In einer Streitsituation zwischen Amy und Robert kommt dieses Thema der Vergewaltigung auf und Amy bekommt eine Ohrfeige.

ich bin jetzt aber au nich eine wo dann sagt okay ja schlag mich ich schlag nich zurück oder so, (.) ähm (2) ja beim Robert ging's ja auch ruckzuck (.) als er mir eine Ohrfeige gegeben hat, (.) bin ich auch voll auf ihn los und hab gesagt ${ }^{\circ}$ wag es noch einnmal ${ }^{\circ}$ also sowas geht gar nich, ((atmet ein)) (727-730)

Nach dieser Ohrfeige wirft Amy ihren Freund aus der Wohnung und schließt diese ab. In diesem Beziehungsstreit dokumentiert sich Amys Verhalten erneut als reaktiv. Sie wartet lange und hofft auf eine Veränderung in der Beziehung. Allerdings ist mit der Ohrfeige eine Grenze bei ihr erreicht, die es ihr erlaubt, aus ihrer bisherigen Situation auszubrechen und diese zu beenden, indem sie Robert aus der Wohnung wirft.

Außerdem zeigt sich in dieser Situation, dass Amy versucht, in Konfliktsituationen deeskalierend zu wirken: 
ich bin halt immer schon (.) diejenige gewesen bevor ss halt (.) wirklich zum Schlag kommt oder so bin ich diejenige immer gewesen ((holt Luft)) die noch vorher raus k- kicken konnte, (722-725)

Gleichzeitig schreckt sie aber auch nicht davor zurück, sich notfalls körperlich zu wehren (»ich bin jetzt aber au nich eine wo dann sagt okay ja schlag mich ich schlag nich zurück oder so, « (727f.). Zudem zeigt sich, dass sie, bevor sie gegenüber anderen Personen körperlich aggressiv wird, zunächst ihre Aggression an Gegenständen auslässt, wie das folgende Zitat zeigt:

ich bin halt auch eine ich schlag gerne Gegenstände, (1) ((atmet ein)) also ich hab (.) bei meinem Ex sein (.) Kl- Kleiderschrank hab ich total (.) kurz und klein geschlagen, ((holt Luft)) ich hab drei SMS gefunden bei ihm im Handy, ((atmet ein)) und ne Telefonrechnung von achthundert Euro, (2) ja und des war alles auf einem Tag (769-773)

Insgesamt werden hier bezüglich des unterliegenden Orientierungsrahmens zwei Aspekte deutlich: Zum einen reagiert Amy auf körperliche Grenzverletzungen unmittelbar und konsequent. Damit wird ihre Grenze sichtbar, an der sie aus dem Zustand der Akzeptanz in Aktion tritt. Zum anderen zeigt sich in ihrer Haltung in Konfliktsituationen ein deeskalierendes Verhalten, indem sie ihre Wut und Aggression auf Gegenstände lenkt und zunächst nicht gegen Personen richtet.

Für ihre Reaktion, den Freund aus der Wohnung geworfen zu haben, schämt sich Amy vor ihrer Tochter:

die Kleine kam dann raus Mama des kannst du nich machen der kann doch nich draußen bleiben, und ja:=ich war total=ich wär am liebsten in an Loch, (1) wie kannst du nu:r u:nd (1) ((holt Luft) ja, (.) (120-123)

Hier dokumentiert sich Amys Verhalten im Umgang mit Konflikten. Es zeigt sich, dass sie über keine Problemlösestrategien verfügt; das Erlebte wird verschwiegen, nicht thematisiert. Hier zeigt sich auch eine Parallele zu Amys Aufwachsen: Sie erlebte häufig Gewaltsituationen, in die ihre Mutter verstrickt war, was auf die generationale Weitergabe von Erfahrungen und deren Umgang damit verweist.

\section{Sexualisierte Gewalt}

\section{Sexualisierung der Welt}

ja immer dieses (1) $d$ - $f$ - ah Sexual-Sexuis-Sexuistische des immer überall immer alles dreht sich nur um dieses Sex und ich (1) krieg da voll meine Aggressionen, also es gibt nix anderes auf dieser Welt wie nur Sex=Sex=Sex=Sex, ich kann's nich mehr (.) ich bin so (.) richtig bäh davon, weil das wirklich nix Tolles is,, (132-136) 
In dieser Passage thematisiert Amy ihre Erfahrungen mit Sexualisierungen, denen sie »überall« begegnet und die für sie ein allgegenwärtiges Thema darstellen. Ihre abschließende Aussage bewertet die Bedeutung des Themas, der sexualisierten Welt, für sie persönlich: »und für mich gibt's wichtigere Dinge, schönere Dinge, (1) aber sowas is (.) ganz schlimm, (1)« (137f.). Amys Bewertung der Allgegenwärtigkeit von Sex als »richtig bäh « bringt ihre negativen Erfahrungen in diesem Bereich deutlich zum Ausdruck. Der Verweis, es sei "ganz schlimm« (138), deutet die Intensität der negativen Erfahrungen an.

Der darauffolgende Abschnitt, in dem sie schildert, wie sie sich mit einer Freundin über dieses Thema unterhält und sie fragt »wann hat's denn bei dir angefangen« (140), zeigt, welches Gewicht bzw. welchen Raum dieses Thema im Leben Amys - und dem ihrer Freundin - hat. Aufgrund des vorausgegangenen Kontextes kann davon ausgegangen werden, dass Amy mit ihrer Frage auf den Beginn sexualisierter Gewalt abzielt. Der Satz beinhaltet kein Subjekt; damit wird auch kein Täter angesprochen, von dem die Gewalt ausgehen könnte. Weiter ist die Frage nicht terminiert, sondern spricht einen lang anhaltenden Zustand an, der die These von Gewalt als Normalzustand unterstreicht. In der Art und Weise, wie Amy hier fragt, wird deutlich, dass sexualisierte Gewalt einen Normalzustand in ihrem Leben darstellt.

Amy führt nicht näher aus, was sie unter »Sexual - Sexuis- Sexuistische« (133) versteht. Deutlich wird ihre Suche nach einem passenden Begriff für das, was sie meint. Auch wenn sie dies nicht weiter ausführt, wird doch klar, dass sie Sexualisierungen - welcher Art auch immer - als allgegenwärtig erlebt und dass Sex diesen nennt sie explizit - ebenfalls eine gewisse Relevanz in ihrem Leben bislang gespielt hat, wenn auch in negativer Hinsicht. Sexualität wird nicht als etwas Positives erlebt.

In Bezug auf den hier unterliegenden Orientierungsrahmen wird deutlich, dass es sich um einen Zugang zur Gestaltung von Beziehungen bzw. zum Eingehen von Beziehungen handelt, der von dem Bedürfnis und der Suche nach Liebe, Geborgenheit und Nähe getragen ist. In der Akzeptanz und dem In-Kauf-Nehmen der sexuellen Bedürfnisse ihrer Partner zeigt Amy ein angepasstes Verhalten. Für sie ist Sex auch in Partnerschaften negativ konnotiert, da sie diesen immer als negativ und unangenehm erfahren hat. Gleichzeitig kennt sie kein anderes Modell für eine Beziehung, was in ihren ersten negativen Erfahrungen mit Sexualität und deren Fortsetzung durch ihre gesamte Biografie begründet liegt.

Sexueller Missbrauch

Möglicher Missbrauch der Tochter Eines Tages entdeckt Amy Fotos von ihrer Tochter auf dem PC ihres Freundes Robert. Amys Reaktion auf ihre Entdeckung besteht im sofortigen Rauswurf von Robert aus der gemeinsamen Wohnung. Sie sucht sich 
anschließend eine eigene Wohnung, in die sie wenige Wochen vor dem Interviewtermin eingezogen ist. Sie evaluiert diese Passage abschließend mit den Worten »es fängt (2) bisschen langsam an bergauf zu laufen« (71f.).

ich hatte jetzt (.) vor (1) drei vier Tagen war ich mit der Kleinen essen und sie hat dann wieder sowas gesa:gt so (.) wo mich dann zum $\mathrm{Na}$ :chdenken bringt vielleicht war da doch was oder (1) will sie's mir nich sa-((stößt auf)) aber ich komm nich aus ihr raus=also ich will $\mathrm{j}$ - ich hab auch verboten bekommen sie jetzt damit zu konfrontieren von wegen hat da war da was des sel jenes (.) des kommt erst mit der Zeit, ich soll einfach sie reden lassen u:nd immer ihr di- des Gefühl geben (.) du kannst reden mit mi:r (.) und ${ }^{\circ} \mathrm{ja}:^{\circ}$ (.) Schuldgefühle mach ich mir, (1) dass ich des alles überhaupt hinkrieg ${ }^{\circ}$ und $^{\circ}$ (.) warum ich nich früher gegangen bin, (1) ((holt Luft)) (72-81)

Amys Erzählung ist durch Umschreibungen und Satzabbrüche geprägt. Es fällt ihr offensichtlich schwer, über ihre Ängste zu sprechen und das eigentliche Thema den möglichen sexuellen Missbrauch - zu benennen. Der Hinweis, sie habe »verboten bekommen sie jetzt damit zu konfrontieren«, deutet auf therapeutische Unterstützung hin. Amy äußert Schuldgefühle, weshalb sie ihren Partner nicht bereits früher verlassen hat, und hegt Zweifel, ob sie alles schaffen wird. Insgesamt dokumentiert sich eine verantwortungsvolle Haltung Amys gegenüber ihrer Tochter, die durch eine erwachsene Sicht auf diese geprägt ist. Erneut zeigt sich das Muster, welches immer nach einer Trennung einsetzt: Amy hat zu diesem Zeitpunkt genug von Männern (»von Männern bin ich jetzt total (2) also ganz schlecht also« (81f.)).

Eigene Missbrauchserfahrungen Amy erzählt, im Alter von vier Jahren in Kanada sexuell missbraucht worden zu sein. Unklar bleibt, ob sich Amy an den Missbrauch erinnern kann oder ob sie bspw. durch ihre Therapie mit Erinnerungen in Kontakt kommt.

ich bin als ahm vierjähriges Mädchen auch missbraucht worden im Kanada, (2) (141-142)

Hinweise auf Traumatisierungen zeigen sich in der auf das obige Zitat folgenden Passage durch erzählerische Lücken, die zu einer Inkonsistenz in der Erzählung führen. Zudem vermischen sich die Erzählstränge zu Amys eigenen Kindheitserfahrungen mit denen, in denen sie ihren Umgang mit dem Verdacht auf einen möglichen Missbrauch der Tochter thematisiert.

Der Hinweis, sie sei »auch« missbraucht worden, schafft die Parallelität $\mathrm{zu}$ Amys Gegenwart und zu dem Thema, das sie zum Zeitpunkt des Interviews am meisten beschäftigt: dem möglichen Missbrauch ihrer Tochter. Amy beschreibt das von ihr momentan gefühlte Durcheinander in ihrem Kopf herrsche: »Ich krieg des einfach nich hin zusammen (.) wie kann man nem kleinen Ding sowas antun ob- 
wohl man es selber angetan bekommen hat (144f.). Hier wird deutlich, dass Amy den Grund für Roberts Verhalten, Fotos von ihrer Tochter zu erstellen, zu finden versucht. Gerade vor dem Hintergrund, dass er selbst als Kind vergewaltigt wurde, scheint ihr sein Verhalten umso unbegreiflicher.

Die Fragen, die sie hierbei aufwirft, sind eingebettet in argumentativ-bewertende Stellungnahmen in Implizitform, die überzeitlich formuliert sind und sich an den Vorstellungsgehalten des gegenwärtigen theoretischen und bewertenden Orientierungssystems orientieren (vgl. Schütze 1987, S. 149). Damit erzielt Amy eine Distanz zu den vergangenen Ereignissen und zeigt außerdem Empathie sich selbst gegenüber.

Sie beschreibt weiter, wie schwierig es für sie ist, mit dem Vorfall zurechtzukommen. Immer wieder erlebt Amy mit ihrer Tochter Situationen, in denen es Hinweise gibt, die bei ihr die Frage aufwerfen, ob es zu einem sexuellen Missbrauch kam. Ihre Aussage, Robert selbst "sagt er hat niemals wirklich was gemacht« (154), deutet daraufhin, dass sie ihn zur Rede gestellt hat.

Amy springt in dieser Sequenz erzählerisch zwischen ihren Erinnerungen an den eigenen erlebten Missbrauch als Kind und der heutigen Situation des möglichen Missbrauchs ihrer Tochter hin und her. Die aktuellen Ereignisse wühlen sie umso mehr auf, da ihre eigenen Erinnerungen angetriggert werden. So ist es auf den ersten Blick meist nicht klar, über welches der beiden Ereignisse sie spricht. Durch ein konsequentes sequenzanalytisches Vorgehen (und zum Teil mithilfe von Kontextwissen aus anderen Passagen, z.B. die Vergewaltigung Roberts als Kind) gelingt es, die Erfahrungsaufschichtungen voneinander zu differenzieren.

Amys Aussage "weil ich weiß (nich) meine Mutter hat zu mir nur gesagt $\mathrm{i}$ - ich bin heim sie is heim gekommen ich hab's ihr sofort an den Kopf geknallt (1) ${ }^{\circ} \mathrm{des}$ da das und das und das war ${ }^{\circ}$ (155-157) bezieht sich auf die Situation, in der sie unmittelbar nach dem erlittenen Missbrauch ihrer Mutter davon erzählt. Durch die Verwendung der kausalen Konjunktion »weil« stellt sie eine Differenz zwischen ihrer Beziehung zu ihrer Tochter und der Beziehung zwischen ihr und ihrer Mutter her. In der Folge erläutert sie, dass sie selbst ihren Partner geliebt habe - im Gegensatz zu ihrer Mutter, die den Missbraucher nicht geliebt hat -, und spekuliert, ob sich ihre Tochter Mia ihr möglicherweise aus diesem Grund nicht in Bezug auf den Missbrauch anvertraut habe. Für Amy spielt diese Differenz eine wichtige Rolle, denn wenn sie mit ihrer Vermutung Recht hätte, würde das für sie bedeuten, eine größere Schuld auf sich geladen zu haben. Der Missbrauch ihrer Tochter wäre nach dieser Logik weit schlimmer als ihr eigener. Durch die erzählte und damit erinnerte Vermischung der beiden Fälle wird hier insgesamt deutlich, dass Amy ihre eigenen Missbrauchs-Traumatisierungen auf ihre Tochter projiziert. Zudem kann von einer Retraumatisierung Amys ausgegangen werden. 
Professionelle Hilfe und Unterstützung

Amy erfährt im Laufe ihrer Biografie immer wieder professionelle Hilfe und Unterstützung. Sie thematisiert diese Hilfeerfahrungen nicht direkt. Stattdessen fließen sie in Form von Hintergrundkonstruktionen in ihre Erzählung ein und bleiben eher angedeutet und vage. Dennoch wurden sie als Kernthemen identifiziert, da sie eine zentrale Funktion in Amys Leben einnehmen.

Jugendberufshilfe: Ausbildung und Mutterkurs Über Standpunkt, einen Träger der Jugendberufshilfe, beginnt Amy eine Ausbildung zur Schreinerin, die sie jedoch nach einiger Zeit wieder abbricht:

dann (1) ((holt Luft)) hab ich die Schreinerausbildung abgebrochen, durch Mobbing von meinem Chef, hab dann durch Standpunkt versucht noch zu probieren ob ich irgendwo anders reinkomm, (43-46)

Wie der Kontakt zwischen Amy und Standpunkt zustande kam, bleibt im Interview unerwähnt. Es kann aber davon ausgegangen werden, dass Amy durch die Begleitung von Standpunkt beim Übergang von Schule in Ausbildung neben der Vermittlung eines Ausbildungsplatzes auch ganzheitliche Unterstützung erfahren hat. Nach Abbruch ihrer Ausbildung versucht sie noch einen Wechsel zu einer anderen Ausbildungsstelle, was dann jedoch aufgrund ihrer Schwangerschaft nicht gelingt.

Nach der Geburt ihrer Tochter möchte Amy wieder arbeiten und wendet sich zunächst ans Arbeitsamt:

dann bin ich zum Arbeitsamt hab gesagt hier bin ich, will arbeiten (.) mir egal was und dann haben se mich wieder in den Eurojob reingesteckt und (.) dann wurd des alles so:: (.) nich schleichend aber so radikal diese Treppe auf jede Stufe immer mehr ((holt Luft)) (924-927)

Hier wird deutlich, wie sich Amys Situation in dieser Zeit verschärft. Mit der Metapher der Treppe versucht sie zu beschreiben, wie sie ihre Situation erlebte, die von einer enormen Zunahme an Belastungen gekennzeichnet war. In dieser Zeit kommt Anke, eine Mitarbeiterin von Standpunkt, auf Amy zu und bietet ihr Unterstützung an:

dann kam die Anke und hat gemeint ja: (1) wie sieht's aus Mutterkurs und so, und ja klar hier bin ich, (1) ja:,//okay//(2) und so, ich find's halt immer schön auch zu wissen dass jemand da is egal wenn was is,//mmh//ich kann immer hingehen oder (.) nachfragen, so wie jetzt ne (.) Ersatzmama sag ich einfach und Proble:m, und sie sagt auch einmal hallo $>$ s reicht jetzt oder so: und ja://mmh//(2) ${ }^{\circ} \mathrm{doch}^{\circ}$ (1) (927-932) 
Im »Mutterkurs «" erfährt Amy Hilfe und Unterstützung in ihrer Situation als alleinerziehende Mutter. Zwei Aspekte werden hier von Amy hervorgehoben: Zum einen wird die Tatsache als positiv erlebt, dass es eine Ansprechpartnerin gibt, die für alle Sorgen, Nöte, Fragen und Themen da ist. Zum anderen vermittelt die Sozialarbeiterin aber auch das Gefühl, für Amy da zu sein. Die Formulierung »dass jemand da is egal wenn was is « bringt die emotionale Komponente des Beziehungsverhältnisses zum Ausdruck. Über die Aufnahme in den Mutterkurs gelingt es Amy schließlich, ihre Ausbildung wiederaufzunehmen, die sie in wenigen Monaten abschließen wird.

\section{Caritas}

ham wir am zwölften ham wir einen Termin mit ihm, (1) u:nd mit der Caritas, da warn wir jetzt schon öfters weil er mich ja auch angeklagt hat wegen Sorgerecht und wegen Umgangsrecht, ((holt Luft)) u:nd ${ }^{\circ} \mathrm{ja}$, der kann ruhig machen, $\% / \mathrm{mmh} / /$ jetzt hab ich den Termin veranlasst um ihm mal ein bisschen (.) nich die Augen zu öffnen weil die kann man nich mehr bei ihm öffnen=des geht einfach nich mehr=is schon zu lang her=wo ich sag (.) die Hoffnung is schon längst gestorben, nur einfach dass es Caritas mitkriegt dass ich irgendwas auf der Hand ha:be wenn's wirklich soweit kommt (.) (und) wir müssen wieder vor Gericht,//mmh//wo ich sagen kann ja: und jetzt reicht's mir, (1) (259-267)

Da der Vater von Amys Tochter Mia Amy wegen des Sorge- und Umgangsrechts »angeklagt« hat, hat Amy einen Termin bei der Caritas vereinbart, um sich rechtzeitig abzusichern, sollte es zu einem Gerichtsverfahren kommen. Hier dokumentiert sich Amys aktives Handeln, indem sie vorausschauend plant und sich rechtzeitig (fachliche) Hilfe holt. Auch hier zeigt sich, wie sie entschlossen versucht, ihr Leben selbstbestimmt zu gestalten. Sie kämpft hier für die gemeinsame Zukunft mit ihrer Tochter.

Therapie Zum Zeitpunkt des Interviewserhält Amy Unterstützung durch eine Therapeutin. Wie das folgende Zitat zeigt, ist sie durch die jüngsten Erfahrungen an einen Punkt gelangt, der es ihr unmöglich macht, weiterhin allein zurechtzukommen:

9 Über den Jugendberufshilfeträger Standpunkt besucht Amy nach der Ceburt ihrer Tochter einen Vorbereitungskurs für Teilzeitausbildung. In diesem Kurs erhält sie Unterstützung bei der Suche nach geeigneten Ausbildungsplätzen und wird durch spezielle Qualifizierungsangebote auf die Ausbildung vorbereitet. Außerdem erhält sie Hilfe bei der Organisation der Kinderbetreuung und bei allen Fragen, die Familie und Ausbildung betreffen. Diese Informationen habe ich von der pädagogischen Fachkraft Anke erhalten, die den Kontakt zwischen Amy und mir hergestellt hat. 
ja: is schwierig (2) sehr schwierig, (1) ich versteh's einfach nich ich kann das, ich (.) versuch den Sinn irgendwie aber da gibt's kein Sinn (1) mein ich bin in Therapie auch seitdem//mmh//und lass mir da helfen weil ich selbst komm damit einfach nich klar//mmh//, sowas is No-Go des geht gar nich (86-89)

Durch die Therapie kommt ein Reflexionsprozess in Gang, der Amy hilft, einen Teil ihrer Biografie zu verstehen - und davon ausgehend vielleicht auch zu lernen, mit den gemachten Erfahrungen zu leben.

\subsubsection{Argumentative Stellungnahmen}

Zum Abschluss ihrer biografischen Stegreiferzählung setzt Amy zu einem längeren Fazit an: Sie resümiert die Lebensphase ab dem Zeitpunkt, an dem sie sich mit Jungen eingelassen hat, als eine Zeit, in der sie durchweg »betrogen und belogen « (283) wurde. Die Zusammenfassung »das jo: war's eigentlich mit den Männern« (285) macht deutlich, dass sie ihrem Resümee nichts hinzuzufügen hat. Gleichzeitig wird nochmals verdeutlicht, dass sie sich auf die gesamte Spanne von Beziehungsphasen bezieht: von Beginn an mit Jungen bis hin zu Beziehungen mit Männern im Erwachsenenalter. Ihre negativen Erfahrungen ziehen sich dabei durch die gesamten Lebensphasen hindurch. Ihre Enttäuschung kommt in den Aussagen »reicht auch $(285)$, » ${ }^{\circ}$ is echt genug ${ }^{\circ}$ « (286) und »die Hoffnung is @@gestor@ben « (286f.) zum Ausdruck. Dennoch bleiben ein letzter Rest Hoffnung, dass irgendwann in Zukunft eine Beziehung wieder möglich sein kann (»vielleicht irgendwann« (287)), und auch der Wunsch nach einer - schönen - Beziehung: »ich mein klar ich hätt's gern anderst « (291f.).

In der folgenden theoretischen Aktivität zieht Amy Parallelen zwischen dem Leben ihrer Mutter und ihrem eigenen Leben. Sie bezeichnet die Zusammenhänge als eine Art »Lebenskreis« (294), in dem sich Erfahrungen wiederholen. In Abgrenzung zu den eigenen negativen Erfahrungen und denen ihrer Mutter sieht sie ihre Tochter, für die sie keinesfalls eine Wiederholung der Erfahrungen möchte. Möglicherweise liegt darin auch der Motor für Amys Bestrebungen, ein besseres Leben für sich und vor allem für ihre Tochter zu erreichen.

Rückblickend stellt sie fest, wie schwierig es war, sich durchzukämpfen. Ihr Fazit »aber jetzt hab ich mich durchgeboxt « (306) zeigt, dass sie ihre jetzige Lebensphase als Meilenstein wertet und zuversichtlich der Zukunft entgegenblickt.

\subsubsection{Zusammenfassung}

Erleben von Gewalt in Paarbeziehungen Amy berichtet zwar in einzelnen Passagen des Interviews von konkreten Situationen, in denen sie bspw. Gewalt in Form von Bedrohungen oder einer Ohrfeige erhalten hat. Viele Erfahrungen, insbesondere sexualisierte Gewalterfahrungen, bleiben jedoch in ihren Erzählungen implizit. Wie die Rekonstruktion der Einzelfallanalyse zeigt, ist es für Amy gar nicht erforder- 
lich, Gewalterlebnisse als solche zu beschreiben, da das Erleben von Gewalt für sie Normalität bedeutet. Dies hat sie von Geburt an so erlebt.

Umgang mit erlebter Gewalt, Bewältigungsstrategien und Ressourcen In der Rekonstruktion zeigt sich, dass Amy ihre Wut sowie ihre Aggressionen, die sie gegen ihren Partner verspürt, gegenüber Gegenständen ausagiert; so tritt sie bspw. einen Kleiderschrank kaputt und zerstört auch mehrere Laptops ihres Freundes. Auf verbaler Ebene tritt sie ihren Partnern mit der klaren Botschaft gegenüber, sich gegebenenfalls auch körperlich zu wehren.

Hinsichtlich der Mobilisation von Kräften in existenziell bedrohlichen Situationen zeigt Amy ein enormes Potenzial, dem eine konkrete Zukunftsorientierung zugrunde liegt. Dabei ist es ihr jedoch nicht möglich, frühzeitig und perspektivisch ihr Leben zu planen und entsprechende Entscheidungen zu fällen.

Beziehungsmuster und Beziehungsgestaltung Amy hat innerhalb ihres Orientierungsschemas explizite Vorstellungen, was das Zusammenleben als Paar und Familie anbelangt. Da Amy mit 20 Jahren eine Tochter bekommt, lassen sich ihre Beziehungsvorstellungen nicht von ihren Familienvorstellungen trennen, sondern sind miteinander verwoben. Damit Familie und Beziehung gelingen kann, bedarf es eines Partners, bei dem sie sich geliebt, wohl und geborgen fühlt. Ihre Vorstellungen versucht sie mit verschiedenen Partnern zu verwirklichen, sie wird jedoch jedes Mal enttäuscht.

Amys Bedürfnis nach Liebe und Geborgenheit zeigt sich auf konjunktiver Ebene im Modus Operandi, als permanente Suche nach Zugehörigkeit über Beziehung, und prägt damit wesentlich ihren Orientierungsrahmen. Dabei hat sie keine konkrete Vorstellung, was eine erwachsene Paarbeziehung ausmacht, bzw. sie kennt kein Modell, das ihr Orientierung bietet. Insgesamt werden ihre Beziehungserfahrungen, bis zum Zeitpunkt des Therapiebeginns, nicht reflektiert. Sie hat keine Idee, dass sie eine Beziehung erst mal langsam angehen lassen könnte, abwartend, austestend. Stattdessen geht sie eine Beziehung ein, von der sie nach kurzer Zeit enttäuscht wird, um sofort die nächste zu beginnen, von der sie hofft, dass diese besser wird. Diese Austauschbarkeit der Männer spiegelt sich auch in Amys Erzählung wider, in der diese nicht namentlich erwähnt, sondern immer wieder pauschal genannt werden.

Durch ihre Aktivität (Beziehungen anfangen und beenden, Männer aus der Wohnung werfen) bleibt sie handlungsfähig und nicht ohnmächtig wie früher. Diese Aktivität suggeriert Macht und Kontrolle über die Männer, die Beziehungen, die Situation und verhindert, (zurück) in die passive Rolle des Opfers gedrängt zu werden. Zudem handelt es sich um eine Beziehungsgestaltung, die das vertraute Drama immer wieder reproduziert. In diesem vertrauten Erleben kennt sich Amy aus und weiß, was zu tun ist. Dies vermittelt ihr Gefühle von Sicherheit und Kontrolle. 
Dabei zieht sich durch Amys Biografie hindurch ein Muster: Am Ende einer Beziehung stellt Amy immer wieder fest, dass sie keinen Mann mehr will bzw. braucht. Doch dann lernt sie wieder jemanden kennen und hofft, mit diesem ihre Beziehungswünsche realisieren zu können. Diese Suche nach Zugehörigkeit innerhalb von Beziehungen ist die zentrale Orientierung, die die Fallstruktur Amys prägt.

Gewalt(-Beziehungen) und Sozialisation In der Rekonstruktion des Interviews mit Amy zeigt sich, dass sich ihr Gewalterleben durch die gesamte Biografie hindurchzieht. So erfährt sie bereits als Vierjährige sexuellen Missbrauch sowie Gewalt auf der Eltern-Kind-Ebene, indem Amy von beiden Eltern Schläge bekommt.

Hinsichtlich ihrer familiären Sozialisation ist Amys Aufwachsen geprägt durch ein chaotisches Umfeld und die Alkoholsucht ihrer alleinerziehenden Mutter. Der Vater ist nach der Rückkehr Amys nach Deutschland nicht mehr präsent. Die Suchterkrankung der Mutter wirkt sich dominant auf das Aufwachsen aus, indem Amy eine frühe Parentifizierung erlebt. Zudem erlebt sie häufig wechselnde Männerbekanntschaften oder Beziehungen der Mutter. Bezüglich Amys ersten Beziehungserfahrungen zeigt sich eine frühe Kontaktaufnahme im Alter von 12 bzw. 13 Jahren zu deutlich älteren Männern. Für sie liegt diesen Kontakten die Suche nach Nähe und Geborgenheit sowie nach Anerkennung und Wertschätzung zugrunde, die in der biografischen Erzählung mit dem Fehlen der Vaterfigur begründet wird. In der Vielzahl der folgenden Männerbekanntschaften fällt die Parallele zum Beziehungsverhalten der Mutter auf.

\subsubsection{Zofia - »irgendwann ging mir das: [...] soweit dass ich dann wirklich auch für mich entschieden hab [...] und jetzt wirklich geh« (114-116)}

\subsubsection{Fallporträt}

Zofia wächst mit ihrer Schwester und ihren Eltern in einer Großstadt in der Tschechoslowakei auf. Die Mutter des Vaters lebt ebenfalls bei der Familie. Die Kinder werden tagsüber in einer Kinderkrippe betreut, da beide Eltern in Vollzeit erwerbstätig sind. Die Wochenenden verbringt die Familie regelmäßig bei den Großeltern auf dem Land, sodass Zofia viel Zeit mit Spielen im Freien bzw. im Wald verbringt. Sie erinnert sich vor allem an den großen Garten, in dem verschiedene Gemüsesorten und Früchte wachsen. Zofia bezeichnet ihre Kindheit als eine sehr schöne Zeit. Sie fühlt sich in ihrer Heimatstadt verwurzelt und schätzt die Abwechslung zwischen Stadt- und Landleben. Belastend, vor allem für die Mutter, ist die Alkoholsucht des Vaters, der »sehr früh« (22) zu trinken beginnt. Zofia erinnert sich, dass ihre Mutter ihren Vater immer wieder in Kneipen suchen musste, und erlebte ihre Mutter häufig in großer Sorge um ihn. Zudem beschreibt sie ihre Mutter als co-abhängig und finanziell abhängig von deren Mann. Aufgrund der Religio- 
sität der Großmutter geht die Familie sonntags regelmäßig in die Kirche, und die Kinder werden dazu angehalten, regelmäßig den Rosenkranz zu beten und zur Beichte $z u$ gehen. Für Zofia bedeuten diese Rituale ein Muss, das lediglich eine lästige Pflichterfüllung darstellt.

Als Zofia neun Jahre alt ist, beschließen ihre Eltern nach Deutschland zu emigrieren, um ihren Kindern eine bessere Zukunft zu ermöglichen. Zofia und ihrer Schwester gegenüber erzählen die Eltern jedoch zunächst, sie würden einen zweiwöchigen Urlaub in Deutschland machen, sodass Zofia erst in Deutschland damit konfrontiert wird, dass die gemachte Reise ohne Rückreise bleiben wird.

Die Familie lebt zunächst in Übergangsheimen, wodurch Zofia viel Kontakt zu anderen ausländischen Kindern hat. Sie zieht anfänglich zweimal innerhalb von Deutschland um, was für Zofia bedeutet, sich immer wieder auf ein neues soziales Umfeld einzulassen. Als die Familie schließlich in einer Stadt bleibt, findet Zofia schnell Zugang zu Freund_innen. Sie lernt zügig Deutsch, ist motiviert in der Schule und hat in allen Fächern sehr gute Noten. In der Schule macht sie verschiedene Diskriminierungserfahrungen; so lassen ihre Mitschüler_innen sie bspw. nicht am Vergleich der Hausaufgaben teilhaben. Auch die Klassenlehrerin stellt sie regelmäßig vor der Klasse bloß und gibt ihr schließlich, trotz ihrer guten Noten, lediglich die Empfehlung für die Realschule. Auch dort ist Zofia zunächst eine sehr gute Schülerin. Schulisch wie sportlich ist sie außerordentlich ehrgeizig. Sie tritt in einen Sportverein ein, intensiviert das Leistungsturnen und verbringt viel Zeit im Training und auf Wettkämpfen. Mit Beginn der Pubertät kommt es zunehmend zu Konflikten zu Hause. Zofia hat häufig Wutausbrüche und fühlt sich hin- und hergerissen zwischen dem strengen Erziehungsstil des Vaters und der eher liberal erziehenden Mutter, die ihr viel Freiheit lässt. Mit 13 Jahren beginnt sie, eine Magersucht und eine Bulimie $\mathrm{zu}$ entwickeln. Als sie 15 Jahre alt ist, verlässt sie den Sportverein. Sie sehnt sich nach einem normalen Jugendalter und möchte vor allem »einfach nur (1) JUNGS kennenlernen« (79).

Mit 17 Jahren hat sie ihren ersten Freund, in den sie zwar nicht verliebt ist, mit dem sie aber ausprobieren möchte, wie es sich anfühlt, einen Freund zu haben. Ihr Wunsch nach einer Beziehung wird durch die Tatsache verstärkt, dass bereits viele Freundinnen einen Freund haben oder hatten. Mit 19 Jahren kommt Zofia aufgrund ihrer Essstörung mehrere Monate stationär in eine Klinik. Dort verliebt sie sich zum ersten Mal richtig und beginnt eine Beziehung mit einem Mann, wegen dem sie ihren Klinikaufenthalt auch abbricht. Nach einem knappen Jahr scheitert die Beziehung, was für Zofia eine große Enttäuschung darstellt. Sie fühlt sich alleingelassen, erfährt aber Rückhalt bei ihren Eltern, wo sie sich immer wieder in ähnlichen Situationen zurückzieht. Neben ihrer Essstörung beginnt Zofia, verschiedene Drogen zu konsumieren und landet schließlich nach einem unkontrollierten Exzess im Krankenhaus. 
Es folgen zwei weitere Beziehungen, in denen Zofia mühsam darum kämpft, eine ausgeglichene und gleichberechtigte Beziehung zu führen.

Während des Freiwilligen Sozialen Jahres (FSJ), das sie nach der Schule beginnt, lernt sie einen Mann kennen, in den sie sich zunächst verliebt. Sie beginnen eine Beziehung, wobei es bald zu Abhängigkeitsmechanismen und Machtkämpfen kommt. Zofia entscheidet sich für eine ambulante Psychotherapie, um ihre in dieser Zeit immer stärker werdende Bulimie in den Griff zu bekommen. Die Beziehung mit ihrem Freund belastet sie stark. Er bedroht sie, ist ihr gegenüber körperlich gewalttätig und betrügt sie mit anderen Frauen. Zofia erfindet Geschichten, um ihre Blessuren und blauen Flecken gegenüber ihren Freund_innen und ihrer Familie zu erklären und ihren Freund zu schützen. Sie entwickelt in dieser Zeit eine Alkoholsucht, wobei die Intervalle, in denen sie trinkt, immer kürzer werden.

Zofia absolviert eine Ausbildung zur Erzieherin. Als sie wider Erwarten einen Studienplatz an einer Dualen Hochschule erhält, sieht sie ihre Chance, sich, auch finanziell, von einem Mann unabhängig zu machen. Sie nimmt den Platz an und trennt sich von ihrem Freund. Nach dem Umzug und dem Beginn des Studiums hat Zofia noch einmal eine neue Beziehung, die neun Monate dauert. Auch in dieser Beziehung macht sie sich viele Vorwürfe und sieht die Verantwortung bei sich, wenn es innerhalb der Beziehung nicht gut läuft. Nach der Trennung zieht sie in eine WG, wo sie versucht, sich selbst als wertvoll zu erfahren - unabhängig von einem Mann und der Bestätigung durch ihn.

\subsubsection{Analyse der Erzählstruktur}

Zofia strukturiert die Erzählung ihrer Lebensgeschichte zunächst chronologisch, indem sie die Erzählsegmente durch zeitliche Grenzziehung in biografische Abschnitte unterteilt. Die Themen, die sie hierbei anspricht, lassen sich wie folgt bündeln: Kindheit in der Tschechoslowakei, Emigration, Schulzeit in Deutschland. Ab dem biografischen Zeitpunkt der Pubertät strukturiert sie ihre Erzählung anhand thematischer Zusammenhänge: Anderssein, Abfolge der Paarbeziehungen, Studium. Hier wird der unmittelbare Bezug auf die wissenschaftliche Fragestellung offensichtlich. Nach Beendigung der biografischen Stegreiferzählung fällt die Dominanz argumentativer Stellungnahmen auf, bei denen die gegenwärtige, teilweise aber auch damalige bewertende Haltung der Erzählerin (Schütze 1987, S. 138) gegenüber dem erzählten Leben zum Ausdruck kommt. Damit wird deutlich, dass Zofia ihre Biografie intensiv reflektiert. Deutungsmuster werden aus verschiedenen weltanschaulichen und kulturellen Orientierungen sowie wissenschaftlichen, alltagsweltlichen und politischen Wissensbeständen entnommen (vgl. Lüders/Meuser 1997 und Meuser/Sackmann 1991 in Lucius-Hoene/Deppermann 2002, S. 130). Zofias Erzählung ist durchzogen von Reflexionen über ihr Leben, was vermutlich einerseits durch die Auseinandersetzung mit ihrer Biogra- 
fie innerhalb therapeutischer Settings und andererseits durch die Beschäftigung mit psychologischen und soziologischen Theorien innerhalb ihres sozialwissenschaftlichen Studiums verstärkt wurde. So zeigen sich an verschiedenen Stellen in der lebensgeschichtlichen Erzählung Niederschläge solcher psychologischer und soziologischer Theorien wie bspw. das Rekurrieren auf das Individualisierungstheorem, nach dem Frauen heute selbstverständlich arbeiten und Kinder erziehen können (626ff.), sowie die Übernahme von Deutungsmustern aus der Psychotherapieerfahrung wie bspw. Zofias Reflexionen über ihr Suchtpotenzial, das sich zunächst in einer Bulimie und später im Missbrauch von Alkohol zeigt und welches sie als Bewältigungsstrategie (695) interpretiert.

\subsubsection{Herkunftsfamilie und Bedingungen des Aufwachsens}

Zofia beginnt ihre Lebensgeschichte mit der Benennung ihres Geburtslandes Tschechoslowakei und dem Jahr ihrer Geburt. Es folgt sogleich eine Beschreibung ihrer Kindheit, die "ganz nett« bzw. "ne ganze tolle Kindheit« war. Auch die Wochenenden, die die in der Großstadt lebende Familie auf dem Land bei dem Großvater verbringt, sind »immer sehr schön super«. Es dokumentiert sich hier, dass Zofia den Beginn ihrer Lebensgeschichte zunächst räumlich verortet.

\section{Eingangsnarration}

Okay (1) gut (1) ((holt Luft)) also: Aufgewachsen bin i:ch oder geboren bin ich in der Tschechoslowakei, ((räuspert sich)) 1983 ((holt tief Luft)) a:::::hm die Kindheit verlau-verlief eigentlich ganz nett ich hatte: ne ganze tolle Kindheit soweit ich mich erinnern kann ahm wir hatten ((schluckt)) in ner Croßstadt gelebt waren jedes Wochenende aufm Land bei meinem Opa also s war immer sehr schön super (1) ahm (2) ja:::, (2) (16-21)

In dieser ersten Eingangssequenz wirken Zofias Bewertungen etwas überbetont. Zum einen nehmen sie in Bezug auf die anderen Aussagen relativ viel Raum ein. Zum anderen scheint die Aussage »die Kindheit verlau- verlief eigentlich ganz nett» zu den folgenden Äußerungen »ich hatte: ne ganz tolle Kindheit « und »s war immer sehr schön super « nicht zu passen. Verstärkt wird der Eindruck, dass hier mühsam ein positives Bild der Kindheit hergestellt wird, indem erstens die Aussage "ganz nett« durch die Fortführung "ganz toll« gesteigert wird, zweitens durch die Anschlussäußerung »soweit ich mich erinnern kann« eine Einschränkung des Gesagten vorgenommen wird und drittens die Intention der Aussage "s war immer sehr schön« durch die Ergänzung »super« nochmals verstärkt werden soll. Durch diese Überbetonung wird deutlich, dass Zofia versucht, ein positives Bild der Kindheit zu evozieren. Dieser Konstruktionsprozess erscheint mühsam, Aussagen werden revidiert bzw. ergänzend verbessert. 
Vor dem Hintergrund der folgenden Sequenz werden die Ambivalenzen in der Darstellung plausibel: Zofia versucht zunächst, ein positives Bild ihrer Kindheit zu zeichnen. Mit der Erwähnung ihres Vaters als Alkoholiker wird dieses positive Bild jedoch relativiert und das Bemühen bzw. Ringen um eine positive Beschreibung der Kindheit wird nachvollziehbarer - wenn auch noch nicht erklärbar ist, weshalb Zofia hier so agiert.

Durch die Präambel »an was [...] ich mich erinnern kann« (21f.) kündigt Zofia die Bedeutung des nun zu Erzählenden an und berichtet von ihrem Vater, der »früh« (22) Alkoholiker wurde. Diesen Zustand erinnert sie als belastende Situation für ihre Mutter. Sowohl die Großmutter als auch die Mutter konfrontieren den Vater mit seiner Sucht, was dieser wiederum »natürlich« (24) ablehnt. Zofia vermutet eine Co-Abhängigkeit seitens ihrer Mutter, die sie sich auch durch deren finanzielle Abhängigkeit vom Vater erklärt.

In diesem Abschnitt wird die räumliche Verortung der vorausgehenden Sequenz nicht fortgeführt. Stattdessen thematisiert Zofia die Alkoholsucht ihres Vaters. Dabei kann davon ausgegangen werden, dass Zofias Kindheit wesentlich durch das Trinken des Vaters beeinflusst wurde.

Einen einschneidenden Bruch in Zofias Biografie stellt der Entschluss ihrer Eltern dar, nach Deutschland zu emigrieren. In einer Hintergrundkonstruktion verweist sie darauf, dass beide zum Zeitpunkt der Entscheidung noch sehr jung waren. Konkretisiert wird das Alter der Mutter, die damals »Ende zwanzig Anfang dreißig« (30f.) war. Zofia und ihre Schwester werden über diesen Entschluss der Eltern nicht informiert. Stattdessen wird ihnen mitgeteilt, dass die Familie zwei Wochen Urlaub in Deutschland machen wird. Die Tatsache, dass die Kinder nicht einbezogen bzw. nicht informiert werden, verstärkt den biografischen Bruch. Eine Vorbereitung auf die bevorstehende Veränderung, eine Auseinandersetzung damit sowie ein Verabschieden von Freund_innen usw. werden unmöglich gemacht.

Zofia beschreibt den Übergang mit den Worten »ja:: des war dann irgendwie (.) aber ich hab's ziemlich schnell vergessen« (38f.). Darin dokumentiert sich Zofias Fähigkeit, sich schnell neuen Situationen anzupassen. Auch dass Zofia zügig Deutsch lernt und in der vierten Klasse in eine reguläre Grundschulklasse integriert wird, belegt ihre Anpassungsfähigkeit. Gleichzeitig taucht ein neues Thema in der Passage auf:

\section{Normalität}

da:nn ahm hab ich auch relativ schnell deutsch gele:::- gelernt=ich war ziemlich offen sehr offenes lebendiges Kind, hamn auch die Deutschlehrer gesagt ich wurde dann in die zweite in der dritten oder in der vierten Klasse dann integriert in ne Gy-ganz normale Crundschulklasse, (44-48) 
Mit dem Verweis auf die »normale« Klasse betont Zofia ihre Erfahrung des Andersseins oder Ausgegrenzt-Seins, da sie sich oft als nicht dazugehörig empfindet. Sie führt diese Erfahrungen jedoch zunächst nicht weiter aus, sondern erzählt stattdessen, ein »offenes lebendiges« Kind gewesen zu sein, was auch von den Lehrern bestätigt wurde.

Hier dokumentiert sich Zofias Bestreben, sich mit hoher Bereitschaft so gut wie möglich in ihre neue Welt einzufügen bzw. sich diese anzueignen. Die Konkretisierung der Handlung erfolgt dabei anhand eines positiven Horizonts, der mit der Beschreibung ihres Naturells, ein offenes und lebendiges Kind zu sein, sowie der Tatsache, dass sie schnell die deutsche Sprache lernt, ausgeführt wird. Dem wird ein negativer Horizont gegenübergestellt, der auf den Diskriminierungserfahrungen in der Grundschule durch die Mitschüler_innen gründet. Das Enaktierungspotenzial besteht demnach auf einer intrinsischen Motivation, sich an die neue Situation anzupassen und sich dafür zu engagieren, was allerdings durch äußere Bedingungen, wie das ausgrenzende Verhalten der Mitschüler_innen, erschwert wird. Insgesamt wird deutlich, dass Zofia eine hohe Anpassungsbereitschaft zeigt, zielstrebig das >Dazuzugehören $<$ zu verwirklichen.

Die Betonung Zofias, nie »gehänselt oder gemobbt« (53f.) worden zu sein, steht im Widerspruch zu den genannten Diskriminierungserfahrungen. Vielleicht unterscheidet Zofia aber auch zwischen Mobbing und Diskriminierung: Sie fühlt sich diskriminiert, was sie jedoch als nicht so schlimm definiert wie Mobbing. Damit würde sich dieselbe idealisierende Darstellungsart, wie Zofia sie auch in der Schilderung über ihr Aufwachsen präsentiert, zeigen.

Zofia zieht mit ihrer Familie in der darauffolgenden Zeit einige Mal um, wodurch sich ihr soziales Umfeld immer wieder ändert. Dadurch potenzieren sich auch die Anforderungen an sie, sich immer wieder zu integrieren. Die Aussage »ich musst dann schon immer so $n$ bisschen mein soziales Umfeld auch ah loslassen« (56f.) belegt Zofias Umgang mit den damit verbundenen Herausforderungen. Sie lässt es nicht zu, dass diese Herausforderungen zu groß werden, und versucht, sie damit für sie bewältigbar erscheinen zu lassen.

Sowohl in der neuen Schule als auch im Verein, in den sie in Stadt 1 eintritt, findet Zofia »sehr sch:nell« (58) Zugang zu Mitschüler_innen sowie anderen Kindern und Jugendlichen. Hier scheint sie anzukommen, und es dokumentiert sich wieder ihre Haltung als aktiv Handelnde, die sich einbringen will und dies auch tut.

Zofia schließt diese Sequenz mit der Konklusion ab: »und es war alles eigentlich alles okay« (60). Dieser Abschluss in Form einer positiven Bewertung verweist auf ein gutes Ende der bis dahin geschilderten biografischen Etappe und bringt das Gefühl zum Ausdruck, angekommen zu sein.

Insgesamt zeigt sich, dass Zofias Kindheit von vielen Brüchen, wie der Emigration nach Deutschland oder durch mehrere Umzüge bedingte Wohnortwech- 
sel, geprägt ist. Dadurch muss Zofia ihre gerade neu gewonnenen Sozialkontakte ständig wieder aufgeben. Neben diesen Verlusterfahrungen macht sie zusätzlich Diskriminierungserfahrungen in der Schule. Dennoch dokumentieren sich in den Erzählungen über diese Zeit ein starker Wille sowie der Ehrgeiz, die Situationen zu meistern bzw. schaffen zu wollen. Zofia zeigt hier ein hohes Maß an Energie, sich in neue Situationen einzufinden und sich diesen anzupassen.

\subsubsection{Kernthemen des Interviews}

Anderssein

Anderssein

aber irgendwann hat's angefangen ((holt Luft)) dass ich mich an bisschen auch ahm (1) ab- setzen wollte ich wollt mich ahm (1) ich wollt (.) anders sein als die anderen ganz einfach gesagt, ich wollt anders sein und des hat so mit dreizehn angefangen (60-63)

Mit dem Gliederungsmarkierer »aber irgendwann hat's angefangen« leitet Zofia sprachlich eine neue Erzähleinheit ein. Auch wenn der Begriff »irgendwann« relativ unbestimmt ist, kündigt sich damit eine neue zeitliche Situierung an. Sie konkretisiert im darauffolgenden Satz, was genau begonnen hat: Zofia möchte sich von anderen Mitschüler_innen und Freund_innen »ab- setzen« und präzisiert nochmals: Sie möchte »anders sein als die anderen«. Sie datiert den Beginn dieser Entwicklung auf ihr 14. Lebensjahr, in dem sie beginnt, sich zurückzuziehen. Zu diesem Zeitpunkt setzt auch die Entwicklung einer Essstörung ein.

In einer argumentativen Passage versucht sie, ihr Verhalten hinsichtlich der Essstörung zu erklären. Durch die starke Fixierung auf das Äußere des Körpers möchte sie nach außen ihre Stärke signalisieren. Sie bringt es abschließend nochmals auf den Punkt: Die Abgrenzung erfolgt durch das Erbringen sportlicher Leistung trotz des körperlichen Dünnseins.

Der Beginn der Entwicklung einer Essstörung stellt einen biografischen Einschnitt, eine Art Wendepunkt, dar. Der Wunsch, sich von anderen abzugrenzen und anders sein zu wollen als diese, ist zentral in Zofias Lebensgeschichte. Dies versucht sie, mit 13 Jahren über die Entwicklung einer Essstörung zu sverwirklichen<. Der positive Horizont liegt darin, anderen die eigene Stärke, trotz Dünnsein sportliche Leistung bringen zu können, zu demonstrieren. Der Gegenhorizont begründet sich auf der anderen Seite darin, dass sich Zofia auch als »total zu schwach" erlebt. Dies kann sie jedoch sich und anderen gegenüber nicht eingestehen.

An die initiale Äußerung, mit der zugleich das Thema der Textsequenz festgehalten werden kann (»ich mich an bisschen auch ahm (1) ab- setzen wollte«), schließen sich fortführend Äußerungen in Form einer Validierung an, die die erste Äußerung bestätigt und konkretisiert (»ich wollt (.) anders sein als die anderen«); 
darauf folgt eine weitere ratifizierende Äußerung (»ich wollt anders sein«), die das zuvor Gesagte bestätigt.

In der Entwicklung der Essstörung bzw. im Dünnwerden dokumentiert sich ein Widerstand, indem der Körper als eigenes Revier markiert wird, über das selbst bestimmt wird. Dadurch erfährt Zofia Stärke und Macht. ${ }^{10}$

Die hier skizzierten Elemente deuten damit auf einen Orientierungsrahmen hin, innerhalb dessen Zofia ihren Körper als Autonomiezentrum einsetzt. Der subjektive Sinn der Körperstrategie besteht darin, dass über den Körper sowohl die Anpassung an die Anforderungen des Aufwachsens als auch ein Widerstand dagegen ausgetragen werden. Insgesamt wird jedoch Zofias Streben nach Autonomie deutlich.

Interesse an Jungs und das erste Verliebtsein

Interesse an Jungs

und dann kam so die Pubertät und da eben das Interesse für die Jungs: (1) dann hab ich meinen Verein irgendwann mit fünfzehn sechzehn glaub irgendwie aufgegeben aufgrund der des ah strengen Leistungsdruck ich hab dann immer nur Wettkämpfe gehabt ich wollt einfach mal mein Jugendalter leben und wollt (2) einfach nur (1) JUNCS kennenlernen da hat's dann so angefangen irgendwie mit fünfzehn sechzehn ((holt Luft)) (75-80)

Zofia erwähnt zu Beginn dieser Passage, dass ihr Leben "ganz gut« (75) verläuft, bis sie 14 bzw. 15 Jahre alt ist. Dann jedoch kommt es mit Beginn der Pubertät zu einem entscheidenden Wendepunkt, als sie beginnt, sich für Jungen zu interessieren. Eine weitere wesentliche Veränderung in ihrem Leben bringt ihr Entschluss mit sich, ihren Verein zu verlassen. Sie begründet diese Entscheidung in einer Hintergrundkonstruktion, in der sie den herrschenden Leistungsdruck sowie zahlreiche Wettkämpfe als Erklärung ausführt. Sie hat das Bedürfnis, Jugendliche sein zu dürfen und »einfach nur (1) JUNGS « kennenzulernen. Die Wiederholung des Wortes »einfach« betont den formulierten Wunsch. Das Wort »nur« verweist darauf, dass dieses Anliegen in Zofias Augen angemessen ist und nicht etwa zu viel verlangt, was auf einen Konflikt mit ihrem Vater, der in erster Linie den Leistungssport befördert, hindeutet. Sie beendet ihre Ausführung durch den Schlusskommentar »da hat's dann so angefangen irgendwie mit fünfzehn sechzehn«, der nochmals den zu dieser Zeit stattfindenden Wendepunkt bestätigt.

10 Vgl. dazu Barbara Staubers Ausführungen zur selbstbestimmten Seite von Körperinszenierungen, die eben nicht unfrei machen, sondern zum Ausdruck bringen, dass Mädchen sich nicht vereinnahmen lassen wollen und sich in vermeintlich selbstgewählten Regeln bewegen (vgl. Stauber 2012). 
Insgesamt dokumentiert sich hier, dass Zofia im Alter von etwa 15 Jahren beginnt, sich von ihren Eltern, abzugrenzen, was durch das Austreten aus dem Verein und der damit verbundenen Beendigung des Leistungssports vor allem auch eine Abgrenzung gegenüber dem Vater bedeutet. Der Beginn der Pubertät, das damit beginnende Interesse für Jungen sowie Zofias Wunsch nach Normalität, also eine »normale« Jugendliche sein zu wollen, führen zu ihrer Entscheidung, aus dem Sportverein auszutreten und den Leistungssport zu beenden.

Damit deuten die skizzierten Elemente der biografischen Konstruktion Zofias einen Orientierungsrahmen an, der durch autonomes Handeln geprägt ist: Sie setzt sich gegen ihren Vater durch, indem sie den Leistungssport aufgibt, und verfolgt ihre eigenen Interessen, um eine »normale« Jugendliche zu sein.

\section{Das erste Verliebtsein}

ich hatte mich siebzehn ei- meinen ersten Freu:nd aber es war eher so ausprobieren, (.) es war nich so ah ich war total verliebt das kam dann mit neunzehn nach zwei Jahren nachdem ich dem dann wirklich so den Korb gegeben@hab@aber hab ich dann irgendwie gesagt ah hab ich jemand anders kennengelernt des war aber zu der Zeit wo ich in der Kli:nik war, (2) also ich war in der Klinik wegen (.) meiner Essstörung (2) hier in XX viereinhalb Monate und hatte mal Ausgang über Weihnachten und da hab ich ihn kennengelernt und der hat mich dann voll (.) umgehauen, das waren so die ersten Cefühle so die erste große Liebe, ((holt Luft)) ging dann neun Monate (2) (81-90)

Indem Zofia ihre erste Paarbeziehung mit 17 Jahre unter der Prämisse eingeht, eine Beziehung einfach mal testen zu wollen, auch wenn sie nicht ausnahmslos verliebt ist, bringt sie die Relevanz, eine Beziehung zu haben bzw. sich in einer solchen erfahren zu können, zum Ausdruck. Durch die Abgrenzung zur darauffolgenden Beziehung, der ein totales Verliebtsein vorausgeht, dokumentiert sich Zofias Vorstellung von Beziehung: Sich total zu verlieben gilt für sie als relevante Voraussetzung für eine Beziehung.

Während eines insgesamt viereinhalb Monate dauernden Klinikaufenthaltes, in der sich Zofia wegen ihrer Essstörung behandeln lässt, lernt sie einen Mann kennen, den sie als »die erste große Liebe« bezeichnet. Dass dieser Mann sie »voll (.) umgehauen « hat, deutet auf ein intensives Erleben - im Unterschied zu ihrer ersten Beziehung - hin. Sie führt diese Zeit jedoch nicht weiter aus, sondern beendet ihre Erzählung mit dem Hinweis, dass die Beziehung insgesamt neun Monate dauerte. Ihre Ausführung »und er hat dann ah« (90) bricht sie ab und fährt stattdessen mit der Begründung fort, er sei mit ihrer Essstörung nicht zurechtgekommen, da diese eine Überforderung für ihn darstellte. Auffällig ist hier, dass sie den begonnenen Satz, der in seiner Fortsetzung vermutlich die Trennung seitens des Freundes beinhaltete, nicht ausführt. Als Trennungsgrund gibt sie ihre Essstörung an. Dabei 
konkretisiert sie ihre Erklärung durch den Aspekt der Überforderung. Dies deutet darauf hin, dass sie ihren (Ex-)Freund in Schutz nimmt: Sie legitimiert die Trennung dadurch, dass sie ihm zugesteht, mit ihrer Essstörung überfordert zu sein.

Die Trennung nimmt sie zum damaligen Zeitpunkt stark mit. Aus heutiger Perspektive evaluiert Zofia diese Erfahrung als eine, die sie bis heute prägt. Zum einen ist dies die Erfahrung des Alleingelassen-Werdens, zum anderen die damit verbundene Kränkung. Diese Erfahrung scheint sich in Zofias Leben zu wiederholen.

Unterschiedliche Modi in der Gestaltung von Paarbeziehungen

\section{Unterwerfung und Füreinanderda-Sein}

ja:: und dann kam wieder nochmal $\mathrm{n}$ (.) ah $\mathrm{t}$ - also wieder ein neuer Versuch mit nem neuen Freund, (3) und ich bin ((räuspert sich)) (2) weil ich andere Ansätze hatte=ich hab dann irgendwie gemerkt ich muss mich durchsetzen in ner Beziehung was ich in der ersten Beziehung nicht gemacht hab da hab ich dann immer gedacht ich muss mich eher so unterwerfen und irgendwie ((holt Luft)) hab da irgendwie das noch nicht rausgehabt wie es irgendwie is überhaupt (.) Kompromisse zu schließen und ich hab immer gedacht man muss füreinander da sein, (1) (95-103)

Es folgt ein weiterer Versuch mit einem neuen Partner. Die Verwendung des Begriffs »Versuch« deutet dabei ein eher technokratisches, vielleicht auch pragmatisches und weniger emotionales oder intimes Vorgehen an. Verstärkt wird dieser Eindruck durch die einleitenden Worte »und dann kam wieder nochmal n $n$ Versuch, die weniger auf die Anbahnung einer romantischen Beziehung als vielmehr auf den Versuch, eine funktionierende Beziehung zu gestalten, hinweisen.

Zofia bricht ihre Erzählung ab und evaluiert in einer theoretisch-reflektierenden Stellungnahme ihr Verhalten in der Beziehung und macht gleichzeitig ihre Sichtweise dessen deutlich, was notwendig ist, damit die Beziehung gelingen kann. Während ihrer »ersten Beziehung« (sie meint damit ihren zweiten Freund) ist Zofia der Auffassung, sich unterordnen zu müssen. Zentral ist hier außerdem für sie, »füreinander da zu sein «. Während der erstgenannte Aspekt eine, wenn auch selbst gewählte, Anforderung an sie darstellt, zielt der zweite auf einen gegenseitigen Anspruch. Die Annahmen weisen auf ein eher internal motiviertes, uneigennütziges Umsorgen des Anderen hin.

Als Fazit führt Zofia an, dass ihr zu dieser Zeit die Fähigkeit, Kompromisse zu schließen, noch fehlt. Damit drückt sie gleichzeitig aus, dass sie es aus heutiger Perspektive für wichtig erachtet, innerhalb der Beziehung Kompromisse finden zu können.

Aufgrund dieser Erfahrungen zieht sie nach der ersten Beziehung die Bilanz, sich mehr durchsetzen zu müssen. 
Insgesamt fällt auf, dass Zofia in beiden Sequenzen mit einer Erzählung beginnt, diese jedoch nach kurzer Zeit unterbricht und mit Argumentationen fortfährt. In diesen Ausführungen beschäftigt sie sich mit der Frage, weshalb die Beziehungen nicht hielten bzw. auseinandergingen. Ihr Fokus ist mehr auf die Schwierigkeiten innerhalb der Beziehungen und die Trennungsgründe und weniger auf den Verlauf oder das Gelingen gerichtet.

Hinsichtlich Zofias Vorstellungen über die eigene Rolle innerhalb einer Paarbeziehung wird in dieser Sequenz ein Verlauf von sich verändernden Vorstellungen angekündigt. Dabei zielten die Erfahrungen mit dem ersten Freund eher auf ein Ausprobieren. Sie werden im weiteren Verlauf des Interviews auch nicht als Beziehung klassifiziert. In Bezug auf ihre ersten Beziehungserfahrungen mit einem festen Freund wird deutlich, dass sich hier tradierte Normvorstellungen wiederfinden, wie sie Zofia in ihrer Herkunftsfamilie auf der elterlichen Beziehungsebene erlebt hat. Diese Normvorstellungen umfassen ein Beziehungsverständnis, das von Unterordnung geprägt ist.

\section{Wunsch nach Akzeptanz und Wertschätzung}

und der zweite Freund [...] man (.) entwirft halt ne Illusion da war zwar des Verliebtsein da so- aber es war nie so dass ich ((holt Luft)) dass er mich halt irgendwie auch ern- ich hab immer das Gefühl gehabt er will immer seine S- Bedürfnisse über meine so hin- (2) wegsetzen=und wo ich mich dann wider- (.) setzt hab ((holt Luft)) da kam's dann dass er halt mich ahm (2) genau er hat mich (2) ja in meinem verbalen Verständnis dann immer so $\mathrm{n}$ bisschen plattgemacht dass er dann immer des umgedreht hat was ich gesagt hab ((holt Luft)) und praktisch so:: hingedreht hat dass er dann im Recht stehen konnte und mich (.) und und ich im Endeffekt es ging mir nicht ums Recht (oder so) es ging eigentlich mehr darum dass man sich (.) wertschätzen und dass man sich akzeptieren soll, (1) (103-114)

Obwohl Zofia verliebt ist, bestätigt sich ihr Wunschbild, das sie von dieser Beziehung hat, nicht. Sie hat das Gefühl, ihr Freund wolle seine Bedürfnisse über die ihren stellen und sich durchsetzen. In dem Moment, wo sie sich dem widersetzt, kommt es zu verbalen Auseinandersetzungen, bei denen Zofia sich zurückgesetzt fühlt. Die Konfrontationen enden damit, dass ihr Freund am Ende Recht behält. Zofia betont, ihr gehe es nicht ums Rechthaben, vielmehr seien ihr gegenseitige Wertschätzung und Akzeptanz wichtig.

Hier zeigen sich innerhalb Zofias Orientierungsschemas sehr explizite Vorstellungen hinsichtlich des Umgangs miteinander in einer Paarbeziehung. Die reziproke Wertschätzung und Anerkennung sieht sie als elementar für die Basis einer Beziehung an. 


\section{Trennung und Ohrfeige}

irgendwann ging mir das: (.) eigentlich wirklich so (1) soweit dass ich dann wirklich auch für mich entschieden hab ich hab=ha- hatt ich meinen Streit und jetzt wirklich geh, ((holt Luft)) und dann (.) hat er aber=also dann is er ich ich hab des dann eigentlich recht heimlich gemacht, na indem er arbeiten war hab ich dann einfach des ganze Zeug weil wir hatten teilweise auch zusammen gewohnt, (1) (weil) ich meinen ganzen Kram bei ihm hatte und dann hab ich mein ganzes Zeug rausgenommen wo er arbeiten war und dann ((holt Luft)) kam er zurück und dann war ahj war das schon so dass er das irgendwie ich mein des war ja für ihn wahrscheinlich auch nich des war er war nicht (.) informie::rt und ich habs nicht mehr ausgehalten und ich hab das für mich entschieden und dann kam dann schon so irgendwie erstmal (.) mhmh sag ich mal so ne Ohrfeige kam da erstmal und dann halt die Sachen alle aus dem Fenster rausschmeißen, (1) er hat mich nicht aufgehalten ich bin gegangen, (114-127)

Zofia kommt an den Punkt, an dem sie für sich entscheidet, sich aus der Beziehung zu lösen. Dass sie die Trennung »heimlich« vollzieht, lässt darauf schließen, dass sie die Auseinandersetzung mit ihrem Freund scheut. Während er bei der Arbeit ist, holt sie ihre Sachen, die sie noch bei ihm hat, ab. Ihr Freund kommt jedoch zu diesem Zeitpunkt zurück und es kommt zu einer Ohrfeige. Außerdem wirft er Zofias Sachen aus dem Fenster.

In einer Hintergrundkonstruktion erklärt sie sein Verhalten damit, dass er schließlich nichts von ihren Absichten wusste, was einer Entschuldigung nahekommt. In dieser Argumentation macht sie auch deutlich, dass sie sich bewusst für diese Art der Trennung entschieden hat, da die Situation für sie nicht mehr zu ertragen war. In dieser Argumentation wird Zofias innere Zerrissenheit deutlich: Einerseits erkennt sie die problematische Seite an der Art ihrer Trennung, die ihrem Freund jede Gelegenheit entzieht, sich dazu zu äußern. Andererseits sieht sie für sich nur die Möglichkeit, sich so von ihm zu lösen; vermutlich aus Angst davor, von ihm bedroht oder angegriffen zu werden, was dann tatsächlich doch passiert, als sie von ihm überrascht wird.

Nach der Trennung fasst Zofia den Schluss, ihr »Leben irgendwie auch finanziell irgendwie« (128f.) zu gestalten. Hier dokumentiert sich ihr Wunsch nach finanzieller Eigenständigkeit und Unabhängigkeit. In ihrer weiteren Ausführung verschiebt sich allerdings der Fokus auf die Suche nach einer Tätigkeit, die sie interessiert. Bereits zu einem früheren Zeitpunkt nutzt sie die Gelegenheit, über ein Praktikum zu prüfen, ob ihre Interessen eher im künstlerischen oder sozialen Bereich liegen. Den Prozess der Orientierung hinsichtlich ihrer Interessen beschreibt sie als »lange [...] Suche« (131). Rückblickend hält sie fest, dass sich Vieles einfach ergeben hat, ohne dass sie vorab konkrete Vorstellungen dazu hatte. 
Insgesamt dokumentiert sich hier in verschiedenen Ausprägungen Zofias Bedürfnis nach Autonomie: Hinsichtlich ihrer Paarbeziehung trifft sie die Entscheidung, sich zu trennen, als sie erkennt, dass ihre Bedürfnisse und Vorstellungen nach einer gleichberechtigten Gestaltung der Beziehung nicht verwirklicht werden können. Auch bezüglich ihrer zukünftigen Lebenssituation spielt die finanzielle Unabhängigkeit (die möglicherweise in Zusammenhang mit den Erfahrungen im Elternhaus steht) eine wichtige Rolle. Dabei ist ihr jedoch auch wichtig, einen Beruf zu erlernen, der ihr nicht nur die Möglichkeit der finanziellen Unabhängigkeit bietet, sondern darüber hinaus auch eine Chance auf Selbstverwirklichung darstellt.

Abhängigkeit

\section{Verliebtsein}

dann hab ich dann an Praktikum gemacht ein FS] und da hab ich dann meinen dritt-en Freund dann kennengelernt und da hat dann wieder s gleiche eigentlich (.) irgendwie ich hab mich immer (.) verlie::bt (.) es war immer schön ((räuspert sich)) und ahm des war für mich auch immer die Vorstellung überhaupt ahm ne Beziehung einzugehen das halt eben erst mal so ne (.) dass erst mal ne Verliebt(.) seinphase da sein muss oder dass es mich komplett umhauen muss wie man so sagt so ne rosarote Brille, (136-145)

Während ihres FSJ lernt Zofia ihren dritten Freund kennen. Sie verliebt sich in ihn und erläutert in einer biografischen Orientierungstheorie, dass das Vorhandensein von Verliebtheitsgefühlen für sie eine Grundbedingung darstellt, um überhaupt eine Beziehung einzugehen.

Über ihre dritte Beziehung äußert sich Zofia zunächst argumentativ und beschreibend aus der Retrospektive: Die Beziehung ist von »Abhängigkeitsmechanismen« (149) und »Machtkampf « (149) geprägt, sodass nach drei Jahren für beide klar ist, dass die Beziehung eigentlich nicht mehr funktioniert. Dennoch fällt die Trennung beiden so schwer, dass die Beziehung ein weiteres, viertes Jahr dauert.

Nach diesem Vorgriff auf das Ende der Beziehung thematisiert Zofia die Dynamik der Beziehung: Sie bezeichnet sich als diejenige, die »immer gefordert hat « (150) und ihre Position klar vertritt. Als problematisch beschreibt sie die Ignoranz ihres Freundes. Zofias Äußerung »der wird dann irgendwann doch merken wenn ich ah [...] wenn ich ihm ein Ultimatum stell« (153-155) macht deutlich, dass sie darauf hofft, ihr Freund könnte sich diesbezüglich verändern. Sie selbst geht davon aus, dass man, wenn man jemanden liebt, auch die Bereitschaft zeigen muss, für die Beziehung zu »kämpfen« (159) und zu »arbeiten« (159).

Zofia hat zu diesem Zeitpunkt das Bedürfnis, "selber etwas ändern« (164) zu wollen, und beginnt auf eigenen Wunsch hin eine ambulante Therapie wegen ihrer Bulimie. In der Betonung, dass dies ihre eigene Entscheidung war, dokumentiert 
sich erneut Zofias Handlungspraxis, selbst aktiv zu werden und an sich zu arbeiten, um Veränderungen zu erzielen. Durch ihren Therapeuten erfährt sie »Rückhalt« (166), was darauf schließen lässt, dass sich ihre psychische Situation stabilisiert. Gleichzeitig betont sie, kein Abhängigkeitsverhältnis zu ihm aufgebaut zu haben. In der Betonung ihrer eigenen Unabhängigkeit wird Zofias großes Bedürfnis nach Selbständigkeit und Autonomie deutlich.

In der Therapie kann sie einerseits das »rauslassen « (169), was in der Beziehung nicht möglich ist. Gleichzeitig wird ihr »Beachtung (170) entgegengebracht, die sie in ihrer Beziehung vermisst. Die Nicht-Beachtung durch ihren Freund erlebt Zofia als mangelnde Wertschätzung und Anerkennung ihrer Person. Sie erläutert in der darauffolgenden Argumentation, dass ihr Freund Zofias Bedürfnis nach Austausch nicht teilt und auf ihre Bitten darum mit Schweigen oder Hinauszögern reagiert.

\section{Rauschtrinken}

und dann hat man halt einfach immer angestaut man hat des angestaut ((holt Luft)) und irgendwann hat sich des so $\mathrm{n}(2)$ ich glaub dann hat sich meine Suchtpotenzial in so $\mathrm{n}$ Alkohol- ah trinken also um dieses Ganze irgendwie einfach zu fressen hab ich Alkohol getrunken (175-178)

Diese Erfahrungen der Nicht-Anerkennung, vermutlich auch verbunden mit Gefühlen des Nicht-geliebt-Werdens, führen dazu, dass Zofia mit dem Trinken anfängt, um, wie sie selbst formuliert, »vergessen einfach um vergessen« (181) zu können.

Die Abstände zwischen dem Trinken werden kürzer, und es kommt zur Eskalation innerhalb der Beziehung:

\section{Eskalation und körperliche Übergriffe}

und die Intervalle werden immer kürzer und dann eskaliert's und dann kam's so dass er mir angedr-also dass er mir gedroht hat ((holt Luft)) und dass er mich dann ahm schon körperlich auch angegangen also dass er mich ahm (.) er is körperlich ge- walttätig geworden ((holt Luft)) aber ah meistens halt auch in so ner Ekstase und dann hat er sich sich damit immer entschuldigt ((schluckt)) (182-187)

Zofias Freund droht ihr - wobei sie dies nicht näher ausführt - und wird ihr gegenüber auch körperlich gewalttätig. Zofia erklärt sein Verhalten damit, dass sich dieses im Rahmen einer Ekstase abspielt, und ergänzt, »also es tat ihm auch wirklich leid weil er danach auch selber in Tränen ausgebrochen is«. Beide sind sich nach solchen Eskalationen darüber einig, dass sie dies gemeinsam "gestaltet« (190) haben und damit auch die gemeinsame Verantwortung dafür tragen.

Hier dokumentiert sich Zofias Bestreben, gemeinsam mit ihrem Freund handlungsfähig zu bleiben. Sie sieht die Gewalt ihres Freundes nicht zwingend als un- 
vermeidlich an, da für sie die Tatsache, geliebt zu werden, auch gleichzeitig bedeutet, verletzt zu werden. Dies zeigt sich auch darin, dass sie ihm keine Schuld zuschreibt und ihm letztlich verzeiht.

In dieser Passage zeigt sich erneut, dass Zofia innerhalb ihres Orientierungsschemas die explizite Vorstellung von Verliebtsein als Voraussetzung und Basis für eine gemeinsame Beziehung hat. Es dokumentiert sich außerdem Zofias Bestreben nach Handlungsfähigkeit innerhalb ihrer Beziehung. Dies führt auch dazu, dass sie zum Teil die Verantwortung für das gewalttätige Verhalten ihres Freundes übernimmt. Diese Übernahme der Verantwortung hat für Zofia die Funktion inne, sich handlungsmächtig und nicht ohnmächtig zu fühlen.

Zofia sucht nach Worten für ihre weitere Erzählung (»und dann hab war ich das halt hatt ich das halt so weitergesponnen« (203f.)). Was sie genau erzählen möchte, bleibt zunächst unklar und wird lediglich mit »das« (204) und »es« (205) umschrieben. Das nicht Benannte dauert etwa ein Jahr lang und tritt immer wieder auf. Zofia versucht zunächst die Häufigkeit genauer zu benennen und äußert, »es waren bestimmt fünf Mal wo er mich ordentlich auch (2) also ((räuspert sich))« (206f.); danach bricht der Satz ab. An dieser Stelle wird deutlich, wie schwierig es für sie ist, das tatsächlich Vorgefallene zu benennen. Ein Grund dafür kann einerseits Scham sein; andererseits kann das Umschreiben der Erfahrungen auch als Schutz vor den "gewaltigen" Bildern eingesetzt werden, indem so verhindert wird, dass Zofia von ihren Erinnerungen überflutet wird. Bevor sie weiter darauf eingeht, fügt sie zunächst eine Erklärung für das Verhalten ihres Freundes ein, dass dieser »einfach seine Kontrolle verloren hat« (208). An dieser Stelle wird deutlich, was zuvor nur vermutet werden konnte: dass Zofia von ihrem Freund massive körperliche Gewalt erfahren hat und nach Worten ringt, diese zu beschreiben. Der Hinweis auf die Überlegung einer Strafanzeige macht weiter deutlich, dass es sich bei diesen Gewalttaten auch um ein strafrechtlich zu verfolgendes Verhalten handelt. Die Formulierung »ich zeig ihn jetzt schon an« (210) zeigt, dass Zofia nicht mehr weit davon entfernt war, dies auch zu tun. Es kommt jedoch zu keiner Anzeige, da Zofia Sorge hat, sie zerstöre damit das Leben ihres Freundes, vor allem vor dem Hintergrund, dass er einen sozialen Beruf ausübt. Ein weiterer Grund, ihn nicht anzuzeigen, besteht darin, dass sie über sich selbst sagt, keine Rache üben zu wollen.

Insgesamt wird in diesem Segment deutlich, dass Zofia hier über das Ende der Beziehung spricht. Hier ist sie bereits an dem Punkt angelangt, an dem sie keine Perspektive mehr in der Beziehung sieht.

In Bezug auf den unterliegenden Orientierungsrahmen wird deutlich, dass für Zofia die Ausübung von Gewalt erklärbar ist und zwar in Form eines Kontrollverlustes, den ihr Freund erleidet, und dem, innerhalb dieses Erleidens, ebenfalls eine Opferrolle zukommt. Zofia unterscheidet dabei nicht deutlich zwischen Recht und Unrecht resp. Opfer oder Täter. Sie übernimmt ebenfalls Verantwortung für das 
gewaltaktive Handeln ihres Freundes - nicht nur vor sich selbst, sondern auch nach außen, indem sie auf eine Anzeige verzichtet, da diese ihm beruflich schaden würde.

\section{Vertrauensbruch}

irgendwann ahm kam des aber auch soweit dass ahm er dann halt irgendwie mdes Mädchen au mal mit nach Hause gebracht hat wo ich gedacht hab woa was is @jetzt los@und dann war des für mich natürlich ein Vertrauensbruch (218-220)

Zofia absolviert die Ausbildung zur Erzieherin, ihr Freund etwa zeitgleich die Ausbildung zum Krankenpfleger. Die Bemerkung, beide arbeiten anschließend in ihren Berufen, weist auf die Einstellung gewisser Routinen innerhalb der Beziehung hin. Diese werden unterbrochen, als ihr Freund eine Affäre mit einer anderen Frau beginnt. Für Zofia bedeutet das einen massiven Vertrauensbruch. Diese Vorfälle lösen neue Auseinandersetzungen aus, bei denen es zum Schlagabtausch (»Pingpong Schlag« (221)) mit gegenseitigen Vorwürfen kommt und in denen sich die Situation »hochgeschaukelt« (226) hat.

\section{Trennung}

und bei ihm war das so wahnhaft dann ah wo ich gegangen bin letztendlich ahm nach der Ausbildung hab ich diesen Studienplatz bekommen, (227-228)

Bevor Zofia die Trennung näher erläutert, erzählt sie in einer ausführlichen Hintergrundkonstruktion von ihrer Überraschung über den Erhalt des Studienplatzes sowie von ihrer Motivation hinsichtlich ihrer beruflichen Ausbildung, die sie zu der Entscheidung befähigt, sich von ihrem Freund $\mathrm{zu}$ trennen. Auffällig ist, wie Zofia versucht, das Verhalten ihres Freundes zu beschreiben, ohne es konkret $\mathrm{zu}$ benennen: Die Umschreibung »bei ihm war das so wahnhaft« bezieht sich auf den Zeitpunkt ihres Auszugs bzw. ihrer Trennung von ihm, als sie einen Studienplatz erhält. Mit diesem hatte sie, aufgrund ihrer Noten, nicht gerechnet, auch nicht damit, überhaupt jemals zu studieren. Sie hatte sich bereits $\mathrm{zu}$ einem früheren Zeitpunkt, nach Abschluss der Fachhochschulreife, beworben, jedoch keinen Platz erhalten. Die Entscheidung, anschließend eine Ausbildung zu machen, war für Zofia eng damit verbunden, ihr Leben abzusichern - vor allem auch vor dem Hintergrund, dass sie "nie irgendwie finanziell von nem MANN abhängig sein« wollte (235). In der Zusage für den Studienplatz erkennt sie für sich die Chance, dieses Ziel zu verwirklichen.

Hier dokumentiert sich der Modus einer Fokussierung der eigenen Handlungsmöglichkeit im Sinne einer Unabhängigkeit, insbesondere hinsichtlich der materiellen Eigenständigkeit gegenüber einem Beziehungspartner. Indem Zofia den Stu- 
dienplatz annimmt, zeigt sie Bildungsaspiration. Hier wird ihr Streben nach Autonomie deutlich.

\section{Eskalation}

da kam bei ihm so dieser (2) ah ich glaub so dieser (1) Kurz::: also da kam so diese Angst vor dem alleine Dasein und dann hat er mich (.) echt verfolgt in der Wohnung und mich nicht gehen lassen also der hat mich und schon alleine der der Blick war dann oft so (.) ((atmet ein 2)) wo ich ganz genau gewusst hab: So, jetzt (.) ist der Schalter rum (.) und jetzt musst du (.) kucken wie du am besten mit der Situation umgehst (245-250)

Als Zofia die Zusage zu ihrem Studienplatz erhält und ihren Freund über die Annahme des Studienplatzes informiert, kommt es zu einer letzten eskalierenden Situation. Die darauffolgende Kurzschlussreaktion ihres Freundes erklärt Zofia damit, dass ihre Information über ihre Studienabsichten bei ihrem Freund »Angst vor dem alleine Dasein« auslöst. Was dann folgt, beschreibt Zofia sehr detailliert: Ihr Freund verfolgt sie in der Wohnung und lässt sie nicht gehen. An seinem Blick, den sie kennt, kann sie abschätzen, in welcher Verfassung er ist.

\section{Strategien}

es war am Anfang so, dass ich einfach nur noch (1) laut wurde und dann ha- hat's aber nicht besser gemacht dann wurde es nur noch schlimmer (.) und irgendwann hab ich dann versucht ähm (.) aber wenn ich ruhig war und gar nicht geantwortet hab dann hat ihn des aber genau so (.) ah (.) also des war ich hab nie wirklich die Methode rausbekommen wie ich denn damit jetzt am besten umgehen kann damit er nicht ausartet, (.) also damit er nicht ausflippt (251-256)

Zofia beschreibt, wie sie die ersten Male, als ihr Freund ihr gegenüber gewalttätig wird, »laut« wird, was jedoch dazu führt, dass die Situation noch mehr eskaliert. Weiter beschreibt sie, dass sie versucht, ruhig zu bleiben und ihm nicht $\mathrm{zu}$ antworten, dies jedoch genauso wenig ihren Freund von seinen Übergriffen abhält. Ihr Resümee, nie die passende Strategie gefunden zu haben, bestätigt erneut, dass sie einen eigenen Anteil sowie eine eigene Verantwortung für die Ausübung von Gewalt innerhalb der Beziehung sieht.

Sie schätzt es für sie beide als das Beste ein, dass sie ihren Weg weitergegangen ist und die Beziehung sich nicht fortgesetzt hat.

An dieser Stelle dokumentiert sich erneut der Modus der Fokussierung auf das eigene Handeln. Indem Zofia verschiedene Strategien anwendet, um herauszufinden, welche die geeignete ist, ihren Freund vor seinem Kontrollverlust zu bewahren, versucht sie, selbst handlungsfähig zu bleiben. Damit schreibt sie sich selbst auch eine aktive Rolle zu; sie empfindet sich den Gewaltausbrüchen ihres Freundes 
nicht hilflos ausgesetzt und fühlt sich so auch nicht in der Rolle des Opfers. Stattdessen sieht sie sich als Teil der (gewaltvollen) Auseinandersetzungen und schreibt sich selbst auch Verantwortung daran zu.

\subsubsection{Argumentative Stellungnahmen}

Schlussevaluation der Stegreiferzählung

Gegen Ende der biografischen Stegreiferzählung führt Zofia in einer längeren Evaluation ihrer Beziehungsbiografie ihre diesbezüglichen Erfahrungen aus.

\section{Vorwürfe}

ich hab mir immer so viele Vorwürfe gemacht dass ich so viel einfach irgendwie auch falsch gemacht hab ich hab auch immer in jeder Beziehung dann versucht etwas neu zu gestalten und konnt mich eigentlich gar nicht so richtig auf mich (.) irgendwie (.) ich konnt meine meine eigenen Gefühle oder mein ich hab immer gesagt das muss ähm das is irgendwie so vorgegeben so muss es irgendwie funktionieren (.) (316-321)

Zofia beschreibt, sich in Beziehungen allgemein Vorwürfe gemacht zu haben und viele Fehler begangen zu haben. Worin diese Fehler bestanden, führt sie nicht weiter aus. Die Konsequenz bzw. Lernerfahrung ist für sie jedoch, in jeder Beziehung etwas neu gestalten zu wollen. Den Satz »ich konnt meine eigenen Gefühle« bricht sie ab. Die darauffolgende Formulierung »ich hab immer gesagt das muss ähm das is irgendwie so vorgegeben so muss es irgendwie funktionieren" spiegelt einen eher funktionalen Charakter der Beziehung wider. Vor diesem Hintergrund lässt das Nicht-Ausgesprochene des vorausgegangenen Satzes vermuten, dass es in dieser Beziehung für Zofia nicht genügend Platz für Emotionalität gibt.

Hinsichtlich des hier unterliegenden Orientierungsrahmens wird deutlich, dass Zofia in ihrer Vorstellung, wie eine Beziehung funktionieren kann bzw. muss, einen Plan hat, nach dem sie ihr Handeln ausrichtet. Sie hat also eine Vorstellung, wie Beziehung funktionieren kann, und nach dieser Vorstellung handelt sie bzw. versucht sie, die Beziehung zu gestalten. Dabei begreift sie ihre Beziehungserfahrungen als einen Lernprozess, indem sie in jeder neuen Beziehung versucht, Dinge zu verändern und Neues auszuprobieren. Gleichzeitig scheinen emotionale Bedürfnisse ihrerseits zurückgestellt werden zu müssen, was sie dann auch als Mangel innerhalb der Beziehung erlebt. Ihre Vorstellung, eine Beziehung gelinge, wenn sie sich unterordne, funktioniert nicht mehr. Sie weiß aber auch nicht, welche anderen Modi der Beziehungsgestaltung in Fragen kommen könnten. Dies dokumentiert sich deutlich in ihrer allgemeinen Vorstellung von >Beziehung als Arbeit<, als Leistung. Damit zeigt sich ein weiteres wesentliches Moment: Zofia sieht sich selbst als verantwortlich für das Scheitern oder Gelingen der Beziehung an. 


\section{Selbsterfahrung}

irgendwann wo ich mich mehr kennengelernt hab hab ich dann auch schon aber=dann versucht mich einzu-wow @.@//@.@//äh mich zu entdecken und zu erfahren aber es war (nich) (.) war nicht einfach (321-323)

Zofias Vorgehen bzw. ihr Verhalten in Beziehungen steht auch in Abhängigkeit zu dem Prozess ihrer Selbsterfahrung, durch den sie sich mehr und mehr kennenlernt. Sie versucht, sich »zu entdecken und zu erfahren«, was auf mehr Rückbesinnung auf ihre eigenen Gefühle und Bedürfnisse schließen lässt.

Ihre abschließende Bemerkung, dass dies nicht leicht gewesen sei, macht den mühsamen Prozess dieses Sich-neu-Ausprobierens in Beziehungen deutlich. Auch hier erlebt sie ihre Bemühungen eher anstrengend und schwierig.

Biografische Orientierungstheorie

In der folgenden biografischen Orientierungstheorie, die möglicherweise auch Ergebnis eines durch therapeutische Erfahrung angeregten Reflexionsprozesses ist, nimmt Zofia Bezug auf ihren außerordentlich leistungsorientierten Vater und stellt einen Zusammenhang zwischen den diesbezüglichen Erfahrungen in ihrer Kindheit und ihren Vorstellungen und ihrem Verhalten in Beziehungen her:

${ }^{\circ}$ und ${ }^{\circ}$ in meiner Kindheit hatt ich vielleicht einfach von meinem Vatter vielleicht noch () und des dann einfach auf das vielleicht zurückgeht dass mein Vatter sehr leistungs-orientiert war (.) ähm teilweise auch irgendwie meine Kindheit ja auch v e r p a s s t hat also er war schon n liebevoller Vater also ich möcht ihn jetzt irgendwie nicht nur ins schlechte Licht äh rücken ((atmet ein)) er hat äh sich sehr darum bemüht dass wir auch sehr viel Sport machen aber es war eher immer in dem Sinne so (.) »ich bin n stolzer Vater« und nicht »welche Bedürfnisse hat mein Kind « (.) sag ich jetzt einfach aus (.) dem was ich jetzt irgendwie auch reflektieren (und) kann und ähm er war sehr streng was halt des Lernen anging und dann ((räuspert sich)) gibt's gab's da auch schon mal ne Ohrfeige und so es war ${ }^{\circ}$ damals halt auch noch in der Kindheit irgendwie so (1) Maßregelung ähm ja//hmhm//des war der Vatter halt der durfte das (.) genau (1) ((atmet ein)) (324-336)

Auch wenn sie ihren Vater als liebevoll bezeichnet und ihn nicht negativ darstellen möchte, äußert sie, durch seine Forderungen, viel Sport zu betreiben, einen wesentlichen Teil ihrer Kindheit verpasst zu haben. Damit verbunden ist auch die erfahrene Anerkennung des Vaters bezogen auf gute sportliche Leistungen (und damit nicht auf das Kind an sich), wobei persönliche Bedürfnisse des Kindes nachgeordnet wurden oder keine Rolle spielten.

An dieser Stelle berichtet Zofia auch von Ohrfeigen und weiteren Maßregelungen durch ihren Vater, die allein durch die Tatsache, dass sie vom Vater ausgeübt wurden, legitimiert waren. 
Hier dokumentiert sich die biografische Verwurzelung von dem Bedürfnis bzw. der Suche nach Anerkennung, die nur durch Erbringung von Leistung erfahrbar wird. Diese Erfahrungen erklären auch die Schwierigkeit, einen Selbstwert zu entwickeln, der es erlaubt und ermöglicht, sich selbst, unabhängig von Leistung, zu lieben.

Theorie zum eigenen Selbst

ja und seit drei Jahren hab ich jetzt keinen Freund mehr und wie gesagt das fällt mir auch schwer Beziehungen jetzt inzwischen einzugehen und (.) mich fallen zu lassen und äh fahl äh Ver äh Vertrauen aufzubauen (336-339)

Zofia lebt nun seit drei Jahren als Single. In einer Theorie zum eigenen Selbst schildert sie aus heutiger Sicht, wie es ihr inzwischen schwerfällt, Beziehungen einzugehen, vor allem Vertrauen zu entwickeln. Die erlebten Erfahrungen haben sich so manifestiert, dass sie, ihrer Meinung nach unbewusst, eine Beziehung nicht zulassen kann, da sie sich letztlich nie sicher sein kann, ob ihr "sowas« (341) wie die erlebten Gewalterfahrungen nicht noch einmal widerfahren könnte.

In einem Nachklapp ergänzt sie, dass sie trotz allem sehr stolz auf sich ist, »immer wieder den Absprung geschafft« (346) zu haben. Besonders ihre Familie sieht sie in diesem Zusammenhang als die Instanz, die sie besonders darin unterstützt hat. Die Einschränkung »auch wenn sie mehrmals [...] an mich den Appell gestellt hat geh « (349f.), also die Trennung von ihrem Freund eingefordert hat, macht nochmals deutlich, dass Zofia dies auch aus heutiger Sicht nicht als akzeptabel empfindet. Dennoch schmälert dies die oben genannte Unterstützung nicht.

aber letztendlich glaub ich selbst (.) ja (.) also wenn man dann einfach ne gewisse Grenze erreicht hat und überschritten hat und einfach nur noch (.) ähm (1) einfach diese Beziehung nur noch diesen Lebensinhalt darstellt (1)//(zustimmend) hm//und wenn man dann erkennt dass es eben nicht das alles ist (.) sondern einfach auch dass äh:m ma:n (.) alleine äh sich äh wertvoll erfahren kann und nicht immer diese Bestätigung auf Seiten vom Mann oder von seinem Partner braucht (350-357)

Zofia urteilt aus heutiger Perspektive, dass in der Vergangenheit Grenzen überschritten wurden und dass es problematisch ist, wenn eine Beziehung zum alleinigen Lebensinhalt wird. Nach der Erfahrung, sich auch selbst als wertvoll erleben zu können und unabhängig von der Bestätigung eines Mannes oder Partners zu sein, orientiert sich Zofia aktuell wieder mehr an anderen sozialen Beziehungen. Dann wird es auch nicht mehr notwendig, sich »anzupassen« (364) und zu fragen »wie soll ich denn jetzt sein« (364), damit die Paarbeziehung funktioniert. 
Normative Anforderungen

irgendwann wollt ich hab ich gedacht ich krieg des alles alleine hin auch und so ich brauch da niemand und (.) es wird ja einem so vermittelt und so dass dass du heut irgendwie alles als Frau so schlucken kannst, und du gehst arbeiten du gehst (2) ja am besten erziehst du nebenher noch Kinder und studierst vielleicht noch nebenher=es gibt doch super Alpha-Mamis und so die=es wird ja einem irgendwie auch so vermittelt aber ich bin ja nicht=das is zu viel, (.) da bist du einfach überfordert also, ich find ich finds vollkommen in Ordnung wenn man sagt ich leb ich entscheide mich für den Vollzeit-(.) Beruf Mama, (3) und es gibt halt ähm (4) und ich war damals nämlich auch so dass ich gedacht hab ich muss alles schaffen, (625-634)

Zofia beschreibt rückblickend einen zeitlichen Punkt in ihrer Biografie, an dem sie dachte, alles alleine schaffen zu können. Sie bezieht sich auf gesellschaftliche normative Bilder, die das Bild der omnipotenten Frau suggerieren. Aus ihrer heutigen Perspektive reflektiert sie die Unmöglichkeit dieses Bildes. In diesem Zusammenhang führt Zofia auch ihr »Problem per-fek-tionistisch zu sein« (635f.) an.

\subsubsection{Zusammenfassung}

Erleben von Gewalt in Paarbeziehungen Zofia erlebt in ihrer dritten Partnerschaft im Alter von ca. 21 Jahren Gewalt, in der sich eine Dynamik zeigt, die Leonore Walker als »Gewaltspirale« (Walker 1983) bezeichnet. Nach Phasen des Spannungsaufbaus folgen die körperlichen Übergriffe, die im Nachhinein von ihrem Freund entschuldigt werden.

Umgang mit erlebter Gewalt, Bewältigungsstrategien und Ressourcen Zofia zeigt neben aktiven auch passive Bewältigungsstrategien, insbesondere im direkten Umgang mit der erlebten Gewalt. Auf der aktiven Ebene versucht sie, die Beziehung zu gestalten, indem sie bspw. Forderungen stellt. Auf die erlebte Gewalt reagiert sie mit passiven Strategien, mit denen sie vor allem die Gewalt entschuldigt und nicht nur ihren Freund, sondern vor allem auch sich als verantwortlich für den Gewaltausbruch ansieht. Außerdem versucht sie, die erlebte Gewalt vor ihrer Familie und ihren Freund_innen zu verheimlichen. Insgesamt ist sie jedoch sehr bemüht, innerhalb ihrer Beziehung handlungsfähig zu bleiben und sich nicht ohnmächtig der Gewalt des Freundes auszuliefern, was ihr Streben nach Autonomie auch innerhalb einer Gewaltbeziehung zum Ausdruck bringt.

Eine weitere Bewältigungsstrategie im Umgang mit der erlebten Gewalt zeigt sich bei Zofia im Trinken, wobei die Intervalle zwischen den einzelnen Phasen des Rauschtrinkens im Laufe der Beziehung immer kürzer werden. 
Schließlich stellt für Zofia die professionelle Unterstützung durch einen Therapeuten eine Ressource dar, auf die sie zurückgreift und die ihr dabei hilft, sich zu stabilisieren.

Durch das für sie unerwartete Angebot eines Studienplatzes sieht sie eine Chance auf Unabhängigkeit und Autonomie. Diese Bildungsoption ermöglicht ihr den Ausstieg aus der zu diesem Zeitpunkt gewaltvollen Beziehung.

Beziehungsmuster und Beziehungsgestaltung Im Interview mit Zofia wird deutlich, dass sie innerhalb ihrer ersten Beziehungen versucht herauszufinden, wie Beziehung funktioniert. Dabei geht sie von einem ideell-egalitären Beziehungskonzept aus, das auf der Vorstellung einer partnerschaftlichen Liebe beruht. Sie probiert im Laufe verschiedener Beziehungen unterschiedliche Modi der Beziehungsgestaltung aus: Unterwerfung, Durchsetzung, Machtkampf bzw. Kampf um reziproke Anerkennung und Wertschätzung.

Dabei entwickelt Zofia innerhalb ihres Orientierungsschemas sehr explizite Vorstellungen von Verhaltensnormen innerhalb einer Paarbeziehung. Demnach sieht sie Verliebtsein als Grundvoraussetzung für das Eingehen einer Beziehung an. Im Verlauf ihrer Biografie und in Auseinandersetzung mit ihren verschiedenen Erfahrungen formuliert sie als weitere Grundvoraussetzung die gegenseitige Anerkennung, gegenseitigen Respekt sowie die Fähigkeit, Kompromisse einzugehen.

Der Ausgangspunkt ihrer biografischen Orientierung ist die Fokussierung auf die eigenen Steuerungsmöglichkeiten innerhalb von Beziehung sowie die Ausrichtung an einer diesbezüglichen persönlichen Weiterentwicklung. Dementsprechend ist das Zentrum des hier vorliegenden Orientierungsrahmens die konsequente Gestaltung der Beziehung, die nur über ausreichende Leistung zu vollbringen ist. Dabei ist das Ziel dieser Handlungspraxis das Erreichen einer eigenen Autonomie innerhalb einer gleichberechtigten und von Anerkennung geprägten Beziehung.

Zofia hat in ihrer Vorstellung, wie eine Beziehung funktionieren kann bzw. muss, einen Plan, nach dem sie ihr Handeln ausrichtet. Dabei begreift sie ihre Beziehungserfahrungen als einen Lernprozess, nach dem sie in jeder neuen Beziehung versucht, »etwas neu zu gestalten« (318). Gleichzeitig scheinen emotionale Bedürfnisse ihrerseits zurückgestellt werden zu müssen, was sie dann auch als Mangel innerhalb der Beziehung erlebt.

Deutlich dokumentiert sich somit die Vorstellung von >Beziehung als Arbeit<, als Leistung, als Gestaltung. Wird diese nicht ausreichend erbracht, kommt es innerhalb der Beziehung zu Problemen oder sie scheitert. Damit zeigt sich ein weiteres wesentliches Moment: Zofia sieht sich selbst als verantwortlich für das Scheitern oder Gelingen der Beziehung an. Hier dokumentiert sich die biografische Verwurzelung von dem Bedürfnis bzw. der Suche nach Anerkennung, die nur durch Erbringung von Leistung erfahrbar wird. 
Zofia zeigt damit auf der Ebene des kommunikativen Wissens, hinsichtlich ihres Orientierungsschemas, klare Vorstellungen von Beziehungen. Hier formuliert sie auch die Notwendigkeit eines autonomen Lebens, insbesondere in finanzieller und materieller Hinsicht. Auf der Ebene des Orientierungsrahmens, der die Bearbeitung und den Umgang mit den genannten normativen Vorstellungen von Beziehung aufzeigt, zeigt sich jedoch immer wieder die Abhängigkeit vom Partner als Teil der Handlungsorientierung. Dieser Orientierungsrahmen fungiert hier als Möglichkeitsraum, innerhalb dessen die Beziehungsgestaltung auf die Erfahrung von Einssein und Ganzheitlichkeit in der Beziehung zielt.

Des Weiteren zeigt sich das Thema Abhängigkeit innerhalb Zofia Biografie auch in Bezug auf Krankheit: So wurde deutlich, dass sich in der Essstörung und Alkoholsucht Abhängigkeitsverhältnisse widerspiegeln.

Gewalt(-Beziehungen) und Sozialisation Zofias Kindheit und Jugend ist durch eine Vielzahl von Brüchen geprägt. Als emigriertes Kind macht sie zunächst über einen langen Zeitraum die Erfahrung, als Außenseiterin wahrgenommen zu werden. Möglicherweise liegt hierin auch ein Teil ihres Normalitätsstrebens begründet. Mit dem Austritt aus dem Leistungssport, der mit dem Entzug der Anerkennung und Aufmerksamkeit des Vaters verbunden ist, zeigt sich bspw. dieses Streben nach Normalität. Auch die Entwicklung einer Essstörung kann spiegelbildlich für die konträren (gesellschaftlichen) Erwartungen, mit denen sie sich auseinandersetzen muss, angesehen werden.

Zofia zeigt in ihrem Streben nach Autonomie ein hohes Maß an Bildungsaspiration. Diese ist außerdem verbunden mit der Möglichkeit der Erfahrung von Selbstwert, den sie während ihrer Kindheit und Jugend nur erschwert aufbauen konnte. Die Anerkennung durch ihren Vater erfolgte nur in Abhängigkeit mit guter sportlicher Leistung; Emigration, Mobbing in der Schule sowie viele Umzüge erschwerten ebenfalls den Aufbau eines gesunden Selbstwerts.

Bezüglich generationaler Transmissionsprozesse zeigt sich bei Zofia aufgrund ihrer sozialisatorischen Erfahrung, in der sie ihre Mutter abhängig von ihrem Vater erlebte, eine starke Motivation, unabhängig, vor allem in ökonomischer Hinsicht, zu leben.

In ihren Paarbeziehungen ringt Zofia um ihre Autonomie und Gleichberechtigung. Über ihre Bildungsaspiration setzt sie ihr Ziel um, finanziell unabhängig zu bleiben. Allerdings gerät sie, insbesondere in ihrer letzten Beziehung, in eine emotionale Abhängigkeit, die dazu führt, dass das Gewaltgeschehen über einen langen Zeitraum auftritt. 


\subsubsection{Daria - "wenn er handgreiflich wird geht's schon mal gar nich« (587f.)}

\subsubsection{Fallporträt}

Daria wird in der Ukraine geboren und emigriert im Alter von drei Jahren mit ihren Eltern nach Deutschland, sodass sie sich nicht - oder kaum - an die Zeit dort erinnern kann. Daria besucht mit ihren Eltern in den Ferien alle zwei Jahre die Familie in der Ukraine. Sie hat insbesondere zu ihrer dort lebenden zwei Jahre älteren Cousine ein sehr enges Verhältnis. Die Familie wohnt zuerst bei Darias Oma, wo es aufgrund der großen Wohnung zunächst Platz für alle gibt. Anschließend erfolgen ein Umzug in eine nahe gelegene Stadt sowie ein weiterer Umzug innerhalb dieser Stadt. Daria hat keine Erinnerungen an die Zeit des Ankommens in Deutschland. An ihre Kindergartenzeit denkt sie mit gemischten Gefühlen. Nach der Grundschule wechselt Daria auf die Hauptschule. Wichtig für sie sind insbesondere ihre Freund_innen, mit denen sie Dinge unternimmt und sich allgemein viel mit ihnen draußen aufhält. Immer wieder kommt es auch zu Auseinandersetzungen mit Mitschüler_innen, mit denen sie sich prügelt. Als Daria 15 Jahre alt ist, trennen sich ihre Eltern und Daria bleibt bei ihrer Mutter. Sie hat weiterhin regelmäßig Kontakt zu ihrem Vater, sieht ihn häufig und telefoniert fast täglich mit ihm.

Nach der Trennung reduzieren sich die regelmäßigen Fahrten in die Ukraine. Erschwerend kommt hinzu, dass Darias Cousine inzwischen in Russland lebt und gemeinsame Treffen in der Ukraine aufgrund unterschiedlicher Urlaubsregelungen schwierig zu organisieren sind. Eine weitere Schwierigkeit besteht darin, dass Daria nicht mehr fließend russisch spricht, ${ }^{11}$ weshalb die Kommunikation mit ihrer Cousine über das Telefon erschwert wird. Dies führt zu einer Distanzierung in der Beziehung der beiden.

Daria wechselt nach der Hauptschule auf die Berufsschule, da sie noch schulpflichtig ist, und absolviert anschließend ein Praktikum von acht Monaten in einem Pflegeheim. Sie wird als Auszubildende übernommen und besucht die Gewerbliche Schule, wo sie eine Ausbildung zur Altenpflegerin beginnt.

Im Verlauf der gesamten Schulzeit ist für Daria das Thema Freundschaft zentral.

Mit 15 Jahren geht sie ihre erste Paarbeziehung ein. Nach etwa vier Monaten kommt es zu zwei Situationen, in denen Darias Freund ihr gegenüber körperlich gewalttätig wird. Sie trennt sich daraufhin von ihm, was er jedoch nicht akzeptiert. Er beginnt, sie massiv zu bedrohen und zu stalken. Etwa ein Jahr später enden die Bedrohungen.

Zum Zeitpunkt des Interviews ist Daria 18 Jahre alt und hat einen neuen festen Freund.

11 In der Ukraine wird sowohl Russisch als auch Ukrainisch gesprochen. 


\subsubsection{Analyse der Erzählstruktur}

Im Interview mit Daria gilt es, insbesondere den Einstieg ins autobiografisch-narrative Interview näher zu betrachten. Nach anfänglicher Herstellung von Sprachlosigkeit kann durch die konsequente Anwendung von narrativen Detaillierungsfragen die Aufhebung der Sprachlosigkeit unterstützt werden.

Die Interviewerin gibt zunächst einen offenen Erzählstimulus, indem sie die interviewte Person auffordert, ihre gesamte Lebensgeschichte zu erzählen. Die Interviewte fragt daraufhin nochmals rück und äußert, dass es ihr schwerfalle, zu beginnen.

I: Von daher würde ich dich jetzt einfach bitten, dass du mir deine ganze Lebensgeschichte erzählst,//okay//alles was dir dazu einfällt, und ich würde dich auch erst mal gar nicht unterbrechen ich mach mir en paar Notizen und komm darauf später zurück.

D: Okay also soll ich einfach erzählen

I: Alles was dir einfällt genau.

D: Okay des is schwer jetzt so@anzufangen@ sich weiß gar nicht was ich sagen solk//mmh//oaa (1) gut (3)) (1-11)

Diese Situation in Form eines Hin und Her, zwischen erneuter Aufforderung und dadurch erzeugter Sprachlosigkeit, dauert etwa zwei Minuten.

Viele Jugendliche, und vermutlich auch Daria, hatten noch nie die Gelegenheit, über ihre Biografie im Ganzen zu sprechen. Damit hatte sie auch nicht die Möglichkeit zu erfahren, dass andere sich für ihre Erfahrungen interessieren und diese für erzählenswert halten. Wurden Erfahrungen bislang niemandem erzählt und damit auch nicht sprachlich übertragen, können sie nur teilweise oder mühsam erinnert werden (vgl. Rosenthal u.a. 2006, S. 205).

In der vorliegenden Eingangssequenz fällt zunächst auf sprachlicher Ebene die Hilflosigkeit der Interviewerin auf, die sich durch die Wiederholung der Bitte, »alles« zu erzählen, zeigt. Die Intention der Interviewerin, einen möglichst offenen Rahmen zu gestalten, indem die Interviewte dazu angeregt wird, ganz nach ihren eigenen Relevanzsetzungen das Interview zu beginnen, fruchtet hier in keinster Weise. Im Gegenteil erzeugt die Interviewerin damit immer wieder neu die Sprachlosigkeit der Biografin.

Nachdem die Interviewerin erkannt hat, dass sie durch ihre offenen Erzählimpulse Sprachlosigkeit hervorruft, anstatt den Erzählbeginn zu unterstützen, ändert sie ihre Fragetechnik und stellt eine konkret formulierte Frage:

I: Mmh okay, ahm (2) gibt's (1) vielleicht ein Ereignis oder ne Situation wo du sagst des is des Früheste an was ich mich erinnern kann? (41-43)

Nach dieser Aufforderung, sich an das frühestmögliche Ereignis zu erinnern und von diesem zu erzählen, kann Daria ihre Erzählung beginnen. Auch im weiteren 
Verlauf des Interviews bewährt sich die konsequente Anwendung von Fragen, die zu Erzählungen von Lebensphasen oder einzelnen Situationen auffordern. Daria gewinnt dadurch den Eindruck, dass sich die Interviewerin tatsächlich für ihre Geschichte und ihre Erfahrungen interessiert und das Erzählte nicht negativ bewertet.

Bei der Betrachtung, wie Daria Themenabfolge und -darbietungen organisiert, fällt auf, dass sich drei große Themen im Interview finden lassen: Familie, Peers, Paarbeziehung. Zudem wird im Interview deutlich, dass ihre Perspektive sowie ihr Involvement ${ }^{12}$ im Laufe der Erzählung - je nach Thema - wechseln. Der Blick auf diese Wechsel ermöglicht eine Analyse des Zugangs zur subjektiven Bedeutsamkeit verschiedener Themen (Lucius-Hoene/Deppermann 2002, S. 228).

In bestimmten Passagen, in denen sie ihre Gewalterfahrung innerhalb der Paarbeziehung thematisiert, lässt sich zunächst festhalten, dass diese im Modus der Erzählung dargelegt werden:

jetzt zum Beispiel an seinem Geburtstag waren wir in so ner Bar und da bin ich ihm anscheinend a bissel zu laut geworden und dann hat er mich so am Hals gepackt und so gegen die Wand gedrückt (1) und hat halt gemeint ja ich soll jetzt endlich die Fresse halten, weil ich den, weil ich den nerv weil ich einfach viel zu laut bin//mmh//und ja: dacht ich so okay ich sag jetzt nix mehr und dann hab ich irgendwann mal gesagt er soll mich einfach loslassen, weil's mir einfach irgendwann zu viel geworden is, weil (1) ja:, aber des hat halt keiner gesehen gehabt des war so: (2) so ums Eck sozusagen, die saßen alle da vorne, da ging's so ums Eck und ich war dort, und dann hat er mich halt dort gegen die Wand so gedrückt und deswegen hat des keiner wirklich mitgekriegt (523-532)

Bereits in der Verwendung dieser Textsorte drückt Daria ihre subjektive Perspektive aus und stellt das Erlebte nicht nur deskriptiv dar. Bezüglich ihrer Perspektive und des Involvements zeigt sie aber eine hohe Distanzierung gegenüber ihrem erzählten Selbst und den geschilderten Erfahrungen. Dies zeigt sich in der Art und Weise, wie sie die Ereignisse rahmt, indem sie eher beiläufig und bagatellisierend über ihre Erfahrungen spricht. Die Szenen werden nur kurz und knapp erzählt. Emotionen seitens Daria werden nicht geschildert. Gleichzeitig kommt ihre implizite Bewertung des Verhaltens ihres Freundes zum Ausdruck und spiegelt ihre Positionierung als Erzählerin wider.

Im Gegensatz dazu findet sich in den Passagen zu gewaltaktiven Auseinandersetzungen in der Peergroup eine hohe persönliche und emotionale Beteiligung. Die Szenen werden ausführlich und detailliert dargestellt und zu einem gewissen Teil

12 »Involvement«(Tannen 1989) meint die dargestellte und zum Ausdruck gebrachte emotionale und vergegenwärtigte Beteiligung des Erzählers/der Erzählerin (vgl. Lucius-Hoene/Deppermann 2002, S. 228). 
affektiv nacherlebt, woraus eine Identifikation der Erzählerin mit dem früheren Selbst abgeleitet werden kann:

ich wa:r mal mit einer, also einer aus meiner Klasse, ah keine Ahnung die saßen so weit weg von uns und wir saßen auf der Treppe und dann hamn sie die ganze Zeit voll Krach gemacht und keine Ahnung so irgendwas so gegen so Holzdinger geschlagen, und dann hab ich erstmal gesagt so ja kannst du bitte mal aufhören weil des einfach aufgeregt hat, na hat die uns extra weiter provoziert und hat immer weitergemacht, na hab ich n paar Mal gesagt sa mal jetzt reicht's dann irgendwann mal und dann bin ich irgendwann mal auch ausgerastet, hab gesagt jetzt (1) also auf die () Art oder so sie soll jetzt damit aufhören//mmh//dann hat die angefangen irgendwie ja:: deine Mu::tter und bla:, hat irgendwie angefangen meine Eltern zu beleidigen und so (1) und dann ähm bin ich irgendwann mal ausgerastet und dann bin ich zu der hi:n und dann hab ich gesagt was soll die Scheiße und keine Ahnung, (1) dann sind wir durch die ganze Schulhaus gerannt, na hab ich irgenirgendwann mal am Ende des Tages meine Sachen im ganzen Schulhaus so eingesammelt, meine Ketten mein Schal alles was so irgendwie unterwegs alles so rumgeflogen is, //mmh//ja: da hamn wir uns schon ziemlich in die Haare gekriegt (298-313)

Grund für Darias unterschiedliche Handlungspraxis in Gewaltsituationen mit ihrem Freund bzw. mit Mitschülerinnen, die sich hier auf der Ebene der Darstellung wiederfinden lässt, ist möglicherweise, dass die erlebte Gewalt durch ihren Freund bislang nicht verarbeitet ist. In Darias Haltung wird deutlich, dass ihr Freund kein Recht hat, sie so zu behandeln. Dies kommt auch in der impliziten Bewertung zum Ausdruck. Dass er sie dennoch würgt und schlägt, wirkt auf Daria wie ein Schock, der bis heute nicht verarbeitet ist und sich hier auf sprachlicher Ebene zeigt. Im Gegensatz dazu sind die gewaltaktiven Auseinandersetzungen unter Mädchen für Daria Teil ihrer alltäglichen Normalität. Hier ist sie nicht in einer passiven, sondern in einer aktiven Rolle, in der sie selbst bestimmt, auch körperlich in Auseinandersetzung zu gehen. Damit sind diese Erlebnisse auch erzählbar(er).

In anderen thematischen Passagen zu Peers, in denen Daria über Erfahrungen innerhalb ihrer Peergroup berichtet, verschwindet die Ich-Perspektive teilweise komplett und sie berichtet ausschließlich aus der Perspektive des Wir der Gruppe. Dazu wurde die Interpretationshypothese entwickelt, dass Daria eine starke Orientierung nach außen zeigt.

Sie sieht sich als Teil der Klasse, des sozialen Zusammenhangs, in dem sie sich bewegt, und weniger als eigenständige Person.

\subsubsection{Herkunftsfamilie und Bedingungen des Aufwachsens}

Daria wird in der Ukraine geboren und wächst dort die ersten drei Lebensjahre mit ihren Eltern auf. Sie hat keine Geschwister. Als Daria drei Jahre alt ist, emigrieren 
ihre Eltern nach Deutschland. An diese Zeit kann sie sich kaum erinnern. Daria besucht mit ihren Eltern regelmäßig im Sommer die Familie in der Ukraine.

\section{Ukraine}

${ }^{\circ}$ ja: ${ }^{\circ}(3)$ ja die Zeit dort war schon schö:n (1) $/ / \mathrm{mmh} / /^{\circ} \mathrm{ja}$, is ${ }^{\circ}$ nich so wie hier da kennt sich jeder//mmh//und da (2) da sammelt man sich halt a-irgendwie an einem Tisch oder so//mmh//und dann macht man halt da seine Party oder so//mmh//da geht man nich so weg//mmh//so wie hier//mmh//(1) ${ }^{\circ}$ 's viel besser ${ }^{\circ} / / j a / /(4){ }^{\circ} \mathrm{ja}^{\circ}$ (87-91)

In dieser Bewertung, in der Daria das Leben in der Ukraine mit dem Leben in Deutschland vergleicht, werden ihrerseits eine Abgrenzung von Deutschland und eine Zugehörigkeit zu formuliert. Die positive Bewertung gründet auf dem sozialen Zusammenhalt, den Daria dem Leben in der Ukraine zuschreibt, im Unterschied zum Ausgehen in Deutschland, das für sie mit einer höheren Unverbindlichkeit und Anonymität verbunden ist.

Hier zeigt sich bereits, was im Verlauf der Interviewanalyse noch deutlicher markiert werden wird: Für Daria sind der soziale Zusammenhalt und das Aufgehobensein in einer sozialen Gruppe elementar.

Darias Erzählung über die in der Ukraine verbrachten Ferien und die Beziehung zu ihrer Cousine bestätigen Darias Bedürfnis nach Zugehörigkeit, die sich hier innerhalb familialer bzw. verwandtschaftlicher Beziehungen dokumentiert:

\section{Ferien in der Ukraine}

Also meine Cousine is (1) zwei: Jahre älter als ich und, aber des war jetzt nich so des Problem dass sie dann gesagt hat ne ich bin da mit meinen Freunden und du kannst dir dann welche in deinem Alter suchen so war des nich also wir waren immer zusammen, haben irgendwas zusammen gemacht weil wir uns sowieso so selten sehen und dann haben wir öfters auch mal bei Freunden im Ga:rten geschlafen oder so und haben dort unsere eigene@Party@gemacht//mmh//und ja:mit meiner Cousine versteh ich mich, (110-117)

Der Verweis darauf, dass ihre Cousine zwei Jahre älter ist als sie selbst, und der anschließende argumentative Einschub, dass der Altersabstand kein Problem sei, in dem Sinne, dass ihre Cousine deshalb möglicherweise nichts mit ihr zu tun haben wolle, zeigen Darias Sorge, möglicherweise nicht dazuzugehören. Im Gegenteil waren die beiden »immer zusammen « und haben alles gemeinsam unternommen, wenn sie die Ferien miteinander verbracht haben. Der Verweis auf gelegentliche Übernachtungen bei Freunden im Garten, mit denen eine »eigene @Party@« veranstaltet wird, greift die oben beschriebene Handlungspraxis auf, nach der in der Ukraine eigene Feste und Partys initiiert werden, die bei Freund_innen zu Hause 
oder in deren Garten stattfinden und damit einen engen sozialen, familiären Kontext darstellen. Des Weiteren kommt in dieser Beschreibung auch zum Ausdruck, wie positiv Darias Erinnerungen an diese Zeit sind.

In dieser Gegenüberstellung von Unternehmungen und Partys im privaten Umfeld in der Ukraine versus den in Deutschland stattfindenden Veranstaltungen im öffentlichen Bereich, dokumentiert sich Darias eigene Verortung im familiären Raum.

Ja: also in die Ukraine gehen wir eigentlich se:Iten [...] uuaa ja und meine Cousine seh ich dann jetzt wahrscheinlich mein Gott in zwanzig Jahren wieder oder so (1) $(65-74)$

Daria beschreibt, dass sie inzwischen nur noch selten in die Ukraine fahren. Nach der Scheidung ihrer Eltern fährt sie nur noch mit ihrer Mutter dorthin, wobei diese inzwischen ungern in die Ukraine reist, da es schwierig ist, eine zeitliche Passung zwischen den Urlauben der Mutter, der Oma in der Ukraine und der inzwischen in Russland lebenden Cousine zu finden. Daria bedauert dies. Außerdem geht sie von keiner Veränderung des momentanen Zustandes aus.

\section{Sprachbarrieren}

ein Problem is halt dass ich nich mehr so gut Russisch kann@(.)@ (ja) deswegen können wir auch//okay//nich so, also ich mag des dann nich zu telefonieren mit ihr zum Beispiel, weil ich des einfach nicht mehr so gut kann wie früher,//mmh//außer wenn wir jetzt in die Ukraine gefahren sind so nach ner Woche oder so konnt ich des dann schon wieder richtig//mmh//aber so:: jetzt, zum Telefonieren oder so wenn ich Geburtstag hab dann rufen die mal an und dann weiß ich gar nicht was ich sagen soll, dann sag ich immer nur »ja: mmh okay« und dann leg ich @immer auf@oder so (120-128)

Erschwert wird der Kontakt u.a. durch die reduzierten Besuche in der Ukraine, was wiederum dazu führt, dass Daria zunehmend weniger Russisch spricht, was für sie die Kommunikation mit ihrer Cousine, bspw. über das Telefon, erheblich erschwert.

Die erste Zeit in Deutschland ist geprägt durch einige Umzüge. Daraus lässt sich ableiten, dass Daria mehrmals mit Abschiednehmen und Eingewöhnungen in ein neues Umfeld konfrontiert wird. Wie sie damit umgeht, bleibt allerdings offen, da sie hierzu nichts weiter thematisiert. Als Zwischenevaluation hält sie fest »am Anfang war alles okay« (145). Die Scheidung der Eltern bedeutet die Zäsur dieses Zustandes. Der Vater zieht aus, während Daria bei der Mutter bleibt. 


\section{Kindergarten I}

ja also die Kindergartenzeit daran kann ich mich schon erinnern weil ich dann in der Grundschule auch wieder fast mit den gleichen Leuten zusammen war, dann in der Hauptschule waren's wieder die gleichen Leute und also irgendwie waren wir immer alle zusammen//okay//also vom Ki-Kindergarten an bis zu:r Grundschule dann bis zur Hauptschule wieder//mmh//und ja:: so Kindergarten und ${ }^{\circ} \mathrm{ja}{ }^{\circ}$ (1) gab’s schon so gute und schlechte Zeiten (156-161)

An die Zeit im Kindergarten kann sich Daria gut erinnern, weil sie die Zeit dort mit den gleichen Kindern verbringt, mit denen sie dann auch in der Grundschule und später in der Hauptschule ist. Das verbindende Moment hier in der Beschreibung der gesamten Kindheit, sind die "gleichen Leute«, also die anderen Kinder, die Peers, die Freund_innen, mit denen hier die institutionellen Abläufe gemeinsam durchlaufen und geteilt werden.

Hier zeigt sich erneut die Bedeutung von Zugehörigkeit bzw. der Beziehung zu den Peers für Daria.

\section{Kindergarten II}

bei uns wars jetzt nich so gut, wei:I (1) die immer ein gezwungen habn dass du isst und wenn du ss jetzt nicht isst dann darfst du nicht nach Hause gehen//mmh//und, dann kam auch oft meinen Mutter und hat gemeint ja wenn sie des jetzt nich essen will dann kann sie ja wohl gehen und dann//mmh//gabs immer so Stress also bei uns warn die da schon so (2) streng im Kindergarten//mmh//so von wegen du musst jetzt essen (1) (166-172)

Es gibt auch negative Erinnerungen an die Kindergartenzeit, die sich auf das Erziehungsverhalten der Erzieherinnen und die Interaktion mit diesen beziehen. Daria schildert hier eine sich wiederholende Situation, die ihr eindrücklich in Erinnerung geblieben ist. In der Schilderung wird deutlich, dass die Essenssituationen für Daria mit Zwang und Drohungen verbunden sind. Positiv erlebt sie hier ihre Mutter, die sie als unterstützend und zu ihr haltend erfährt.

Über die Grundschulzeit berichtet Daria, sie sei "ganz oka:y« (173) gewesen. Mit Beginn der Hauptschule verändert sich allerdings die bis dahin unbeschwert scheinende Zeit.

\section{Hauptschule}

erst in der Hauptschule ist es dann so geworden, also da gab es, schon so, also es gab auch Momente wo wirklich die ganze Klasse gegen einen war, und (1) weil dann Leute immer gemeint haben sie müssten irgendwas über einen erzählen und dann hieß es ja der hat gelo:gen und des stimmt nich und dann warn die immer 
gegen ein (1)//mmh//u:nd (2) ja:, dann gab's auch wieder Tage wo dann irgendwie alle wieder zusammengehalten haben, (2) und dann, oder wo sich die Klasse zweigeteilt hat und dann gab's auch voll den Krieg, (174-180)

Daria macht hier offensichtlich neue Erfahrungen der Ausgrenzung bzw. des Ausgegrenzt-Werdens und begründet das Entstehen solcher Situationen damit, dass andere Lügen über sie verbreiten. Im Gegenzug dazu gibt es aber auch Phasen, in denen sie sich in der Klassengemeinschaft aufgehoben fühlt. Wie sich Daria innerhalb der Klasse im Umgang mit diesen Konflikten verhält, wird im Interview nicht deutlich.

In dieser Erzählung zeigt sich, wie Daria innerhalb des Umbaus der sozialen Beziehungen während des frühen Jugendalters Beziehungsfähigkeit testet, indem sie soziale Beziehungen aufnimmt, aufrechterhält und ggf. wieder aufgibt. Dabei spielt die Cliquenzugehörigkeit im innerschulischen Bereich für sie eine große Rolle.

Gegen Ende der Hauptschulzeit kommt es zur Trennung der Eltern:

\section{Scheidung der Eltern}

ja: als meine Eltern sich scheiden lassen hamn war des für mich schon hart//mmh//(2) des is jetzt drei Jahre her//mmh//(2) ${ }^{\circ}$ ja des is nich so lange her ${ }^{\circ} / / \mathrm{mmh} / /$ und ja des war für mich echt hart am Anfang aber (1) is irgendwo auch besser so weil, die haben sich ne Zeitlang einfach nur noch gestritten (2)//mmh//und des hat mich dann halt auch genervt weil ich mir dann immer des Geschrei anhören musste (226-231)

Hinsichtlich ihrer Bewertung der Trennung äußert sich Daria ambivalent. Einerseits beschreibt sie, dass die Zeit zu Beginn »echt hart « war, andererseits findet sie die Trennung auch gut, da ihre Eltern viel gestritten haben. Dass sie diesen Streitigkeiten nicht mehr ausgesetzt ist, empfindet sie als entlastend. Daria wohnt seither bei ihrer Mutter und beschreibt, einen guten Kontakt zu ihrem Vater zu haben. Sie sieht ihn häufig und telefoniert fast täglich mit ihm, was auf ein gutes VaterTochter-Verhältnis hindeutet.

Ihr Verweis »deswegen geht's ${ }^{\circ}$ eigentlich ${ }^{\circ}$ « (234) zeigt, dass die Situation insgesamt für sie inzwischen gut tragbar ist. Daria schließt die Sequenz mit der Bemerkung $a b$, dass sich auch ihre Eltern inzwischen wieder gut verstehen. 


\subsubsection{Kernthemen des Interviews ${ }^{13}$ \\ Peers}

In Darias Erzählungen über ihre Schulzeit fällt auf, dass sie diese durchgängig mit dem Fokus auf Freundschaftsbeziehungen darstellt. So bezieht sie sich zunächst in ihrer Erzählung zur Hauptschulzeit auf die »Abschluss(-zeit) und die Feier und die Fahrt« (55), wodurch der Zusammenhalt der Klasse betont wird.

\section{Abschlussfahrt nach Tschechien}

dann aber am Ende des Jahr- also am Ende der neunten Klasse dann, als wir nach

(1) Tschechien gefahren sind, (1)//mmh//da wars dann wieder besser (1)//mmh//ja da hamn wir alle zusammen mal gefeiert und so und ja:, also des war schon gut (1)//mmh//(1) des würd ich gern nochmal'@machen@//mmh//mit der Klasse, also des war schon ganz gut//ja//was Schönes (3) (181-185)

Die vor Beginn der Abschlussfahrt stattfindenden Konflikte innerhalb der Klasse spielen nun kaum mehr eine Rolle, da vermutlich angesichts des Schulendes und des damit verbundenen Neubeginns die Klassengemeinschaft enger erlebt wird.

Die positive Bezugnahme auf die soziale Gemeinschaft in der Klasse setzt sich auch in der Darstellung auf die Zeit in der Gewerblichen Schule fort, die Daria zum Zeitpunkt des Interviews besucht. In der Aussage »da: (1) war's auch am Anfang so da hamn sich erst mal alle voll gut verstanden« (186f.) wird dies ebenfalls deutlich. Daria beschreibt hier ihre momentane Gefühlslage als »traurig« (57), da »jetzt alle gehen« (58). Hier wird ein relevantes Thema deutlich: nämlich Abschied nehmen und das Ende von gemeinsam verbrachter Zeit mit anderen. Es dokumentiert sich, wie oben bereits angedeutet, die Bedeutung eines positiven Zusammenhalts unter Freund_innen für Daria.

\section{Gruppenbildung (Gewerbliche Schule)}

dann gab's halt auch mal so mit der Klasse Stress weil (1) di:e so, ss war auch so zwei Cruppen, hier warn die einen und da die anderen und des kam uns dann immer so vor als ob die einen über die anderen lästern und dann gab's halt auch voll

13 Im Unterschied zu den Falldarstellungen der anderen Interviews, in denen die Kernthemen des Interviews überwiegend in chronologischer Reihenfolge des biografischen Verlaufs dargestellt werden, wird in diesem Kapitel eine andere Form gewählt: Die Präsentation der Inhalte erfolgt anhand einer thematischen Bündelung. Grund dafür ist, dass es beim vorliegenden Interview zu keiner biografischen Stegreiferzählung kam (vgl. Analyse dazu in Kap. 7.1.4.2 Analyse der Erzählsituation) und die Erzählerin insgesamt wenig über den biografischen Verlauf erzählt. Deshalb wurden die zentralen Themen, die die Erzählerin setzt, aufgegriffen und schwerpunktmäßig in die Analyse einbezogen. 
den Streit, und (1) irgendwann mal stand eine ganz alleine da weil sie die-, wegen ihr die ganze Scheiße passiert is und dann hamn die die alle voll, was heißt fertiggemacht, wir hamn ihr halt die Meinung gesagt und die hat gemeint ja des juckt mich alles nich und stand halt da und hat so getan als ob sie nicht interessiert (1) hat halt aber irgendwann mal angefangen zu heu:len und (1) keine Ahnung//mmh//(2) aber dann nach ner Zeit hamn wir des auch wieder vergessen und dachten ja mein Cott $^{\circ}$ was solls so isses halt $/ / \mathrm{mmh} / / \mathrm{ja}$ : und jetzt halt traurig dass (2) die Hälfte weg is (2)//ja//ja: (2) (200-210)

Während der Zeit auf der Gewerblichen Schule spielt die Cliquenbildung innerhalb der Klasse eine große Rolle: Es kommt zu Konflikten in der Klasse, die sich in zwei rivalisierende Gruppen teilt.

Daria schildert dazu eine konkrete Situation, in der eine Mitschülerin, die sie als Verantwortliche für die stattgefundenen Streitigkeiten und Auseinandersetzungen sieht, dann »irgendwann mal [...] ganz alleine« dastand und fertiggemacht wurde. In Darias Darstellung des anderen Mädchens, die sie in Distanz zu sich selbst setzt, dokumentiert sich Darias eigene Positionierung innerhalb ihrer Gruppe, wobei sie froh zu sein scheint, dem Gros der Klasse anzugehören.

\section{Unwichtige Leute}

@(.)@unwichtig (2) ja:, also mir sind Leute unwichtig die nich ehrlich sind//mmh//davon gibt's ja genug und die so, also die halt einfach falsch sind, die dann (1) vor dir so tun als ob des so voll die Freunde sind und hinter deinem Rücken halt schlecht über einen reden (1)//mmh//sind mir sehr@unwichtig@//mmh//davon hat's auch n paar, [...] und dann stehst du einfach da und fühlst dich so=(plötzlich) sind alle gegen dich//mmh//wo du gar nicht weißt um was es geht (241-253)

Daria greift hier aus der Nachfrage der Interviewerin, die nach Wichtigem und Unwichtigen in Darias Biografie fragt, den Aspekt Unwichtiges heraus und erklärt, ihr seien »Leute unwichtig, die nich ehrlich sind«. Sie führt aus, bereits häufiger Stress mit anderen gehabt zu haben, die hinter ihrem Rücken Unwahrheiten über sie erzählt haben. Dabei fällt erneut das Fehlen der Ich-Perspektive auf; stattdessen spricht Daria hier in der zweiten Person Singular über ihre Gefühle (»dann stehst du einfach da und fühlst dich so«). Diese Erfahrung ist für Daria mit Gefühlen von Ausgrenzung und Orientierungslosigkeit verbunden.

Hinsichtlich Darias Orientierungsschemas wird deutlich, dass sie konkrete Vorstellungen vom Umgang innerhalb von Freundschaften hat und Ehrlichkeit dabei für sie eine große Rolle spielt. 
Gewaltaktive Auseinandersetzungen unter Mädchen

\section{Schlägereien auf der Hauptschule}

also in der Hauptschule hab ich mich jetzt auch Mal, n paar Mal geschlagen @(.)@ mit $n$ paar aus meiner Klasse, aber des war halt einfach weil (1) zu viele Mädchen und dann gab's immer voll den Zickenkrieg und dann hamn die einen so voll die Scheiße angefangen zu erzählen und, (1) ja:: des hat alles überhaupt nicht gestimmt obwohl die des eigentlich alle selber so gesagt hamn über die eine Person, und dann, ja: gab's halt immer so Stress und irgendwann mal hat's, hat es einfach mit ner Schlägerei geendet//mmh//und ja::, hat ich auch Elterngespräche//mmh//und alles und (1) ja: is halt immer so (2)//mmh//(2) ah ich glaub des hat jeder schon mal durchgemacht @sowas@ also jetzt vielleicht nich unbedingt mit ner Schlägerei aber $/ / \mathrm{mmh} / /^{\circ}$ einfach mit dem ganzen Zicken- (1) terror $^{\circ}$ (258-269)

Auf Nachfrage der Interviewerin, ob sie eine konkrete Situation dazu schildern könne, beschreibt Daria Situationen auf der Hauptschule, in denen sie sich mehrfach mit anderen Mädchen geprügelt hat. Als Grund für die Schlägereien führt sie an, dass »zu viele Mädchen« da sind, was immer zu »Zickenkrieg« führt, der dann in einer Schlägerei endet.

Für Daria sind Schlägereien als Folge von Meinungsverschiedenheiten unter Mädchen normal. Diese Normalität kommt auf sprachlicher Ebene wie folgt zum Ausdruck: Nach Darias Auffassung kennen alle Mädchen Situationen, in denen sich Mädchen gegenseitig stressen (»Zicken- (1) terror«), und nicht alle, aber doch manche dieser Situationen enden auch bei anderen - und nicht nur bei Daria - in körperlichen Auseinandersetzungen.

In Bezug auf den unterliegenden Orientierungsrahmen zeigt sich hinsichtlich Darias Handlungspraxis eine gewisse kämpferische Perspektive, aus der heraus sie sich gegenüber Herabsetzungen wehrt bzw. ihren eigenen Ruf oder den ihrer Freundinnen verteidigt.

\section{Drohung eines Mädchens}

ähm da war ja, also so ne Grenze zwischen Hauptschule und da war die Sonderschule,//mmhh//und wir durften ja nie rüber, [...] und da hat mal eine aus der Sonderschule gemeint ja ich mach mich die ganze Zeit anscheinend an ihren Freund ran und was weiß ich was und dann dacht ich so hä ne eigntlich ja nich, und da gabs halt dann voll den Stress und dann war, hat die mir die ganze Zeit gedroht so von wegen wenn ich dich mal seh und dann hau ich dir eine rein und keine Ahnun-, aber dazu is es nie gekommen//mmhh//und mittlerweile versteh ich mich eigentlich auch (1) recht gut mit ihr, und hab dann auch gesagt also ne eigentlich stimmt des ja nich//mmh//und jetzt is es ihr eigentlich auch egal,//mmh//des war halt damals so da waren wirnoch@klein@(275-288) 
Daria erzählt hier eine konkrete Situation, als sie auf der Hauptschule ist und von einer anderen Schülerin einer benachbarten Schule vorgeworfen bekommt, sie "mache« sich an deren Freund »ran«. Daraus folgt "voll Stress« zwischen beiden Mädchen und Daria wird von dem anderen Mädchen bedroht. Hier dokumentiert sich, dass Daria schnell in Situationen gerät, in denen Mädchen sich stressen bzw. streiten. Genauso schnell, wie der Konflikt entsteht, scheint er sich aber auch wieder aufzulösen, und häufig vertragen sich die Mädchen dann auch wieder. Die beschriebene Situation deutet auf ein Umfeld, in dem eine gewisse Gewaltbereitschaft vorhanden ist. Deutlich wird dies auf sprachlicher Ebene (»wenn ich dich mal seh und dann hau ich dir eine rein«), die eine aggressive Grundhaltung erkennen lässt.

In einer eigentheoretischen Argumentation erklärt sich Daria die Konflikte auch damit, dass sie noch »@klein@«waren, woraus sich folgern lässt, dass sie diese Form der körperlich ausgetragenen Konflikte einer bestimmten Phase der Pubertät zuschreibt, die mit zunehmendem Alter und persönlicher Entwicklung nachlässt.

\section{Gewaltaktive Auseinandersetzung I}

Ja:: ähm (1) ich wa:r mal mit einer, also einer aus meiner Klasse, ah keine Ahnung die saßen so weit weg von uns und wir saßen auf der Treppe und dann hamn sie die ganze Zeit voll Krach gemacht und keine Ahnung so irgendwas so gegen so Holzdinger geschlagen, und dann hab ich erstmal gesagt so ja kannst du bitte mal aufhören weil des einfach aufgeregt hat, na hat die uns extra weiter provoziert und hat immer weitergemacht, na hab ich n paar Mal gesagt sa mal jetzt reicht's dann irgendwann mal und dann bin ich irgendwann mal auch ausgerastet, hab gesagt jetzt (1) also auf die () Art oder so sie soll jetzt damit aufhören//mmh//dann hat die angefangen irgendwie ja:: deine Mu::tter und bla:, hat irgendwie angefangen meine Eltern zu beleidigen und so (1) und dann ähm bin ich irgendwann mal ausgerastet und dann bin ich zu der hi:n und dann hab ich gesagt was soll die Scheiße und keine Ahnung, (1) dann sind wir durch die ganze Schulhaus gerannt, [...]//mmh//ja: da hamn wir uns schon ziemlich in die Haare gekriegt//mmh//und dann, (1) ja: dann durften wir erstmal nich in Unterricht rein, (mussten wir) uns irgendwie erstmal, also versuchen darüber zu re:den, des hat aber nich geklappt, des war auch klar//mmh//dass des nicht klappt (298-316)

Daria berichtet von einer weiteren Situation, in der sie sich von einer Mitschülerin provoziert fühlt und, nachdem sie diese mehrfach dazu aufgefordert hat, aufzuhören, sich mit ihr "ziemlich in die Haare« kriegt. Die Schule reagiert zunächst mit Unterrichtsverbot und fordert die beiden Mädchen auf, den Konflikt zu besprechen, was jedoch nicht funktioniert. 
Daria ist, nach mehreren Versuchen, den Konflikt verbal zu klären, durchaus bereit, sich körperlich mit dem anderen Mädchen auseinander zu setzen. Für sie - und ihr Umfeld - scheinen solche Situationen und der beschriebene Umgang damit normal, fast alltäglich zu sein. Eine Konfliktklärung scheint ihr unwichtig oder egal zu sein (»des hat nicht geklappt, des war auch klar«). Auch als die Schule um ein Elterngespräch bittet, scheint Daria gelassen zu bleiben. Hier zeigt sich, dass Daria für die Reaktionen der Schule kein Verständnis hat bzw. diese für sie nicht nachvollziehbar sind (»ja was sollen meine Eltern groß zu, oder dazu sagen, ja: is halt passiert «(317f.). Möglicherweise verurteilen Darias Eltern die gewaltaktiven Auseinandersetzungen ihrer Tochter nicht; oder sie bewerten sie durchaus als negativ, handeln dies aber mit ihrer Tochter allein und nicht im Kontext der Schule aus. Letzteres würde für eine Haltung der Eltern sprechen, die ihre Tochter als Person unterstützt, nicht aber deren Verhalten. In jedem Fall scheint Daria keine allzu großen negativen Erziehungskonsequenzen seitens ihrer Eltern zu befürchten. Damit dokumentiert sich erneut, dass Daria ihr eigenes Verhalten als normal und angemessen bewertet.

\section{Gewaltaktive Auseinandersetzung II}

ja: und dann (1) mit ner anderen auch mal ähm (2) keine Ahnung also die hatte mit einer aus unserer Klasse Stress und irgendwie stand ich dann eher zu ihr weil ich mehr Kontakt zu ihr hatte und dann hat sie angefangen mich zu beleidigen, hab ich natürlich zurückbeleidigt so wie des halt meistens is, dann sind wir mitten im Unterreicht irgendwie aufeinander los und dann ist die@Lehrerin@ dazwischen, hat die auch noch eine abgekriegt (1) des war halt dann schlecht für sie dass sie dazwischengegangen is@(.)@mmh aber des war halt so, des hat, du hast ga:r nichts gesehen, weil alle so, sind oben drauf gesprungen und wollten uns auseinandernehmen//mmh//und dann ist die Lehrerin noch dazwischen ang-, sind nur noch Fäuste geflogen und dann hat die halt auch eine abbekommen (319-330)

In der folgenden Situation bestätigt sich Darias Haltung, in Stresssituationen auch schnell mal gewaltaktiv zu (re-)agieren: Während des Unterrichts beginnt sie eine Schlägerei mit einer Mitschülerin. Dass die Lehrerin einen Schlag abbekommt, als sie die Mädchen trennen möchte, beschäftigt Daria nicht weiter (»des war dann halt schlecht für sie dass sie dazwischengegangen ist«).

Daria scheint Konflikte aus der Perspektive des Ehrkampfes zu deuten, wonach »der Einsatz physischer Gewalt ein angemessenes Mittel darstellt, um ihren Durchsetzungswillen zu demonstrieren und die verletzte Ehre wiederherzustellen « (Equit 2011, S. 11). Gewalt wird dabei positiv bewertet und als gewaltaffiner Ehrenkodex bezeichnet. Dabei werden die kollektiven Normen und Werte der Peergroup geteilt. Es kann davon ausgegangen werden, dass die von Mädchen ausgeübte Ge- 
walt in den Peer-Kontexten eine anerkannte Form weiblichen Durchsetzungsvermögens darstellt (vgl. ebd.).

Dass sich Daria heute mit Mitschüler_innen gut versteht, mit denen sie früher Ärger hatte, zeigt erneut die entdramatisierende Haltung Darias gegenüber den stattgefundenen Auseinandersetzungen.

Insgesamt dokumentiert sich in dieser Sequenz Darias Bereitschaft, Konflikte auch gewaltaktiv auszutragen, wenn sie sich provoziert fühlt oder beleidigt wird. Für sie ist es auch als Mädchen selbstverständlich, sich zu prügeln. Ihrer Handlungspraxis liegt ein Ehrenkodex zugrunde, der kollektiv von der Peergroup geteilt wird. Diesem zufolge stellt der Einsatz physischer Gewalt einerseits ein anerkanntes Mittel dar, um verletzte Ehre wiederherzustellen andererseits wird er als anerkannte Form weiblichen Durchsetzungsvermögens angesehen.

Der erste Freund

\section{Beginn der Beziehung}

als ich mal mit m- meinem Ex-Freund zusammen ${ }^{\circ}$ war $\% / / m m h / /$ des war jetzt nich so: ne tolle Zeit//mmh//, am Anfang war alles so schön und gut und, so die ersten Monate aber dann ja: hat's, (2) hat's einfach gar nich mehr geklappt wir hamn uns einfach, wir hamn nur noch gestritten und (1) es hieß dann jedes Mal ja kannst du kommen kannst du kommen ich musste immer von hier bis nach Stadt 1 fahren, ist ja schon ziemlich weit weg das heißt über Stadt 2 und was weiß ich $/ / \mathrm{mmh} / /$ und irgendwann mal is es halt auch a- arsch teuer geworden weil jedes Mal mit dem Zug da hinzufahren und dann ja:, keine Ahnung gab's halt auch oft Streit und, (1) (514-523)

Daria beginnt die Erzählung zur Beziehung mit ihrem Ex-Freund bagatellisierend mit dem Duktus der Beiläufigkeit (»mal«). Bevor sie auf die Beziehung näher eingeht, bewertet sie in einem Abstract die gesamte Beziehung als "nich so: ne tolle Zeit«.

Den Beginn, die ersten Monate der Beziehung, beschreibt Daria allerdings als »schön und gut« bis es dann »nich mehr geklappt« hat. Es kommt zu vielen Streitereien. Zudem fragt ihr Freund, der recht weit weg wohnt, Daria oft, ob sie ihn besuchen kommt. Darias Bemerkung »ich musste immer von hier bis nach Stadt 1 fahren « macht eine gewisse Schieflage in der Beziehung deutlich. Sie ist diejenige, die den weiten Weg auf sich nimmt, um sich treffen zu können, was zudem noch recht viel Geld kostet. Aushandlungen scheinen nicht möglich; stattdessen ist sie diejenige, die das Gefühl hat, fahren zu müssen.

Hier dokumentiert sich Darias Bereitschaft, zunächst in die Beziehung zu investieren, indem sie zum größten Teil die Fahrten auf sich nimmt. Es scheint keine gleichberechtigte Besuchspraxis stattzufinden; stattdessen nimmt Daria viel Zeit und Geld in Kauf, um Treffen mit ihrem Freund zu ermöglichen. Demnach 
scheint es auch keine Aushandlungspraxis zu geben, wie ein gleichberechtigtes Beziehungsmodell aussehen kann. Damit deutet sich bereits in den Anfängen der Beziehung ein Ungleichgewicht auf Kosten Darias an, an dem sie sich zwar stört, das sie aber trotzdem akzeptiert. Weiter wird deutlich, dass sie sich, in dieser Absicht, in die Beziehung zu investieren, den Wünschen ihres Freundes unterordnet.

\section{Gewaltsituation Kneipe}

jetzt zum Beispiel an seinem Geburtstag waren wir in so ner Bar und da bin ich ihm anscheinend a bissel zu laut geworden und dann hat er mich so am Hals gepackt und so gegen die Wand gedrückt (1) und hat halt gemeint ja ich soll jetzt endlich die Fresse halten, weil ich den, weil ich den nerv weil ich einfach viel zu laut bin//mmh//und ja: dacht ich so okay ich sag jetzt nix mehr und dann hab ich irgendwann mal gesagt er soll mich einfach loslassen, weil's mir einfach irgendwann zu viel geworden is, weil (1) ja:, aber des hat halt keiner gesehen gehabt des war so: (2) so ums Eck sozusagen, die saßen alle da vorne, da ging's so ums Eck und ich war dort, und dann hat er mich halt dort gegen die Wand so gedrückt und deswegen hat des keiner wirklich mitgekriegt//mmh//sonst denk ich wären's schon, also war auch ne Freundin von mir dabei die wär auf jeden Fall dazwischengegangen ((schluckt)) (1) (523-534)

Nach Darias Hinweis, es habe zunehmend Streit mit ihrem Ex-Freund gegeben, schildert sie eine konkrete Situation, die sie exemplarisch für solche Auseinandersetzungen anführt: Daria begeht den Geburtstag mit ihrem Ex-Freund und einigen Freund_innen in einer Bar. Sie beschreibt, sie sei ihrem Freund zu laut gewesen, was diesen zudem genervt habe. Außerhalb des Sichtbereichs der anderen packt er Daria am Hals und drückt sie gegen die Wand. Darias erste Reaktion ist, sich vorzunehmen, nichts mehr zu sagen und damit den Wünschen ihres Freundes, leiser zu sein, zu entsprechen. Die Situation scheint anzudauern bzw. in Daria Erinnerung spitzt sie sich zu, denn sie erwähnt »dann hab ich irgendwann mal gesagt er soll mich loslassen, weil's mir einfach zu viel geworden is«.

Bezüglich des hier unterliegenden Orientierungsrahmens dokumentiert sich ein Modus der Fokussierung auf die verbale Ebene zur Lösung der Situation. Daria gelingt es, über den Weg der Kommunikation aus der Situation herauszukommen. Dabei fällt auf, dass sie das Verhalten ihres Freundes nicht wertet. Gleichzeitig wird aber deutlich, dass sie um die ihr widerfahrene Grenzverletzung weiß. Dies wird an ihrem Versuch, das Verhalten ihres Freundes zu erklären, deutlich: Dass sie »anscheinend a bissel zu laut geworden« ist, impliziert ihren Zweifel an der Berechtigung dieses Motivs, das eher als Alibi-Erklärung angesehen werden kann. In dieser Begründung dokumentiert sich auch deutlich Darias Unrechtsbewusstsein bzw. die Bewertung des Übergriffs. 
Hinsichtlich der Dynamik innerhalb der Beziehung zeigt sich außerdem die Zunahme von Macht und Gewalt innerhalb der Beziehung, indem Darias Freund sie hier auch körperlich angreift.

Ein weiteres wichtiges Thema in dieser Passage ist die Rolle der Freund_innen. Daria ist sich sicher, dass ihre Freundin ihr geholfen hätte, wenn diese den Übergriff mitbekommen hätte. Dass sich Daria der Hilfe ihrer Freundin sicher ist, zeigt deren Bedeutung für Daria - im Sinne der Beistandschaft und des möglichen Schutzes. In ihrer Vorstellung kann sie sich gemeinsam mit der Freundin gegen den Übergriff ihres Freundes wehren.

Weiter wird deutlich, dass sich die Gewaltsituation im öffentlichen Raum ereignet, der hier keinen Schutz bedeutet. Auch Freund_innen, die nicht unmittelbar in der Situation dabei sein, sich aber in der Nähe befinden, bedeuten keinen Schutz.

\section{Gewaltsituation Auto}

und (1) dann warn wir auch mal mim Auto unterwegs und wir saßen hinten, also ich saß hinten und er saß vorne und dann hab ich ihn die ganze Zeit gerufen und er hat mich nich gehört irgendwie, dann hab ich dem so ähm auf die Schulter irgendwie geklopft und dann is er irgendwie voll ausgerastet und draußen standen auch voll viele Freunde von dem wahrscheinlich musste der sich da irgendwie extrem cool fühlen, hat sich dann umgedreht und, wollte mir eigentlich die Faust ins Gesicht schlagen hat mich aber dann a-, f, zum Clück nur hier getroffen ((zeigt auf Dekolleté)), aber ss hat auch also n paar Tage echt wehgetan hier, (1) und dann hab ich irgendwann mal gesagt okay mir reicht's jetzt dann hab ich mit ihm Schluss gemacht und ((schluckt oder trinkt)) (1) (534-542)

Daria setzt ihre Erzählung fort, in der sie eine weitere Situation schildert: Während einer Autofahrt mit ihrem Freund spricht sie ihn immer wieder an, ohne dass er sie hört resp. hören will. Als sie ihm daraufhin auf die Schulter klopft, um seine Aufmerksamkeit zu erreichen, »is er irgendwie voll ausgerastet «. Er dreht sich um und will Daria mit der Faust ins Gesicht schlagen, erwischt sie allerdings am Dekolleté.

Im Anschluss an die in der vorausgehenden Passage explizierte Gewaltsituation findet auch die hier erzählte in einem öffentlichen Rahmen statt. Auch hier sucht Daria nach Erklärungen für das Verhalten ihres Freundes, das sie sich dadurch erklärt, dass dieser sich vor seinen Freunden, die in diesem Moment um das Auto herumstehen, als »irgendwie extrem cool« präsentieren will.

\section{Trennung}

und ich dacht so okay wenn er mir ge- also wenn er handgreiflich wird geht's schon mal gar nich//mmh//weil mein Gott ja okay da war's halt nur mal an die Wand gedrückt und mal ne Faust gegen $\mathrm{Kr}$ - also hier nicht ins Gesicht leider, 
${ }^{\circ}$ wahrscheinlich ${ }^{\circ}$, also für ihn war's wahrscheinlich echt schade dass er mich nich im Gesicht getroffen hat, aber da denk ich auch so okay wenn's jetzt zwei Mal vorkam jetzt auch noch $\mathrm{n}$ drittes Mal vorkommt wenn's ihm dann wieder nich passt oder ja:,//mmh//(2) ja am Anfang war's echt okay aber dann am Ende hat ich einfach kein Bock mehr (587-594)

Nach der wiederholten Erfahrung körperlicher Gewalt kommt Daria an einen Punkt, an dem ihr klar wird, dass sie die Beziehung so nicht weiterleben möchte. Sie trennt sich von ihrem Freund.

In dieser Passage dokumentiert sich bezüglich Darias Handlungspraxis, dass sie ihre Entscheidung reifen lässt. Sie lässt sich Zeit, handelt nicht impulsiv oder anlassbezogen, sondern bezieht ihren Entschluss, sich zu trennen, auf eine Reihe von Auslösern.

In ihrer eigentheoretischen Erklärung für die Trennung fällt auf, dass Daria aus der Perspektive ihres Ex-Freundes spricht: Sie formuliert hier seine Gedanken, macht dabei aber ihre eigenen Verletzungen nicht zum Thema. Gleichzeitig argumentiert sie nicht aus einem sicheren Standpunkt heraus, sondern verharmlost erneut ihre Erfahrungen (z.B. »hat ich einfach kein Bock mehr«).

\section{Stalking}

hat er mich monatelang noch bedroht, hat mich jedes Mal angerufen also zu Hause aufm Handy ich bin aber nie ans Handy gegangen dann hat er immer nachts zu Hause bei mir angerufen, (544-547)

Nach der Trennung von ihrem Freund beginnt dieser, Daria über SMS, Handy, Telefon, Internet (Skype oder ICQ) »überall« (624) zu stalken und zu bedrohen. Er möchte, dass Daria wieder zu ihm zurückkommt. Die Situation hält monatelang an. Bei Anrufen zu Hause bedroht er nicht nur Daria, sondern auch ihre Mutter, wenn diese ans Telefon geht.

Vor dem Hintergrund, dass sich die hier geschilderten Erfahrungen des Gestalkt- und Bedroht-Werdens zeitlich an einem Punkt ereignen, an dem sich Daria für die Trennung von ihrem Freund entschieden hat, fällt in dieser Passage auf, dass sie erstmals ihre eigene Position benennt, indem sie nicht ans Telefon geht. Gleichzeitig führt sie die Mutter in der Rolle als bestärkende Unterstützerin ein, die sich solidarisch mit ihrer Tochter zeigt.

\section{Unterstützung durch Freund_innen}

und dann hat halt immer ne Freundin bei mir geschlafen ich konnt's einfach nich allein weil ich immer Schiss hatte dass er jetzt irgendwie die Tür aufreißt und dann steht er plötzlich da//mmh//weil ((schluckt)) irgendwie in der Zeit hab ich dem alles zugetraut (602-605) 
Da ihre Mutter in dieser Zeit häufig bei ihrem neuen Partner übernachtet, hat Daria Angst, nachts alleine zu Hause zu sein. Sie traut sich nicht, ihrer Mutter davon zu erzählen, da sie nicht will, dass diese sich Sorgen macht. Allerdings vertraut sie sich ihren Freundinnen an, sodass immer eine von ihnen bei ihr übernachtet, wenn die Mutter nicht da ist.

Bezüglich des unterliegenden Orientierungsrahmens dokumentiert sich, dass Daria ihre Schutzbedürftigkeit erkennt und sich bei ihren Freundinnen Hilfe und Unterstützung holt. Damit zeigt sich eine Umkehr in der Solidarisierung: Mit Ende der Beziehung werden soziale Netzwerke für Daria wieder wichtig, da sie beschützend wirken, was während der Zeit der Beziehung zu ihrem Freund nicht der Fall war.

Daria hat Angst, dass ihr Freund plötzlich und unerwartet vor ihrer Tür steht; sie traut ihm in dieser Zeit alles zu:

\section{Bedrohung}

und, irgendwann mal hat er mich die ganze Zeit bedroht und ich hab so geheult dass ich keine Luft mehr gekriegt hab und dann musste ich mich übergeben weil des für mich, einfach es, irgendwann mal zu viel geworden, (605-608)

Die Situation spitzt sich zu bis zu dem Punkt, an dem sich Daria so sehr bedroht fühlt, dass sie vor lauter Angst und Weinen keine Luft mehr bekommt und sich übergeben muss.

Nach drei Monate erzählt sie ihrer Mutter von diesen Bedrohungen. An dieser Stelle erwähnt sie auch, über eine polizeiliche Anzeige nachgedacht zu haben. Sie verwarf den Gedanken jedoch wieder, da sie sich sicher war, eine Anzeige würde nichts bringen - und die Sache möglicherweise eher noch schlimmer machen.

Hier dokumentiert sich Darias Auffassung, an dem Zustand, bedroht zu werden, können weder sie noch eine Anzeige etwas verändern. Damit zeigt sich weiter eine Haltung der Anpassung an die Situation, in der sie die passive Rolle innehat und sich lediglich zur Reaktion gezwungen sieht.

\section{Morddrohung und Verfolgung}

und ja dann warn wir aufm Jahrmarkt in Stadt 2 mit ner Freundin zu zweit und dann hab ich den da gesehen ((schluckt)) (1) und da hat er mir schon davor SMS(e) geschrieben ja wenn ich dich da seh ich bring dich um und keine Ahnung hat mich wirklich mit $m$ Messer verfolgt dort (1) (550-553)

Im Folgenden schildert Daria eine konkrete Situation, in der sich das Bedrohungsszenario zuspitzt und seinen Höhepunkt erreicht. Gemeinsam mit einer Freundin besucht sie einen Jahrmarkt. Bereits im Vorfeld erhält sie per SMS Drohungen 
ihres Ex-Freundes, sollte er sie dort sehen, würde er sie umbringen. Als sie ihm begegnet, verfolgt er sie mit einem Messer.

\section{Unterstützung durch Freunde}

bis ich da an Kumpel getroffen hab und des dem erzählt hab und dann hat er Freunde geholt und hat gesagt »ja jetzt ahm gehn wir mal dahin und fragen mal was sein Problem is, « und dann hat er natürlich plötzlich voll die kleine Fresse gehabt und hat halt gemeint ha nee ich hätte der doch gar nichts getan und was weiß ich was, (553-558)

Daria wendet sich an einen Freund und berichtet ihm, verfolgt und bedroht $\mathrm{zu}$ werden. Dieser konfrontiert, gemeinsam mit einigen Freunden, Darias Ex-Freund, worauf dieser sich zurückzieht. Daria ist sich in ihrem Resümee sicher, dass die Situation ohne die Unterstützung ihrer Freunde für sie ungut geendet hätte.

Bezüglich des unterliegenden Orientierungsrahmens werden zwei Aspekte deutlich: Zum einen holt sich Daria aktiv die Unterstützung durch Freunde. Diese Handlungspraxis beruht auf ihrer Erfahrung der Verlässlichkeit von Freundschaften sowie der Beistandschaft, die sie durch diese wiederholt biografisch erfährt. Zum anderen dokumentiert sich in ihrem Willen, auf dem Jahrmarkt zu bleiben, auch ihre Haltung, nicht davonzulaufen bzw. sich zu verstecken, um sich zu schützen. Sie handelt aktiv und autonom.

\subsubsection{Argumentative Stellungnahme}

Im folgenden argumentativen Segment schildert Daria die Veränderung des Beziehungsverlaufs. Dabei kommt ihre aus heutiger Sicht bewertende und theoretisch reflektierende Stellungnahme zum Ausdruck: »Aber dann mit der Zeit hat es mich einfach alles genervt « (586f.). Die Handgreiflichkeiten ihres Freundes bewertet sie - aus heutiger Perspektive und damit im Nachhinein - als etwas, das »schon mal gar nicht« geht. Gleichzeitig bagatellisiert sie die Vorfälle. Mit den Formulierungen »halt nur mal an die Wand gedrückt « (588f.) und »mal ne Faust« (589) verharmlost Daria die Übergriffe ihres Freundes, indem sie deren Einmaligkeit betont. Gleichzeitig kommt in der Sequenz ihre Sorge zum Ausdruck, dass ihr Freund, nachdem er zweimal übergriffig wurde, dies auch erneut werden könnte. Diese Überlegungen veranlassen sie dazu, über eine Trennung nachzudenken.

Argumentationen setzen an strittigen oder erklärungsbedürftigen Positionen an (vgl. Lucius-Hoene/Deppermann 2002, S. 253). In der vorliegenden Argumentation wird Darias Position deutlich, indem sie sich von der Position ihres ExFreundes abgrenzt, der Gewalt als legitimes Mittel zur Durchsetzung seiner Interessen ansieht. Ihre eigene Position erwähnt sie zwar nicht explizit, diese kann aber in der Abgrenzung implizit herausgelesen werden. Demnach stellt das gewaltakti- 
ve Verhalten für Daria eine klare Grenzüberschreitung dar, die sie perspektivisch nicht in Kauf nehmen will.

\section{Angst vor Wiederholung}

des war halt eigentlich die Zeit so ${ }^{\circ}$ wo ich denk ${ }^{\circ}$ da hab ich auch, also am Anfang so, dacht ich so nee kein Freund mehr und so//mmh//is echt nich so, also, hat ich halt irgendwo auch Angst davor dass es wieder so wird oder so aber jetzt mittlerweile bin ich mit meinem Freund zwei Jahre zusammen,//mmh//klar streiten wir auch und so aber jetzt nich so dass er mich dann irgendwie bedroht oder sowas, des gar $\mathrm{nich} / / \mathrm{mmh} / /(1)(560-566)$

In dieser biografischen Eigentheorie reflektiert Daria ihre biografische Entwicklung ab dem Zeitpunkt der Trennung von ihrem Ex-Freund bis heute. Es wird deutlich, dass die gemachten Erfahrungen physischer und psychischer Gewalt dazu führen, dass Daria zunächst Angst vor einer neuen Beziehung hat, aus Sorge, sie könnte ähnliche Erfahrungen erneut machen. Dass sie sich schließlich unmittelbar auf eine neue Beziehung einlässt, gibt Aufschluss über ihre identitätsrelevante Einstellung und Selbstpositionierung, die es ihr offensichtlich ermöglichen, neue - positive - Erfahrungen in Paarbeziehungen zu machen.

\subsubsection{Zusammenfassung}

Erleben von Gewalt in Paarbeziehungen Daria erlebt in ihrer ersten Paarbeziehung im Alter von 15 Jahren zwei körperliche Übergriffe durch ihren Freund, bei denen er sie einmal an die Wand drückt und würgt und das andere Mal mit der Faust schlägt. Nach der Trennung erlebt sie psychische Gewalt durch ihren Ex-Freund, indem dieser sie stalkt und über diverse soziale Medien bedroht.

Umgang mit erlebter Gewalt, Bewältigungsstrategien und Ressourcen Daria reagiert in beiden Situationen schockiert und passiv; sie wehrt sich auf verbaler Ebene bzw. in der zweiten geschilderten Situation gar nicht. Nach dieser zweiten Erfahrung steht für sie fest, dass sie innerhalb dieser Beziehung mit fortsetzenden Gewaltausübungen durch ihren Freund zu rechnen hat und trennt sich daraufhin von diesem. Dass Daria sich hier umgehend trennt, zeigt, dass sie über einen gesunden Selbstwert verfügt. In dieser Situation ist sie autark. Die hier vorliegende beziehungsbiografische Konstruktion beruht auf dem Motiv der Autonomie. Daria gelingt etwas, was viele gewaltbetroffene Frauen jahrelang nicht schaffen: Sie hat nach der zweimaligen Erfahrung körperlicher Übergriffe keine Hoffnung, dass die Gewalt durch ihren Freund aufhört. Sie überlegt nicht, was sie ändern müsste, damit die Gewalt nicht eskaliert. Für sie ist die Frage nach dem Grund seines Verhaltens zwar relevant, gleichzeitig steht für sie aber auch fest, dass ihr Freund sich nicht ändern 
wird und sie mit einem erneuten Übergriff zu rechnen hätte. Damit zeigt Daria eine klare Haltung gegenüber Grenzüberschreitungen.

Hinsichtlich der Bedrohungen durch ihren Freund nach der Trennung wendet sie sich zunächst an ihre Freundinnen und bittet diese um Unterstützung, bspw. indem diese bei Daria übernachten. Nach einiger Zeit vertraut sie sich auch ihrer Mutter an.

Insgesamt wird in der Rekonstruktion deutlich, dass die Peers für Daria eine wesentliche Ressource darstellen, auf die sie zurückgreifen kann und die sie als Unterstützung auch in sehr belastenden und bedrohlichen Lebenssituationen erlebt.

Beziehungsmuster und Beziehungsgestaltung Hinsichtlich ihrer Beziehungsvorstellungen strebt Daria nach einer egalitären Beziehung, die frei von Übergriffen ist. Sie geht mit 15 Jahren ihre erste Paarbeziehung ein. Dabei zeigt sich von Beginn an eine Asymmetrie, indem Daria diejenige ist, die viel Zeit und Geld investiert und häufig zu ihrem Freund fährt, damit sich beide sehen können. Als es dann zu den Übergriffen kommt, reagiert sie umgehend mit der Trennung.

Gewalt(-Beziehung) und Sozialisation Darias Jugendphase ist geprägt vom Zusammensein mit Peers, wobei insbesondere gewaltaktive Auseinandersetzungen unter Mädchen eine wichtige Rolle spielen, bei denen Daria sich ihren Platz in der Clique erkämpft. Interessant dabei ist, dass Gewalt in Paarbeziehungen für Daria eine klare Grenzüberschreitung darstellt, während gewaltaktive Auseinandersetzungen unter Mädchen für sie »normal«bzw. eher alltäglich sind.

$\mathrm{Zu}$ ihren Eltern hat sie ein gutes Verhältnis, das sich auch nach der Scheidung der beiden fortsetzt. Die gute Beziehung zu ihren Eltern bietet für Daria eine sichere Basis, aufgrund derer sie ein gesundes Selbstwertgefühl entwickeln konnte, das sie letztlich auch dazu befähigt, ihre Grenzen wahrzunehmen.

\subsubsection{Alina - »ich hab immer gedacht ja okay, er liebt mich« (61)}

\subsubsection{Fallporträt}

Alina wird als erste Tochter ihrer Eltern geboren. Ihre Mutter ist 19 Jahre alt, als sie mit Alina schwanger wird. Sie trennt sich nach einem Jahr Ehe von ihrem Mann, durch den sowohl sie als auch Alina körperliche Gewalt erfahren. Nach der Trennung ziehen Alina und ihre Mutter zu den Großeltern. Diese betreiben eine Gaststätte, in der Alina aufwächst. Alina hat anfänglich noch regelmäßigen Kontakt zu ihrem Vater, was jedoch, als Alina drei Jahre alt ist, auf ihren Wunsch hin eingestellt wird. Zwischen den Eltern kommt es im Laufe der Jahre immer wieder zu Streitigkeiten wegen des Unterhalts. Nach einigen Jahren hat Alinas Mutter einen 
neuen Partner, der bereits eine Tochter hat. Mit ihm zusammen bekommt Alinas Mutter noch eine weitere Tochter.

Nach der Grundschule besucht Alina die Hauptschule, wo sie die siebte Klasse wiederholt. In dieser Zeit hat sie im Alter von 15 Jahren eine Beziehung mit Daniele, der ihr nach einiger Zeit verbietet, sich mit Freundinnen zu treffen, und sie über verschiedene Medien »terrorisiert« (110). Nach etwa einem halben Jahr kommt es zur Trennung von Daniele und Alina beginnt eine Beziehung mit Tim. Nachdem diese Beziehung nach einigen Monaten endet, kommt sie mit Christoph zusammen. Die Beziehung ist erneut geprägt von Verboten durch Christoph, sowie von Streit. Nach einer eskalierenden Situation, in der Alina Unterstützung durch Tim erhält, nähern sich beide wieder einander an. Bis heute sind Alina und Tim ein Paar.

Nach der Hauptschule besucht Alina ein Jahr lang die hauswirtschaftliche Schule. Sie absolviert verschiedene Praktika in Pflegeheimen und bewirbt sich auf Ausbildungsstellen. Sie wechselt auf die Gewerbliche Schule, wo sie durch die Unterstützung einer Lehrerin einen Ausbildungsplatz vermittelt bekommt.

Mit 17 Jahren nimmt sie Kontakt zu ihrem leiblichen Vater auf, der inzwischen mit einer neuen Partnerin und einer weiteren Tochter zusammenlebt. Nach kurzer Zeit kommt es jedoch zu Auseinandersetzungen, und der Kontakt bricht wieder ab.

\subsubsection{Analyse der Erzählstruktur}

Bei der Betrachtung der Gesamtstruktur fällt der Beginn der biografischen Stegreiferzählung auf, der symbolisch für das gesamte Interview steht. Alina wurde von mir im Vorfeld über mein Forschungsinteresse informiert und auch darüber, dass ich mich für ihre gesamte Lebensgeschichte interessiere. Dennoch kommt es zu einer Irritation, als ich den offenen Erzählstimulus formuliere:

\section{Erzählstimulus und Beginn der biografischen Stegreiferzählung}

I: Dann würd ich dich einfach bitten, dass du mir deine Lebensgeschichte erzählst//mmh//und zwar alles was dir einfällt//von meinen Beziehungen//nee von deinem ganzen Leben//oha okay//mmh.

A: Also soll ich anfangen, von ganz klein an?

I: Cenau zum Beispiel und ich

A: also

I: unterbrech dich auch erst mal gar nich//mmh//mach mir vielleicht $\mathrm{n}$ paar Notizen auf die komm ich später//mmh//zurück//okay//genau. (1-12)

Meinen offenen Erzählstimulus, in dem ich Alina auffordere, ihre gesamte Lebensgeschichte zu erzählen, unterbricht sie und fragt nach, ob sie von ihren Beziehungen erzählen soll. Ich verneine und validiere meine Erzählaufforderung, indem ich Alina nochmals bitte, von ihrem »ganzen Leben« zu erzählen. Sie fragt erneut nach, 
ob sie an dem Punkt beginnen soll, an dem sie ganz klein war, was ich bestätige. Alina beginnt daraufhin sofort mit »also«, während ich noch weiterspreche und die Erzählaufforderung beende. Alina ratifiziert die Aufforderung mit »okay«.

An dieser Stelle zeigt sich, dass jede Äußerung von den Interaktionsteilnehmerinnen für genau den Kontext, der im gegebenen Gesprächsmoment vorliegt, formuliert wird: Alina geht hier von den im Voraus ausgebauten Wissens- und Beziehungsstrukturen aus. Sie weiß, dass ich mich für das Thema Paarbeziehungen interessiere und dafür ein Interview mit ihr führen möchte. Auf diesen vorab mitgeteilten Sachverhalten baut sie den Beginn ihrer Erzählung auf. Als ich meine Erzählaufforderung zu Ende bringe, ist sie kurz irritiert, da ich sie bitte, mir ihre gesamte Lebensgeschichte zu erzählen. Auch wenn dies im Vorfeld so von mir angekündigt war, scheint diese Information nicht mehr präsent zu sein, weshalb sich Alina nochmals rückversichert.

Alina beginnt daraufhin zügig mit ihrer Stegreiferzählung. Dabei kann sie sich zunächst an der für sie neuen Erzählaufforderung orientieren, indem sie mit Erzählungen zu ihrer Kindheit einsetzt. Nach der darstellerischen Gliederung in Segmente durch die zeitliche Grenzziehung in biografische Abschnitte (Aufwachsen bei den Großeltern, Grundschule, Hauptschule) wechselt sie in den Modus der Darstellung durch thematische Zusammenhänge, die auf die Forschungsfrage abzielen (erster Freund, darauffolgende Beziehungen), bis zum Ende der biografischen Stegreiferzählung. Sie bezieht sich also erneut auf das Thema der Beziehungen, von dem sie zunächst ausging, auf das sie sich eingestellt hatte und das sie offensichtlich auch als Schwerpunkt präsentieren möchte. Im Laufe des immanenten Nachfrageteils des Interviews taucht ein weiteres relevantes Thema auf, das durch zeitgedehntes und ausführliches Erzählen auffällt und auf »innere, gedankliche oder emotionale Vorgänge (Lucius-Hoene/Deppermann 2002, S. 119) verweist, »die einen Bezug zur voranschreitenden Handlung besitzen« (ebd.): das Thema der Mädchenfreundschaften. Wie sich in der Analyse des Interviews dann auch rekonstruieren lässt, sind die Verortung in Freundschaften mit Mädchen sowie die Verortung der eigenen Paarbeziehung ein wesentliches biografisches Thema, das sich bereits in der Eingangsnarration sowie im Verlauf des Interviews auch deutlich innerhalb der Erzählstruktur widerspiegelt.

\subsubsection{Herkunftsfamilie und Bedingungen des Aufwachsens Beginn der Stegreiferzählung}

Also (2) meine Oma und mein Opa die hamn ne Gaststätte (2) und da bin ich von Kind auf aufgewachsen, (1) meine Eltern die hamn sich getrennt, oder die warn verheiratet meine Mutter hat mit neunzehn geheiratet, und die hamn sich ja, warn aber nur ein Jahr zusammen (1) und auch nur ein Jahr verheiratet und dann gings lo:s, da mit meinen Eltern, also er hat meine Mutter geschlagen und mich (14-18) 
Alina beginnt ihre Erzählung mit dem Verweis, bei ihren Großeltern, die eine Gaststätte betreiben, aufgewachsen zu sein. Im weiteren Verlauf erläutert sie, wie es zu dieser Konstellation gekommen war. Ihre Mutter hatte mit 19 Jahren geheiratet. Die Beziehung dauerte jedoch nur ein Jahr, dann trennten sich die Eltern. Alina holt in ihrer Erzählung aus, um die Gründe für die Trennung darzulegen: Ihr Vater habe ihre Mutter geschlagen und auch sie selbst. Er sei oft betrunken nach Hause gekommen und die Situation sei zunehmend »ausgeartet« (21). Die Mutter zieht danach mit Alina zu deren Großeltern. In der Folge kommt es zu Anschuldigungen von Alinas Vater gegenüber der Mutter. Bei der oben geschilderten Erzählung kann es sich, angesichts Alinas Alter, nur um Berichte handeln, die ihr erzählt wurden, und nicht um eigene Erinnerungen. Dennoch lässt die Bezugnahme auf die Großeltern gleich zu Beginn der Stegreiferzählung vermuten, dass diese außerordentlich wichtige Bezugspersonen für Alina darstellen.

\section{Intergenerationale Gewalt und Gewalt auf der Elternebene}

s=ging halt darum warum er so zu mir war und zu meiner Mutter und er hat halt gesagt er hätte das nicht gemacht und (.) meine Mutter hat Bilder gehabt wo sie so aussah was er mit ihr gemacht hat und alles (1)

Die Benennung der gewaltvollen Geschichte zwischen ihren Eltern wird von Alina in einem lakonischen Duktus erzählt. Daran wird deutlich, dass sie sich die Geschichte nicht zu eigen gemacht hat. Für eine 18-Jährige scheint die Art der Erzählung nicht adäquat. Vielmehr entspricht ihre Art des Darüber-Sprechens einer Erzählung, die aus dem kindlichen Erleben heraus erfolgt und in der weder Dramatik noch Emotionen zum Vorschein kommen.

\section{Aufwachsen bei den Großeltern}

und wir hatten Ziegen Hasen Pferden also wir hatten schon alle Tiere wo's gab oder wo's gibt (1)//mmh//und wir waren da, jeden Tag war ich eigentlich bei meiner Oma weil mein Opa war für mich wie mein Ersatzvater, am Vatertag hab ich keine Briefe für meinen Vater geschrieben sondern für meinen Opa (.) also (.) war das immer so (.) ja dann war ich jeden Tag eigentlich bei meinem Opa (.) hatte dort meine Freunde (1) und während meine Mutter arbeiten war hab ich dort meine Hausaufgaben gemacht (1) damals hat meine Oma noch gelebt und dann ham wir zum Beispiel an Weihnachten oder so ham wir immer Plätzchen gebacken und alles (341-348)

Alina beschreibt zunächst die äußeren Umstände, die ihr Aufwachsen bei den Großeltern rahmen. Alina ist jeden Tag bei diesen und leitet an dieser Stelle argumentativ in die Begründung über, nach der ihr Opa für sie wie ein Vater 
war. Dass Alina hier das Fehlen ihres leiblichen Vaters thematisiert, zeigt ihre Beschäftigung damit.

In ihrer Beschreibung führt sie das Bild der harmonischen Familie weiter aus: Während die Mutter arbeitet, erledigt sie bei ihrer Oma die Hausaufgaben, an Weihnachten werden gemeinsam Plätzchen gebacken, auch ihre Freund_innen befinden sie im dortigen Umfeld.

\subsubsection{Kernthemen des Interviews}

Erste Beziehungserfahrungen

ja und dann (3) kam Grundschule und alles (2) dann kam mein erster Freund in der Crundschule@(.)@ahm @(.)@, des war glaub in der vierten Klasse, ja: in der vierten Klasse war des ${ }^{\circ}$ dann hat ich da meinen ersten Freund ${ }^{\circ}$ ja nur zwei Wochen @(.)@ aber ich kenn den heute noch und wir reden also oft von früher, (36-40)

Während der Grundschule hat Alina ihren ersten Freund. Durch die Verwendung des Begriffs "mein erster Freund« wird die Intimbeziehung sprachlich markiert. Die Beziehung dauert allerdings nur kurz, sie wird auch nicht weiter ausgeführt. Alinas Ergänzung, dass sie sich mit diesem Jungen auch heute noch gut versteht und Kontakt zu ihm hat, macht deutlich, dass Freundschaft und Akzeptanz durch andere für sie einen hohen Stellenwert haben.

ja und dann kam ich in die Hauptschule (2) und in der siebten (1) oder ${ }^{\circ}$ sechsten $^{\circ}$, irgendwann so hab ich halt auch $n$ Freund, aber es war auch nich so weil, ich war früher $n$ bisschen arg schüchtern@(.)@ und dann warn wir glaub zwei Monate zusammen (40-43)

Als Alina auf die Hauptschule kommt, hat sie in der sechsten oder siebten Klasse einen Freund. Ihre Formulierungen »irgendwann« und »halt auch n Freund« machen eine gewisse Notwendigkeit deutlich, einen Freund zu haben; möglicherweise auch, um innerhalb der Peergroup dazuzugehören. Ihre Beschreibung »aber es war auch nich so weil, ich war früher $\mathrm{n}$ bisschen arg schüchtern« deutet darauf hin, dass die Beziehung für sie früh oder zu früh war und sie eigentlich noch kein Bedürfnis nach einem intimen Kontakt hatte. Die Beziehung endet nach zwei Monaten, da ihr Freund eine Beziehung mit einem anderen Mädchen eingeht (»ja dann (3) war bei uns eigentlich relativ schnell Schluss weil er hatte eine aus einer

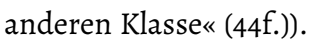

Alina schließt die Sequenz erzählerisch ab mit dem Verweis, dass auf ihrer Schule generell viele Wechsel in Beziehungen stattfinden.

Hier dokumentiert sich Alinas Konformität mit Gleichaltrigen. Es ist für sie wichtig, einen Freund zu haben, um bei ihren Freund_innen gut anzukommen. Den Referenzrahmen bildet die Schulklasse, in der (intime und sexuelle) Beziehun- 
gen gelebt werden. Für Alina stellt sich die Frage, ob sie hinsichtlich ihres sozialen Status unter den Peers mithalten kann.

Ein neuer Junge kommt in die Klasse, der Alina Liebesbriefe schreibt. Sie geht irgendwann mit ihm aus, es kommt aber nicht zum Austausch von Intimitäten (»wir haben nichts gemacht oder so, gar nichts, nicht geküsst Händchen gehalten gar nichts« (48f.)). Als ihr Freund plötzlich eine andere Freundin hat, erfährt Alina dies nicht von ihm. Sie führt auch nicht aus, ob diese Erfahrung für sie verletzend war. Dagegen fügt sie hier erneut an, dass sie sich auch mit diesem Jungen heute noch gut versteht. Dies bestätigt die obig formulierte Vermutung, dass für Alina Anerkennung unter Peers ein besonders bedeutsames Thema zu sein scheint.

Fester Freund

\section{Kennenlernen I}

und dann (2) hatte ich nen Freund (3) des war n Italiener (.) n Sizilianer (2) und Sizilianer sind sowas wie (1) sag jetzt mal Türken (1) wo ihre Frauen, also beleidigen (.) ihnen nichts erlauben gar nichts (53-55)

Die Erzählung über ihre erste längere Beziehung beginnt Alina mit einem Abstract, in dem sie ihren Freund als Italiener, genauer Sizilianer, einführt. Dabei enthält der hier formulierte Abstract auch eine evaluative Bedeutung, indem Alina Sizilianer als eine Menschengruppe generalisiert, die Frauen beleidigt und ihnen alles verbietet. Dass Alina diese Beschreibung bereits ganz zu Beginn der von ihr begonnen Erzählung einfügt, deutet darauf hin, dass sie die benannten Beleidigungen und Verbote selbst erlebt hat.

\section{Kennenlernen II}

Ja (.) also er war auch auf der Hauptschule [...] dann haben wir auch schon (immer mal) in Facebook geschrieben oder halt in Kwick (.) un=dann hat er (sich halt also mich) auf=m Schulhof immer so angesprochen dann ham wir aufm Schulhof meistens zusammen gechillt (1) u:nd dann war ja []... so Jogginghosen-Zeit und ich hatte irgendwie die auffälligste weil meine rot war, und durch das hat der mich halt (.) ja (.) und dann hat der mich angesprochen (474-480)

Alina kennt Daniele aus der Schule. Sie haben zunächst Kontakt über Facebook und Kwick. Irgendwann spricht Daniele Alina auf dem Schulhof an. Hier zeigt sich, dass Alina eher in eine passive Rolle schlüpft, indem sie abwartet, bis sie angesprochen wird.

Sie chillen gemeinsam auf dem Schulhof. Alina erwähnt, dass sie eine auffällige rote Jogginghose trägt und von Daniele auf diese angesprochen wird. Daniele und Alina sind zunächst befreundet und werden dann auch »beste Freunde« (481). 
Daniele verliebt sich erst in Alinas beste Freundin, und deren Freund verliebt sich wiederum in Alina. Nach einiger Zeit kommt es zu einem »Frauentausch« (483).

Alinas Formulierung »weil die zwei ja dann (.) getauscht ham« (ebd.) impliziert erneut ihre eher zurückhaltende, passive Rolle in dem ganzen Geschehen. Sie überlässt den Jungen den aktiven Part. Alinas Erzählung, wie es dazu kommt, dass sie und Daniele ein Paar werden, ist sehr kurz und allgemein gehalten:

\section{Treffen}

also er hat mich meis- also aufm Schulhof hat der mich gefragt (1) dann wir ham uns gesehn (1) (warn) wir eigentlich nur morgens (.) er hat mir Hallo gesagt dann is er zu seinen Kumpels gegangen (.) bei der Pause war er auch wieder bei sei- bei seinen Kumpels außer mittags ham wir auch manchmal aber (1) ja sonst ham wir nur Wochenende irgendwas gemacht, (2) (484-488)

Sie sehen sich morgens auf dem Schulhof, er begrüßt sie, geht dann aber zu seinen Freunden, mit denen er außerdem die Pausen verbringt. Selten sehen sich Alina und Daniele nachmittags. Ihr Hinweis, sich über die Treffen in der Schule hinaus nur an den Wochenenden getroffen zu haben, deutet auf einen weniger intensiven Kontakt zwischen den beiden hin. Sie ergänzt, sich hin und wieder auch nachmittags auf dem »Skatingplatz« (507) getroffen zu haben.

Insgesamt zeigt sich, dass Alina das Kennenlernen in dieser Sequenz ohne den Verweis auf Beleidigungen und die Herkunft ihres Freundes erzählt. Vielmehr scheint es ihr zu gefallen, dass er sie aufgrund ihres trendigen Kleidungsstils anspricht. Weiter dokumentiert sich in dieser Passage Alinas Orientierung, sich mit ihrem Freund an den von ihm präferierten Orten zu treffen. Damit bestätigt sich die Vermutung, dass sie sich insgesamt, was die Häufigkeit und Orte der Treffen angeht, nach ihrem Freund richtet und ihre eigenen Wünsche ihm gegenüber entweder nicht formuliert oder diese erst gar nicht entstehen.

Im Folgenden schildert Alina eine konkrete Situation, in der sie zu Daniele nach Hause eingeladen wird.

Gewalterfahrungen

\section{Abwertung im Kreise der Peers}

dann hat er, warn wir immer mit seinen Kumpels und hat gesagt ja: »Alina is voll () was will die und so « keine Ahnung hat mich halt vor (den) Kumpels immer voll schlecht gemacht, (62-64)

Alinas Freund diskreditiert sie vor seinen Freunden. Sowohl er als auch seine Freunde bezeichnen sie als »Fotze« (69) und zeigen ihr gegenüber obszöne Gesten. Ihre Aussage, sie habe sich »nich so angesprochen gefühlt« (68) macht ihren 
Umgang damit deutlich: Sie versucht die Beleidigungen nicht auf sich zu beziehen bzw. lässt sie an sich abprallen.

\section{Verbote}

Wir saßen mal mittags an der Schulstraße und ich meine Mädels warn eigentlich (.) einmal im Monat oder=zweimal im Monat immer $n$ Mädelsabend gemacht $/ / \mathrm{mhm} / /$ (.) u:nd in der Zeit hat er gesagt »du gehst da nich hin weil die anderen Mädels können ja was Schlimmes über mich sagen«(536-539)

Alina verbringt ein- bis zweimal im Monat einen Abend zusammen mit ihren Freundinnen. Daniele verbietet ihr, dort hinzugehen mit der Begründung, diese könnten sich negativ über ihn äußern. Alina hält sich zu Beginn an die Verbote, beschließt dann aber, sich heimlich mit ihren Freundinnen zu treffen. Als Daniele dies mitbekommt, kommt es zum Streit zwischen ihm und Alina, bei dem er sie anschreit. Dieses Verhalten seinerseits verstärkt sich im Beisein von Danieles Freunden. Dagegen ist er »friedlich distanziert« (543), wenn er mit Alina allein ist.

Im Laufe der Zeit verändert sich jedoch Alinas Handlungspraxis im Umgang mit den Verboten, und sie wählt die Strategie, sich heimlich mit ihren Freundinnen zu treffen. Grundlage für die Wahl dieser Handlungspraxis ist für sie zum einen die Erfahrung, dass Daniele, zumindest wenn sie allein sind, ihr Verhalten vermutlich nicht kritisiert, und zum anderen die Annahme, ihr Freund werde seine Haltung mit der Zeit wieder ändern.

Ein weiterer Grund für ihre Akzeptanz gegenüber Danieles Verboten und ihre Unterwerfung unter diese liegt in der Annahme, dass ihr Freund aus Liebe zu ihr heraus agiert:

aber ich hab immer gedacht ja okay, er liebt mich (61f.)

Eine Auseinandersetzung mit Daniele scheut sie, und die Möglichkeit, selbst zu entscheiden bzw. sich ihm zu widersetzen oder sich zu trennen, kommt zu diesem Zeitpunkt in der Beziehung nicht für sie in Betracht.

In Bezug auf den hier unterliegenden Orientierungsrahmen wird deutlich, dass Alina den Austausch mit ihrem Freund vermeidet und in der Folge sein über sie verfügendes Verhalten akzeptiert. Dabei nimmt ihr Freund nicht die Rolle des Aggressors ein. Im Gegenteil nimmt sie ihn sogar in Schutz, indem sie argumentiert, "weil wir nur Streit hatten« (528), habe ihr Freund sie nicht sehen wollen - obwohl ihr ein Treffen wichtig gewesen wäre.

Damit zeigt sich auch, dass Alina über keine positiven Erfahrungen verfügt, was die Gestaltung von Beziehungen angeht. Sie hat kein Modell, wie eine andere Interaktion innerhalb der Paarbeziehung aussehen könnte. Innerhalb der Beziehung nimmt sie eine passive, abwartende Rolle ein; gleichzeitig sucht sie sich auch einen Freund, der die Rolle des Aktiven übernimmt. 
Alina geht innerhalb der Beziehung in Abhängigkeit, sie vermeidet den Austausch resp. die Auseinandersetzung mit ihrem Freund. Sie ist trotz vielfältiger Anlässe, die ihr Freund ihr bietet und die sie empören, nicht in der Lage, die Bindung $\mathrm{zu}$ ihm abzubrechen und sich zu trennen. Sie scheint auf die emotionale Anerkennung und Zuwendung, die sie innerhalb der Beziehung bekommt, angewiesen zu sein und akzeptiert daher auch Abwertungen und Verbote.

\section{Ohrfeige}

also (.) er wollt halt dass ich mit ihm schlaf (1) aber ich wollte das (nich)//mhm//und da (wir=ja) eh nur die ganze Zeit echt im Bett gelegen weil er einfach nichts anderes machen wollte (.) [...] hat der angefangen mich so $\mathrm{n}$ bisschen auszuziehen na=hab ich gesagt »ich will das nich «//mhm//dann hat er mir erst mal so ne leichte Backpfeife gegeben hat gesagt »du machst des jetzt« und dann hab ich gesagt »weißt du was hier is die Tür du kannst gehen« (889-896)

Alina berichtet von einer konkreten Situation, in der Daniele mit ihr schlafen will, sie das jedoch nicht möchte. Zunächst stellt sie den Kontext dar, der darin besteht, dass sie mit Daniele im Bett liegt, so wie sie es die meiste Zeit ihrer Beziehung machen, da Daniele dies so will.

In dieser konkreten Situation beginnt Daniele, Alina auszuziehen, und signalisiert ihr damit, mit ihr schlafen zu wollen. Alina erwidert, dass sie das nicht möchte, woraufhin Daniele sie verbal und körperlich, indem er ihr eine Ohrfeige versetzt, unter Druck setzt. Daraufhin fordert Alina ihn auf zu gehen. Sie selbst geht zu ihrer Mutter und berichtet ihr von dem Vorfall, woraufhin diese Daniele aus der Wohnung wirft.

Für den hier unterliegenden Orientierungsrahmen zeigt sich erneut, dass Alina in dieser Beziehung weitestgehend die Rolle der anpassungsfähigen Freundin einnimmt, die sich den Wünschen des Freundes unterordnet. Allerdings wird hier auch eine Grenze sichtbar, an der Alina sich nicht mehr anpasst. Als Daniele ihr gegenüber körperlich übergriffig wird, signalisiert sie deutlich diese Grenze, indem sie ihn aus der Wohnung wirft. Außerdem holt sie sich Unterstützung bei ihrer Mutter, was auf ein gutes bzw. vertrauensvolles Verhältnis zwischen den beiden hindeutet und auf das sie hier zurückgreift.

\section{Trennung}

Also ich war ja erst immer so (.) ja: lassen wir ihn machen, er beruhigt sich schon (.) dann ging das ja mit seinen Mädels los (.) und dann hab ich gedacht da-, dann hab ich: dann hat er mir Fasching verbieten wollen da hab ich gesagt »das geht schon mal fünf mal nich « (.) und das wollte er aber nich akzeptiern un=dann hab ich gesagt das geht nich mehr, un=dann hab ich ihm angerufn (.) na hab ich gesagt 
»du ganz ehrlich, ich kann das nich mehr ich will nich mehr weil so und so« (.) »aha also machst du Schluss « dann hab ich gesagt »ja « dann sagt er »ok bevor du Schluss ma-Schluss machst mach ich Schluss du Fotze « und hat einfach aufgelegt (550-557)

Im Laufe der Beziehung häufen sich verschiedene Verhaltensweisen bei Daniele, die Alina ihre Einstellung hinsichtlich der Beziehung ändern lassen. So flirtet er bspw. mit anderen Mädchen und untersagt Alina die Treffen mit ihren Freundinnen. Als er ihr allerdings verbieten will, auf die Fastnacht zu gehen, die für Alina eine große Bedeutung hat, ist für sie eine deutliche Grenze erreicht. Sie trennt sich daraufhin von Daniele. Da dieser es offensichtlich nicht ertragen kann, als der Verlassene dazustehen, versucht er, ihr zuvorzukommen, und formuliert die Trennung, indem er sie noch zusätzlich als »Fotze« beschimpft.

Nach der Trennung bekommt Alina wieder Nachrichten von Daniele, er würde sie vermissen. Als sie ihm gegenüber erneut die Trennung deutlich macht, beschimpft er sie erneut mit obszönen Ausdrücken.

Rückblickend bewertet Alina ihr Verhalten damals mit den Worten »ich war einfach blind verliebt « (519f.). Auch in der folgenden Bemerkung, Daniele habe nur mit seinen kiffenden Freunden rumgehangen, kommt zum Ausdruck, dass sich Alina etwas anderes in der Beziehung wünschte.

\section{Digitale Gewalt}

und dann hat's angefangen dass er mich terrorisiert hat und alles und keine Ahnung (.) so ging's halt los und dann irgendwann hat er's dann mal gecheckt aber keine Ahnung wann, aber er hat's dann gecheckt und hat mich in Ruhe gelassen, (1) dann hat er wieder geschrieben in Facebook hat er mir so Texte geschrieben () es tut mir leid und er braucht mich [...] da hab ich gesagt nee also ich will das nicht mehr weil, für mich kommt sowas nicht infrage und keine Ahnung ss geht einfach nicht mehr, und dann kam wieder du Fotze du Schlampe dies das jenes (109-116)

Daniele akzeptiert die Trennung zunächst nicht und versucht, Alina zurückzugewinnen, indem er sie über Facebook kontaktiert. Als Alina ihm ihre Haltung erneut bestätigt, beschimpft er sie als "Fotze« und »Schlampe«. Dieses Muster setzt sich einige Wochen fort und endet, als Alina ihn in den sozialen Medien sperrt. Bezüglich des unterliegenden Orientierungsrahmens wird deutlich, dass Alina in ihrer Handlungspraxis hinsichtlich der Trennung von ihrem Freund konsequent bleibt. Auch als dieser beginnt, sie über den Weg der digitalen Medien zu terrorisieren, weiß sie sich zu helfen, indem sie ihn sperrt. 


\section{Die eigenen Peers}

Im Interview mit Alina wird deutlich, dass sie sich zwischen ihrer Freundin und ihrem Freund positionieren muss.

\section{Rat der besten Freundin}

ich hab auf meine beste Freundin nie gehört so in der Art weil sie hat immer gesagt »mach Schluss mit ihm das bringt nichts « und ich hab immer gesagt »was laberst du« weil ich hab meine Freundin, also sie war früher mein Ein und Alles weil sie hat mir aus n paar Scheißn schon rausgeholfen, und dann hab ich eher mich auf mein Freund konzentrierte statt auf sie und hab eher auf das gehört was mein Freund gesagt hat damals und nicht auf sie (91-96)

Alinas beste Freundin, die ihr bereits mehrfach aus verschiedenen Schwierigkeiten herausgeholfen hat, rät Alina immer wieder, sich zu trennen. Alina fühlt sich jedoch, im Unterschied zu früher, ihrem Freund näher und distanziert sich von den Ratschlägen ihrer Freundin. Aus heutiger Sicht bereut sie ihr Verhalten.

Hier dokumentiert sich Alinas Orientierung hin zu ihrem Freund und weg von der ehemals besten Freundin. Damit wird die Herausforderung, die sich im Jugendalter zum ersten Mal und neu stellt, sich im Zuge der ersten Beziehungen zwischen dem Freund und den Peers neu ausrichten müssen, deutlich. Gerade hinsichtlich des Status innerhalb der Peer-Gruppe spielt das Eingehen oder Innehaben einer Liebesbeziehung eine bedeutende Rolle.

Eine neue Beziehung

Auf einem Fastnachtsumzug wird Alina von einem Jungen, Tim, angesprochen, der sie nach ihrem Namen fragt. Sie unterhalten sich und schreiben sich ab diesem Zeitpunkt über die sozialen Medien Nachrichten. In einem Fastnachtsritual werden sie symbolisch verheiratet, woran beide einen großen Spaß haben. Sie nutzen die Symbolik auch in ihrer weiteren Kommunikation, in der sie sich bspw. mit »Ehemann« (131) anschreiben. Nach einiger Zeit haben beide jedoch nicht mehr nur Spaß zusammen, sondern es kommt zu vielen Auseinandersetzungen, da beide sehr eifersüchtig sind, wenn der oder die andere sich mit einem anderen Mädchen oder Jungen unterhält. Alina und Tim führen ihre Beziehungskonstellation als loses Arrangement fort. Sie treffen sich zunehmend abends bei ihm, und sie übernachtet schließlich bei ihm. Auf Tims Frage, ob sie denn nun ein Paar seien, reagiert Alina zunächst distanziert und lehnt dies ab. Ihre Begründung »weil ich ja einfach davor noch ne Beziehung hatte« (145) deutet auf ihre Einstellung hin, es müsse eine bestimmte Zeit vergangen sein, bis sie eine neue Beziehung eingehen könne. Bestätigt wird diese Lesart durch die folgende Äußerung »und dann irgendwann hab ich gesagt ja jetzt sind wir zusammen, (145f.). 
Die in dieser Passage skizzierte biografische Konstruktion weist auf einen Orientierungsrahmen hin, innerhalb dessen die Paarbeziehung als normatives heterosexuelles Beziehungsmodell gedacht wird, in dem Treue als Grundvoraussetzung für die Beziehung angesehen wird. Bezüglich des unterliegenden Orientierungsschemas zeigt sich die an normativen Regeln ausgerichtete Vorstellung, nach einer Beziehung dürfe nicht zu schnell die nächste erfolgen. Verbunden ist damit möglicherweise die Assoziation, wer sich (zu) schnell in eine neue Beziehung begibt, gilt als sexuell aktiv und wird als »Flittchen « abgewertet.

Alina beschreibt die folgende Zeit als »echt ne schöne Zeit« (147). Sie und Tim verbringen gemeinsam mit Tims Eltern einen zweiwöchigen Urlaub. Es wird deutlich, dass Alina die Beziehung zu Tims Eltern sehr wichtig ist. Sie stellt Tims Eltern als »voll lieb« (148) dar. Alina erfährt durch Tims Mutter Zuwendung, indem diese auf Alina zugeht und sich mit ihr unterhält. Alina beschreibt diese Erfahrung mit den Worten »dann hab ich mich schon gefühlt als wär ich zu Hause ganz ehrlich« (156f.).

Hier dokumentiert sich Alinas Bedürfnis nach Nähe, Zuwendung und Angenommensein, die sie in Tims Familie erfährt. Um dieses Bedürfnis zu erreichen, engagiert sich Alina, indem sie sich aktiv in das Familienleben einbringt.

\section{Trennung}

und dann gab's aber für uns ne Zeit (1) >s war letztes Jahr gab's für uns ne Zeit (1) wir haben nur gestritten wegen egal wegen was ich hab dem noch en Buch gemacht im Urlaub und danach geht's los wir ham nur gestritten dann ham wir gesagt »is glaub mal besser wenn jetzt Schluss is erstmal« (157-161)

Nach dem gemeinsamen Urlaub kommt es zunehmend zu Auseinandersetzungen. Dabei investiert Alina zunächst noch in die Beziehung, indem sie ein Buch für Tim gestaltet, das sie ihm schenkt. Sie trennen sich jedoch nach einiger Zeit in gegenseitigem Einvernehmen, nachdem sie sich nur noch streiten.

Alina fällt es schwer, sich von Tim zu lösen. Ihr Freund Christoph, der für Alina ein guter Kumpel ist, ist in dieser Zeit »immer da und wollte immer was mit mir machen« (162f.). Diese Unterstützungsbereitschaft könnte auch darauf hindeuten, dass er sich möglicherweise von Alina angezogen fühlte oder in sie verliebt war. Alina kommt dann doch mit ihm zusammen, sieht die Funktion der Beziehung allerdings in der Ablenkung, die ihr diese von Tim bietet. Mit der Zeit gelingt ihr die Ablösung von Tim, worüber Alina sehr »froh« (169) ist.

In dieser zunächst als vertrauensvoll beschriebenen Beziehung kommt es dennoch von Beginn an zu Stress, insbesondere auch dadurch, dass Christoph ihr Dinge verbietet. In der folgenden Passage dokumentiert sich, dass sich Alinas Haltung gegenüber Verboten jedoch geändert hat: 


\section{Umgang mit erneuten Verboten}

mit Christoph hab ich auch voll oft gestresst weil er mir halt auch Sachen verboten hat und ich lass mir nichts mehr verbieten weil daraus hab ich einfach gelernt (1) $(172-174)$

Auf der Ebene der konkreten Handlungspraxis zeigt sich Alinas veränderter Umgang mit Verboten darin, dass es zu Auseinandersetzungen kommt und sie Verbote nicht mehr akzeptiert. Dennoch gibt es Hinweise auf eine emotionale Belastung (»)s war mir einfach wieder zu viel« (176f.)), die zum Entschluss führt, sich zu trennen. Verstärkt wird die Entscheidung seitens Alinas vermutlich auch dadurch, dass sie »Tim einfach wieder öfters gesehen« (171f.) hat.

\section{Signifikante Andere}

In der Analyse des Interviews mit Alina lassen sich wesentliche Personen bestimmen, die als signifikante Andere eine wichtige Rolle in Form von Orientierung und Unterstützung für Alina spielen. Dies sind ihre Mutter und ihr Großvater. Im Folgenden soll anhand ausgewählter Zitate deren Bedeutung für Alina rekonstruiert werden.

Alina schildert an mehreren Stellen im Interview die Ressource, in schwierigen Situationen auf ihre Mutter zurückgreifen zu können.

\section{Unterstützung durch die Mutter}

Auf jeden Fall reden//mhm//also nich schweigen so wie andere des tun weil ((holt kurz Luft)) ne Freundin von mir die (.) ihr Freund hat sie auch oft geschlagen und sie hat nich darüber geredet//mhm//und des hats (.) weiß nich des (2) ich zum Beispiel ich bin n Mensch ich rede mit meiner Mutter über alles meine Mutter is für mich wie: wie meine beste Freundin=weil mit ihr kann ich=über alles reden=meine=Mutter redet mit mir über alles (.) und, reden einfach mit irgendjemand reden und nich alles in sich reinfressen (1020-1026)

In dieser Passage sowie in den Passagen 889-898 und 255-258 (vgl. Darstellung weiter oben) dokumentiert sich Alinas Orientierung an der Unterstützung durch die Mutter. Damit wird deutlich, dass deren Ratschläge bzw. Interventionen hinsichtlich Alinas Beziehungsverhalten für diese von Bedeutung sind.

Auch Thematisierungen der Mutter zu Alinas Beziehungen nimmt sie ernst:

ham uns drei Wochn nicht gesehn weil er mich nich sehn wollte weil wir nur Streit hattn aber keine Ahnung wieso (un=da) hat meine Mutter gesagt des kann (ich mir) doch nich weiterhin antun »weil (.) du bist doch nich glücklich, «ja un=dann gings dann los ${ }^{\circ}$ dass ich dann nich ${ }^{\circ}$ mehr wollte (527-530) 
Diese Anmerkungen der Mutter regen bei Alina eine Reflexion ihrer Beziehungserfahrungen an und führen am Ende zu einer Änderung ihrer Handlungsorientierung. Sie ist hier bspw. nicht mehr bereit, alles innerhalb ihrer Beziehung zu akzeptieren, und denkt z.B. über eine Trennung nach.

\section{Der Großvater}

und wir waren da, jeden Tag war ich eigentlich bei meiner Oma weil mein Opa war für mich wie mein Ersatzvater, am Vatertag hab ich keine Briefe für meinen Vater geschrieben sondern für mein Opa (.) also (.) war des immer so (.) ja dann war ich jeden Tag eigentlich bei meim Opa (.) (342-345)

Alina führt ihre Großeltern bereits im ersten Satz ihrer biografischen Stegreiferzählung mit dem Verweis ein, bei diesen aufgewachsen zu sein. Dabei nimmt ihr Großvater eine gesonderte Stellung ein, indem sich Alina in der Beschreibung ihres Aufwachsens ausschließlich auf den Großvater bezieht und seine Rolle als Vaterersatz thematisiert: "mein Opa war für mich mein Ein und Alles« (350). Auch im weiteren biografischen Verlauf bleibt die Bedeutung des Großvaters erhalten, was sich darin zeigt, dass Alina ihm ihren Freund vorstellt: »und dann hab ich ihn halt vorgestellt mein Opa war am Anfang schon so mhh« (851f.).

\subsubsection{Argumentative Stellungnahme}

Im Folgenden werden argumentative Textpassagen analysiert, die Reflexionen über die eigenen Erfahrungen in Paarbeziehungen beinhalten und die damit für die Forschungsfrage dieser Arbeit immanent sind.

haa weiß nich hätt ich mein Freund nich ich glaub ich würds auf Jungs weiß nich () (1) ich hab auch schon mal gedacht bei ihm er is genau so weil Jungs warn in dem Moment alle gleich für mich, weil ja: (1) [...] Also weiß nich (1) der eine hat mir was verboten der andre hat mir was [...] verboten die warn alle so gleich und=da hab ich gedacht mein Freund wär auch so [...] Wegen dem ich hab mich (1) ich konnt mich gar nich so einlassen auf die Beziehung weil=ich immer so Schiss hatte er=is genau so zu mir aber (.) er is das krasse Gegenteil [...] wir reden über alles sobald uns was stört sagen wir das wenn ich zickig bin dann lässt er mich halt zicken (1) sowas hab ich noch nie gekannt (822-847)

In dieser Passage wird deutlich, dass Alina ihre aktuellen Beziehungserfahrungen mit Tim als Folie nutzen kann, um ihre Erwartungen an eine Beziehung bzw. die Bewertung der Beziehungen zu Daniele und Christoph zu reflektieren. Ihre Reflexion basiert dabei auf der für sie damals gültigen Annahme, dass alle Jungs gleich seien. Diese Annahme wird in der folgenden Aussage revidiert. Mit Tim macht sie eine ganz neue Erfahrung mit ihrem Freund, nämlich darüber zu sprechen, wenn sie oder ihn etwas stört. Auch, dass ihr Freund sie so annimmt, wie sie ist (»wenn 
ich zickig bin dann lässt er mich halt zicken«), stellt für sie eine neue Qualität und Erfahrung in einer Beziehung dar.

Zuletzt noch eine Anmerkung zu den geschlechterbezogenen und kulturellen Generalisierungen, die Alina immer wieder in argumentativen Einschüben verwendet: Indem Alina bestimmte Freunde oder Bekannte einer Kultur zuordnet (»Sizilianer«, »Türken«, »Kurden«), eröffnet sie einen Begründungszusammenhang, in welchem sie versucht, ihre Beziehungserfahrungen einzuordnen. Dabei greift sie auf allgemeine Pauschalisierungen und Generalisierungen zurück. Das gleiche Prinzip wendet sie auch auf die Gender-Dimension an:

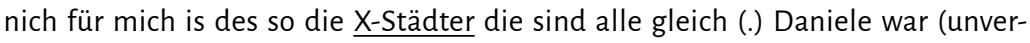
ständlich 1) Christoph war (unverständlich 1) die waren beide so gleich (177-179)

Solchen Generalisierungen können zwei Aspekte zugrunde liegen: Zum einen gewinnt nicht der individuelle Andere an Wirkmächtigkeit, was bedeutet, dass es nicht erforderlich ist, sich mit dieser konkreten Person auseinanderzusetzen. Und zum anderen wird eine Distanz zu sich selbst hergestellt, indem die eigene Handlungspraxis bzw. die eigenen Handlungsoptionen nicht thematisiert werden (müssen). Stattdessen wird der Blick ausschließlich auf das Gegenüber resp. die anderen gerichtet.

\subsubsection{Zusammenfassung}

Erleben von Gewalt in Paarbeziehung Alina erlebt innerhalb ihrer ersten längeren Beziehung, die sie im Alter von 15 Jahren mit Daniele eingeht, verschiedene Formen von Gewalt. Zum einen erfährt sie bereits zu Beginn der Beziehung soziale Gewalt durch Verbote sowie Abwertung und Demütigungen, indem Daniele sie im Kreise seiner Peers beleidigt und bloßstellt. Als er eines Tages mit ihr schlafen möchte, nötigt er sie sexuell dazu und wendet körperliche Gewalt an. Nach der Trennung erfährt Alina über die sozialen Medien Beschimpfungen.

In einer weiteren Beziehung (mit Christoph) macht Alina erneut die Erfahrung, dass ihr Freund ihr verbieten will, auszugehen. Auch hier wird sie nach dem Ende der Beziehung über die sozialen Medien weiter »terrorisiert«.

Umgang mit erlebter Gewalt, Bewältigungsstrategien und Ressourcen In ihrer ersten Beziehung mit Daniele reagiert Alina auf die Verbote, sich mit ihren Freundinnen zu treffen, indem sie nicht in Auseinandersetzung mit ihm geht, sondern sich heimlich mit ihren Freundinnen trifft. Sie zeigt sich ihm gegenüber damit angepasst und scheinbar unterordnend. In ihrer Entscheidung, sich dennoch mit ihren Freundinnen zu treffen, liegt jedoch ein widerständiges Moment, das sie für sich als Strategie nutzt, teilweise selbstbestimmt zu handeln.

Bezüglich der Abwertungen, die sie im Laufe der Beziehungen erfährt, ließ sich in der Falldarstellung der Umgang bzw. die Bewältigung damit in Form von 
Ignoranz rekonstruieren. Dabei wird das Problem an sich nicht gelöst, sondern aktiv verdrängt.

Im Gegensatz zu den geschilderten Erfahrungen der Kontrolle sozialer Kontakte sowie der psychischen Gewalt durch Demütigung und Abwertung setzt Alina in der Situation, in der sie sexuell unter Druck gesetzt wird, deutlich eine Grenze und wirft ihren Freund aus der Wohnung. Außerdem wendet sie sich an ihre Mutter und sucht dort Unterstützung. In der Folge dieses Ereignisses trennt sie sich von ihrem Freund.

Bezüglich ihres Umgangs mit digitaler Gewalt zeigt sich, dass es Alina durch ihre klare Haltung, mit der sie ihre Trennung weiterhin vertritt, sowie das Sperren ihres Ex-Freundes mit der Zeit gelingt, die digitalen Beschimpfungen zu unterbinden.

Eine wesentliche Ressource, auf die Alina immer wieder zurückgreift, stellt die Beziehung zu ihrer Mutter dar. Ihre Mutter ist Ratgeberin, Freundin, Unterstützerin. Sie mischt sich immer wieder in Alinas Beziehungen ein, indem sie ihr ihre Eindrücke zu Alinas Beziehungen rückmeldet. Alina erlebt deren Unterstützung als hilfreich und fordert diese auch immer wieder ein.

Beziehungsmuster und Beziehungsgestaltung In der Rekonstruktion des Interviews mit Alina konnte deutlich gezeigt werden, dass Alina zunächst eine Beziehung mit einem Jungen eingeht, um ihren Status innerhalb der Peergroup zu erhalten. Dabei kostet sie das Eingehen der Beziehung Überwindung, da sie schüchtern ist, was auch den weiteren Verlauf der Beziehung bestimmt. Die Konformität mit Gleichaltrigen ist für sie jedoch wichtig; in Alinas Fall stellt die Schulklasse den Referenzrahmen dar, in dem intime und sexuelle Beziehungen gelebt werden.

In der darauffolgenden Beziehung zu Daniele zeigt sich Alinas Anpassung an die Wünsche ihres Freundes, was besonders hinsichtlich der Häufigkeit und der Wahl der Orte, an denen sie sich treffen, deutlich wird.

Die Akzeptanz bzw. Ignoranz, mit der Alina auf die erfahrenen Abwertungen reagiert, zeigen ihre Abhängigkeit innerhalb der Beziehung. In ihrer passiven Rolle überlässt sie die Entscheidungen (bspw. wo und wann sie sich treffen) ihrem Freund. Sie lässt die Beschimpfungen und Abwertungen ihres Freundes in Gegenwart seiner Freunde über sich ergehen. Sie vermeidet die Auseinandersetzung mit ihm. Wie in der Analyse rekonstruiert, liegt dieser Handlungspraxis ein großes Bedürfnis nach Anerkennung und Zuwendung zugrunde, das dazu führt, sich innerhalb der Beziehung in eine (emotionale) Abhängigkeit zu begeben und - wenn auch nicht alles, so doch recht lange - vieles zu akzeptieren.

In der Rekonstruktion des biografischen Verlaufs wird außerdem deutlich, dass es Alina schwerfällt, allein zu sein. Sie ist ab dem Zeitpunkt ihrer ersten Beziehungserfahrung dauerhaft in Beziehung, auch wenn diese wechseln, was auf eine unsichere, emotional-abhängige Art verweist. 
Alina erfährt innerhalb ihrer Beziehungen immer wieder die Rückmeldung, bedeutungslos und wertlos zu sein. Verbote, sich mit ihren Freundinnen zu treffen, akzeptiert sie, da sie der Auffassung ist, sie geschehen aus Liebe zu ihr. Weitere Erklärungsmodelle, die sie anführt, sind heteronormativ-biologistisch oder kulturalisierend. In Beziehung zu sein bzw. einen Freund zu haben, verschafft ihr außerdem in der Peergroup einen gewissen sozialen Status.

Hinsichtlich geschlechterbezogener Inszenierungen in Paarbeziehungen zeigt sich, dass in Alinas Beziehungsmuster eher präskriptive Vorstellungen über männliche Dominanz und weibliche Unterordnung zu finden sind. Demnach wird auch aggressives Verhalten von Männern (im Falle Alinas bezieht sich das auf die ausgesprochenen Verbote, Demütigungen und Abwertungen) eher als Teil des maskulinen Verhaltens akzeptiert. Allerdings kann sie ihre Grenze, in der Situation, in der sie sexuell unter Druck gesetzt wird, erfolgreich durchsetzen.

Gewalt und Sozialisation Da Alina noch sehr klein war, als sich ihre Mutter von ihrem Vater trennte, hat sie keine eigenen Erinnerungen an diese Zeit. Sie weiß jedoch aus Erzählungen, dass ihr Vater sowohl der Mutter als auch ihr selbst gegenüber gewalttätig war. Die Mutter besitzt Fotos, auf denen die Gewalt dokumentiert ist. Damit erlebt Alina traditional-hierarchische Geschlechterkonstruktionen in ihrer Herkunftsfamilie. Obwohl sie im Kindesalter zunächst regelmäßig Kontakt zu ihrem Vater hat, scheint sie die Beziehung zu ihm nicht als positiv zu erleben. Ihre Großeltern, vor allem aber der Großvater, bei denen sie aufwächst, sind für Alina wichtige Beziehungspersonen. Sie berichtet, ihren Großvater wie einen Vater erlebt zu haben.

Alina erlebte also bereits im familiären Kontext Gewalt sowohl auf der Elternebene (häusliche Gewalt) als auch auf intergenerationaler Ebene (Vater gegenüber Alina). Diese Erfahrung bildet eine Grundlage, auf der Alina die Bewältigung von Gewalt erlernt. Dabei ist die Wahl der Bewältigungsform von Vorbildern abhängig. Darüber hinaus orientieren sich Menschen an Beziehungsstrukturen einer partnerschaftlichen Beziehung, die sie als Kind kennengelernt haben. Durch die beschriebenen Erfahrungen in der Herkunftsfamilie ist Gewalt für Alina zumindest teilweise normalisiert. Aus Erzählungen weiß sie, dass sich ihre Mutter nach einem Jahr Ehe und der Erfahrung körperlicher Gewalt von ihrem Partner trennt. Die sozialisatorisch bedingte Erfahrung von Trennung als Möglichkeit zur Bewältigung bzw. Beendigung von Gewalt innerhalb der Paarbeziehung wendet Alina in ihrer eigenen Beziehung an.

Weitere Themen Weiter wird deutlich, dass für Alina der Umbau sozialer Beziehungen eine zusätzliche Herausforderung darstellt. So muss sie sich einerseits zwischen ihrem Freund und ihren Freund_innen sowie ihrer Familie positionieren. Bei Sorgen und Kummer mit ihrem Freund thematisiert Alina diese nicht mit ihm selbst, 
sondern mit ihren Freundinnen. Bei den Bedrohungen durch ihren Ex-Freund holt sich Alina Unterstützung bei ihrer Freundin.

Im Interview mit Alina wird außerdem ein weiteres Thema deutlich: die Bedeutung von Medien. Es zeigt sich, dass es bei der Anbahnung von Beziehungen zunächst leichter zu sein scheint, über soziale Medien miteinander in Kontakt zu treten. Gleichzeitig erfährt sie gerade auch über diese Gewalt, indem sie bedroht und beleidigt wird.

Letztlich wird im biografischen Verlauf auch ein Wandlungsprozess deutlich: In ihrer aktuellen Beziehung macht Alina die Erfahrung, dass es möglich ist, Dinge auch innerhalb der Beziehung zu thematisieren. Außerdem erfährt sie, so angenommen $\mathrm{zu}$ werden, wie sie ist.

\subsubsection{Jasmin - "wenn's dann nich gepasst hat hab ich Schläge gekriegt und des war eigentlich jeden Tag so« (312f.)}

\subsubsection{Fallporträt}

Jasmin wird als älteste Tochter geboren. Es folgen noch zwei jüngere Geschwister aus der Beziehung ihrer Eltern. Aus der vorausgehenden Ehe ihres Vaters hat Jasmin außerdem noch vier Halbgeschwister, zu denen sie jedoch kaum Kontakt hat. Jasmin beschreibt ihre Kindheit als "nicht so rosig« (2of.), da ihr Vater schwerer Alkoholiker war. Dennoch berichtet sie über ihn, dass er sich bis zur Geburt der jüngsten Schwester viel »um uns Kinder« (53) gekümmert hat. Der Zeitpunkt der Geburt der jüngsten Schwester stellt einen Wendepunkt innerhalb der Familie dar: Der Vater beginnt, »relativ viel Alkohol« (55) zu trinken. Die folgenden Jahre sind geprägt von massiven Gewalterfahrungen durch den Vater innerhalb der Familie. Jasmin wird früh Zeugin häuslicher Gewalt. Auch sie und ihre Geschwister erleben massive physische und psychische Gewalt durch den Vater. Als Jasmin neun Jahre alt ist, trennt sich die Mutter von ihrem Partner und flüchtet mit ihren Kindern ins Frauenhaus. Die Familie lebt in großer Angst vor dem Vater, da dieser versuchte, Jasmins Mutter umzubringen, und in den Besuchskontakten mit seinen Kindern droht, diese zu entführen. Als Jasmin 13 Jahre alt ist, stirbt ihr Vater, was eine große Erleichterung für sie und ihre Geschwister bedeutet.

Eine wichtige Rolle in Jasmins Kindheit spielt ihre Großmutter, bei der sie viel Zeit verbringt und bei der sie sich aufgehoben und geborgen fühlt. Als Jasmin zehn Jahre alt ist, stirbt ihre Großmutter.

Über ihre Schulzeit berichtet Jasmin wenig. Ihre Mutter hat in dieser Zeit viele wechselnde Partner, und die Familie zieht, auch um vor dem Vater zu flüchten, häufig um, was für Jasmin eine große Anzahl an Brüchen hinsichtlich ihres sozialen Umfeldes bedeutet. Sie beschreibt sich selbst als Außenseiterin, die wenig Freund_innen hat, und berichtet von einigen Situationen, in denen sie in gewalt- 
aktive Auseinandersetzungen mit ihrer Lehrerin oder den Jungen in ihrer Klasse verwickelt ist.

Im Kreise ihrer Peergroup beginnt Jasmin nach dem Tod ihres Vaters zunächst zu kiffen. In der Folge konsumiert sie Koks, Ecstasy und letztlich auch Heroin. Während ihrer Jugendzeit schließt sie sich zunächst einer rechtsradikalen Gruppe an, die sie nach einiger Zeit wieder verlässt, um sich der Punkszene anzuschließen. Als Jasmin 16 oder 17 Jahre alt ist, suizidiert sich ein befreundetes Paar von ihr. Diese schmerzhafte Erfahrung führt dazu, dass Jasmin den Absprung von den Drogen schafft.

Sie zieht in eine eigene Wohnung und beginnt eine Ausbildung zur Bäckereifachverkäuferin. In dieser Zeit lernt sie ihren ersten Freund kennen, mit dem sie schließlich auch zusammenwohnt und insgesamt zwei Jahre zusammen ist. Jasmin beendet ihre Ausbildung und beginnt, in einem Imbiss zu arbeiten. Dort lernt sie einen Mann kennen, der zwölf Jahre älter ist als sie und in den sie sich verliebt. Sie trennt sich von ihrem Freund und zieht direkt bei ihrem neuen Freund ein. Die Beziehung zu ihm entwickelt sich rasant zu einer machtvollen Gewaltbeziehung, in der es schnell zu einer Zuspitzung der ausgeübten Gewalt gegenüber Jasmin kommt.

Bei der Arbeit lernt sie schließlich einen Mann kennen, der sie darin unterstützt, sich aus der gewaltvollen Beziehung zu lösen. Zum Zeitpunkt des Interviews lebt Jasmin mit ihrem Freund in einer gemeinsamen Wohnung.

\subsubsection{Analyse der Erzählstruktur}

Im Interview mit Jasmin fällt die äußerst kurze Stegreiferzählung auf, die lediglich knapp vier Minuten dauert. Dieses Erzählsegment beginnt Jasmin zunächst mit einer zeitlichen Grenzziehung in den biografischen Abschnitt "meine Kindheit» (20), wechselt dann jedoch die Gliederungsform, indem sie dazu übergeht, ihre Erzählung nach thematischen Zusammenhängen zu strukturieren (Gewalt durch den Vater; die Rolle der Großmutter; Zugehörigkeit zu jugendkulturellen Szenen; erste Beziehungserfahrung; Gewaltbeziehung; aktuelle Paarbeziehung). Dabei deutet sie diese Themen eher an, führt sie maximal in wenigen Sätzen aus und beendet ihre Stegreiferzählung mit dem Gliederungsmarkierer »sonst weiß ich gar nich was ich jetzt @erzählen@ soll«(47). Die Stegreiferzählung ist außerdem geprägt durch emotionsloses Erzählen von dramatischen Situationen, fragmentarischem Erzählen, temporalen Sprüngen und der Bagatellisierung von erlittenem Leid (vgl. Rosenthal u.a. 2006, S. 34), was auf traumatische Erfahrungen hindeutet.

Bereits nach diesen ersten vier Minuten hatte ich Hinweise auf einen äußerst problembelasteten und ausgesprochen krisenhaften biografischen Verlauf, der massive Gewalterfahrungen aufweist. Entscheidend waren für mich dabei die Hinweise, die darauf deuteten, dass Jasmin bereits seit ihrer Kindheit häufig trau- 
matisierenden Lebenssituationen ausgesetzt war. Dies veranlasste mich, meine Nachfragen, die ich zunächst anhand meiner Notizen strukturierte, hochsensibel anzugehen, um im weiteren Verlauf der Gesprächsführung offen zu bleiben und mögliche Erzählwiderstände oder Erinnerungsschwierigkeiten rechtzeitig erkennen zu können, gleichzeitig aber auch nicht unbeabsichtigt zur Institutionalisierung des Verschweigens von Gewalterfahrungen beizutragen (vgl. ebd., S. 33; vgl. Kap. 7.6).

Im folgenden Interviewverlauf versuchte ich, Jasmin mithilfe einer konsequenten narrativen Gesprächsführung zur Generierung von Erzählungen zu unterstützen. Jasmin entfaltete erzählerisch anschließend ihre Biografie anhand der nachgefragten Themen. Dabei wurden nicht alle, aber einzelne Passagen erneut zeitlich gerafft dargestellt und ähnlich der Darstellungsweise in der Stegreiferzählung durch sachliche und emotionslose Formulierungen gekennzeichnet. An diesen Stellen hakte ich nicht weiter nach, um Retraumatisierungen und Dissoziationen zu vermeiden.

Gegen Ende des Interviews führte sie eine längere Bilanzierung ihrer Lebensgeschichte an, die vermutlich durch den aktuellen Aufenthalt in einer psychiatrischen Tagesklinik zum Zeitpunkt des Interviews unterstützt und beeinflusst wurde.

\subsubsection{Herkunftsfamilie und Bedingungen des Aufwachsens Erzählstimulus}

I: Ähm keine Fragen mehr (.) von dir dann starten wir (.) okay also ich ähm würd dich jetzt einfach bitten dass du mir deine ganze Lebensgeschichte erzählst (.) ]: $\mathrm{mhm}$

I: Alles was dir einfällt (.) du kannst anfangen wo du möchtest und ich würd dich erstmal gar nicht unterbrechen

]: Des heißt eigentlich von Kindheit auf an

I: Genau mhm (.) ich mach mir ein paar Notizen einfach nebenher auf die komm ich dann nochmal später zurück (.) ja

J: Alles klar

I: Okay (2-18)

Die Interviewerin gibt einen offenen Erzählstimulus, in dem sie die interviewte Person auffordert, ihre gesamte Lebensgeschichte zu erzählen. An der Stelle, an der die Interviewerin äußert, die Interviewte könne lebensgeschichtlich an dem Punkt anfangen, an dem sie möchte, setzt die Interviewte ein und versichert sich rück »das heißt eigentlich von Kindheit auf an«. Die Interviewerin bestätigt dies.

Mit ihrer Nachfrage vergewissert sich Jasmin, ob die Interviewerin ihre Lebensgeschichte von »Kindheit auf an« hören möchte. Möglicherweise auch, ob sie wirklich dazu bereit ist. 


\section{Beginn der Stegreiferzählung}

Ja@(.)@ überleg gerade wo ich anfangen soll (.) ja meine Kindheit war eigentlich nicht so rosig mein Vater war schwerer Alkoholiker (.)//mmh//hat uns alle: geschlagen, oft geschlagen, meine Mutter vergewaltigt ((atmet ein)) (20-22)

Jasmins kurzes Auflachen zu Beginn der Stegreiferzählung könnte auf eine Unsicherheit hindeuten. Das Explizieren ihrer Gedanken »überleg gerade wo ich anfangen soll« validiert den Eindruck ihrer Unsicherheit, wie sie ihre biografische Erzählung beginnen soll. Nach einer kurzen Pause beginnt sie mit einer evaluativen Bewertung ihrer Kindheit, die sie als "nicht so rosig« beschreibt, und fügt direkt die Erklärung dafür an, dass ihr Vater Alkoholiker war.

Hier dokumentiert sich in Jasmins Rückblick auf ihre Kindheit, dass sie angesichts der (anzunehmenden) schwierigen und belastenden Erlebnisse mit einem alkoholkranken Vater ihre Kindheit zunächst nicht als schwer beschreibt, sondern ihre Bewertung mit dem eingeschobenen Wort "so« abmildert. Damit relativiert und verharmlost sie die belastenden Erlebnisse während ihrer Kindheit, da diese sonst vermutlich kaum auszuhalten wären.

Anfangssätze repräsentieren meistens bestimmte Lebensthematiken oder Strategien der Identitätskonstruktion (Lucius-Hoene/Deppermann 2002). In Jasmins Erzählung zeigen sich hier Erfahrungen von Gewalt und Angst, die bereits in früher Kindheit erlebt werden. Sowohl die elterliche Beziehung als auch die Beziehung des Vaters zu den Kindern ist von Gewalt geprägt. Als Kind ist Jasmin den Übergriffen des Vaters ausgeliefert. Die Mutter scheint sie nicht schützen zu können, sondern ist selbst von Gewalt durch ihren Partner betroffen.

Jasmin erzählt aus ihrer heutigen Sicht, retrospektiv und zusammenfassend, über den Erlebenszusammenhang >Kindheit<, der geprägt war durch die Schläge des Vaters, die Vergewaltigung(en) der Mutter durch den Vater und schließlich die große Angst vor einer Entführung durch den Vater, nachdem sich die Mutter von ihm getrennt hatte und ins Frauenhaus geflüchtet war. Jasmins Bekräftigung, die Angst wäre »ständig« (24) und »lang« (ebd.) da gewesen, verdeutlichen den Zustand und die Atmosphäre, in der sie aufgewachsen ist.

\section{Tod der Großmutter}

dann ist irgendwann der einzige Halt wo wir eigentlich alle hatten war unsere Oma die ist dann gestorben (.) (24-25)

Als einschneidendes biografisches Ereignis kann der Tod der Großmutter angesehen werden. Sie wird von Jasmin als die einzige Person beschrieben, bei der sich Jasmin und ihre Geschwister (»wir«) sicher und geborgen fühlten.

Drei Jahre später stirbt der Vater, was für Jasmin und ihre Familie erneut einen einschneidenden Wendepunkt bedeutet. Ihre Worte »Gott sei Dank (.) ab dem 
Zeitpunkt war die Angst einfach weg« (26f.) machen die große Erleichterung deutlich.

In diesem Segment erzählt Jasmin in wenigen knappen Sätzen die wesentlichen Ereignisse, die ihre Kindheit stark prägen. Dabei fällt auf, dass es neben den permanenten Angstzuständen, ausgelöst durch die Bedrohung durch den Vater, eine wichtige und positive Person in Jasmins Kindheit gibt, ihre Oma, durch die sie Halt und Unterstützung erlebt. Diese bildet ein positives Gegenüber zu den belastenden Ereignissen.

\section{Jugendszenen}

ja und dann fings bei mir an dass ich mich in sämtliche Sachen @geflüchtet@ hab von Drogen, über Alkohol die falschen Freunde, Naziszene Punk Cothics sowas immer wieder tendierend (2) (27-29)

Nach dem Tod des Vaters beginnt für Jasmin ein neuer Lebensabschnitt, der jedoch nicht die Befreiung widerspiegelt, die nach dem Wegfall der Bedrohung durch den Vater angenommen werden könnte, sondern eine Flucht in verschiedene Bewältigungsstrategien wie »Drogen, über Alkohol die falschen Freunde, Naziszene Punk Gothics .

Ein weiterer biografischer Einschnitt findet zur gleichen Zeit statt, als sich ein Paar, mit dem Jasmin sehr eng befreundet ist, mit einer Überdosis Heroin das Leben nimmt. Nach diesem einschneidenden biografischen Erlebnis führt Jasmin einen Entzug durch, womit ihr der »Absprung von den Drogen« (211) gelingt. Darin dokumentieren sich Jasmins Wille und ihre Entscheidung, in ihre Zukunft zu investieren und ihr Leben diesbezüglich zu verändern.

Mit dem Absprung von den Drogen beginnt Jasmin, sich zu ritzen. Erneut relativiert sie die belastende Zeit mit den Worten »also relativ bewegende Zeit« (216), um emotional nicht zu dicht an die erlebten Gefühle zu kommen. Rückblickend bewertet sie diese Phase in ihrem Leben als eine, in der sie viel »mitgemacht« (217) und »durchgemacht« (ebd.) hat.

Jasmin setzt ihre Erzählung fort und berichtet, in dieser Zeit eine eigene Wohnung, zwei Katzen und einen Hund gehabt zu haben. Einerseits verweist sie damit auf ein gewisses Maß an Normalität in dieser Zeit, andererseits betont sie auch die damit verbundene Verantwortung (»und dann noch« (219); »das volle Programm« (220)). Zur gleichen Zeit absolviert sie auch die Ausbildung zur Bäckereifachverkäuferin, die sie auch abschließt, »trotz, Drogen trotz allem aber ich habe es durchgezogen« (222).

Insgesamt dokumentiert sich hier, dass Jasmin in der Lage ist, enorme Kräfte $\mathrm{zu}$ mobilisieren, die es ihr ermöglichen, neue Handlungsstrategien zu entwickeln. Diese befähigen sie wiederum, ihre Situation verändern zu können. 


\subsubsection{Kernthemen des Interviews \\ Drogenkonsum \\ Drogenkarriere}

Ja einfach hab ich ich hab halt schon Freunde gehabt (.) da war dann halt mal ((seufzt 2)) da mal kiffen ((atmet aus)) da war ich schon mit meinem erstbesten Freund eigentlich zusammen (.) zu der Zeit ja da war da mal kiffen da mal kiffen dann hat man (.) gekokst Ecstasy äh ja die typischen Rausch- äh=Rauschzustände gehabt (.) einfach irgendwo (2) $n$ Halt gesucht in den Drogen (.) und des war einfach so da gings mir gut wenn ich des genommen hab und alles andre hab ich vergessen (1) ja und des ging eigentlich relativ weit also wir waren am Schluss dann schon irgendwo bei Heroin also (2) (194-201)

Jasmin antwortet auf die Nachfrage der Interviewerin zu den Dingen, in die sie sich geflüchtet habe, mit einer zeitlich gerafften Beschreibung. Die Sequenz ist, wie auch die biografische Stegreiferzählung, erneut durch Jasmins sachliche und emotionslose Formulierungen gekennzeichnet.

Der Verweis »hab ich halt schon Freunde gehabt«, mit denen Jasmin kifft, deutet darauf hin, dass Jasmin über ihre Peerbeziehungen in Kontakt mit Haschisch kommt. Sie deutet eine Beziehung mit einem Jungen an, der zunächst ihr bester Freund war. Sie greift diese Erzähllinie jedoch nicht wieder auf, sodass unklar bleibt, wie die Beziehung weiter verlief. Vom gelegentlichen Kiffen verändert sich der Drogenkonsum hin zur Einnahme von Koks und Ecstasy. In einer eingeschobenen eigentheoretischen Erklärung benennt Jasmin die Suche nach Halt in den Drogen als Grund für deren Einnahme. Sie beschreibt die Wirkung der Drogen als Zustand, in dem es ihr gut geht und sie alles vergessen kann. Hier dokumentiert sich der Konsum von Drogen als Bewältigungsversuch, mit negativen, belastenden Erfahrungen umzugehen.

Der Verweis »des ging eigentlich relativ weit also wir waren am Schluss dann schon irgendwo bei Heroin« zeigt, dass sich Jasmin über die Steigerung der Drogen, bis hin zu Heroin, bewusst ist. Gleichzeitig deutet der Verweis auf einen "Schluss« darauf hin, dass diese Phase beendet zu sein scheint.

In der Überleitung zur folgenden Sequenz, in der Jasmin die Zugehörigkeit $\mathrm{zu}$ verschiedenen jugendkulturellen Szenen beschreibt (»Nazi Punk ich hab alles @durchgemacht@«(202)), dokumentiert sich ebenfalls Jasmins Suche nach Zugehörigkeit und Halt.

Peergroup und Zugehörigkeit zu jugendkulturellen Szenen Rechte Szene

Die erste war war ich rechts halt so relativ rechtsradikal eingestellt (2) grad durch falsche Freunde des heißt Türken zusammengeschlagen öh (.) ((ausatmend)) ja* 
(.) des was man sich einfach unter rechtsradikal vorstellt auf Demos und lauter so Scheiß mitgemacht (492-495)

In die rechtsextreme Szene kam Jasmin aus heutiger Perspektive durch »falsche Freunde«. In ihrer im Interview vorgenommenen Bewertung über diese Zeit, in der sie »lauter so Scheiß mitgemacht« hat, zeigt sich ihre heutige Distanz gegenüber der rechten Szene.

\section{Punkszene}

hab dann jemand kennengelernt, Bekannte Freundin (1) bin dann so in die Punkszene reingerutscht (.) wo es mir eigentlich sehr gefallen hat muss ich sagen (.) und dann halt auch immer aufgefallen kurze Kleider Haare gefärbt (.) Springerstiefel so: schwarz geschminkt//mmh//aber ich muss sagen es war eigentlich relativ schöne Zeit ich kann darüber nichts Schlechtes verloren klar des was mit Drogen war [...] des war vielleicht nich s Positive, aber ich sag einfach die Freunde was ich damals hat hab ich heut noch (495-502)

Über eine Freundin schließt Jasmin sich der Punkszene an, in der sie sich wohlfühlt. Vor allem die Möglichkeit der Selbstinszenierung durch das Tragen kurzer Kleider und das Färben der Haare macht Jasmin Spaß. Sie bewertet diese Zeit als schöne Zeit, einzig die Drogen, die sie damals nahm, schränken die positiven Erlebnisse ein. Zudem ist sie mit den Freund_innen aus dieser Zeit auch heute noch befreundet, was für die Stabilität und Konstanz dieser Freundschaften spricht.

Hier dokumentiert sich, dass die Beschäftigung mit dem Äußeren, sich zu inszenieren bzw. bewusst zu stylen, Jasmin Freude bereitet. Ein weiterer relevanter Aspekt stellt der Kontext der Peergroup dar, in den die Handlungspraxis des Stylings eingebunden ist und der als positiv erlebt wird. Hier gibt es einen Raum in Jasmins Leben, der positiv besetzt wird, was einerseits in der Erzählung darüber, andererseits aber auch in ihrer Erinnerung an diese Zeit zum Ausdruck kommt.

\section{Wechsel der Szenen}

und s war halt so Punk Cothics so gemischt bei mir immer ich konnt mich nich wirklich entscheiden ich war heut mal das morgen mal das (502-504)

Sie ist mal mehr in der Punk- mal mehr in der Gothicszene unterwegs. Der Wechsel entsteht dadurch, dass Jasmin sich für keine eindeutige Zugehörigkeit entscheiden kann. Jasmin beschreibt, dass sie in dieser Zeit viel Alkohol und Drogen konsumiert, "auch viel Scheiß gemacht also nix irgendwie Schlimmes« (540f.), und es bedauert, die Zeit damals nicht noch mehr genossen zu haben.

Auf der Suche nach Zugehörigkeit zu einer Gruppe bzw. Szene dokumentiert sich Jasmins generelle Suche nach Zugehörigkeit. Vor allem innerhalb der Punksze- 
ne macht Jasmin die Erfahrung, dass es Spaß macht, sich entsprechend zu stylen. Über äußerliche Merkmale, wie gefärbte Haare oder das Tragen von kurzen Kleidern, wird die Zugehörigkeit nach außen hin deutlich markiert und nach innen erlebt. Darüber hinaus sind die Freundschaften, die sich hier bilden, für Jasmin bedeutsam.

\section{Erste Beziehungserfahrung}

Ihre erste Beziehungserfahrung führt Jasmin im Interview nicht weiter aus. Lediglich in einem knappen Satz erwähnt sie ihre erste Beziehung, die sie als »eigentlich ne relativ normale Beziehung «(30) beschreibt.

Auf diese Beziehung folgt eine dreijährige Beziehung mit einem Freund, mit dem Jasmin »eigentlich alles« (31) teilt. Sie haben eine gemeinsame Wohnung, gemeinsame Haustiere.

\section{Erster Freund}

Ja,wir waren @eigentlich@ am Anfang relativ glücklich [...] ja es lief von Anfang alles super [...] wir haben uns eigentlich verhalten wie $n$ achtzigjährig- äh achtzig Jahre verheiratetes Ehepaar so in der Art (2) sin dann auch ausgezogen nach Stadt 1 eigene Wohnung (2) ja, da auch viel Scheiß miteinander gemacht er hatte mal Ausbildung wieder abgebrochen wieder angefangen (1) war am Anfang war des alles schön also die ersten, zwei zweieinhalb Jahre, so wie man sich eigentlich die erste richtige feste Beziehung vorstellt, (242-252)

Jasmin beschreibt die erste Zeit mit ihrem Freund als »relativ glücklich«. Sie vergleicht sich und ihren Freund außerdem mit einem alten Ehepaar, was auf eine gewisse Stabilität, Normalität, geteilten Alltag sowie Routinen in der Beziehung verweist. Der Umzug in eine andere Stadt und in eine gemeinsame Wohnung verdeutlicht dies.

Dagegen deutet die Beschreibung "auch viel Scheiß miteinander gemacht" mehr auf das jugendtypische Verhalten hin und steht dem zuvor gezeichneten Bild des alten Ehepaars diametral entgegen. Auch dass ihr Freund Ausbildungen abbricht und wieder anfängt, verweist auf unstete Zeiten. Erneut betont Jasmin, dass die ersten zwei bis zweieinhalb Jahre schön waren, "so wie man sich eigentlich die erste richtige und feste Beziehung vorstellt«.

und dann hats irgendwann damit angefangen dass ich das Kiffen nich mehr wollte und er es immer heimlich gemacht hat mich angelogen hat, dann: hat er für die Miete ich war bei meinen Eltern gemeldet, dass es nicht auffällt Arbeitsamt praktisch (2) und dann hat er das Celd verkifft und dann war kein Geld mehr da: und dann mit anderen Weibern: (252-256) 
Eine Wendung tritt ein, als Jasmin das Kiffen ihres Freundes nicht mehr akzeptieren möchte und er dies daraufhin heimlich praktiziert. Er »verkifft« das Geld, das für die Miete vorgesehen ist, und beteiligt sich nicht am Haushalt. Ihren Hinweis auf andere Frauen führt sich nicht weiter aus, lässt aber vermuten, dass ihr Freund sich anderen Frauen zugewendet oder sie betrogen hat. Insgesamt leben sich die beiden nach Jasmins Beschreibung zunehmend auseinander.

Gewaltbeziehung

Es folgt eine Beziehung mit einem Mann, der Jasmin »schwer misshandelt« (34). Sie fasst die Beziehung in wenigen Sätzen wie folgt zusammen:

das heißt von: blauer Hüfte über blaue Augen Nase angebrochen Messer im Rücken stecken gehabt (3) ((seufzt)) ja mit dem Kopf auf n Boden geschlagen (2) s volle Programm mich eingesperrt durfte nicht mehr raus durfte mich nicht mehr schminken keinen Ausschnitt mehr anziehen, hätte Kopftuch tragen sollen, kochen putzen wenn das nicht gemacht war dann hab ich abends Prügel gekriegt wenn ich nach Hause gekommen bin (2) ja, (34-39)

Hier fällt auf, dass Jasmin ausschließlich beschreibend und ohne Beteiligung von Emotionen über die Misshandlungen in ihrer Beziehung berichtet. Möglicherweise sind die mit diesen Erfahrungen verbundenen Gefühle nicht zugänglich bzw. Jasmin schützt sich, bewusst oder unbewusst, vor einer Dissoziation (vgl. auch die Überlegungen zu Traumatisierungen in Kap. 8.1.2.2).

In der folgenden Passage erzählt Jasmin von der Trennung von ihrem ersten Freund und dem Übergang der alten in die neue Beziehung. Nach Beendigung ihrer Ausbildung jobbt Jasmin bei einem Imbiss. Dort lernt sie ihren zukünftigen Freund kennen.

ja und dann hab ich mein (2) also, also ${ }^{\circ}$ Ex-Freund ${ }^{\circ}$ kennengelernt $/ / \mathrm{mmh} / /$, und ja na is meine Beziehung halt voll ganz $n$ Bach runter weil der is dann: über (.) Sylvester [...] zu Kumpels, is dort fremdgegangen//mmh//[...], ja klar ich in der Zeit hier gewesen dann mit dem Typ da was angefangen gehabt ich bin aber nicht fremdgegangen also:, ja ((atmet aus)) und irgendwann ((atmet aus)) bin ich dann wo ich des mitgekriegt hab ausgezogen, (262-268)

Als Jasmin erfährt, dass ihr Freund sie betrogen hat, scheint dies der Anlass für die endgültige Trennung zu sein. Sie zieht aus der gemeinsamen Wohnung aus und direkt zu ihrem neuen Freund. Hier dokumentiert sich, dass Jasmin einen schnellen Wechsel von der einen in die nächste Beziehung vollzieht, der sich durch die räumliche Verortung - sie zieht direkt bei ihrem neuen Freund ein - noch potenziert. Möglicherweise ist sie aber auch aufgrund ihrer prekären Lebenssituation und der mangelnden bzw. nicht vorhandenen Unterstützung durch ihre Mutter gezwun- 
gen, direkt bei ihrem neuen Freund einzuziehen, da die Finanzierung einer Miete durch sie allein nicht möglich ist.

Hinsichtlich ihres Ex-Freundes fügt Jasmin dessen weiteren biografischen Verlauf an: "und dann ging bei ihm halt alles bergab« (268). Sie bemüht sich, die gemeinsamen Katzen zu vermitteln, da diese von ihrem Ex-Freund nicht mehr angemessen versorgt werden. An dieser Stelle dokumentiert sich Jasmins Verantwortungsgefühl, das sie gegenüber den gemeinsamen Haustieren zeigt und das sie veranlasst, aktiv zu handeln.

Den Übergang in ihre neue Beziehung beschreibt sie mit den Worten »also ich hab n kompletten Sprung in n neues Leben gemacht von heut auf morgen « (274f.). Damit wird nochmals die bedeutsame Veränderung, die diese Entscheidung für Jasmins Leben bedeutet, hervorgehoben.

In einer abschließenden Evaluation bewertet Jasmin die Beziehung als eine »so wie man sich es am Anfang oder das erste Mal richtig verliebt sein vorstell « (276f.). Hier zeigen sich Jasmins normative Vorstellungen hinsichtlich des Eingehens einer Beziehung, wonach das Sich-Verlieben eine Voraussetzung für eine Beziehung darstellt.

In der auf die Nachfrage der Interviewerin folgende Erzählung ${ }^{14}$ umreißt Jasmin die gesamte gewaltgeprägte Beziehung, die neben klar formulierten Gliederungsmarkierern zu Beginn (»da kann ich* viel erzählen da kann ich Bücher drum @schreiben@« (300); »AM ANFANG war alles schön« (301)) und am Ende (»und dann hab ich meinen jetzigen Freund kennengelernt (...) und der hat mir da eigentlich rausgeholfen« (351f.)) einen deutlichen Spannungsaufbau beinhaltet.

\section{Die erste Ohrfeige}

((ausatmend)) da kann ich* viel erzählen da kann ich Bücherdrum @schreiben@ (2), AM ANFANG war alles schön (3) also wirklich, äh er war ganz nett es: ich hab auch nie n Problem gehabt dass er 31 war ((seufzt)) des hat dann bei einer ganz komischen Situation angefangen wir saßen im, Auto (2) und gegenüber war n jüngerer Mann der mir zu gegrinst hat ich hab dann halt @zurückgegrinst@dann hab ich das erste Mal ne Ohrfeige gekriegt//mmh//also da hat mir fast den Kopf aufs Armaturenbrett gehauen, und dann hat er immer gesagt»]a:: Entschuldigung und tut mir leid und war nicht so gemeint« (2) ja, ich hab's halt geglaubt (2) und ((atmet aus)) und dann war er irgendwann so weit halt ja, zusammen einkaufen gegangen ich sag ja es war alles gut () ich hab dann immer gesagt die Ohrfeige des war $\mathrm{n}$ Ausrutscher des passiert nich mehr, (300-310)

14 Die hier interpretierte Passage unterscheidet sich formal auffällig vom Rest des Interviews. Sie ist geprägt durch äußerst detaillierte Erzählungen und Beschreibungen. Außerdem fällt die lange und ausführliche Behandlung eines Themas, nämlich die Gewaltbeziehung, auf. 
Den Beginn der Beziehung beschreibt Jasmin als »schön«, ihr Freund ist "ganz nett«, und der Altersabstand von gut zehn Jahren stört sie nicht. Als Jasmin beginnt, den Verlauf der Beziehung, und damit von der Gewaltspirale, die recht bald einsetzt, zu erzählen, umschreibt sie diese Entwicklung mit den Worten »des hat dann bei ner ganz komischen Situation angefangen«.

Im Zusammenhang mit der Geschichte von der ersten Ohrfeige weist Jasmin auf die Entschuldigung ihres Freundes hin, mit der sie gleichzeitig die Beziehung zu ihm legitimiert. Jedes Mal, wenn ihr Freund sie schlägt, folgen Entschuldigungen seinerseits. Jasmin sagt über sich selbst, sie habe ihm dies geglaubt. Außerdem geht sie zu Beginn davon aus, dass die Ohrfeige ein »Ausrutscher« war.

Hier dokumentiert sich Jasmins Akzeptanz der Übergriffe. Sie glaubt den Entschuldigungen und hofft damit auf positive Veränderungen. Ihre Aussage »sag ja es war alles gut « macht die Diskrepanz zwischen dem Wunsch nach einer gelingenden Beziehung ohne Gewalt und der tatsächlichen angespannten und gewaltgeprägten Stimmung in der Beziehung deutlich.

\section{Kontrolle}

und dann fings eigentlich an er ist abends heimgekommen wenn die Wohnung nich sauber war (2) hat kontrolliert arg kontrolliert unterm Vitrine unterm Sofa (3) und wenn's dann nich gepasst hat hab ich Schläge gekriegt und des war eigentlich jeden Tag so und irgendwann hat er mir dann eingeredet dass ich selber schuld dran bin, des hab ich ihm halt irgendwann abgenommen (2) (310-315)

Die Dynamik spitzt sich zu, als Jasmins Freund anfängt, sie zu kontrollieren. Wenn er abends nach Hause kommt, prüft er verschiedene Dinge, z.B. ob Jasmin sauber genug geputzt hat, ob der Kühlschrank leer ist oder ob sie rechtzeitig das Essen gekocht hat. Sie hat nach einiger Zeit selbst das Gefühl, dazu beizutragen, dass sie körperliche Gewalt erfährt. Sie glaubt, mit ihrem Verhalten ihren Freund provoziert $\mathrm{zu}$ haben.

An dieser Stelle zeigt sich, dass Jasmin aus der Retrospektive erzählt. Ihre Erfahrungen sind überarbeitet, indem sie zusammenfassend und evaluierend erzählt werden. Sie kann sie im Heute, aus der Position heraus zu wissen, wie alles gekommen ist, darbieten. Damit dokumentiert sich, dass Jasmin zum Zeitpunkt des Interviews andere Sichtweisen auf die gemachten Erfahrungen entwickelt hat und diese Beziehung anders bewertet als zum damaligen Zeitpunkt.

Jasmin verliert ihren Job im Imbiss, als ihr Freund eine Auseinandersetzung mit ihrem Chef beginnt. Als sie beginnt, in einer Kneipe zu jobben, kommt es zu einem weiteren Wendepunkt in der Beziehung; »dann fing der Horror eigentlich erst richtig an« (320f.). Wenn sie nicht pünktlich zu einer bestimmten Uhrzeit zu Hause ist, wird sie verprügelt (»hab ich Dresche gekriegt« (325)). 


\section{Zuspitzung}

dann fings an [...] wenn ich nich zu Hause war das heißt wenn ich um elf Feierabend hatte, und ich fünfzehn nach elf nich daheim war, hab ich Dresche gekriegt, und $s$ war dann irgendwann mal so weit dass er mich mit dem Kopf auf den Boden geschlagen hat (1) mit $n$ Arbeitsschuhen auf mich eingetreten hat (2) mir $n$ Kopf gegen Kühlschrank und sowas (3) ${ }^{\circ} \mathrm{ja}^{\circ}$ (2) (321-327)

Im Verlauf der Beziehung nimmt die Gewalt sowohl bezüglich der Häufigkeit als auch der Intensität zu. Jasmin wird laufend kontrolliert, ihr Freund ruft alle fünf Minuten an oder fährt bei ihrer Arbeitsstelle vorbei, um zu sehen, ob sie mit »fremden Männern« (333) redet.

Es folgt eine Schlüsselsituation, von der Jasmin einleitend erwähnt, dass nicht einmal Anna ${ }^{15}$ davon weiß, da Jasmin »zu viel Angst gehabt« (329) hat, ihr davon zu erzählen. Hier fällt auf, dass Jasmin - was selten während ihrer Erzählung passiert - ein Gefühl benennt. Dabei deutet die Tatsache, dass nicht einmal Anna von der im Folgenden erzählten Situation weiß, auf zweierlei hin: Zum einen kann davon ausgegangen werden, dass sich Jasmin ansonsten häufig der Streetworkerin anvertraut und diese eine wichtige Bezugsperson für sie ist. Zum anderen grenzt sich die Erfahrung, um die es hier geht, von allen anderen Erfahrungen ab, da sich Jasmin diesbezüglich nicht an Anna wendete.

\section{Eskalation}

ja und dann gabs die Situation, ich hab nich gleich die Einkaufstüten ausgeräumt, wenn wir einkaufen warn (5) u:nd ja dann hat er mich geschlagen und dann hab ich des Messer aus der Schublade genommen und hab zu ihm gesagt wenn er mich nich in Ruhe lässt (3) dann: steche ich ihn ab, ja, und dann hat er sich beruhigt gehabt, ja und ich bin dann ins Schlafzimmer gegangen hab bloß noch gemerkt wie was im Rücken sticht ich hab nich gewusst was los is (1) ich bin dann so zwischen Flur und Wohnzimmer zusammengebrochen und, ja ab da weiß ich nich mehr viel, ich weiß dann dass ich aufgewacht bin, mein Hund hatte sein Kopf auf meinem Bauch liegen, und ich hab sie neben mich hingelegt und hab gesehen dass alles voller Blut is so, und dann hab ich erst gedacht er hat meinem Hund irgendwas getan (.) bin ich so aufgestanden und hab gemerkt bei mir im Rücken, dass es brennt (2) im Rücken also () so lange Narbe jetzt am Rücken aber ich bin nich zum Arzt ich hab's einfach, zugetapet mit Panzerklebeband damals (1) dass es einigermaßen nich blutet, ja, (334-348)

15 Anna ist die Streetworkerin, die Jasmin bereits seit einigen Jahren kennt. Über Anna wurde auch der Kontakt zwischen Jasmin und mir hergestellt. Sie war am Interviewtermin anwesend, jedoch nicht im Cespräch dabei. 
Die geschilderte Situation stellt aufgrund mehrerer Aspekte eine Art Schlüsselsituation dar: Erstens wehrt sich Jasmin zum ersten Mal gegen die Schläge. Sie nimmt ein Messer und droht ihrem Freund, sollte er sie nicht in Ruhe lassen, würde sie ihn attackieren. Zweitens eskaliert die Gewalt an diesem Punkt vollends, als ihr Freund, nachdem sie sich umgedreht hat, ihr das Messer von hinten in den Rücken sticht. Jasmin wird daraufhin bewusstlos. Als sie wieder zu sich kommt, liegt ihr Hund bei ihr. Sie versorgt sich selbst, indem sie die Wunde mit Klebeband zuklebt. Drittens beinhaltet diese Situation ein weiteres Novum, nämlich die Inkaufnahme des Freundes, Jasmin mit dem Stich zu töten. Die Beziehung dauert noch ein weiteres halbes Jahr, in der es zu weiteren Misshandlungen kommt.

Bezüglich des hier unterliegenden Orientierungsrahmens zeigt sich eine absolute Ohnmacht Jasmins gegenüber den Gewaltausübungen ihres Freundes. Sie ist seinen Übergriffen vollkommen ausgesetzt und lässt diese über sich ergehen. Sie vertraut sich niemandem an, auch nicht der Streetworkerin, zu der sie ansonsten eine vertrauensvolle Beziehung unterhält. Der Verlauf der Beziehung spiegelt das wider, was allgemein als Gewaltspirale bezeichnet wird: den Verlauf einer Gewaltdynamik, die sich mit der Zeit immer mehr zuspitzt und aus der es immer schwieriger wird auszubrechen. Selbst als Jasmin lebensbedrohlich verletzt wird, versucht sie, die Situation allein zu bewältigen. Dies zeigt die enorme Isolation, die sie mit der Zeit innerhalb der Beziehung bzw. durch diese erfährt.

Eine neue Beziehung: Der aktuelle Freund

und dann hab ich meinen jetzigen Freund kennengelernt//mmh//und der hat mir eigentlich da rausgeholfen//mmh//(351-352)

Als Jasmin ihren jetzigen Freund kennenlernt, schafft sie es, sich aus der Gewaltbeziehung zu lösen. Hier dokumentiert sich die Unterstützung durch einen sog. signifikant Anderen (vgl. Kap. 9.2).

\section{Kennenlernen}

aber des hat sich dann gezogen gehabt also er war noch verheiratet, also is verheiratet (.) [...] ja und irgendwann hat er sich dann von seiner Frau getrennt, dann hat man sich getroffen//mmh//sind miteinander weggegangen oft, dann bin ich damals zu: meiner: Mu:tter gezogen weil er gesagt hat wenn ich nicht ausziehe will er nix mehr mit mir zu tun haben weil er sich des nicht anschaut wie ich immer mit blauen Flecken und sowas (2) ja (1) und dann sind wir irgendwann zusammen gekommen (357-364)

Jasmin lernt ihren heutigen Freund »auch auf Arbeit« (357) kennen und bezieht sich damit auf die vorausgegangene Beziehung mit ihrem Ex-Freund, den sie ebenfalls auf ihrer Arbeit kennenlernt. Im Gegensatz zu Jasmins bisherigen Beziehungen 
dauert es jedoch eine gewisse Zeit, bis sie ein Paar werden. Ihr Freund ist zunächst noch verheiratet, trennt sich jedoch von seiner Frau, und erst dann kommt es zu gemeinsamen Treffen mit Jasmin. Jasmin zieht zu ihrer Mutter, da ihr (zukünftiger) Freund dies von ihr fordert: »weil er gesagt hat wenn ich nich auszieh will er nix mehr mit mir zu tun haben«. Jasmins Freund ist derjenige, der die Misshandlung benennt und rahmt sowie Empathie zeigt. Indem er sagt, dass er sich die Blutergüsse »nicht anschaut «, drückt er damit erstens aus, dass diese nicht richtig sind bzw. nicht sein dürften, womit er indirekt seine Positionierung gegen Gewalt zum Ausdruck bringt. Zweitens äußert er, dass das Ansehen der Blutergüsse für ihn unerträglich ist. Damit nimmt er Jasmin außerdem gewissermaßen die Gefühlsleistung $a b$, da sie das selbst offensichtlich nicht kann. Nach einiger Zeit werden Jasmin und ihr neuer Freund ein Paar, woraufhin Jasmin dann recht schnell bei ihm einzieht.

Hier dokumentiert sich, dass Jasmin und ihr neuer Freund sich zunächst häufig treffen und oft miteinander ausgehen, bevor sie ein Paar werden. Dies ist eine deutlich andere, langsamere Art des Beziehungsbeginns. Indem sie sich Zeit lassen, schaffen beide die Möglichkeit, sich besser kennenzulernen und jeweilige Ziele und Vorstellungen hinsichtlich einer Paarbeziehung auszuloten. Jasmin zieht auf seine Forderung hin bei ihrem Ex-Freund aus und zu ihrer Mutter. Damit dokumentiert sich, dass es für sie vermutlich schwierig war, sich aus eigener Kraft von dem sie misshandelnden Freund zu trennen. Mit der Unterstützung ihres neuen Freundes bewältigt sie die Trennung von ihrem Ex-Freund. Jasmin bewertet die bislang gemeinsam verbrachte Zeit als eine, in der sie »eigentlich immer glücklich war« (365). Zum Zeitpunkt des Interviews dauert die Beziehung bereits ein Jahr lang. Der Verweis darauf, es sich in der gemeinsamen Wohnung "gemütlich gemacht« (383) zu haben, zeigt ihr aktives Engagement hinsichtlich der Gestaltung ihrer aktuellen Lebenssituation

Jasmins Ergänzung »fühl mich eigentlich auch wohl [...] und [...] hilft mir auch viel über mein Ex-Freund wegzukommen « (387f.) bezieht sich zunächst auf die Beziehung mit ihrem aktuellen Freund und in der Fortsetzung der Äußerung auf ihren Aufenthalt in der Tagesklinik, welchen sie als unterstützend und hilfreich in der Verarbeitung und Bewältigung der letzten Beziehungserfahrungen erlebt.

Professionelle Unterstützung

Jasmin erfährt im Laufe ihrer Biografie immer wieder professionelle Hilfe und Unterstützung: zum einen über einen längeren Zeitraum von vermutlich einigen Jahren durch die Mobile Jugendarbeit, zum anderen ist sie zum Zeitpunkt des Interviews in einer psychiatrischen Tagesklink. Ihre Kontakte zur Mobilen Jugendarbeit thematisiert sie nicht direkt. Stattdessen fließen sie in Form von Hintergrundkon- 
struktionen in ihre Erzählung mit ein. Dennoch wurden sie als Kernthemen identifiziert, da sie eine zentrale und konstante Funktion in Jasmins Leben einnehmen.

\section{Streetwork}

trotz, Drogen trotz allem aber ich hab's durchgezogen//mmh//(.) ja (5) meine Ausbildung war halt auch nicht leicht, aber ich sag ja es hat geklappt de:n Willen hab ich gehabt dazu (2)//mmh//(10) und dann hab ich zu der Zeit Anna kennengelernt//mmh//bisschen später nochmal (ne) Drogen (Sache) sie hat nich mehr viel mitgekriegt s Kiffen hat sie noch mitgekriegt, da:nn kennt sie noch mein ExFreund, ja (2) zu der ${ }^{\circ}$ Zeit hab ich Anna kennengelernt ${ }^{\circ}$, grad durch meinen ExFreund auch $\mathrm{n}$ Thomas//mmh//(2) ja wo ich dann regelmäßig hingegangen bin dann haben wir irgendwann Vertrag aufgesetzt @wegen@ dem selbstverletzenden Verhalten dass ich mich vorher melden soll,//mmh//des eigentlich bis jetzt immer gut geklappt hat (222-231)

In dieser Sequenz wird deutlich, dass Jasmin Anna in der Zeit kennenlernt, als sie gerade dabei ist, ihre Ausbildung abzuschließen. Jasmin ist zu dieser Zeit 16 oder 17 Jahre alt. Es kommt zu regelmäßigen Kontakten zwischen Jasmin und der Streetworkerin (»wo ich dann regelmäßig hingegangen bin«). Der Hinweis auf den Abschluss eines gemeinsam vereinbarten Vertrags zwischen Jasmin und Anna bezüglich des Ritzens deutet auf eine mittel- bis längerfristig bestehende Unterstützung hin, der ein entsprechendes Vertrauensverhältnis zugrunde liegen muss, damit diese Hilfe überhaupt möglich ist. Der Verweis auf das Funktionieren des Vertrages zeigt, dass die einzelfallorientierte Hilfe der Streetworkerin von Jasmin als hilfreich erlebt wird. Dabei kann davon ausgegangen werden, dass die über den langen Zeitraum von fast sieben Jahren konstante und verlässliche persönliche Beziehung zu Anna für Jasmin eine wertvolle Unterstützung und Begleitung bedeutet.

\section{Tagesklinik}

Mh, ich war zuerst immer bei Anna hat immer gesagt ich soll mir n Psychologen suchen oder so, aber ich wollt des nie [...] und dann hab ich gesagt jetzt gehts hier ja auch nich weiter ich brauch Hilfe//mmh//, [...] ja und dann hamn wir gesagt ich geh in die Tagesklinik jetzt bin ich [...] hier (390-406)

Hinsichtlich des Zugangs zur Tagesklinik beschreibt Jasmin, dass Anna ihr immer wieder vorschlug, einen Psychologen aufzusuchen, was Jasmin zunächst ablehnte. Sie gelangt allerdings mit der Zeit selbst an einen Punkt, an dem sie das Gefühl hat »jetzt gehts hier ja auch nich weiter«.

Durch die Unterstützung in der Tagesklinik versucht Jasmin, ihr Leben bzw. ihre Erlebnisse »in Griff zu kriegen« (46). Damit wird im Umkehrschluss auch deut- 
lich, dass sie das Gefühl gehabt haben muss, oder immer wieder noch hat, dass ihr Leben ihr entgleitet, sie es nicht im Griff hat.

Jasmin bilanziert ihre momentane Situation hinsichtlich der professionellen Hilfe und Unterstützung, die sie in der Tagesklinik erfährt, als wohltuend. Angesichts ihrer vergangenen biografischen Geschichte, in der sie überwiegend auf sich allein gestellt war und vieles zu einem frühen Zeitpunkt selbstständig bewältigen musste, scheint die Tatsache, Hilfe nun »zulassen« (411) zu können, ein großer Schritt zu sein. Die Unterstützung, die sie in der Tagesklinik erfährt, wirkt sich vor allem auch auf ihr "Selbstbewusstsein« (412) aus. Hier dokumentiert sich, dass es einen Wendepunkt gab, an dem Jasmin sich eingestanden hat, Unterstützung zu benötigen und sie sich auf diese auch einlassen konnte.

\subsubsection{Argumentative Stellungnahme}

Im folgenden Kapitel wird der Blick auf die Rekonstruktion der Theorien gelenkt, die Jasmin systematisch über ihre Handlungspraxis entfaltet. Dabei fällt auf, dass Jasmin zu Beginn des Interviews kaum im Modus der Argumentation berichtet. Erst im weiteren Verlauf des Interviews tauchen zunehmend einzelne Argumentationen in Form von Hintergrundkonstruktionen auf. Offenbar ermöglichte das biografisch-narrative Interview Jasmin mit der Zeit »ein nachdenkliches Innehalten gegenüber der Alltagspraxis oder in der retrospektiven Rekonstruktion von den eigenen Handlungs- und Erleidensverwobenheiten [...], um sich unplausible oder gar unverständliche Erlebnisse erklären zu können« (Schütze 1987, S. 138).

Im Folgenden wurden zwei Zitate ausgewählt, die jeweils in einen Kontext eingebettet sind, in dem Jasmin zu einer Reflexion des Erzählvorgangs innehält. Sie spiegeln die gegenwärtige theoretische Haltung von Jasmin wider.

\section{Sich Hilfe holen I}

ich würde es nur jedem raten dass er sich Hilfe holt wenn irgendwas is//mmh//aber egal was ich sehe es mittlerweile ob des in der Beziehung is ich hol mir jetzt auch Hilfe für meine Beziehung wir haben morgen Paargespräch//mmh//wo ich einfach sag des sind Leute die sich damit auskennen//mmh//(4) ja//mmh//(10) des is ganz hilfreich hier (3) ich sag ja ich rat's eigentlich jedem//mmh//vor allem rat ich den meisten jungen Frauen, weil des Schlimmste was ich in meinem Leben durchgemacht hab war des mit meinem Ex-Freund (2) (419-426)

Jasmin schließt hier ihre Bilanzierung mit einem Rat ab, der sich vor allem an junge Frauen richtet: Generell empfiehlt sie, sich Hilfe und Unterstützung zu holen. Auch für ihre aktuelle Paarbeziehung nutzt sie die Möglichkeit, indem sie sich in einem Paargespräch Hilfe holt. In dem allgemeinen Ratschlag, der sich an junge Frauen wendet, dokumentiert sich Jasmins Bereitschaft, sich auf professionelle Hilfe einzulassen und diese zuzulassen. Ihre Erfahrungen sind positiv und sie erlebt die 
Unterstützung als hilfreich, weshalb sie diese weiterempfiehlt. In der Annahme professioneller Hilfe dokumentiert sich Jasmins Engagement, in ihre Zukunft zu investieren.

\section{Sich Hilfe holen II}

also des is so das Hilfreiche vor allem sich Hilfe suchen//mmh//(.) würd ich jederzeit wieder machen//mmh//auch grad so Mobile Jugendarbeit oder wenn irgendwas is Ansprechpartner brauchst, ja, des würd ich halt au anders machen jetzt//mmh//hätt ich mir früher hätt ich mir keine Hilfe geholt da hätt ich gesagt »kannst de alles allein « aber mittlerweile sag ich [...] egal ob du mit deinem Freund Theater hast ob er dich schlägt ((atmet ein)) oder familiär oder sonst irgendwas//mmh//ja (807-815)

In dieser rückblickenden Bewertung hält Jasmin fest, wie hilfreich sie die Unterstützung in den letzten Jahren durch die Mobile Jugendarbeit erlebt hat. Gleichzeitig wird der Wandel deutlich, den sie diesbezüglich durchgemacht hat: Sie hält zunächst fest, dass sie früher der Meinung war, ihr Leben und die damit verbundenen Schwierigkeiten und Herausforderungen allein meistern zu müssen. Im Gegensatz dazu vertritt sie heute die Auffassung, sich gegebenenfalls - auch frühzeitig - Unterstützung zu holen.

\subsubsection{Zusammenfassung}

Erleben von Gewalt in Paarbeziehung Jasmin erlebt in ihrer zweiten Beziehung Gewalt durch ihren Partner, wobei die erlebte Gewalt Teil eines komplexen Misshandlungssytems ist. Verschiedene Handlungs- und Verhaltensweisen ihres Freundes zielen darauf ab, Macht und Kontrolle über sie zu gewinnen. Die physischen und psychischen Gewalttaten, die Jasmin erfährt, sind darauf ausgerichtet, Abhängigkeiten aufzubauen und ihr Selbstvertrauen sowie ihre Handlungsspielräume zu untergraben. Die Gewaltdynamik, die sich in der Analyse des Interviewmaterials rekonstruieren ließ, ist mit der sogenannten »Gewaltspirale« (Walker 1979) vergleichbar, nach der nach Phasen des Spannungsaufbaus der Gewaltausbruch folgt, welcher wiederum von beiden Seiten entschuldigt wird und damit zu einer Entlastung des Gewaltausübenden führt. Außerdem zeigt sich, dass es im Laufe der Beziehung zu einer Steigerung der Gewaltausbrüche kommt und diese an Schwere und Häufigkeit zunehmen.

Umgang mit erlebter Gewalt, Bewältigungsstrategien und Ressourcen Hinsichtlich des Umgangs mit der erlebten Gewalt werden im Interview ausschließlich passive Strategien angesprochen. So entschuldigt Jasmin das gewalttätige Verhalten ihres Partners und sucht die Ursachen bzw. die Schuld bei sich selbst. Sie wendet vermeidende Strategien der Gewaltbewältigung an, indem sie bemüht ist, die 
Wünsche ihres Freundes zu erfüllen (die Wohnung zu putzen, pünktlich zu Hause zu sein). Indem sie auf diese Art und Weise versucht, den Forderungen ihres Freundes nachzukommen, begibt sie sich in eine Position der Unterordnung, die gleichzeitig eine Machtsteigerung des Partners bedingt. Nicht zuletzt stellt das Ertragen der erlebten Gewalt eine passive Strategie der Gewaltbewältigung dar.

Die Unterstützung durch Außenstehende bedeuten für Jasmin eine wesentliche Ressource bei der Bewältigung ihrer Erfahrungen. Zum einen geschieht dies durch den langjährigen Beistand der Streetworkerin, zum anderen durch einen jungen Mann, der sie als Außenstehender auf die von ihm beobachtete Gewalterfahrung anspricht und mit dessen Hilfe es Jasmin letztlich gelingt, sich aus der Misshandlungsbeziehung zu lösen.

Beziehungsmuster und Beziehungsgestaltung In ihrer ersten Paarbeziehung macht Jasmin zunächst die Erfahrung einer gewissen Normalität. Diese gerät ins Ungleichgewicht, als ihr Freund das für die Miete eingeplante Haushaltsgeld für den Kauf von Drogen verbraucht. Damit geht auch eine Ungleichverteilung der Rollen innerhalb der Beziehung einher, bei der Jasmin diejenige ist, die die Hauptverantwortung trägt. In der darauffolgenden Beziehung zeigen sich gleich zu Beginn eine ungleiche Rollenverteilung sowie die Entwicklung einer von Abhängigkeit gekennzeichneten gewaltgeprägten Beziehung.

(Gewalt-)Beziehung und Sozialisation Jasmin erlebt bereits in ihrer frühesten Kindheit Gewalt sowohl auf intergenerationaler Ebene (physische und psychische Gewalt durch den Vater) als auch auf transgenerationaler Ebene (häusliche Gewalt). Dabei übernimmt sie als älteste Tochter bereits früh Verantwortung für ihre beiden jüngeren Geschwister und versucht, diese vor dem gewalttätigen Vater zu schützen. Ihre Mutter erlebt sie als hilflos und ohnmächtig. Nach Buddenberg (1983) zeigt sich hier ein typisches transgenerationales Interaktionsmuster hinsichtlich der Partnerwahl von Frauen, die in ihrer Herkunftsfamilie häusliche Gewalt miterlebt haben:

»Da die Frau in ihrer Herkunftsfamilie häufig parentifiziert wurde oder gegenüber jüngeren Ceschwistern Mutterfunktionen ausüben mußte, sucht sie sich einen sich stark und überlegen fühlenden Mann, der ihr erlaubt, hilfsbedürftig und unselbständig zu sein.«(Buddeberg 1983, S. 274)

Die Wahrnehmung geschlechtsspezifischer Elternrollen sowie das Miterleben häuslicher Gewalt beeinflussen die eigenen Geschlechterkonstruktionen. Bei Jasmin lässt sich eine patriarchal-traditionale Verteilung der Geschlechternormen rekonstruieren, die innerhalb der dargestellten Gewaltbeziehung bis hin zur totalen Unterordnung führt. In dieser Beziehung erlebt sie das, was sie von zu Hause kennt: einen gewaltausübenden Partner, der ihr gegenüber stark und überlegen 
ist und ihr dadurch erlaubt, hilfsbedürftig und ohnmächtig zu sein. Jasmin sieht die Gewalt seitens ihres Freundes als Ausdruck von Liebe an, denn sie hat in ihrer Sozialisation früh gelernt, dass geliebt $\mathrm{zu}$ werden gleichzeitig auch bedeutet, verletzt $\mathrm{zu}$ werden.

Weitere Themen Ein wesentliches Thema, das sich im Interview mit Jasmin zeigt, ist die Zugehörigkeit zu verschiedenen Jugendkulturen, die als Ort der Anerkennung und Zugehörigkeit fungieren und die Möglichkeiten zur Selbstinszenierung bieten.

Die Zugehörigkeit zu verschiedenen (jugendkulturellen) Szenen (Drogenszene, Naziszene, Punkszene) deuten auf Lösungs- und Bewältigungsversuche, die Jasmin wählt. Dabei fällt auf, dass es sich teilweise um aggressive Milieus handelt, was darauf hindeuten könnte, dass Jasmin zunächst Zugehörigkeit in Milieus sucht, die von Gewalt und Aggressivität geprägt sind und damit Parallelen zu ihrem familiären sozialisatorischen Umfeld aufweisen. Sie erlebt hier das, was sie auch aus ihrer Kindheit kennt.

Insgesamt zeigt der Fall Jasmin, dass die Erfahrung der Welt als unsicherer Ort zu einer totalen Abhängigkeit, bis hin zur Unterwerfung, führen kann. Da Jasmin nie die Erfahrung von Sicherheit und Vertrauen gemacht hat, sucht sie Sicherheit durch eine Person, die ihr sagt, was sie tun soll.

\subsection{Typengenese: Praktiken und Handlungsorientierungen im Umgang mit Gewalt}

Für die Generierung von Typen werden in der Literatur zwei Wege vorgeschlagen: Entweder werden bereits zu einem frühen Beginn der Auswertung Textpassagen fallübergreifend anhand eines gemeinsamen Themas miteinander verglichen oder es werden zunächst ausführliche Einzelfallanalysen durchgeführt, um Vergleichsdimensionen für die Verallgemeinerung von Besonderheiten der Fälle zu identifizieren (vgl. Kelle/Kluge 2010, S. 85). Für die vorliegende Forschungsarbeit wurde der Weg der ausführlichen Einzelfalldarstellung gewählt. Im Zuge der Fallbeschreibungen wurden die Orientierungsrahmen rekonstruiert, die die Fälle konstituierten, und zwar so, wie sie prozesshaft im Rahmen einer Erzählung entfaltet wurden (vgl. Bohnsack 2010a, S. 141). »Dabei bleibt die Gesamtcharakteristik des Falles, die (individuelle oder kollektive) >Weltanschauung $<$ die in ihrer >Totalität « unterschiedliche Erlebnisschichten oder Erfahrungsräume ... umfasst, in ihrer Komplexität oberster Bezugspunkt der Interpretation und Darstellung« (ebd.). Anhand der in diesen Einzelfallanalysen herausgearbeiteten Kernthemen konnten Vergleichsdimensionen gewonnen werden, die zum Ziel haben, das Datenmaterial zu strukturieren. Mithilfe der vergleichenden Kontrastierung konnten dann Ähnlichkeiten und Unterschiede ermittelt werden, aus denen letztendlich die Typolo- 
gie entwickelt wurde (vgl. Kelle/Kluge 2010, S. 85). Die grundlegende Perspektive ist eine praxeologische (Bohnsack 2007b), indem habituelles Handeln, konjunktive Erfahrung und Orientierungsmuster im Fokus der Analyse stehen. Praxeologische Typenbildung ${ }^{16}$ unterscheidet sich dabei von der Typenbildung des Common Sense (Bohnsack 2007b, S. 226) durch »die genetische Perspektive auf praktisches Handeln, die nicht allein nach dem Handlungsentwurf, sondern auch nach dessen Entstehung (Weil-Motiv) und den Orientierungsrahmen fragt « (Franz/Griese 2010, S. 275f.). Mithilfe der komparativen Sequenzanalyse wurden dann einzelfallübergreifend (kollektive) Erfahrungen und Orientierungen (Bohnsack u.a. 2007, S. 21) rekonstruiert.

In der Auswertung der vorliegenden Fallstudien wurden Handlungspraktiken und Orientierungen junger Frauen im Umgang mit Gewalt während der ersten eigenen Paarbeziehungen rekonstruiert. Durch den Vergleich der Fälle sowie deren zugrunde liegenden Orientierungsrahmen konnten drei Typen identifiziert werden, die im Folgenden charakterisiert werden.

Für die sinngenetische Typenbildung stellt sich die Frage nach den Unterscheidungsmerkmalen (Bohnsack 2007b, S. 236) hinsichtlich des Umgangs mit Beziehungsgewalt bzw. nach deren Bewältigung. Der sinngenetischen Typenbildung liegen in der vorliegenden Arbeit vier Dimensionen zugrunde, auf denen die drei generierten Typen basieren:

1. eine generelle biografische Perspektive auf den Lebenszusammenhang der jeweiligen Erzählerin und damit auch auf ihre Herkunftsfamilie,

2. der Blick auf subjektive Vorstellungen zu Paarbeziehungen und auf Vorstellungen zu gesellschaftlicher Normalität (Ebene der Orientierungsschemata),

3. die dritte Dimension, die sich aus dem Forschungsinteresse der Arbeit ergibt und die den Orientierungsrahmen im Hinblick auf den Umgang mit den erlebten Gewalterfahrungen in den Blick nimmt (Bewältigungsstrategien), sowie

4. die Neuorientierung, die sich aus dem Orientierungsrahmen nach Beendigung der Gewaltbeziehung ergibt bzw. ergeben kann.

Alle vier Dimensionen liefern Hinweise auf den Umgang mit Beziehungsgewalt bzw. auf Bewältigungsmuster, die sich in habitualisierten Stilen zeigen und die sich zu Typen verdichten lassen. Dabei werden die Sinnmuster des handlungsleitenden Wissens, also die Orientierungsrahmen bzw. der Habitus, die als homologes Muster an verschiedenen Fällen identifizierbar sind, von den fallspezifischen Besonderheiten gelöst.

16 Zur Abgrenzung dieses rekonstruktiven Verständnisses von Typenbildung von anderen Ansätzen der Typenbildung sowie zur von Bohnsack vorgeschlagenen Verbindung von Typenbildung des Common Sense einerseits und praxeologischer Typenbildung andererseits vgl. auch Bohnsack $2010 b$. 
Die im Folgenden dargestellten Typen sind also nicht identisch mit den in Kapitel 8.1 dargestellten Einzelfällen. Vielmehr stellt ein Typus stilisierte Ansichten, Orientierungen und Aspekte von Einzelfällen dar. Die Typen bezeichnen die in den Fällen rekonstruierten Handlungsoptionen und stellen damit Handlungspraktiken dar.

Grundlage für die folgende Genese von Typen bildeten sechs Fälle, zwei pro Typus.

\subsubsection{Der Typus »Pertinenz « - »die Liebe, die ich immer gesucht habe « ${ }^{17}$}

In der folgenden Beschreibung des Typus »Pertinenz« wird der Typus insbesondere anhand der Fälle Anna und Amy ausgewiesen, es werden aber auch andere Fälle herangezogen, in denen sich in der fallübergreifenden komparativen Analyse ein gemeinsamer Orientierungsrahmen identifizieren ließ.

Allgemeine Beschreibung des Typus

Nach diesem Sinnmuster werden Paarbeziehungen gestaltet und ausgeformt. Dabei dienen Beziehungen häufig der unmittelbaren Bedürfnisbefriedigung. Umgekehrt zeigt sich auf Seiten der Frauen ein Handlungsmuster, nach dem sie sich als leicht verfügbar anbieten. Auch der Umgang mit Cewalt innerhalb der Paarbeziehung strukturiert sich nach diesem handlungsleitenden Wissen. Demnach bestehen Grenzziehungen gegenüber wechselseitigen Nutzungsrechten und Verfügbarkeiten, diese werden aber nicht kommunikativ ausgehandelt, sondern jeweils gewalttätig gesetzt.

Frauen dieses Typus nehmen bereits zu einem frühen Zeitpunkt intime Beziehungen zu deutlich älteren Männern auf und zeigen sich sexuell aktiv. Das Beziehungskonzept dieser Frauen ist eng verknüpft mit einer Familienorientierung, was sich (in den hier vorliegenden Fällen) durch frühe Schwangerschaft(en), die Orientierung an wesentlich älteren Männern und die hohe Akzeptanz gegenüber Verhaltensweisen in der Gestaltung der Beziehung, die eigentlich nicht gutgeheißen werden, zeigt.

Hinsichtlich des Umgangs mit Gewalt innerhalb der Paarbeziehung zeigt sich, dass Frauen dieses Typus ein gewisses Maß an Cewalt akzeptieren, solange die erlebte Gewalt den Zugang zu Zugehörigkeit ermöglicht. In Abgrenzung zum Typus »Abhängigkeit«, bei dem die Gewaltdynamik zu einer Zunahme an Häufigkeit und Schwere der Cewalt führt, beenden Frauen des Typus »Pertinenz« die Beziehung vor Einsetzen eines Kreislaufs der Gewalt.

17 Das Zitat bezieht sich auf eine Aussage Amys: »und die Zuneigung und die Liebe hab ich halt immer gemei::nt« (Amy, Z. 52f.). Im Kontext der Sequenzanalyse wurde die Verwendung des Wortes »gemeint « in der Bedeutung von »gesucht« interpretiert. 
Bezüglich der Neuorientierung (die zum Zeitpunkt des Interviews ausgemacht werden konnte) zeigte sich, dass es Frauen dieses Typus gelingen kann, sich durch Aktivierung eigener Ressourcen sowie mithilfe professioneller Unterstützung aus der Gewaltbeziehung zu lösen.

Biografische Perspektive In den Fällen Anna und Amy zeigen sich Parallelen hinsichtlich der Bedingungen des Aufwachsens. Sowohl Amy als auch Anna wachsen mit ihrer alleinerziehenden Mutter in einem chaotischen Umfeld auf. Der Vater ist wenig oder gar nicht präsent. Bei beiden wirkt sich die Suchterkrankung der Mutter (Alkohol bzw. Drogen) dominant auf das Aufwachsen der jeweiligen Töchter aus, indem es zu einer frühen Parentifizierung kommt. Zudem erleben beide Mädchen häufig wechselnde Männerbekanntschaften oder Beziehungen der Mutter. Hier teilen Anna und Amy den konjunktiven Erfahrungsraum Familie hinsichtlich seiner milieuspezifischen Besonderheiten. Im Vergleich zu Amy macht Anna jedoch auch positive bildungsspezifische Erfahrungen, die die Voraussetzungen für die in ihrer Biografie zentral werdenden Bildungsaspirationen bilden.

Subjektive Vorstellungen Hinsichtlich ihres Beziehungskonzeptes zeigen beide Frauen eine starke familiale Orientierung, die sich in frühen Schwangerschaften zeigt sowie in lang andauernden differenten Versuchen, als Familie zusammenzuleben. Innerhalb ihres Orientierungsschemas haben sowohl Anna als auch Amy explizite Vorstellungen davon, wie eine Beziehung gestaltet werden und welche Normen innerhalb einer Beziehung eingehalten werden sollten. Dabei werden bei Anna die Vorstellung, es in einer Beziehung langsam angehen lassen, und der Wunsch nach gemeinsamen Unternehmungen als Paar sowie mit Freund_innen deutlich. Im Gegensatz dazu zeigt sich bei Amy mehr der Wunsch nach einem klassisch-normativen Familienleben, das sich vor allem in der Gestaltung eines gemeinsamen häuslichen Alltags zeigt. In beiden Fällen zeigt sich eine Diskrepanz zwischen dem Idealbild von Familie, das hier insbesondere durch Zugehörigkeit, vermittelt durch Kinder und Verbundenheit, charakterisiert ist. Die Realität gestaltet sich diesbezüglich aber anders und muss daher in ihren Unterschieden immer wieder neu in Einklang mit dem Idealbild gebracht werden. Dies geschieht entweder durch Trennung, neue Beziehungsversuche oder durch die Bestimmung von Punkten (z.B. indem Erwartungen formuliert oder Bedingungen gestellt werden), die erwarten lassen, dass sich die Beziehung doch in Richtung des Idealbildes gestaltet.

Umgang mit erlebten Gewalterfahrungen Hinsichtlich des Umgangs mit Gewalt zeigen sowohl Anna als auch Amy eine große Toleranz gegenüber groben Verhaltensweisen sowie der Akzeptanz von ungewollten sexuellen Intimitäten innerhalb der Beziehung. Auch bei Alina zeigt sich dieser Orientierungsrahmen, wenn auch abge- 
schwächt, in Form von Akzeptanz gegenüber den Entscheidungen ihres Freundes, wann und wo gemeinsame Treffen stattfinden.

Dagegen stellt die Ausübung physischer Gewalt bei Frauen dieses Typus eine deutliche Grenze dar. Die Reaktionen darauf können differieren. Während Anna zwar schockiert ist, zeigt sie gegenüber ihrem Freund keine Reaktion. Im Gegensatz dazu reagiert Amy auf die Androhung körperlicher Gewalt mit einer Gegendrohung bzw. auf die Ausübung von Gewalt mit dem Rauswurf ihres Freundes aus der gemeinsamen Wohnung. Außerdem agiert sie ihre Wut in erster Linie gegenüber Gegenständen aus.

Die Frauen dieses Typus zeigen sich nicht generell als gewaltbereit oder gewaltaktiv. An bestimmten Grenzen reagieren die Frauen aber auch entweder mit eigener Gewaltaktivität oder mit Gewalt gegenüber Gegenständen.

Neuorientierung Die Fokussierung einer Familienorientierung zeigt sich hier als zentrale Strategie, das Idealbild von Familie und damit auch von gelingender Partnerschaft zu verwirklichen. Die Ausrichtung auf Familie setzt sich auch nach der Trennung von einem gewaltausübenden Partner fort - entweder in der Suche nach einem neuen Partner oder aber, wenn es zu einer Abkehr von Versuchen, mit einem Partner zusammenzuleben kommt, im primären Zusammenleben mit Kind(ern). Mit dieser Fokussierung sowie mit Unterstützung professioneller Hilfe (Familienhilfe, sozialpädagogischer Unterstützung innerhalb der Jugendberufshilfe, Kinderund Jugendpsychotherapie bzw. Therapie) gelingt es den Frauen, sich aus gewaltvollen Beziehungen zu lösen

\subsubsection{Der Typus "Autonomie" - »sich allein als wertvoll erfahren ohne die Bestätigung des Partners « ${ }^{18}$}

Die Beschreibung des Typus "Autonomie« wird vor allem anhand der Fälle Zofia und Daria ausgewiesen. Aber auch hier werden wieder andere Fälle herangezogen, in denen sich in der fallübergreifenden komparativen Analyse ein gemeinsamer Orientierungsrahmen identifizieren lässt.

18 Das Zitat wurde in Anlehnung an ein Originalzitat von Zofia, der besseren Lesbarkeit wegen, verkürzt. Im Original lautet es: »dass äh:m ma:n (.) alleine äh sich äh wertvoll erfahren kann und nicht immer diese Bestätigung auf Seiten vom Mann oder von seinem Partner braucht« (Zofia, Z. 355-357) 
Allgemeine Beschreibung des Typus

Für den Typus »Autonomie« ist charakteristisch, dass die den übergreifenden Orientierungsrahmen kennzeichnende Fokussierung auf Autonomie durch ein Zusammenspiel mehrerer Faktoren der Beziehungsgestaltung hervorgebracht wird.

Biografisch zeigt sich, dass bei Frauen dieses Typus, während des Aufwachsens mindestens ein Elternteil als stärkend und unterstützend erlebt wurde.

Hinsichtlich der subjektiven Vorstellungen von Beziehungen ist es für Frauen dieses Typus wichtig, eine egalitäre Beziehung zu führen. Dies gestaltet sich auf emotionaler Ebene in gegenseitiger Anerkennung und dem Entgegenbringen von Respekt aus. Auf der interaktiven Ebene wird ein gleichberechtigtes Einbringen in die Beziehung erwartet, bspw. hinsichtlich der Investition von Zeit und Celd. Beide Beziehungspartner_innen werden dabei als autonome Beteiligte angesehen, wobei sich die Autonomie insbesondere auch auf die berufliche und finanzielle Unabhängigkeit vom Partner bezieht.

Im Umgang mit und in der Bewältigung von Cewalterfahrungen innerhalb der Paarbeziehung zeigen diese Frauen aktive Strategien, indem sie Forderungen hinsichtlich Änderungswünschen an den Partner stellen und so versuchen, eine gemeinsame Beziehungsgestaltung umzusetzen. Eine weitere aktive Bewältigungsstrategie stellt bei Frauen dieses Typus die Trennung von ihrem Freund bzw. Partner nach wiederholter Cewaltausübung dar. Außerdem zeigen sich Frauen dieses Typus kompetent, sich Hilfe und Unterstützung innerhalb ihres sozialen Umfeldes oder ggf. auch professionelle Hilfe zu holen.

Biografische Perspektive Den Fällen Zofia und Daria ist gemeinsam, dass beide im Kindesalter nach Deutschland immigrieren und mit deutlichen Brüchen innerhalb ihrer Biografie konfrontiert sind. Beide Frauen wachsen in ihren Kernherkunftsfamilien auf, wobei sich die Eltern von Daria scheiden lassen, als Daria 15 Jahre alt ist. Sie hält jedoch weiterhin einen engen und guten Kontakt zum Vater, während sie bei der Mutter wohnt.

Mit Blick auf die Biografie zeigt sich bei Zofia bereits im Jugendalter die Herausbildung eines Habitus, der auf Autonomie ausgerichtet ist. So verlässt sie ihren Sportverein, um ein selbstbestimmteres Leben führen zu können, und nimmt damit einen Bruch in der Beziehung zu ihrem Vater in Kauf, der mit dem Verlust seiner Anerkennung verbunden ist. Dieser Entscheidung zugrunde liegt das Bedürfnis, sich mit Jungen ungehindert treffen zu können.

Die Entwicklung eines autonomen Habitus zeigt sich auf weiteren biografischen Ebenen. So spiegelt sich auch in der Entwicklung einer Essstörung - wie bei Zofia der Fall - das Streben nach Autonomie wider, indem über die selbstbestimmte Inszenierung des Köpers ein Teil von Autonomie gewahrt wird, der es ermöglicht, in vermeintlich selbstgesetzten Regeln zu agieren. Bei Daria zeigt sich 
die biografische Herausbildung eines autonomen Typus im Kontext ihrer Peers, innerhalb dessen sie in Form gewaltaktiver Auseinandersetzungen unter Mädchen ihre autonome Position in der Mädchenclique erwirbt.

Bei diesem Typus zeigt sich zudem eine enorme Bildungsaspiration (vgl. Anna, Amy, Zofia), die sich in einem intensiven Bestreben zeigt, den formalen Bildungsweg (gut) zu absolvieren (Schulabschluss bei Schwangerschaft, Ausbildung als Alleinerziehende, Studium im Anschluss an Berufsausbildung).

Subjektive Vorstellungen Hinsichtlich des Beziehungskonzeptes ist es Frauen dieses Typus wichtig, dass die Beziehung egalitär ausgerichtet ist. Die Egalität bezieht sich dabei einerseits auf die emotionale Ebene, was sich in gegenseitigem Respekt, Anerkennung und Wertschätzung zeigt, und andererseits auf eine interaktive Ebene, bei der es darum geht, dass sich beide gleichermaßen bezüglich zeitlicher und finanzieller Investitionen einbringen.

Bei Zofia wird dies deutlich, indem sie von Beziehung zu Beziehung unterschiedliche Modi der Beziehungsgestaltung ausprobiert, immer mit dem Ziel, eine gegenseitige Wertschätzung und Akzeptanz als Basis der Beziehung zu erreichen. Im Interview mit Daria wird die zweite Ebene deutlich, die zeigt, dass sich beide Partner in einer Beziehung gleichberechtigt an der Investition von Zeit und Geld beteiligen sollten.

Des Weiteren dokumentiert sich der egalitäre Anspruch, den Frauen dieses Typus in ihren Beziehungen verfolgen, auch in Bezug auf die autonome Ausübung der eigenen Berufsausbildung bzw. -tätigkeit. Damit einher geht die Auffassung, sich ein finanziell unabhängiges Leben aufbauen zu können.

Umgang mit Gewalt Hinsichtlich des Umgangs mit Gewalt zeigen sich bei Frauen dieses Typus vor allem aktive Bewältigungsstrategien: Diese umfassen eine gewisse Spanne und reichen von Strategien innerhalb der Beziehung, aktive Forderungen an den Partner zu stellen, sein Verhalten zu verändern, bis hin zur umgehenden Trennung nach einer Gewalterfahrung. Letztere erfolgt dabei im Bewusstsein, dass auf eine einmalige Ausübung von Gewalt auch mit weiteren Gewaltausübungen zu rechnen ist. Des Weiteren sind Frauen dieses Typus in der Lage sich Hilfe zu holen. Dies erfolgt im direkten sozialen Umfeld und kann sich auf Familienangehörige oder den Freundeskreis beziehen.

Neuorientierung Eine Neuorientierung zeigt sich bei diesem Typus nach erfolgreicher Beendigung der Gewaltbeziehung dahingehend, dass die Frauen durch selbstinitiatives Handeln, das Ergreifen spontaner Bildungsgelegenheiten und/oder die Annahme von professioneller Hilfe einen Wandlungsprozess durchlaufen, der ihnen neue Beziehungsmöglichkeiten eröffnet. 
Die rekonstruierte Funktion von Bildung zur Befreiung aus einengenden Rahmenbedingungen sowie zur Ermöglichung eines selbstbestimmten Lebens geht einher mit der Fokussierung auf den Abschluss einer Berufsausbildung. In Annas Fall setzt sich die Bildungsaspiration mit dem Anschluss eines Studiums fort. Dieser Orientierungsrahmen findet sich auch bei Zofia. Über diesen Weg, sowie mit Unterstützung professioneller Hilfe, war es Anna möglich, sich aus der Gewaltbeziehung, aber auch von dem Muster der wechselnden Beziehungspartner zu lösen. Amy ist zum Zeitpunkt des Interviews ebenfalls dabei, ihre Ausbildung abzuschließen. Der Umzug in eine eigene Wohnung, die therapeutische Begleitung sowie ihre formulierte Absicht, zunächst Abstand von Männern zu gewinnen, deuten auf einen Neuanfang hin.

\subsubsection{Der Typus "Abhängigkeit" - "und wenns dann nicht gepasst hat hab ich Schläge gekriegt « ${ }^{19}$}

Die Beschreibung dieses Typus bezieht sich insbesondere auf die Fälle Alina und Jasmin.

Allgemeine Beschreibung des Typus

Der Typus »Abhängigkeit« ist dadurch charakterisiert, dass sich die den übergreifenden Orientierungsrahmen kennzeichnende Abhängigkeit aufallen Ebenen der Beziehungsgestaltung manifestiert.

Hinweise auf die biografische Herausbildung dieses Musters ergeben sich vor dem Hintergrund von Cewalterfahrungen in der Herkunftsfamilie. Sowohl das Miterleben häuslicher Cewalt auf Elternebene als auch das Erleben von Cewalt auf ElternKind-Ebene wurden hier rekonstruiert.

Das Beziehungskonzept der Frauen dieses Typus ist gekennzeichnet durch eher traditionale Geschlechtsnormen, die auf Differenz, Komplementarität und Asymmetrie verweisen. Das heißt, dass eher geschlechtstypische Erwartungen an den Partner gestellt werden, die sich an traditionelle Vorstellungen von Ceschlecht anlehnen.

Hinsichtlich des Umgangs mit Cewalt innerhalb der Paarbeziehung zeigen die Frauen vor allem passive Strategien der Bewältigung. Diese werden in Form von Bagatellisierung, Verheimlichung und Verdrängung der Cewalt deutlich. Die betroffenen Frauen fühlen sich selbst verantwortlich und suchen die Ursachen bei sich selbst. Die erlebte Cewalt ist eingebettet in verschiedene Formen der Machtausübung durch den Partner/die Partnerin, die die Unabhängigkeit, das Selbstvertrauen und die Handlungsoptionen der Frauen unterminieren und so Abhängigkeiten aufbauen. Merkmal

19 Zitat von Jasmin, Z. $312 f$. 
für die Cewaltdynamik innerhalb dieses Typus ist die klassische Gewaltspirale (vgl. Walker 1983).

Biografische Perspektive Frauen des Typus »Abhängigkeit« haben meist in ihrer Herkunftsfamilie Gewalt auf der Elternebene und/oder der Eltern-Kind-Ebene erlebt. Der Vater war entweder nicht präsent oder wurde als massiver Gewalttäter erlebt.

In den Interviews mit Frauen, in denen sich dieser Typus besonders stark zeigt, nehmen die Peers eine noch bedeutungsvollere Rolle ein als ohnehin schon. Über die Einbindung in Cliquen mit Gleichaltrigen oder die Anbindung an jugendkulturelle Szenen wird die Zugehörigkeit ${ }^{20}$ erlebt, die meist angesichts der prekären Verhältnisse in der Herkunftsfamilie fehlt. Neben den Peers wurden im biografischen Verlauf jedoch auch die Bedeutung weiterer signifikanter Anderer rekonstruiert, denen für die individuelle Entwicklung außerhalb der (Kern-)Familie eine wichtige Funktion zukommt. So spielen zum Beispiel die Großeltern bzw. die Großmutter bei Alina und Jasmin oder die Streetworkerin, ebenfalls bei Jasmin, eine zentrale Rolle. Diese signifikanten Anderen im verwandtschaftlichen Umfeld, aber auch die außerfamilialen signifikanten Anderen, handeln an Eltern statt, und ihre Interventionen zeigen eine biografische Wirkung in dem Sinne, dass die Biografie ohne sie anders verlaufen wäre (vgl. Hildebrand 2007).

Subjektive Vorstellungen Das Beziehungskonzept von Frauen des Typus »Abhängigkeit« kann als (eher) traditionelles Beziehungskonzept bezeichnet werden, in dem recht strikte Geschlechtsnormen für Männer und Frauen bestehen. Bei Alina wird dies in ihrer passiven Rolle während der Kontaktaufnahme, aber auch innerhalb der Beziehungsgestaltung deutlich, indem sie sich nach den Wünschen und Vorgaben ihres Freundes richtet, wann und wo sie sich treffen und welche Unternehmungen sie machen. Bei Jasmin findet sich noch eine Steigerung hiervon, indem ihr allein die Aufgaben der Haushaltsführung zukommen. Gleichzeitig wird der Partner als derjenige angesehen, der das Dominante verkörpert.

Umgang mit Gewalt Bezüglich des Umgangs mit Gewalt reagieren Frauen dieses Typus vor allem mit passiven Strategien der Gewaltbewältigung. Dies zeigt sich einerseits in der Bagatellisierung (vgl. Alina) sowie der Verdrängung der erlebten Gewalt. Es findet auch keine (verbale) Auseinandersetzung innerhalb der Beziehung über die Beziehung statt. Stattdessen entschuldigen Frauen die erlebte Gewalt durch ihren Partner und suchen die Ursachen der Gewalt bei sich selbst (vgl. Zofia, Jasmin). Auch wenden sie vermeidende Strategien an, um die Gewaltausbrüche ihres

20 Im Unterschied zum Typus »Pertinenz«, bei dem Zugehörigkeit über die (wechselnden) Beziehungen mit Männern zu leben versucht wird, bezieht sich die Zugehörigkeit hier auf unterschiedliche Zusammenhänge mit Gleichaltrigen. 
Partners abzumildern (vgl. Zofia, Jasmin), indem sie bspw. große Mühen darauf verwenden, das Essen rechtzeitig zu kochen und zu servieren oder die Wohnung sauber zu putzen, um keinen Anlass für einen Gewaltausbruch zu bieten (vgl. Jasmin). Letztlich besteht auch eine Strategie darin, die erlebte Gewalt zu ertragen und damit zu leben bzw. sie zum eigenen Leben als dazugehörig zu betrachten. Häufig werden gewaltvolle Erfahrungen nach außen verheimlicht.

Neuorientierung In beiden Ankerfällen (Alina und Jasmin) wird zum Zeitpunkt des Interviews eine Neuorientierung sichtbar: Die jungen Frauen führen jeweils eine Beziehung, die sie als positiv und wohltuend beschreiben. Bei Alina konnte im biografischen Verlauf ein Wandlungsprozess rekonstruiert werden, der deutlich macht, dass es ihr zunehmend möglich ist, ihre Anliegen auch innerhalb der Beziehung zu thematisieren. In ihrer aktuellen Beziehung macht sie außerdem die Erfahrung, so angenommen und akzeptiert zu werden, wie sie ist. Auch bei Jasmin lässt sich zum Zeitpunkt des Interviews eine Neuorientierung ausmachen, die sich einerseits ebenfalls durch die

Unterstützung ihres aktuellen Freundes sowie andererseits durch die professionelle Unterstützung in der Tagesklinik begründet. ${ }^{21}$

Die folgende Abbildung macht den Modus Operandi des Umgangs mit Gewalt innerhalb der drei rekonstruieren Typen deutlich:

Abbildung 4: Modus Operandi des Umgangs mit Gewalt

\begin{tabular}{|c|c|c|c|c|}
\hline & & $\begin{array}{c}\text { Typus } \\
\text { »Pertinenz } ~\end{array}$ & $\begin{array}{c}\text { Typus } \\
\text { »Autonomie } «\end{array}$ & $\begin{array}{c}\text { Typus } \\
\text { »Abhängigkeit« }\end{array}$ \\
\hline & 䒕 & $\begin{array}{l}\text { Suche nach } \\
\text { Zugehörigkeit und } \\
\text { Anerkennung }\end{array}$ & $\begin{array}{l}\text { Fokussierung auf } \\
\text { Autonomie }\end{array}$ & Abhängigkeit \\
\hline 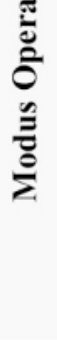 & 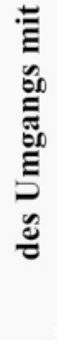 & $\begin{array}{l}\text { Akzeptanz von } \\
\text { Gewalt solange sie } \\
\text { Zugang zu } \\
\text { Zugehörigkeit } \\
\text { ermöglicht }\end{array}$ & $\begin{array}{l}\text { Aktive } \\
\text { Bewältigungs- } \\
\text { strategien im } \\
\text { Umgang mit } \\
\text { Gewalterfahrungen } \\
\text { (Verhandlung, } \\
\text { Trennung) }\end{array}$ & $\begin{array}{l}\text { »Gewaltspirale« } \\
\text { Passive } \\
\text { Bewältigungs- } \\
\text { strategien } \\
\text { (Bagatellisieren, } \\
\text { Verheimlichung, } \\
\text { Verdrängung) }\end{array}$ \\
\hline
\end{tabular}

21 Hier muss berücksichtigt werden, dass für die Teilnahme an einem Interview die Voraussetzung bestand, dass die Cewaltbeziehung abgeschlossen ist und sich die Frauen aktuell in keiner Cewaltbeziehung befinden. 



\section{Diskurstheoretische Betrachtungen}

Inspiriert durch neuere Auseinandersetzungen mit diskurs- und subjekttheoretischen Perspektiven am Ende meines Forschungsprozesses, aus denen sich ein kritisch-reflexiver und dekonstruktiver Umgang mit biografischen Texten ableitet, soll im folgenden Kapitel die Inszenierung der Lebensgeschichte durch eine ssubjekttheoretische Brille< betrachtet werden, wie es auch Nadine Rose (2012) als letzten Analyseschritt vorschlägt (vgl. Rose 2012, S. 120).

Ziel dieser Überlegungen stellt die methodologische Auseinandersetzung, in Ergänzung $\mathrm{zu}$ den in Kapitel 7.5 dargestellten Reflexionen, dar, indem Adressierungs- und Re-Adressierungspraktiken in den Blick genommen werden. ${ }^{1}$ Ein weiteres Ziel besteht darin, mittels einer performativ-dekonstruktiven Perspektive Möglichkeiten von Handlungsfähigkeit zu rekonstruieren.

Wie in Kapitel 3 ausgeführt, sind lebensgeschichtliche Darstellungen als »situativ und rhetorisch vermittelte Konstruktionsleistungen der Erzählenden zu verstehen« (ebd., S. 119). Dabei geht es um die Frage, auf welche Weise die Erzählerin resp. Biografin »sich im Interview inszeniert findet (performativ), aber auch welchen diskursiven Normen diese Darstellung folgt (dekonstruktiv)« (ebd.). Biografische Texte werden also als Inszenierungen begriffen und als solche interpretiert. Rose sieht in der spezifischen Erzählsituation - die in der vorliegenden Arbeit durch das biografisch-narrative Interview erzeugt wurde - einen Anlass für die performative Analyse dieser lebensgeschichtlichen Texte: Mit dem Erzählstimulus wird die Bühne für die jeweilige Erzählerin eröffnet. Der Text wird also »nur aufgrund eines spezifischen situativen Anlasses (und zugrunde liegenden Forschungsinteresses) entworfen und erzählt ... und damit als notwendig selektive Perspektive auf das potentiell erzählbare Leben zu einem bestimmten Zeitpunkt verstanden" (ebd., S. 119, Herv. im Original). Wird die Erzähldarstellung als Inszenierung verstanden, lässt sie sich auch daraufhin betrachten, "wie und mit welchen Effekten

\footnotetext{
1 Mit diesen Überlegungen und Reflexionen am Ende des Forschungsprozesses lässt sich auch eine Veränderung der Forschungsperspektive und des Bildungsprozesses der Autorin rekonstruieren, der keinesfalls abgeschlossen ist, sondern vielmehr am Anfang steht. In Anbetracht dessen erscheint es auch sinnvoll, dass die dargestellte Auseinandersetzung am Ende der Arbeit, und nicht in Kapitel 6.5, platziert wird.
} 
in ihr Figuren, Handlungen, Evaluationen etc. aufeinander bezogen werden, also daraufhin, welche Dramaturgie diese Ausgestaltung zeigt (ebd.). Nicht zuletzt machen die Rede und Interpretation eines biografischen Textes als Inszenierung deutlich, "dass die analytischen Aussagen zu diesem biografischen Text sich einzig auf das Inszenierte und dessen Effekte beziehen, ohne ein gleichsam dahinter stehendes Subjekt erreichen zu können« (ebd., S. 120).

Genau an der Stelle, an der betrachtet wird, wie etwas gesagt wird, zeigt sich eine Parallele zur dokumentarischen Methode, die in der vorliegenden Arbeit als $\mathrm{Me}$ thode zur Rekonstruktion von subjektiven Handlungs- und Deutungsmustern und ihrer biografischen Einbettung in subjektive Relevanzsysteme ausgewählt wurde. Die dokumentarische Methode zeichnet sich »durch eine methodologisch begründete ... Hinwendung zur Praxis des sozialen Handelns« (Bohnsack 2012, S. 120) aus. Der Weg zur Praxis dieses sozialen Handelns führt über das diese Praxis orientierende sogenannte implizite oder inkorporierte Wissen. In der empirischen Analyse dieses dokumentarischen Sinngehalts wird die geschilderte Erfahrung als Dokument einer Orientierung rekonstruiert, die die erzählte Erfahrung strukturiert (vgl. Nohl 2009, S. 8). Dabei wird in den Blick genommen, wie und in welchem Orientierungsrahmen ein Thema bearbeitet wird. Die Frage nach der Art und Weise, mit der ein Thema bearbeitet wird, verweist ebenso auf formale und semantische Aspekte, weshalb die Semantik des Textes von seiner formalen Konstruktion auch nicht zu trennen ist (vgl. ebd., S. 47). Aus diesem Grund greift die dokumentarische Interviewinterpretation die Textsortentrennung auf, wie sie von Fritz Schütze in der Narrationsstrukturanalyse entwickelt worden ist. Bezüglich der Semantik greift sie dann auf ihre eigenen Mittel der komparativen Sequenzanalyse zurück (vgl. ebd.) (vgl. auch Kap. 7.4 zu den Auswertungsschritten).

Vor dem dargestellten theoretischen Hintergrund, nach dem biografische Texte als Texte zu verstehen sind, die an Diskursen partizipieren und sie weiterleiten, bieten sich in Bezug auf die vorliegende Arbeit folgende Fragestellungen an:

1. Wie werden über die Partizipation an Diskursen immer schon vorfindbare Kategorien der (Selbst-)Beschreibung aufgegriffen und ggf. transformiert (vgl. Rose 2012, S. 120)?

Biografieforschung wird hierbei genutzt, um diskursive Machtwirkungen auf die im Text inszenierten Subjekte zu dekonstruieren (vgl. ebd.). Dazu werden konkrete Adressierungspraktiken als empirisch zugänglicher Cegenstand für eine Analyse von Subjektivationsprozessen genutzt. Für die vorliegende Arbeit wurden folgende Elemente für die Analyse ausgewählt: das Anschreiben an potenzielle Interviewpartnerinnen, der Erzählstimulus sowie die Erzählstruktur in den erhobenen biografisch-narrativen Interviews. Dies wird in Kapiteln 9.1 dargestellt. 
2. Wo werden dominante Diskurse unterlaufen und wo zeigen sich (damit) Phänomene von Widerstand?

Die aufgezeigte performativ-dekonstruktive Perspektive ermöglicht es außerdem, Handlungsfähigkeit analytisch zu untersuchen. Deshalb werden im Anschluss anhand der Konzepte des biografischen Eigensinns und der Biografizität Phänomene von Widerständigkeit in den Blick genommen. Die Möglichkeiten widerständigen Handelns im Sinne eines Unterlaufens dominanter Diskurse deutlich zu machen, bedeutet auch, ein Stück weit eine neue Perspektive auf Cewalt gegen Frauen vorzuschlagen. Dies hat ein verändertes oder zumindest erweitertes Verständnis von Cewalt zur Folge. Dieser Teil wird in Kapitel 9.2 bearbeitet.

Bezüglich der dieser Arbeit zugrunde liegenden Forschungsfrage, die nach den Praktiken und Handlungsorientierungen junger Frauen im Umgang mit Gewalt während der ersten eigenen Paarbeziehungen fragt, sind die genannten Fragestellungen zentral, weil sie neben der methodologischen Reflexion der Datenerhebung und den daraus resultierenden Daten resp. den narrativen Texten die Perspektive auch auf die Möglichkeiten von Handlungsfähigkeit richten. Diese lassen sich durch diese Art der Rekonstruktion auch auf theoretischer Ebene erklären.

Im letzten Kapitel dieser Arbeit (Kap. 9.3) werden angesichts des relativ jungen Bewusstseins über die Thematik der Gewalt in jungen Paarbeziehungen aus den dargestellten Ergebnissen Schlussfolgerungen für sozialpädagogische Unterstützung gewaltbetroffener jugendlicher und junger Frauen gezogen.

\subsection{Ergebnisteil I: Adressierungen und Readressierungen}

\subsubsection{Exkurs zur Subjekttheorie Judith Butlers}

Im Folgenden sollen mit Bezug auf einige aktuelle Adaptionen Judith Butlers im erziehungswissenschaftlichen Diskurs knapp die Grundzüge der Butler'schen Subjekttheorie ${ }^{2}$ nachgezeichnet werden. Die Subjektkonzeption Butlers bietet sich gerade deshalb an, weil sie »Subjekte als in existentieller Weise abhängig von anderen sowie den Normen der sozialen Welt entwirft« (Rose 2012, S. 113, Herv. im Original). Zudem knüpfen die in Kapitel 9.2 dargestellten Überlegungen zu (Re-)Adressierungsprozessen an diese an.

2 Butler betont, dass »das Subjekt « nicht gleichzusetzen sei mit »der Person« oder »dem Individuum«. Stattdessen verweise die Cenealogie des Subjekts als kritische Kategorie darauf, dass das Subjekt »als sprachliche Kategorie aufzufassen ist, als Platzhalter, als in Formierung begriffene Struktur«(Butler 2001, S. 15). 
Butler bezieht sich in ihren Überlegungen zu Subjektivierungsprozessen auf die Ausführungen von Luis Althusser und Michel Foucault und verbindet in ihren Überlegungen deren sozialphilosophische Theorien der Macht mit »dem Diskurs der Psychoanalyse« (Butler 2001, S. 22). Butler geht es in ihrem Konzept der Subjektivation darum,

»die grundlegende Ambivalenz der Subjektkonstitution herauszuarbeiten, die darin besteht, dass die Konstitution des Subjekts sowohl das Unterworfensein durch die Macht als auch Subjektwerdung im Sinne der Entstehung von (potentiell widerständiger) Handlungsfähigkeit bedeutet. Paradigmatisch zugespitzt lässt sich diese Ambivalenz der Subjektkonstitution in der Beschreibung dessen nachvollziehen, was Butler im Anschluss an Althusser als `Anrufungく bezeichnet. Ein Individuum wird demzufolge zum Subjekt, indem es von einer Instanz der Macht als Subjekt angerufen wird und darauf reagiert, indem es sich dieser Macht unterwirft.«(Koller 2014, S. 24, Herv. im Original)

Subjektivierung stellt also einen paradox machtvollen Vorgang dar und »bezeichnet den Prozeß des Unterworfenwerdens durch Macht und zugleich den Prozeß der Subjektwerdung« (Butler 2001, S. 8). Ausgangspunkt für die eine Seite dieses Prozesses, die Unterwerfung unter die Macht, ist für Butler die affektive Bindung des Kindes an seine frühesten Bezugspersonen, in der sich die grundlegende Angewiesenheit des Kindes auf andere und seine Anfälligkeit für Macht begründen (vgl. Koller 2014, S. 24). Damit es überhaupt zu einer Subjektwerdung kommen kann, muss »das Verhaftetsein in seinen ursprünglichen Formen ... verleugnet werden« (Butler 2001, S. 13). Die andere Seite der Subjektivation besteht darin, dass das Subjekt mit der Unterwerfung unter die Macht Handlungsfähigkeit gewinnt: "Eine auf das Subjekt ausgeübte Macht ist die Unterwerfung, doch eine vom Subjekt angenommene Macht, eine Annahme, die das Instrument des Werdens dieses Subjekts ausmacht« (Butler 2001, S. 16). Entscheidend ist also, dass »diese Handlungsfähigkeit sich potentiell auch gegen die Macht richtet, d.h. sich als Widerstand bzw. Auflehnung gegen die Macht artikulieren kann« (Koller 2014, S. 25).

Wesentlich in Butlers Konzept der Subjektivierung ist ihre Idee von der Hervorbringung von Subjekten im Diskurs, wobei sie sich direkt auf Foucaults Theorie der »diskursiven Erzeugung des Subjekts« bezieht. Nach Butlers Vorstellung konstituieren sich Subjekte im Rahmen von Diskursen und in der Unterwerfung unter bestimmte Diskurse. Butler versteht Diskurse als "produktive, Erkenntnisse und Wahrheiten generierende Zusammenhänge« (Rose 2012, S. 114). Diskurse bezeichnen »die Bedingungen der Möglichkeit, dass ein Gegenstand (in diesem Fall: ein Subjekt) überhaupt in Erscheinung treten kann. Ein solches Verständnis geht über die Auffassung von einem Diskurs als einzelner Äußerung oder $>$ Rede $<$... insofern hinaus, als ein Diskurs mit Foucault eher als gleichsam hinter den Aussagen liegendes Regelsystem verstanden werden kann« (ebd.). Regelhafte und wiederholende 
Aussagen werden innerhalb von Diskursen so miteinander verknüpft, dass in ihnen bestimmte Gegenstände und Wahrheiten über die Gegenstände hervorgebracht werden können (vgl. ebd.). Diskurse sind darüber hinaus aber nicht nur als Aussagen über einen Gegenstand zu verstehen, sondern sie sind auch ein »sprachlich artikuliertes Ordnungsgefüge, das Subjekte in ihrem jeweiligen Sein konstituiert « (ebd., S. 115). So schlägt Butler vor, »Subjekte als >Folgeerscheinungen von Diskursen zu denken und als Effekte von >Regeln<, die sie zwar nicht determinieren, aber doch in ihren Handlungsmöglichkeiten einschränken« (Rieger-Ladich 2012, S. 63).

Mit Bezug auf Luis Althusser stellt Butler fest, dass Subjektivierung immer im Kontext kultureller Interpellation, der >Anrufung < innerhalb einer diskursiven Ordnung, entsteht (vgl. Reckwitz 2010, S. 92). Durch Adressierungspraktiken werden "Subjekte gewissermaßen in ihre soziale Existenz hineingerufen« (Althusser 1977, zit. in Rose 2012, S. 116). Nach Butler sorgt die Psyche der Subjekte, die leidenschaftliche Verhaftung, dafür, dass "das fragliche Individuum sich umwendet, die Stimme als an sich adressiert annimmt und damit auch die durch diese Stimme bewirkte Unterordnung und Normalisierung akzeptiert« (Butler 2001, S. 11). Das Subjekt sieht sich also selbst angerufen und gliedert sich so in Diskurse ein. Dennoch entwirft Butler dieses diskursiven Normen unterworfene Subjekt als ein handlungsfähiges Subjekt. Dabei versteht sie Handlungsfähigkeit »nicht als Gegenteil von Beherrschung oder Unterwerfung, sondern als aus der Beherrschung abgeleitete und deshalb notwendig auch begrenzte Ermächtigung eines Subjekts im Diskurs« (Rose 2012, S. 116). Nach Butlers Verständnis beruhen Normen und Ordnungen auf Performativität, also dem ständigen Wiederholen und Bestätigen dieser Normen. Und genau hier liegt das Moment, in dem Handlungsfähigkeit entstehen kann. Diese Handlungsfähigkeit besteht darin, dass Begriffe nicht vollständig oder richtig wiederholt werden (vgl. ebd., S. 117) oder »für andere Zwecke fehlangeignet werden« (Butler 2006, S. 226). An diese Überlegungen knüpft Kapitel 9.2 an, in dem die Perspektive auf widerständiges und eigensinniges Handeln der befragten jungen Frauen gerichtet wird, um davon ausgehend die Fragen nach Aspekten des Wandels und der Persistenz von Gewalt zu beleuchten.

\subsubsection{Adressierung}

Im Folgenden sollen nun konkrete Adressierungspraktiken näher analysiert werden, wie sie innerhalb der vorliegenden Forschungsarbeit in Form des Anschreibens (vgl. Anlage) und in der Erzählaufforderung als konkrete Vorgänge der Ansprache von jemandem als jemand zu finden sind. Die Analyse der Erzählstruktur zeigt auf der performativen Ebene auf, wie sich auf der Ebene der Praxis des Erzählens neben den Adressierungspraktiken auch Re-Adressierungspraktiken zeigen.

Da das Anschreiben an sich kein Interaktionsgeschehen darstellt, bleibt die Analyse auf Adressierungspraktiken durch mich als Forscherin begrenzt. D.h. es 
liegen keine Re-Adressierungen durch die Interviewpartnerinnen vor, die einer Interpretation unterzogen werden könnten. Ich halte die Analyse der im Anschreiben enthaltenen Anrufungen dennoch für wichtig, da diese von allen späteren Interviewpartnerinnen gelesen wurden und davon ausgegangen werden kann, dass die erfahrenen Adressierungen einen Einfluss auf das Interview und die darin enthaltenen Selbstpräsentationen haben. Dagegen werden bei der Rekonstruktion des Interviewbeginns neben den Adressierungspraktiken auch Re-Adressierungen bei der Analyse berücksichtigt.

Ich beziehe mich bei der Interpretation auf die Adressierungsanalyse nach Rose und Ricken (2018), die das Verständnis von Adressierung im Butlerschen Sinn um »eine grundsätzliche Struktur in und von Interaktion wie Kommunikation « (ebd., S. 166, Herv. im Original) erweitert haben. ${ }^{3}$ So konstatieren die Autor_innen, dass »Adressierung als mehrfach rekursives soziales Phänomen konzipiert und untersucht werden müsste, so dass die Frage nach konkreten Adressierungen immer auch die Frage nach den Antworten auf solche Adressierung bzw. Re-Adressierung aufruft« (ebd., S. 167).

Vor diesem Hintergrund verstehen sie Adressierungen als

»konkrete, explizite, aber auch implizite Ansprachen von jemandem (als jemand) und zugehörige Antworten und Re-Adressierungen der Adressierten, die darin selbst wiederum zu Adressierenden werden, als zentrale Dimension und durchgängiges Moment von sozialen Praktiken« (Rose/Ricken 2018, S. 167).

Bei der Analyse der vorfindbaren Adressierungen lehne ich mich an vier Dimensionen in oder an Adressierungsprozessen an, wie sie von Rose und Ricken (2018, S. 168) vorgeschlagen werden:

1. Selektion und Reaktion: Rekonstruktion der Auswahl als Angesprochene_r und der Reaktion der Signale auf diese Auswahl.

2. Definition und Normation: Rekonstruktion der in Adressierungen etablierten situativen Ordnungen und Rahmungen.

3. Positionen und Relationen: Rekonstruktion der unterschiedlichen Positionierungen und Positionszuweisungen zu sich, zu den anderen und zur Welt.

4. Valuation: Rekonstruktion der expliziten und impliziten Wertzuschreibungen in Adressierungen.

3 Rose und Ricken entwickelten die Adressierungsanalyse im Rahmen des DFG-geförderten Forschungsprojektes »Die Sprachlichkeit der Anerkennung. Empirische Rekonstruktion von Adressierungsprozessen im Sekundarschulunterricht« (vgl. Rose/Ricken 2018, S. 161). Während hier der Bedeutung von Anerkennung für Subjektivationsprozesse in einem pädagogischen Kontext, nämlich Schule, nachgegangen wird, beziehe ich mich bei der Analyse von Adressierungen auf einen Forschungskontext, konkret die Situation der Erhebung von Daten. 
In dem von mir formulierten Anschreiben an potenzielle Interviewpartnerinnen im Rahmen der Datenerhebung (vgl. Kap. 7.3 zur Datenerhebung) finden sich verschiedene Elemente, die als Ausdruck der Interpellation bzw. Anrufung (vgl. Butler 2001, S. 101) angesehen werden können. Im Folgenden soll nun gezeigt werden, wie solche Anrufungen im Rahmen von Diskursen Subjekte in ihre soziale Existenz hineinrufen und sie so erzeugen.

Zunächst ist $\mathrm{zu}$ bemerken, dass das Anschreiben mit dem Briefkopf der entsprechenden Universität versehen wurde, was dem Vorhaben zunächst eine formale, institutionelle Zugehörigkeit vermittelt. Die namentliche Vorstellung meiner Person als Diplom-Pädagogin (und bewusst nicht als Sozialwissenschaftlerin oder Forscherin) intendiert eine Nähe zur Praxis sozialer Arbeit und weniger zum Feld der Forschung und Wissenschaft. Sowohl diese Begrifflichkeit wie auch der Begriff »Doktorarbeit « (statt Promotion oder Dissertation) waren bewusst gewählt. Über diese Wahl der Adressierung sollten sich potenzielle Interviewpartnerinnen eher angesprochen fühlen, indem die verwendeten Begrifflichkeiten Alltagsnähe symbolisieren und verständlich eingesetzt wurden.

Mit der Benennung der Thematik »Gewalt in Beziehungen« sowie der Konkretisierung "mit jungen Frauen Interviews führen, um so zu erfahren, wie sie Gewalt erlebt haben« taucht zweimal der nicht unproblematische Begriff Gewalt auf. Problematisch deshalb, weil Gewalt einerseits ein emotional und moralisch aufgeladenes Thema darstellt und es andererseits keine einheitliche Definition von Gewalt gibt bzw. Gewalt subjektiv sehr unterschiedlich definiert wird. Indem der Gewaltbegriff verwendet wird, werden die angesprochenen Frauen bereits als Frauen adressiert, die - in welcher Form auch immer - Gewalt erlebt haben. Gleichzeitig besteht in der Konstruiertheit und Uneindeutigkeit von Gewalt als sozialem Phänomen aber auch eine Chance: Indem im Anschreiben nicht näher definiert wird, was unter Gewalt zu verstehen ist, bleibt es jeder Leserin des Schreibens überlassen, für sich selbst zu definieren, ob sie sich mit ihren jeweiligen Erfahrungen angesprochen fühlt oder nicht. Der Rahmen wird also bewusst offengehalten.

Denkbar wäre außerdem, dass sich manche Frauen mit der Benennung des Erleidens von Gewalt als Opfer angesprochen fühlen. Dies kann zur Folge haben, dass durch eine solche Adressierung als Opfer die Lebensgeschichte unter dieser Perspektive dargestellt wird und sich diese Frauen in und über die Ebene der Darstellung als Opfer subjektivieren.

Etwas abgeschwächt wird die Gewaltthematik in der im Anschreiben erfolgten Zusammenfassung. Hier ist abgemildert nicht mehr von Gewalt, sondern von »schwierigen « Erfahrungen die Rede. Dadurch wird die Beschreibung des Forschungsvorhabens erweitert, und Frauen, die sich selbst nicht unbedingt als gewaltbetroffen beschreiben würden, können sich ebenfalls angesprochen fühlen. In der folgenden Formulierung des Ziels der Arbeit wird deutlich gemacht, dass die Studie, zu der die Adressierten möglicherweise beitragen, sinnvolle Auswirkun- 
gen hat, indem die Ergebnisse hilfreich und unterstützend für Beratungsstellen sind. ${ }^{4}$ Potenzielle Teilnehmerinnen werden hier also als Beteiligte adressiert, die die Möglichkeit haben, mit ihrem Wissen und ihren Erfahrungen einen Beitrag zur Verbesserung bestehender (Beratungs-)Verhältnisse zu leisten. Daran knüpft auch die Adressierung als Expertin im letzten Teil des Anschreibens an. Die explizite Verwendung des Expertinnen-Begriffs adressiert die Leserin als Expertin ihrer Lebensgeschichte, ihrer Erfahrungen.

Insgesamt werden mit dem vorliegenden Anschreiben potenziell an der Forschung Beteiligte v.a. in Bezug auf zwei Aspekte angesprochen: zum einen hinsichtlich der bestehenden Forschungsthematik, in der nach dem Erleben von Gewalt gefragt wird; zum anderen hinsichtlich ihrer Expertinnen-Rolle in Bezug auf das zugrunde liegende Thema.

In der Folge bedeutet das für mich als Forscherin, im Blick zu haben, wie diese Adressierungen aufgenommen wurden und welche Re-Adressierungen daraufhin im Interview erfolgten. Die analytische Fokussierung auf Adressierungsprozesse ermöglicht es, neben der Frage, wie jemand von wem angesprochen wird und wie er oder sie dann damit umgeht bzw. re-adressiert, auch danach zu fragen, »wer man in diesen Adressierungen und Re-Adressierungen wird bzw. zu wem man gemacht wird und sich selbst macht «(Rose/Ricken 2018, S. 160), also danach, wie sich Menschen als Subjekte eine Individualität und Identität aneignen. Die Adressierungsanalyse nimmt so den konkreten Vollzug von Subjektivierungsprozessen in den Blick. Rose und Ricken (2018, S. 168) benennen folgende Fragen, die es adressierungstheoretisch zu klären gilt:

1. Wie wird jemand vom wem als wer angesprochen bzw. explizit oder implizit adressiert?

2. $\mathrm{Zu}$ wem wird der- oder diejenige dadurch von wem und vor wem gemacht, zu wem macht er oder sie sich selbst, und inwieweit bestimmt ihre oder seine re-adressierende Reaktion darauf den Prozess mit?

Diese Fragen werden im Hinblick auf die Analysen der Eingangssequenz sowie hinsichtlich der Analyse der Erzählstruktur, die für alle Interviews durchgeführt wurden, im Folgenden näher beleuchtet. Dabei beziehe ich mich immer wieder auf die Einzelfallanalysen, in denen häufig die Interpretation der Eingangssequenz

4 Dass ein Forschungsvorhaben den Beteiligten möglichst viele Gelegenheiten bieten soll, Forschung für sich selbst als etwas Positives zu erleben, und dass erkennbar sein sollte, wo die Studie, zu der sie beitragen, sinnvolle Auswirkungen hat, ist eine von drei Anforderungen an Forschung, wie sie Julia Downs u.a. (2014) in ihrem Ethikmodell vorschlagen (vgl. Hagemann-White 2016, S. 28f.). 
bereits dargestellt wurde. In jedem Interview verwendete ich den gleichen Erzählstimulus:

Ich möchte Dich bitten, mir Deine gesamte Lebensgeschichte zu erzählen, alle Erlebnisse, die Dir einfallen. Ich werde Dich erst einmal nicht unterbrechen und nur einige Notizen machen und später darauf zurückkommen.

Die Erzählaufforderung knüpft zunächst an die im Anschreiben formulierte Adressierung als Expertin der eigenen Lebensgeschichte sowie der eigenen Erfahrungen, diese zu berichten, an. In den durchgeführten Interviews wird der Erzählstimulus unterschiedlich aufgegriffen. So finden sich in manchen Interviews hinsichtlich der Erzählstruktur neben einem flüssigen Erzählstil (Anna, Amy, Zofia, Alina, Angela) ein eher bedächtiges nachdenkliches Erzählen (Emira, Jasmin) bis hin zu anfänglicher Sprachlosigkeit, in der ein Erzählen zunächst nicht möglich ist (Daria). Die im Anschreiben explizit formulierte Adressierung als Expertin, die sich innerhalb der Erzählaufforderung in impliziter Weise wiederholt, wird also nicht gleichermaßen von den interviewten Frauen aufgegriffen. Dies führt mich zur zweiten der oben genannten Fragen, bei der es sich anbietet, die performative Kontextuierung (vgl. Bohnsack 2010c, S. 304) der erzählten Lebensgeschichte in den Blick zu nehmen. Denn auf der performativen Ebene, die sich in der Praxis des Erzählens widerspiegelt, können sprachliche Elemente beobachtet werden, die es ermöglichen, Aussagen darüber zu machen, wie die befragten jungen Frauen zu jemand gemacht werden bzw. zu wem sie sich selbst machen. Bei der Betrachtung aller Interviews zeigen sich auf der Ebene der performativen Kontextuierung insgesamt drei verschiedene Praxen des Erzählens: Die erste Art zu sprechen bzw. zu erzählen deutet auf eine gesellschaftlich erfahrene Tabuisierung von Gewalt hin (v.a. Anna, Emira). Dies zeigt sich bspw. im thematischen Nachfragen der Interviewten, über die die Haltung der Interviewerin zum Thema Gewalt abgefragt wird (vgl. Kap. 8.1.1.2). Der Intention, eine solche Haltung abzufragen, liegt sehr wahrscheinlich die Erfahrung der Tabuisierung von Gewalt zugrunde, die sich z.B. in der antizipierten Überforderung von Gesprächspartner_innen zeigen kann. Die Erzählerin sichert sich hier ab, ob und inwieweit sie von ihren Erzählungen berichten kann. Aufgrund der bisherigen Erfahrung, dass Gewalt (in bestimmten Zusammenhängen der Lebensgeschichte) tabuisiert wurde, positioniert sich die Erzählerin hier als Subjekt, das handlungsaktiv für sich die Positionen, für sie als Erzählerin und für die Interviewerin als Zuhörerin, auslotet. Im ausgewählten Beispiel reagiert die Interviewerin einerseits mit der Betonung der Freiwilligkeit der Erzählung und unterstreicht damit die Entscheidungsmacht der Erzählerin. Andererseits signalisiert sie ihre Bereitschaft zuzuhören, wodurch sie erneut die Adressierung der Interviewten als Expertin bestätigt. Zudem würde eine grundsätzliche Vermeidung von schwierigen Lebenssituationen mit dazu beitragen, dass gesellschaftliche und soziale Tabuisierungsprozesse aufrechterhalten werden. 
Die zweite Art zu sprechen weist auf erfahrene Traumatisierungen in der bisherigen Lebensgeschichte (v.a. Amy, Jasmin, Emira) hin. Wie vor allem in Kapitel 8.1.2.2 und 8.1.6.2 aufgezeigt, werden über die Darstellung von Brüchen, über erzählerische Leerstellen oder immer wieder auftauchende Inkonsistenzen in der Erzählung in einigen Interviews Hinweise auf traumatische Erfahrungen deutlich. Durch die Wahrnehmung dieser Spuren von Traumatisierungen durch mich als Interviewerin findet eine (wenn auch implizite) Adressierung der befragten Frauen als »möglicherweise oder vermutlich traumatisiert «statt. Als Konsequenz im Umgang mit solchen Hinweisen ist es erforderlich, auf ein Repertoire an Handlungsmöglichkeiten (z.B. In-Kontakt-Bleiben mit der Interviewpartnerin, Ausstieg aus dem Thema oder Pausen anbieten) zurückgreifen zu können. Immer aber erfordert eine Nachfrage ein Abwägen darüber, ob diese eine heilsame Integration der Erfahrungen unterstützen kann oder ob sie retraumatisierend wirkt. Für die befragten Frauen, die sich zu einem Interview bereit erklärten, bietet die Erzählung der eigenen Lebensgeschichte zum einen Chancen für das Selbstverstehen sowie die Möglichkeit, dass (erste) heilsame Prozesse einsetzen und das Interview so positive Auswirkungen auf die Einzelnen haben kann (vgl. Rosenthal 2002, S. 6). Eine längere Erzählung über das eigene Leben ermöglicht es, eigene Relevanzkategorien zu setzen, wodurch Erinnerungsprozesse unterstützt werden und häufig bereits Prozesse des Selbstverstehens stattfinden (vgl. ebd., S. 13). Zum anderen bietet die lebensgeschichtliche Erzählung die Möglichkeit zur Reorganisation der bisherigen Sicht auf das Leben und kann bspw. dazu führen, dass die rückblickende Perspektive stärker die Gestalt eines aktiven und autonomen Lebens annimmt (vgl. ebd., S. 14). Nicht zuletzt kann das Erzählen der gesamten Lebensgeschichte auch da$\mathrm{zu}$ beitragen, ein Gefühl der Kontinuität für die eigene Biografie zu bekommen, indem es durch das Erzählen zum notwendigen Zusammensetzen von einzelnen Erinnerungsfragmenten kommt, was zu einer Integration von schwierigen oder traumatischen Erlebnissen in den Gesamtzusammenhang der Lebensgeschichte beiträgt (vgl. ebd., S. 15).

Meines Erachtens enthalten die Praktiken einer narrativen Gesprächsführung Adressierungsformen, die die Interviewpartnerinnen als kompetente Erzählerinnen ihrer Lebensgeschichte anrufen resp. ansprechen. Indem sie sich als solche adressieren lassen, gewinnen sie die Möglichkeit, sich entsprechend als Expertin ihrer Lebensgeschichte $\mathrm{zu}$ zeigen (zu inszenieren) und sich in dieser Rolle wiederum adressierend an ihr Gegenüber, die Interviewerin, zu richten. Ein großer Vorteil, nicht nur, aber gerade auch bei Interviews, in denen es um Gewalt geht, liegt darin, dass die benannten Adressierungs- und Re-Adressierungsprozesse es ermöglichen, das von vorneherein in der Interviewsituation angelegte asymmetrische (Macht-)Verhältnis auszugleichen.

Die dritte Art erzählerischer Praxis verweist darauf, dass die Interviewpartnerinnen es nicht gewohnt sind, ihre Biografie zu erzählen. Möglicherweise hat- 
ten sie bislang auch nicht die Gelegenheit dazu. Damit verbunden kann auch die fehlende Erfahrung sein, dass ihre Erlebnisse von anderen für relevant oder erzählenswert erachtet werden (v.a. Daria, Emira). Auf der textlichen Ebene zeigt sich dies in einem stockenden Erzählfluss, langen und vielen Pausen, die deutlich machen, wie schwer es fällt, ins Erzählen zu kommen. Auch hier gilt, dass eine narrative Gesprächshaltung den Befragten das Gefühl vermittelt, dass sich die Interviewerin tatsächlich für ihre persönlichen Erlebnisse interessiert ohne diese dabei negativ zu bewerten (vgl. Rosenthal u.a. 2006, S. 205f.). So kann bspw. eine anfängliche Sprachlosigkeit aufgehoben werden. Wie oben ausgeführt, finden sich in diesen Gesprächssituationen ebenfalls die genannten Adressierungs- und Re-Adressierungsprozesse, die auf die Rolle der Expertin sowie auf eine ausgeglichene Balance im Rollenverhältnis während der Gesprächssituation fokussieren.

Abschließend kann festgehalten werden, dass sich zum einen in den dialogischen, verbalen, aber besonders auch nonverbalen Interaktionen während der Erhebungssituation, aber auch zum anderen bereits zuvor (im Anschreiben) Adressierungs- und Re-Adressierungspraktiken zeigen, die auf den weiteren Prozess, im vorliegenden Fall also den weiteren Verlauf des Interviews, Auswirkungen haben. So können Adressierungspraktiken, die auf die Rolle der Expertin fokussieren, dazu beitragen, dass sich die befragten Frauen als Expertinnen ihrer Lebensgeschichte positionieren. Darin liegt gleichzeitig auch die Chance, Bedingungen für ein erstes heilsames Erzählen zu schaffen. Werden dagegen Adressierungen, die stark auf das Merkmal "gewaltbetroffen" setzen, aufgenommen, führt dies dazu, dass eine Biografie stark unter dieser Positionierung dargestellt wird. Dabei gilt es, sich bewusst zu machen, dass das Aufgreifen bestimmter Adressierungen zu Positionierungen führt. Positionierungen meinen "das, was die*der Einzelne daraus `macht « (Spies 2018, S. 544), im Unterschied zur Position, die im gesellschaftlichen Diskurs angeboten wird. Dabei bedeutet das Machen keine bewusste Entscheidung; auch wenn Individuum und Struktur zwar miteinander verwoben sind, ist das Individuum der Struktur nicht vollständig unterworfen (vgl. ebd.).

Wenn Butler Subjekt als ein Subjekt denkt, das auf die Bestätigung seines Status durch sein Gegenüber resp. durch andere angewiesen ist (vgl. Butler 2001), und dieser Gedanke auf die Situation eines Forschungssettings, konkret der Datenerhebung mittels biografisch-narrativer Interviews, übertragen wird, lässt sich als weiteres Fazit der oben ausgeführten Reflexionen Folgendes festhalten:

Zunächst zeigt sich, dass die genutzte Forschungsmethode geeignet ist, Prozesse der Subjektivation zu erforschen. Weiter zeigt sich, dass es wichtig ist, Adressierungspraktiken während der Datenerhebung mitzuberücksichtigen, weil sie Einfluss auf die Art sowie den Verlauf des Interviews nehmen. Die resultierenden Überlegungen fanden in diesem Forschungsprozess am Ende statt - und stellen gleichzeitig den Anfang der Fortsetzung der Frage dar, wie sich 
Subjektkonstitutionsprozesse in der Auseinandersetzung bzw. im Umgang mit Gewalt definieren lassen.

\subsection{Ergebnisteil II: Gewalt zwischen Wandel und Persistenz: Widerstandsstrategien und Ansatzpunkte für ein reformuliertes Gewaltverständnis}

Im letzten Kapitel dieser Arbeit wird die Perspektive auf die zentralen Widerstandsstrategien gerichtet, die sich in den biografischen Erzählungen der befragten jungen Frauen rekonstruieren ließen. Dabei konnten drei zentrale Handlungsstrategien ausgemacht werden, die Frauen befähigen, sich aus gewaltgeprägten Beziehungen zu lösen. Die dargestellten Ergebnisse werden anhand der Konzepte des biografischen Eigensinns und der Biografizität theoretisch kontextualisiert. Beide Konzepte stellen eine geeignete Folie dar, um Momente der Widerständigkeit innerhalb gesellschaftlicher Machtverhältnisse und sozialer Ordnungen zu fassen. Widerständigkeit oder Eigensinn wird dabei nicht im Sinne von widerständig gegen Normierungen verstanden, sondern vielmehr im Sinne einer »Eigenlogik und Individualität biografischer Konstruktionen« (Thon 2016, S. 188).

Neben den Strategien des Widerstandes, die eine Veränderung der Gewaltverhältnisse bedingen, wird aber auch in den Blick genommen, welche Aspekte $\mathrm{zu}$ einer Persistenz von Gewalt führen. Im Anschluss an diese Ergebnisse werden Schlussfolgerungen für sozialpädagogische Unterstützung gezogen und dargestellt.

\subsubsection{Beziehungsgestaltung als biografisches Projekt: die Konzepte des biografischen Eigensinns und der Biografizität}

Der poststrukturalistischen Subjektkritik, nach der sich vor allem die Biografieforschung mit dem Vorwurf konfrontiert sieht, ein Subjekt vorauszusetzen oder zu produzieren, das theoretisch bereits dekonstruiert ist (vgl. Kap. 3.3), begegnet Thon (2016) mit einer Neubestimmung von Widerständigkeit, die sie im Konzept des biografischen Eigensinns ${ }^{5}$ ausformuliert. Dabei geht es darum, Ansätze zu entwickeln oder zu denken, die die poststrukturalistische Subjektkritik in biografische Rekonstruktionen integrieren - oder konkreter gesagt, zu fragen, »wie sich biografischer Eigensinn im Verhältnis zu gesellschaftlichen Transformationsprozessen verstehen lässt« (ebd., S. 185). Bereits 1994 hält Dausien fest, dass Biografieforschung einen

5 Christine Thon zeigt an drei empirischen Arbeiten, nämlich denen von Andreas Hanses, Nadine Rose und Tina Spies, auf, dass Beobachtungen von biografischem Eigensinn und seine theoretische Fassung mit Elementen poststrukturalistischer Subjekttheorien gestützt werde können. 
methodologischen und theoretischen Zugang zum Problem der Subjektivität eröffnet,

»ohne dieses jedoch auf die individuell-psychologische Dimension einerseits oder den auf die Interaktionssituation verkürzten sozial-konstruktivistischen Aspekt andererseits zu reduzieren. Biographie als theoretisches Konzept thematisiert die subjektive Aneignung und >Konstruktion`von Gesellschaft ... ebenso wie die gesellschaftliche Konstitution von Subjektivität. (Dausien 1994, S. 152, zit. in Thon 2016, S. 186f.)

Dausien formuliert bereits hier ein nicht vorgängig biografisches Subjekt, sondern die Konstituiertheit von Subjektivität innerhalb gesellschaftlicher Verhältnisse wird als Grundlage des Verständnisses von Biografie angesehen (vgl. ebd., S. 187). Indem Diskontinuitäten, Widersprüchlichkeiten und Ambivalenzen in Biografien rekonstruiert werden, wird die »lineare Normalbiografie als androzentristische Illusion infrage« (ebd.) gestellt. Die Forderung nach einer Subjektorientierung, die Frauen einen Subjektstatus zuerkennt und die widerständiges Handeln und transformatorisches Potenzial ${ }^{6}$ aufzeigt, lässt sich mit dem Konzept der Biografizität fassen (vgl. ebd.):

»Ausgangspunkt ist die Idee, dass biografischer Eigensinn Resultat einer spezifischen und einzigartigen Erfahrungsaufschichtung ist, die auf die weitere Bearbeitung und aktiv zu leistende Integration in vorgefundenen sozialen Strukturen zurückwirkt. Da umgekehrt im Sinne von Biografizität die soziale Wirklichkeit auf ihre Reproduktion durch das biografische Subjekt angewiesen ist, die aber entlang einer im beschriebenen Sinne zu verstehende Eigenlogik stattfindet, können so auch Veränderungen dieser sozialen Realitäten erklärt werden.« (Thon 2016, S. 194)

Das Konzept der Biografizität erweist sich dabei als anschlussfähig an die Überlegungen Butlers zur Reiteration. Denn »mit dem Butler'schen Vokabular kann deutlicher formuliert werden, dass dadurch Räume an den Rändern des Diskurses entstehen können, die für sabweichende < eigensinnige Subjektivitäten in Beschlag genommen werden können« (Thon 2016, S. 195). Demnach setzt die Reproduktion von Normen eine beständige Wiederholung voraus, die aber eben unterlaufen werden können. Und an dieser Stelle schließt auch das Konzept der Biografizität an. Mit

6 Christine Thon weist auf die Anschlussfähigkeit verschiedener Ansätze in der (erziehungswissenschaftlichen) Biografieforschung (vgl. Alheit 1993, Marotzki 1999, Schulze 2002) hin, die ebenfalls von einer Eigensinnigkeit biografischer Konstruktionen ausgehen (vgl. Thon 2016, S. 187). 
dem Begriff der »Biographizität « ${ }^{7}$ (ebd., S. 32) beschreibt Alheit die Rekonstruktion der Vermittlung zwischen Individuum und Struktur:

»Biographizität bedeutet nicht jene empathische Einzigartigkeit des Individuellen [...] Aber sie ist eben auch nicht als schlichter Oberflächenausdruck einer zu Grunde liegenden Struktur zu deuten.«(Alheit 1992, S. 32)

Biografisches Hintergrundwissen verhilft uns demnach als Individuen zurechtzukommen und stellt eine »Ressource für Individualität« (ebd., S. 31) dar. Zum einen, weil das heimliche Wissen von den (noch) nicht gelebten Lebenschancen unsere bewusst verfügbare Selbstreferenz offenhält und uns ermöglicht, dass wir zu uns selbst eine andere Position einnehmen können, ohne den Sinn, der unsere Erfahrung ausmacht, korrigieren zu müssen. Optionen, die wir erkennen, erweitern unsere Handlungsoptionen oder aber schränken sie ein (vgl. Alheit 2006, S. 5). Wir sind also in der Lage, die sogenannten Sinnüberschüsse - die Potenzialität von Erfahrungen - unseres Lebens zu erkennen und damit unseren Bezug zu uns selbst und zur Welt zu verändern. Und auch wenn biografische Alternativen begrenzt sind, bestehen innerhalb der strukturellen Grenzen der Lebenskonstruktionen vielfältige Optionen und damit Handlungsspielräume. Zum anderen ermöglicht biografisches Hintergrundwissen aber auch die Veränderung von Strukturen.

»Die Modifikation individueller Selbst- und Weltbezüge birgt Chancen zur Transformation auch der institutionellen Rahmenbedingungen sozialer Existenz. sStrukturen sind ja zu beträchtlichen Teilen die unbewusst funktionierenden Hintergrundgewissheiten, auf die sich soziale Individuen intuitiv beziehen, wenn sie alltäglich, aber auch wenn sie biografisch agieren. Sobald solche unbefragten Vorannahmen ins Bewusstsein treten und verfügbar werden, ändern sich die Strukturen. ১Ungelebtes Leben< besitzt durchaus soziale Sprengkraft.«(vgl. Alheit 2006, S. 5).

In Bezug auf das, was Beck und Giddens als »Individualisierung « beschreiben, meint das Konzept der Biografizität die »Innenseite« (ebd., S. 6). Sie ist »der Zwang und die Chance zugleich, unser Leben selbst zu gestalten « (ebd.). Die Grundannahme der Biografieforschung ist, dass unser Grundgefühl, das Leben sei planbar, als sogenannte Lebenskonstruktionen in individuelle Sinnstrukturen eingebunden ist. Diese Lebenskonstruktionen sind aber nicht immer bewusst zugänglich und

7 In seinem Beitrag »Identität oder >Biographizität‘? Beiträge der neueren sozial- und erziehungswissenschaftlichen Biographieforschung zu einem Konzept der Identitätsentwicklung« diskutiert Alheit die Komplementarität der Konzepte »Identität« und »Biographie«. Dabei macht er deutlich, dass sich Identität in Biografien als innere Sinnstruktur, als ein generatives Erzeugungsprinzip in einer zeitlichen Perspektive aufzeigen lässt. Diese innere Struktur ist damit als biografische Konstruktion zu verstehen und Identität als biografische Identität (vgl. Alheit 2010, S. 230). damit wird auch deutlich, dass Identität als statisches Konzept überholt ist. 
gewollt im Sinne von Plänen, sondern stehen als "versteckter Sinn« hinter den Strukturen des Lebenslaufs (vgl. Alheit 1992, S. 29f.). Interessant dabei ist, dass wir diese Idee von und über uns selbst »nicht trotz, sondern gerade wegen der strukturellen Begrenzungen unserer sozialen und ethnischen Herkunft, unseres Geschlechts und der Zeit, in der wir leben « (ebd., S. 30, Herv. im Original), haben.

Auf das vorliegende Forschungsinteresse übertragen bedeutet dies, dass die Deutung und Konstruktion von Paarbeziehung sowie die Deutung und Konstruktion von Gewalterfahrungen innerhalb dieser Paarbeziehungen auf der Basis der individuellen Orientierungen stattfinden. Mich interessierte, wie diese individuellen biografischen Konstruktionen entstehen, die die Grundlage für den subjektiven Handlungsrahmen und damit die Beziehungsgestaltung sowie den Umgang mit Gewalt bilden. Wie wird Paarbeziehung konstruiert? Welche Vorstellungen formulieren die jungen Frauen hinsichtlich Paarbeziehungen und welche biografischen Entscheidungen treffen sie? All das ist vor dem Hintergrund gesamtbiografischer Konstruktionen und Sinnhorizonte zu sehen und als Ausdruck biografischen Handelns zu verstehen.

Um verstehen und rekonstruieren zu können, wie sich ein solch biografisches Handeln, das zwar sozial vorstrukturiert ist, aber dennoch individuell konstruiert wird, prozesshaft entwickelt, lohnte es sich, eine Perspektive auf Biografie und Sozialisation einzunehmen. In der vorliegenden Arbeit wurden deshalb die Sozialisationsgeschichten der befragten Frauen (vgl. Kap. 8.1) als Hintergrundfolie betrachtet, auf der subjektive Denk- und Handlungsmuster entstehen, und die Wechselbeziehungen von sozialisatorischen Kontexten bzw. gesellschaftlichen Rahmenbedingungen und biografischem Handeln wurden in den Blick genommen.

Die vorliegende Arbeit thematisiert als einen weiteren Analysestrang Fragen von Handlungsmöglichkeiten und -begrenzungen sowie Fragen zur Reproduktion und Veränderung von Verhaltensweisen, zu Subjektivität und Subjektwerdung. Hier kann Biografie als theoretisches Konzept bzw. eine biografietheoretisch fundierte Subjekttheorie eine lohnenswerte Forschungsperspektive darstellen, mit der Beziehungsorientierungen junger Frauen, die in biografische Konstruktionen eingelassen sind, in den Blick genommen werden können, ohne dass die Subjekte als autonom abgegrenzt angesehen werden. Vielmehr wird den Frauen ein Subjektstatus zuerkannt, und in »alltäglichen Widerstandspotentialen « (Dausien 1994, S. 141) kann »transformatorisches Potenzial alltäglichen Handelns« (Thon 2016, S. 187) deutlich gemacht werden.

Die Frage nach der Eigensinnigkeit meint dabei nicht die Frage nach einer vorgängigen Eigenschaft des Subjekts, sondern sie stellt eine Eigenlogik dar, die sich aus »einer je einzigartigen Abfolge und Gestalt von Erfahrungen« (Dausien 1998, S. 267, zit. in Thon 2016, S. 187) ergibt. Diese Eigenlogik erzeugt eine Individualität, die das Resultat von Aneignungsprozessen der sozialen Welt darstellt (vgl. ebd.): 
»Der Begriff des biografischen Eigensinns erlaubt es also, Phänomene von Widerständigkeit analytisch zu fassen, ohne dabei Subjektivität als solche Quelle von Widerständigkeit zu machen. Eigensinn äußert sich so, wie er biografietheoretisch beschrieben wird, nicht notwendig als widerständig in dem Sinne, dass er sich gegen Normierungen richten oder sich ihnen verweigern würde. Es geht hier eher um die Eigenlogik und Individualität biografischer Konstruktionen.« (Dausien 1998, S. 267, zit. in Thon 2016, S. 188)

Vor diesem Hintergrund wird im Folgenden widerständiges und eigensinniges Verhalten, das sich in den Biografien der befragten Frauen zeigt, in den Blick genommen.

Davon ausgehend wird in einem zweiten Schritt näher beleuchtet, inwiefern aufgrund der rekonstruierten Praktiken und Handlungsorientierungen im Umgang mit Gewalt innerhalb der Paarbeziehung eher von einem Wandel oder von Persistenz der Gewalt gesprochen werden kann.

\subsubsection{Wandel und Persistenz von Gewalt}

Die Frage nach dem Wandel oder der Persistenz im Umgang mit Gewalt in Paarbeziehungen ist verbunden mit Fragen nach der Reproduktion und Transformation von Geschlechterordnungen. Denn mit dem zugrunde liegenden Verständnis von Gewalt, das diese im Kontext von Geschlechterverhältnissen (vgl. Kap. 4.2.3) betrachtet, findet Gewalt innerhalb eines existierenden Machtverhältnisses statt und wird weiter auch als Mittel zur Herstellung von Abhängigkeitsverhältnissen, zur Durchsetzung von Interessen und zur Austragung von Konflikten eingesetzt.

Zur Persistenz von Gewalt Einigkeit besteht heute weitgehend darüber, dass die Ursachen von Gewalt in einer multifaktoriellen Gemengelage zu sehen sind. Knapp zusammengefasst lässt sich sagen, dass

»Cewalt gegen Frauen eng verknüpft ist mit strukturell verankerten Hierarchien zwischen den Geschlechtern und dichotomen, komplementären Konzepten von Männlichkeit und Weiblichkeit. Diese sind kulturell tradiert, werden über unterschiedliche Sozialisationsinstanzen vermittelt und sowohl leiblich als auch psychisch von den Individuen erfahren und aktiv angeeignet.«(Stövesand 2010, S. 92)

Diese Aspekte finden sich in den rekonstruierten Typen der vorliegenden Arbeit wieder, wenn auch in unterschiedlich starker Weise. So zeigt sich insbesondere hinsichtlich der Konzepte von Männlichkeit und Weiblichkeit eine starke Differenzierung zwischen den Typen »Autonomie« und »Abhängigkeit«: Während der erste Typus stark egalitäre Vorstellungen von Paarbeziehung repräsentiert, werden im 
Typus »Abhängigkeit« komplementäre Vorstellungen von Männlichkeit und Weiblichkeit sichtbar.

Weiter wird in den Ergebnissen die Bedeutung von sozialisatorischen Erfahrungen erkennbar. So zeigen aktuelle Forschungsergebnisse, wie in Kapitel 4.2.3 näher ausgeführt, dass die Beziehung zu den eigenen Eltern sowie deren Erziehungsverhalten unterschiedliche Bereiche der Beziehungsqualität in den ersten eigenen Paarbeziehungen beeinflusst (vgl. Walper u.a. 2010). Kindler und Unterstaller (2013) machen mit Bezug auf aussagekräftige Befunde verschiedener (Längsschnitt-)Studien deutlich, dass

»das nachhaltige Erleben eines negativen Modells elterlicher Fürsorge in Form von Misshandlungen oder Vernachlässigung die Fähigkeit zur gewaltfreien Cestaltung eigener Partnerschaften erkennbar beeinträchtigt. Gleiches gilt für das chronische Miterleben von Partnergewalt in der Herkunftsfamilie. Auch eine Lebensgeschichte, in der aggressive und regelverletzende Verhaltensauffälligkeiten ohne geeignete erzieherische und pädagogische Antwort bleiben, stellt einen deutlichen Risikofaktor für spätere Partnergewalt dar. Die Befunde gelten für das Ausüben von Partnergewalt, teilweise aber auch für die Bereitschaft, in einer gewaltförmigen Partnerschaft auszuharren.« (ebd., S. 519)

In der Rekonstruktion der dieser Arbeit zugrunde liegenden Lebensgeschichten zeigen sich intergenerationale Transmissionsprozesse von Gewalt als ein wesentliches Ergebnis für die Persistenz von Gewalt. So haben von den acht befragten Frauen, die innerhalb ihrer ersten oder in einer ihrer ersten Paarbeziehung Gewalt erfahren haben, sechs bereits Gewalt in der Herkunftsfamilie entweder auf der elterlichen Ebene und/oder auf der Eltern-Kind-Ebene erfahren (vgl. dazu in Kap. 7.3.3 die Übersicht zum Sample). In einem Fall (Amy) zeigt sich die Weitergabe von Gewalt über drei Generationen hinweg.

Die Ergebnisse der Einzelfallanalysen machen darüber hinaus auch deutlich, dass nicht nur Gewalt intergenerational weitergegeben wird. Auch der in der Sozialisation erworbene Orientierungsrahmen resp. der Habitus als eine in der Familiengeschichte angelegte Struktur bezüglich der Wahrnehmung, des Denkens und Handelns, zeigt sich in den Biografien in Form von familialen bzw. transgenerationalen Diskursen und Handlungsmustern. So wurden immer wieder Parallelen zwischen Kindheit und Jugend und dem frühen Erwachsenenalter deutlich (z.B. im äußeren Umfeld, Modelle von Partnerschaft). Demnach zeigt sich im Typus »Pertinenz« eine deutliche Parallele zwischen dem Miterleben vieler wechselnder Partnerschaften der Mutter und dem eigenen Beziehungsmodell, nach dem viele kurze Beziehungen zu Männern gelebt werden. Im Typus »Autonomie« zeigt sich dagegen, dass verlässliche und sichere Beziehungserfahrungen in der Herkunftsfamilie die Herausbildung eines autonomen Habitus begünstigen. Im Unterschied dazu zeigen sich Abhängigkeits- und Ohnmachtserfahrungen während der Kind- 
heit tendenziell auch in erneuter Abhängigkeit während der ersten eigenen Paarbeziehung(en).

Dass in allen Fällen die Gewalt in der Beziehung zum Zeitpunkt des Interviews beendet worden war, stellte eine Voraussetzung an potenzielle Interviewpartnerinnen dar und war Teil des Forschungsdesigns. Folglich können keine Aussagen darüber gemacht werden, inwiefern sich möglicherweise eine Persistenz von Gewalt in der jeweiligen Lebensgeschichte fortsetzt.

Interessant ist allerdings auch die Frage, wie die interviewten Frauen es geschafft haben, sich aus der gewaltgeprägten Beziehung zu lösen und was sie dabei als unterstützend erlebt haben. Dies führt zu der Frage nach dem Wandel bzw. der Veränderung von Gewalt und damit auch zur Frage nach den Bedingungen dieses Wandels.

Bedingungen des Wandels: Widerstandsstrategien Im Folgenden sollen Möglichkeiten des Widerstandes sowie dessen Bedingungen aufgezeigt werden. Dazu wurden in der Rekonstruktion der erzählten Lebensgeschichten drei wesentliche Aspekte herausgearbeitet, die solche Bedingungen der Beendigung von Gewalt innerhalb der Paarbeziehung darstellen: 1) das Unterlaufen symbolischer und materieller Geschlechterkonstruktionen, 2) die Anerkennung durch signifikante Andere und 3) Unterstützung durch professionelle Fachkräfte.

1) Das Unterlaufen symbolischer und materieller Geschlechterkonstruktionen Im Hinblick auf den Diskurs um Geschlechternormen lässt sich eine zunehmende Durchlässigkeit von Geschlechtergrenzen konstatieren (vgl. Kap. 2). So zeigt sich auf der Ebene des Alltagswissens ein Bewusstsein um Gleichberechtigung:

»Das alltagsweltliche Differenzwissen, das also, was die Cesellschaftsmitglieder über den Unterschied der Geschlechter und die soziale Bedeutung der Geschlechterdifferenz, über die Geschlechterordnung und das Verhältnis der Geschlechter wissen, ist ... den Strukturen des Ceschlechterverhältnisses und großen Teilen der sozialen Praxis ein ganzes Stück vorausgeeilt. Kulturelle Deutungsmuster und Selbstkonzepte, Geschlechterdiskurse und mit ihnen der diskursfähige, der explizite und explizierbare Teil der Geschlechternormen haben sich erkennbar von den salten S Selbstverständlichkeiten verabschiedet und geschlechtshierarchische Verteilungsasymmetrien sind entschieden begründungsbedürftiger geworden.« (Wetterer 2003, S. 289)

Allerdings hat sich weder das Geschlechterverhältnis als sozialer Strukturzusammenhang noch die soziale Praxis der Mitglieder innerhalb der Gesellschaft nachhaltig verändert. Dies bedeutet, dass sowohl auf der Ebene sozialer Strukturen und Institutionen als auch in den Routinen des Alltagshandelns eine erhebliche Diskre- 
panz zwischen alltagsweltlichem Differenzwissen und latenten Geschlechternormen, die weiterhin das soziale Handeln bestimmen, besteht. (vgl. ebd., S. 289f.)

Die Wirkung solcher latenten Geschlechternormen zeigt sich in den Biografien der befragten Frauen auf unterschiedliche Weise. So wird insgesamt ein hohes Engagement im reproduktiven Bereich deutlich, was sich besonders bei den Frauen mit Kindern noch deutlicher zeigt. Dies differenziert sich in die Übernahme häuslicher Pflichten, die Fürsorge für ein Kind sowie die Unterstützung des Partners aus. Mit Blick auf widerständiges Handeln zeigt sich an dieser Stelle, dass die in verschiedenen Fällen rekonstruierte Bildungsaspiration Frauen dazu befähigt, handlungsmächtig zu werden und sich in der Folge aus der gewaltgeprägten Beziehung zu lösen. Indem bspw. auf der Absolvierung einer Berufsausbildung beharrt oder der angebotene Studienplatz angenommen wird, schaffen sich die Frauen einen Raum, der es ihnen ermöglicht, sich eine eigene berufliche und damit finanziell abgesicherte(re) Zukunft aufzubauen, die sie unabhängig(er) von ihrem Partner werden lässt.

Eine weitere Möglichkeit widerständigen Handelns zeigt sich im Unterlaufen des dominanten Diskurses um Geschlechterkonstruktionen. Die in weiten Teilen den Diskursen um Gewalt und Geschlecht zugrunde liegenden Geschlechterkonstruktionen von der männlichen Verletzungsmächtigkeit und der weiblichen Verletzungsoffenheit können von Frauen »symbolisch und materiell« (Glammeier 2011, S. 20) unterlaufen werden. Denn

»auch (körperliche) Partnergewalt lässt sich als Versuch definieren, grammatikalisch korrekte, vergeschlechtlichte Subjektpositionen der Verletzungsmächtigkeit und Verletzungsoffenheit, der Handlungsmacht und der Handlungsmachtlosigkeit herzustellen.«(Glammeier 2011, S. 21)

So kann die Entdeckung der eigenen, auch körperlichen, Kraft und Stärke dazu führen, dass sich Frauen als handlungsmächtig erleben und sich in der Lage fühlen, sich körperlich zu wehren. Dies zeigt sich besonders in den aktiven Handlungsstrategien im Umgang und der Bewältigung von Gewalterfahrungen innerhalb der Typen »Pertinenz« und »Autonomie«.

2) Die Bedeutung von signifikanten Anderen Bruno Hildenbrand weist mit Bezug auf verschiedene sozialwissenschaftliche Ansätze, insbesondere jedoch mit Bezug auf den Symbolischen Interaktionismus, darauf hin, dass die Familie nicht unbedingt wichtiger ist als die Personen und Einflüsse, die außerhalb der Familie das Individuum prägen. So werden im Symbolischen Interaktionismus soziologische Theorien als Theorien über die signifikanten Beziehungen zwischen Mitgliedern gleicher oder verschiedener Generationen konzipiert (vgl. ebd.). Diese sogenannten signifikanten Anderen kommen und gehen im Laufe des Lebens und ihre Bedeutung nimmt zu oder ab (vgl. Hildenbrand 2007, S. 32). Wie in den Erzählungen der be- 
fragten Frauen deutlich wurde, können signifikante Andere in lebensgeschichtlich kritischen Phasen auftauchen (z.B. stellte Jasmins Großmutter den einzigen Halt für Jasmin in der Zeit dar, in der ihr Vater zu Hause massiv gewalttätig war) und auf biografische Weichenstellungen Einfluss haben (Jasmin trennt sich, als sie ihren aktuellen Freund kennenlernt). Signifikante Andere stellen damit einen Faktor in der Erklärung dar, wie es möglich ist, trotz (schwerer) Belastungen bis hin zu Gewalterfahrungen in der Kindheit später eine gewaltfreie Beziehung zu leben resp. sich von einem gewaltausübenden Partner zu lösen.

In der vorliegenden Arbeit wurde aufgezeigt, wie die während der Sozialisation in Gang gesetzte Dynamik bzw. die dort gemachten Erfahrungen die Aufnahme eigener intimer Beziehungen beeinflussen. Besonders bei schwierigen oder belastenden Erfahrungen in dieser Zeit können signifikante Andere für die individuelle Entwicklung eine bedeutsame Rolle einnehmen. Den in den Einzelfallanalysen rekonstruierten signifikanten Anderen kommt dabei der »Charakter des Hinzukommenden (Ermöglichenden, ... Kompensierenden)« (Hildenbrand 2007, S. 227) $\mathrm{zu}$, die, wenn sich die Akteurinnen darauf einlassen und diese Beziehungen eingehen, Bedingungen von Widerstand, im Sinne einer Veränderung der bestehenden Gewaltverhältnisse, befördern können.

3) Unterstützung durch professionelle Fachkräfte Bei der Auswertung der biografisch-narrativen Interviews zeigte sich, dass die Unterstützung durch professionelle Fachkräfte von Gewalt betroffenen Frauen Handlungsmöglichkeiten aufzeigt, widerständig mit den Bedingungen und Alltagsroutinen umzugehen und damit einen Wandel ihrer Beziehungsverhältnisse zu vollziehen. Im folgenden Kapitel 9.3 soll diese Perspektive auf die Unterstützung betroffener Frauen ausführlicher eingenommen werden.

Als Fazit lässt sich festhalten, dass die Ergebnisse eine erweiterte Perspektive auf Gewalt aufzeigen, die die bisherige (Fach-)Diskussion um die Dimension ergänzt, nach der Frauen nicht nur bzw. nicht ausschließlich als Opfer von Gewalt angesehen werden. ${ }^{8}$ Die rekonstruierten Typen zeigen dabei eine Abstufung dieser Perspektive auf: So zeigt sich im Typus »Abhängigkeit« eher die Herstellung einer Position, in der sich die Frau als Objekt der Gewalt darstellt. Im Gegensatz dazu konzipieren sich Frauen in den Typen »Pertinenz« sowie »Autonomie« als Subjekte der Gewalt. Diese Frauen verstehen sich biografisch nicht per se als »Opfer«. In ihren Erzählungen konnten zudem positive Bilder von eigenwilligen Frauen rekonstruiert werden, die sich handlungsmächtig fühlen und sich gegenüber der erleb-

8 Vgl. dazu die Forschungsarbeit von Sandra Glammeier (2011), die deutlich macht, dass Frauen nicht allein durch erlebte Gewalt geschädigt werden, sondern vor allem durch kulturelle Konstruktionsprozesse. Sie zeigt weiter auf, wie der Herstellungsprozess von Frauen als Objekt der Cewalt unterbrochen werden kann. 
ten Gewalt innerhalb ihrer Beziehung zur Wehr setzen. Insgesamt verdeutlichen diese unterschiedlichen Subjektivierungspraxen die jeweils »eigensinnige Verarbeitung (Alheit 2006, S. 6), die mit den jeweils eigenen Erfahrungen zu tun hat.

Die rekonstruierten differenten Formen des Erlebens und auch Erleidens von Gewalt bewegen sich auf einem Kontinuum, das von Ohnmachts- und Abhängigkeitserfahrungen bis hin zu handlungsmächtigen (Gegen-)

Strategien reicht. Die Ergebnisse der analysierten Bedingungen des Widerstandes bieten somit ergänzende Anschlusspunkte für eine sozialpädagogische Unterstützung sowie für die Prävention von Gewalt.

\subsection{Anschlusspunkte für sozialpädagogische Unterstützung und Ausblick}

Die Ergebnisse der Arbeit bieten Anschlusspunkte für die Praxis Sozialer Arbeit und zeigen, dass sozialpädagogische Unterstützungsangebote einen wichtigen Beitrag leisten können, wenn es um Hilfe und Unterstützung zur Beendigung von Gewalt geht. In den vorliegenden Fällen zeigt sich, dass manche der betroffenen Frauen aufgrund anderer Lebens- oder Problemlagen, also nicht wegen der erfahrenen Gewalt innerhalb der Paarbeziehung, eine sozialpädagogische Hilfe erhielten. Dies waren bspw. die Familienhilfe, Jugendsozialarbeit oder Mobile Jugendarbeit/Streetwork. Innerhalb ihrer Arbeit in den genannten Unterstützungsangeboten erfuhren die Fachkräfte auch von der bestehenden Thematik der Gewalt in den ersten eigenen Paarbeziehungen. Dabei kommt den Fachkräften eine Art Türöffner-Funktion zu, die es ermöglicht, den betroffenen Frauen die für sie notwendige Unterstützung hinsichtlich der Gewalt-Thematik zukommen zu lassen. Dies erfordert allerdings zunächst eine Offenheit sowie fachliches Wissen darüber, dass es bereits im Jugendalter zu Übergriffen und Gewalt innerhalb der ersten Paarbeziehungen kommen kann. Wie in der Einleitung der Arbeit ausgeführt, ist diese Thematik, zumindest in der Bundesrepublik Deutschland, recht neu und wird bislang gesellschaftlich wenig aufgegriffen. Vor dem Hintergrund weiblicher Verdeckungszusammenhänge und der vermeintlichen Gleichberechtigung, die in der Folge dazu führen können, dass sich betroffene junge Frauen nicht zu Wort melden und von der erlebten Gewalt berichten, bedarf es eines spezifischen Wissens darüber, wie die Bedarfe der jungen Frauen sind, um adäquat handeln zu können. Dabei zeigt die vorliegende Arbeit als wichtiges Ergebnis auf, dass sich die jungen Frauen keineswegs durchweg als Opfer erleben oder beschreiben. Gerade weil »Handlungsmacht und Widerstand von Frauen ... bisher kaum Bestandteil der Repräsentationen des Problems der Gewalt gegen Frauen« (Glammeier 2011, S. 21) waren, gilt es, die Arbeit mit von Gewalt betroffenen jungen Frauen um diesen Aspekt zu ergänzen. 
Der Verlauf einzelner Fälle zeigt außerdem auf, dass manche der jungen Frauen keinerlei professionelle Unterstützung bei der Bewältigung bzw. Beendigung der Beziehungsgewalt hatten, diese aber durchaus hilfreich gewesen wäre. Dies macht zum einen deutlich, dass es in allen Arbeitsfeldern, die mit jungen Menschen arbeiten, ein Wissen um die Thematik benötigt. Zum anderen zeigt dies auch, dass Prävention hier eine Form darstellt, die nicht nur im Vorfeld, sondern auch für Betroffene hilfreich sein kann.

Wie weiter oben ausgeführt, stellt sich die Frage "als wer oder was sich das gewalttätige Subjekt setzt und was sein Anderer ... für ihn darstellt - und umgekehrt wäre zu fragen, was es mit dem Subjekt macht, wenn es zwangsweise als Objekt auf einem unverrückbaren Platz positioniert wird « (Rendtorff u.a. 2016, S. 131). Mit Bezug auf diese Frage ergeben sich zwei entscheidende Aufgaben für Soziale Arbeit, wie sie Rendtorff u.a. (2016) formuliert haben und die ich in Bezug auf die Ergebnisse der vorliegenden Arbeit hervorheben möchte:

»Die erste liegt in der Vermittlung der Cewissheit, ein Recht auf Eigenwillen zu haben, und in der Fähigkeit, diesen zu empfinden und ihm Raum zu geben, um sich zu äußern auch als Protest und Widerstand gegen Zumutungen. Die zweite Aufgabe würde darin bestehen, sowohl die Wahrnehmung der Anderen als anders wie auch die Fähigkeit zu stärken, diese Andersheit anzuerkennen, auch da, wo sie mit der eigenen Wahrnehmung und den eigenen Wünschen kollidiert (das ist keine Frage der Moral, sondern letztlich unfreiwilliger Akt, sofern er die Basis des eigenen Anerkanntwerdens bildet). Denn die Empfindung des Rechts des Anderen auf seinen Eigenwillen kann von der potentiellen Opferseite aus gesehen dazu verhelfen, Übergriffe als solche zu erkennen und sich mitzuteilen; und von der potentiellen Täterperspektive aus gesehen kann sie einen Spalt öffnen zwischen dem, was ich will, und dem, was ich nicht sollte, weil der andere es nicht will.« (Rendtorff u.a. 2016, S. 140)

Entscheidend dabei ist also, sich in der Sprecher_innenposition nicht die Definitionsmacht über den/die andere_n anzueignen.

Zudem gilt auch bei Gewalt in jugendlichen und jungen Paarbeziehungen - wie Befunde aus dem Bereich der häuslichen Gewalt zeigen -, dass eine nachhaltige und wirksame Verhinderung von Gewalt nicht nur die Angelegenheit der Gewaltbetroffenen, sondern auch der Gewalttätigen ist (vgl. Hagemann-White/Meysen 2013, S. 561). Dies bedeutet neben der Unterstützung der Frauen die Umsetzung einer konsequenten Täterorientierung in der Intervention, indem dieser in die Verantwortung für sein Verhalten genommen wird. Dies bedeutet auch, sich von der Konzentration auf Trennung als Verantwortung der Frau zu verabschieden (vgl. Glammeier 2011, S. 22).

Insgesamt zeigen die Ergebnisse der vorliegenden Arbeit, dass das gängige Deutungsmuster der Gewalt, die »stets nur gefürchtet, aber nicht verhindert oder 
gestoppt werden« (ebd.) kann, mit dazu beiträgt, dass die Perspektive auf Handlungsmöglichkeiten verdeckt wird, diese jedoch im Sinne der Unterstützungsmöglichkeiten von jungen Frauen dringend bedacht werden müssen.

Die Perspektive auf Biografie als Ergebnis von Subjektivierungspraxen eröffnet dabei den Blick auf Handlungsoptionen und -spielräume, insbesondere auf diejenigen, mit denen diskursive Praxen durchbrochen werden.

Nicht zuletzt braucht es neben fachlichem Wissen, der Weiterqualifizierung zu dem Thema, auch Öffentlichkeitsarbeit, die das Thema Gewalt in jungen Paarbeziehungen sichtbar macht und die die Zielgruppe anspricht. Und es bedarf - wie es auch die Forderung in der Diskussion um »Peer Violence« und Schutzkonzepte ist - einer grundlegenden Auseinandersetzung mit den Positionierungen von Jugendlichen gegenüber (sexualisierten) Übergriffen sowie einer Auseinandersetzung mit dem Jugendbild, jugendkulturellen Positionierungen und Sexualitäten in diesem Lebensalter (vgl. Strahl u.a. 2017, S. 277). 



\section{Literatur}

Albrecht, Günter (2002): Soziologische Erklärungsansätze individueller Gewalt und ihre empirische Bewährung. In: Wilhelm Heitmeyer und John Hagan (Hg.): Internationales Handbuch der Gewaltforschung. Wiesbaden: Westdeutscher Verlag, S. 763-818.

Alheit, Peter (1992): Biographizität und Struktur. In: Peter Alheit, Bettina Dausien, Andreas Hanses und Antonius Scheuermann (Hg.): Biographische Konstruktionen. Beiträge zur Biographieforschung (19), S. 11-36.

Alheit, Peter (2006): »Biografizität« als Schlüsselkompetenz in der Moderne. Vortragsmanuskript, 2006. Online verfügbar unter www.abl-unigoettingen.de/aktuell/Alheit_Biographizitaet_Schluessel_Flensburg200 6.pdf, zuletzt geprüft am 06.11.2017.

Alheit, Peter (2010): Identität oder »Biographizität«? Beiträge der neueren sozialund erziehungswissenschaftlichen Biographieforschung zu einem Konzept der Identitätsentwicklung. In: Birgit Griese (Hg.): Subjekt - Identität - Person? Reflexionen zur Biographieforschung. Wiesbaden: VS Verlag für Sozialwissenschaften, S. 219-249.

Alkemeyer, Thomas/Buschmann, Nikolaus/Michaeler, Matthias (2015): Kritik der Praxis. Plädoyer für eine subjektivierungstheoretische Erweiterung der Praxistheorien. In: Thomas Alkemeyer, Volker Schürmann und Jörg Volbers (Hg.): Praxis denken. Konzepte und Kritik. Wiesbaden: Springer VS, S. 25-50.

Ashley, Olivia S./Foshee, Vangie A. (2005): Adolescent help-seeking for dating violence: prevalence, sociodemographic correlates, and sources of help. In: Journal of Adolescent Health 36 (1), S. 25-31.

Avery-Leaf, Sarah/Cascardi, Michele (2002): Dating violence education: Preventing and early intervention strategies. In: Paul A. Schewe (Hg.): Preventing violence in relationships. Interventions across the live span. Washington DC: American Psychological Association, S. 79-105.

Averdijk, Margit/Müller-Johnson, Katrin/Eisner, Manuel (2011): Sexuelle Viktimisierung von Kindern und Jugendlichen in der Schweiz. Schlussbericht für die UBS Optimus Foundation November 2011. Hg. v. UBS Optimus Foundation. Online verfügbar unter www.optimusstudy.org/fileadmin/user_upload/docu- 
ments/Full_Report_Schweiz/Optimus_Studie_WissenschaftlicherSchlussbericht_2012_d.pdf, zuletzt aktualisiert am 29.08.2013.

Avery-Leaf, Sarah/Cascardi, Michele/O'Leary, K. Daniel/Cano, Annemarie (1997): Efficacy of a Dating Violence Prevention Program on Attitudes Justifying Aggression. In: Journal of Adolescent Health 21 (1), S. 11-17.

Barter, Christine/McCarry, Melanie/Berridge, David/Evans, Kathy (2009): Partner exploitation and violence in teenage intimate relationships. Online verfügbar unter www.nspcc.org.uk/Inform/research/findings/partner_exploitation_and_violence_report_wdf70129.pdf, zuletzt geprüft am 27.09.2017.

Becker-Schmidt, Regina/Brandes-Erlhoff, Uta/Rumpf, Mechthild/Schmidt, Beate (1983): Arbeitsleben - Lebensarbeit. Konflikte und Erfahrungen von Fabrikarbeiterinnen. Bonn: Verlag Neue Gesellschaft.

Beck-Gernsheim, Elisabeth (1983): Vom »Dasein für andere« zum Anspruch auf ein Stück »eigenes Leben«: Individualisierungsprozesse im weiblichen Lebenszusammenhang. In: Soziale Welt 34 (3), S. 307-340.

Beck, Ulrich/Beck-Gernsheim, Elisabeth (1994): Riskante Freiheiten. Individualisierung in modernen Gesellschaften. Frankfurt a.M.: Suhrkamp.

Beck, Ulrich/Beck-Gernsheim, Elisabeth (2015): Das ganz normale Chaos der Liebe. 13. Auflage. Frankfurt a.M.: Suhrkamp.

Bilden, Helga/Dausien, Bettina (2006): »Sozialisation und Geschlecht - Einleitung in eine vielstimmige Diskussion. In: Helga Bilden und Bettina Dausien (Hg.): Sozialisation und Geschlecht. Theoretische und methodologische Aspekte. Opladen: Verlag Barbara Budrich, S. 7-15.

Blättner, Beate/Hintz, Elisabeth/Schultes, Kristin (2016): Gewalttätiges und grenzwertiges Handeln in den ersten Liebesbeziehungen Jugendlicher: ein in Deutschland noch wenig wahrgenommenes Phänomen. In: ZJJ 28 (1), S. 39-42.

Blättner, Petra/Schultes, Kristin/Birk, Amaya/Becher, Anne (2014): Digitale Prävention von Grenzüberschreitungen und Gewalt in Teenagerbeziehungen. In: Deutsche Jugend 62 (12), S. 521-530.

Blättner, Beate/Schultes, Kristin/Hehl, Liselotte/Brzank, Petra (2015): Grenzüberschreitungen und Gewalt in Teenagerbeziehungen. Risiken und Folgen für die Präventionsstrategien. In: Prävention und Gesundheitsförderung 10 (2), S. 173-179.

Blättner, Beate/Schultes, Kristin (2018): Gewalt in Intimbeziehungen Jugendlicher. Entwicklung einer Typologie als Basis für Prävalenzmessungen und Präventionskonzepte. In: Deutsche Jugend 66 (2), S. 72-79.

Blättner, Beate/Schultes, Kristin/Hintz, Elisabeth (2018): Dating Violence - sexuelle Gewalt unter Gleichaltrigen. In: Alexandra Retkowski, Angelika Treibel und Elisabeth Tuider (Hg.): Handbuch Sexualisierte Gewalt und pädagogische Kontexte. Theorie, Forschung, Praxis. Weinheim und Bael: Beltz Juventa, S. 325-332. 
BMFSFJ-Bundesministerium für Familie, Senioren Frauen und Jugend (2006): Siebter Familienbericht. Familie zwischen Flexibilität und Verlässlichkeit. Perspektiven für eine lebenslaufbezogene Familienpolitik. Hg. v. BMFSFJ. Berlin.

BMFSFJ-Bundesministerium für Familie, Senioren Frauen und Jugend (2012): Achter Familienbericht. Zeit für Familie. Familienzeitpolitik als Chance einer nachhaltigen Familienpolitik. Achter Familienbericht. Hg. v. BMFSFJ. Berlin.

Böhnisch, Lothar (2017): Abweichendes Verhalten. Eine pädagogisch-soziologische Einführung. 5. Aufl. Weinheim: Beltz Juventa.

Bohnsack, Ralf (2007): Typenbildung, Generalisierung und komparative Analyse: Grundprinzipien der dokumentarischen Methode. In: Ralf Bohnsack, Iris Nentwig-Gesemann und Arnd-Michael Nohl (Hg.): Die dokumentarische Methode und ihre Forschungspraxis. Grundlagen qualitativer Sozialforschung. 2., erweiterte und aktualisierte Auflage. Wiesbaden: VS Verlag für Sozialwissenschaften, S. 225-253.

Bohnsack, Ralf (2010a): Rekonstruktive Sozialforschung. Einführung in qualitative Methoden. 8., durchgesehene Auflage. Opladen \& Farmington Hills, MI: Verlag Barbara Budrich.

Bohnsack, Ralf (2010b): Die Mehrdimensionalität der Typenbildung und ihre Aspekthaftigkeit. In: Jutta Ecarius und Burkhard Schäffer (Hg.): Typenbildung und Theoriegenerierung. Methoden und Methodologien qualitativer Bildungsund Biographieforschung. Opladen \& Farmington Hills, MI: Verlag Barbara Budrich, S. 47-72.

Bohnsack, Ralf (2010c): Dokumentarische Methode und Typenbildung - Bezüge zur Systemtheorie. In: René John, Anna Henkel und Anna Rückert-John (Hg.): Die Methodologien des Systems. Wie kommt man zum Fall und wie dahinter? Wiesbaden: VS Verlag für Sozialwissenschaften, S. 291-320.

Bohnsack, Ralf (2012): Orientierungsschemata, Orientierungsrahmen und Habitus. Elementare Kategorien der Dokumentarischen Methode mit Beispielen aus der Bildungsmilieuforschung. In: Karin Schittenhelm (Hg.): Qualitative Bildungs- und Arbeitsmarktforschung. Grundlagen, Perspektiven, Methoden. Wiesbaden: Springer Fachmedien, S. 119-152.

Bohnsack, Ralf (2013): Dokumentarische Methode und die Logik der Praxis. In: Alexander Lenger, Christian Schneickert und Florian Schumacher (Hg.): Pierre Bourdieus Konzeption des Habitus. Grundlagen, Zugänge, Forschungsperspektiven. Wiesbaden: Springer VS, S. 175-200.

Bohnsack, Ralf/Loos, Peter/Schäffer, Burkhard/Städtler, Klaus/Wild, Bodo (1995): Die Suche nach Gemeinsamkeit und die Gewalt in der Gruppe. Hooligans, Musikgruppen und andere Jugendcliquen. Opladen: Leske + Budrich.

Bohnsack, Ralf/Nentwig-Gesemann, Iris/Nohl, Arnd-Michael (2007): Einleitung: Die dokumentarische Methode und ihre Forschungspraxis. In: Ralf Bohnsack, Iris Nentwig-Gesemann und Arnd-Michael Nohl (Hg.): Die dokumentarische 
Methode und ihre Forschungspraxis. Grundlagen qualitativer Sozialforschung. 2., erweiterte und aktualisierte Auflage. Wiesbaden: VS Verlag für Sozialwissenschaften, S. 9-27.

Bohnsack, Ralf/Pfaff, Nicole (2010): Die dokumentarische Methode: Interpretation von Gruppendiskussionsverfahren und Interviews. In: Enzyklopädie Erziehungswissenschaft Online (EEO). Fachgebiet Methoden der empirischen Sozialforschung. Hg. v. Sabine Maschke und Ludwig Stecher.

Breitenbach, Eva (2007): Sozialisation und Konstruktion von Geschlecht und Jugend. Empirischer Konstruktivismus und dokumentarische Methode. In: Ralf Bohnsack, Iris Nentwig-Gesemann und Arnd-Michael Nohl (Hg.): Die dokumentarische Methode und ihre Forschungspraxis. Grundlagen qualitativer Sozialforschung. 2., erweiterte und aktualisierte Auflage. Wiesbaden: VS Verlag für Sozialwissenschaften, S. 167-181.

Brown, B. Bradford (1999): »You're going out with who?«. Peer group influences on adolescent relationships. In: Wyndol Furman, B. Bradford Brown und Candice Feiring (Hg.): The development of romantic relationships in adolescence. Cambridge, Mass.: Cambridge University Press, S. 291-329.

Brzank, Petra/Blättner, Beate/Liepe, Katharina (2013): Gewalt in den ersten Liebesbeziehungen unter Jugendlichen. In: Deutsche Jugend 61 (11), S. 473-481.

Buddeberg, Barbara (1983): Kinder mißhandelter Frauen - Struktur und Dynamik von Mißhandlungsfamilien. In: Praxis der Kinderpsychologie und Kinderpsychiatrie 32 (8), S. 273-277.

Burkart, Günter (2018): Soziologie der Paarbeziehung. Eine Einführung. Wiesbaden: Springer VS.

Butler, Judith (1991): Das Unbehagen der Geschlechter. Frankfurt a.M.: Suhrkamp.

Butler, Judith (2001): Psyche der Macht. Das Subjekt der Unterwerfung. Frankfurt a.M.: Suhrkamp.

Butler, Judith (2006): Haß spricht. Zur Politik des Performativen. 5. Auflage. Berlin: Suhrkamp.

BZgA - Bundeszentrale für gesundheitliche Aufklärung (Hg.) (2006): Jugendsexualität 2006. Repräsentative Wiederholungsbefragung von 14- bis 17-Jährigen und ihren Eltern. BZgA. Köln.

BZgA - Bundeszentrale für gesundheitliche Aufklärung (Hg.) (2010): Jugendsexualität. Repräsentative Wiederholungsbefragung von 14- bis 17-Jährigen und ihren Eltern. Aktueller Schwerpunkt Migration. BZgA. Köln.

BZgA - Bundeszentrale für gesundheitliche Aufklärung (Hg.) (2015): Jugendsexualität. Repräsentative Wiederholungsbefragung. Die Perspektiven der 14- bis 25-Jährigen. BZgA. Köln.

Dausien, Bettina (1994): Biographieforschung als »Königinnenweg«? Überlegungen zur Relevanz biographischer Ansätze in der Frauenforschung. In: Angelika Diezinger, Hedwig Kitzer, Ingrid Anker, Irma Bingel und Erika Haas (Hg.): Erfah- 
rung mit Methode: Wege sozialwissenschaftlicher Frauenforschung. Freiburg i.Br.: Kore, S. 129-153.

Dausien, Bettina (1996): Biografie und Geschlecht. Zur biografischen Konstruktion sozialer Wirklichkeit in Frauenlebensgeschichten. Bremen: Donat.

Dausien, Bettina (2006): Geschlechterverhältnisse und ihre Subjekte. Zum Diskurs um Sozialisation und Geschlecht. In: Helga Bilden und Bettina Dausien (Hg.): Sozialisation und Geschlecht. Theoretische und methodologische Aspekte. Opladen: Verlag Barbara Budrich, S. 17-44.

Dausien, Bettina (2008): Biografieforschung: Theoretische Perspektiven und methodologische Konzepte für eine rekonstruktive Geschlechterforschung. In: Ruth Becker und Beate Kortendiek (Hg.): Handbuch Frauen- und Geschlechterforschung. Theorie, Methoden, Empirie. 2. erweiterte und aktualisierte Auflage. Wiesbaden: VS Verlag für Sozialwissenschaften, S. 354-367.

Dausien, Bettina/Thon, Christine/Walgenbach, Katharina (2015): Geschlecht - Sozialisation - Transformation. In: Bettina Dausien, Christine Thon und Katharina Walgenbach (Hg.): Geschlecht - Sozialisation - Transformation. Opladen, Berlin, Toronto: Verlag Barbara Budrich, S. 9-50.

Dausien, Bettina/Walgenbach, Katharina (2015): Sozialisation von Geschlecht Skizzen zu einem wissenschaftlichen Diskurs und Plädoyer für die Revitalisierung einer gesellschaftsanalytischen Perspektive. In: Bettina Dausien, Christine Thon und Katharina Walgenbach (Hg.): Geschlecht - Sozialisation - Transformation. Opladen, Berlin, Toronto: Verlag Barbara Budrich, S. 17-50.

Davis, Antoinette (2008): Interpersonal and Physical Dating Violence among Teens. Hg. v. FOCUS. Views from the National Council on Crime and Delinquency. Online verfügbar unter www.nccdcrc.org/nccd/pubs/2008_focus_teen_dating_violence.pdf, zuletzt geprüft am 08.04.2011.

Denzin, Norman K. (2013): Symbolischer Interaktionismus. In: Uwe Flick, Ernst von Kardorff und Ines Steinke (Hg.): Qualitative Forschung. Ein Handbuch. 10. Aufl. Reinbek bei Hamburg: Rowohlt-Taschenbuch-Verl., S. 136-150.

Dobash, Russell P./Dobash, Emerson, P. (2002): Gewalt in heterosexuellen Partnerschaften. In: Wilhelm Heitmeyer und John Hagan (Hg.): Internationales Handbuch der Gewaltforschung. Wiesbaden: Westdeutscher Verlag, S. 921-941.

Eisner, Manuel (2002): Langfristige Gewaltentwicklung: Empirische Befunde und theoretische Erklärungsansätze. In: Wilhelm Heitmeyer und John Hagan (Hg.): Internationales Handbuch der Gewaltforschung. Wiesbaden: Westdeutscher Verlag, S. 58-80.

Equit, Claudia (2011): Weibliche Emanzipation mit den Fäusten? Perspektiven gewaltaktiver Mädchen und Bedingungen ihres Gewalthandelns. In: Forum Erziehungshilfen 17 (1), S. 10-14. 
Fischer, Wolfram/Kohli, Martin (1987): Biografieforschung. In: Wolfgang Voges (Hg.): Methoden der Biografie- und Lebenslaufforschung. Opladen: Leske + Budrich, S. 25-50.

Flaake, Karin (1998): Weibliche Adoleszenz - Neue Möglichkeiten, alte Fallen? Widersprüche und Ambivalenzen in der Lebenssituation und den Orientierungen junger Frauen. In: Mechthild Oechsle und Birgit Geissler (Hg.): Die ungleiche Gleichheit. Junge Frauen und der Wandel im Geschlechterverhältnis. Opladen: Leske + Budrich, S. 43-65.

Flick, Uwe (2007): Qualitative Sozialforschung. Eine Einführung. Reinbek bei Hamburg: Rowohlt-Taschenbuch-Verl.

Flick, Uwe/Kardorff, Ernst von/Steinke, Ines (2010): Was ist qualitative Forschung? Einleitung und Überblick. In: Uwe Flick, Ernst von Kardorff und Ines Steinke (Hg.): Qualitative Forschung. Ein Handbuch. 10. Aufl. Reinbek bei Hamburg: Rowohlt-Taschenbuch-Verl., S. 13-29.

Foucault, Michel (1991): Governmentality. In: Graham Burchell, Collin Gordon und Peter Miller (Hg.): The Focault Effect. Studies in Governmentality. Chicago: Chicago University Press, S. 87-104.

Franz, Julia/Griese, Birgit (2010): Dokumentarische Methode und Narrationsstrukturanalyse - ein Vergleich. In: Birgit Griese (Hg.): Subjekt - Identität - Person? Reflexionen zur Biographieforschung. Wiesbaden: VS Verlag für Sozialwissenschaften, S. 271-316.

Friebertshäuser, Barbara (1997): Interviewtechniken - ein Überblick. In: Barbara Friebertshäuser und Annedore Prengel (Hg.): Handbuch qualitative Forschungsmethoden in der Erziehungswissenschaft. Weinheim: Juventa, S. 371-395.

Fuchs-Heinritz, Werner (1998): Soziologische Biographieforschung: Überblick und Verhältnis zur Allgemeinen Soziologie. In: Gerd Jüttemann und Hans Thomae (Hg.): Biographische Methoden in den Humanwissenschaften. Weinheim: Beltz, S. 3-23.

Furman, Wyndol/Simon, Valerie A. (1999): Cognitive representations of adolescent romantic relationships. In: Wyndol Furman, B. Bradford Brown und Candice Feiring (Hg.): The development of romantic relationship in adolescence. Cambridge: Cambridge University Press, S. 75-98.

Galtung, Johan (1990): Cultural Violence. In: Journal of Peace Research 3, S. 291-305.

Geissler, Birgit/Oechsle, Mechthild (1996): Lebensplanung junger Frauen. Zur widersprüchlichen Modernisierung weiblicher Lebensläufe. Unter Mitarbeit von Gudrun Braemer. Weinheim: Deutscher Studien Verlag.

Gelles, Richard J. (2002): Gewalt in der Familie. In: Wilhem Heitmeyer und John Hagan (Hg.): Internationales Handbuch der Gewaltforschung. Wiesbaden: Westdeutscher Verlag, S. 1043-1077. 
Giddens, Anthony (1992): The transformation of Intimacy. Sexuality, Love \& Eroticism in modern Societies. Cambridge: Polity Press.

Gildemeister, Regine/Robert, Günther (2008): Geschlechterdifferenzierungen in lebenszeitlicher Perspektive. Interaktion - Institution - Biografie. Wiesbaden: VS Verlag für Sozialwissenschaften.

Gilligan, Carol (1984): Die andere Stimme. Lebenskonflikte und Moral der Frau. München: Piper.

Glammeier, Sandra (2011): Zwischen verleiblichter Herrschaft und Widerstand. Realitätskonstruktionen und Subjektpositionen gewaltbetroffener Frauen im Kampf um Anerkennung. Wiesbaden: VS Verlag für Sozialwissenschaften.

Glass, Nancy/Fredland, Nina/Campbell, Jacquelyn/Yonas, Michael (2003): Adolescent Dating Violence: Prevalence, Risk Factors, Health Outcomes, and Implications for Clinical Practice. In: Journal of Obstetric, Gynecologie and Neonatal Nursing (JOGNN) 32 (2), S. 227-238.

Grob, Alexander/Jaschinski, Uta (2003): Erwachsen werden. Entwicklungspsychologie des Jugendalters. Weinheim: Beltz PVU.

Grossmann, Karin/Grossmann, Klaus E. (2004): Bindungen. Das Gefüge psychischer Sicherheit. Stuttgart: Klett-Cotta.

Hagemann-White, Carol (1992): Strategien gegen Gewalt im Geschlechterverhältnis. Bestandsanalyse und Perspektiven. Pfaffenweiler: Centaurus-Verl.-Ges. (Forschungsberichte des BIS, 4).

Hagemann-White, Carol (2002): Gender-Perspektiven auf Gewalt in vergleichender Sicht. In: Wilhelm Heitmeyer und John Hagan (Hg.): Internationales Handbuch der Gewaltforschung. Wiesbaden: Westdeutscher Verlag, S. 124-149.

Hagemann-White, Carol (2016): Grundbegriffe und Fragen der Ethik bei der Forschung über Gewalt im Geschlechterverhältnis. In: Cornelia Helfferich, Barbara Kavemann und Heinz Kindler (Hg.): Forschungsmanual Gewalt. Grundlagen der empirischen Erhebung von Gewalt in Paarbeziehungen und sexualisierte Gewalt. Wiesbaden: Springer Fachmedien, S. 13-31.

Hagemann-White, Carol/Meysen, Thomas (2013): Partnerschaftsgewalt und familiäre Gewalt gegen Kinder: kohärente, divergenzsensible staatliche Reaktionen auf Menschenrechtsverletzungen - Strategien in und für Europa. In: Barbara Kavemann und Ulrike Kreyssig (Hg.): Handbuch Kinder und häusliche Gewalt. 3., aktualisierte und überarbeitete Auflage. Wiesbaden: Springer Fachmedien, S. 552-573.

Hainz, Michael (2012): Widerhaken der »Individualisierung«: eine Auseinandersetzung mit dem gleichnamigen Theorem Ulrich Becks. Institut für Gesellschaftspolitik an der Hochschule für Philosophie München. Online verfügbar unter www.nbn-resolving.de/urn:nbn:de:0168-ssoar-293957, zuletzt geprüft am 15.12.2017. 
Hanses, Andreas (2013): Das Subjekt in der sozialpädagogischen AdressatInnenund NutzerInnenforschung - zur Ambiguität eines komplexen Sachverhalts. In: Gunther Graßhoff (Hg.): Adressaten, Nutzer, Agency. Akteursbezogene Forschungsperspektiven in der Sozialen Arbeit. Wiesbaden: Springer VS, S. 99-117.

Hartmann, Jutta (2015): Transformatorische Denkbewegungen - Weiterentwicklung von Sozialisationstheorien im Anschluss an Judith Butlers diskurstheoretisches Verständnis von Geschlecht. In: Bettina Dausien, Christine Thon und Katharina Walgenbach (Hg.): Geschlecht - Sozialisation - Transformation. Opladen, Berlin, Toronto: Verlag Barbara Budrich, S. 53-76.

Haubl, Rolf (2003): Riskante Worte. Forschungsinterviews mit Traumatisierten. In: psychosozial 26 (91), S. 63-77.

Hausen, Karin (1976): Die Polarisierung der »Geschlechtscharaktere« - Eine Spiegelung der Dissoziation von Erwerbs- und Familienleben. In: Werner Conze (Hg.): Sozialgeschichte der Familie in der Neuzeit Europas. Stuttgart: ErnstKlett-Verlag, S. 363-393.

Heitmeyer, Wilhelm/Hagan, John (2002): Gewalt. Zu den Schwierigkeiten einer systematischen internationalen Bestandsaufnahme. In: Wilhelm Heitmeyer und John Hagan (Hg.): Internationales Handbuch der Gewaltforschung. Wiesbaden: Westdeutscher Verlag, S. 15-25.

Helfferich, Cornelia (2004): frauen leben. Eine Studie zu Lebensläufen und Familienplanung. 4. Auflage. Unter Mitarbeit von Wilfried Karmaus, Kurt Starke und Konrad Weller. Hg. v. BZgA - Bundeszentrale für gesundheitliche Aufklärung. BZgA. Köln.

Helfferich, Cornelia (2005): Die Qualität qualitativer Daten. Manual für die Durchführung qualitativer Interviews. 2. Auflage. Wiesbaden: VS Verlag für Sozialwissenschaften.

Helfferich, Cornelia/Kavemann, Barbara (2010): Gewalt in Ehe und Partnerschaft: Unterschiede beim Unterstützungsbedarf und bei Beratungsbarrieren und die spezifische Situation von Migrantinnen - Neue Forschungsergebnisse aus Deutschland. BGSS Workshop Documentation »Implementierung von Rechtsnormen: Gewalt gegen Frauen in der Türkei und in Deutschland«. Institut für Sozialwissenschaften, Humboldt-Universität zu Berlin.

Helfferich, Cornelia/Kavemann, Barbara/Kindler, Heinz (2016): Einleitung. In: Cornelia Helfferich, Barbara Kavemann und Heinz Kindler (Hg.): Forschungsmanual Gewalt. Grundlagen der empirischen Erhebung von Gewalt in Paarbeziehungen und sexualisierte Gewalt. Wiesbaden: Springer Fachmedien, S. 1-12.

Helsper, Werner/Kramer, Rolf-Torsten/Thiersch, Sven (2013): Orientierungsrahmen zwischen Kollektivität und Individualität - ontogenetische und transformationsbezogene Anfragen an die dokumentarische Methode. In: Peter Loos, Arnd-Michael Nohl, Aglaja Przyborski und Burkhard Schäffer (Hg.): Dokumen- 
tarische Methode. Grundlagen - Entwicklungen - Anwendungen. Opladen, Berlin, Toronto: Barbara Budrich, S. 111-140.

Herma, Holger (2007): Liebe und Authentizität. Generationswandel in Paarbeziehungen. Wiesbaden: VS Verlag für Sozialwissenschaften.

Hildenbrand, Bruno (2007): Sozialisation in der Familie und Generationenbeziehungen. Die Bedeutung von signifikanten Anderen innerhalb und außerhalb der sozialisatorischen Triade. In: Familiendynamik 32 (32), S. 211-228.

Hirseland, Andreas/Leuze, Kathrin (2010): Jenseits der traditionellen Paarbeziehung? Sozialstrukturelle Bedingungen moderner Beziehungskonzepte. In: Peter A. Berger und Ronald Hitzler (Hg.): Individualisierungen. Ein Vierteljahrhundert »jenseits von Stand und Klasse«? Wiesbaden: VS Verlag für Sozialwissenschaften, S. 191-209.

Hitzler, Ronald/Niederbacher, Arne (2010): Leben in Szenen. Formen juveniler Vergemeinschaftung heute. Wiesbaden: VS Verlag für Sozialwissenschaften.

Hoffmann-Riem, Christa (1980): Die Sozialforschung einer interpretativen Soziologie. Der Datengewinn. In: Kölner Zeitschrift für Soziologie und Sozialpsychologie 32, S. 339-372.

Hradil, Stefan (1997): Differenz und Integration. Gesellschaftliche Zukunftsentwicklungen als Herausforderungen an die Soziologie. In: Stefan Hradil und Deutsche Gesellschaft für Soziologie (DGS) (Hg.): Differenz und Integration: die Zukunft moderner Gesellschaften. Verhandlungen des 28. Kongresses der Deutschen Gesellschaft für Soziologie in Dresden 1996. Frankfurt a.M.: Campus-Verl., S. 39-53.

Hurrelmann, Klaus/Quenzel, Gudrun (2016): Lebensphase Jugend. Eine Einführung in sozialwissenschaftliche Jugendforschung. Weinheim und Basel: Beltz.

Illouz, Eva (2011): Warum Liebe weh tut. Eine soziologische Erklärung. Berlin: Suhrkamp.

Illouz, Eva (2015): Gefühle in Zeiten des Kapitalismus. Frankfurt a.M.: Suhrkamp. Imbusch, Peter (2002): Der Gewaltbegriff. In: Wilhelm Heitmeyer und John Hagan (Hg.): Internationales Handbuch der Gewaltforschung. Wiesbaden: Westdeutscher Verlag, S. 26-57.

Jakob, Gisela (1997): Das narrative Interview in der Biographieforschung. In: Barbara Friebertshäuser und Annedore Prengel (Hg.): Handbuch qualitative Forschungsmethoden in der Erziehungswissenschaft. Weinheim: Juventa, S. $445-458$.

Jensen, Olaf/Welzer, Harald (2003): Ein Wort gibt das andere, oder: Selbstreflexivität als Methode. In: Forum Qualitative Sozialforschung 4 (2), S. 58 Absätze. Online verfügbar unter www.nbn-resolving.de/urn:nbn:deo114-fqs0302320, zuletzt geprüft am 08.07.2017.

Jurczyk, Karin (2001): Individualisierung und Zusammenhalt. Neuformierungen von Geschlechterverhältnissen in Erwerbsarbeit und Familie. In: Margrit 
Brückner und Lothar Böhnisch (Hg.): Geschlechterverhältnisse. Gesellschaftliche Konstruktionen und Perspektiven ihrer Veränderung. Weinheim, München: Juventa, S. 11-38.

Jurczyk, Karin/Keddi, Barbara/Lange, Andreas/Zerle, Claudia (2009): Zur Herstellung von Familie. In: DJI Bulletin Plus 4 (88), S. I-VIII.

Jurczyk, Karin/Lange, Andreas/Thiessen, Barbara (2014): Doing Family als neue Perspektive auf Familien. Einleitung. In: Karin Jurczyk, Andreas Lange und Barbara Thiessen (Hg.): Doing Family. Warum Familienleben heute nicht mehr selbstverständlich ist. Weinheim und Basel: Beltz Juventa, S. 7-48.

Kallmeyer, Werner/Schütze, Fritz (1977): Zur Konstitution von Kommunikationsschemata der Sachverhaltsdarstellung. In: Dirk Wegner (Hg.): Gesprächsanalysen. Vorträge, gehalten anläßlich des 5 . Kolloquiums des Instituts für Kommunikationsforschung und Phonetik, Bonn, 14. - 16. Oktober 1976. Hamburg: Helmut Buske, S. 159-274.

Kalmuss, Debra (1984): The Intergenerational Transmission of Marital Aggression. In: Journal of Marriage and Family 46 (1), S. 11-19.

Kann, Laura/Kinchen, Steve/Shanklin, Shari L./Flint, Katherine H. (2014): Youth Risk Behavior Surveillance - Unites States 2013. In: MMWR 63 (4), S. 1-168.

Keddi, Barbara (2003): Projekt Liebe. Lebensthemen und biografisches Handeln junger Frauen in Paarbeziehungen. Opladen: Leske + Budrich.

Kelle, Udo/Kluge, Susann (2010): Vom Einzelfall zum Typus. 2., überarbeitete Auflage. Wiesbaden: VS Verlag für Sozialwissenschaften.

Kindler, Heinz/Unterstaller, Adelheid (2013): Primäre Prävention von Partnergewalt: Ein entwicklungsökologisches Modell. In: Barbara Kavemann und Ulrike Kreyssig (Hg.): Handbuch Kinder und häusliche Gewalt. 3., aktualisierte und überarbeitete Auflage. Wiesbaden: Springer Fachmedien, S. 513-532.

Kleemann, Frank/Krähnke, Uwe/Matuschek, Ingo (2009): Interpretative Sozialforschung: eine praxisorientierte Einführung. Wiesbaden: VS Verlag für Sozialwissenschaften.

Köberlein, Luzia/Sartingen, Petra/Hahn, Sibylle/Krohe-Amann, Armin/Gaiser, Harald/Toth, Györgyi et al. (2010): Heartbeat - Herzklopfen. Beziehungen ohne Gewalt. Ein Arbeitspaket zur schulischen und außerschulischen Prävention von Gewalt in intimen Teenagerbeziehungen. Hg. v. Der Paritätische. BadenWürttemberg.

Kohlhofer, Birgit/Neu, Regina/Sprenger, Nikolaj (2008): E.R.N.S.T. machen. Sexuelle Gewalt unter Jugendlichen verhindern. Ein pädagogisches Handbuch. Köln: Mebes \& Noack.

Koller, Hans-Christoph (2014): Zur Bedeutung von Butlers Subjekttheorie für die Erforschung biographischer Bildungsprozesse. In: Bettina Rose und Nadine Kleiner (Hg.): (Re-)Produktion von Ungleichheiten im Schulalltag. Judith 
Butlers Konzept der Subjektivation in der erziehungswissenschaftlichen Forschung. Opladen, Berlin, Toronto: Barbara Budrich, S. 21-33.

Krahé, Barbara (2003a): Sexuelle Aggression zwischen Jugendlichen: Prävalenz und Prädikatoren. In: Bundeszentrale für gesundheitliche Aufklärung (Hg.): Wissenschaftliche Grundlagen. Teil 2 - Jugendliche. Köln: BZgA (Forschung und Praxis der Sexualaufklärung und Familienplanung, Bd. 13,2), S. 93-123.

Krahé, Barbara (2003b): Aggression von Männern und Frauen in Partnerschaften: Unterschiede und Parallelen. In: Siegfried Lamnek und Manuela Boatca (Hg.): Geschlecht - Gewalt - Gesellschaft. Opladen: Leske + Budrich (Otto-von-Freising-Tagungen der Katholischen Universität Eichstätt-Ingolstadt, 4), S. 369-383.

Krahé, Barbara (2004): Verbreitungsgrad und Risikofaktoren sexueller Aggression von Frauen. In: Prävention 24 (2), S. 29-31.

Krahé, Barbara (2008a): Sexuelle Aggression und Partnergewalt im Jugendalter. In: Herbert Scheithauer, Tobias Hayer und Kay Niebank (Hg.): Problemverhalten und Gewalt im Jugendalter. Erscheinungsformen, Entstehungsbedingungen, Prävention und Intervention. Stuttgart: Kohlhammer, S. 128-139.

Krahé, Barbara (2008b): Verbreitungsgrad und Risikofaktoren sexueller Aggression bei Jugendlichen und jungen Erwachsenen. In: IzKK-Nachrichten 1/2008, S. 8-13.

Krahé, Barbara (2009): Sexuelle Aggression und Opfererfahrungen unter Jugendlichen und jungen Erwachsenen. Prävalenz und Prädiktoren. In: Psychologische Rundschau 60 (3), S. 173-183.

Krahé, Barbara/Scheinberger-Olwig, Renate (2002): Sexuelle Aggression. Verbreitungsgrad und Risikofaktoren bei Jugendlichen und jungen Erwachsenen. Göttingen: Hogrefe Verl. für Psychologie.

Krahé, Barbara/Scheinberger-Olwig, Renate/Waizenhöfer, Eva (1999): Sexuelle Aggression zwischen Jugendlichen: Eine Prävalenzerhebung mit Ost-WestVergleich. In: Zeitschrift für Sozialpsychologie, Sonderheft , Gewalt in der Gesellschaft, 30 (2-3), S. 165-178.

Kramer, Rolf-Torsten/Helsper, Werner/Thiersch, Sven/Ziems, Carolin (2009): Selektion und Schulkarriere. Kindliche Orientierungsrahmen beim Übergang in die Sekundarstufe I. Wiesbaden: VS-Verlag

Kramer, Rolf-Torsten/Helsper, Werner/Thiersch, Sven/Ziems, Carolin (2013): Das 7.Schuljahr. Wandlungen des Bildungshabitus in der Schulkarriere? Wiesbaden: VS-Verlag.

Kühnlein, Irene/Mutz, Gerd (1996): Psychotherapie als Transformationsprozess: Expertenwissen im Alltagshandeln ehemaliger Klienten. Opladen: Westdeutscher Verlag.

Lamnek, Siegfried (1995): Qualitative Sozialforschung. Band 2. Methoden und Techniken. 3. korrigierte Auflage. Weinheim: Beltz. 
Lamnek, Siegfried/Luedtke, Jens/Ottermann, Ralf/Vogl, Susanne (2012): Tatort Familie. Häusliche Gewalt im gesellschaftlichen Kontext. 3., erweiterte und überarbeitete Auflage. Wiesbaden: VS Verlag für Sozialwissenschaften.

Lange, Andreas (2009): Gestaltungsaufgaben in der Familienbiographie. In: Gerhard Mertens, Winfried Böhm und Volker Ladenthin (Hg.): Handbuch der Erziehungswissenschaft. Band III Familie - Kindheit - Jugend - Gender. Umwelten. Paderborn: Ferdinand Schöningh, S. 437-455.

La Rue, Lisa de/Polanin, Joshua R./Espelage, Dorothy L./Pigott, Terry D. (2014): School-Based Interventions to Reduce Dating and Sexual Violence: A Systematic Review. Hg. v. Campbell Systematic Reviews.

Lenz, Karl (2009): Soziologie der Zweierbeziehung. Eine Einführung. 4. Aufl. Wiesbaden: VS Verlag für Sozialwissenschaften.

Lenz, Karl (2014): Zeit(en) in der alltäglichen Lebensführung von Paaren. In: Karin Jurczyk, Andreas Lange und Barbara Thiessen (Hg.): Doing Family. Warum Familienleben heute nicht mehr selbstverständlich ist. Weinheim und Basel: Beltz Juventa, S. 113-127.

Levy, Barrie (Hg.) (1990): Dating Violence. Young women in danger. Seattle: Seal Press.

Levy, Barrie (2006): In Love and in Danger. Berkeley: Seal Press.

Levy, Barrie/Occhiuzzo Giggans, Patricia (1995): What parents need to know about datig violence. Seattle: Seal Press.

Liz Claiborne, Inc/Teen Research Unlimited (TRU) (2008): Tween and teen dating violence and abuse study. Online verfügbar unter www.loveisrespect.org/wp-content/uploads/2008/07/tru-tween-teen-study-feb-081.pdf, zuletzt geprüft am 08.04.2011.

Loch, Ulrike (2002): Grenzen und Chancen der narrativen Gesprächsführung bei Menschen mit traumatischen Erlebnissen in der Kindheit. In: Doris Schaeffer und Gabriele Müller-Mundt (Hg.): Qualitative Gesundheits- und Pflegeforschung. Bern: Verlag Hans Huber, S. 233-246.

Loch, Ulrike (2007): Gesellschaftliche Konstruiertheit von Gewalterfahrungen und ihren Sinnzuschreibungen. Folgen für die Soziale Arbeit. In: Ingrid Miethe, Wolfram Fischer, Cornelia Giebele, Martina Goblitsch und Gerhard Riemann (Hg.): Rekonstruktion und Intervention. Interdisziplinäre Beiträge zur rekonstruktiven Sozialarbeitsforschung. 4 Bände. Opladen: Verlag Barbara Budrich (Rekonstruktive Forschung in der Sozialen Arbeit), S. 201-211.

Loch, Ulrike (2008): Spuren von Traumatisierungen in narrativen Interviews. [20 Absätze]. In: Forum Qualitative Sozialforschung/Forum: Qualitative Social Research 9 (1). Online verfügbar unter http://nbnresolving.de/urn:nbn:de:0114-fqso801544, zuletzt geprüft am 29.01.2018. 
Loch, Ulrike/Rosenthal, Gabriele (2002): Das narrative Interview. In: Doris Schaeffer und Gabriele Müller-Mundt (Hg.): Qualitative Gesundheits- und Pflegeforschung. Bern: Verlag Hans Huber, S. 221-232.

Lucius-Hoene, Gabriele/Deppermann, Arnulf (2002): Rekonstruktion narrativer Identität. Ein Arbeitsbuch zur Analyse narrativer Interviews. Opladen: Leske + Budrich.

Luhmann, Niklas (1982): Liebe als Passion. Zur Codierung von Intimität. 7. Auflage. Frankfurt a.M.: Suhrkamp.

Maihofer, Andrea (2014): Familiale Lebensformen zwischen Wandel und Persistenz. Eine zeitdiagnostische Zwischenbetrachtung. In: Cornelia Behnke, Diana Lengersdorf und Sylka Scholz (Hg.): Wissen-Methode-Geschlecht: Erfassen des fraglos Gegebenen. Wiesbaden: VS-Verlag, S. 313-333.

Mannheim, Karl (1964a): Beiträge zur Theorie der Weltanschauungsinterpretation. In: Karl Mannheim (Hg.): Wissenssoziologie. Hg. von Kurt H. Wolff. Neuwied: Luchterhand, S. 91-154.

Mannheim, Karl (1964b): Das Problem der Generationen. In: Karl Mannheim (Hg.): Wissenssoziologie. Hg. von Kurt H. Wolff. Neuwied: Luchterhand, S. 509-565.

Mannheim, Karl (1980): Strukturen des Denkens. Frankfurt a.M.: Suhrkamp.

Marotzki, Winfried (1995): Forschungsmethoden in der erziehungswissenschaftlichen Biographieforschung. In: Heinz-Hermann Krüger und Winfried Marotzki (Hg.): Erziehungswissenschaftliche Biographieforschung. Opladen: Leske + Budrich, S. 55-89.

Marotzki, Winfried/Nohl, Arnd-Michael/Ortlepp, Wolfgang (2005): Einführung in die Erziehungswissenschaft. Wiesbaden: VS Verlag für Sozialwissenschaften.

Maschke, Sabine/Stecher, Ludwig (2017): SPEAK! Die Studie. "Sexualisierte Gewalt in der Erfahrung Jugendlicher«. Öffentlicher Kurzbericht. Hg. v. Hessisches Kultusministerium.

Matthiesen, Silja/Dekker, Arne (2015): Sexuelle Schwierigkeiten in heterosexuellen studentischen Beziehungen. In: Zeitschrift für Soziologie der Erziehung und Sozialisation 35. (3), S. 232-251.

Melzer, Wolfgang/Schubarth, Wilfried (2015): Gewalt. In: Wolfgang Melzer, Dieter Hermann, Uwe Sandfuchs, Mechthild Schäfer et al. (Hg.): Handbuch Aggression, Gewalt und Kriminalität bei Kindern und Jugendlichen. Bad Heilbrunn: Verlag Julius Klinkhardt, S. 23-29.

Micus-Loos, Christiane/Plößer, Melanie/Geipel, Karen/Schmek, Marike (2016): Normative Orientierungen in Berufs- und Lebensentwürfen junger Frauen. Wiesbaden: Springer VS.

Ministère de la Communauté francaise/Direction de l'Egalité des Chances (2009): La violance dans les relations amoureuses chez les jeunes ages de 12 a 21 ans. Une étude menée en Communauté francaise de Belgique. Hg. v. Direction de l'Egalité des Chances. Bruxelles. Online verfügbar unterwww.ega- 
lite.cfwb.be/fileadmin/sites/sdec/upload/sdec_super_editor/sdec_editor/documents/violence_envers_femmes/Etude_Violences_dans_les_relations_amoureuses_des_jeunes_2009_01.pdf, zuletzt geprüft am 26.04.2011.

Morgan, David (1996): Family Connections. Cambridge: Polity Press.

Mruck Katja/Mey, Günther (1998): Selbstreflexivität und Subjektivität im Auswertungsprozess biographischer Materialien: zum Konzept einer »Projektwerkstatt qualitativen Arbeitens « zwischen Colloquium, Supervision und Interpretationsgemeinschaft. In: Gerd Jüttemann und Hans Thomae (Hg.): Biographische Methoden in den Humanwissenschaften. Weinheim: Beltz, S. 284-306.

Müller, Ursula/Schröttle Monika (2004): Lebenssituation, Sicherheit und Gesundheit von Frauen in Deutschland. Eine repräsentative Untersuchung zu Gewalt gegen Frauen in Deutschland. Hg. v. Bundesministerium für Familie, Senioren Frauen und Jugend.

Nave-Herz, Rosemarie (Hg.) (2002): Kontinuität und Wandel der Familie in Deutschland. Eine zeitgeschichtliche Analyse. Stuttgart: Lucius \& Lucius (Der Mensch als soziales und personales Wesen, 19).

Nave-Herz, Rosemarie (2004): Ehe- und Familiensoziologie. Eine Einführung in Geschichte, theoretische Ansätze und empirische Befunde. Weinheim, München: Juventa.

Nave-Herz, Rosemarie (2007): Familie heute. Wandel der Familienstrukturen und Folgen für die Erziehung. Darmstadt: Primus.

Nohl, Arnd-Michael (2001): Migration und Differenzerfahrung. Junge Einheimische und Migranten im rekonstruktiven Milieuvergleich. Opladen: Leske + Budrich.

Nohl, Arnd-Michael (2005): Dokumentarische Interpretation narrativer Interviews. Bildungsforschung (Jahrgang 2, 2). Online verfügbar unter www.bildungsforschung.org/Archiv/2005-02/interview/, zuletzt geprüft am 04.07.2008.

Nohl, Arnd-Michael (2007): Komparative Analyse: Forschungspraxis und Methodologie dokumentarischer Interpretation. In: Ralf Bohnsack, Iris NentwigGesemann und Arnd-Michael Nohl (Hg.): Die dokumentarische Methode und ihre Forschungspraxis. Grundlagen qualitativer Sozialforschung. 2., erweiterte und aktualisierte Auflage. Wiesbaden: VS Verlag für Sozialwissenschaften, S. 255-276.

Nohl, Arnd-Michael (2009): Interview und dokumentarische Methode. Anleitungen für die Forschungspraxis. 3. Auflage. Wiesbaden: VS Verlag für Sozialwissenschaften.

Nohl, Arnd-Michael (2016): Innovationen in der dokumentarischen Interpretation narrativer Interviews. In: Sozialer Sinn 17 (2), S. 329-354. 
Oerter, Rolf/Dreher, Eva (2008): Jugendalter. In: Rolf Oerter und Leo Montada (Hg.): Entwicklungspsychologie. 6., vollst. überarb. Aufl. Weinheim: Beltz, S. 271-332.

Ohms, Constance (2008): Das Fremde in mir. Gewaltdynamiken in Liebesbeziehungen zwischen Frauen. Soziologische Perspektiven auf ein Tabuthema. Bielefeld: transcript Verlag.

Peuckert, Rüdiger (2012): Familienformen im sozialen Wandel. 8. Auflage. Wiesbaden: VS Verlag für Sozialwissenschaften.

Pinquart, Martin (2008): Die Entwicklung von romantischen und sexuellen Beziehungen im Jugendalter. In: IzKK-Nachrichten 1/2008, S. 4-6.

Pirog-Good, Maureen A./Stets, Jan E. (Hg.) (1989): Violence in dating relationships. Emerging Social Issues. New York, Westport, Connecticut u.a.: Praeger.

Przyborski, Aglaja/Slunecko, Thomas (2010): Dokumentarische Methode. In: Günther Mey und Mruck Katja (Hg.): Handbuch Qualitativer Methoden in der Psychologie. Wiesbaden: VS Verlag für Sozialwissenschaften, S. 627-642.

Przyborski, Aglaja/Wohlrab-Sahr, Monika (2010): Qualitative Sozialforschung. Ein Arbeitsbuch. 3. Aufl. München: Oldenbourg Wissenschaftsverlag.

Reckwitz, Andreas (2010): Subjekt. 2. Aufl. Bielefeld: transcript Verlag.

Reinhold, Gerd (1992): Soziologie-Lexikon. 2. Auflage. München: Oldenbourg Wissenschaftsverlag.

Rendtorff, Barbara (2015): Zugewinne und Fallen - aktuelle Veränderungen in Geschlechtervorstellungen und ihre Probleme. In: Bettina Dausien, Christine Thon und Katharina Walgenbach (Hg.): Geschlecht - Sozialisation - Transformation. Opladen, Berlin, Toronto: Verlag Barbara Budrich, S. 77-92.

Rendtorff, Barbara/Glammeier, Sandra/Vogelsang, Verena (2016): Bildung - Gewalt - Geschlecht. In: Johannes Bilstein, Jutta Ecarius, Norbert Ricken und Ursula Stenger (Hg.): Bildung und Gewalt. Wiesbaden: Springer Fachmedien, S. 129-143.

Ribeaud, Denis (2015): Entwicklung von Gewalterfahrungen Jugendlicher im Kanton Zürich 1999-2014. Forschungsbericht. Hg. v. Professur für Soziologie ETH Zürich Kriminologische Forschungseinheit.

Ricken, Norbert/Rose, Nadine/Kuhlmann, Nele/Otzen, Anne (2017): Die Sprachlichkeit der Anerkennung. Eine theoretische und methodologische Perspektive auf die Erforschung von »Anerkennung«. In: Vierteljahreszeitschrift für wissenschaftliche Pädagogik 93 (2), S. 193-235.

Rieger-Ladich, Markus (2012): Judith Butlers Rede von Subjektivierung. Kleine Fallstudie zur »Arbeit am Begriff«. In: Norbert Ricken und Nicole Balzer (Hg.): Judith Butler: Pädagogische Lektüren. Wiesbaden: VS-Verlag, S. 57-73.

Rose, Nadine (2012): Subjekt, Bildung, Text. Diskurstheoretische Anregungen und Herausforderungen für biografische Forschung. In: Ingrid Miethe und Hans- 
Rüdiger Müller (Hg.): Qualitative Bildungsforschung und Bildungstheorie. Opladen, Berlin, Toronto: Budrich, S. 111-126.

Rose, Nadine/Ricken, Norbert (2018): Interaktionsanalyse als Adressierungsanalyse - eine Perspektive der Subjektivationsforschung. In: Martin Heinrich und Andreas Wernet (Hg.): Rekonstruktive Bildungsforschung. Zugänge und Methoden. Wiesbaden: Springer Fachmedien, S. 159-175.

Rosenthal, Gabriele (2002): Biographisch-narrative Gesprächsführung: zu den Bedingungen heilsamen Erzählens im Forschungs- und Beratungskontext. In: Psychotherapie und Sozialwissenschaft 4 (3), S. 204-227. Online verfügbar unter www.nbn-resolving.de/urn:nbn:deo168-ssoar-56763, zuletzt geprüft am 12.01.2018.

Rosenthal, Gabriele (2008): Interpretative Sozialforschung. Eine Einführung. 2. korrigierte Auflage. Weinheim, München: Juventa.

Rosenthal, Gabriele/Köttig, Michaela/Witte, Nicole/Blezinger, Anne (2006): Biographisch-narrative Gespräche mit Jugendlichen. Chancen für das Selbst- und Fremdverstehen. Opladen: Verlag Barbara Budrich.

Scherr, Albert (2004): Körperlichkeit, Gewalt und soziale Ausgrenzung in der >postindustriellen Wissensgesellschaftr. In: Wilhelm Heitmeyer und Hans-Georg Soeffner (Hg.): Gewalt: Entwicklungen, Strukturen, Analyseprobleme. Frankfurt a.M.: Suhrkamp, S. 202-223.

Schewe, Paul A. (Hg.) (2002): Preventing violence in relationships. Interventions across the live span. Washington DC: American Psychological Association.

Schittenhelm, Karin (2012): Sampling und die Suche nach fallübergreifender Gültigkeit. Vergleichende Analysen von Statusübergängen zwischen Bildungsabschluss und Arbeitsmarkt. In: Karin Schittenhelm (Hg.): Qualitative Bildungsund Arbeitsmarktforschung. Grundlagen, Perspektiven, Methoden. Wiesbaden: Springer Fachmedien, S. 407-437.

Schwiter, Karin (2011): Lebensentwürfe. Junge Erwachsene im Spannungsfeld zwischen Individualität und Geschlechternormen. Frankfurt, New York: CampusVerlag.

Schütze, Fritz (1977): Die Technik des narrativen Interviews in Interaktionsfeldstudien. Arbeitsberichte und Forschungsmaterialien Nr. 1 der Universität Bielefeld, Fakultät für Soziologie.

Schütze, Fritz (1983): Biographieforschung und narratives Interview. In: Neue Praxis 13 (3), S. 283-293.

Schütze, Fritz (1987): Das narrative Interview in Interaktionsfeldstudien I. Kurseinheit 1.: Studientexte der Fernuniversität Hagen.

Spies, Tina (2009): Diskurs, Subjekt und Handlungsmacht. Zur Verknüpfung von Diskurs- und Biografieforschung mithilfe des Konzepts der Artikulation. [70 Absätze]. In: Forum Qualitative Sozialforschung/Forum: Qualitative Social Research 10 (2). 
Spies, Tina (2018): Biographie, Diskurs und Artikulation. In: Helma Lutz, Martina Schiebel und Elisabeth Tuider (Hg.): Handbuch Biographieforschung. Wiesbaden: Springer Fachmedien, S. 537-547.

Stauber, Barbara (2012): Schlanke Mädchen - kein Problem? Körperinszenierungen zwischen Selbst- und Fremdbestimmung. In: Katharina Zeller, Eva Maria Lohner und Regine Kottmann (Hg.): Wer bin ich? Ich bin wer! Essstörungen verstehen, verhindern und überwinden. Tübingen: dgvt-Verlag, S. 27-37.

Stauber, Barbara (2014): Backspin, Freeze und Powermoves. Zur Gestaltung biografischer Übergänge im jugendkulturellen Bereich. Wiesbaden: Springer VS.

Stauber, Barbara/Walther, Andreas (2013): Junge Erwachsene - eine Lebenslage des Übergangs? In: Wolfgang Schröer, Barbara Stauber, Andreas Walther, Lothar Böhnisch und Karl Lenz (Hg.): Handbuch Übergänge. Weinheim und Basel: Beltz Juventa, S. 270-290.

Stich, Jutta (2005): Annäherung an sexuelle Beziehungen. Empirische Befunde zu Erfahrungs- und Lernprozessen von Jungen. In: Vera King und Karin Flaake (Hg.): Männliche Adoleszenz. Sozialisation und Bildungsprozesse zwischen Kindheit und Erwachsensein. Frankfurt a.M.: Campus-Verlag, S. 163-181.

Strahl, Benjamin/Schröer, Wolfgang/Wolff, Mechthild (2017): »Peer Violence« und Schutzkonzepte. Gewalt im Kontext der Jugendarbeit aus der Sicht von Jugendlichen. In: Diskurs Kindheits- und Jugendforschung 12 (3), S. 277-292.

Strauss, Anselm/Corbin, Juliet (1996): Grounded Theory. Grundlagen qualitativer Sozialforschung. München: Beltz.

Strübing, Jörg (2010): Grounded Theory - ein pragmatischer Forschungsstil für die Sozialwissenschaften. Enzyklopädie Erziehungswissenschaft online. Online verfügbar unter www.erzwissonline.de, zuletzt geprüft am 28.09.2017.

Strübing, Jörg (2014): Grounded Theory. 3., überarbeitete und erweiterte Auflage. Wiesbaden: Springer Fachmedien.

Stövesand, Sabine (2010): Gewalt im Geschlechterverhältnis. Wieso, weshalb, was tun? In: Mechthild Bereswill und Gerd Stecklina (Hg.): Geschlechterperspektiven für die Soziale Arbeit. Zum Spannungsverhältnis von Frauenbewegungen und Professionalisierungsprozessen. Weinheim, München: Juventa, S. 81-102.

Tedeschi, James T. (2002): Die Sozialpsychologie von Aggression und Gewalt. In: Wilhelm Heitmeyer und John Hagan (Hg.): Internationales Handbuch der Gewaltforschung. Wiesbaden: Westdeutscher Verlag, S. 573-597.

Temple, Jeff R./Freeman, Daniel H. (2011): Dating Violence and Substance Use Among Ethnically Diverse Adolescents. In: Journal of Interpersonal Violence 26 (4), S. 701-718.

Thon, Christine (2012): Individualisierte Geschlechterordnungen? Feministische und hegemonieanalytische Kritik eines modernisierungstheoretischen Konzepts. In: Vera Moser und Barbara Rendtorff (Hg.): Riskante Leben? Geschlech- 
terdimensionen reflexiver Modernisierungsprozesse. 8 Bände. Opladen: Barbara Budrich, S. 27-43.

Thon, Christine (2016): Biografischer Eigensinn - widerständige Subjekte? Subjekttheoretische Perspektiven in der Biografieforschung. In: Zeitschrift für Pädagogik 62 (2), S. 185-198.

Walker, Lenore (1983): The battered women syndrome study. In: David Finkelhor, Richard J. Gelles, Gerad T. Hotaling und Murray A. Straus (Hg.): The dark side of families. Current Family Violence Research. Newburs Park, London, New Delhi: Sage, S. 31-48.

Walper, Sabine/Thönissen, Carolin/Wendt, Eva-Verena/Schaer, Markus (2010): Der lange Arm der Familie: Die Paarbeziehungen junger Männer und Frauen im Lichte ihrer Beziehungen zu Mutter und Vater. In: Sabine Walper und Eva-Verena Wendt (Hg.): Partnerschaften und die Beziehungen zu Eltern und Kindern. Befunde zur Beziehungs- und Familienentwicklung in Deutschland. Würzburg: Ergon, S. 289-319.

Weisz, Arlene N./Black, Beverly M. (2009): Programs to reduce teen dating violence and sexual assault. Perspectives on what works. New York, Chichester: Columbia University Press.

Wendt, Eva-Verena (2009): Sexualität und Bindung. Qualität und Motivation sexueller Paarbeziehungen im Jugend- und jungen Erwachsenenalter. Weinheim, München: Juventa.

Wendt, Eva-Verena/Walper, Sabine (2015): Herausforderungen in romantischen und sexuellen Beziehungen Jugendlicher und junger Erwachsener. In: Zeitschrift für Soziologie der Erziehung und Sozialisation 35 (3), S. 227-231.

West, Candace/Zimmerman, Don H. (1987): Doing Gender. In: Gender \& Society 1 (2), S. 125-151.

Wetterer, Angelika (2003): Rhetorische Modernisierung: Das Verschwinden der Ungleichheit aus dem zeitgenössischen Differenzwissen. In: Gudrun-Axeli Knapp und Angelika Wetterer (Hg.): Achsen der Differenz. Gesellschaftstheorie und feministische Kritik II. Münster: Westfälisches Dampfboot, S. 286-319.

WHO/LSHTM (2010): Preventing Intimate Partner Violence and Sexual Violence against Women: Taking Action and Generating Evidence. Hg. v. World Health Organization und London School of Hygiene and Tropical Medicine. Geneva.

Wolfe, David A./Wekerle, Christine/Scott, Katreena (1997): Alternatives to Violence. Empowering youth to develop healthy relationships. Thousand Oaks: Sage.

Zimmermann, Peter/Celik, Fatma (2015): Heilt die Zeit alle Wunden? Der Einfluss von Bindung und emotionaler Verletzungssensitivität auf das emotionale Erleben von Trennungen bei Erwachsenen. In: Zeitschrift für Soziologie der Erziehung und Sozialisation 35 (3), S. 268-284. 


\section{Anhang}

\subsection{A 1 Transkriptionsregeln ${ }^{1}$}

Auf Satzzeichen im Sinne grammatikalischer Regeln wird verzichtet. Kommata werden stattdessen entsprechend hörbarer Interpunktion, das heißt bei kurzem Absetzen gesetzt. Es wird auf die Einteilung in Sätze durch Punkte, Ausrufe- oder Fragezeichen verzichtet, da damit bereits eine Interpretation ist (vgl. Rosenthal u.a. 2006: 121f.) $)^{2}$

1 In Anlehnung an: Bohnsack, Ralf (2006): Rekonstruktive Sozialforschung. Einführung in qualitative Methoden. Opladen: Barbara Budrich, S. 235.

2 Rosenthal, Gabriele u.a. (2006): Biographisch-narrative Gespräche mit Jugendlichen. Chancen für das Selbst- und Fremdverstehen, Opladen: Barbara Budrich. 


\begin{tabular}{|c|c|}
\hline $\begin{array}{l}(.) \\
(1),(2),(3)\end{array}$ & $\begin{array}{l}\text { Gesprächspausen } \\
\text { kurze Pause } \\
\text { Anzahl der Sekunden, die eine Pause dau- } \\
\text { ert }\end{array}$ \\
\hline$\underline{\text { nein }}$ & Betont \\
\hline NEIN & Laut \\
\hline Viell- & Abbruch eines Wortes \\
\hline${ }^{\circ}$ nein ${ }^{\circ}$ & Leise gesprochen \\
\hline @Handy@ & Lachend gesprochen \\
\hline @(.)@ & Kurzes Auflachen \\
\hline Nei::n & $\begin{array}{l}\text { Dehnung einzelner Buchstaben } \\
\text { Die Häufigkeit von :: entspricht der Länge } \\
\text { der Dehnung }\end{array}$ \\
\hline Haben & Gedehnt gesprochenes Wort \\
\hline Und=irgendwie & Schneller Anschluss \\
\hline ((seufzend)) ich habe keine* Ahnung & $\begin{array}{l}\text { Besondere Sprechweise, "markiert das En- } \\
\text { de }\end{array}$ \\
\hline »das mache ich« & $\begin{array}{l}\text { Nachahmung einer direkten Rede/Aussa- } \\
\text { ge einer Person }\end{array}$ \\
\hline (doch) & Unsicherheit bei der Transkription \\
\hline () & Unverständliche Äußerung, je nach Länge \\
\hline $\begin{array}{l}((\text { hustet })) \\
((\text { atmet aus 2)) }\end{array}$ & $\begin{array}{l}\text { Parasprachliche Ereignisse } \\
\text { Parasprachliche Ereignisse und deren } \\
\text { Dauer in Sek. }\end{array}$ \\
\hline$/ / \mathrm{mmh} / /$ & Hörsignal der Interviewerin \\
\hline $\begin{array}{l}\text { Ja so was } \\
\text { nein ich }\end{array}$ & $\begin{array}{l}\text { Überlappung der Redebeiträge } \\
\text { Cleichzeitiges Sprechen ab »so« }\end{array}$ \\
\hline
\end{tabular}




\subsection{A 2 Anschreiben im Rahmen der Datenerhebung}

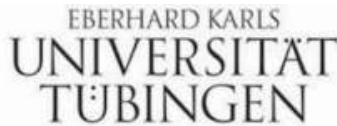

Universiat Tubingen
Institut fur Erziehungnwissenschat Munzpasse 26 72070 Tubingen
Wirtschafts- und Sozialwissenschaftliche Fakultät

Institut fur Erziehungswissenschah

Hallo,

kurz zu meiner Person: ich heiße Eva Maria Lohner, bin Diplom-Pädagogin und beschäftige mich schon seit einigen Jahren mit dem Thema Gewalt in Beziehungen. Zu diesem Thema schreibe ich auch meine Doktorarbeit. Dazu möchte ich mit jungen Frauen interviews fuhren, um so zu erfahren, wie sie Gewalt erlebt haben.

Für dieses Forschungsvorhaben bin ich auf der Suche nach Interviewpartnerinnen, die im Jugendalter schwierige Erfahrungen in ihren Beziehungen gemacht haben und die bereit sind, mir ihre Lebensgeschichte zu erzählen.

Ziel der Arbeit ist es, mehr darüber zu erfahren, wie Jugendliche und junge Erwachsene Gewalt in ihren Paarbeziehungen erleben. Dieses Wissen ist z. B. für Beratungsstellen wichtig, um hilfreich und unterstützend wirken zu können.

Ich unterliege der Schweigepflicht und bin zur Wahrung des Datengeheimnisses verpflichtet. Das bedeutet, dass ich alle Angaben vollständig anonymisiere und keine Rückschlüsse auf Sie oder das von Ihnen Erzahite gezogen werden können.

Wenn Sie sich vorstellen können, von Ihren Erinnerungen und Erfahrungen zu erzählen und damit als Expertin für dieses Thema ein Interview zu geben, freue ich mich sehr, wenn Sie sich bei mir melden.

Falls Sie noch Fragen haben, können wir diese auch telefonisch oder per Email klären.

Herzlichen Dank und viele Grüße

Eva Maria Lohner 



\section{Dank}

Ohne die folgenden Personen gäbe es die vorliegende Arbeit nicht. Deshalb möchte ich ein großes Dankeschön aussprechen an:

- Prof. Dr. Petra Bauer für kritische Auseinandersetzungen, für die großartige und kontinuierliche Unterstützung sowie für alle Ermutigungen im Laufe der Betreuung;

- Prof. Dr. Barbara Stauber für die Begleitung als Zweitbetreuerin, für wertvolle Ratschläge und insbesondere für weiterführende Anregungen bei methodischen Fragen;

- alle Interviewpartnerinnen für ihre Bereitschaft und ihr Vertrauen, mir Einblicke in ihre Lebensgeschichten zu gewähren;

- all diejenigen, die mir Zugang zu Interviewpartnerinnen vermittelt haben und die aus Gründen der Wahrung der Anonymität nicht namentlich genannt werden können;

- Mirjana Zipperle und Eberhard Bolay für vielfältige fachliche und wichtige emotionale Unterstützung;

- Heidi Hirschfeld, Sibylle Walter, Monika Lehmann und Axel Pohl für intensive Diskussionen besonders während der Auswertung und damit auch für das interpretative Weiterkommen sowie für die Möglichkeit des Austauschs zu sämtlichen Fragen der Vereinbarkeit;

- Brigitte-Johanna Kappus, Liane Wacker, Christine Koch, Daniela Kipp, Kerstin Kipp, Margrit und Gerhard Kipp, Lisa Schewe, Johanna Güntner, Marie Güntner, Hanna Smitmans, Susanne Jetter, Petra Sartingen, Michael Hahn, Lucia Pröger, Kristina Ehrlinger, Niklas König und Ilka Engelmann, die mich als Freund_innen, Cousinen und (mehr als >nur $\iota$ ) Nachbar_innen vielfältig unterstützt haben;

- meinen Vater Wolfgang Lohner, besonders für winterliche Kinderbetreuung;

- Andreas Karl Gschwind für sein grafisches Know-How;

- Victoria Stewen, Lisa Vest und Katrin Beer für Transkriptionen, Kopien und Bibliotheksgänge;

- Anja Lochner für die kompetente Unterstützung rund um Sprache; 
- Carl Philipp für alle Ermutigungen in Phasen des Zweifelns und Haderns sowie Helena und Leonard für viel Geduld und fürs Mitmachen und Aushalten! 


\section{Pädagogik}

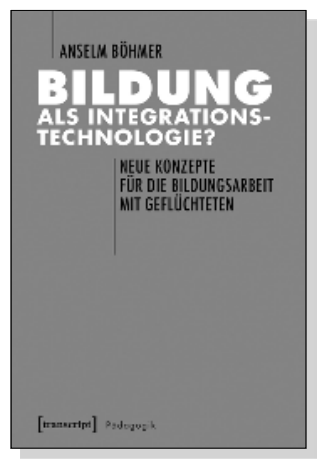

Anselm Böhmer

\section{Bildung als Integrationstechnologie?}

Neue Konzepte für die Bildungsarbeit mit Geflüchteten

2016, 120 S., kart.

$14,99 €(D E), 978-3-8376-3450-1$

E-Book: $12,99 €(D E)$, ISBN 978-3-8394-3450-5

EPUB: $12,99 €(D E)$, ISBN 978-3-7328-3450-1

Nadja Köffler, Petra Steinmair-Pösel,

Thomas Sojer, Peter Stöger (Hg.)

\section{Bildung und Liebe}

Interdisziplinäre Perspektiven

2018, 412 S., kart., 11 SW-Abbildungen

39,99€ (DE), 978-3-8376-4359-6

E-Book: 39,99 € (DE), ISBN 978-3-8394-4359-0

Monika Jäckle, Bettina Wuttig, Christian Fuchs (Hg.)

Handbuch Trauma - Pädagogik - Schule

TRAUMA

PÄDAGOGIK

SCHULE

2017, 726 S., kart., 13 SW-Abbildungen

39,99€ (DE), 978-3-8376-2594-3

E-Book: 39,99€ (DE), ISBN 978-3-8394-2594-7

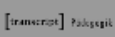




\section{Pädagogik}

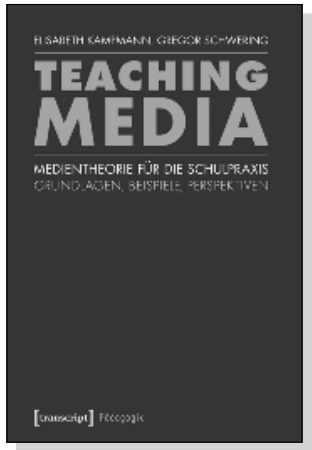

Elisabeth Kampmann, Gregor Schwering

\section{Teaching Media}

Medientheorie für die Schulpraxis -

Grundlagen, Beispiele, Perspektiven

2017, 304 S., kart., 5 SW-Abbildungen

24,99€ (DE), 978-3-8376-3053-4

E-Book: kostenlos erhältlich als Open-Access-Publikation, ISBN 978-3-8394-3053-8

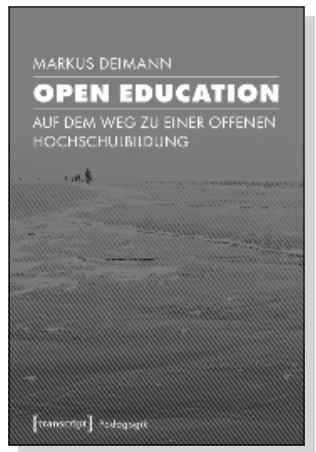

Markus Deimann

\section{Open Education}

Auf dem Weg zu einer offenen Hochschulbildung

Januar 2019, 260 S., kart.

$34,99 €(D E), 978-3-8376-4496-8$

E-Book: 34,99€ (DE), ISBN 978-3-8394-4496-2

Stefan Thomas, Madeleine Sauer, Ingmar Zalewski

Sielan Thom as.
Madet tine Seue

Maderaine Saver,
Ingmar Zalewsk

Unbegleitete minderjährige Geflichtete Inre Lebernasituationen und
Perspestiver in Deutschland

\section{Unbegleitete minderjährige Geflüchtete}

Ihre Lebenssituationen und Perspektiven in Deutschland

2018, 254 S., kart., 26 SW-Abbildungen

29,99€ (DE), 978-3-8376-4384-8

E-Book: kostenlos erhältlich als Open-Access-Publikation, ISBN 978-3-8394-4384-2 